\title{
Analytical Modelling of the Effects of Different Gas Turbine Cooling Techniques on Engine Performance = Analytische Modellierung der Wirkungen von verschiedenen Kuhlungskonfigurationen der Turbinenschaufeln auf der Gasturbinenleistung
}

Selcuk Can Uysal

Follow this and additional works at: https://researchrepository.wvu.edu/etd

\author{
Recommended Citation \\ Uysal, Selcuk Can, "Analytical Modelling of the Effects of Different Gas Turbine Cooling Techniques on \\ Engine Performance $=$ Analytische Modellierung der Wirkungen von verschiedenen \\ Kuhlungskonfigurationen der Turbinenschaufeln auf der Gasturbinenleistung" (2017). Graduate Theses, \\ Dissertations, and Problem Reports. 6856. \\ https://researchrepository.wvu.edu/etd/6856
}

This Dissertation is protected by copyright and/or related rights. It has been brought to you by the The Research Repository @ WVU with permission from the rights-holder(s). You are free to use this Dissertation in any way that is permitted by the copyright and related rights legislation that applies to your use. For other uses you must obtain permission from the rights-holder(s) directly, unless additional rights are indicated by a Creative Commons license in the record and/ or on the work itself. This Dissertation has been accepted for inclusion in WVU Graduate Theses, Dissertations, and Problem Reports collection by an authorized administrator of The Research Repository @ WVU. For more information, please contact researchrepository@mail.wvu.edu. 


\title{
ANALYTICAL MODELLING OF THE EFFECTS OF DIFFERENT GAS TURBINE COOLING TECHNIQUES ON ENGINE PERFORMANCE
}

\author{
Selçuk Can UYSAL \\ Dissertation submitted to the Benjamin Statler College of Engineering at \\ West Virginia University \\ in partial fulfillment of the requirements \\ for the degree of \\ Doctor of Philosophy \\ in \\ Aerospace Engineering
}

\author{
Andrew C. Nix, PhD, Committee Chairperson \\ V'yacheslav Akkerman, PhD \\ Cosmin Dumitrescu, PhD \\ John M. Kuhlman, PhD \\ James B. Black, PhD \\ Mechanical and Aerospace Engineering Department
}

Morgantown, West Virginia

2017

Keywords: Cooled Turbine Modelling, Turbine Losses, Engine Performance Calculation, Detached Eddy Simulations, Anti-Vortex Hole, Turbine Blade Configurations

Copyright 2017 Selcuk Can Uysal 


\section{Abstract \\ ANALYTICAL MODELLING OF DIFFERENT GAS TURBINE COOLING TECHNIQUES ON ENGINE PERFORMANCE}

\section{Selçuk Can UYSAL}

Highest available power or thrust with maximum efficiency and minimum levels of fuel consumption and emissions are the ultimate goals in gas turbine engine design. Advancements in turbine blade and vane cooling techniques can aid in improving engine performance and durability by allowing higher turbine inlet temperatures and providing longer component life. Due to this fact, numerous cooling techniques have been and continue to be developed by researchers and engine manufacturers for internal and external blade cooling.

A wide variety of cooling techniques have been applied to modern engine designs. This necessitates appraising the relative benefits of an individual cooling technique compared to others on their impacts to overall engine performance. Researchers often analyze the performance of new cooling techniques (either isolated or combined with other techniques) through heat transfer experiments and/or Computational Fluid Dynamics (CFD) methods that are valid only on a limited region of a single blade, which is an extremely small region as compared to the actual engine component size. To properly assess the impact of different cooling techniques and configurations, while considering the additional losses associated with the $2^{\text {nd }}$ Law of Thermodynamics, such as exergy loss from blade heat transfer, cooling air friction losses, fluid mixing, etc., a thermodynamic model, that can take heat transfer experiment/CFD data as inputs and use them in the cooled turbine calculations, is needed.

In this research, MATLAB Simulink $®$ was used to develop a cooled engine model for industrial gas turbines and aero-engines. The model consists of uncooled on-design, mean-line turbomachinery design and a cooled off-design analysis in order to evaluate the engine performance parameters by using operating conditions, polytropic efficiencies, material information and cooling system details. The cooling analysis algorithm involves a 2 nd law analysis to calculate losses from the cooling technique applied.

The model is used in a sensitivity analysis that evaluates the impacts of variations in metal Biot number, thermal barrier coating Biot number, film cooling effectiveness, internal cooling effectiveness and maximum allowable blade temperature on main engine performance parameters of aero and industrial gas turbine engines. The model is subsequently used to analyze the relative performance impact of employing Anti-Vortex Film Cooling holes (AVH) by means of data obtained for these holes by Detached Eddy Simulation-CFD Techniques that are valid for engine-like turbulence intensity conditions. Cooled blade configurations with $\mathrm{AVH}$ and other different external cooling techniques were used in a performance comparison study. 


\section{ACKNOWLEDGMENTS}

The author wishes to acknowledge the U.S. Department of Energy, National Energy Technology Laboratories (NETL) and the Oak Ridge Institute of Science and Education (ORISE) Fellowship program, that enabled this research.

The author would also like to thank his mentors in NETL, Dr. James B. Black and Eric Liese, for their continuous support, guidance and technical reviews on each part of the research that helped to give the final form of this thesis.

Author would like to acknowledge the support, technical reviews, and the guidance he received throughout his PhD research in West Virginia University (WVU) to his research advisor Dr. Andrew C. Nix, as well as the help he received during and after his transfer to the PhD program in WVU.

Author would like to thank Dr. V'yacheslav Akkerman, for the Advanced Thermodynamics class that helped the author to be able to gain insight to high level thermodynamics concepts that are used in the derivations carried out in the several parts of this research and for all his reviews to this thesis.

Author's sincere thanks also goes to Dr. John M. Kuhlman for his reviews and insightful suggestions during and after the thesis proposal that helped the author to present the results of this research more effectively. Author would also like to thank Dr. Cosmin Dumitrescu for all his reviews to this thesis.

Finally, the author would like to thank his mother and father for their support throughout his PhD education started in Syracuse University and continued in WVU, which was challenging for most of the time. 


\section{TABLE OF CONTENTS}

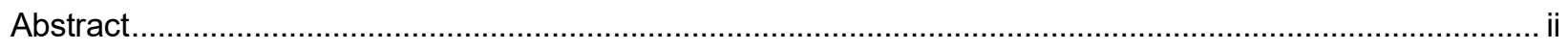

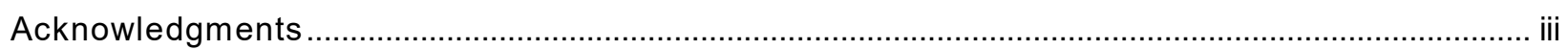

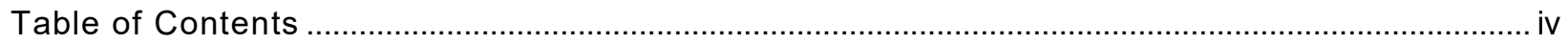

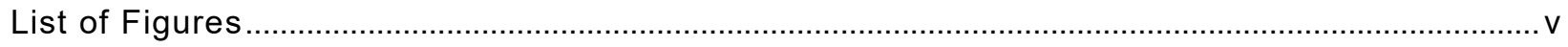

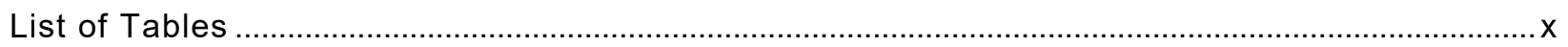

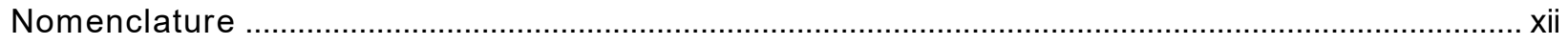

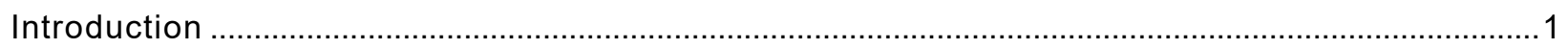

Chapter 1: Development of a Cooled Turbine Model ............................................................... 3

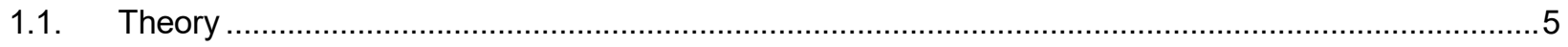

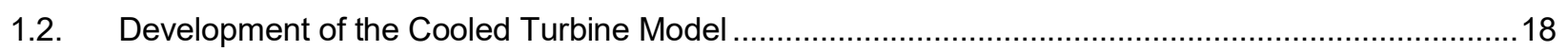

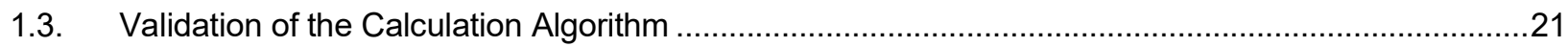

Chapter 2: Development of the Cooled Engine Models ........................................................23

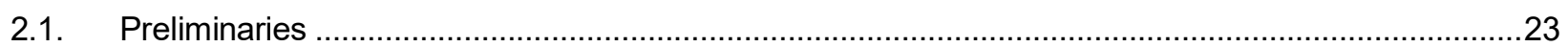

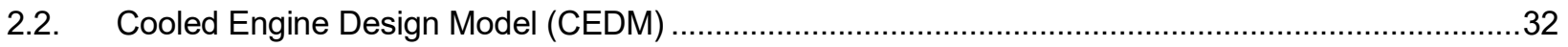

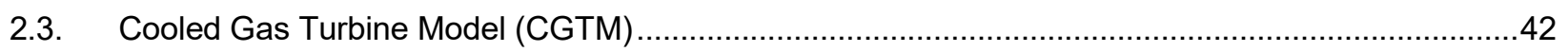

Chapter 3: $\quad$ Sensitivity Analysis on General Cooling Parameters ..........................................61

3.1. Analyzing the Effects of Individual Cooling Parameters..........................................................61

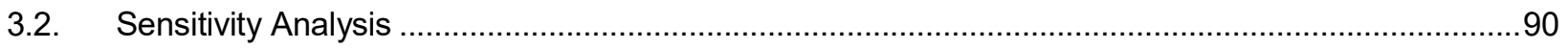

Chapter 4: Using Detached Eddy Simulation Technique to Generate an Advanced Film

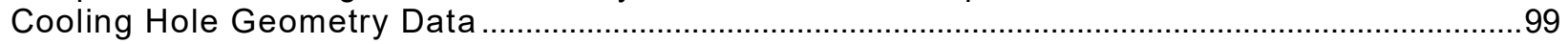

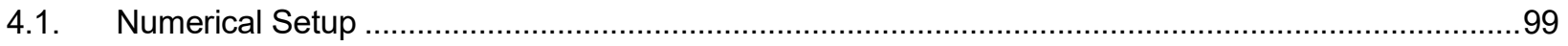

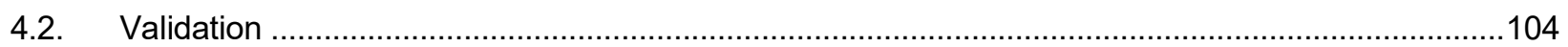

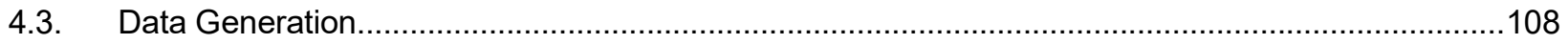

Chapter 5: Determining the Performance of Cooled Gas Turbines Using Different Cooling

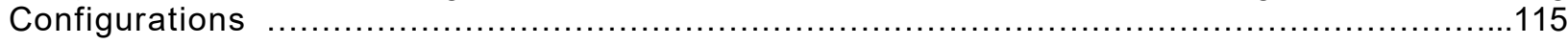

5.1. Literature Review on Advanced Coolant Calculation Methods .................................................115

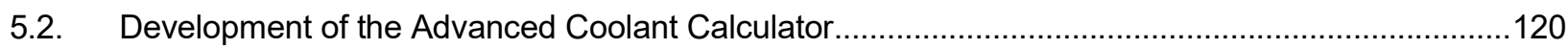

5.3. The Effect of Different Cooling Configurations on Gas Turbine Performance ..................................132

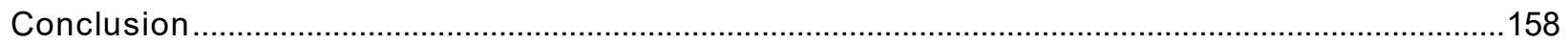

Appendix-A An Algorithm to Find the Coolant Exit Temperatures (and Enthalpies)........................160

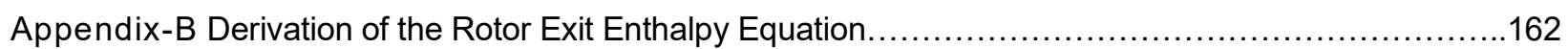

Appendix-C Development of a Compressor Model with the Effect of Coolant Air Extraction on the

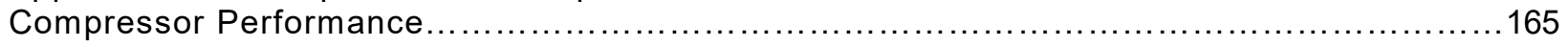

Appendix-D Development of a Transition Cooling Model............................................168

Appendix-E Internal and External Cooling Correlations used in Advanced Coolant Calculator..............170

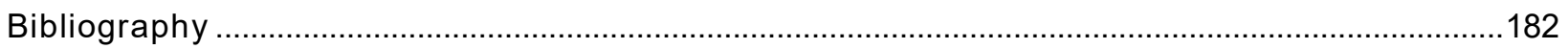

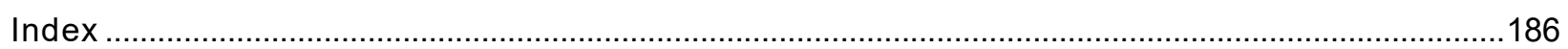

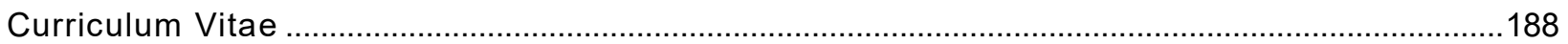




\section{LIST OF FIGURES}

Figure 1: A common flowchart valid for the cooled turbine analysis algorithms found in literature.................1 Figure 2: Mollier Diagram for turbines operating between the same inlet and exit states; isentropic turbine has the highest work output, followed by the uncooled turbine and cooled turbine having the least output due to losses in efficiency.

Figure 3: Assumed cooled turbine stage scheme and mass flow rate relations for the cooling analysis by Young and Wilcock [7], stage indices are not shown in the figure....

Figure 4: 1D Heat Transfer Scheme and temperatures for an internally and externally cooled blade assumed in the derivation of semi-empirical mass flow-rate correlation....................................................... 7 Figure 5: Control Volume definition used for all internal stations; $W_{\text {stage }}=0$ for purge stations and the control volume encompasses the indicated station entirely (1) Figure 6: Cooled Turbine Control Volumes around purge $(P)$, stator $(S)$ and rotor $(R)$ stations that are used in the $2^{\text {nd }}$ Law Analysis shown for a single stage turbine...................................................................... 10 Figure 7: Schematic of the model for stator/rotor entropy generation term calculations from Young and

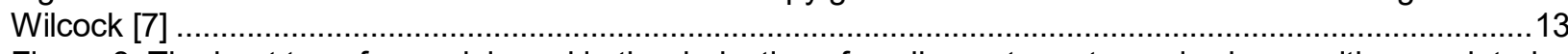
Figure 8: The heat transfer model used in the derivation of cooling entropy terms is shown with associated entropy terms for a blade having internal and external cooling (film cooling) with a TBC layer....................14 Figure 9: Assumed 1D Convection Heat Transfer Analysis that is applied in the derivation of the loss term 15 Figure 10: Control Volume used in the 1D Convection Heat Transfer Analysis that is used to derive the external heat transfer loss term ....................................................................................................... 16 Figure 11: Mixing Kinetic Energy Loss occurs due to mixing of two different flows with different speeds .....17 Figure 12: Coolant Calculator block flowchart that calculates stator-cooling fraction $\left(\varepsilon_{1}\right)$ and rotor-cooling

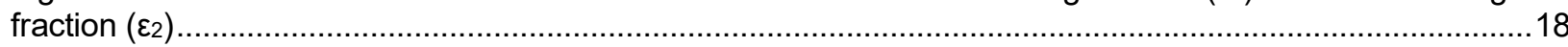
Figure 13: 1st Law Analysis section of the cooling analysis model......................................................... 19 Figure 14: $2^{\text {nd }}$ Law Analysis section of the cooling analysis model.........................................................20 Figure 15: Cooling Analysis Model Flowchart including the sub-models ...............................................20 Figure 16: Comparison of stator entropy rise calculated for each loss source between the CTM and Young and Wilcock [7] ....................................................................................................................22 Figure 17: Comparison of rotor entropy rise calculated for each loss source between the CTM and Young

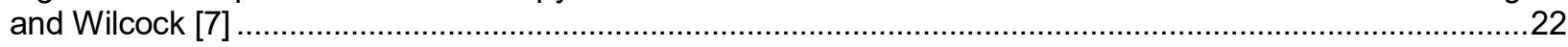

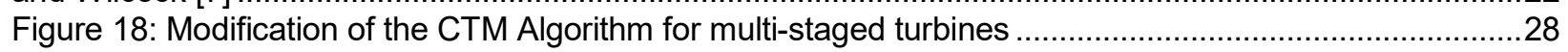
Figure 19: Enthalpy of the pure air, combustion products of methane, and combustion products of kerosene

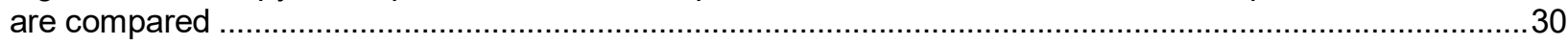
Figure 20: Flowchart for the cooled engine models ......................................................................... 31 Figure 21: Schematic of the turbofan engine configuration, engine station numbering and coolant flow paths

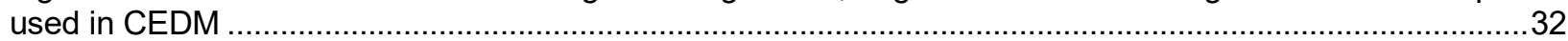

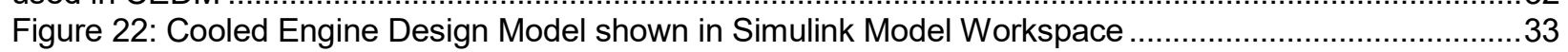

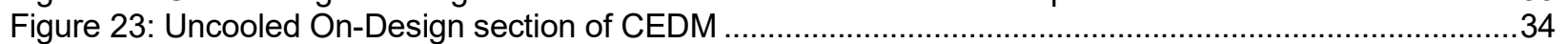
Figure 24: Turbomachinery Design Section of CEDM is the same with EDM...........................................34 Figure 25: Cooled Off-Design section of CEDM including the CTM as a subsystem (shown as "Cooling

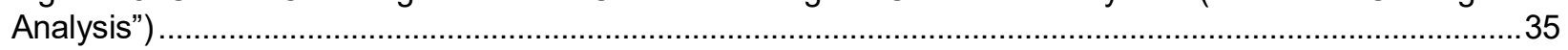
Figure 26: Change in Specific Thrust and Thrust Specific Fuel Consumption calculated for the cooled engine

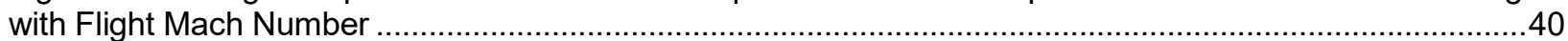
Figure 27: Change in Thrust calculated for the cooled engine with design by-pass ratio .............................40 Figure 28: Change in Thrust and Propulsive Efficiency calculated for the cooled engine with Turbine Inlet

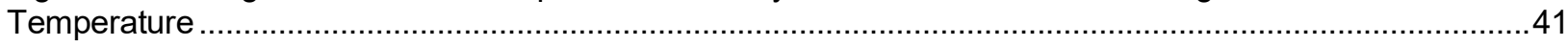
Figure 29: Schematic of the industrial gas turbine configuration, engine station numbering and coolant flow

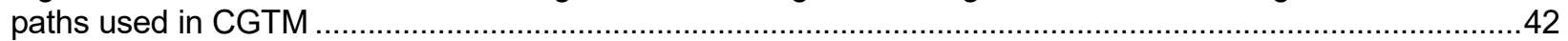
Figure 30: Transformation from aero-engine to industrial gas turbine modelling is illustrated.....................44 Figure 31: Calculated power output is compared for the uncooled gas turbine running with methane fuel ...48 Figure 32: Calculated power specific fuel consumption output is compared for the uncooled gas turbine


Figure 33: Thermal Efficiency output is compared for the uncooled gas turbine running with methane fuel .49 
Figure 34: Siemens SGT6-8000H case compressor blade height comparison ........................................50

Figure 35: Siemens SGT6-8000H case turbine blade height comparison............................................51

Figure 36: GE7HA.02 case compressor blade height comparison .....................................................52

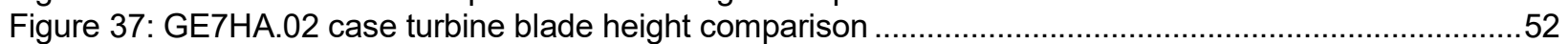

Figure 38: M501 J case compressor blade height comparison .............................................................53

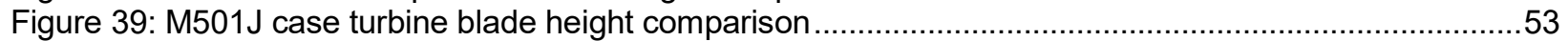

Figure 40: Change in Thermal Efficiency and Power Specific Fuel Consumption calculated for the cooled

engine with the turbine inlet temperature ............................................................................................... 58

Figure 41: Temperature-entropy chart for a gas turbine; low ambient temperature case shown in orange,

high ambient temperature case is shown in green

Figure 42: Change in Power and Thermal Efficiency calculated for the cooled engine with the ambient

temperature

. .59

Figure 43: Change in Power and Thermal Efficiency calculated for the cooled engine with the back-pressure

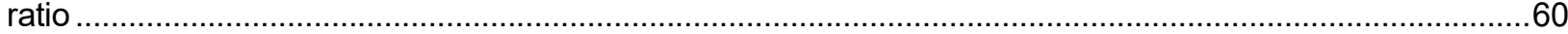

Figure 44: Change in the engine thrust when the metal Biot number is increased ...................................63

Figure 45: Change in the thrust specific fuel consumption when the metal Biot number is increased ...........63

Figure 46: Change in the thermal efficiency when the metal Biot number is increased................................64

Figure 47: Change in the total coolant fractions when the metal Biot number is increased.........................64

Figure 48: Change in the shaft power delivered when the metal Biot number is increased .........................65

Figure 49: Change in the heat rate when the metal Biot number is increased .........................................65

Figure 50: Change in the thermal efficiency when the metal Biot number is increased...............................66

Figure 51: Change in the total coolant flow rates when the metal Biot number is increased........................66

Figure 52: Change in the engine thrust when the TBC Biot number is increased...........................................67

Figure 53: Change in the thrust specific fuel consumption when the TBC Biot number is increased ...........68

Figure 54: Change in the thermal efficiency when the TBC Biot number is increased ..............................68

Figure 55: Change in the total coolant fractions when the TBC Biot number is increased ...........................69

Figure 56: Change in the shaft power delivered when the TBC Biot number is increased ..........................69

Figure 57: Change in the heat rate when the TBC Biot number is increased ..........................................70

Figure 58: Change in the thermal efficiency when the TBC Biot number is increased .................................70

Figure 59: Change in the total coolant fractions when the TBC Biot number is increased ..........................71

Figure 60: Change in the engine thrust when the maximum allowable blade metal temperature is increased

Figure 61: Change in the thrust specific fuel consumption when the maximum allowable blade metal

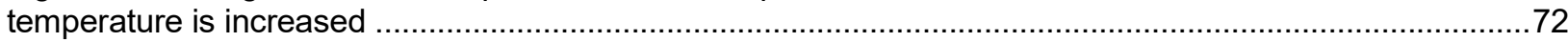

Figure 62: Change in the thermal efficiency when the maximum allowable blade metal temperature is

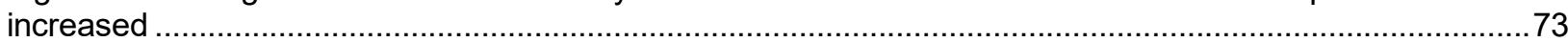

Figure 63: Change in the total coolant flow rates when the maximum allowable blade metal temperature is

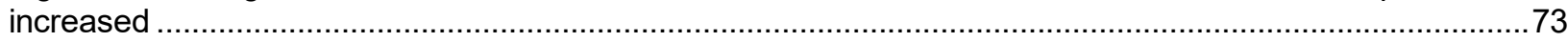

Figure 64: Change in the shaft power delivered when the maximum allowable blade metal temperature is

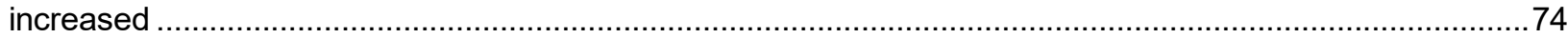

Figure 65: Change in the heat rate when the maximum allowable blade metal temperature is increased ....74

Figure 66: Change in the thermal efficiency when the maximum allowable blade metal temperature is

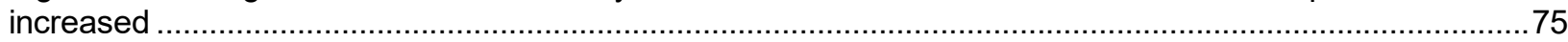

Figure 67: Change in the total coolant flow rates when the maximum allowable blade metal temperature is

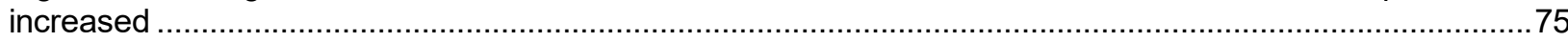

Figure 68: Change in the engine thrust when the internal cooling flow effectiveness is increased ...............76

Figure 69: Change in the thrust specific fuel consumption when the internal cooling flow effectiveness is

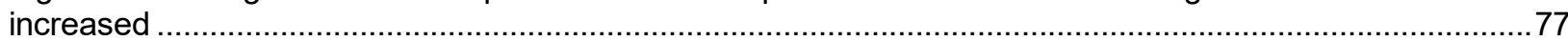

Figure 70: Change in the thermal efficiency when the internal cooling flow effectiveness is increased.........77

Figure 71: Change in the total coolant flow rates when the internal cooling flow effectiveness is increased.78

Figure 72: Change in the shaft power delivered when the internal cooling flow effectiveness is increased ..78

Figure 73: Change in the heat rate when the internal cooling flow effectiveness is increased .....................79

Figure 74: Change in the thermal efficiency when the internal cooling flow effectiveness is increased.........79

Figure 75: Change in the coolant flow rates when the internal cooling flow effectiveness is increased.........80

Figure 76: Change in the engine thrust when the blade adiabatic film cooling effectiveness is increased ....81

Figure 77: Change in the thrust specific fuel consumption when the blade adiabatic film cooling

effectiveness is increased..... 
Figure 78: Change in the thermal efficiency when the blade adiabatic film cooling effectiveness is increased

Figure 79: Change in the coolant flow rates when the blade adiabatic film cooling effectiveness is increased

Figure 80: Change in the shaft power delivered when the blade adiabatic film cooling effectiveness is

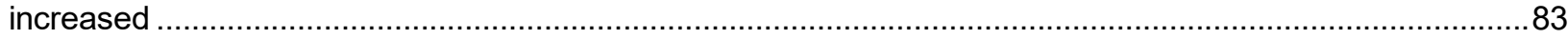

Figure 81: Change in the heat rate when the blade adiabatic film cooling effectiveness is increased ...........84

Figure 82: Change in the thermal efficiency when the blade adiabatic film cooling effectiveness is increased

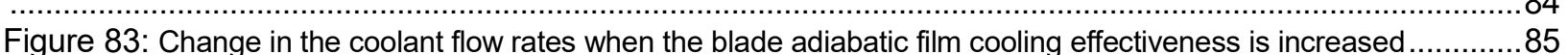

Figure 84: Definition of the effective film cooling flow angle is shown schematically ....................................86

Figure 85: Change in the engine thrust when the effective film cooling flow angle is increased ...................86

Figure 86: Change in the thrust specific fuel consumption when the effective film cooling flow angle is increased ........87

Figure 87: Change in the thermal efficiency when the effective film cooling flow angle is increased.............87

Figure 88: Change in the engine thrust when the blowing ratio is increased ...........................................89

Figure 89: Change in the thrust specific fuel consumption when the blowing ratio is increased ...................89

Figure 90: Change in the thrust specific fuel consumption when the blowing ratio is increased ...................90

Figure 91: Sensitivity of engine thrust on the general cooling parameters ..............................................92

Figure 92: Sensitivity of thrust specific fuel consumption on the general cooling parameters ......................93

Figure 93: Sensitivity of the total coolant flow fraction on the general cooling parameters ............................93

Figure 94: Sensitivity of thermal efficiency on the general cooling parameters .............................................94

Figure 95: Sensitivity of shaft power delivered on the general cooling parameters .......................................95

Figure 96: Sensitivity of heat rate on the general cooling parameters ...................................................96

Figure 97: Sensitivity of total coolant flow rates on the general cooling parameters ...................................96

Figure 98: Sensitivity of thermal efficiency on the general cooling parameters ...........................................97

Figure 99: Half of the used solution geometry is shown for the AVH case, conventional hole case has the

same geometry except for the side holes............................................................................................100

Figure 100: Grid density is increased in both turbulence models to decide over the performance of the models. The response of increasing mesh quality was better in k- $\omega$ SST model ....................................102

Figure 101: Centerline cooling effectiveness predictions with different time steps are compared with

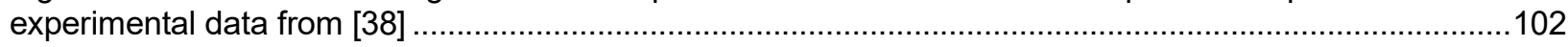

Figure 102: Adaptive meshing with temperature gradients increased mesh concentration in the film cooling

flow region ............................................................................................................................... 103

Figure 103: Grid Independence tests made with adaptive meshes are compared with experimental data

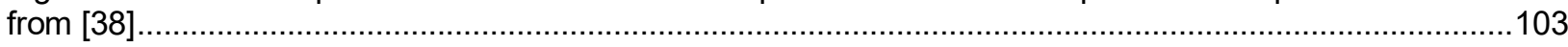

Figure 104: Effectiveness distribution obtained with the experimental conditions given in Table 30 for the

conventional hole shows the expected cooling effectiveness distribution ..............................................104

Figure 105: Temperature distribution plot for the center plane allows to identify the flow separation and

reattachment regions to explain the fluctuations in the effectiveness predictions .....................................105

Figure 106: Centerline effectiveness data obtained for conventional hole DES simulation is compared with

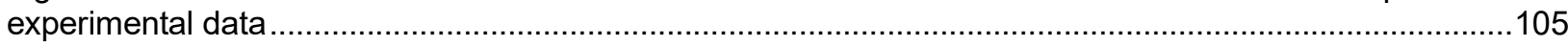

Figure 107: Grid Independence tests made with adaptive meshes for AVH cases .................................107

Figure 108: Span averaged effectiveness from DES simulations was compared with the experimental data

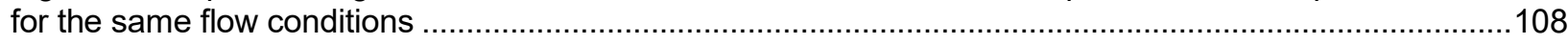

Figure 109: Velocity contours on the conventional hole solution domain mid-plane are compared for three

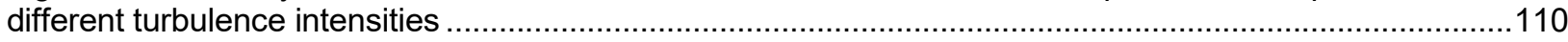

Figure 110: Temperature distribution on the adiabatic wall surface is compared for three different turbulence intensities for the conventional hole case ......................................................................................111

Figure 111: Span averaged cooling effectiveness data from DES simulations for high blowing ratio, high

turbulence intensity and large length scale conditions for conventional hole geometry.............................111

Figure 112: Velocity contours on the AVH solution domain mid-plane are compared for three different

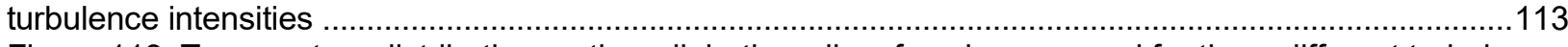

Figure 113: Temperature distribution on the adiabatic wall surface is compared for three different turbulence

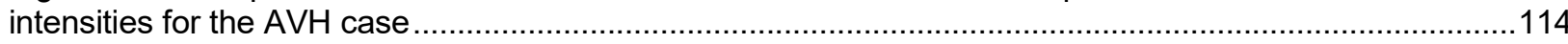

Figure 114: Span averaged cooling effectiveness data from DES simulations for high blowing ratio, high

turbulence intensity and large length scale conditions for AVH geometry ..............................................114 
Figure 115: The differential area considered in the derivation of advanced coolant calculation methods is shown with the blade coordinates ....................................................................................................116

Figure 116: The projection onto the $x-y$ plane of the differential area used in the derivations ....................116 Figure 117: The projection onto the $x-z$ plane of the differential area used in the derivations .....................117 Figure 118: The smallest analysis element is a strip with length $z_{n}$ which is equal to the lateral hole spacing. Depending on the user-input cooling configuration, number of holes and the shown internal cooling type could be different. 120

Figure 119: Flowchart of the advanced coolant calculator ................................................................123 Figure 120: Internal and External Cooling Types that are included in the advanced coolant calculator .......124 Figure 121: Assumed blade sections for internal cooling calculations .................................................124 Figure 122: Flowchart of the Internal Cooling sub-system is shown with possible internal cooling types that

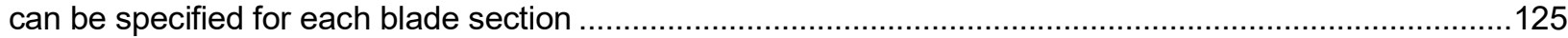

Figure 123: Schematic view of the assumed external cooling scheme ...............................................127

Figure 124: Schematic view of the assumed external cooling scheme ..................................................127 Figure 125: Flowchart of the external cooling sub-system that calculates average adiabatic effectiveness for

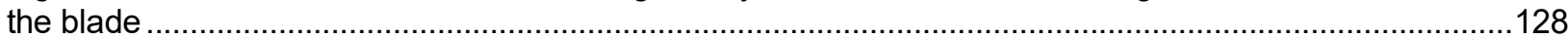

Figure 126: Schematic view of the cooled turbine blade modelled in advanced cooling calculator .............133 Figure 127: Comparing the span-averaged film cooling effectiveness values of different hole geometries used in the analysis...

Figure 128: Total coolant fractions are compared for different film cooling hole geometry cases of the aero-

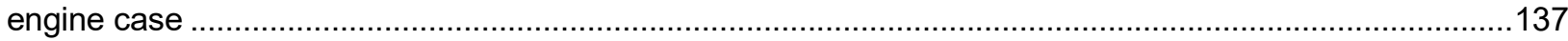
Figure 129: Specific Thrust values are compared for different film cooling hole geometry cases of the aero-

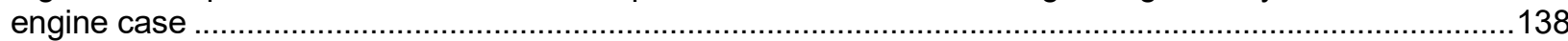
Figure 130: Thrust Specific Fuel Consumptions are compared for different film cooling hole geometry cases

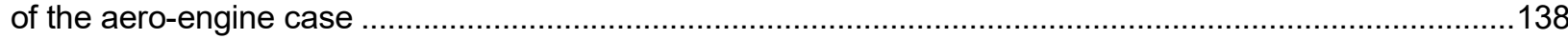
Figure 131: Thermal efficiencies compared for different film cooling hole geometry cases of the aero-engine

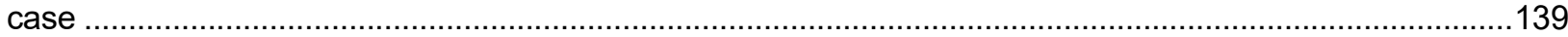
Figure 132: Total coolant fractions are compared for different film cooling hole geometry cases of the


Figure 133: Shaft power values are compared for different film cooling hole geometry cases of the industrial

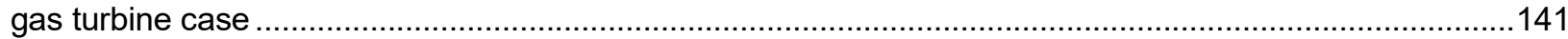
Figure 134: Heat rates are compared for different film cooling hole geometry cases of the industrial gas

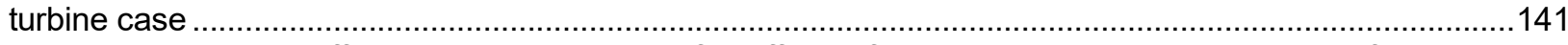
Figure 135: Thermal efficiencies are compared for different film cooling hole geometry cases of the industrial gas turbine case .142 Figure 136: Mid-Chord Rib cooling performance of the analysis cases are compared by using their

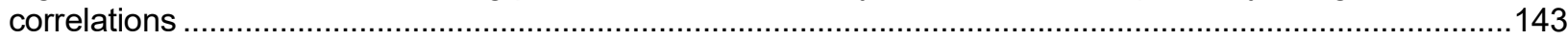
Figure 137: Total coolant fractions are compared for different rib cooling cases of the aero-engine case ..144 Figure 138: Specific thrust values are compared for different rib cooling cases of the aero-engine case ...144 Figure 139: Thrust Specific Fuel Consumption values are compared for different rib cooling cases of the aero-engine case

Figure 140: Thermal Efficiencies are compared for different rib cooling cases of the aero-engine case .....145 Figure 141: Total coolant fractions are compared for different rib cooling cases of the industrial gas turbine

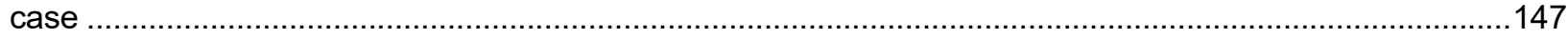
Figure 142: Shaft power values are compared for different rib cooling cases of the industrial gas turbine

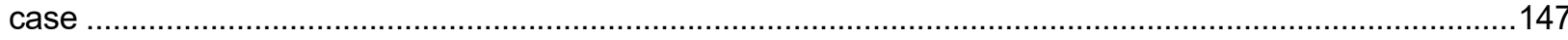
Figure 143: Heat Rate values are compared for different rib cooling cases of the industrial gas turbine case

Figure 144: Thermal Efficiencies are compared for different rib cooling cases of the industrial gas turbine case ...148

Figure 145: Trailing edge pin-fin cooling performance of the analysis cases are compared by using their

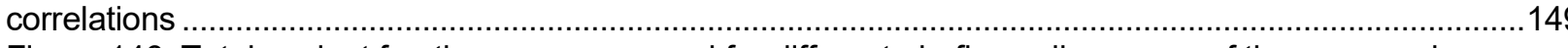
Figure 146: Total coolant fractions are compared for different pin-fin cooling cases of the aero-engine case 150

Figure 147: Specific thrust values are compared for different pin-fin cooling cases of the aero-engine case 
Figure 148: Thrust Specific Fuel Consumption values are compared for different pin-fin cooling cases of the

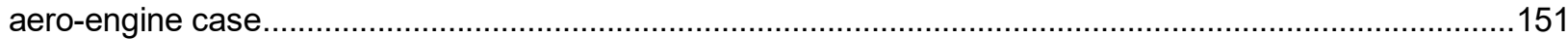
Figure 149: Thermal efficiencies are compared for different pin-fin cooling cases of the aero-engine case 151 Figure 150: Total coolant fractions are compared for different pin-fin cooling cases of the industrial gas turbine case..... 152

Figure 151: Shaft power values are compared for different pin-fin cooling cases of the industrial gas turbine

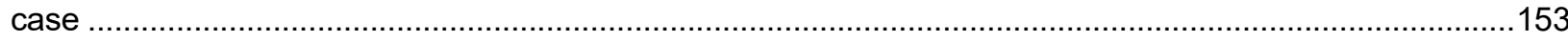
Figure 152: Heat rate values are compared for different pin-fin cooling cases of the industrial gas turbine

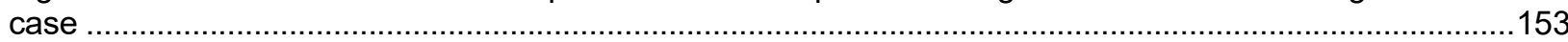
Figure 153: Thermal efficiencies are compared for different pin-fin cooling cases of the industrial gas turbine

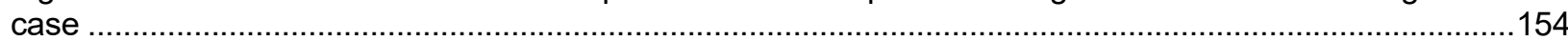
Figure 154: Specific thrust and thermal efficiencies obtained by increasing the number of film cooling rows are compared for aero-engine blade 155 Figure 155: Changes in thrust specific fuel consumption and total coolant fractions when the number of film cooling rows are increased are compared for aero-engine blade ........................................................ 155 Figure 156: Shaft power delivered and heat rates obtained by increasing the number of film cooling rows are

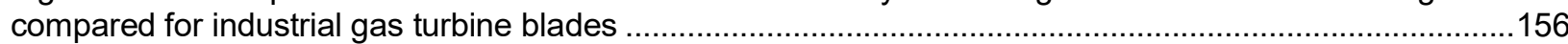
Figure 157: Thermal efficiency and coolant fractions when the number of film cooling rows are increased are



Figure C 1: Compressor modelling approach employed in the compressor model with losses ..................165 Figure C 2: Control Volume boundaries encompasses the coolant extraction region that is after the stator of

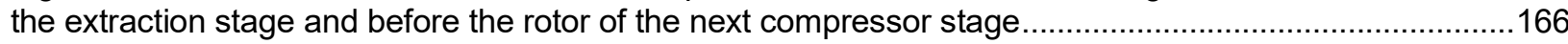

Figure D 1: Control Volume used in the transition cooling model development is shown with the engine station numbers used in CGTM.

Figure E 1: Schematic view of the parallel $90^{\circ}$ ribs with correlation input parameters.................................... 170

Figure E 2: Schematic view of the parallel angled ribs with correlation input parameters .............................. 171

Figure E 3: Schematic view of the parallel V-Shaped ribs with correlation input parameters ........................ 172

Figure E 4: Schematic view of the criss-crossed ribs with correlation input parameters ............................... 173

Figure $\mathrm{E} 5$ : Discrete $\mathrm{V}$-Shaped ribs differ from the parallel $\mathrm{V}$-Shaped ribs with an offset between two legs of the rib [55] .............................................................................................................................................. 174 Figure E 6: Plot-digitization of the experimental data by Wright et al. [55] given for non-rotating case is used

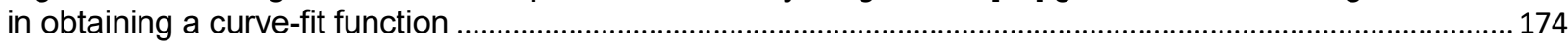

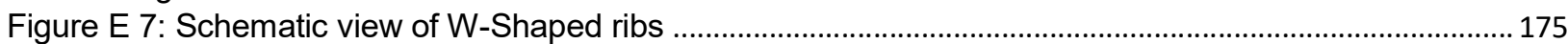
Figure E 8: Plot-digitization of the experimental data by Wright et al. [55] given for non-rotating case is used in obtaining a curve-fit function ..................................................................................................................... 176 Figure E 9: Schematic view of the inline impingement geometry is shown with used input parameters in the correlation

Figure E 10: Film cooling hole types used in the comparisons are shown schematically with their top and

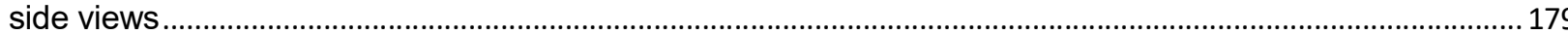

Figure E 11: Span averaged film cooling effectiveness database generated by using the correlation by Colban et al. [60]

Figure E 12: Span averaged film cooling effectiveness database used from Shih and $\mathrm{Na}$ [61] 180 181 


\section{LIST OF TABLES}

Table 1: Definitions of used cooled turbine station numbering and mass flow rate indexing.......................... 6

Table 2: Known (or fixed) parameters in the semi-empirical method are given with their definitions ...............7

Table 3 Validation of the coolant calculator and 1st Law analysis results are done with the Young and

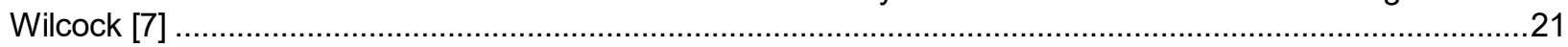

Table 4: Methane combustion properties were validated with the enthalpy scenarios for 5 different fuel-to-air

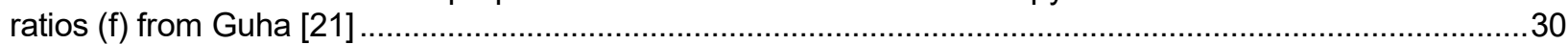

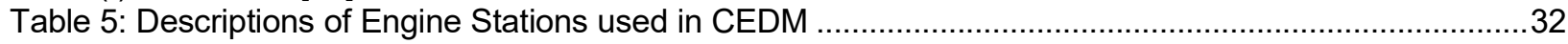

Table 6: Descriptions of Component Performance Parameters used in CEDM..........................................33

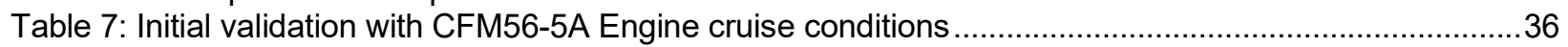

Table 8: Inputs for the CFM56-5A Engine case specific test case ...........................................................

Table 9: Result comparison of case specific test for the uncooled CFM-56A case .....................................38

Table 10: Result comparison of case specific test for the cooled CFM-56A case.......................................39

Table 11: Descriptions of Engine Stations used in CEDM ................................................................42

Table 12: Descriptions of Component Performance Parameters used in CGTM........................................43

Table 13: Descriptions of Component Performance Parameters used in CGTM.........................................45

Table 14: Results of certain cycle parameters are compared for uncooled industrial gas turbine using

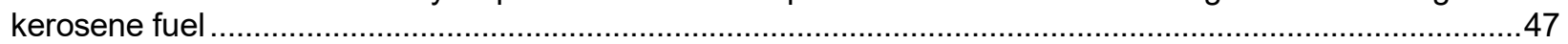

Table 15: Results of certain cycle parameters are compared for uncooled gas turbine using methane fuel 47

Table 16: Assumed material information used in component sizing calculations of CGTM for SGT6-8000H

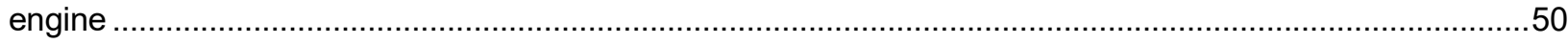

Table 17: Assumed material information used in component sizing calculations of CGTM for GE7HA.02

engine.

Table 18: Assumed material information used in component sizing calculations of CGTM for M501J engine

Table 19: Initial validation result comparison with Siemens SGT6-8000H industrial gas turbine ..................54

Table 20: Initial validation result comparison with GE7HA.02 industrial gas turbine ....................................54

Table 21: Initial validation result comparison with M501J industrial gas turbine ........................................55

Table 22: Inputs generated for the generic $\mathrm{H}$-Class engine scenario for case-specific tests ........................56

Table 23: Case Specific Test result comparison for generic H-Class engine case ......................................56

Table 24: Cooling parameters used in the Sensitivity Analysis are shown with their reference values and change intervals

Table 25: Results of the parameter dependency study for the aero-engine case .....................................91

Table 26: Results of the parameter dependency study for the industrial gas turbine case ...........................91

Table 27: Results of the parameter dependency study for the industrial gas turbine case ...........................98

Table 28: Boundary types that are applied to several sections of the solution domain................................100

Table 29: Flow Conditions at the solution domain boundaries ..........................................................101

Table 30: Flow Conditions used in DES validations made with experimental conventional hole data from [39]

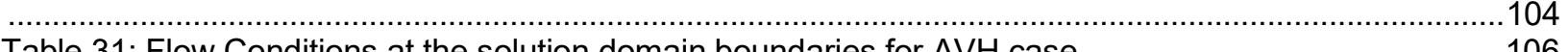

Table 31: Flow Conditions at the solution domain boundaries for AVH case ..........................................106

Table 32: Flow variables set at the boundaries for data generation of conventional hole under engine-like

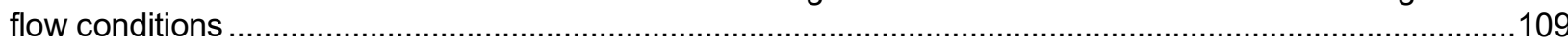

Table 33: Flow variables set at the boundaries for data generation of AVH under engine-like flow conditions

Table 34: Used correlations, their references and corresponding internal cooling schedule indices ...........126

Table 35: Used correlation/film cooling data, their references and corresponding external cooling type index

Table 36: Inputs used in the validation of the new coolant calculation algorithm with semi-empirical formula

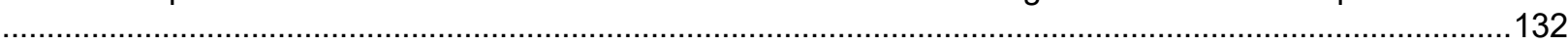

Table 37: Comparison of the coolant fraction calculations by the new algorithm with the semi-empirical



Table 38: User Inputs for Cooling Analysis with Advanced Coolant Calculator ........................................133

Table 39: Blade Cooling Configurations used for Analysis...................................................................134 
Table 40: Cooling Analysis inputs used in High By-Pass Turbofan Engine cases ....................................135

Table 41: Cooling Analysis inputs used in the industrial gas turbine case .............................................135

Table 42: Variations in the blade film cooling and internal cooling flow effectiveness with different film cooling configurations

Table 43: Variations in the blade film cooling and internal cooling flow effectiveness with different film cooling configurations

Table 44: Variations in the mid-chord Stanton number and cooling flow effectiveness with different rib cooling configurations

Table 45: Variations in the mid-chord Stanton number and cooling flow effectiveness with different rib cooling configurations

Table 46: Variations in the trailing edge Stanton number and cooling flow effectiveness with different pin-fin

cooling configurations

Table 47: Variations in the mid-chord Stanton number and cooling flow effectiveness with different pin-fin cooling configurations 


\begin{tabular}{|c|c|c|c|}
\hline A & Area & w & wall \\
\hline$C_{p}$ & Specific Heat at constant pressure & & \\
\hline d & Hydraulic Diameter & & \\
\hline$d_{h}$ & Film Cooling Hole Diameter & & \\
\hline e & Polytropic Efficiency & & \\
\hline$f$ & Fuel-to-Air Ratio (at burner) & Other letters & \\
\hline g & Gravitational Constant & $\alpha$ & Convection HT Coefficent \\
\hline $\mathrm{h}$ & Enthalpy (specific) & $\beta$ & Bleed Air \\
\hline i & Cooled Turbine Stage Number & Y & Specific Heat Ratio \\
\hline $\mathrm{k}$ & $\begin{array}{l}\text { Conduction Heat Transfer } \\
\text { Coefficient }\end{array}$ & $\varepsilon$ & Effectiveness \\
\hline M & Mach Number & $\epsilon$ & Coolant Fraction \\
\hline $\mathrm{n}$ & Polytropic Exponent & $\eta$ & Efficiency \\
\hline $\mathrm{P}$ & Pressure & & \\
\hline $\mathrm{R}$ & Universal Gas Constant & $\mathrm{v}$ & Specific Volume \\
\hline $\mathrm{s}$ & Entropy (specific) & $\pi$ & $\begin{array}{l}\text { Pressure Ratio of the } \\
\text { component }\end{array}$ \\
\hline$Q(q)$ & Heat Flux & $\rho$ & Density \\
\hline $\mathrm{t}$ & Wall Thickness & $\Sigma(\sigma)$ & Loss (entropy rise) \\
\hline $\mathrm{T}$ & Temperature & & \\
\hline $\mathrm{u}$ & Internal Energy (specific) & $\mathrm{T}$ & $\begin{array}{l}\text { Temperature Ratio of the } \\
\text { component }\end{array}$ \\
\hline V & Velocity & $\varphi$ & Effective Injection Angle \\
\hline W (w) & Work & $\psi$ & Transition Cooling \\
\hline $\begin{array}{l}\text { Z } \\
\text { Subscripts }\end{array}$ & Height & $\stackrel{\omega}{\varnothing}$ & $\begin{array}{l}\text { Rotational Speed } \\
\text { Equivalence Ratio }\end{array}$ \\
\hline aw & Adiabatic wall & & \\
\hline bw & Blade wall & \multicolumn{2}{|c|}{ Heat Transfer and Aerodynamics } \\
\hline c & Cooled (thermodynamic properties) & $\mathrm{Bi}$ & Biot Number \\
\hline $\mathrm{cl}$ & Coolant & $\operatorname{Pr}$ & Prandtl Number \\
\hline comb & Combustor & $\mathrm{Nu}$ & Nusselt Number \\
\hline ext & External & $\operatorname{Re}$ & Reynolds Number \\
\hline g & Gas side & St & Stanton Number \\
\hline $\mathrm{g}^{*}$ & Gas side with comb. Pattern factor & Abbreviations & \\
\hline & internal & $\mathrm{HR}$ & Heat Rate \\
\hline met (or m) & metal & HT & Heat Transfer \\
\hline$p$ & purge & PSFC & $\begin{array}{l}\text { Power Specific Fuel } \\
\text { Consumption }\end{array}$ \\
\hline $\mathrm{r}$ & rotor & TBC & Thermal Barrier Coating \\
\hline rc & Rotor cooling & TSFC & $\begin{array}{l}\text { Thrust Specific Fuel } \\
\text { Consumption }\end{array}$ \\
\hline $\mathrm{s}$ & stator & Constants & \\
\hline $\mathrm{sc}$ & Stator cooling & $\mathrm{K}_{\text {cool }}$ & Cooling Coefficient \\
\hline surf & surface & $\mathrm{K}_{\text {comb }}$ & Combustion Pattern Factor \\
\hline $\mathrm{t}$ & Total (thermodynamic properties) & Kswirl & Swirl coefficient \\
\hline uc & uncooled & Ktemp,gain & $\begin{array}{l}\text { Temperature gain of coolant } \\
\text { within supply channels }\end{array}$ \\
\hline
\end{tabular}




\section{INTRODUCTION}

The ultimate goal in engine design is to produce the highest available power or thrust the maximum efficiency with minimum fuel consumption and emissions. Turbine blade cooling has a well-known impact to increase the performance of both aero-engines and industrial gas turbines through higher allowable combustor exit temperatures and to provide longer engine life. Numerous cooling techniques have been and continue to be developed by researchers and engine producers for internal and external disk and blade cooling.

Through the development of gas turbines, there is now a wide variety of cooling techniques applicable to certain engine designs. This requires the need of assessing the relative benefits of a certain cooling technique compared to another. In the last few decades, specific focus on such comparison was to directly compare CFD or experimental performance data that are valid only on a limited region of a blade. To be able to compare the impact of different cooling techniques on the overall engine performance, a thorough thermodynamic model of the gas turbine including the turbine cooling effects needs to be used. Therefore, the focus of this research is to develop a tool that can use experimental or CFD data on several internal and external cooling techniques to calculate their corresponding impact on the entire engine performance.

Several researchers have developed thermodynamic models for cooled turbine analysis in recent years. One of the most detailed models is developed by Consonni [1], with other prominent ones being as GASCAN by El-Marsi [2], CPF by NASA [3], TURBOMATCH by Lallini et al. [4] and IGCC by Li et al. [5]. The latter two models employ a typical aero-engine or industrial gas turbine cycle calculation coupled with a cooled turbine model. TURBOMATCH varied by also including a turbomachinery algorithm. In general, cooled turbine analysis models contain a coolant fraction calculation sub-routine followed by a $1^{\text {st }}$ Law Analysis, which involves a continuity and energy balance for each stage component. The analysis is then completed with estimations of cooling related losses (e.g. exergy loss from blade heat transfer, cooling air friction losses, fluid mixing, etc.) through a pressure loss correlation term [6], or in advanced models with a detailed $2^{\text {nd }}$ Law Analysis. Entropy generation is calculated by a relevant heat transfer and momentum analysis, which is further used in a $2^{\text {nd }}$ Law analysis to calculate the associated pressure losses. The overall process is illustrated in Figure 1.
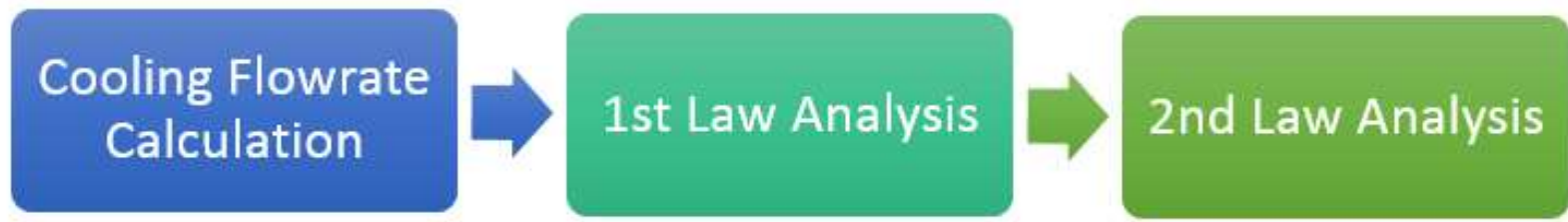

Figure 1: A common flowchart valid for the cooled turbine analysis algorithms

A detailed analysis of a cooled turbine involving the $2^{\text {nd }}$ Law losses was proposed by Young and Wilcock [7], which also includes the rotational effects and the stage loading information in rotor equations. This model is used and cited by several advanced cooled gas turbine models developed by Lallini et al. [4], Horlock and Wilcock [8], and Torbidoni and Horlock [9].

These models can provide an overall insight on how a certain class of cooling techniques influences the engine performance, for instance in obtaining the effect of increasing the film cooling effectiveness on shaft power. However, if the effect on the engine performance by a specific film cooling technique needed to be 
analyzed, these models cannot provide enough information alone. Therefore, to perform additional analysis, the model by Young and Wilcock [7] was chosen and specific correlations for several cooling techniques were incorporated into the algorithm to include the effects of aerodynamic and heat transfer losses associated with these techniques. The resulting modified algorithm is then combined with another modified engine performance analysis algorithm, which includes the effects of air extraction on the compressor to assess the performance of different cooling concepts through the cycle parameters.

Developed cooled engine models are then used in a sensitivity analysis that shows the relative impact strengths of several cooling parameters. Based on the results obtained from that analysis, advanced film cooling techniques are considered as a potential cooling technology improvement and in this research, the impact of using an Anti-Vortex Hole geometry instead of using a conventional hole geometry as the external cooling method is studied by developing an advanced coolant calculation method, which can compute the cooling fractions and several other coolant thermodynamic properties based on the applied cooling configuration. The differences between the external cooling methods are mainly from the differences in the film cooling span averaged effectiveness data and these data for the considered hole geometries were generated for engine-like flow conditions by using Detached Eddy Simulation CFD techniques, after validating the numerical setup with experimental data for the considered hole geometries.

The contributions of the current research are distinguished from the results of existing analyses in the literature [1-9] by including the effects of coolant extraction on the compressor performance, utilizing more information from turbomachinery in the cooling analysis, allowing users to select different cooling techniques on different turbine stages, utilizing the $2^{\text {nd }}$ Law Analysis method by Young and Wilcock [7] in upper level models, and including correlations for several internal and external cooling techniques to form a bridge between the experimental and CFD cooling analyses and the engine performance calculations. 


\section{CHAPTER 1: DEVELOPMENT OF A COOLED TURBINE MODEL}

The turbine thermodynamics is often represented by means of the Mollier diagram (enthalpy vs. entropy), which is formed by the constant pressure lines, that indicates the thermodynamic states. Consider three different turbines operating between the same inlet and exit pressures indicated by $P_{1}$ and $P_{2}$, respectively; namely the first one undergoing an isentropic expansion process, the second one undergoing an actual expansion process without turbine cooling and the third one undergoing an actual expansion process with turbine cooling. The Mollier diagram representing these processes is presented in Figure 2.

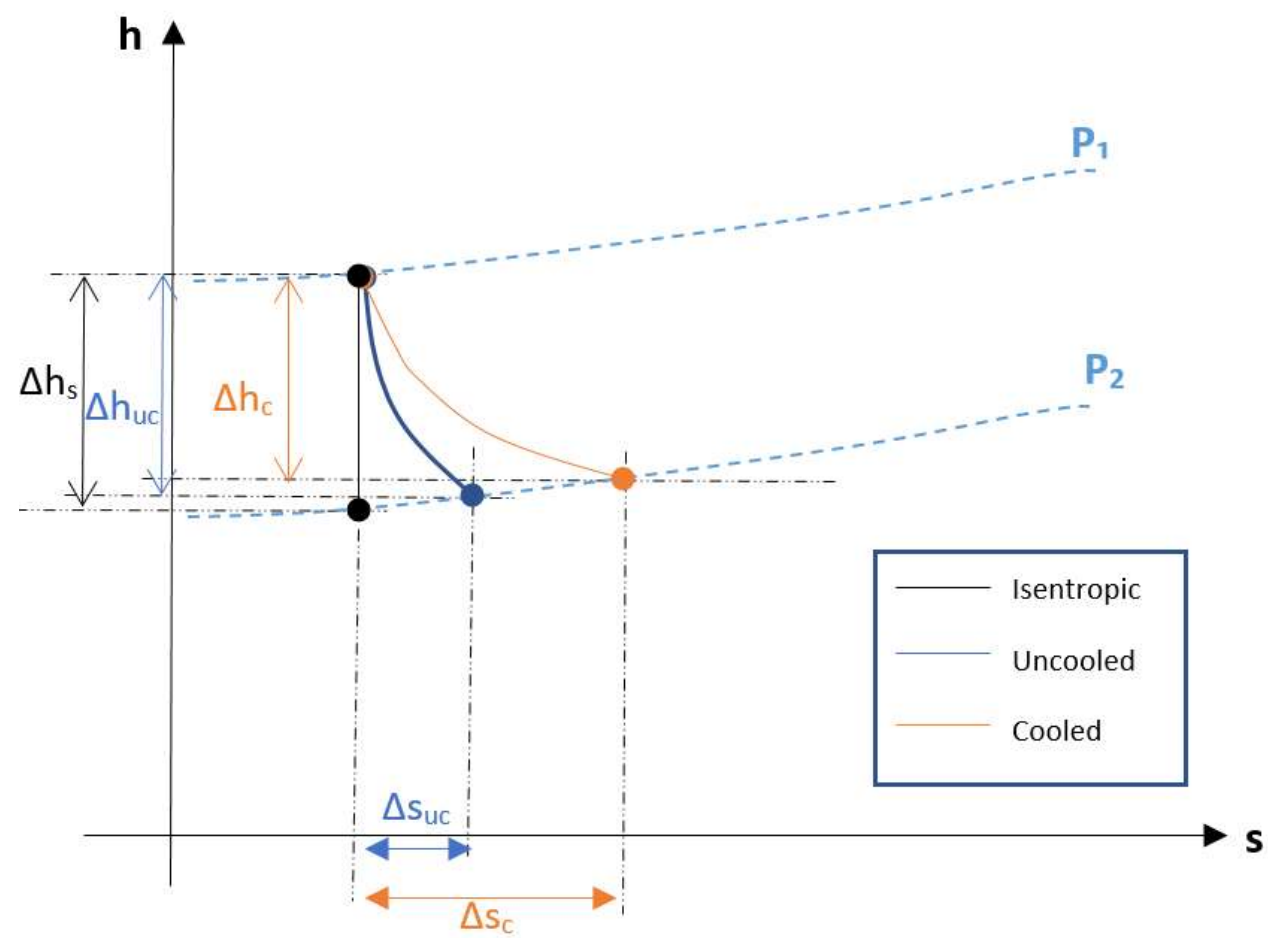

Figure 2: A Mollier diagram for turbines operating between the same inlet and exit states; isentropic turbine has the highest work output, followed by the uncooled turbine and cooled turbine having the least output due to efficiency losses

By having the highest enthalpy $\operatorname{drop}\left(\Delta \mathrm{h}_{\mathrm{s}}\right)$ without an increase in entropy $(\Delta \mathrm{s}=0)$, the isentropic process has the highest work output between the indicated thermodynamic states. Actual turbines are undergoing a polytropic process, with the polytropic efficiency for the uncooled turbine $e_{t}$ and for the cooled turbine $e_{t, c}$, each are being less than $100 \%$. The values of these polytropic efficiencies are affected by the increase in entropy during a process, and it is expected that $e_{t, c}$ to be lower than $e_{t}$ due to the existence of additional losses related to turbine cooling.

Assuming the ideal gas behavior for the working fluid, the polytropic expansion in the actual turbines (uncooled and cooled) are described with Equation(s) (1).

$$
\frac{T_{t 2}}{T_{t 1}}=\left(\frac{P_{t 2}}{P_{t 1}}\right)^{e_{t} \frac{\gamma_{t}-1}{\gamma_{t}}}
$$




$$
\frac{T_{t 2}{ }^{\prime}}{T_{t 1}}=\left(\frac{P_{t 2}{ }^{\prime}}{P_{t 1}}\right)^{e_{t, c^{\prime}} \frac{\gamma_{t}-1}{\gamma_{t}}}
$$

In Equation(s) (1), $\gamma_{t}$ is the specific heat ratio and assumed to be the same for the uncooled and cooled expansion processes. Cooled turbine exit thermodynamic state is indicated with 2', which differs from the uncooled turbine exit state 2 due to pressure losses related to turbine cooling process.

A differential analysis is required for a complete study of the polytropic expansion processes considered. The differential form of the $1^{\text {st }}$ Law of Thermodynamics for open systems is given in Equation (2).

$$
\delta q-\delta w=d u
$$

In Equation (2), $q$ is the specific heat transfer from the turbine to surroundings, $w$ is the specific boundary and shaft work in the turbine and $u$ is the specific internal energy. The enthalpy is conventionally defined with Equation (3).

$$
\begin{gathered}
h=u+P v \\
d h=d u+P d v+v d P
\end{gathered}
$$

In Equation (3), $\mathrm{P}$ is the pressure and $v$ is the specific volume (or inverse density), which is the inverse of the density. The differential form of the $2^{\text {nd }}$ Law of Thermodynamics is given in Equation (4).

$$
T d s=\delta q
$$

For the expansion process in the turbine, $\delta w$ is given as in Equation (5).

$$
\delta w=P d v
$$

Inserting the heat and work terms from Equations (4) and (5), respectively to Equation (2), and using the definition of enthalpy from Equation (3) to replace internal energy term in Equation (2), will result in the fundamental relation given in Equation (6), which is valid for both turbine processes considered.

$$
T d s=d h-v d P
$$

For an ideal gas, $d h=c_{p}(T) d T$, where $\mathrm{c}_{\mathrm{p}}$ is the specific heat at constant pressure and $P v=R T$ from ideal gas law. In Equation (6), replacing the enthalpy term with $c_{p}(T) d T$ and the specific volume with $R T / P$ from the ideal gas law results in Equation (7), which can be integrated between the inlet and exit states.

$$
T d s=c_{p}(T) d T-R T \frac{d P}{P}
$$

To obtain a relationship between the change in entropy (losses) with the turbine inlet and exit thermodynamic states (work), Equation (7) is integrated between inlet (State 1) and exit (State 2 or 2'). This procedure results in Equation (8); which has turbine temperature and pressure ratios, that are also gas turbine engine performance parameters. 


$$
s_{2}-s_{1}=c_{p} \ln \left(\frac{T_{t 2}}{T_{t 1}}\right)-R \ln \left(\frac{P_{t 2}}{P_{t 1}}\right)
$$

A relation between the specific heats, universal gas constant $(R)$ and the ratio of specific heats $\left(\gamma_{t}\right)$ is given in Equation (9).

$$
\frac{\gamma_{t}}{\gamma_{t}-1}=\frac{c_{p}}{R}
$$

Replacing the temperature ratio of Equation (8) with the definition of the polytropic efficiency given in Equation(s) (1), and using the relation given in Equation (9), results in a more compact form that relates the entropy change to the pressure ratio, as given in Equation (10) for the uncooled turbine.

$$
\Delta s_{u c}=s_{2}-s_{1}=R\left(e_{t}-1\right) \ln \left(\frac{P_{t 2}}{P_{t 1}}\right)
$$

Similarly, for the cooled turbine, the relation between the turbine pressure ratio and the entropy change is given with Equation (11).

$$
\Delta s_{c}=s_{2 \prime}-s_{1}=R\left(e_{t, c}-1\right) \ln \left(\frac{P_{t 2 \prime}}{P_{t 1}}\right)
$$

The pressure ratio of the cooled turbines $(\pi)$ is therefore affected by the entropy increase ( $\Delta$ s terms), and the polytropic efficiencies and they can be obtained after re-arranging Equations (10) and (11) which results in the relations in Equation(s) (12).

$$
\begin{gathered}
\pi_{t, u c}=\frac{P_{t 2}}{P_{t 1}}=e^{\frac{\Delta s_{u c}}{R\left(e_{t}-1\right)}} \\
\pi_{t, c}=\frac{P_{t 2 \prime}}{P_{t 1}}=e^{\frac{\Delta s_{c}}{R\left(e_{t, c}-1\right)}}
\end{gathered}
$$

Here, the polytropic efficiencies are not independent of the entropy changes that are encountered during the processes. This dependency requires a detailed analysis of the turbine expansion stages such as purge flows, stators, and rotors, and is given in the following sub-sections.

\subsection{Theory}

Assume a cooled turbine stage that is formed sequentially by a purge flow before the stator, a cooled stator stage, another purge flow in between the stator and rotor, and a cooled rotor stage. This sequence is described as shown in Figure 3. 


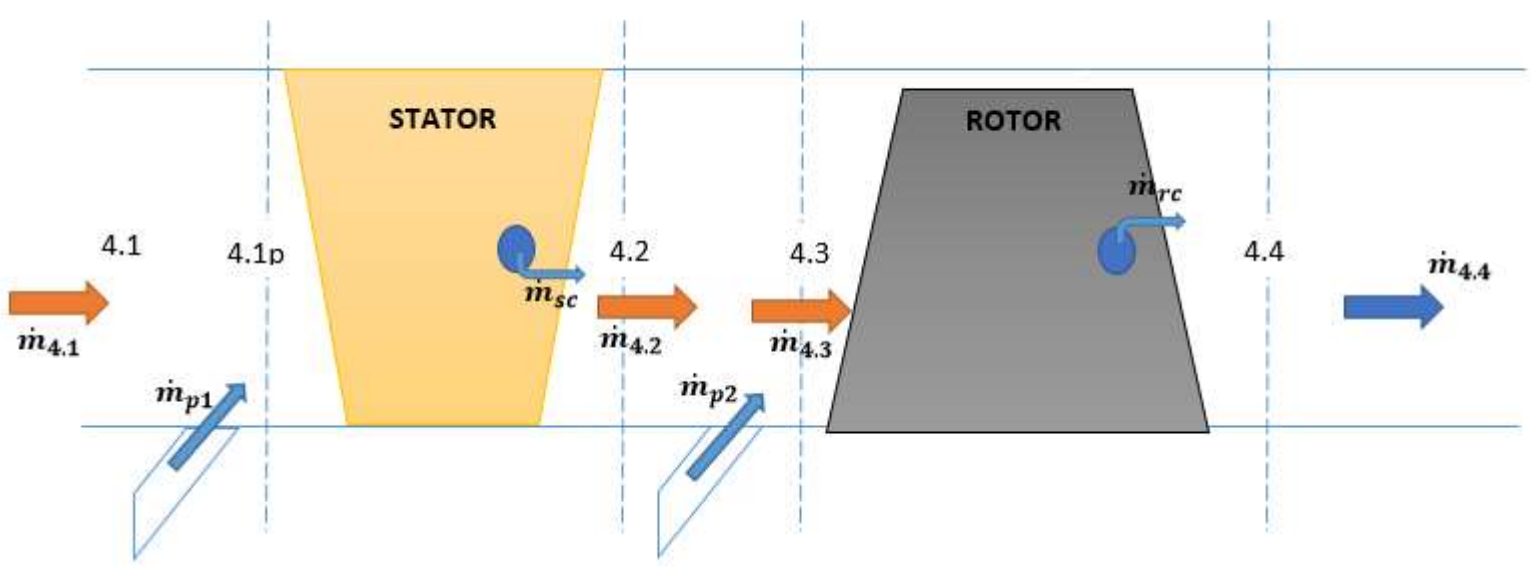

Figure 3: Assumed cooled turbine stage scheme and mass flow rate relations for the cooling analysis by Young and Wilcock [7], stage indices are not shown in the figure

In Figure 3 and throughout the thesis, for each cooled turbine stage "i" the station number and mass flow rate indexing given in Table 1 will be used. Station numbering follows the general gas turbine station numbering and Station 4 corresponds to the entrance of the turbine section.

Table 1: Definitions of used cooled turbine station numbering and mass flow rate indexing

\begin{tabular}{|c|c|c|c|}
\hline Station Numbers & Definition & Mass Flow rate Index & Definition \\
\hline $4.1 \mathrm{i}$ & Stage Entry of $\mathrm{i}^{\text {th }}$ stage & $\dot{m}_{4.1 i}$ & $\begin{array}{l}\text { Stage Entry Hot Gas Mass flow } \\
\text { rate }\end{array}$ \\
\hline $4.1 \mathrm{pi}$ & Station after first purge & $\dot{m}_{s c}$ & $\begin{array}{l}\text { Stator Coolant Flow (from all } \\
\text { types of applied external cooling } \\
\text { techniques) }\end{array}$ \\
\hline $4.2 \mathrm{i}$ & Stator Exit & $\dot{m}_{p 1,2}$ & Purge Flows \\
\hline 4.3i & $\begin{array}{l}\text { Station after second purge } \\
\text { and Rotor Entry }\end{array}$ & $\dot{m}_{r c}$ & $\begin{array}{l}\text { Rotor Coolant Flow (from all } \\
\text { types of applied external cooling } \\
\text { techniques) }\end{array}$ \\
\hline $4.4 i$ & $\begin{array}{l}\text { Rotor Exit (Stage Exit of } i^{\text {th }} \\
\text { stage) }\end{array}$ & $\dot{m}_{4.4 i}$ & $\begin{array}{l}\text { Cooled flow exiting from the } i^{\text {th }} \\
\text { stage }\end{array}$ \\
\hline
\end{tabular}

For the mass flow rates within the cooled turbine stage, relations given in Equation(s) 13 are valid.

$$
\begin{gathered}
\dot{m}_{4.2 i}=\dot{m}_{4.1 i}+\dot{m}_{s c}+\dot{m}_{p 1} \\
\dot{m}_{4.3 i}=\dot{m}_{4.1 i}+\dot{m}_{s c}+\dot{m}_{p 1}+\dot{m}_{p 2} \\
\dot{m}_{4.4 i}=\dot{m}_{4.1 i}+\dot{m}_{s c}+\dot{m}_{p 1}+\dot{m}_{r c}+\dot{m}_{p 2}
\end{gathered}
$$

The mass flow rates found by using Equation(s) (13) correspond to the hot gas mass flow rates $\left(\dot{\boldsymbol{m}}_{\boldsymbol{g}}\right)$, that will be used to calculate the coolant fractions $\left(\dot{\boldsymbol{m}}_{s c}\right.$ and $\left.\dot{\boldsymbol{m}}_{r c}\right)$ at each cooled turbine stage. 


\subsubsection{Calculation of Coolant Fractions (Semi-Empirical Method)}

In this method, it is assumed that the parameters given in Table 2 are known a priori. In general, these parameters are known either from material limitations, the technology level of applied internal and external cooling techniques and from other thermodynamic analysis modules that output this information.

Table 2: Known (or fixed) parameters in the semi-empirical method, are given with their definitions

\begin{tabular}{|c|c|c|}
\hline Parameter & Definition & Expression \\
\hline $\operatorname{Ttg}^{*}$ & $\begin{array}{l}\text { Gas Side Temperature (includes comb. } \\
\text { Pattern factor) }\end{array}$ & $T_{t g *}=T_{t g}+K_{c o m b} \Delta T_{c o m b}$ \\
\hline$T_{b, \max }$ & $\begin{array}{l}\text { Maximum Allowable } \text { Blade } \\
\text { Temperature }\end{array}$ & \\
\hline$\varepsilon_{f c}$ & Adiabatic Film Cooling Effectiveness & $\varepsilon_{f c}=\frac{T_{t g}-T_{t a w}}{T_{t g}-T_{t c l, x}}$ \\
\hline$\varepsilon_{c}$ & Internal Flow Cooling Effectiveness [7] & $\varepsilon_{c}=\frac{T_{t c l, x}-T_{t c l, i}}{T_{m, i n t}-T_{t c l, i}}$ \\
\hline$B i_{m}$ & Metal Biot Number [7] & $B i_{m}=\frac{\alpha_{g} t_{m e t}}{k_{m e t}}$ \\
\hline$B i_{T B C}$ & Thermal Barrier Coating Biot Number [7] & $B i_{T B C}=\frac{\alpha_{g} t_{T B C}}{k_{T B C}}$ \\
\hline
\end{tabular}

The assumed heat transfer scheme together with the temperatures used in Table 2 to derive the coolant mass-flow rate relation is shown in Figure 4 for the case of an internally and externally cooled blade with Thermal Barrier Coating (TBC) $)^{\S}$ application.

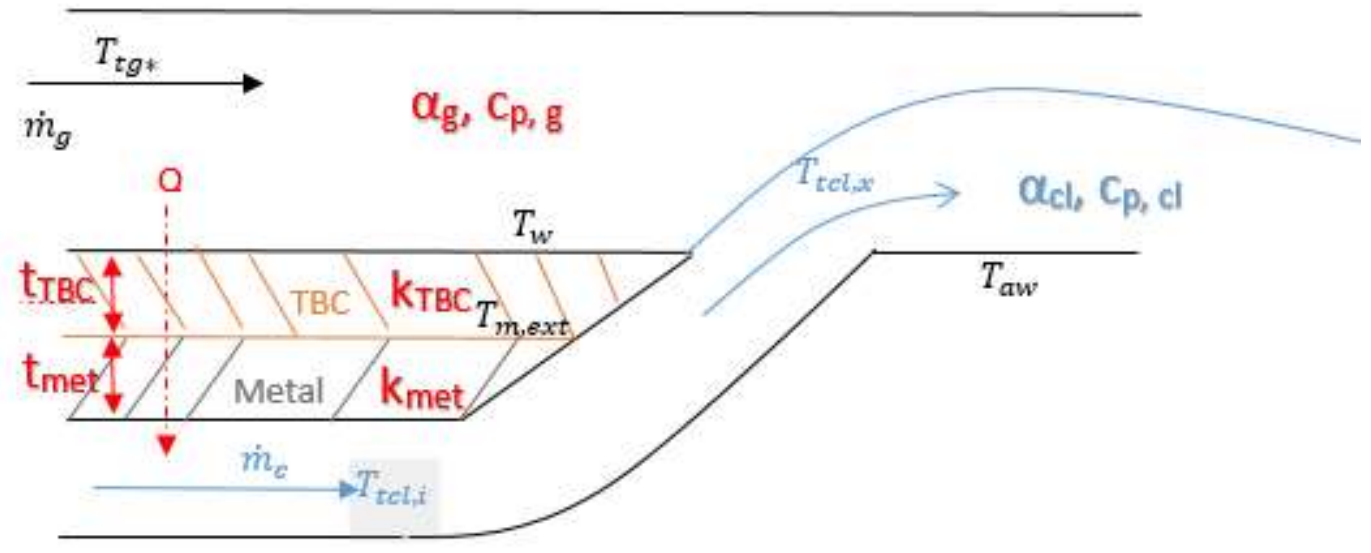

Figure 4: 1D Heat Transfer Scheme and temperatures for an internally and externally cooled blade assumed in the derivation of semi-empirical mass flow-rate correlation

§Thermal Barrier Coating: A coating layer formed by using advanced ceramic materials that have low conduction rates, applied on blade metal walls to provide additional protection. 
The model uses a semi-empirical approach to calculate the required coolant flows by using the formula obtained from writing a heat transfer resistance network for the heat flux " $Q$ " shown in Figure 4. The resulting equation is given in Equation (14) for a cooled blade having both internal and external cooling with a TBC layer [9].

$$
\frac{\dot{m}_{c}}{\dot{m}_{g}}=\frac{K_{\text {cool }}}{\left(1+B i_{\text {total }}\right)}\left\{\frac{\varepsilon_{0}-\varepsilon_{f c}\left(1-\varepsilon_{c}\left(1-\varepsilon_{0}\right)\right)}{\varepsilon_{c}\left(1-\varepsilon_{0}\right)}\right\}
$$

In Equation (14), Bitotal is the total Biot number $\$$ calculated for the metal and TBC materials (hot-gas side based), $\varepsilon_{0}$ is the overall cooling effectiveness for the blade, $\varepsilon_{f c}$ is the film cooling effectiveness, and $\varepsilon_{c}$ is an internal flow cooling effectiveness. $\mathrm{K}_{\mathrm{cool}}$ is a constant representing the ratio of internal to external heat transfer, which changes depending on the applied cooling configuration and the level of cooling technology. The definition of the overall cooling effectiveness is given in Equation (15) and includes a "combustion pattern factor" first considered by Consonni [1] and implemented similarly by Young and Wilcock [7], $\mathrm{K}_{\text {comb }}$ which accounts for the temperature fluctuations due to rotation of the hot gas and suggested in references [1] and [7] to be taken as 0.1 for stators and 0.05 for rotors of the first turbine stage.

$$
\varepsilon_{0}=\frac{\left(T_{t g}+K_{c o m b} \Delta T_{c o m b}\right)-T_{b, \max }}{\left(T_{t g}+K_{c o m b} \Delta T_{c o m b}\right)-T_{c l, i n}}
$$

Once the overall cooling effectiveness is known, total Biot number (Bitotal) in Equation (13) can be calculated with Equation (16).

$$
B i_{\text {total }}=B i_{T B C}+B i_{m}\left(\frac{\varepsilon_{0}-\varepsilon_{f c}}{1-\varepsilon_{0}}\right)
$$

In Equation (14), $\mathrm{K}_{\text {cool }}$ is a constant parameter that accounts for the internal and external Stanton numbers§§, the flow-turning angle across the stage, and the solidity of the cooled blade [10], which reflects the blade passage geometry information to the calculations. If the cooled blade does not use one of the techniques considered in Equation (13), the same formula can be used by eliminating the terms related to the unused technique. This approach is used in calculating the required coolant fractions that will be used in the later sections of the model. Calculation of the coolant fractions will be revisited and calculated in an iterative fashion when the internal and external cooling correlations are added to the model in Chapter 5.

\subsubsection{Calculation of Enthalpies (1 ${ }^{\text {st }}$ Law Analysis)}

A $1^{\text {st }}$ Law Analysis is made for each cooled turbine internal station (first purge region, stator, intermediate purge region and rotor), to obtain the enthalpies at the station exit, which are required in the later parts of cooled turbine calculations that determine other thermodynamic properties such as the total temperatures, total pressures, and entropies.

$\S$ Biot Number: A dimensionless quantity used in heat transfer named after physicist Jean Baptiste Biot, which gives a simple index of the ratio of the heat transfer resistances inside of and at the surface of a body.

$\S \S$ Stanton Number: A dimensionless quantity used in forced convection heat transfer analysis that measures the ratio of heat transferred into a fluid to the thermal capacity of fluid 
The control volume used in this analysis use a stationary frame of reference, which is a frame that is attached to the engine nacelle (or casing) and has zero rotation, and is defined in Figure 5.

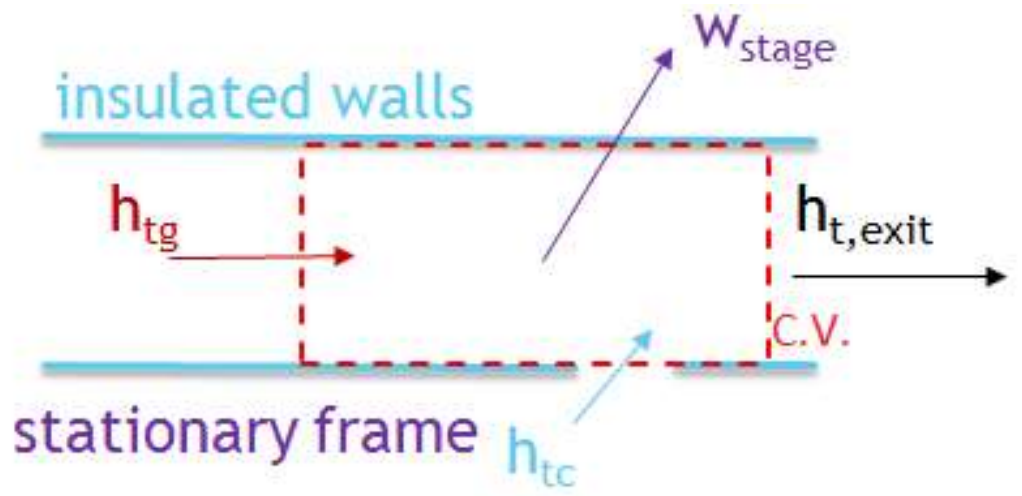

Figure 5: Control Volume definition used for all internal stations; $W_{\text {stage }}=0$ for purge stations and the control volume encompasses the indicated station entirely

For all internal stations, with the control volume considered in Figure 5 for steady flow conditions, the $1^{\text {st }}$ Law of Thermodynamics was used to derive enthalpy equations is in the form given in Equation (17).

$$
\dot{Q}_{\text {station }}-\dot{W}_{\text {station }}=\sum_{\text {st.ext }} \dot{m}_{\text {st.exit }}\left(h_{t, \text { st.ext }}+\frac{V_{\text {st.ext }}^{2}}{2}+g z_{\text {st.ext }}\right)-\sum_{\text {st.in }} \dot{m}_{\text {st.in }}\left(h_{t, \text { st. in }}+\frac{V_{\text {st.in }}^{2}}{2}+g z_{\text {st.in }}\right)
$$

In a gas turbine, the relative height differences between the internal stations are very small therefore the potential energy terms $\left(\mathrm{gz}_{\mathrm{x}}\right)$ can be neglected. For the stators and purge stations, the kinetic energy change from inlet to exit can be neglected. Wall insulation allows the out-of-wall heat flux term $\left(\dot{Q}_{\text {station }}\right)$ to be ignored for all stations, and except for the rotors, $\dot{W}_{\text {station }}=0$. Therefore, using Equation (17) for the purge station before stator (Station 4.1pi) would yield the station exit enthalpy equation given in Equation (18).

$$
h_{t 4.1 p i}=\frac{\left(\dot{m}_{4.1 i}\right) h_{t 4.1 i}+\dot{m}_{p 1} h_{t, p 1 i}}{\left(\dot{m}_{4.1 i}+\dot{m}_{p 1}\right)}
$$

Similarly, the enthalpy equation for the stator exit station (Station 4.2i) can be obtained as in Equation (19).

$$
h_{t 4.2 i}=\frac{\left(\dot{m}_{4.1 i}+\dot{m}_{p 1}\right) h_{t 4.1 p i}+\dot{m}_{s c} h_{t s c, x}}{\dot{m}_{4.1 i}+\dot{m}_{s c}+\dot{m}_{p 1}}
$$

The purge coolant enthalpies are known from the coolant extraction stage properties at the compressor (with a predetermined coolant heat addition from the supply pipes). For the stator stage, the coolant exit enthalpy $\left(h_{\text {tsc, } x}\right)$ is known from internal and external cooling efficiencies and can be determined with a thermodynamic property calculator that uses the coolant exit temperature $\left(T_{t c l, x}\right)$. An algorithm to find the coolant exit temperatures with the known parameters of Table 2 is developed and given in Appendix-B.

The enthalpy equation for the rotor stations is derived by considering the rotational effects, and should be transformed to the non-rotating reference frame attached to the engine nacelle (or casing) in order to be used in cycle calculations. The details of the derivation of this equation, which involves 1D continuity and rothalpy balance, is provided in Appendix-B. By using the transformations from relative to static enthalpy, including all 
swirl effects within a constant parameter ( $\left.\mathrm{K}_{\text {swirl }}\right)$ by also considering the effect of stage degree of reaction $\left({ }^{0} R_{t i}\right)$ and relating the power generated in the rotor stage with the associated stage loading $(\psi)$, the relation given in Equation (20) is obtained for the cooled rotor station exit enthalpy.

$$
h_{t 4.4 i} \cong \frac{h_{t c l, i}-\frac{\left(K_{s w i r l}-{ }^{o} R_{t}\right)}{\psi}\left(h_{t 4.3 i}+\frac{\dot{m}_{r c}}{\dot{m}_{4.3 i}} h_{t c l, i}\right)}{1-\frac{\left(K_{s w i r l}-{ }^{o} R_{t}\right)}{\psi}\left(1+\frac{\dot{m}_{r c}}{\dot{m}_{4.3 i}}\right)}
$$

The derivation given in Appendix-B is reproduced for this work independently and could be different than the derivation procedure of Young and Wilcock [7] but Equation (20) has the same form with the rotor station exit enthalpy equation used by Consonni [1].

It should be noted that, in Equations (18), (19) and (20), the coolant flow rates are known from the coolant flow rate correlation used from Section 1.1.1, and the enthalpies on the right side of the equations are known from the previous station calculations.

\subsubsection{Calculation of Entropies (2 ${ }^{\text {nd }}$ Law Analysis)}

In the $2^{\text {nd }}$ Law Analysis of the cooled turbine, entropies due to several loss factors are determined. These factors are mainly due to aerodynamic losses, frictional losses, and cooling losses. In this context, pressure and temperature losses related to cooling of a turbine will only be analyzed and the remainder of the loss resources are assumed to be included in the polytropic efficiency term that is used in the cycle calculations.

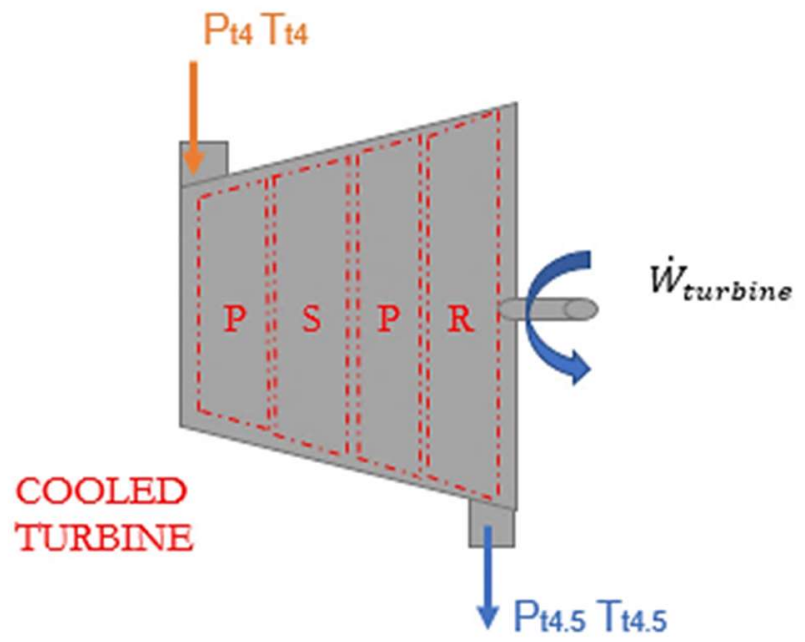

Figure 6: Cooled Turbine Control Volumes around purge $(P)$, stator $(S)$ and rotor $(R)$ stations that are used in the $2^{\text {nd }}$ Law Analysis shown for a single stage turbine

For each control volume in the cooled turbine of Figure 6 with steady flow, $2^{\text {nd }}$ law of thermodynamics is in the form given as in Equation (21) [11].

$$
0=\sum_{j} \frac{\dot{Q}_{j}}{T_{j}}+\dot{m}\left(s_{\text {station,in }}-s_{\text {station,ext }}\right)+\dot{\Sigma}_{\text {station }}
$$


The summation term in Equation (21) represents the overall heat transfer from the turbine to an external reservoir, and if the turbine external walls are well insulated, this term is eliminated and the compact form shown in Equation (22) is obtained.

$$
\sigma_{\text {station }}=\left(s_{\text {station, }, \text { in }}-s_{\text {station,ext }}\right)=\Delta s
$$

In Equation (22), $\sigma_{\text {station }}$ is the overall specific entropy rise across the indicated turbine station per unit mass flow rate, which can belong to a purge, stator or rotor station. For each stage station, the entropy rise $\Delta s$ can be found as in Equation (23) by integrating Equation (7) between the inlet and exit of each station.

$$
\sigma_{\text {station }}=s^{0}\left(T_{\text {station.ext }}\right)-s^{0}\left(T_{\text {station.in }}\right)-R \ln \left(\frac{P_{\text {station.ext }}}{P_{\text {station.in }}}\right)
$$

In Equation (23), $\mathrm{s}^{0}$ indicates the entropy rise from absolute zero to indicated temperature and defined as given in Equation (24a). Its value can be found with Equation (24b) by using the reduced pressures $\left(P_{r}=P / P_{c}\right)$, which is the pressure value of the thermodynamic state divided by the critical pressure of that species $\left(P_{c}\right)$. Reduced pressure information is tabulated for most of the working fluids considered in gas turbines [12].

$$
\begin{aligned}
& s^{0}(T)=\int_{0}^{t} c_{p}(T) \frac{d T}{T} \\
& s^{0}(T)=R \ln \left(P_{r}(T)\right)
\end{aligned}
$$

Re-arranging Equation (23) yields an expression that can be used to calculate turbine station pressure ratio as in Equation (25).

$$
\frac{P_{\text {station.ext }}}{P_{\text {stati in }} \text {.in }}=e^{\frac{\Delta s_{\text {station }}^{0}-\sigma_{\text {station }}}{R}}
$$

In Equation (25), $\Delta s_{\text {station }}^{0}=s^{0}\left(T_{\text {station.ext }}\right)-s^{0}\left(T_{\text {station.in }}\right)$ and can be found from using Equation(s) (24) for each turbine station. The pressure ratio of a cooled turbine stage with the station numbering shown in Figure 3 can be found with the product formula shown in Equation (26).

$$
\pi_{\text {stage }, i}=\frac{P_{t 4.4 i}}{P_{t 4.1 i}}=\frac{P_{t 4.1 p i}}{P_{t 4.1 i}} \frac{P_{t 4.2 i}}{P_{t 4.1 p i}} \frac{P_{t 4.3 i}}{P_{t 4.2 i}} \frac{P_{t 4.4 i}}{P_{t 4.3 i}}
$$

Each station pressure ratio can be found from Equation (25) applied between consecutive stage stations. Using Equation (25) to obtain the cooled stage pressure ratio will result in Equation (27), which is valid for the entire cooled turbine stage.

$$
\pi_{\text {stage }, i}=\frac{P_{\text {t4.4i }}}{P_{t 4.1 i}}=e^{\frac{\Delta s_{\text {purge }, 1}^{0}-\sigma_{\text {purge }, 1}}{R}} e^{\frac{\Delta s_{\text {stator }}^{0}-\sigma_{\text {stator }}}{R}} e^{\frac{\Delta s_{\text {purge }, 2}^{0}-\sigma_{\text {purge }, 2}}{R}} e^{\frac{\Delta s_{\text {rotor }}^{0}-\sigma_{\text {rotor }}}{R}}
$$




$$
\pi_{\text {stage }, i}=\frac{P_{t 4.4 i}}{P_{t 4.1 i}}=e^{\frac{\left(\Delta s_{\text {purge }, 1}^{0}+\Delta s_{\text {stator }}^{0}+\Delta s_{\text {purge }, 2}^{0}+\Delta s_{\text {rotor }}^{0}\right)-\left(\sigma_{\text {purge }, 1}+\sigma_{\text {stator }}+\sigma_{\text {purge }, 2}+\sigma_{\text {rotor }}\right)}{R}}
$$

Using the definition of $\Delta s^{0}$ for each station reduces the first term in the exponent to the difference between inlet and exit states of the stage and using $\sigma_{\text {stage }, i}$ for the second term in the exponent gives the compact pressure ratio equation given in Equation (28).

$$
\pi_{\text {stage }, i}=\frac{P_{t 4.4 i}}{P_{t 4.1 i}}=e^{\frac{\left(s^{0}\left(T_{t 4.4 i}\right)-s^{0}\left(T_{t 4.1 i}\right)\right)-\sigma_{\text {stage }, i}}{R}}
$$

In Equation (28), $\sigma_{\text {stage }, i}$ represents the overall specific stage entropy rise and it is basically the summation of station entropy rises, which is shown in Equation (29).

$$
\sigma_{\text {stage }, i}=\sigma_{\text {purge }, 1}+\sigma_{\text {stator }}+\sigma_{\text {purge }, 2}+\sigma_{\text {rotor }}
$$

The first exponential term of Equation (28) can be calculated by using an appropriate thermodynamic table for the flowing media, and $\mathrm{R}$ is the universal gas constant. However, calculation of $\sigma_{\text {stage, } i}$ is not straightforward and needs a higher-level calculation procedure that involves the entropy generation due to all possible types of component losses.

For the purge stations, the entropy rise is attributed to mixing losses only and can be easily found by applying a mixing analysis on the control volume shown in Figure 4. Assuming no-pressure loss in purge flow mixing, Equation (23) gives the purge station entropy rise as given in Equation(s) (30).

$$
\begin{aligned}
& \dot{\Sigma}_{\text {purge }, 1}=\dot{m}_{4.1 i} s_{4.1 i}-\left(\dot{m}_{p 1} s_{p, 1}+\left(\dot{m}_{4.1 i}+\dot{m}_{p 1}\right) s_{4.2}\right) \\
& \dot{\Sigma}_{\text {purge }, 2}=\left(\dot{m}_{4.2}+\dot{m}_{s c}\right) s_{4.2}-\left(\dot{m}_{p 2} s_{p, 2}+\left(\dot{m}_{4.3}\right) s_{4.3}\right)
\end{aligned}
$$

For the stator and rotor stations, an advanced calculation methodology, which is applied the same for both component types, is given by Young and Wilcock [7]. In this methodology, the entropy rise across the rotor $\left(\dot{\Sigma}_{r}\right)$ or stator $\left(\dot{\Sigma}_{S}\right)$ is assumed to be formed by the linear combination of basic and cooling contributions and given in Equation (31).

$$
\dot{\Sigma}_{S / r}=\dot{\Sigma}_{s / r, b a s i c}+\dot{\Sigma}_{s / r, \text { cool }}
$$

In Equation (31), and throughout the rest of this chapter subscript "s/r" indicates that the loss term is valid for both stator and rotor stages. The first term is the basic loss, which is an inevitable parameter because it is generated due to the irreversible expansion of the working fluid. The second term is solely related to cooling related losses, and it is assumed to be formed by several sub-contributions, depending on the applied cooling technology and is illustrated schematically in Figure 7. Each entropy term will be explained in detail, in the following sub-sections. 


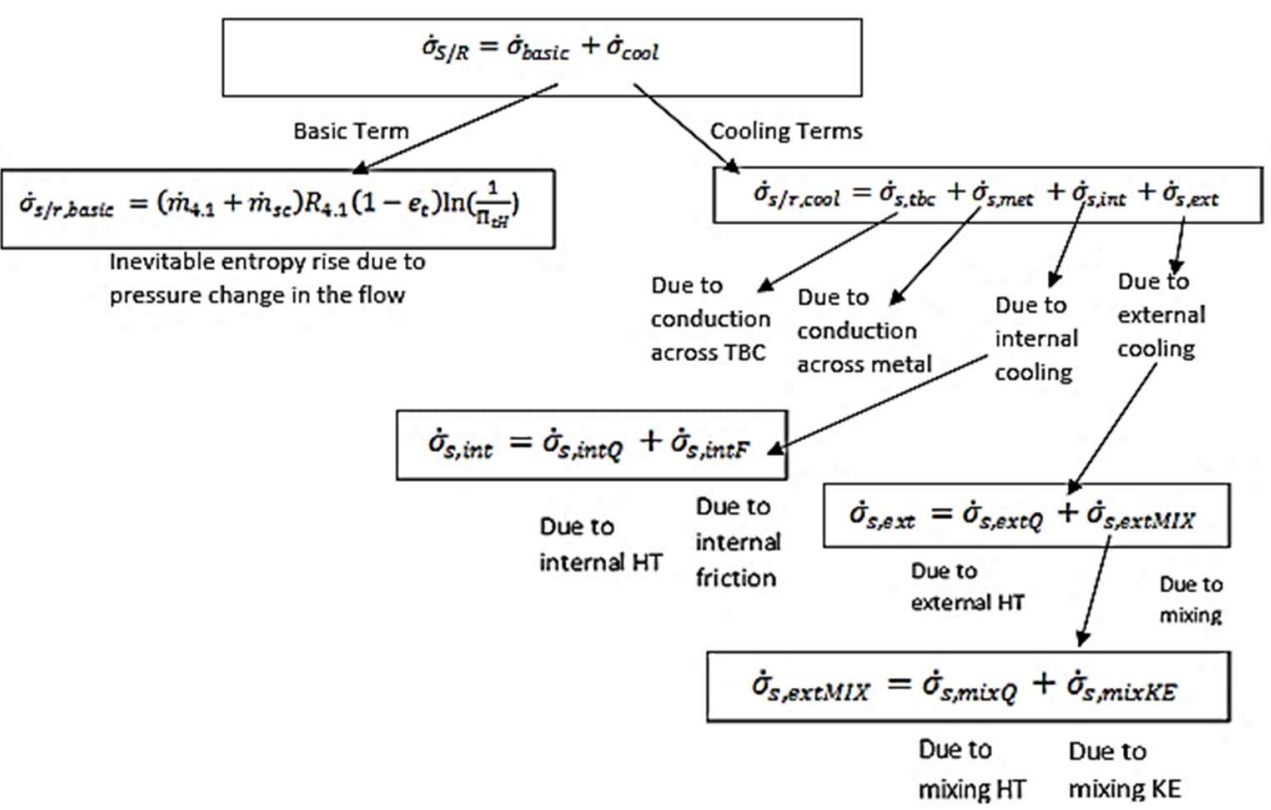

Figure 7: Schematic of the model for stator/rotor entropy generation term calculations from Young and Wilcock [7]

\subsubsection{Basic Loss Term $\left(\dot{\Sigma}_{s / r, b a s i c}\right)$}

This is the basic rate of entropy change of the uncooled component due to irreversible expansion of the working fluid. This term includes all secondary losses that are not related to the cooling of the turbine, and it is given in Equation(s) (32).

$$
\begin{aligned}
& \dot{\Sigma}_{s, \text { basic }}=\left(\dot{m}_{4.1 p i}\right) R\left(1-e_{t, \text { basic }}\right) \ln \left(\left(\frac{P_{t 4.1 p}}{P_{t 4.2 i}}\right)_{\text {uncooled }}\right) \\
& \dot{\Sigma}_{r, \text { basic }}=\left(\dot{m}_{4.2 i}\right) R\left(1-e_{t, \text { basic }}\right) \ln \left(\left(\frac{P_{t 4.3 i}}{P_{t 4.4 i}}\right)_{\text {uncooled }}\right)
\end{aligned}
$$

In Equation(s) (32), the pressure ratios are for the same turbine stage without cooling, and et,basic indicates the uncooled turbine polytropic efficiency, which is higher than cooled turbine polytropic efficiency (et,c) because it does not include cooling losses. The effect of cooling losses on the stage pressure ratio is added to calculations through the $\dot{\Sigma}_{s / r, \text { cool }}$ term.

\subsubsection{Cooling Loss Term $\left(\dot{\Sigma}_{s / r, \text { cool }}\right)$}

Depending on the applied cooling technology, this term is formed as a linear combination of several loss terms as shown in Equation (33).

$$
\dot{\Sigma}_{s / r, \text { cool }}=\dot{\Sigma}_{s / r, t b c}+\dot{\Sigma}_{s / r, m e t}+\dot{\Sigma}_{s / r, i n t}+\dot{\Sigma}_{s / r, e x t}
$$

The assumed 1D heat transfer model used for the cooled blade and the associated entropy terms of Equation (33) from [7] are shown in Figure 8. 


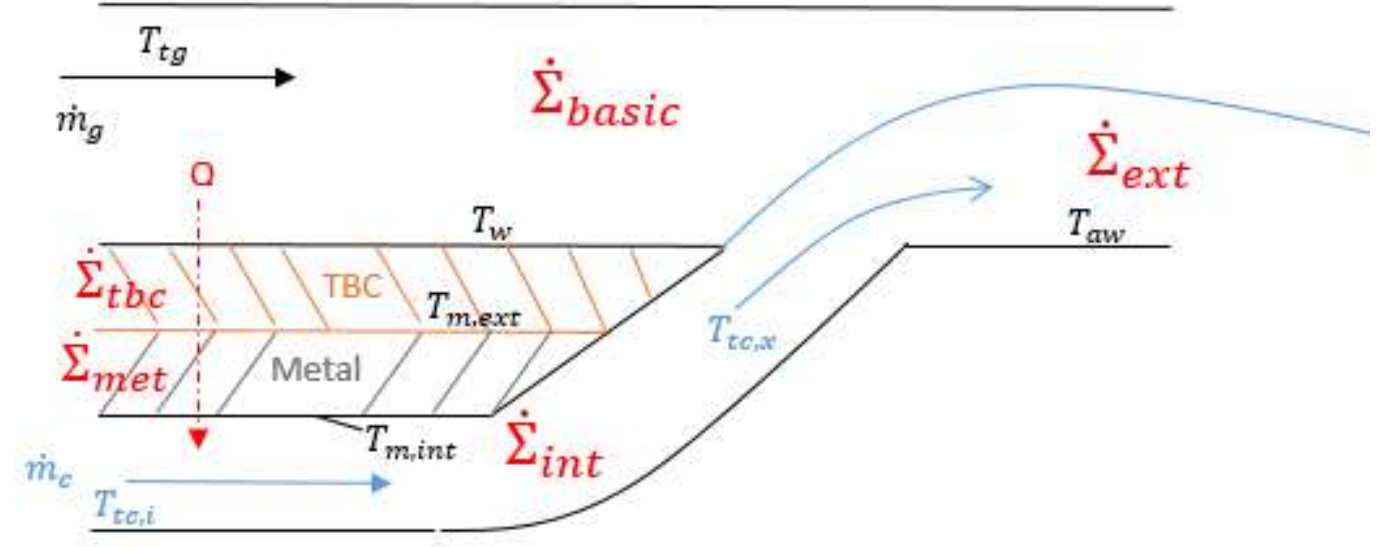

Figure 8: The heat transfer model used in the derivation of cooling entropy terms is shown with associated entropy terms for a blade having internal and external cooling (film cooling) with a TBC layer

\subsection{TBC Conduction Heat Transfer Loss Term $\left(\dot{\Sigma}_{s / r, t b c}\right)$}

This loss occurs due to the temperature difference across the TBC layer ( $T_{w}$ and $T_{m \text {,ext }}$ of Figure 8 ) and can be derived by using a conduction heat transfer analysis across the TBC layer. The term is defined as in Equation (34) by Young and Wilcock [7].

$$
\dot{\Sigma}_{t b c}=\dot{Q}\left(\frac{1}{T_{m, e x t}}-\frac{1}{T_{w}}\right)
$$

In Equation (34), the heat transfer rate $\dot{Q}$ can be found from Equation (35).

$$
\dot{Q}=\frac{k_{t b c}}{t_{t b c}} A_{\text {surf }}\left(T_{w}-T_{m, e x t}\right)
$$

Using a TBC material with lower thermal conductivity or applying a thicker layer of TBC would reduce the heat transfer rate and result in a reduction of this loss term.

\subsection{Blade Metal Conduction Heat Transfer Loss Term $\left(\dot{\Sigma}_{s / r, \text { metal }}\right)$}

This loss occurs due to the temperature difference across the two sides of the blade metal $\left(T_{m \text {,int }}\right.$ and $T_{m, \text {,ext }}$ of Figure 8) and can be derived by using a conduction heat transfer analysis across the blade metal. The term is defined as in Equation (36) by Young and Wilcock [7].

$$
\dot{\sigma}_{m e t}=\dot{Q}\left(\frac{1}{T_{m, i n t}}-\frac{1}{T_{m, e x t}}\right)
$$

The heat transfer rate $\dot{Q}$ of Equation (36) can be found from Equation (37).

$$
\dot{Q}=\frac{k_{m e t}}{t_{m e t}} A_{\text {surf }}\left(T_{m, e x t}-T_{m, i n t}\right)
$$


This loss term can also be considered as an inevitable loss term, since there will always be a temperature difference on the two sides of the blade wall. Reduction of this loss is possible with low thermal conductivity materials.

\subsection{Internal Cooling Losses $\left(\dot{\Sigma}_{s / r, i n t}\right)$}

This is the rate of entropy generation between the coolant supply location (usually at the blade hub) and the blade internal channels. This loss is assumed to be formed by a linear combination of a convection heat transfer and fluid friction loss term and given in Equation (38).

$$
\dot{\Sigma}_{\text {int }}=\dot{\Sigma}_{\text {int }, Q}+\dot{\Sigma}_{\text {int }, F}
$$

\subsection{Internal Heat Transfer Losses $\left(\dot{\Sigma}_{\text {int }, Q}\right)$}

These losses are due to the temperature and pressure difference between the supply and the blade internal cooling channel network. A 1D convection heat transfer analysis for pipe flows is made on the control volume shown in Figure 9 in the derivation of this loss term.

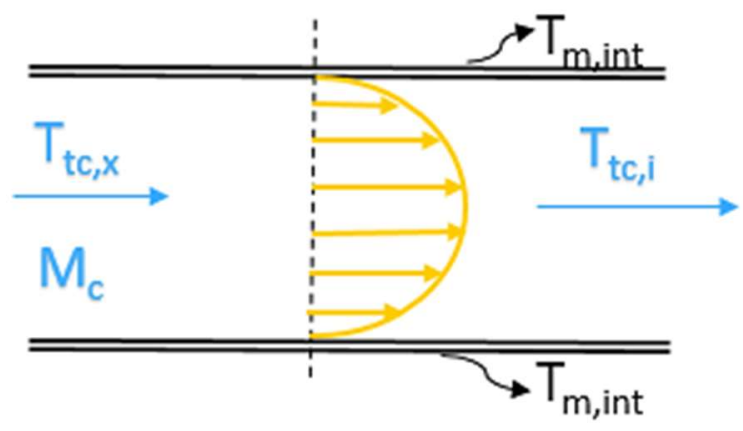

Figure 9: Assumed 1D Convection Heat Transfer Analysis that is applied in the derivation of the loss term

Derivation results in the loss term given in Equation (39) from Young and Wilcock [7].

$$
\dot{\Sigma}_{i n t Q}=\dot{m}_{c l} c_{p, c l}\left[\left(1+\frac{\gamma_{c l}-1}{2} M_{c l}^{2}\right) \ln \left(\frac{T_{t c l, x}}{T_{t c l, i}}\right)-\frac{T_{t c l, x}-T_{t c l, i}}{T_{m, i n t}}\right]
$$

Reduction of this loss term can be done with a good internal cooling geometry design, which has high level of efficiency.

\subsection{Internal Friction Losses $\left(\dot{\Sigma}_{\text {int }, F}\right)$}

This term includes all frictional losses generated as the coolant fluid flows through the supply pipes of the cooling system. If the internal cooling system supplies coolant to the external cooling system, then this term also includes the effects of Boundary Layer friction.

A heat transfer boundary layer analysis for 1D pipe flow is made on the same control volume of Figure 9. Derivation results in the loss term, that includes terms related to pipe friction and Boundary Layer friction as shown in Equation (40). 


$$
\dot{\Sigma}_{i n t F}=\dot{m}_{c l} c_{p, c l}\left[\ln (\underbrace{T_{t c l, x}}_{t c l l, k})-\left(1+\frac{\gamma_{c l}-1}{2} M_{c l}^{2}\right) \ln \left(\frac{T_{t c l, x}}{T_{t c l, i}}\right)\right]-\dot{m}_{c} R_{c} \ln \left(\frac{P_{t c, l x}}{P_{t c l, i}}\right)
$$

\section{Related to}

pipe friction

\section{Related to}

A possible remedy to reduce losses associated with this source, is to use higher momentum flux ratio in the external cooling system to speed up the coolant exit flow, and using smoother channels to reduce frictional heating.

\subsection{External Cooling Losses $\left(\dot{\Sigma}_{s / r, \text { ext }}\right)$}

These losses stem from the heat transfer to the coolant flow and the mixing of the coolant flow with the mainstream hot gas. It should be noted that, the Boundary Layer friction of external cooling flow is already included in $\dot{\Sigma}_{\text {intF }}$ term, therefore this term includes the external cooling losses other than the boundary layer friction effects. This loss is assumed to be formed by a linear combination of an external heat transfer and a mixing loss term and given in Equation (41) by Young and Wilcock [7].

$$
\dot{\Sigma}_{\text {ext }}=\dot{\Sigma}_{\text {ext }, Q}+\dot{\Sigma}_{\text {ext,MIX }}
$$

\subsection{External Heat Transfer Loss $\left(\dot{\Sigma}_{\text {ext }, Q}\right)$}

This entropy generation is due to the convection heat transfer occurring between the coolant flow and the hot mainstream gas, starting from the exit of the cooling holes. The derivation of this term is made with a convection heat transfer analysis on the control volume shown in Figure 10.

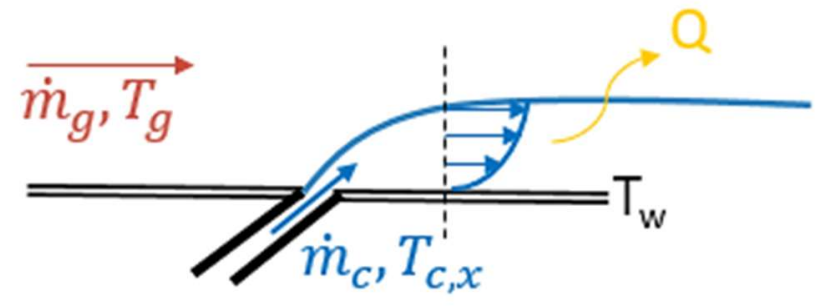

Figure 10: Control Volume used in the 1D Convection Heat Transfer Analysis that is used to derive the external heat transfer loss term

Derivation results in the loss term given in Equation (42) by Young and Wilcock [7].

$$
\dot{\Sigma}_{\text {extQ }}=\dot{Q}\left(\frac{1}{T_{w}}-\left(1+\frac{\gamma_{g}-1}{2} M_{g}^{2}\right) \frac{1}{T_{t g *}}\right)
$$

The heat transfer rate $\dot{Q}$ of Equation (42) is given in Equation (43) from [7].

$$
\dot{Q}=\alpha_{g} A_{\text {surf }}\left(T_{t g *}-\varepsilon_{f c}\left(T_{t g *}-T_{t c, x}\right)-T_{w}\right)
$$


From Equation (43), having a higher film cooling efficiency reduces this loss, as well as having a lower wall temperature by having a high internal cooling efficiency. In the cooling configurations that do not use external cooling, this loss term is eliminated.

\subsection{Mixing Losses $\left(\dot{\Sigma}_{\text {ext,MIX }}\right)$}

This loss occurs due to the injection of the coolant flow into the mainstream and is caused by the dissipation of thermal and kinetic energies during this mixing process. It is modelled as the linear combination of losses for the heat transfer and the change in kinetic energy, and given in Equation (44) from Young and Wilcock [7].

$$
\dot{\Sigma}_{\text {extMIX }}=\dot{\Sigma}_{\text {mixQ }}+\dot{\Sigma}_{\text {mixKE }}
$$

In several cooled turbine models without a $2^{\text {nd }}$ Law Analysis this is the type of loss that is modelled by Shapiro Theory to include the cooling pressure losses in cycle calculations,

\subsection{1 Mixing Heat Transfer Losses $\left(\dot{\Sigma}_{\text {ext,MIXQ }}\right)$}

This loss occurs due to the thermal dissipation from the hot gas to the coolant fluid during the mixing process. The approximate equation is obtained from the momentum and energy equations of mixing flow heat transfer analysis and is given in Equation (45) from Young and Wilcock [7].

$$
\dot{\Sigma}_{\text {mixQ }}=\dot{m}_{c l} c_{p, c l}\left[\ln \left(\frac{T_{g *}}{T_{c l, x}}\right)-\left(1-\frac{T_{c l, x}}{T_{g *}}\right)\right]
$$

In the cooling configurations that do not use external cooling, this loss term is eliminated.

\subsection{2 Mixing Kinetic Energy Loss $\left(\dot{\Sigma}_{\text {ext,MIXKE }}\right)$}

This term covers the entropy rise due to the loss of kinetic energy normal to the blade surface, as the highspeed coolant flow mixes with the slower mainstream flow as shown in Figure 11.

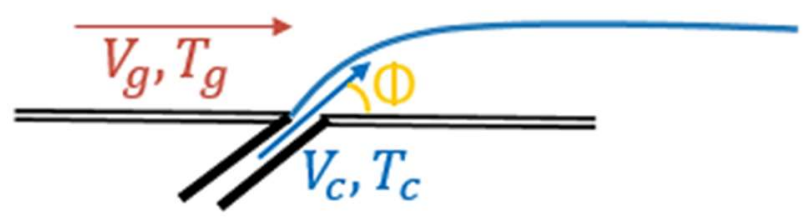

Figure 11: Mixing kinetic energy loss occurs due to mixing of two different flows with different speeds

In Figure 11, the angle $(\Phi)$ represents an effective fluid injection angle, which might be different than geometrical hole incline angle depending on the cooling hole design. By using the notations from Figure 10, this loss term is given by Equation (46) from Young and Wilcock [7]. 


$$
\dot{\Sigma}_{m i x, K E}=\dot{m}_{c l}\left[\frac{V_{g *}{ }^{2}+V_{c l}{ }^{2}-2 V_{c l} \cos \phi}{2 T_{g *}}\right]
$$

Using a film cooling hole that provides lower effective injection angle, lower penetration height for a fixed momentum ratio would be beneficial in reducing such losses.

\subsection{Development of the Cooled Turbine Model}

A cooled turbine algorithm to calculate the thermodynamic states at the cooled turbine exit station, is developed by using the cooled turbine theory discussed in Section 1.1. The algorithm is then integrated into upper level models that are using it to calculate engine performance parameters. The developed algorithm consists of three main sections namely, the coolant calculator, $1^{\text {st }}$ Law Analysis and $2^{\text {nd }}$ Law Analysis; each named after the relevant theory used from Section 1.1.

\subsubsection{Coolant Calculator (Semi-Empirical Method)}

The coolant calculator block is responsible for the coolant fraction calculations for each stage component, and is based on the semi-empirical approach in Section 1.1.1. The cooling type for each component is known from the user inputs, and the appropriate correlation method is selected per this schedule. Internal and external Stanton numbers are used as constants in this section. The flowchart of this section is given in Figure 12.

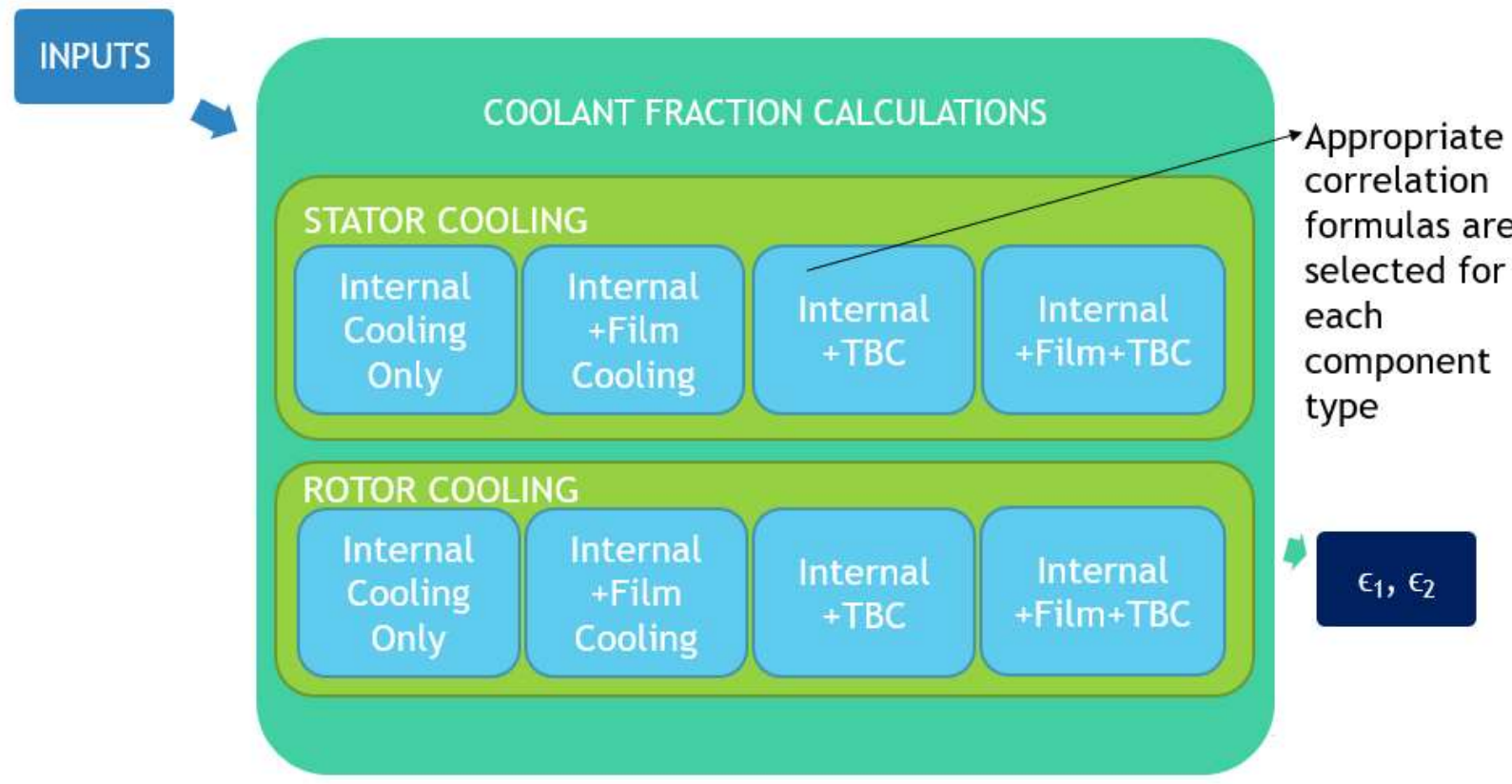

Figure 12: Coolant Calculator block flowchart that calculates stator-cooling fraction $\left(\varepsilon_{1}\right)$ and rotor-cooling fraction ( $\left.\varepsilon_{2}\right)$ 


\subsection{2. $1^{\text {st }}$ Law Analysis}

The first law analysis calculates the enthalpy drop due to cooling and mixing with the equations derived in Section 1.1.2. This sub-routine also includes thermodynamic property tables for the pure air and fuel combustion products to obtain the total temperatures needed in the remaining sections of the analysis. Coolant fractions for each cooled turbine component is used from the outputs of the coolant calculator block. The flowchart is given in Figure 13.

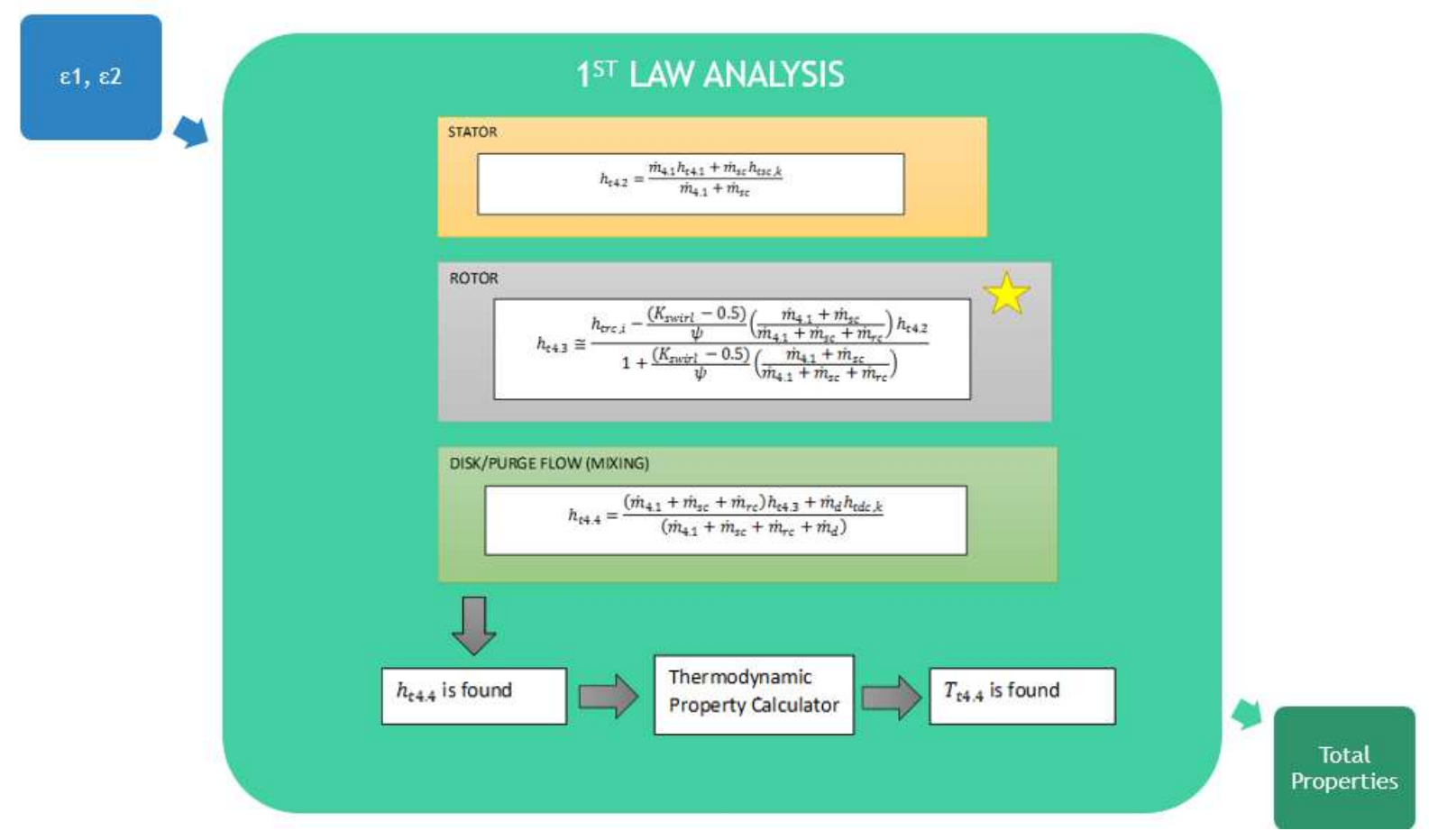

Figure 13: 1st Law Analysis section of the cooling analysis model

\subsection{3. $2^{\text {nd }}$ Law Analysis}

This sub-routine calculates the entropy rise across the stage per the approach summarized in Section 1.1.3. Required coolant mass flow rates and temperatures are used from the results of coolant calculator and $1^{\text {st }}$ Law Analysis subroutines, respectively. The flowchart for this section is given in Figure 14. 


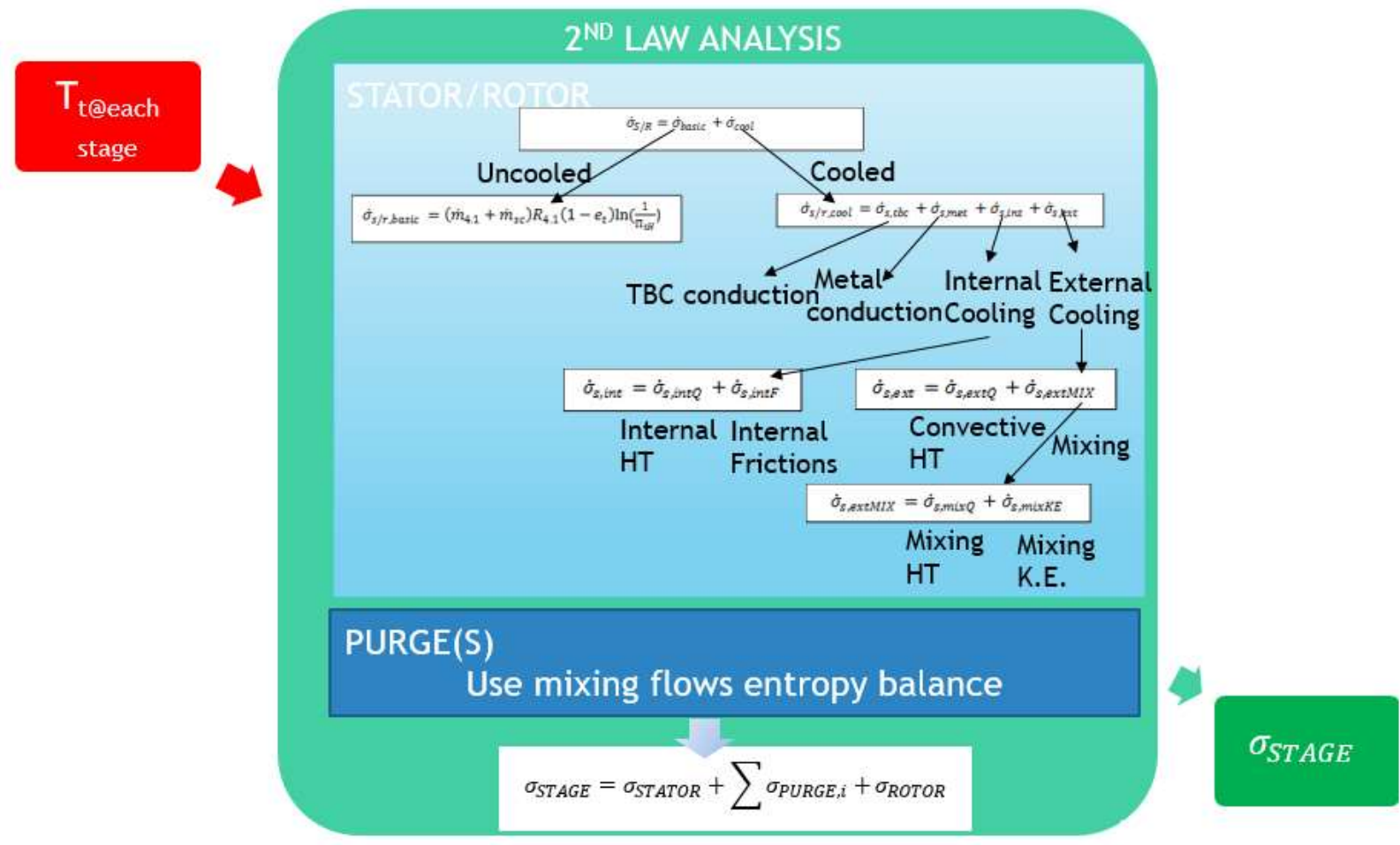

Figure 14: $2^{\text {nd }}$ Law Analysis section of the cooling analysis model

By using the three subroutines based on the analysis by Young and Wilcock [7], the cooling analysis algorithm is developed as shown in Figure 15. It contains two nested loops with the inner loop performing the cooling analysis for each stage component. The outer loop repeats the process for each turbine stage and calculates the cooled turbine exit values such as total enthalpy, total exit temperature, and total exit pressure, which are used in cycle calculations.

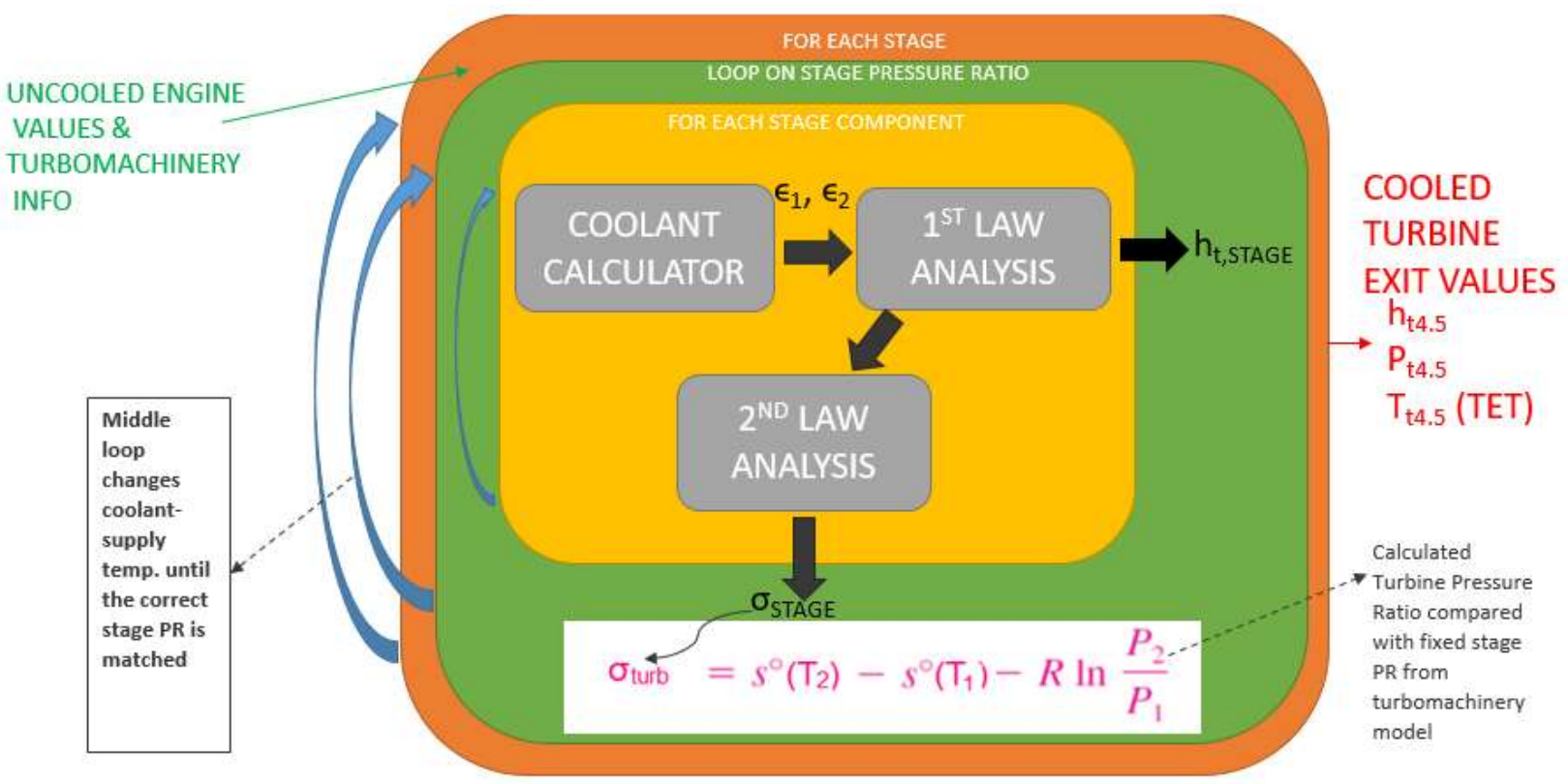

Figure 15: Cooling Analysis Model Flowchart including the sub-models 
The defined algorithm was modelled in MATLAB/Simulink® to use it within the Engine Design Model (EDM) developed by Uysal [13].

\subsection{Validation of the Calculation Algorithm}

The standalone Cooled Turbine Model (CTM) that uses the algorithm with the details given in Section 1.2 was verified with the example case results from Young and Wilcock [7] for a single staged turbine using pure air in both hot gas and coolant sides. Validations are carried out for the coolant calculator and 1 st Law Analysis sections together, and for the $2^{\text {nd }}$ Law Analysis separately. Selected model output parameters from the example case in [7] was used in direct comparison of the results obtained by CTM.

\subsubsection{Validation of the Coolant Calculator and $1^{\text {st }}$ Law Analysis Sub-Systems}

Mass flow rates and component exit total temperatures are the main parameters shown in that comparison. In Table 3, close estimations in cooling fractions show the correctness of the coolant fraction calculations, and close total temperature results show the correctness of the enthalpy equations derived ( $1^{\text {st }}$ Law) as used in the model. Small discrepancies are due to the differences in values used from the thermodynamic property tables of pure air.

Table 3 Validation of the coolant calculator and 1st Law analysis results are done with the Young and Wilcock [7]

\begin{tabular}{|l|l|l|}
\hline Parameter & CTM & Young and Wilcock [7] \\
\hline Stator & \multicolumn{2}{|l|}{} \\
\hline Cooling Fraction & 0.145 & 0.145 \\
\hline Exit Total Temperature (K) & 1598 & 1603 \\
\hline Rotor & \\
\hline Cooling Fraction & 0.050 & 0.049 \\
\hline Exit Total Temperature (K) & 1484 & 1487 \\
\hline Calculated Pressure Ratio & 0.41 & 0.42 \\
\hline
\end{tabular}

\subsubsection{Validation of the $2^{\text {nd }}$ Law Analysis Sub-System}

This part of the validation was carried out by using the graph from Young and Wilcock [7], that provides the changes in entropy terms for different values of the $K_{\text {cool }}$ parameter of Equation (14). The graph from [7] shows the entropy rise from given loss source regardless of their relative amount of contributions to overall entropy rise across the cooled turbine. This graph was plot digitized for a fixed $\mathrm{K}_{\text {cool }}$ value of 0.045 and units were converted to BE units for this work. Comparison of the results of the $2^{\text {nd }}$ Law analysis sub-system of CTM are then made with these plot-digitized values. The results comparison of individual entropy terms from CTM with the plot digitized values from [7] for stator stage are shown in Figure 16. 


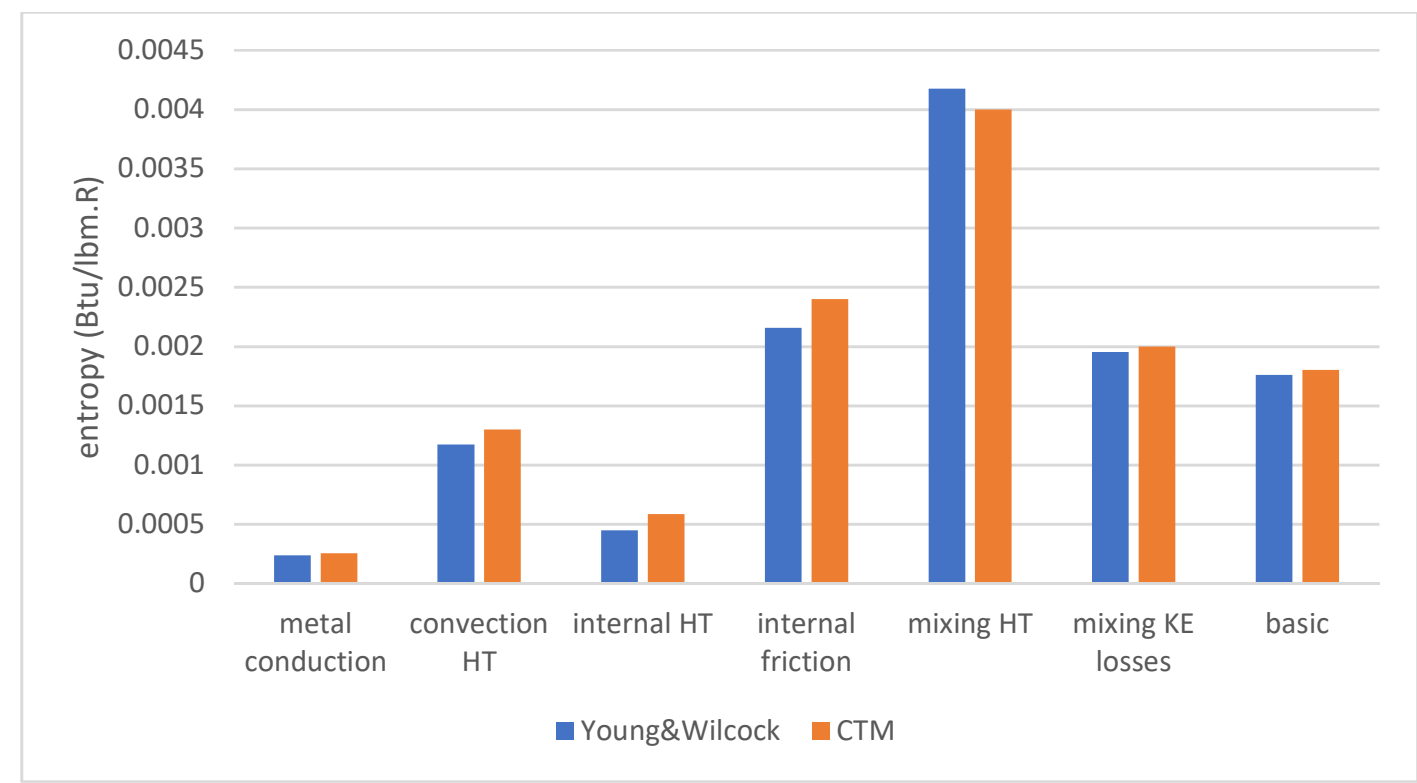

Figure 16: Comparison of stator entropy rise calculated for each loss source between the CTM and Young et al. [7]

For the same cooled turbine stage, individual entropy terms of the rotor are compared in Figure 17.

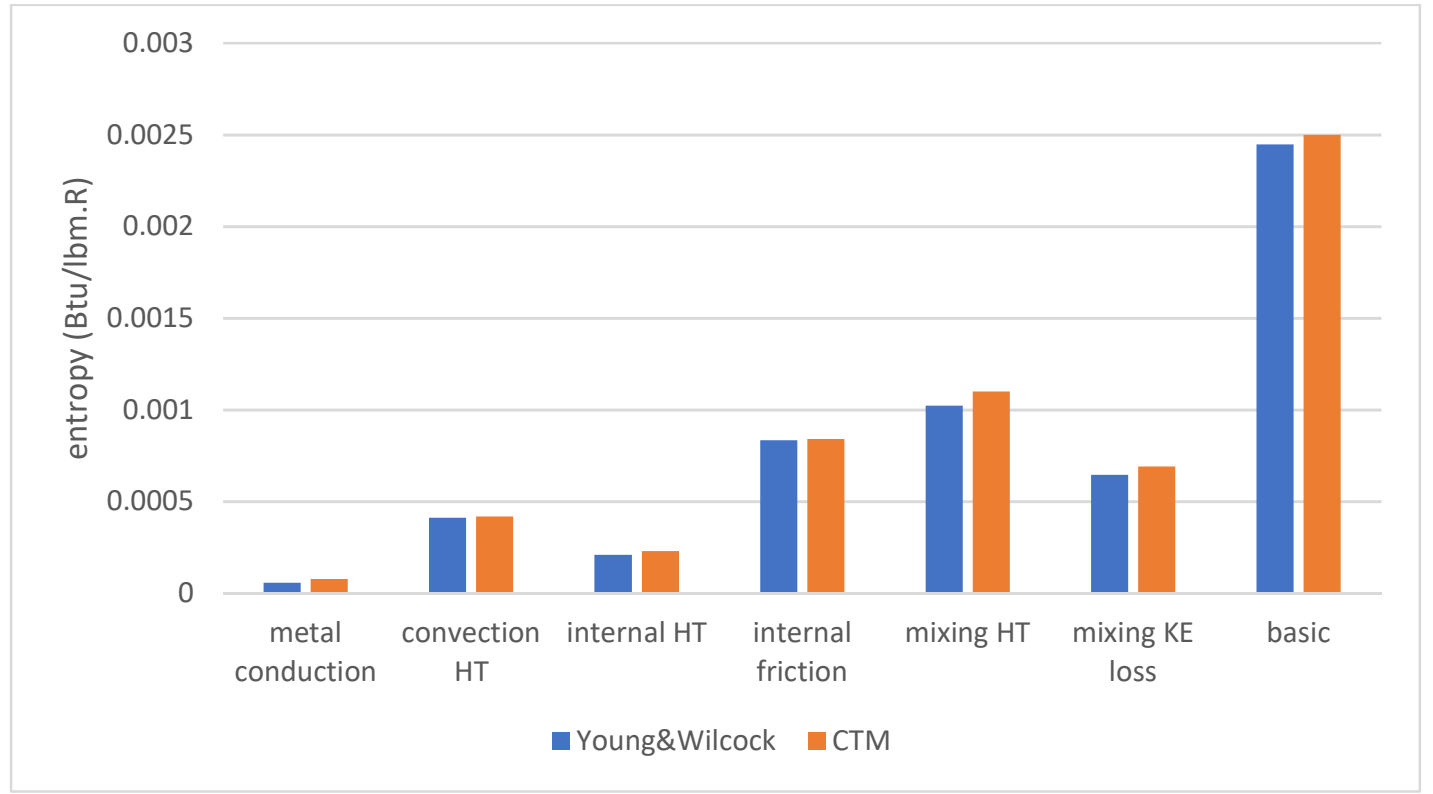

Figure 17: Comparison of rotor entropy rise calculated for each loss source between the CTM and Young et al. [7]

The differences in results were within the range of error associated from plot digitizing the data [7], and from the differences in values used from the thermodynamic property tables of pure air.

As can be seen from Figure 16 and Figure 17, there is a good agreement between the CTM and the results from Young and Wilcock [7]. Similar trends in the ranking of the loss terms are observed for both rotors and stators, except for the basic loss terms. Basic loss is higher in the rotors due to having higher pressure ratio across the rotor stage than the stator stage. Considering the fact that high entropy causes more deviation from the maximum allowable turbine work (through increased exergy), reducing these loss terms with better cooling design will result in higher gas turbine efficiency. 


\section{CHAPTER 2: DEVELOPMENT OF THE COOLED ENGINE MODELS}

In this part of the research, cooled engine models were developed in MATLAB Simulink® to perform the cycle calculations that calculate engine performance parameters. Cooled engine models are based on the Engine Design Model (EDM) [13] developed for a high by-pass turbofan engine. The EDM is an aerothermal engine performance calculation model that includes on-design, off-design, and a turbomachinery design section for high, mid and low by-pass turbofan engines. The model is based on the theory given by Mattingly [12], which is based on the conservation of mass and energy of the flow across the gas turbine components. The model assumes a steady-state quasi-1D flow with variable specific heat, and uses mean-line calculations with free vortex design in the turbomachinery section. Polytropic efficiencies include the effects of pressure and aerodynamic losses in compressor sections and cooling losses in the turbine sections. The EDM was validated both with AEDSys Software [12] and published engine data for CFM-56 and GE90 engines.

In EDM, coolant flows were assumed to be mixed with the main flow through "coolant mixers", which are located before the high pressure turbine first stage stator vane and between the last high pressure turbine rotor and before the first stator vane of low pressure turbine. The amounts of coolant flows were taken from the user inputs. In the new models, cooling calculations are made within a sub-system named "HPT Cooling Analysis" that includes the Cooled Turbine Model (CTM) Algorithm and calculates the cooled turbine exit thermodynamic states.

The turbofan engine version of the cooled engine model was named "Cooled Engine Design Model (CEDM)", and the industrial gas turbine version was named "Cooled Gas Turbine Model (CGTM)".

\subsection{Preliminaries}

Integration of the Cooled Turbine Model algorithm into engine cycle performance calculations is done through the development of "HPT Cooling Analysis" sub-system, which has the following considerations:

- CTM model should allow multi-stage cooling configurations

- The coolant supply temperature ( $T_{\mathrm{tcl}}$, extr $)$ information for each cooled turbine stage should be from appropriate compressor stage, which in practice is determined by the pressure values of compressor stages

- The coolant supply temperatures ( $T_{t c l, ~ i n}$ ) should be obtained by the multiplication of the compressor stage temperature with a gain factor representing the thermal heating and frictional losses of the coolant flow from the compressor to turbine cooling supply location

- Cooled turbine's pressure ratio should be calculated and compared to uncooled turbine's pressure ratio for consistency of calculations

- Outputs of the algorithm should be the stage exit thermodynamic states (total enthalpy, total temperatures and pressures) and total coolant flow fractions that can be used directly in the engine cycle calculations 


\subsubsection{Calculating the Coolant Supply Temperatures ( $\left.T_{t c l, ~ e x t r}\right)$}

In EDM, mean-line turbomachinery design section calculates the pressure and temperature information of each rotating turbomachinery component by using flow turning angles and flow speeds [13]. For the compressor section, this information can be used to extract correct coolant supply temperatures that will be used within the cooled turbine analysis. In order to accomplish this, an algorithm is developed that reads the total pressures at each compressor stage, compares these with total pressures at each turbine stage and determines which compressor stages will be used to extract the coolants. The algorithm decides to pull the coolant from a certain compressor stage if the stage pressure is greater than the corresponding turbine stage pressure. Once the coolant extraction stages are known, the coolant total temperatures at the extraction points $\left(T_{\mathrm{cl}, \mathrm{extr}}\right)$ are used from the stage total temperature information, which are already calculated in the turbomachinery sub-system.

From the coolant extraction point at the compressor to the coolant injection points at the turbine blades, the coolant temperature usually increases due to frictional effects within the supply channels and a small amount of heat transfer from the combustor chamber. This heat addition is modelled as a constant gain multiplier (Ktemp, gain) as given in Equation (47), which has a value that will be found later within the cooling analysis loops.

$$
T_{t c l, i n}=K_{t e m p, g a i n} \cdot T_{t c l, e x t r}
$$

Therefore, the coolant supply temperature $\left(T_{\mathrm{tcl}}\right.$, in $)$ at the turbine blades will have different values in different cooled turbine stages, if the coolants are extracted from different compressor stages. The pressure loss of the coolant supply channels was modelled with another gain parameter that is multiplied with the compressor stage pressures and included in the algorithm that is used in determining the coolant extraction stages.

\subsubsection{Extension of the CTM to be used in Multi-Stage Turbines}

In the case of the multi-staged cooled turbines, calculation of the stage pressure ratio through the use of CTM Algorithm described in Section 1 requires further considerations. In a study that makes use of cooled turbine entropy increase in a more effective thermodynamic efficiency representation by Horlock and Torbidoni [14], application of the cooled turbine modelling with $2^{\text {nd }}$ Law analysis by Young and Wilcock [7] was made and an outer loop on turbine exit temperatures was suggested by the authors to compute the cooled turbine thermodynamic states. However, given that the algorithm in this study was starting with an estimation of turbine exit temperature, solving the $1^{\text {st }}$ Law Analysis equations was done in the reverse order given in Figure 12.

Analysis of the same solution technique with the order considered in CTM showed that the required iterative solution can also be done by changing the coolant supply temperature $\left(T_{t c l}\right.$, in) instead and calculating the respective turbine exit temperature. When the $2^{\text {nd }}$ Law (given in Equation (23)) is balanced, the iterations are stopped and a respective turbine exit temperature is calculated. Here, the iteration temperature refers to the temperature at the end of the coolant supply lines originating from the compressor, and this is done through changing the constant gain multiplier ( $K_{\text {temp, gain }}$ ) value of Equation (47). This temperature gain is expected to be within $1-4 \%$ for both aero and industrial gas turbines.

The extended CTM algorithm contains 3 nested loops, of which the outer and innermost ones being the same as with the original CTM algorithm for a single stage, shown in Figure 15. The middle loop determines $\mathrm{K}_{\text {temp, }}$ gain, which is reduced by $0.1 \%$ in every loop until matching the cooled stage pressure ratio. 
Matching of the cooled turbine's stage pressure ratio with the uncooled turbine is made in the outer loop and it uses a relation that is found by using the turbine pressure ratio relations obtained in Equation(s) (12), which assumes constant pressure ratio across the stage. With this assumption and using the theory in Section 1.1, $\Delta s$ terms of Equation (12) becomes as given in Equation(s) (48).

$$
\begin{gathered}
\Delta s_{u c}=\sigma_{\text {basic }} \\
\Delta s_{c}=\sigma_{\text {basic }}+\sigma_{\text {cool }}
\end{gathered}
$$

Then for the cooled turbine's stage pressure ratio, Equation (12b) can be re-written as in Equation (49).

$$
\pi_{t, c i}=\frac{P_{t 4.4 i}}{P_{t 1}}=e^{\frac{\sigma_{\text {basic }}+\sigma_{\text {cool }}}{R\left(e_{t, c}-1\right)}}=e^{\frac{\sigma_{\text {basic }}}{R\left(e_{t, c}-1\right)}} e^{\frac{\sigma_{c o o l}}{R\left(e_{t, c}-1\right)}}
$$

For the uncooled turbine's pressure ratio, using Equation (12a) with Equation (48a) result in the expression given in Equation (50).

$$
\pi_{t, u c i}=\frac{P_{t 4.4 i, u c}}{P_{t 1}}=e^{\frac{\sigma_{\text {basic }}}{R\left(e_{t}-1\right)}}
$$

Multiply and divide the first exponent of Equation (49) with (et-1) and re-arrange to obtain Equation (51).

$$
\begin{gathered}
\pi_{t, c i}=e^{\frac{\sigma_{\text {basic }}\left(e_{t}-1\right)}{R\left(e_{t, c}-1\right)\left(e_{t}-1\right)}} e^{\frac{\sigma_{c o o l}}{R\left(e_{t, c}-1\right)}}=e^{\frac{\sigma_{\text {basic }}\left(e_{t}-1\right)}{R\left(e_{t}-1\right)\left(e_{t, c}-1\right)}} e^{\frac{\sigma_{c o o l}}{R\left(e_{t, c}-1\right)}} \\
\pi_{t, c i}=\left(e^{\frac{\sigma_{b a s i c}}{R\left(e_{t}-1\right)}}\right)^{\frac{\left(e_{t}-1\right)}{\left(e_{t, c}-1\right)}} e^{\frac{\sigma_{c o o l}}{R\left(e_{t, c}-1\right)}}
\end{gathered}
$$

In Equation (51), the term in parenthesis is the uncooled turbine's stage pressure ratio (from Equation (50)). Replacing this term and re-arranging Equation (51) gives a relation between the stage pressure ratios of the cooled and uncooled turbine stages as in Equation (52).

$$
e^{\frac{\sigma_{c o o l}}{R\left(e_{t}-1\right)}} \pi_{t, u c i}=\Delta \pi \cdot \pi_{t, u c i}=\pi_{t, c i} \frac{\left(e_{t, c}-1\right)}{\left(e_{t}-1\right)}
$$

In Equation (52), $\Delta \pi=e^{\frac{\sigma_{\text {cool }}}{R\left(e_{t}-1\right)}}$ represents a change in the stage pressure ratio due to turbine cooling. A study by Esgar and Ziemer [15] on the effects of cooling on aero-engine performance states that there is an expected pressure drop for the cooled turbine due to the cooling losses which yields a reduction in turbine exit pressure and the thrust. Same study indicates that the amount of this pressure loss and the effect on the turbine performance might be different for different engine operating conditions (turbine inlet temperatures, fuel fraction, etc.).

Quantification of this pressure loss is needed to define the outermost loop of the extended CTM algorithm. The example case used in the validation made in Section 1.3 uses inputs valid for recent gas turbine technology and maximum $\sigma_{\text {cool }}$ is expected in stator stages due to being the first component of the stage that is exposed to the highest gas temperatures. Using $\sigma_{c o o l}$ from stator and assuming the uncooled turbine stage 
polytropic efficiency within $[0.89,0.98]$ interval for recent gas turbine technology, the maximum expected loss value should be $5 \%$ of the uncooled turbine pressure ratio.

In Equation (52), the exponent of the cooled turbine's stage pressure ratio, $\frac{\left(e_{t, c}-1\right)}{\left(e_{t}-1\right)}$, is still an unknown parameter. For a polytropic expansion in a turbine with polytropic efficiency $e_{t}$ for the uncooled case and $e_{t, c}$ for the cooled case, the polytropic exponents ( $\mathrm{n}$ in $P v^{n}=$ Constant) are as given in Equation(s) (53) [16].

$$
\begin{aligned}
& \frac{n_{u c}-1}{n_{u c}}=e_{t} \frac{\gamma_{t}-1}{\gamma_{t}} \\
& \frac{n_{c}-1}{n_{c}}=e_{t, c} \frac{\gamma_{t}-1}{\gamma_{t}}
\end{aligned}
$$

The polytropic exponents of Equation(s) (53) satisfies the relations given in Equation(s) (54) for the stage inlet (4.1i) and stage exit (4.4i, uc and 4.4i for uncooled and cooled, respectively) stations from the definition of polytropic processes.

$$
\begin{aligned}
P_{t 4.1 i} v_{4.1 i}^{n, u c} & =P_{t 4.4 i, u c} v_{4.4 i, u c}^{n, u c}=\text { Constant }_{1} \\
P_{t 4.1 i} v_{4.1 i}^{n, c} & =P_{t 4.4 i} v_{4.4 i}^{n, c}=\text { Constant }_{2}
\end{aligned}
$$

Definition of differential work given in Equation (5) can be integrated as in Equation(s) (55) to obtain the work due to expansion in the uncooled and cooled stages.

$$
\begin{gathered}
W_{u c}={ }_{4.1 i} W_{4.4 i, u c}=\int_{4.1 i}^{4.4 i, u c} P d v \\
W_{c}={ }_{4.1 i} W_{4.4 i}=\int_{4.1 i}^{4.4 i} P d v
\end{gathered}
$$

Using pressure values in the integrations from Equation(s) (54) and replacing the constants from Equation(s) (54) after the integrations, will result in the work expressions given in Equation(s) (56).

$$
\begin{gathered}
W_{u c}=\frac{P_{t 4.4 i, u c} v_{4.4 i, u c}-P_{t 4.1 i} v_{4.1 i}}{1-n_{u c}} \\
W_{c}=\frac{P_{t 4.4 i} v_{4.4 i}-P_{t 4.1 i} v_{4.1 i}}{1-n_{c}}
\end{gathered}
$$

A relation between the uncooled and cooled stage works can be found by adding and subtracting $P_{t 4.4 i} v_{4.4 i}$ to the nominator of Equation (56a) and re-arranging gives the following relations.

$$
\begin{gathered}
W_{u c}=\frac{P_{t 4.4 i, u c} v_{4.4 i, u c}-P_{t 4.1 i} v_{4.1 i}+P_{t 4.4 i} v_{4.4 i}-P_{t 4.4 i} v_{4.4 i}}{1-n_{u c}} \\
W_{u c}=\frac{P_{t 4.4 i} v_{4.4 i}-P_{t 4.1 i} v_{4.1 i}}{1-n_{u c}}+\frac{P_{t 4.4 i, u c} v_{4.4 i, u c}-P_{t 4.4 i} v_{4.4 i}}{1-n_{u c}}
\end{gathered}
$$


Since the stage exit pressures of the cooled and uncooled turbines are close (differ max. 5\%), assuming $P_{t 4.4 i, u c} \cong P_{t 4.4 i}$ allows the second term to be neglected. Using Equation (56b) on the first term gives a relation between the uncooled and cooled stage work as given in Equation (57).

$$
\begin{gathered}
W_{u c}=\frac{\left(1-n_{c}\right) W_{c}}{1-n_{u c}} \\
\frac{1-n_{u c}}{1-n_{c}}=\frac{W_{c}}{W_{u c}}=K_{H T}
\end{gathered}
$$

In Equation (57), $\mathrm{K}_{\mathrm{HT}}$ is the ratio of cooled to uncooled stage work. Using the definition of polytropic coefficients from Equation(s) (53) and noting that $\gamma_{t}<1.5$ for the working fluids considered in gas turbines, an approximation given in Equation (58) can be found for the $\frac{\left(e_{t, c}-1\right)}{\left(e_{t}-1\right)}$ term of Equation (52).

$$
\frac{\left(1-e_{t, c}\right)}{\left(1-e_{t}\right)} \cong \frac{W_{c}}{W_{u c}}=K_{H T}
$$

This approximation is also used in commercial gas turbine performance calculation software [17], in cooled turbine pressure ratio calculations from uncooled turbine pressure ratio information. Using the definition frm Equation (58) gives the pressure ratio comparison equation for the outermost loop of extended CTM algorithm as in Equation (59).

$$
\Delta \pi \cdot \pi_{t, u c i}=\pi_{t, c i} K_{H T}
$$

For looping purposes, a tolerance value (named as "tol") is defined as $\Delta \pi=1-$ tol. Using the cooled turbine pressure ratio from CTM algorithm (i.e. from Equation (28)) and uncooled turbine stage pressure ratio from uncooled turbine cycle calculations, result in the final form of pressure ratio comparison as given in Equation (60).

$$
(1-t o l) . \pi_{t, u c i}=e^{\frac{\left(\left(s^{0}\left(T_{t 4.4 i}\right)-s^{0}\left(T_{t 4.1 i}\right)\right)-\sigma_{s t a g e, i}\right) K_{H T}}{R}}
$$

When Equation (60) is balanced, stage calculations are finalized, $1^{\text {st }}$ and $2^{\text {nd }}$ Laws are balanced, and the following stage calculations start with calculated previous stage temperature value. The extended algorithm is shown schematically in Figure 18. 


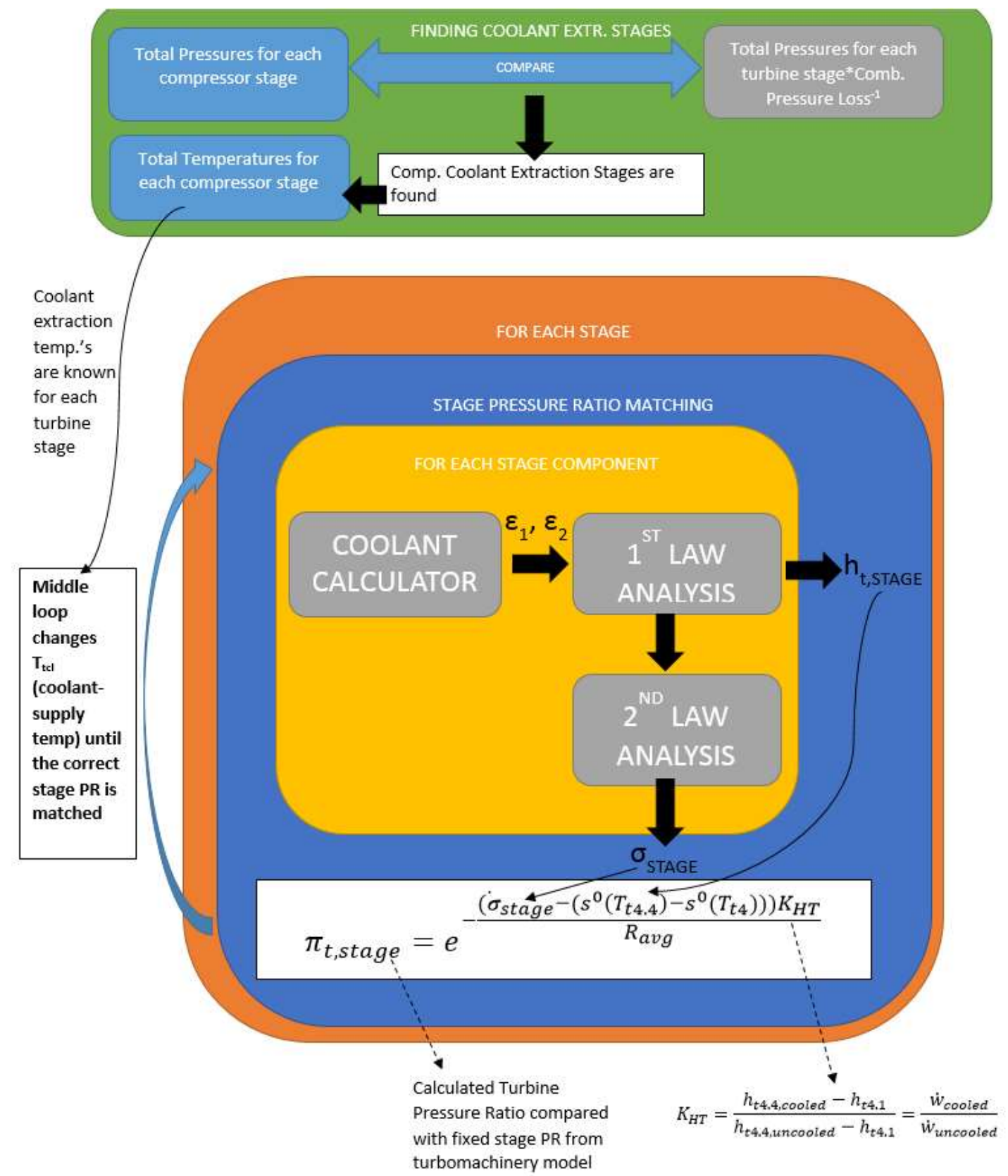

Figure 18: Modification of the CTM Algorithm for multi-staged turbines

\subsubsection{Generating Methane Combustion Property Table Data}

Development of the cooled industrial gas turbine version of cooled engine models (CGTM), requires the use of methane combustion products thermodynamic properties throughout the model. CEDM and its baseline model EDM [13] uses kerosene combustion product thermodynamic property calculators, named as F-AIR sub-models. These sub-models include tables for thermodynamic properties such as reduced pressure, enthalpy, entropy, specific heats and the ratio of specific heats of kerosene-air combustion products for various fuel-to-air ratio conditions. By specifying the fuel-to-air ratio $(f)$ and a known thermodynamic property (either temperature, enthalpy, reduced pressure, or entropy) these sub-models can calculate other thermodynamic properties. 
Using kerosene combustion property tables and changing the Lower Heating Value (LHV) of the fuel to match methane combustion is a solution. However, several resources [18] claim that such an approach may lead to additional errors in individual component performance estimations up to $8 \%$. Therefore, use of correct combustion product information is important to eliminate additional errors in industrial gas turbine calculations.

In order to be in accordance with the input/output parameters used in EDM [13], the same properties (enthalpy, reduced pressure, entropy, $c_{p}$, and $\mathrm{y}$ ) for methane combustion had to be produced. The same reference reaction conditions were used for methane combustion from Mattingly [12] and Chappel and Cockshutt [18] to generate methane combustion tables. For the combustion reaction, a low reference pressure value ( 2 psia was used for kerosene combustion [12]) is often used in generating those tables to get perfect gas properties for combustion products, which is the general assumption for the working fluid in cycle calculations.

The step-by-step procedure to generate combustion property tables is described as follows:

1) A complete methane combustion reaction was written as follows in terms of equivalence ratios (ø), where $\varnothing$ is defined as the ratio of actual $(f)$ to stochiometric fuel-to-air ratio $\left(f_{\mathrm{s}}\right)$ as given in Equation (61). For all equivalence ratios, stochiometric fuel-to-air ratio for methane combustion of 0.058 is used.

$$
\emptyset=\frac{f}{f_{s}}
$$

Four different fuel-to-air ratio $(f)$ conditions $(0.0169,0.0338,0.0507$ and 0.0577$)$ were obtained by changing these equivalence ratios. Standard Air is assumed in determining $\mathrm{O}_{2}(\mathrm{~g})$ and $\mathrm{N}_{2}(\mathrm{~g})$ concentrations. The molar fractions of the products parametrized with the equivalence ratio of the assumed lean combustion for this analysis is shown as follows:

\begin{tabular}{lllllll} 
& $\mathrm{CH}_{4}(\mathrm{~g})+$ & $2 \mathrm{O}_{2}(\mathrm{~g})+$ & $\mathrm{N}_{2}(\mathrm{~g})$ & $\rightarrow$ & $\mathrm{CO}_{2}(\mathrm{~g})+$ & $2 \mathrm{H}$ \\
& $\mathrm{O} O(\mathrm{~g})+\mathrm{N}_{2}(\mathrm{~g})$ \\
$\mathrm{S}:$ & $1 \mathrm{~mol}$ & $(2 / \varnothing) \mathrm{mol}$ & $(7.56 / \varnothing) \mathrm{mol}$ & & & \\
$\mathrm{R}:$ & $-1 \mathrm{~mol}$ & $-2 \mathrm{~mol}$ & - & $+1 \mathrm{~mol}$ & $+2 \mathrm{~mol}$ & $+(7.56 / \varnothing) \mathrm{mol}$ \\
\hline $\mathrm{F}:$ & 0 & $((2 / \varnothing)-2) \mathrm{mol}$ & - & $1 \mathrm{~mol}$ & $2 \mathrm{~mol}$ & $(7.56 / \varnothing) \mathrm{mol}$
\end{tabular}

2) GASEQ Software [19] was used to obtain molar fractions of $\mathrm{CO}_{2}, \mathrm{H}_{2} \mathrm{O}, \mathrm{N}_{2}, \mathrm{O}_{2}$ for each case of equivalence ratios obtained in Step 1 for the methane combustion at constant pressure ( $P_{\text {ref }}=2$ psia).

3) REFPROP Software [20] was used to generate thermodynamic tables, by specifying "new mixture "cases using the molar fractions for combustion products obtained in Step 2. Isoproperty tables were generated for the reaction reference pressure ( $2 \mathrm{psia})$, for the temperature range of $300^{\circ} \mathrm{R}-4000^{\circ} \mathrm{R}$.

4) The values obtained for enthalpy and entropy in Step 3 are not with respect to specific reference values. The reference values obtained from GASEQ Software [19] (step 2) was used to obtain the final enthalpy and entropy values that will be used in "F-AIRng sub-models" that are used in the CGTM to obtain required thermodynamic properties with specified input parameters. 
With the generated values, the enthalpy of combustion products of methane was compared with kerosene-air combustion for the same fuel-to air ratio (0.0169) in Figure 19.

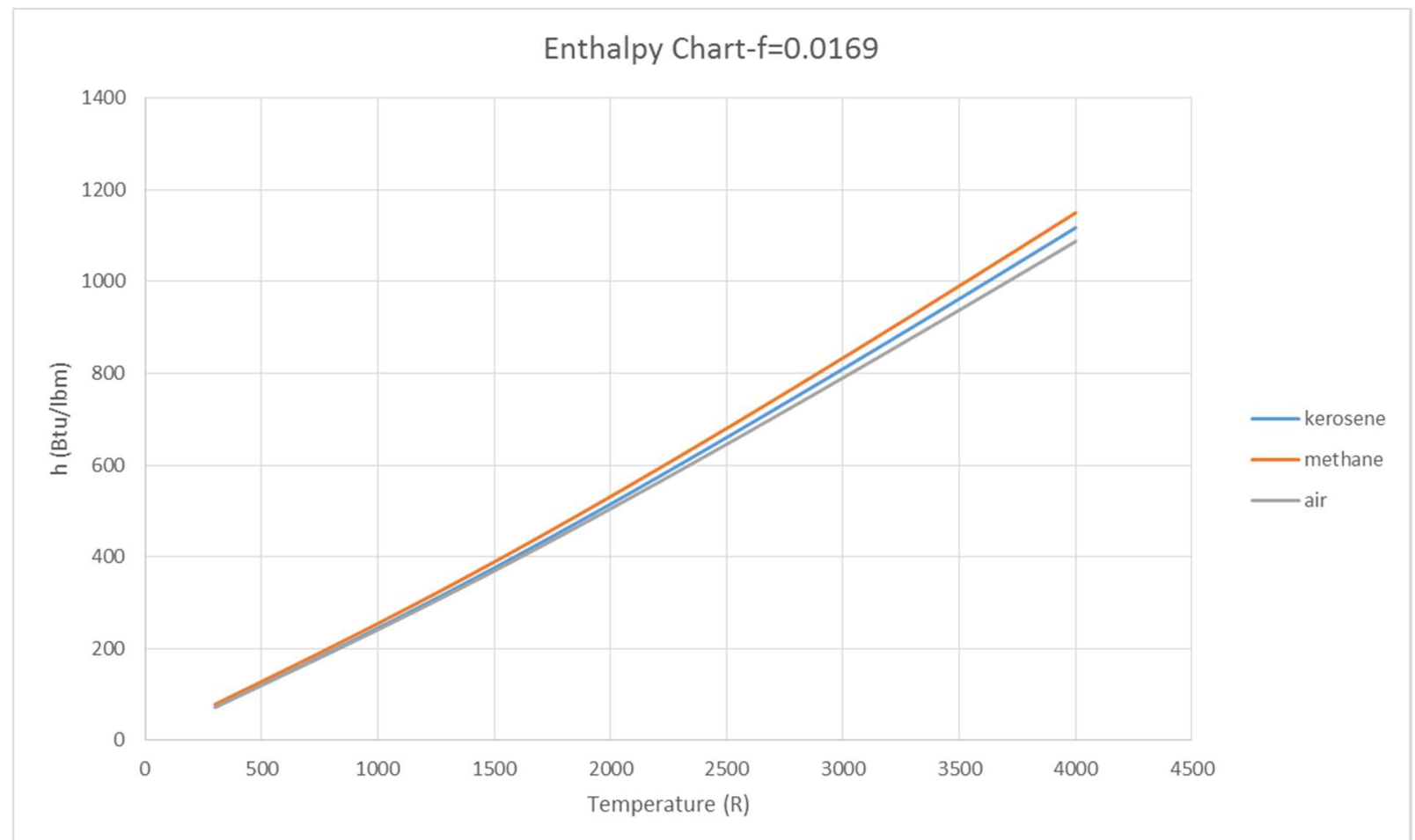

Figure 19: Enthalpy of the pure air, combustion products of methane, and combustion products of kerosene are compared

In Figure 19, methane combustion gives the highest enthalpy for each temperature, as expected (due to higher reaction enthalpy).

Calculated results were compared with the validation data of Guha [21] given for the calculated enthalpy differences $(\Delta h)$ between two specified temperatures for different fuel-to-air ratios for low and high temperature ranges. The validation was done using the same temperature differences in the new F-AIRng calculators, and calculating the corresponding enthalpy differences $(\Delta \mathrm{h})$. The comparison showed agreement with the compared data from [21], and is given in Table 4.

Table 4: Methane combustion properties were validated with the enthalpy scenarios for 5 different fuel-to-air ratios (f) from Guha [21]

\begin{tabular}{|l|l|l|l|}
\hline $\begin{array}{l}\text { Low Temperature Test } \\
\left(\mathbf{T}_{\mathbf{1}}=\mathbf{1 0 8 0} \mathbf{~}^{\mathbf{R}}, \mathbf{T}_{\mathbf{2}}=\mathbf{1 8 0 0} \mathbf{~} \mathbf{~}^{\mathbf{R}}\right)\end{array}$ & $\mathbf{\Delta h}$ (F-AIRng) & $\mathbf{\Delta h}[\mathbf{2 1}]$ & \%Difference \\
\hline $\mathbf{f = 0 . 0 1}$ & $0.452 \mathrm{MJ}$ & $0.450 \mathrm{MJ}$ & $0.44 \%$ \\
\hline $\mathbf{f = 0 . 0 2}$ & $0.464 \mathrm{MJ}$ & $0.461 \mathrm{MJ}$ & $0.65 \%$ \\
\hline $\mathbf{f = 0 . 0 3}$ & $0.474 \mathrm{MJ}$ & $0.471 \mathrm{MJ}$ & $0.64 \%$ \\
\hline $\mathbf{f = 0 . 0 4}$ & $0.485 \mathrm{MJ}$ & $0.481 \mathrm{MJ}$ & $0.83 \%$ \\
\hline $\mathbf{f = 0 . 0 5}$ & $0.495 \mathrm{MJ}$ & $0.491 \mathrm{MJ}$ & $0.81 \%$ \\
\hline
\end{tabular}


Table 4 continued: Methane combustion properties were validated with the enthalpy scenarios for 5 different fuel-to-air ratios (f) from Guha [21]

\begin{tabular}{|c|c|c|c|}
\hline $\begin{array}{l}\text { High Temperature Test } \\
\left(T_{1}=2160^{\circ} R, T_{2}=3600{ }^{\circ} R\right)\end{array}$ & $\Delta \mathrm{h}$ (F-AIRng) & $\Delta \mathrm{h}[21]$ & \%Difference \\
\hline$f=0.01$ & $1.009 \mathrm{MJ}$ & $1.005 \mathrm{MJ}$ & $0.40 \%$ \\
\hline$f=0.02$ & $1.042 \mathrm{MJ}$ & $1.034 \mathrm{MJ}$ & $0.77 \%$ \\
\hline$f=0.03$ & $1.072 \mathrm{MJ}$ & $1.063 \mathrm{MJ}$ & $0.84 \%$ \\
\hline$f=0.04$ & $1.100 \mathrm{MJ}$ & $1.092 \mathrm{MJ}$ & $0.73 \%$ \\
\hline$f=0.05$ & $1.128 \mathrm{MJ}$ & $1.117 \mathrm{MJ}$ & $0.98 \%$ \\
\hline
\end{tabular}

\subsubsection{Determining the Flowchart of the Cooled Engine Models}

In the cooled engine models, the cooled engine performance is treated as an off-design point, that is, a performance cycle modelling approach is used. The cooled engine performance calculation requires results from the uncooled engine in order to:

- Have reasonable initial values that are required in the cooling analysis (such as the number of turbine stages, stage loading, combustor exit temperature and fuel-to air ratio)

- Reduce the calculation time (the cold section (fan-LPC-HPC up to burners) of the uncooled and cooled engines are the same)

- Compare the change in performance when the cooling is applied (as in extended CTM algorithm)

The design point of this performance cycle is therefore, the uncooled engine's performance under the same operating conditions with the cooled engine. Based on this approach, the general flowchart of the cooled engine models should consist of an uncooled on-design and a turbomachinery design together with a cooled off-design section, that includes the extended cooling analysis model developed in Section 2.1.2.

Cooled turbine calculations require parameters from the uncooled engine cycle calculations and turbomachinery design. To obtain this information, the on-design and turbomachinery design sections of EDM are retained in the cooled engine models. A schematic of the cooled engine models with their major subsystems is shown in Figure 20.

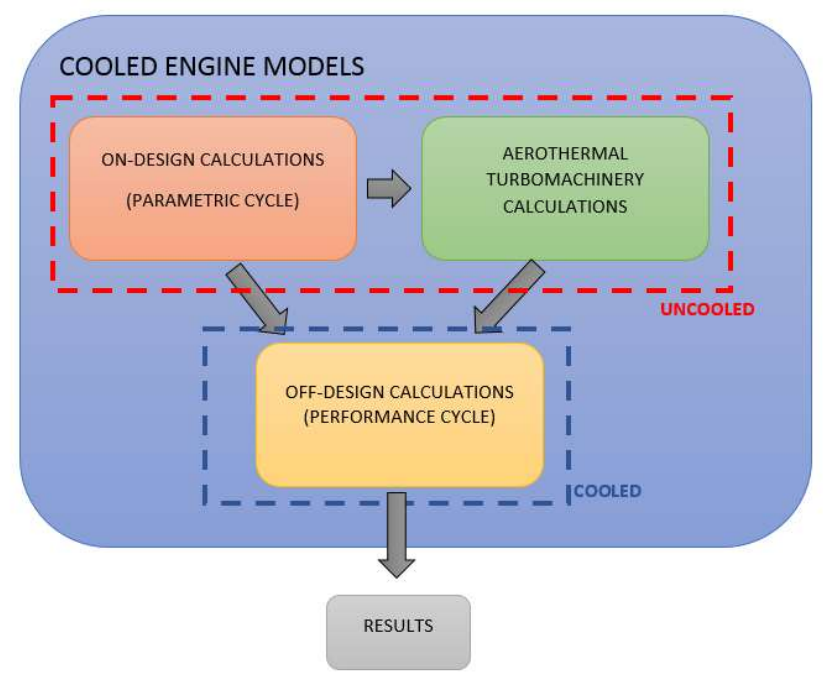

Figure 20: Flowchart for the cooled engine models 


\subsection{Cooled Engine Design Model (CEDM)}

In this section, a cooled engine model for a high by-pass turbofan engine with separate exhausts and dualspool configuration is developed. The engine station numbering used in this model complies with the engine station numbering format used by Mattingly [12], and are shown with the assumed coolant flow paths in Figure 21.

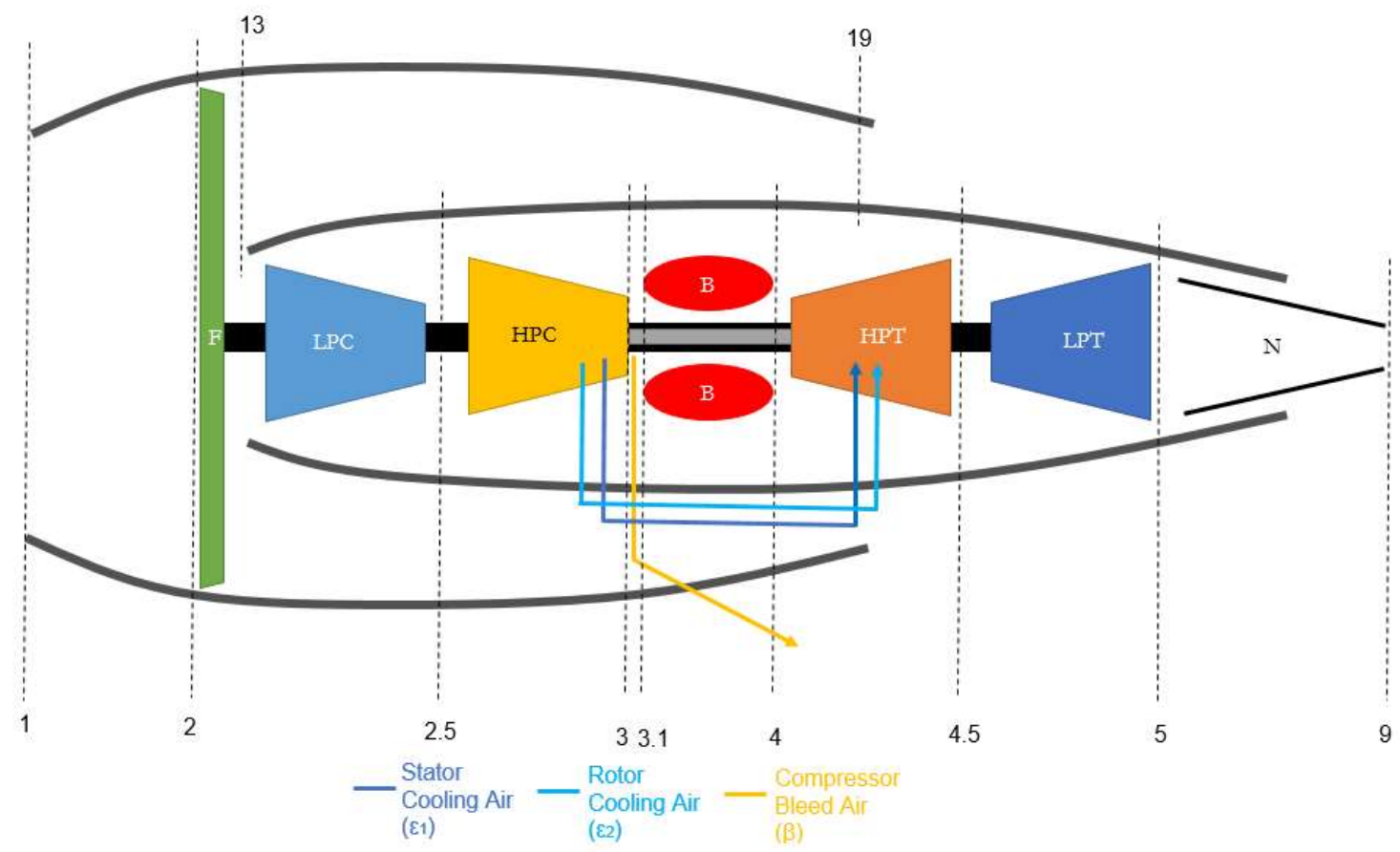

Figure 21: Schematic of the turbofan engine configuration, engine station numbering and coolant flow paths used in CEDM

The descriptions of the engine stations used in CEDM are given in Table 5.

Table 5: Descriptions of Engine Stations used in CEDM

\begin{tabular}{|l|l|}
\hline $\begin{array}{l}\text { Station Number } \\
\mathbf{1}\end{array}$ & $\begin{array}{l}\text { Component Description } \\
\text { Inlet, Diffuser Entry }\end{array}$ \\
\hline Primary Stream & \\
\hline $\mathbf{2}$ & $\begin{array}{l}\text { Diffuser Exit, LPC inlet } \\
\text { LPC Exit, HPC Inlet }\end{array}$ \\
\hline $\mathbf{3}$ & HPC exit, Coolant and/or Bleed Air Extraction \\
\hline $\mathbf{3 . 1}$ & Combustor Inlet \\
\hline $\mathbf{4}(\mathbf{4 . 1 )}$ & Combustor Exit, HPT Inlet \\
\hline $\mathbf{4 . 5}(\mathbf{4} \mathbf{4})$ & HPT exit, LPT inlet \\
\hline $\mathbf{5}$ & LPT exit, Primary Exhaust Inlet \\
\hline $\mathbf{9}$ & Primary Exhaust Exit \\
\hline $\mathbf{S e c o n d a r y ~ S t r e a m ~}$ & \\
\hline $\mathbf{2}$ & Fan Inlet \\
\hline $\mathbf{1 3}$ & Fan Exit, Secondary Exhaust Inlet \\
\hline $\mathbf{1 9}$ & Secondary Exhaust Exit \\
\hline
\end{tabular}


Performance parameters that are used to represent the performance of the indicated engine components are listed in Table 6. Unless otherwise specified, their values are computed from the ratio of total-to-total pressure $(\pi)$ and temperature $(\mathrm{T})$ ratios of the inlet to the exit station of indicated component.

Table 6: Descriptions of Component Performance Parameters used in CEDM

\begin{tabular}{|l|c|c|}
\hline Component & Pressure Ratio & Temperature Ratio \\
\hline Inlet & $\pi_{0}=P_{t 0} / P_{0}$ & $\tau_{0}=T_{t 0} / T_{0}$ \\
\hline Diffuser (D) & $\pi_{d}=P_{t 2} / P_{t 1}$ & $\tau_{d}=T_{t 2} / T_{t 1}$ \\
\hline Primary Stream & & \\
\hline Low Pressure Compressor (LPC) & $\pi_{c L}=P_{t 2.5} / P_{t 2}$ & $\tau_{c L}=T_{t 2.5} / T_{t 2}$ \\
\hline High Pressure Compressor (HPC) & $\pi_{c H}=P_{t 3} / P_{t 2.5}$ & $\tau_{c H}=T_{t 3} / T_{t 2.5}$ \\
\hline Combustor (B) & $\pi_{b}=P_{t 4} / P_{t 3}$ & \\
\hline High Pressure Turbine (HPT) & $\pi_{c H}=P_{t 4.5} / P_{t 4}$ & $\tau_{t H}=T_{t 4.5} / T_{t 4}$ \\
\hline Low Pressure Turbine (LPT) & $\pi_{c L}=P_{t 5} / P_{t 4.5}$ & $\tau_{t L}=T_{t 5} / T_{t 4.5}$ \\
\hline Primary Exhaust Nozzle (N) & $\pi_{n}=P_{t 9} / P_{t 5}$ & $\tau_{n}=T_{t 9} / T_{t 5}$ \\
\hline Secondary Stream & & \\
\hline Fan (F) & $\pi_{f}=P_{t 13} / P_{t 2}$ & $\tau_{f}=T_{t 13} / T_{t 2}$ \\
\hline Secondary Exhaust Nozzle (NF) & $\pi_{n f}=P_{t 19} / P_{t 13}$ & $\tau_{n f}=T_{t 19} / T_{t 13}$ \\
\hline
\end{tabular}

\subsubsection{Development of the Model}

After obtaining satisfactory results from the validation of CTM Algorithm and extending the algorithm to multistage cooling cases, the Engine Design Model [13] was modified to include a cooling analysis. The CEDM is a turbofan engine with on and off-design cycle analysis model that has the extended Cooled Turbine algorithm (Section 2.1.2) used as a subsystem of cooled engine calculations, and was modelled as shown in Figure 22.

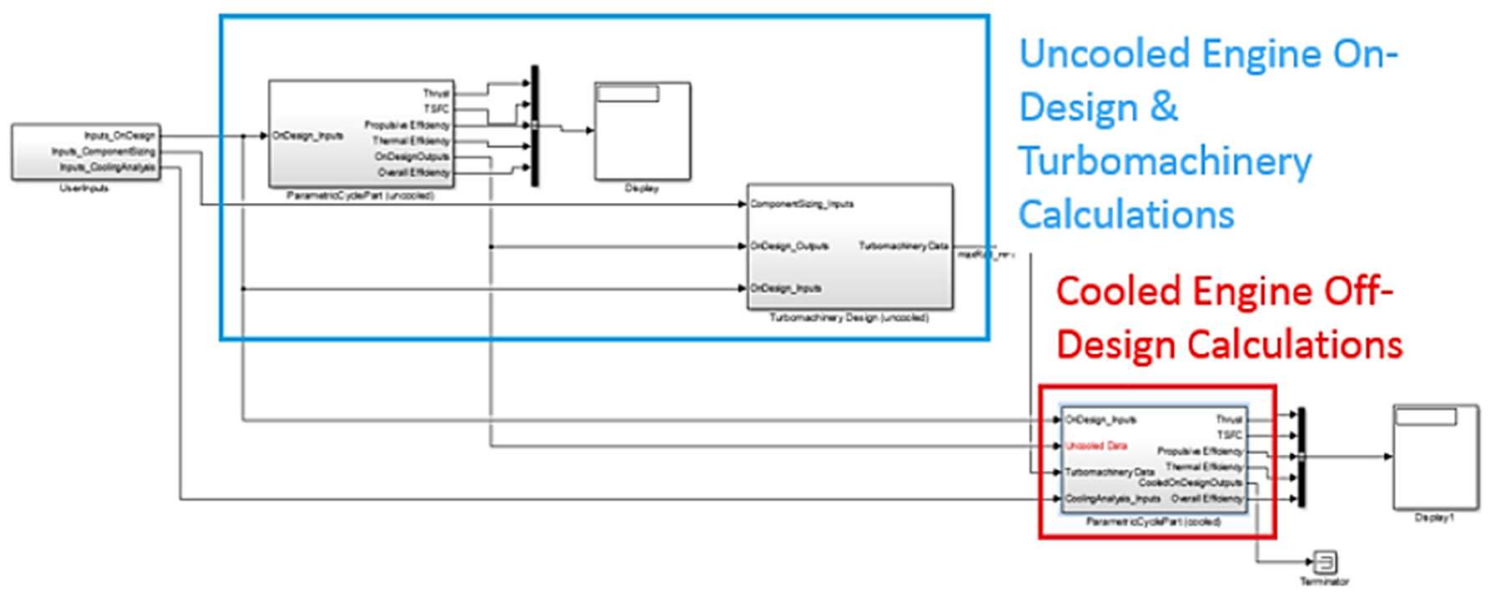

Figure 22: Cooled Engine Design Model shown in Simulink Model Workspace

The original model from EDM [13] was used in the uncooled on-design model by removing the "coolant mixer approach" from the original model. The flowchart of this section is given in Figure 23. 




Figure 23: Uncooled On-Design section of CEDM

This model has the same sub-models as EDM, except the utilization of coolant flows. The turbomachinery design section of EDM [13] was unchanged, and held the same in the CEDM. Calculation of turbomachinery information is made here by using mean-line flow equations obtained from 2D continuity and energy balance across each stage. This section is required for the geometrical and stage parameters that are used in the cooling analysis for the following parameters: stage pressure ratio, stage temperature ratio, total temperatures and pressures at each stage exit location, number of turbine stages, flow speeds and flow angles at each stage, blade solidity and 2D blade geometry. The flowchart of this section is given in Figure 24 .

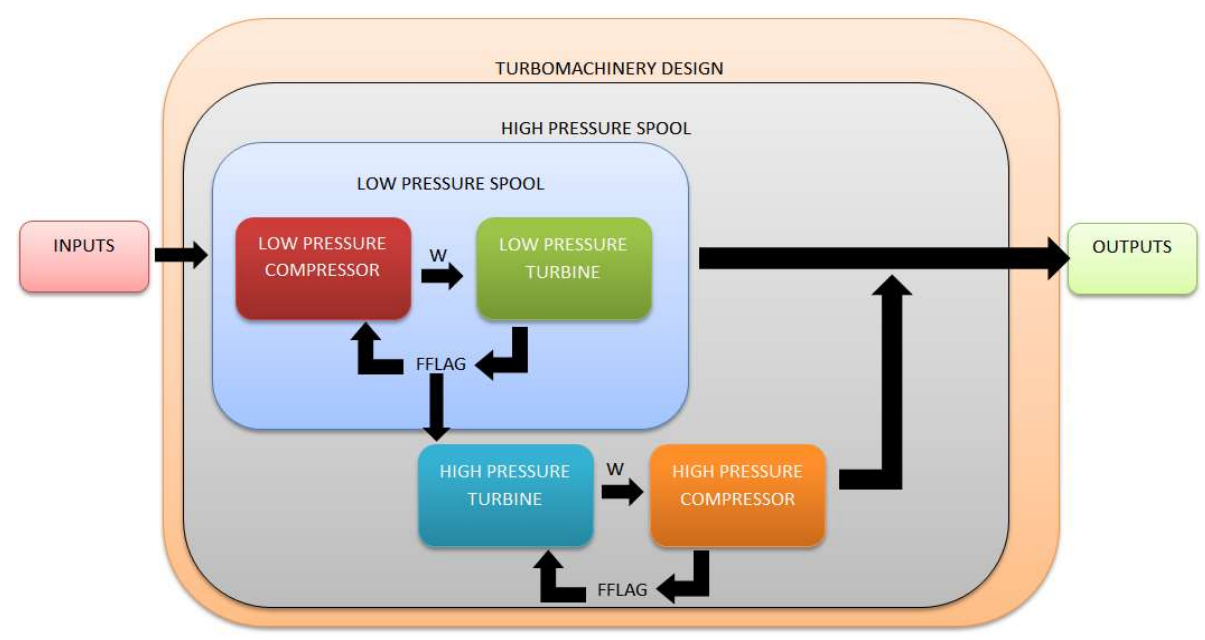

Figure 24: Turbomachinery Design Section of CEDM is the same as EDM

In order to reduce the amount of calculations to reduce overall execution time per simulation, the cold section calculations are used directly from the uncooled section in the cooled off-design analysis. The flowchart of cooled off-design section is given in Figure 25. 


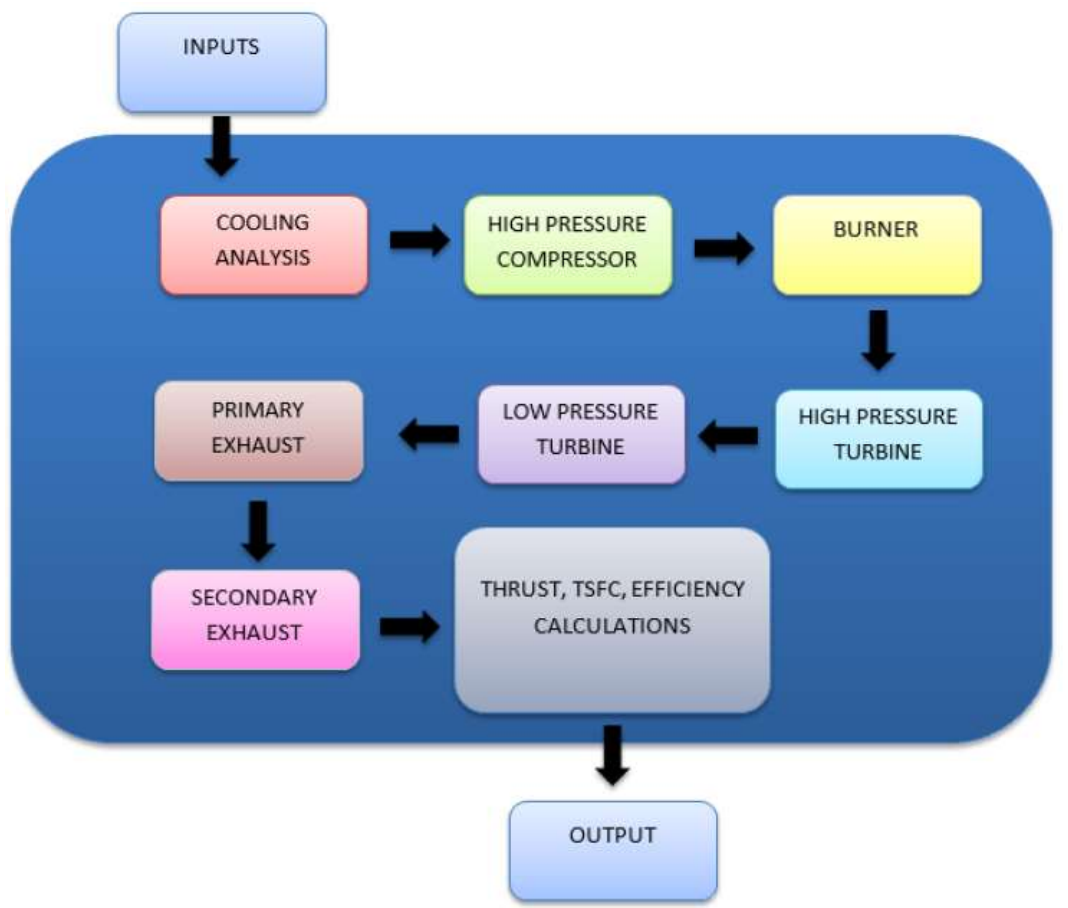

Figure 25: Cooled Off-Design section of CEDM including the CTM as a subsystem (shown as "Cooling Analysis")

Cooled Off-Design section calculations start with cooling analysis sub-model, which uses uncooled engine compressor supply pressure and temperatures to calculate the cooled engine's coolant fractions. These fractions are then used in the high-pressure compressor subsystem to include the effect of coolant extraction on compressor performance. A complete thermodynamic analysis including $1^{\text {st }}$ Law and $2^{\text {nd }}$ Law analyses with assumptions is made to develop the high pressure compressor model of the cooled engine calculations. Assumptions and further details on the development of this compressor model is explained in Appendix-C.

High Pressure Turbine sub-system uses the coolant fractions obtained in the "cooling analysis section" together with calculated entropy rise to calculate the cooled turbine's performance parameters. Including the effects of turbine cooling requires a change in the calculation of high pressure turbine pressure ratio $(\pi t \mathrm{t})$; unlike for the uncooled high pressure turbine case, the cooled turbine's polytropic efficiency is not a user input and there is no direct method to compute it from other cycle parameters.

$\mathrm{K}_{\text {HT }}$ parameter from Equation (58) used in pressure looping is an approximation to the ratio of polytropic efficiencies and using this value directly to compute cooled turbine's polytropic efficiency, and calculating $\pi_{\mathrm{t} H}$ from Equation (1) could result in errors in the estimating the cooled high pressure turbine performance.

Although not directly computed, extended CTM algorithm calculates the effect of the reduction in the polytropic efficiency as a reduction in the stage pressure ratio by using Equation (28). For successive high pressure turbine stages, the overall cooled turbine pressure ratio can be found by multiplying the cooled stage pressure ratios as shown in Equation (62).

$$
\pi_{t H, \text { cooled }}=\frac{P_{t 4.4}}{P_{t 4.1}}=\prod_{i=1}^{\# \text { of HPT Stages }} \pi_{\text {stage }, i}=\left(\frac{P_{t 4.4}}{P_{t 4.1}}\right)_{1}\left(\frac{P_{t 4.4}}{P_{t 4.1}}\right)_{2}\left(\frac{P_{t 4.4}}{P_{t 4.1}}\right)_{3} \ldots
$$


If there is no pressure loss between the cooled turbine stages (i.e. $P_{t 4.4, i}=P_{t 4.1, i+1}$ ) and using Equation (28) for each cooled turbine stage (i.e. using from the outputs of "Cooling Analysis" sub-system), high pressure turbine pressure ratio, that will be used in performance cycle calculations, can then be expressed as in Equation (63).

$$
\pi_{t H, \text { cooled }}=e^{-\frac{\left(\sigma_{t u r b}-\left(s^{0}\left(T_{t 4.4}\right)-s^{0}\left(T_{t 4}\right)\right)\right)}{R_{\text {avg }}}}
$$

Using the pressure ratio calculated from Equation (63) and calculating the cooled turbine temperature ratio from the Cooling Analysis outputs by using Equation (64), concludes the cooled turbine performance analysis.

$$
\tau_{t H, \text { cooled }}=\frac{T_{t 4.4, \text { cooled }}}{T_{t 4.1}}
$$

\subsubsection{Validation of the Model}

An initial validation of the cooled engine model was made with published CFM56-5A high by-pass turbofan engine data [22]. This initial validation helps to determine whether CEDM is giving acceptable and logical results when the cooling analysis is integrated. The comparison of published engine data from [22] with CEDM results is made in Table 7.

\begin{tabular}{|c|c|c|}
\hline CFM56-5A & CEDM & Published Data [22] \\
\hline Thrust (Ibf) & 23506 & 23700 \\
\hline Fuel Consumption (lbm/hr) & 0.6089 & 0.5967 \\
\hline EGT $\left.{ }^{0} \mathrm{C}\right)$ & 861 & 855 \\
\hline No. of HPC/ HPT Stages & $9 / 1$ & $9 / 1$ \\
\hline
\end{tabular}

Table 7: Initial validation with CFM56-5A Engine cruise conditions

HPT cooling is assumed to be an internal+ TBC+ Film cooling type. Inputs related to cooling analysis were used from typical high technology levels $\left(\varepsilon_{\mathrm{fc}}=0.35, \varepsilon_{\mathrm{c}}=0.7\right.$ and $\left.\mathrm{Bi}_{\mathrm{tbc}}=0.3\right)$ from Young and Wilcock [7]. An actual engine may have different configuration and efficiencies, which may explain the differences in the comparison. Similar results for thrust and fuel consumption indicate that the cycle calculations with the cooling analysis return results in the correct range. The yielded exhaust gas temperature is directly related with the correctness of the cooling analysis, and the number of stages is related with the exactness of the turbomachinery design section.

Further validations are carried out by using Gas Turb12 Software from GasTurb GmbH [17]. This is an engine performance and design analysis software used widely by academia and industry. The software allows users to input their estimated coolant flow rates at different sections of the turbine, in order to include the effects of cooling on performance calculations. Validation with cooling analysis is therefore made using the values of coolant flow rates obtained from the cooling analysis section of the CEDM. Validations are done with two types of tests that are named as case specific and continuity tests.

Case specific tests are defined as giving the same input list to both CEDM and GasTurb12, and then comparing the selected model output parameters directly. Continuity tests aim to observe the differences in cooled engine performance prediction with CEDM and GasTurb12 over a range of input parameters. The results obtained from these validation tests are given in detail in the following subsections. 


\subsubsection{Case Specific Tests}

A generic example case was generated based on CFM56-5A Engine properties from [22] at a cruising altitude at $33000 \mathrm{ft}$ with the test inputs given in Table 8 .

Table 8: Inputs for the CFM56-5A Engine case specific test case

\begin{tabular}{|c|c|}
\hline Name of the Parameter & Value of the Parameter \\
\hline \multicolumn{2}{|l|}{ Ambient Conditions } \\
\hline Flight Mach Number $\left(M_{0}\right)$ & 0.8 \\
\hline Flight Altitude [ft] & 33000 \\
\hline Engine Mass Flow Rate $\left(\dot{m}_{0}\right)[\mathrm{lbm} / \mathrm{s}]$ & 938.9 \\
\hline \multicolumn{2}{|l|}{ Cycle Input Parameters } \\
\hline By-Pass Ratio $(\alpha)^{\S}$ & 6 \\
\hline Bleed Air Fraction $(\beta)[\%]$ & 3.0 \\
\hline Fan Pressure Ratio $\left(\pi_{f}\right)$ & 1.55 \\
\hline Low Pressure Compressor Pressure Ratio ( $\left.\pi_{\mathrm{cL}}\right)$ & 1.55 \\
\hline High Pressure Compressor Pressure Ratio $\left(\pi_{c H}\right)$ & 17.097 \\
\hline Diffuser Pressure Ratio $\left(\pi_{d}\right)$ & 0.99 \\
\hline Combustor Pressure Ratio $\left(\pi_{b}\right)$ & 0.95 \\
\hline Nozzle Pressure Ratio $\left(\pi_{n}\right)$ & 0.99 \\
\hline Fan Nozzle Pressure Ratio ( $\left.\pi_{n f}\right)$ & 0.99 \\
\hline Combustor Efficiency $\left(\eta_{b}\right)$ & 0.99 \\
\hline High Pressure Spool Mechanical Efficiency $\left(\eta_{\mathrm{mH}}\right)$ & 0.99 \\
\hline Low Pressure Spool Mechanical Efficiency $\left(\eta_{\mathrm{mL}}\right)$ & 0.99 \\
\hline High Pressure Spool Power Takeoff Efficiency $\left(\eta_{\mathrm{mPH}}\right)$ & 0.99 \\
\hline Low Pressure Spool Power Takeoff Efficiency ( $\left.\eta_{\mathrm{mPL}}\right)$ & 1.0 \\
\hline Fan Polytropic Efficiency $\left(e_{f}\right)$ & 0.93 \\
\hline Low Pressure Compressor Polytropic Efficiency $\left(\mathrm{e}_{\mathrm{cL}}\right)$ & 0.91 \\
\hline High Pressure Compressor Polytropic Efficiency $\left(e_{c H}\right)$ & 0.91 \\
\hline High Pressure Turbine Polytropic Efficiency $\left(\mathbf{e}_{t H}\right)$ [uncooled] & 0.93 \\
\hline Low Pressure Turbine Polytropic Efficiency $\left(e_{\mathrm{tL}}\right)$ & 0.93 \\
\hline Fuel Heating Value (hPR $[$ Btu/lbm] [kerosene fuel] & 18400 \\
\hline High Pressure Turbine Inlet Temperature $\left(T_{t 4}\right)\left[{ }^{0} R\right]$ & 2768.7 \\
\hline \multicolumn{2}{|l|}{ Cooling Analysis Inputs } \\
\hline Blade Metal Biot Number (Bim) & 0.16 \\
\hline TBC Biot Number (Bitbc) & 0.35 \\
\hline Internal Flow Cooling Effectiveness $\left(\varepsilon_{c}\right)$ & 0.7 \\
\hline Film Cooling Effectiveness ( $\varepsilon_{\mathrm{cc}}$ ) & 0.4 \\
\hline Maximum Allowable Blade Metal Temperature $\left(T_{b}, \max \right)\left[{ }^{0} R\right]$ & 1961 \\
\hline Purge Fraction [\%] & 0.1 \\
\hline Cooling Configuration & Internal + Film + TBC \\
\hline Film Cooling Effective Injection Angle (deg) & 30 \\
\hline
\end{tabular}

First comparison with GasTurb12 was made for the uncooled engine case, in order to identify the differences in computations arising from the differences in component modelling, thermodynamic property tables and possible additional loss parameters modelled in GasTurb12. The results for the uncooled engine case is compared in Table 9. 
Table 9: Result comparison of case specific test for the uncooled CFM-56A case

\begin{tabular}{|c|c|c|c|c|c|}
\hline Parameter & Definition & GasTurb12 & CEDM & Unit & $\%$ Difference \\
\hline$\pi_{r}$ & Inlet Pressure Ratio & 1.525 & 1.525 & - & 0 \\
\hline$T_{\mathbf{r}}$ & Inlet Temperature Ratio & 1.128 & 1.128 & - & 0 \\
\hline$\pi_{d}$ & Diffuser Pressure Ratio & 0.99 & 0.99 & - & 0 \\
\hline$T_{d}$ & Diffuser Temperature Ratio & 1 & 1 & - & 0 \\
\hline$\eta_{\mathrm{cL}}$ & LPC Isentropic Eff. & 0.904 & 0.902 & - & 0.27 \\
\hline$T_{c L}$ & LPC Temperature Ratio & 1.148 & 1.148 & - & 0 \\
\hline$\eta_{\mathrm{cH}}$ & HPC Isentropic Eff. & 0.842 & 0.842 & - & 0 \\
\hline $\mathbf{T}_{\mathrm{cH}}$ & HPC Temperature Ratio & 2.441 & 2.485 & - & 1.81 \\
\hline$f$ & Burner fuel-to air ratio & 0.0250 & 0.0249 & - & 0.57 \\
\hline$\eta_{\mathrm{tH}}$ & HPT Isentropic Eff. & 0.939 & 0.941 & - & 0.25 \\
\hline$T_{\text {th }}$ & HPT Temperature Ratio & 0.777 & 0.749 & - & 3.64 \\
\hline$\pi_{\mathrm{tH}}$ & HPT Pressure Ratio & 0.282 & 0.292 & - & 3.67 \\
\hline$\eta_{\mathrm{tL}}$ & LPT Isentropic Eff. & 0.937 & 0.939 & - & 0.22 \\
\hline$\pi_{\mathrm{tL}}$ & LPT Pressure Ratio & 0.389 & 0.401 & - & 3.11 \\
\hline $\mathbf{T}_{\mathbf{t L}}$ & LPT Temperature Ratio & 0.823 & 0.798 & - & 2.98 \\
\hline$P_{t 9} / P_{0}$ & $\begin{array}{l}\text { Primary Exhaust Pressure } \\
\text { Ratio }\end{array}$ & 4.342 & 4.409 & - & 1.55 \\
\hline$T_{t 9}$ & Exhaust Temperature & 1771 & 1739 & ${ }^{0} \mathrm{R}$ & 1.80 \\
\hline Pt18/P0 & $\begin{array}{l}\text { Secondary Exhaust } \\
\text { Temperature Ratio }\end{array}$ & 2.316 & 2.316 & - & 0 \\
\hline$V_{18} / V_{9}$ & $\begin{array}{l}\text { Secondary Stream Velocity } \\
\text { Ratio }\end{array}$ & 0.541 & 0.551 & - & 1.85 \\
\hline $\mathbf{T}$ & Thrust & 15517 & 15268 & Ibf & 1.60 \\
\hline TSFC & $\begin{array}{l}\text { Thrust Specific Fuel } \\
\text { Consumption }\end{array}$ & 0.685 & 0.692 & $\mathrm{lbf} /(\mathrm{lbm}-\mathrm{hr})$ & 0.99 \\
\hline$\eta_{p}$ & Propulsive Efficiency & 72.63 & 72.95 & $\%$ & 0.44 \\
\hline
\end{tabular}

The uncooled engine result comparison in Table 9 demonstrates differences in basic cycle calculations between CEDM and GasTurb12. The 3-4\% difference in certain result parameters can be explained with the differences in air and kerosene combustion thermodynamic property tables used in CEDM and additional internal loss parameters defined for some intermediate stations in GasTurb12.

With the cooling analysis inputs from Table 8 , CEDM calculates $4.08 \%$ of coolant flow for stator cooling and $2.85 \%$ of coolant flow for rotor cooling for the generic test case. These values are input to GasTurb12 and the results for cooled engine case are compared in Table 10.

The differences between results from the two software analyses were within $3 \%$, which is the same for the uncooled case. The integration of the cooling analysis to the system did not yield any additional differences and cooled engine performance prediction is still in the acceptable tolerance limits. This difference can be explained from the variances in air and kerosene combustion thermodynamic property calculations, and possible additional internal loss parameters [12] defined for fans in GasTurb12. 
Table 10: Result comparison of case specific test for the cooled CFM-56A case

\begin{tabular}{|c|c|c|c|c|c|}
\hline Parameter & Definition & GasTurb12 & CEDM & Unit & \%Difference \\
\hline$\pi_{r}$ & Inlet Pressure Ratio & 1.525 & 1.525 & - & 0 \\
\hline$T_{\mathbf{r}}$ & Inlet Temperature Ratio & 1.128 & 1.128 & - & 0 \\
\hline$\pi_{d}$ & Diffuser Pressure Ratio & 0.99 & 0.99 & - & 0 \\
\hline$T_{d}$ & Diffuser Temperature Ratio & 1 & 1 & - & 0 \\
\hline$\eta_{\mathrm{cL}}$ & LPC Isentropic Eff. & 0.904 & 0.902 & - & 0.265 \\
\hline $\mathbf{T}_{\mathrm{CL}}$ & LPC Temperature Ratio & 1.148 & 1.149 & - & 0.110 \\
\hline$\eta_{\mathrm{cH}}$ & HPC Isentropic Eff. & 0.842 & 0.842 & - & 0.012 \\
\hline $\mathbf{T}_{\mathrm{cH}}$ & HPC Temperature Ratio & 2.441 & 2.485 & - & 1.805 \\
\hline$f$ & Burner fuel-to air ratio & 0.0250 & 0.0248 & - & 0.721 \\
\hline$\eta_{\mathrm{tH}}$ & HPT Isentropic Eff. & 0.939 & 0.941 & - & 0.234 \\
\hline$T_{\text {tH }}$ & HPT Temperature Ratio & 0.742 & 0.732 & - & 1.414 \\
\hline$\pi_{t H}$ & HPT Pressure Ratio & 0.264 & 0.267 & - & 1.403 \\
\hline$\eta_{\mathrm{tL}}$ & LPT Isentropic Eff. & 0.937 & 0.939 & - & 0.213 \\
\hline$\pi_{\mathrm{tL}}$ & LPT Pressure Ratio & 0.369 & 0.380 & - & 3.091 \\
\hline$T_{t L}$ & LPT Temperature Ratio & 0.812 & 0.787 & - & 3.081 \\
\hline$P_{t 9} / P_{0}$ & $\begin{array}{l}\text { Primary Exhaust Pressure } \\
\text { Ratio }\end{array}$ & 3.851 & 3.867 & - & 0.403 \\
\hline$T_{t 9}$ & Exhaust Temperature & 1668 & 1676 & ${ }^{0} \mathrm{R}$ & 0.441 \\
\hline Pt18/P0 & $\begin{array}{l}\text { Secondary Exhaust } \\
\text { Temperature Ratio }\end{array}$ & 2.316 & 2.316 & - & 0.016 \\
\hline$V_{18} / V_{9}$ & $\begin{array}{l}\text { Secondary Stream Velocity } \\
\text { Ratio }\end{array}$ & 0.557 & 0.543 & - & 2.532 \\
\hline $\mathbf{T}$ & Thrust & 14960 & 14765 & lbf & 1.305 \\
\hline TSFC & $\begin{array}{l}\text { Thrust Specific Fuel } \\
\text { Consumption }\end{array}$ & 0.663 & 0.667 & lbf/(lbm-hr) & 0.664 \\
\hline$\eta_{p}$ & Propulsive Efficiency & 73.3 & 73.2 & $\%$ & 0.191 \\
\hline
\end{tabular}

\subsubsection{Continuity Tests}

In order to observe the differences in cooled engine performance prediction with CEDM and GasTurb12 over a range of input parameters, these tests were conducted. In these tests, following input parameters are selected and changed within a predetermined interval:

- Flight Mach Number

- By-Pass Ratio

- Turbine Inlet Temperature

Selected engine performance outputs are compared in the following sub-sections.

\subsubsection{1. $\quad$ Flight Mach Number}

In the case of a high by-pass turbofan engine, increasing the flight Mach number increases the inlet speed, which results in a decrease in net thrust due to increased momentum drag. Flight Mach number was changed from 0.65 to 0.85 at constant altitude while other input parameters were fixed to the values presented in Table 8. The change in specific thrust is compared in Figure 26. 


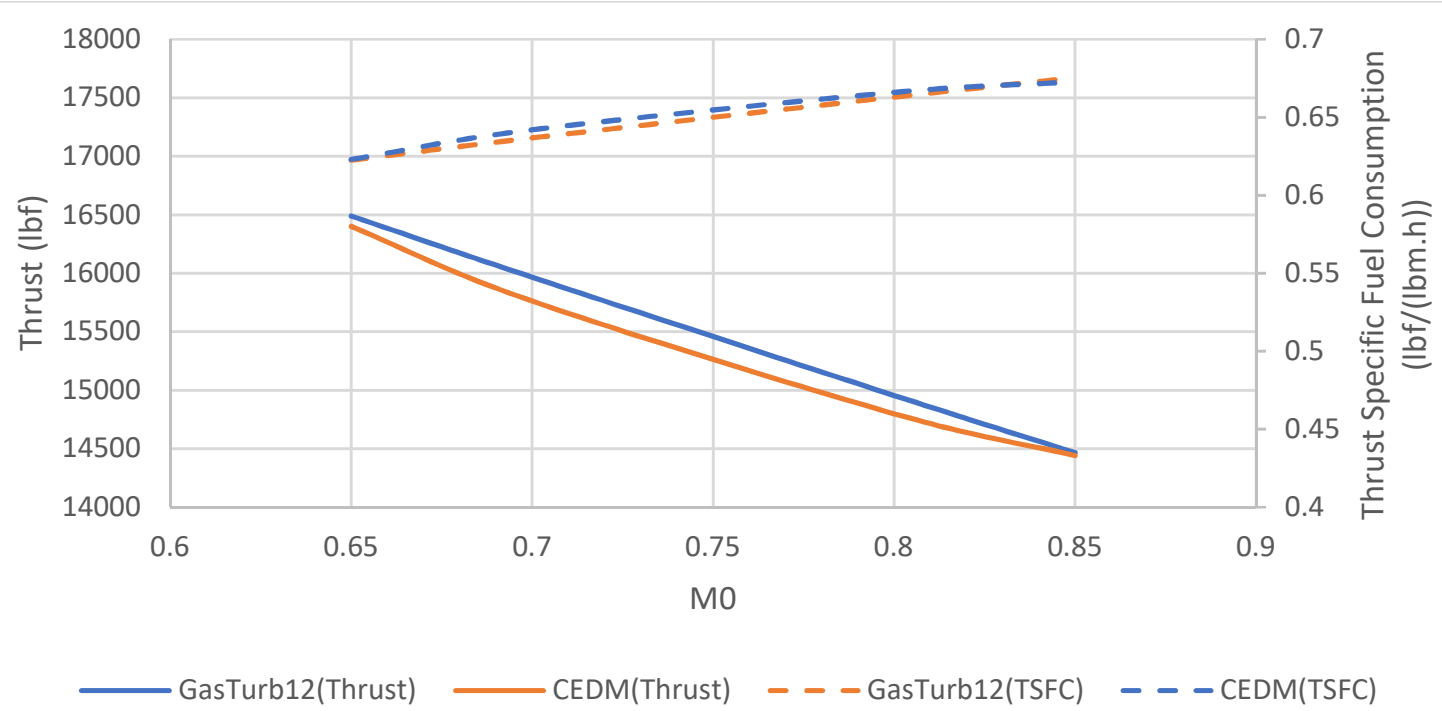

Figure 26: Change in Specific Thrust and Thrust Specific Fuel Consumption calculated for the cooled engine with Flight Mach Number

The difference between the predictions in thrust and thrust specific fuel consumption are within $1 \%$ and $0.5 \%$, respectively.

\subsection{Engine By-Pass Ratio}

In a turbofan engine design given a fixed fan pressure ratio, increasing the engine by-pass ratio reduces the fuel consumption due to reduction in the core air flow rate [12]. However, this change also reduces the amount of air flow entering to core engine, which results in a decrease in engine thrust. This well-known effect is used in this verification test by changing the design by-pass ratio from 4 to 8 (published by-pass ratio of engine is 6 [22]). The respective change in thrust and thrust specific fuel consumption are compared in Figure 27.

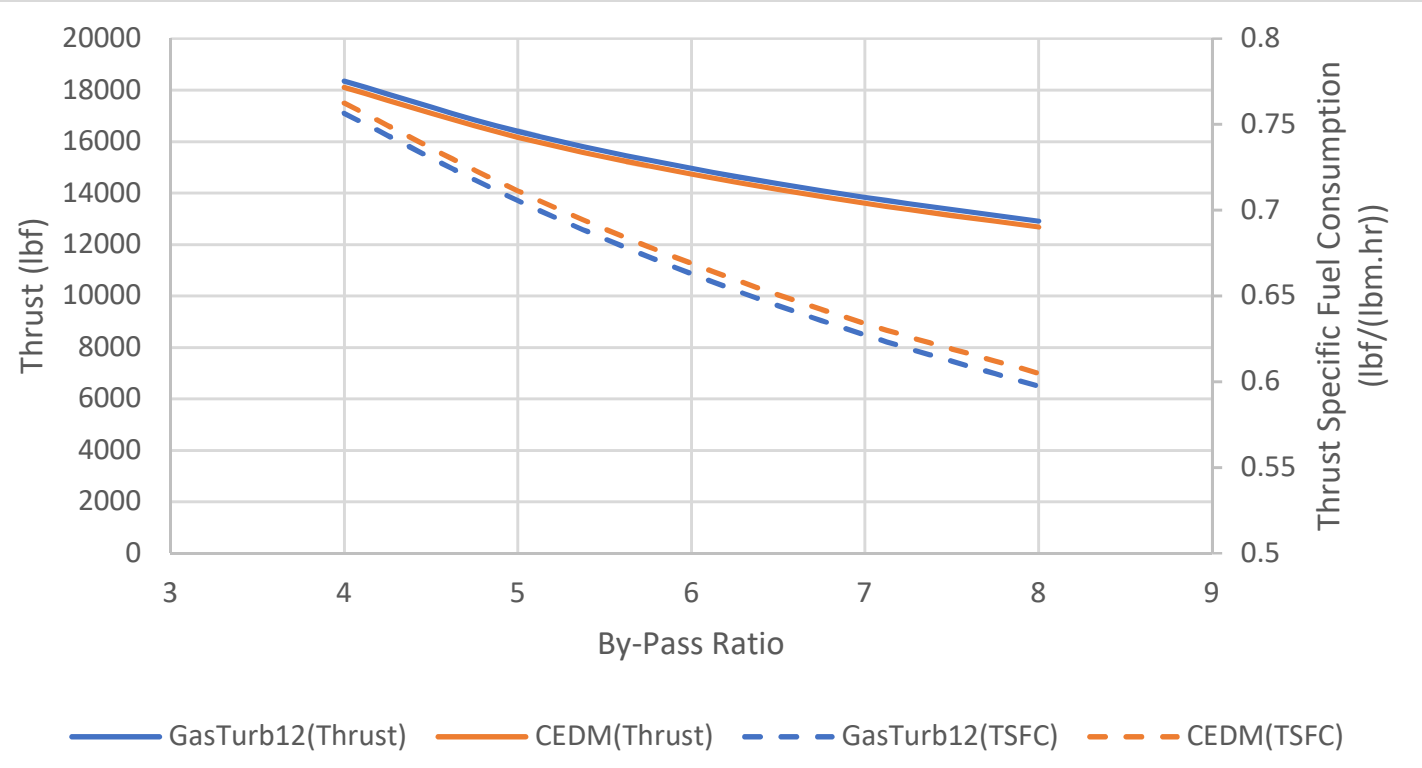

Figure 27: Change in Thrust calculated for the cooled engine with design by-pass ratio 
The difference between predictions in thrust and thrust specific fuel consumptions are within $1.2 \%$ and $0.5 \%$, respectively.

\subsection{Turbine Inlet Temperature $\left(T_{t 4}\right)$}

As the turbine inlet temperature (or combustor exit temperature) is increased, it is expected for a turbofan engine to produce more thrust due to increased turbine work. In order to observe the change in performance with respect to the turbine inlet temperature, this temperature is increased from $2469{ }^{\circ} \mathrm{R}$ to $3669^{\circ} \mathrm{R}$ (published turbine inlet temperature was $2769{ }^{\circ} \mathrm{R}$ [22]). Results obtained from CEDM and GasTurb12 for thrust and propulsive efficiency parameters are compared in Figure 28.

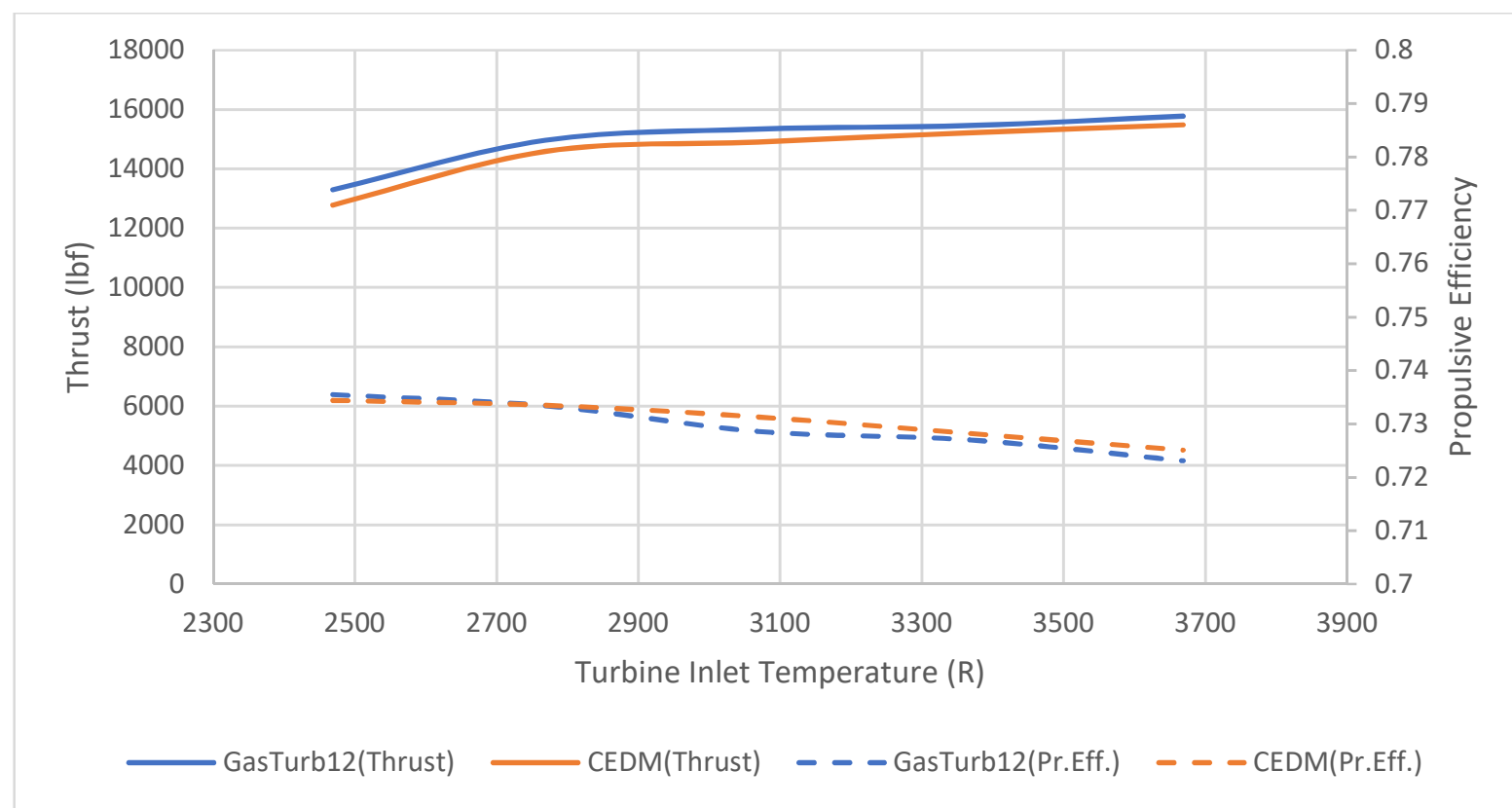

Figure 28: Change in Thrust and Propulsive Efficiency calculated for the cooled engine with Turbine Inlet Temperature

The difference in thrust and propulsive efficiency predictions between CEDM and GasTurb12 in Figure 28 are within $2.5 \%$ and $1 \%$, respectively. As the turbine inlet temperature is increased, due to the increase in required turbine cooling flows, the thrust curve slope is less positive after $\sim 2750{ }^{\circ} \mathrm{R}$ because of increased turbine losses and compressor work. Reduction rate in propulsive efficiency also increased after this temperature value. In turbofan engine design, this point corresponds to an optimum design point. 


\subsection{Cooled Gas Turbine Model (CGTM)}

In this section, a cooled engine model for industrial gas turbines with single spool configuration is developed through a transformation process of CEDM, of which the details of this transformation are explained in following sub-sections. The engine station numbering used in this model complies with the engine station numbering format used for CEDM and are shown for CGTM with the assumed coolant flow paths in Figure 29.

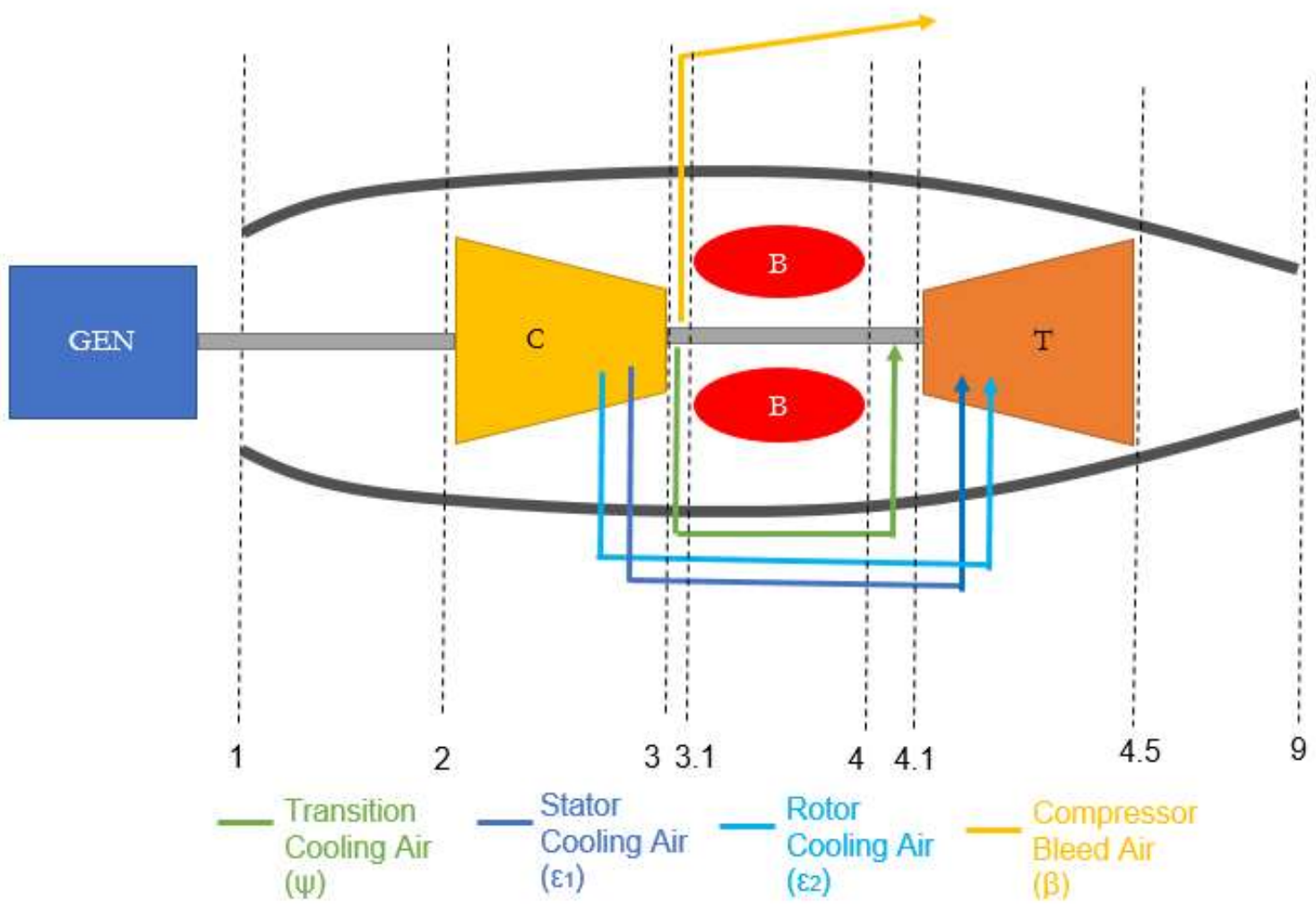

Figure 29: Schematic of the industrial gas turbine configuration, engine station numbering and coolant flow paths used in CGTM

The descriptions of the engine stations used in CGTM are given in Table 11.

Table 11: Descriptions of Engine Stations used in CEDM

\begin{tabular}{|l|l|}
\hline Station Number & Component Description \\
\hline $\mathbf{1}$ & Inlet, Diffuser Entry \\
\hline $\mathbf{2}$ & Diffuser Exit, Compressor inlet \\
\hline $\mathbf{3}$ & Compressor exit, Coolant and Bleed Air Extraction \\
\hline $\mathbf{3 . 1}$ & Combustor Inlet \\
\hline $\mathbf{4}$ & Combustor Exit, Transition Cooling Inlet \\
\hline $\mathbf{4 . 1}$ & Transition Cooling Exit, Turbine Inlet \\
\hline $\mathbf{4 . 5}(\mathbf{= 4 . 4 )}$ & Turbine Exit, Exhaust Inlet \\
\hline $\mathbf{9}$ & Exhaust Exit \\
\hline
\end{tabular}

Performance parameters that are used to represent the performance of the indicated engine components are listed in Table 12, which uses the same definitions with CEDM. 
Table 12: Descriptions of Component Performance Parameters used in CGTM

\begin{tabular}{|l|c|c|}
\hline Station Number & Pressure Ratio & Temperature Ratio \\
\hline Inlet & $\pi_{0}=P_{t 0} / P_{0}$ & $\tau_{0}=T_{t 0} / T_{0}$ \\
\hline Diffuser (D) & $\pi_{d}=P_{t 2} / P_{t 1}$ & $\tau_{d}=T_{t 2} / T_{t 1}$ \\
\hline Compressor (C) & $\pi_{c}=P_{t 3} / P_{t 2}$ & $\tau_{c}=T_{t 3} / T_{t 2}$ \\
\hline Combustor (B) & $\pi_{b}=P_{t 4} / P_{t 3}$ & \\
\hline Transition (CM) & $\pi_{c m}=P_{t 4.1} / P_{t 4}$ & $\tau_{c m}=T_{t 4.1} / T_{t 4}$ \\
\hline Turbine (T) & $\pi_{t}=P_{t 4.5} / P_{t 4.1}$ & $\tau_{t}=T_{t 4.5} / T_{t 4.1}$ \\
\hline Primary Exhaust Nozzle (N) & $\pi_{n}=P_{t 9} / P_{t 5}$ & $\tau_{n}=T_{t 9} / T_{t 5}$ \\
\hline
\end{tabular}

\subsubsection{Development of the Model}

Development of the industrial gas turbine version of the CEDM required several additional considerations. Removing some of the turbofan engine components, adjusting the turbine and exhaust cycle calculations to a land based gas turbine, and changing performance parameter definitions were the first modifications. Usage of natural gas as the fuel in power applications also brought the requirement to have appropriate thermodynamic property tables to be used in the calculations on and after the burner section. Finally, the turbomachinery design section from EDM/CEDM also needed to be modified per industrial gas turbine design principles.

\subsubsection{Modifications to Cycle Calculations}

According to Soares [71], industrial gas turbines are similar to turbojet aero-engines, in which zero thrust is produced. In an aero-engine, the major part of the net power generated from the engine is converted to engine thrust, which gives the required propulsive force to the aircraft. Whereas in an industrial gas turbine, the goal is to convert the entire net power from the engine to shaft power, which drives the generator, without producing thrust. This principle design difference determines the transformation of the aero-engine cycle calculation procedure of EDM/CEDM to a land-based gas turbine.

By equating the turbojet thrust equation from Mattingly [12] to zero and solving for other turbojet cycle relations accordingly, the constraint on exhaust nozzle pressure ratio can be found as given in Equation (65).

$$
\gamma_{9} M_{9}^{2}=\frac{P_{0}}{P_{9}}-1
$$

For land-based gas turbines, the exit Mach number is very low and is typically assumed constant, e.g., 0.05 . [23] The constraint in Equation (65) also fixes the turbine pressure ratio, for a constant exhaust exit to inlet pressure ratio $\left(\mathrm{P}_{9} / \mathrm{P}_{0}\right)$, which is the case for an industrial gas turbine. Deriving the industrial gas turbine component performance equations that are used in CGTM was made through the usage of industrial gas turbine cycle analysis given by Saravanamutto et al. [16] Walsh and Fletcher [23].

The Simulink model of cooled Gas Turbine Model (CGTM) was generated from the CEDM, by removing or by-passing aero-engine specific components such as Fan, Low Pressure Compressor, Low Pressure Turbine and Secondary Exhaust. The flowcharts given for the on-design, and off-design for CEDM in FiguresFigure 23 and Figure 25 are therefore the same for CGTM, except for the aero-engine specific components. The transformation procedure from CEDM is illustrated in Figure 30. 

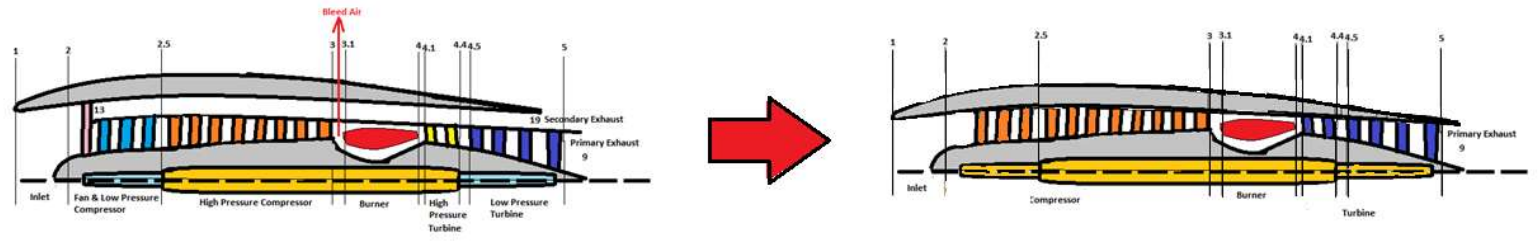

Figure 30: Transformation from aero-engine to industrial gas turbine modelling is illustrated

In the industrial gas turbine case, due to having multi-stage cooling in the turbine, it is often required to extract the coolants from intermediate compressor stages, whereas in the case of an aero-engine coolants are mostly extracted only from the last compressor stage due to having a single staged cooled turbine. Therefore, the effect of coolant extraction on the compressor performance is usually more apparent in industrial gas turbine performance. In order to include this effect on the cooled performance calculations, the compressor model developed in Appendix-C is also used in CGTM.

The calculation procedure developed for the cooled High Pressure Turbine in Section 2.2.1 is also used here to obtain the cooled gas turbines pressure ratio and an additional input parameter was added to the user input list as being the "back-pressure ratio", which is the pressure ratio $\mathrm{P}_{9} / \mathrm{P}_{0}$ of Equation (65).

Transition cooling is another cooling technique that is frequently applied in modern industrial gas turbines, for them to reach higher combustor exit temperatures (and increase power) without having a substantial increase in the turbine inlet temperature. A thermodynamic transition cooling model is developed for CGTM by using a "coolant mixer" approach, of which the details, assumptions and derived equations are provided in AppendixD. This section assumes a fixed gas temperature reduction of $200{ }^{\circ} \mathrm{R}$, and calculates the fraction of the transition cooling air $(\Psi)$, that is extracted after the last stator of the compressor (i.e. Station 3.1).

The definitions of gas turbine performance parameters were used from Walsh and Fletcher [23] and GasTurb12 [17]. Shaft Power Delivered, Heat Rate (HR), Power Specific Fuel Consumption, and Thermal Efficiency were determined to be the main outputs of the model to assess the engine performance, and their definitions are given in Equations (66) -(69).

$$
\begin{gathered}
\text { Power }=\dot{m}_{0}\left(\dot{w}_{\text {turbine }} \cdot \eta_{\text {mech }}-\dot{w}_{\text {compressor }}\right) \eta_{\text {gen }} \\
\text { Heat Rate }=\frac{3600 \cdot \dot{m}_{f} \cdot h_{P R}}{\text { Power }(k W)} \\
\text { PSFC }=\frac{\dot{m}_{0} \cdot f_{0} \cdot 3600}{\text { Power }} \\
\eta_{t h}=\frac{\text { Power }}{\dot{m}_{0} \cdot f_{0} \cdot h_{P R}}
\end{gathered}
$$

In Equation (66), $\eta_{\text {mech }}$ is the shaft mechanical efficiency and $\eta_{g e n}$ is the generator efficiency. The definition of Heat Rate in Equation (67) uses power units converted to $\mathrm{kW}$, whereas other performance parameters uses Btu for the power units. In Equations (67) and (69), hPR is the LHV of the fuel used, and $f_{0}$ is the overall fuel-to air ratio. PSFC in Equation (68) stands for Power Specific Fuel Consumption and $\eta_{\text {th }}$ is the thermal efficiency.

The inputs required by CGTM to perform the intended calculations, are listed in Table 13. 
Table 13: Descriptions of Component Performance Parameters used in CGTM

\begin{tabular}{|c|c|c|}
\hline Input Parameter & Definition & Unit \\
\hline \multicolumn{3}{|c|}{ Operating Conditions } \\
\hline $\mathbf{P}_{0}$ & Ambient Pressure & psia \\
\hline $\mathbf{T}_{0}$ & Ambient Temperature & Rankine \\
\hline$\dot{\mathbf{m}}_{0}$ & Inlet Mass Flow rate & $\mathrm{lbm} / \mathrm{s}$ \\
\hline \multicolumn{3}{|l|}{ Fuel Properties } \\
\hline $\mathbf{h}_{\mathrm{PR}}$ & LHV of the fuel used & Btu/lbm \\
\hline \multicolumn{3}{|c|}{ Engine Parameters-Pressure Ratios } \\
\hline $\boldsymbol{\Pi}_{\mathrm{cH}}$ & Compressor Pressure Ratio & - \\
\hline$\pi_{d}$ & Diffuser Pressure Ratio & - \\
\hline$\pi_{b}$ & Combustor Pressure Ratio & - \\
\hline$\pi_{n}$ & Nozzle Pressure Ratio & - \\
\hline \multicolumn{3}{|c|}{ Engine Parameters-Polytropic Efficiencies } \\
\hline $\mathbf{e}_{\mathrm{cH}}$ & Compressor Polytropic Efficiency & - \\
\hline $\mathbf{e}_{\mathrm{tH}}$ & Turbine Polytropic Efficiency & - \\
\hline \multicolumn{3}{|c|}{ Engine Parameters-Component Efficiencies } \\
\hline$\eta_{\mathbf{b}}$ & Combustor Efficiency & - \\
\hline$\eta_{m H}$ & Shaft Mechanical Efficiency & - \\
\hline \multicolumn{3}{|c|}{ Engine Parameters-Other } \\
\hline $\mathbf{T}_{\mathrm{t} 4}$ & Turbine Inlet Temperature & Rankine \\
\hline$P_{9} / P_{0}$ & Engine Back Pressure Ratio & - \\
\hline \multicolumn{3}{|c|}{ Component Sizing } \\
\hline$\rho_{b, c o m p}$ & Compressor Blade Material density & slug/ft ${ }^{3}$ \\
\hline$\sigma_{b, c o m p}$ & Compressor Blade Material Tensile Stress & psi \\
\hline$\rho_{\mathrm{d}}$ & Disk/Shaft Material density & slug/ft ${ }^{3}$ \\
\hline$\sigma_{\mathrm{d}}$ & Disk/Shaft Material Tensile Stress & psi \\
\hline$\rho_{b, t u r b}$ & Turbine Blade Material density & slug/ft ${ }^{3}$ \\
\hline$\sigma_{b, t u r b}$ & Turbine Blade Material Tensile Stress & psi \\
\hline $\boldsymbol{\omega}_{\text {shaft }}$ & Shaft Rotational Speed & rpm \\
\hline \multicolumn{3}{|l|}{ Cooling Analysis } \\
\hline$[M]_{4 \times n}$ & Cooling Schedule (matrix) & - \\
\hline $\mathrm{Bi}_{\mathrm{m}}$ & Biot Number (metal) & - \\
\hline$B \mathbf{i}_{\text {TBC }}$ & Biot Number (TBC) & - \\
\hline $\mathbf{T}_{\mathbf{b}, \max }$ & Max. Blade Temperature & Rankine \\
\hline$\eta_{\mathrm{fc}}$ & Film Cooling Effectiveness & - \\
\hline$\eta_{c}$ & Internal Cooling Effectiveness & - \\
\hline MFR & Momentum Flux Ratio (Film Cooling) & - \\
\hline$\Phi$ & Effective Coolant Injection Angle (Film Cooling) & deg \\
\hline purge $_{1}$, purge $_{2}$ & Purge Flow Fractions (wrt. mainstream) & $\%$ \\
\hline
\end{tabular}




\subsubsection{Modifications to Turbomachinery Design Calculations}

The turbomachinery design section of the program contributes to cooling analysis in several ways, the most important contribution is determination of the number of stages, stage loadings, the velocity triangles and temperature and pressure information for each uncooled stage. The cooling analysis uses this information in the calculation of coolant flow fractions (see Section 1.1). Also, if CGTM is wanted to run in the design mode, this section will give information about how the new engine will be sized with the given design parameters.

The turbomachinery design model of the CGTM was initially modelled by using the high pressure spool section of the EDM/CEDM [13] (see Figure 24 for the flowchart). The low pressure spool section of the original model was entirely removed. Next, changing some of its parameters was necessary in order to tune its calculations for an industrial gas turbine. The changes were made in accordance to some major design differences from aero-engines [24], listed as follows:

- Slower shaft speeds (i.e. lower rpm)

- Lower internal flow speeds

- Lower compressor pressure ratios

- Lower Aspect Ratio in Turbine blades

- Constant disk diameter

- Longer blades

- Lower stage loadings (to reduce exit flow swirl) *

- Higher degrees of reaction per stage*

Calculations based on Mattingly [12] in the CEDM for the high pressure spool (see Figure 24 and Uysal [13]) were modified with the design equations from Wilson and Korakianitis [25] to obtain the design in the CGTM. The methodology used in aircraft engines was also modified here to make a constant rotational shaft speed design, which is a fixed parameter for industrial gas turbines due to the usage of a connected generator and its requirements to produce the electrical power at a certain frequency.

Aside from usage of different materials, it was also found that the disk shape factors, compressor loss factors, and turbine stage loadings can be lower in industrial gas turbines than typical values used for aero-engines.

\subsubsection{Validation of the Model}

Validations of CGTM cycle calculations were carried out in three phases: in the first phase, uncooled engine cycle calculations were validated with case specific and continuity tests, and in the second phase the modified turbomachinery design section, which is using uncooled cycle results as inputs, is validated from open-access turbomachinery data. In the last phase, cooled cycle calculations were validated with another round of casespecific and continuity tests that are performed in a similar way that is done for CEDM.

\subsubsection{Uncooled Cycle Calculations \\ 2.3.2.1.1. Case Specific Tests}

In this phase, an uncooled gas turbine running with kerosene fuel was generated with the inputs from example case from Walsch and Fletcher [23] was used and the same input set (for parameters see Table 13) were entered to both CGTM and GasTurb12 [17]. The reasoning behind this first test is to understand the amount 
of difference that could occur due to the differences in used methane combustion property tables, which are generated for CGTM in Section 2.1 and has a limited literature for validation. A case specific test with the same procedure used in aero-engine case was made. The case specific test results of the uncooled engine using kerosene fuel is given in Table 14.

Table 14: Results of certain cycle parameters are compared for uncooled industrial gas turbine using kerosene fuel

\begin{tabular}{|l|l|l|l|l|l|}
\hline Parameter & Definition & GasTurb12 & $\begin{array}{l}\text { CGTM } \\
\text { (uncooled) }\end{array}$ & Unit & \%Difference \\
\hline $\mathbf{m}_{\mathbf{r}}$ & Inlet Pressure Ratio & 1 & 1 & - & 0 \\
\hline $\mathbf{T}_{\mathbf{r}}$ & Inlet Temperature Ratio & 1 & 1 & - & 0 \\
\hline $\mathbf{m}_{\mathbf{d}}$ & Diffuser Pressure Ratio & 1 & 1 & - & 0 \\
\hline $\mathbf{T}_{\mathbf{d}}$ & Diffuser Temperature Ratio & 1 & 1 & - & 0 \\
\hline $\mathbf{n}_{\mathbf{c}}$ & Compressor Isentropic Eff. & 0.825 & 0.825 & - & 0 \\
\hline $\mathbf{T}_{\mathbf{c}}$ & Compressor Temperature Ratio & 2.581 & 2.640 & - & 2.300 \\
\hline $\boldsymbol{f}$ & Burner fuel-to air ratio & 0.01886 & 0.01903 & - & 0.901 \\
\hline $\boldsymbol{\eta}_{\mathbf{t}}$ & Turbine Isentropic Eff. & 0.923 & 0.934 & - & 1.192 \\
\hline $\mathbf{T}_{\mathbf{t}}$ & Turbine Temperature Ratio & 0.525 & 0.526 & - & 0.153 \\
\hline $\mathbf{m}_{\mathbf{t}}$ & Turbine Pressure Ratio & 0.0516 & 0.0516 & - & 0 \\
\hline $\mathbf{P}_{\mathbf{t g}} / \mathbf{P}_{\mathbf{0}}$ & Exhaust Pressure Ratio & 1.0016 & 1.0016 & - & 0 \\
\hline $\mathbf{T}_{\mathbf{t g}}$ & Exhaust Temperature & 1324 & 1327 & $0 \mathrm{R}$ & 0.205 \\
\hline $\mathbf{P W S D}$ & Power & 38221 & 38403 & $\mathrm{hp}$ & 0.476 \\
\hline $\mathbf{P S F C}$ & Power Specific Fuel Consumption & 0.3721 & 0.3736 & lbm/(hp.hr) & 0.403 \\
\hline $\boldsymbol{\eta}_{\mathbf{t h}}$ & Thermal Efficiency & 0.3679 & 0.3662 & - & 0.002 \\
\hline
\end{tabular}

Having a maximum $2.3 \%$ difference, which is in the same range with CEDM case-specific tests, in the cycle calculations between GasTurb12 [17] and CGTM that the industrial gas turbine cycle modification from CEDM is handled correctly. Another uncooled engine case specific test is made with changing the fuel type to methane/natural gas and using the same inputs for other input parameters of Table 13. Comparison with GasTurb12 results of this case is given in Table 15.

Table 15: Results of certain cycle parameters are compared for uncooled gas turbine using methane fuel

\begin{tabular}{|l|l|l|l|l|l|}
\hline Parameter & Definition & GasTurb12 & $\begin{array}{l}\text { CGTM } \\
\text { (uncooled) }\end{array}$ & Unit & \%Difference \\
\hline $\mathbf{m}_{\mathbf{r}}$ & Inlet Pressure Ratio & 1 & 1 & - & 0 \\
\hline $\mathbf{T}_{\mathbf{r}}$ & Inlet Temperature Ratio & 1 & 1 & - & 0 \\
\hline $\mathbf{m}_{\mathbf{d}}$ & Diffuser Pressure Ratio & 1 & 1 & - & 0 \\
\hline $\mathbf{T}_{\mathbf{d}}$ & Diffuser Temperature Ratio & 1 & 1 & - & 0 \\
\hline $\mathbf{n}_{\mathbf{c}}$ & Compressor Isentropic Eff. & 0.825 & 0.825 & - & 0 \\
\hline $\mathbf{T}_{\mathbf{c}}$ & Compressor Temperature Ratio & 2.580 & 2.640 & - & 2.310 \\
\hline $\mathbf{f}$ & Burner fuel-to air ratio & 0.016644 & 0.01635 & - & 1.766 \\
\hline $\mathbf{n}_{\mathbf{t}}$ & Turbine Isentropic Eff. & 0.922 & 0.939 & - & 1.788 \\
\hline $\mathbf{T}_{\mathbf{t}}$ & Turbine Temperature Ratio & 0.525 & 0.518 & - & 1.477 \\
\hline $\mathbf{\pi}_{\mathbf{t}}$ & Turbine Pressure Ratio & 0.0516 & 0.0516 & - & 0 \\
\hline
\end{tabular}


Table 15 continued: Results of certain cycle parameters are compared for uncooled gas turbine using methane fuel

\begin{tabular}{|l|l|l|l|l|l|}
\hline Parameter & Definition & GasTurb12 & $\begin{array}{l}\text { CGTM } \\
\text { (uncooled) }\end{array}$ & Unit & \%Difference \\
\hline $\mathbf{P}_{\mathbf{t}} / \mathbf{P}_{\mathbf{0}}$ & Exhaust Pressure Ratio & 1.0016 & 1.0016 & - & 0 \\
\hline $\mathbf{T}_{\mathbf{t g}}$ & Exhaust Temperature & 1324 & 1306 & ${ }^{\circ} \mathrm{R}$ & 1.333 \\
\hline $\mathbf{P W S D}$ & Power & 39298 & 39984 & $\mathrm{hp}$ & 1.746 \\
\hline $\mathbf{P S F C}$ & $\begin{array}{l}\text { Power Specific Fuel } \\
\text { Consumption }\end{array}$ & 0.319 & 0.311 & $\mathrm{lbm} /(\mathrm{hp} . \mathrm{hr})$ & 2.662 \\
\hline $\mathbf{\eta}_{\mathbf{t h}}$ & Thermal Efficiency & 0.373 & 0.383 & - & 0.01 \\
\hline
\end{tabular}

Switching from kerosene fuel to methane fuel in an uncooled engine is expected to reduce the fuel fraction, due to having higher LHV for methane fuel than kerosene fuel. Without turbine cooling, a reduction in exhaust temperature is therefore expected, which is calculated as $21^{\circ} \mathrm{R}$ by CGTM. It is expected to have a difference less than $3 \%$ in hot-section calculations due to possible differences in used thermodynamic property tables, which occurred between the results of CGTM and GasTurb12 within this tolerance level.

\subsection{Continuity Test}

In order to observe the response of CGTM to a change in turbine inlet temperature, this input parameter was changed from $2120^{\circ} \mathrm{R}$ to $2920{ }^{\circ} \mathrm{R}$ for the methane fuel case. For an uncooled gas turbine, it is expected to have a linear increase in power due to linearly increased turbine work output with no changes in compressor power demand. Results for generated power from the uncooled engine is compared with GasTurb12 in Figure 31.

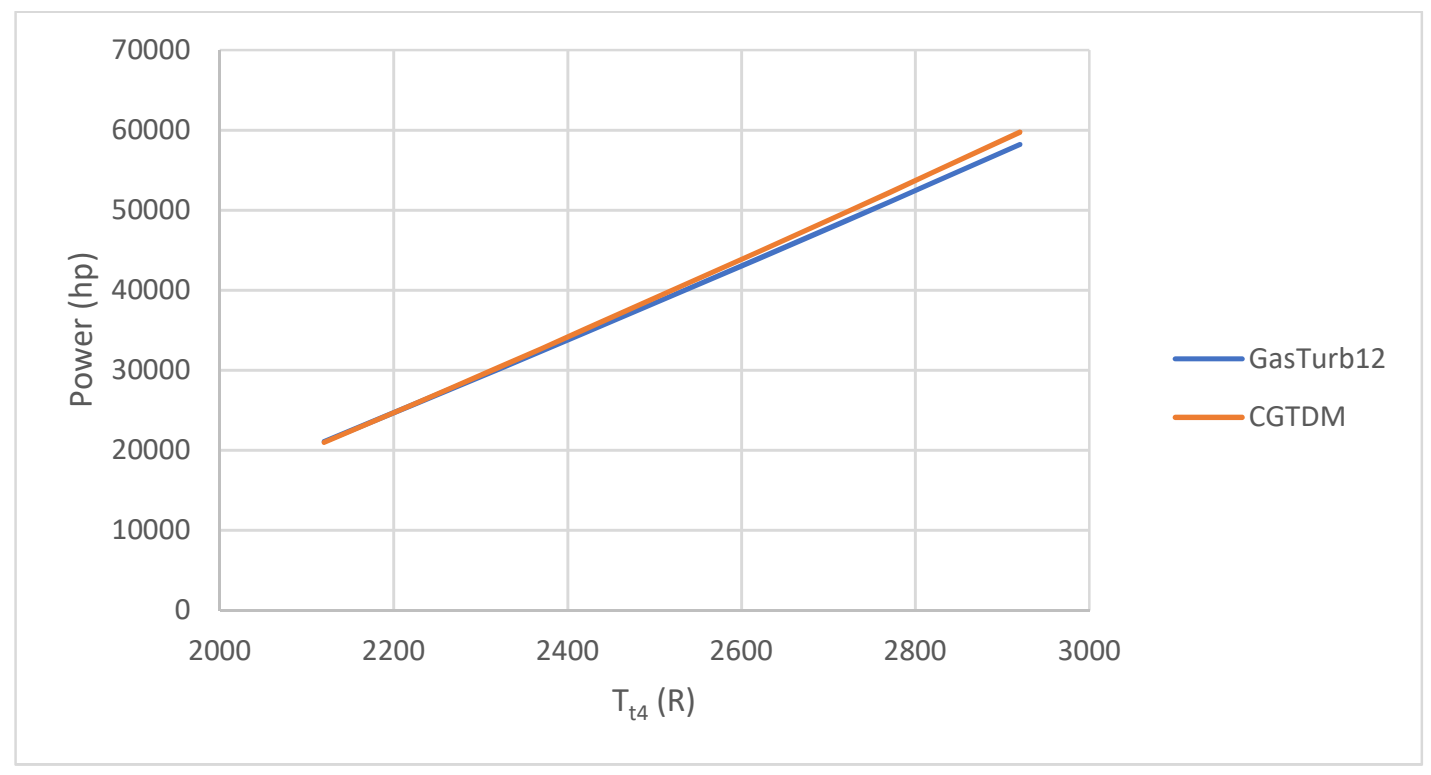

Figure 31: Calculated power output is compared for the uncooled gas turbine running with methane fuel

The difference in results in Figure 31 is within $2 \%$, which is consistent with the case-specific tests. Power Specific Fuel consumption, however, is expected to reduce asymptotically due to increased fuel consumption while the power is increasing linearly when the turbine inlet temperature is increased. Corresponding power specific fuel consumption results are shown in Figure 32. 


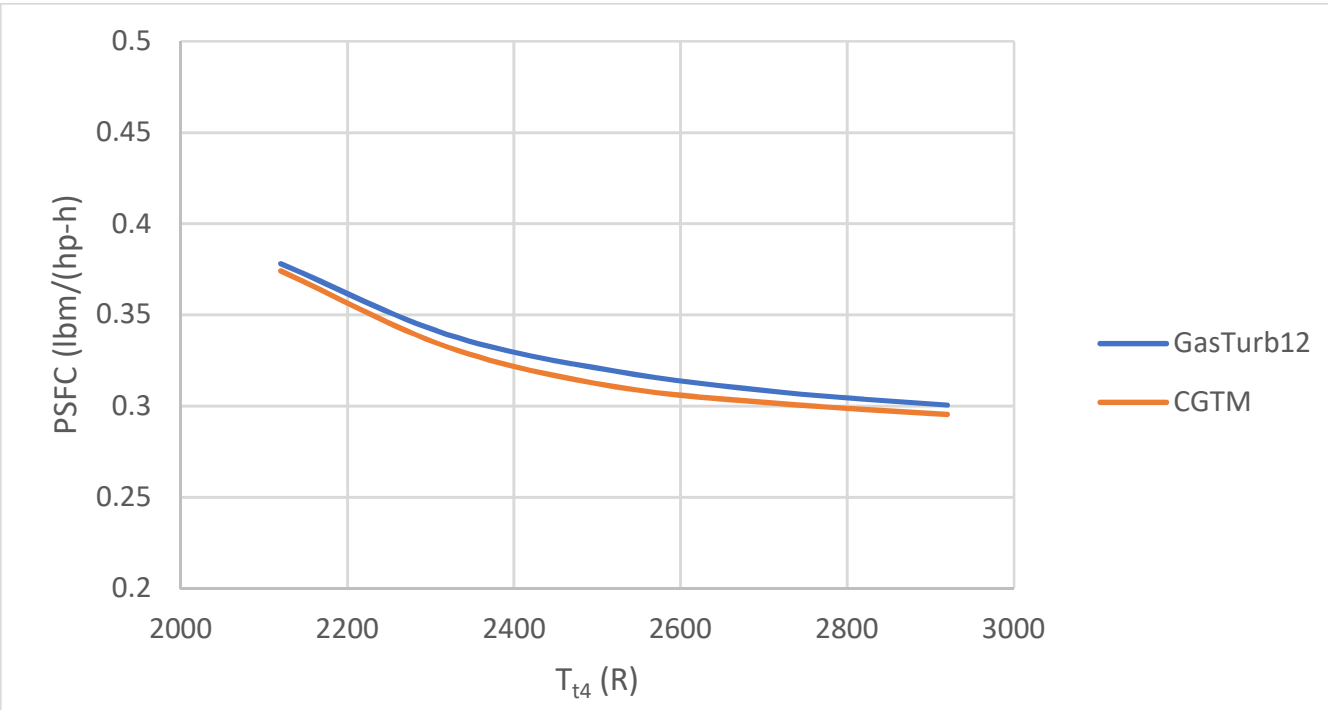

Figure 32: Calculated power specific fuel consumption output is compared for the uncooled gas turbine running with methane fuel

The difference in results in Figure 32 is within $3 \%$. Due to the increase in fuel consumption, thermal efficiency is expected to increase asymptotically. Calculated thermal efficiencies are compared in Figure 33.

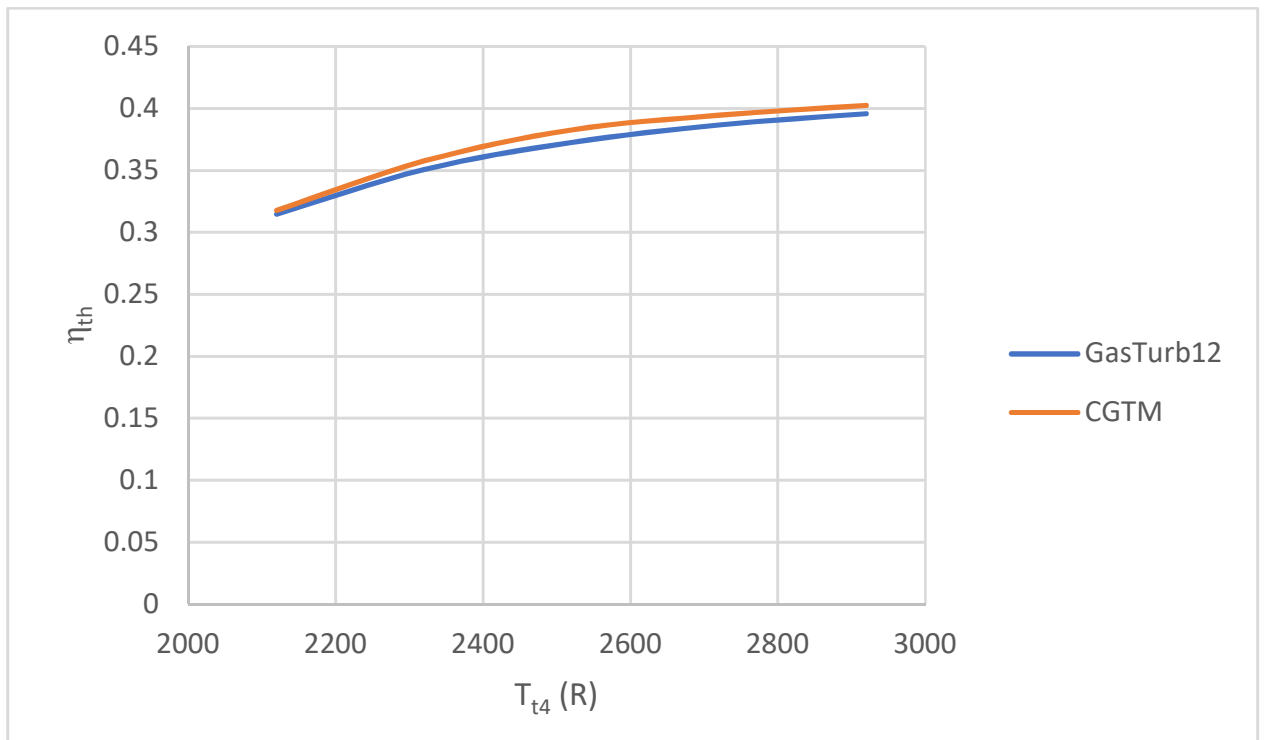

Figure 33: Thermal Efficiency output is compared for the uncooled gas turbine running with methane fuel

The difference in results in Figure 33 is within $2 \%$, which are within acceptable limits and the same expected trends were observed with CGTM and GasTurb12 for the entire input range.

\subsubsection{Turbomachinery Design Calculations}

Validation of the modified mean-line turbomachinery design section was carried out for the CGTM with available $\mathrm{H}$-Class industrial gas turbine data by using blade heights, number of stages, and disk dimensions as the comparison parameters. Required validation data was obtained by plot digitizing of publicly available 
engine cut-views, that are showing all stage components of the engine. In the validation, rotor stages were used in comparison and the results of the validations are given in the following sub-sections named after the used engine data.

\subsection{Siemens SGT6-8000H}

A sketch that is available to be used in plot-digitizing is obtained from [26] for Siemens SGT6-8000H engine. By using the overall engine dimensions from [27], plot-digitizing of rotating components as well as the blade and disk heights could be made.

For the common materials used in industrial gas turbines, the resource from Muktinutalapati [28] was used, in which provides a thorough information on the common materials used in various sections of industrial gas turbines. The materials shown in Table 16 were assumed for SGT6-8000H engine per this resource, which might be different than the ones used in the actual engine.

Table 16: Assumed material information used in component sizing calculations of CGTM for SGT6-8000H engine

\begin{tabular}{|l|l|}
\hline Component & Material Name \\
\hline Compressor Blades & Titanium 6A14V \\
\hline Shaft/Disk & UDIMET 720 \\
\hline Turbine Blades & Rene 77 \\
\hline
\end{tabular}

With the material information from Table 16 used for material tensile stress and density information, turbomachinery data from CGTM was obtained and compared with the ones from plot-digitization. Compressor blade heights are compared in Figure 34.

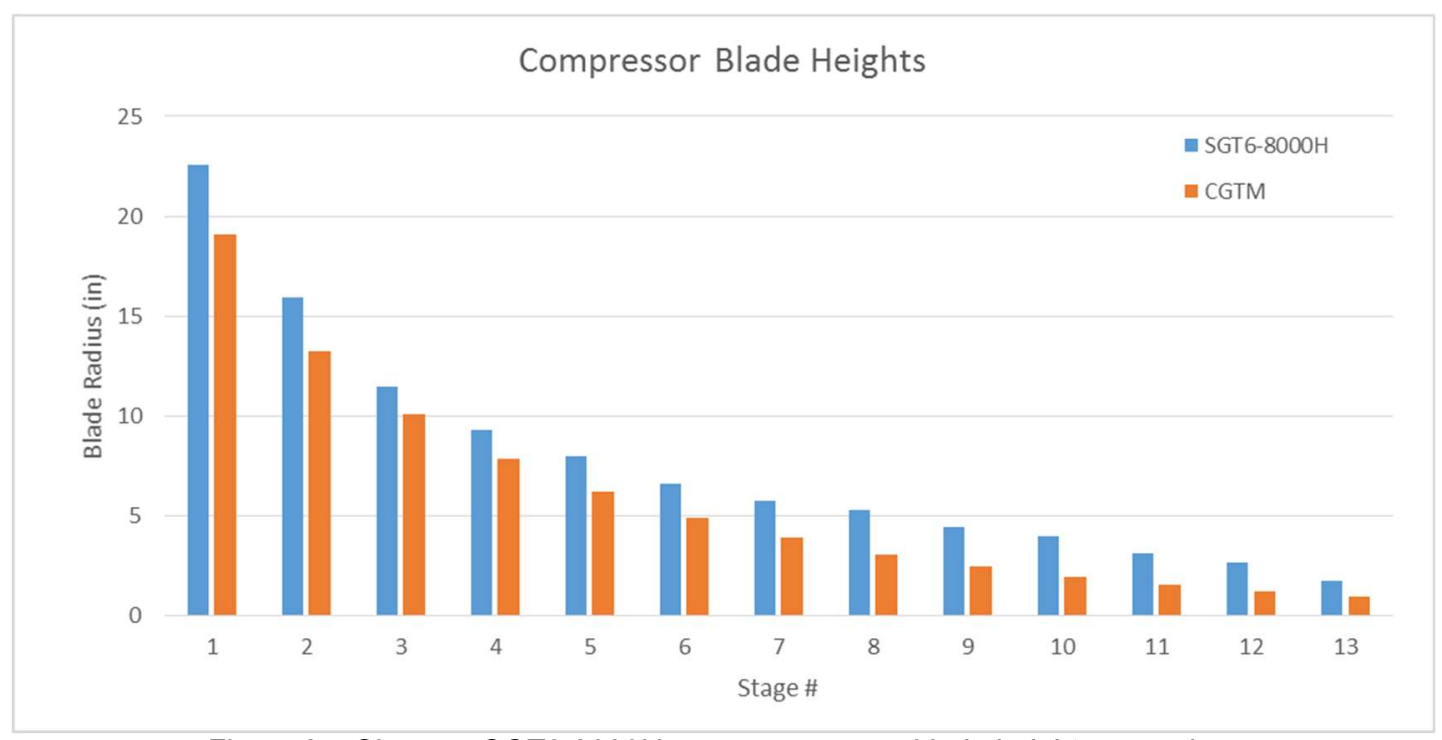

Figure 34: Siemens SGT6-8000H case compressor blade height comparison

Turbine blade heights are compared in Figure 35. 


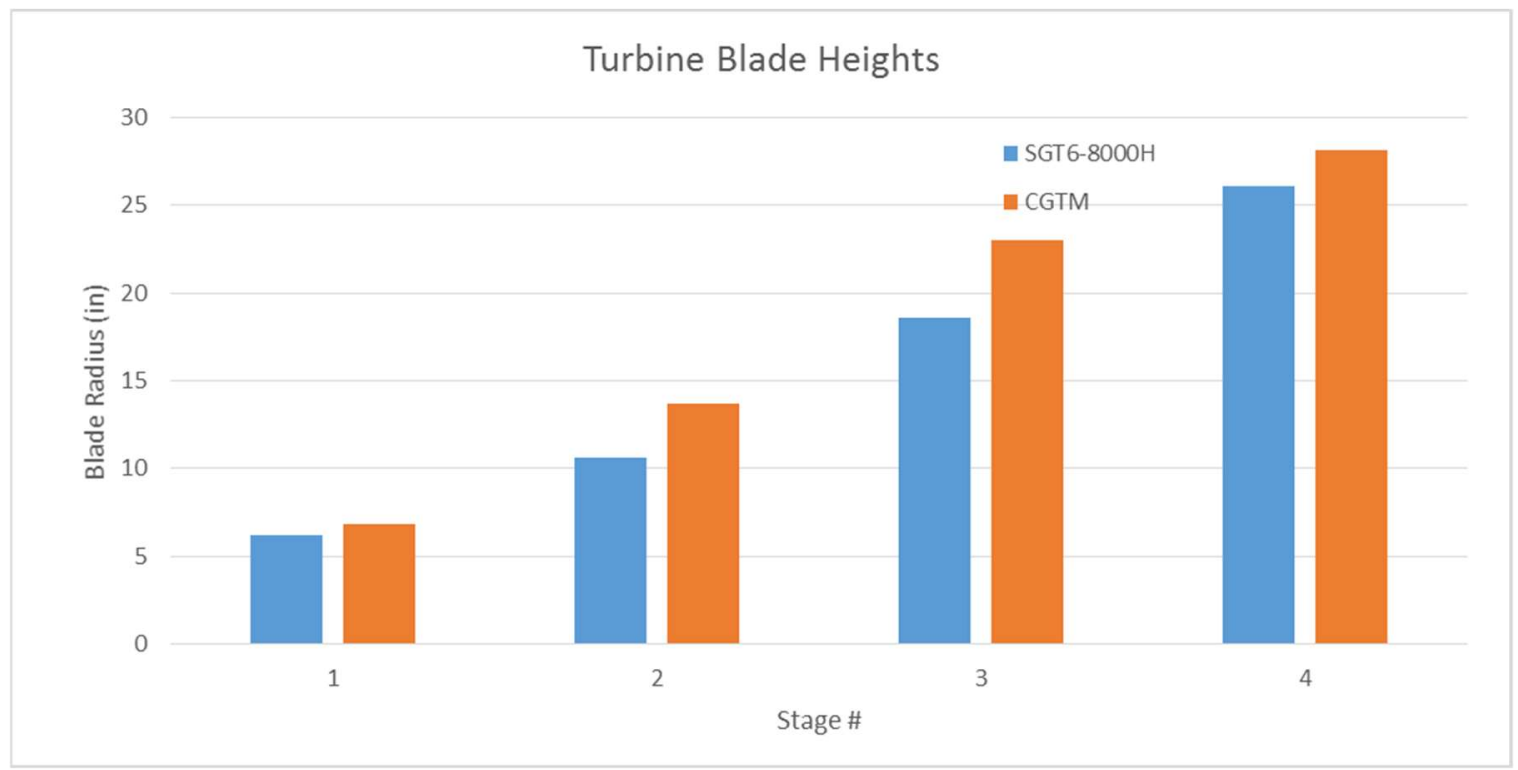

Figure 35: Siemens SGT6-8000H case turbine blade height comparison

The rotational shaft speed is fixed from the user inputs as 3600rpm. By considering the errors from the plot digitization process and the obvious differences from 3D blade/disk design procedures, it was concluded that a reasonable turbomachinery design was obtained.

\subsection{General Electric GE7HA.02}

Similar validation process made in the previous section is carried out for GE7HA.02 engine by using the engine image from [29] and overall dimensions from [27]. The materials shown in Table 17 were assumed for this engine from Muktinutalapati [28] to retrieve the tensile stress levels and densities of the materials that are used in calculations, which might be different than the ones used in the actual engine.

Table 17: Assumed material information used in component sizing calculations of CGTM for GE7HA.02 engine

\begin{tabular}{|l|l|}
\hline Component & Material Name \\
\hline Compressor Blades & Stainless Steel 17-4Ph \\
\hline Shaft/Disk & Inconel 706 \\
\hline Turbine Blades & Rene 77 \\
\hline
\end{tabular}

With the assumed materials, turbomachinery data from CGTM was obtained and compared with the ones from plot-digitization. Compressor blade heights are compared in Figure 36. 


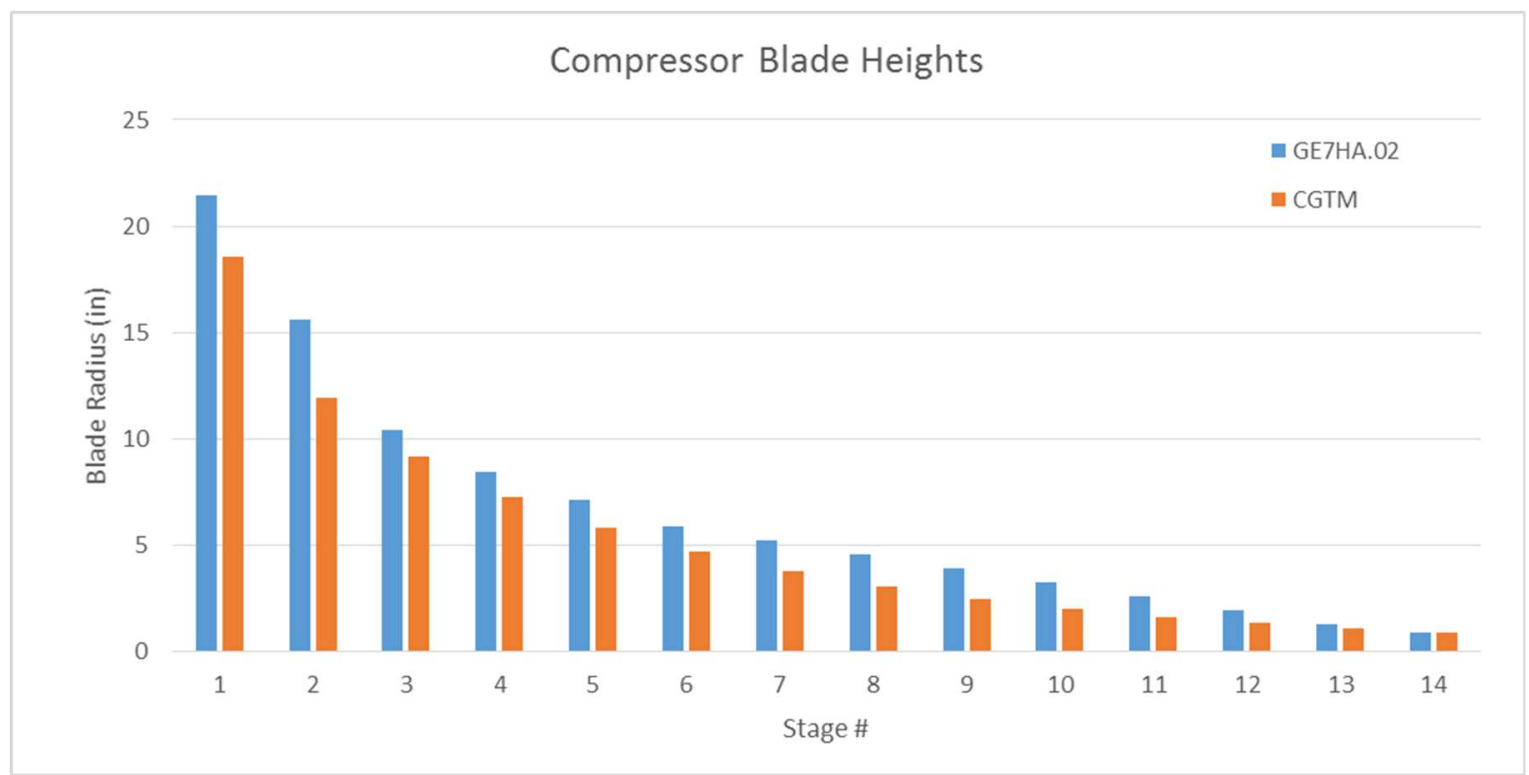

Figure 36: GE7HA.02 case compressor blade height comparison

Turbine blade heights are compared in Figure 37.

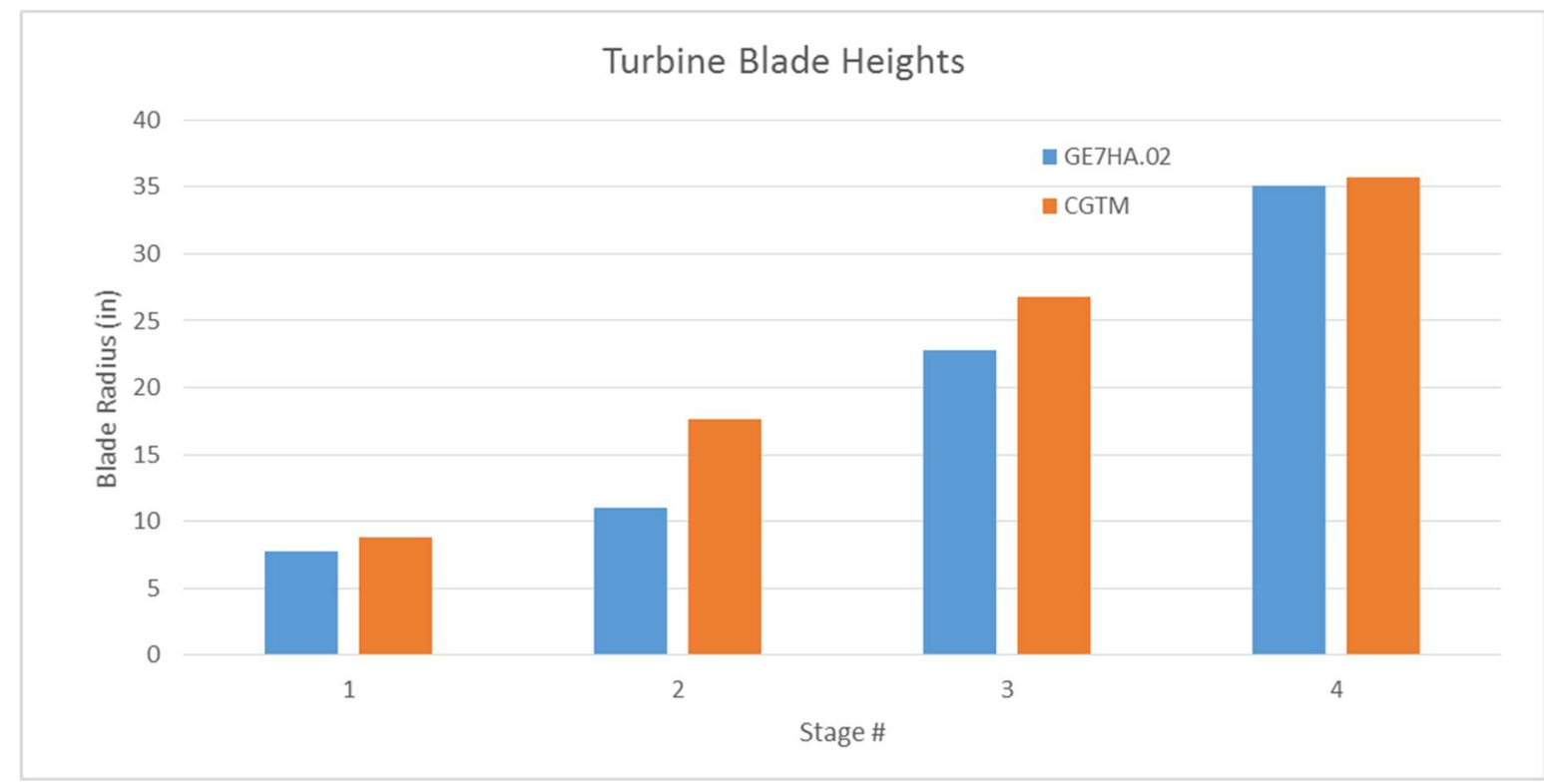

Figure 37: GE7HA.02 case turbine blade height comparison

As for the previous case, the compared compressor and turbine dimensions are vulnerable to plot-digitization errors, and a one-to-one match between CGTM calculated dimensions and the measured dimensions is not expected. This is due to $3 \mathrm{D}$ blade/disk design procedures and additional structural design limitations that cannot be addressed in 2D aerothermal design procedures. However, obtaining representative component dimensions, are important help to obtain more realistic results from CGTM.

\subsection{Mitsubishi-Hitachi Heavy Industries- M501J}

Another validation process is carried out for MHI M501J engine by using the engine image from [30] and overall dimensions obtained from [27]. The materials in Table 18 were assumed for M501J engine from [28] 
and tensile stress levels of the materials obtained from open literature. The resource by Yuri et al. [31] indicates MGT1400 and MGT2400 materials that were used for stator and rotor blades of the turbine but published material property information of these materials was not in the enough detail to be used in CGTM, therefore blade Rene-77 is assumed to be a representative alternative.

Table 18: Assumed material information used in component sizing calculations of CGTM for M501J engine

\begin{tabular}{|l|l|}
\hline Component & Material Name \\
\hline Compressor Blades & Titanium 6A1-4V \\
\hline Shaft/Disk & M152 Stainless Steel \\
\hline Turbine Blades & Rene 77 \\
\hline
\end{tabular}

With the assumed materials, turbomachinery data from CGTM was obtained and compared with the ones from plot-digitization. Compressor blade heights are compared in Figure 38.

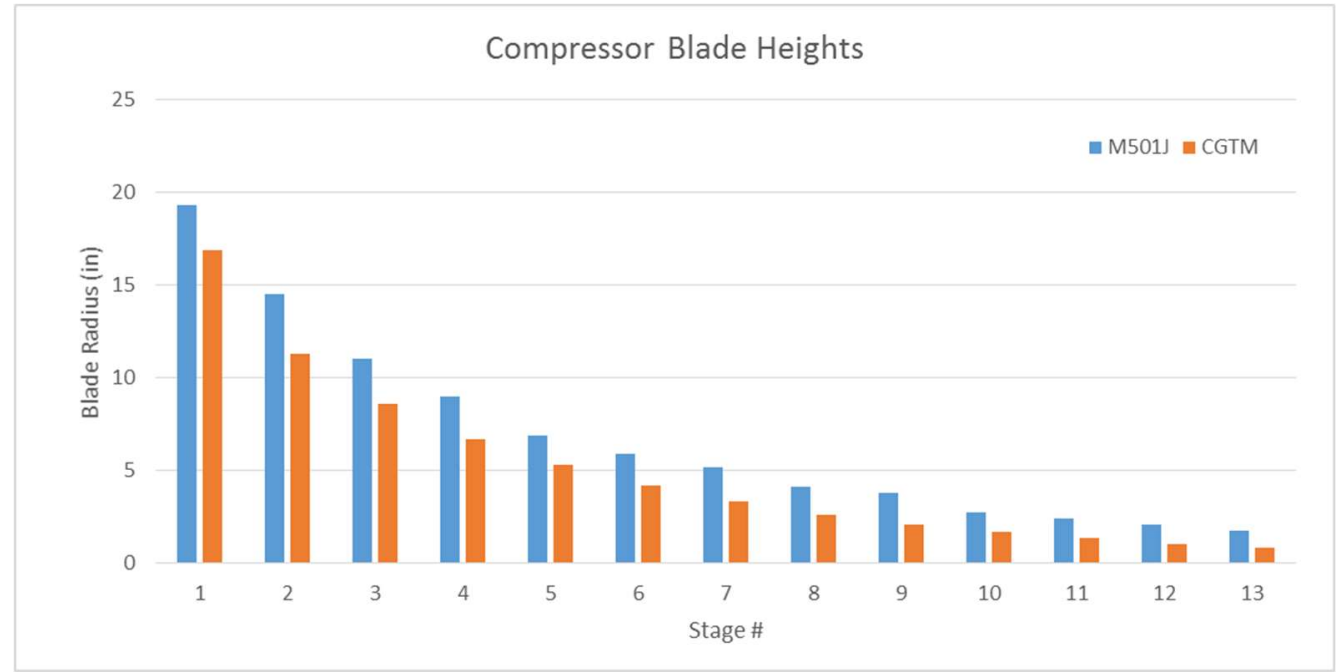

Figure 38: M501J case compressor blade height comparison

Turbine blade heights are compared in Figure 39.

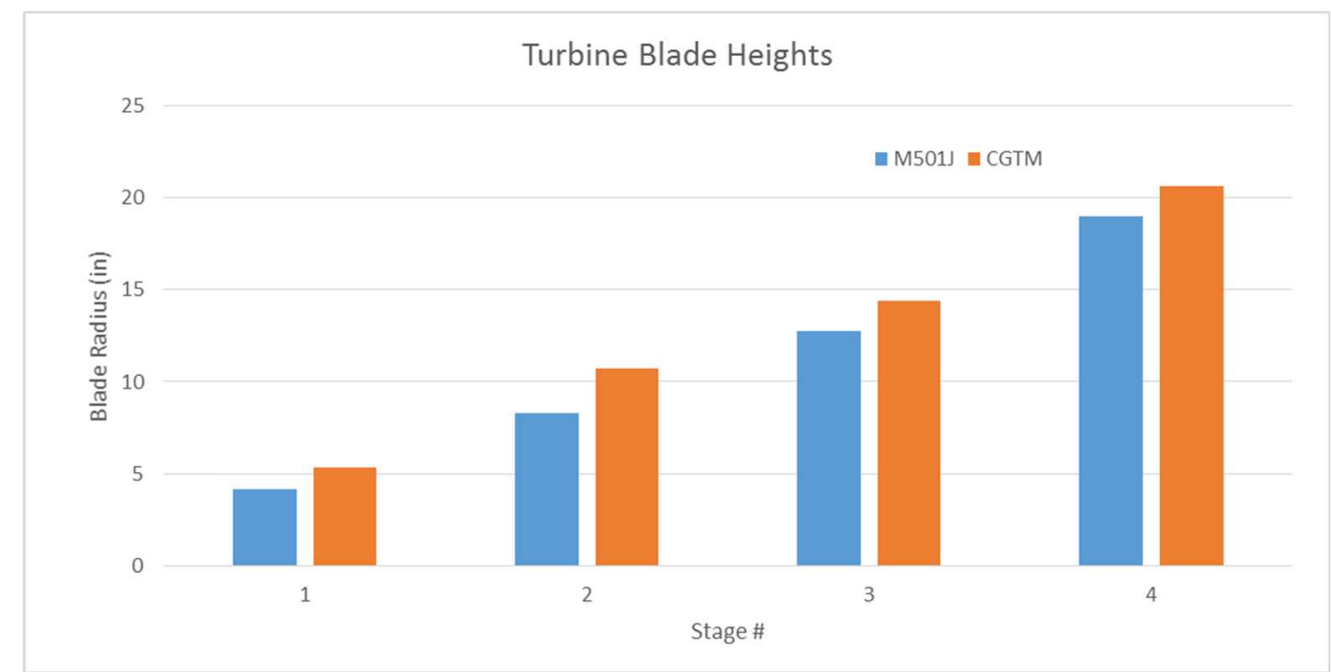

Figure 39: M501J case turbine blade height comparison 
By considering the errors from the plot digitization process and the obvious differences from 3D blade/disk design procedures, it was concluded that reasonable turbomachinery info was obtained for cooling analysis calculation purposes.

\subsubsection{Cooled Cycle Calculations}

An initial validation with published engine performance data for the $\mathrm{H}$-Class industrial gas turbine engines considered in Section 2.3.2.2 from [27] was completed with an assumed cooling scheme from published views of the turbine sections of these engines. Same cooling schedule was assumed for all three engines as being Stages 1 and 2 to be cooled by Internal and Film Cooling with a TBC application, Stage 3 to be cooled by internal cooling with a TBC application, and the final stage to be cooled by internal cooling only without a TBC.

For SGT6-8000H Engine, purge flows were assumed to be $0.5 \%$ of the core flow. The maximum blade temperature was taken to be $1100 \mathrm{~K}\left(1961^{\circ} \mathrm{R}\right)$. The turbine inlet temperature was taken to be $2600{ }^{\circ} \mathrm{F}(3058$ $\left.{ }^{0} \mathrm{R}\right)$ [27]. Cooling air and bleed air were calculated by the CGTM to be taken from Stages 7, 10 and 13 respectively. With these values, the CGTM calculated the results as shown in Table 19.

Table 19: Initial validation result comparison with Siemens SGT6-8000H industrial gas turbine

\begin{tabular}{|c|c|c|}
\hline Siemens SGT6-8000H & CGTM & Published Value [27] \\
\hline $\begin{array}{l}\text { Total Coolant } \\
\text { (includes purges) }\end{array}$ & $14 \%$ & - \\
\hline Power & $297 \mathrm{MW}$ & $296 \mathrm{MW}$ \\
\hline Thermal Efficiency & $40 \%$ & $40 \%$ \\
\hline Heat Rate & $8530 \mathrm{Btu} / \mathrm{kWh}$ & 8530 Btu/kWh \\
\hline Exhaust Temperature & $1156^{\circ} \mathrm{F}$ & $1160^{\circ} \mathrm{F}$ \\
\hline Shaft Speed & $3600 \mathrm{rpm}$ & 3600 rpm \\
\hline Stage \# (comp./turbine) & $13 / 4$ & $13 / 4$ \\
\hline
\end{tabular}

Another general validation was made with GE7HA.02 engine, with maximum blade temperature to be $1100 \mathrm{~K}$ $\left(1978{ }^{\circ} \mathrm{R}\right)$, turbine inlet temperature to be $2800 \mathrm{OF}\left(3258^{\circ} \mathrm{R}\right)$ [27] and purge flows to be $0.5 \%$ of the core flow. Cooling air were calculated by the CGTM to be taken from Stages 10, 12 and 14 . The comparison of the calculated key performance parameters for GE7HA.02 is presented in Table 20.

Table 20: Initial validation result comparison with GE7HA.02 industrial gas turbine

\begin{tabular}{|l|l|l|}
\hline GE7HA.02 & CGTM & Published Value [29] \\
\hline $\begin{array}{l}\text { Total Coolant } \\
\text { (includes purges) }\end{array}$ & $18 \%$ & - \\
\hline Power & $347 \mathrm{MW}$ & $346 \mathrm{MW}$ \\
\hline Thermal Efficiency & $42.2 \%$ & $42.2 \%$ \\
\hline Heat Rate & $8078 \mathrm{Btu} / \mathrm{kWh}$ & $8080 \mathrm{Btu} / \mathrm{kWh}$ \\
\hline Exhaust Temperature & $1155^{\circ} \mathrm{F}$ & $1153{ }^{\circ} \mathrm{F}$ \\
\hline Shaft Speed & $3600 \mathrm{rpm}$ & $3600 \mathrm{rpm}$ \\
\hline Stage \# (comp./turbine) & $14 / 4$ & $14 / 4$ \\
\hline
\end{tabular}

Another general validation was made with M501J engine, with maximum blade temperature assumed to be $1140 \mathrm{~K}\left(2051^{\mathrm{O}} \mathrm{R}\right)$, turbine inlet temperature taken to be $2916{ }^{\circ} \mathrm{F}\left(3389{ }^{\circ} \mathrm{R}\right)$ from [27]. Cooling air were calculated by the CGTM to be taken from Stages 8, 11 and 15. The comparison of the calculated key performance parameters for M501J is presented in Table 21 . 
Table 21: Initial validation result comparison with M501J industrial gas turbine

\begin{tabular}{|l|l|l|}
\hline M501J Foolant & CGTM & Published Value [27] \\
\hline $\begin{array}{l}\text { Total } \\
\text { (includes purges) }\end{array}$ & $20 \%$ & - \\
\hline Power & $324 \mathrm{MW}$ & $327 \mathrm{MW}$ \\
\hline Thermal Efficiency & $41.0 \%$ & $41.0 \%$ \\
\hline Heat Rate & $8324 \mathrm{Btu} / \mathrm{kWh}$ & $8325 \mathrm{Btu} / \mathrm{kWh}$ \\
\hline Exhaust Temperature & $1172^{\circ} \mathrm{F}$ & $1176{ }^{\circ} \mathrm{F}$ \\
\hline Shaft Speed & $3600 \mathrm{rpm}$ & $3600 \mathrm{rpm}$ \\
\hline Stage \# (comp./turbine) & $15 / 4$ & $15 / 4$ \\
\hline
\end{tabular}

Comparing the values obtained by the CGTM to the published data shows good agreement. The differences are explicable with possible differences between the assumed and actual cooling configuration, efficiencies of cooling methods used in the CGTM, and additional heat losses in the actual engine.

With the same cooling technology assumed for 3 engines, the required coolant rates to cool the turbine to the same exhaust temperature $\left(\sim 1165^{\circ} \mathrm{F}\right)$ should be proportional to the shaft power produced by the engine and the turbine inlet temperature and the results obtained by CGTM followed this expected trend.

It should be noted here that, there is no published information about the exact cooling techniques used in these engines, therefore the differences from the published data is also including the differences from the exact cooling technique, maximum blade temperatures, Biot numbers and cooling system efficiencies. The last three cooling analysis parameters were used from the "advanced cooling technology parameters" used in the study by Wilcock et al. [32], in which the same cooling analysis methodology described in Section 1.1 is employed. In that study, "advanced" level of cooling technology corresponds to the expected technology level around 2010-2015, which is the production year interval of the 3 engines considered. There will also be differences from the exact values in component polytropic efficiency, shaft mechanical efficiency and generator efficiency values used in CGTM calculations which might cause differences from the exact values in component performance parameters.

Due to not being able to validate individual component performance parameters calculated by CGTM with open-access data, a round of case specific tests and continuity tests were performed for CGTM with the same methodology used for CEDM validations. Details of the validation process for the cooled cycle calculations are given in detail in the following sub-sections.

\subsection{Case Specific Test}

For the case specific test made for cooled gas turbine calculations, a generic $\mathrm{H}$-Class engine input set was generated by using published values for compressor pressure ratio, turbine inlet temperature, engine mass flow rates, and cooling configuration from [26], [27], [29] and [31] and other parameters used from recent technology levels from Mattingly [12], Saravanamutto et al. [16] and Wilcock et al. [32]. Cooled Gas Turbine Model Input list is given in Table 22 with the values used for case specific test validations made with GasTurb12 [17] using the coolant fractions calculated by CGTM. 
Table 22: Inputs generated for the generic H-Class engine scenario for case-specific tests

\begin{tabular}{|c|c|}
\hline Name of the Parameter & Value of the Parameter \\
\hline Ambient Pressure (psia) & 14.696 \\
\hline Ambient Temperature $\left[{ }^{0} \mathrm{R}\right]$ & 529.67 \\
\hline Engine Mass Flow Rate $\left(\dot{m}_{0}\right)[\mathrm{lbm} / \mathrm{s}]$ & 1500 \\
\hline Compressor Pressure Ratio $\left(\pi_{\mathrm{cH}}\right)$ & 23 \\
\hline Diffuser Pressure Ratio $\left(\pi_{d}\right)$ & 0.99 \\
\hline Combustor Pressure Ratio $\left(\pi_{b}\right)$ & 0.97 \\
\hline Nozzle Pressure Ratio $\left(\pi_{n}\right)$ & 0.95 \\
\hline Combustor Efficiency $\left(\eta_{b}\right)$ & 0.98 \\
\hline Shaft Mechanical Efficiency $\left(\eta_{\mathrm{m}}\right)$ & 0.995 \\
\hline Generator Efficiency ( $\left.\eta_{\text {gen }}\right)$ & 0.99 \\
\hline Compressor Polytropic Efficiency $\left(\mathbf{e}_{c}\right)$ & 0.9 \\
\hline Turbine Polytropic Efficiency $\left(e_{t}\right)$ & 0.91 \\
\hline Fuel Heating Value $\left(h_{P R}\right)[B t u / l b m]$ & 21397.2 \\
\hline High Pressure Turbine Inlet Temperature $\left(T_{t 4}\right)\left[{ }^{0} R\right]$ & 3150 \\
\hline \multicolumn{2}{|l|}{ Cooling Inputs } \\
\hline $\mathrm{Bi}_{\mathrm{m}}$ & 0.17 \\
\hline $\mathbf{B i}_{\mathrm{tbc}}$ & 0.32 \\
\hline$\varepsilon_{\mathrm{c}}$ & 0.71 \\
\hline$\varepsilon_{\mathrm{fc}}$ & 0.4 \\
\hline $\mathbf{T}_{\mathbf{b}, \max }$ & $1983^{\circ} \mathrm{R}$ \\
\hline Purge Fractions (\%of mainstream) & 0.5 \\
\hline Cooling Configuration & $\begin{array}{l}\text { [Internal+Film+TBC; } \\
\text { Internal+Film+TBC; } \\
\text { Internal+TBC; } \\
\text { Internal Only] }\end{array}$ \\
\hline
\end{tabular}

Comparison of the results obtained from CGTM and GasTurb12 for this input case is shown in Table 23.

Table 23: Case Specific Test result comparison for generic H-Class engine case

\begin{tabular}{|l|l|l|l|l|l|}
\hline Parameter & Definition & GasTurb12 [17] & $\begin{array}{l}\text { CGTM } \\
\text { (cooled) }\end{array}$ & Unit & \%Difference \\
\hline $\mathbf{m}_{\mathbf{r}}$ & Inlet Pressure Ratio & 1 & 1 & - & 0 \\
\hline $\mathbf{T}_{\mathbf{r}}$ & Inlet Temperature Ratio & 1 & 1 & - & 0 \\
\hline $\mathbf{m}_{\mathbf{d}}$ & Diffuser Pressure Ratio & 1 & 1 & - & 0 \\
\hline $\mathbf{T}_{\mathbf{d}}$ & Diffuser Temperature Ratio & 1 & 1 & - & 0 \\
\hline $\mathbf{n}_{\mathbf{c}}$ & Compressor Isentropic Eff. & 0.867 & 0.867 & - & 0 \\
\hline $\mathbf{T}_{\mathbf{c}}$ & Compressor Temperature Ratio & 2.603 & 2.667 & - & 2.42 \\
\hline $\boldsymbol{f}$ & Burner fuel-to air ratio & 0.0289 & 0.0290 & - & 0.52 \\
\hline $\mathbf{n}_{\mathbf{t}}$ & Turbine Isentropic Eff. & 0.944 & 0.944 & - & 0 \\
\hline $\mathbf{T}_{\mathbf{t}}$ & Turbine Temperature Ratio & 0.495 & 0.489 & - & 1.21 \\
\hline $\mathbf{m}_{\mathbf{t}}$ & Turbine Pressure Ratio & 0.0497 & 0.0492 & - & 1.01 \\
\hline $\mathbf{P}_{\mathbf{t g}} / \mathbf{P}_{\mathbf{0}}$ & Exhaust Pressure Ratio & 1.06 & 1.05 & - & 0.94 \\
\hline & & & & & \\
\hline
\end{tabular}


Table 23 continued: Case Specific Test result comparison for generic H-Class engine case

\begin{tabular}{|l|l|l|l|l|l|}
\hline Parameter & Definition & GasTurb12 [17] & $\begin{array}{l}\text { CGTM } \\
\text { (cooled) }\end{array}$ & Unit & \%Difference \\
\hline $\mathbf{T}_{\mathbf{t 9}}$ & Exhaust Temperature & 1613 & 1597 & ${ }^{0} \mathrm{R}$ & 0.99 \\
\hline PWSD & Power & 463788 & 476594 & $\mathrm{hp}$ & 2.76 \\
\hline HR & Heat Rate & 8241 & 8201 & Btu/kW.h & 0.49 \\
\hline $\mathbf{\eta}_{\text {th }}$ & Thermal Efficiency & 41.6 & 41.6 & $\%$ & 0 \\
\hline
\end{tabular}

In the industrial gas turbine case, the differences between the results obtained by the CGTM and GasTurb12 for the component performance parameters are lower, mainly due to fewer engine components that have specific pressure or temperature loss models used in GasTurb12 [17]. The differences were occurred within the same levels (less than $3 \%$ ) as in the uncooled case specific tests, which is within acceptable limits.

\subsection{Continuity Tests}

In order to observe the differences in cooled engine performance prediction with CGTM and compare with GasTurb12 over a range of input parameters, these tests were conducted. In these tests, the generic $\mathrm{H}$-Class engine case was used and following parameters were selected as test variables:

- Turbine Inlet Temperature $\left(T_{t 4}\right)$

- Ambient Temperature $\left(\mathrm{T}_{0}\right)$

- Back-Pressure Ratio $\left(\mathrm{P}_{9} / \mathrm{P}_{0}\right)$

Test variables were changed within a pre-determined interval and values of the other input parameters were used from Table 22.

\subsection{Turbine Inlet Temperature}

For fixed coolant flow rates, as the turbine inlet temperature is increased, both turbine work output and burner fuel consumption are expected to increase. However, the effect of these factors is not equal and cannot compensate each other. Explicit form of the thermal efficiency from Equation (69), is shown in Equation (70), and this form will be used to analyze the expected trends in this continuity test.

$$
\eta_{t h}=\frac{\left(\dot{w}_{t}-\dot{w}_{c}\right)}{f\left(1-\beta-\epsilon_{s}-\epsilon_{r}\right) h_{P R}}
$$

In Equation (70), $\beta$ represents the compressor bleed air ratio, $f$ represents burner fuel-to-air ratio and $\epsilon$ represents the turbine total cooling air fractions for stator and rotors. Turbine work is defined as the total specific enthalpy difference between the inlet and exit states of the turbine.

Fuel to air ratio is defined and used in CGTM as in Equation (71)

$$
f=\frac{h_{t 4}-h_{t 3}}{\eta_{b} \cdot h_{P R}-h_{t 4}}
$$

In equation (71) $\eta_{\mathrm{b}}$ is the isentropic burner efficiency, and the engine station numbering is from Figure 29. 
Increasing the turbine inlet temperature has a well-known enhancing effect on the shaft power delivered and thermal efficiency. However, due to the increased coolant requirements to cool the turbine blades to a fixed lower temperature (fixed by the blade material used) the performance curves will have an optimum point as also observed in the turbofan engine case. Following the $2^{\text {nd }}$ Law analysis, the mixing heat transfer loss term will logarithmically increase as the turbine inlet temperature is increased, resulting in increased losses in higher turbine inlet temperatures. Increase in this loss will reduce the rate of increase in turbine power generation after a certain turbine inlet temperature, whereas the rate of increase in fuel consumption is lower. From Equation (70), this situation should cause a parabolic trend for the thermal efficiency as the turbine inlet temperature is increased.

Figure 40 shows the comparison made for thermal efficiency and power specific fuel consumption when the turbine inlet temperature is increased from $2660^{\circ} \mathrm{R}$ to $3460^{\circ} \mathrm{R}$.

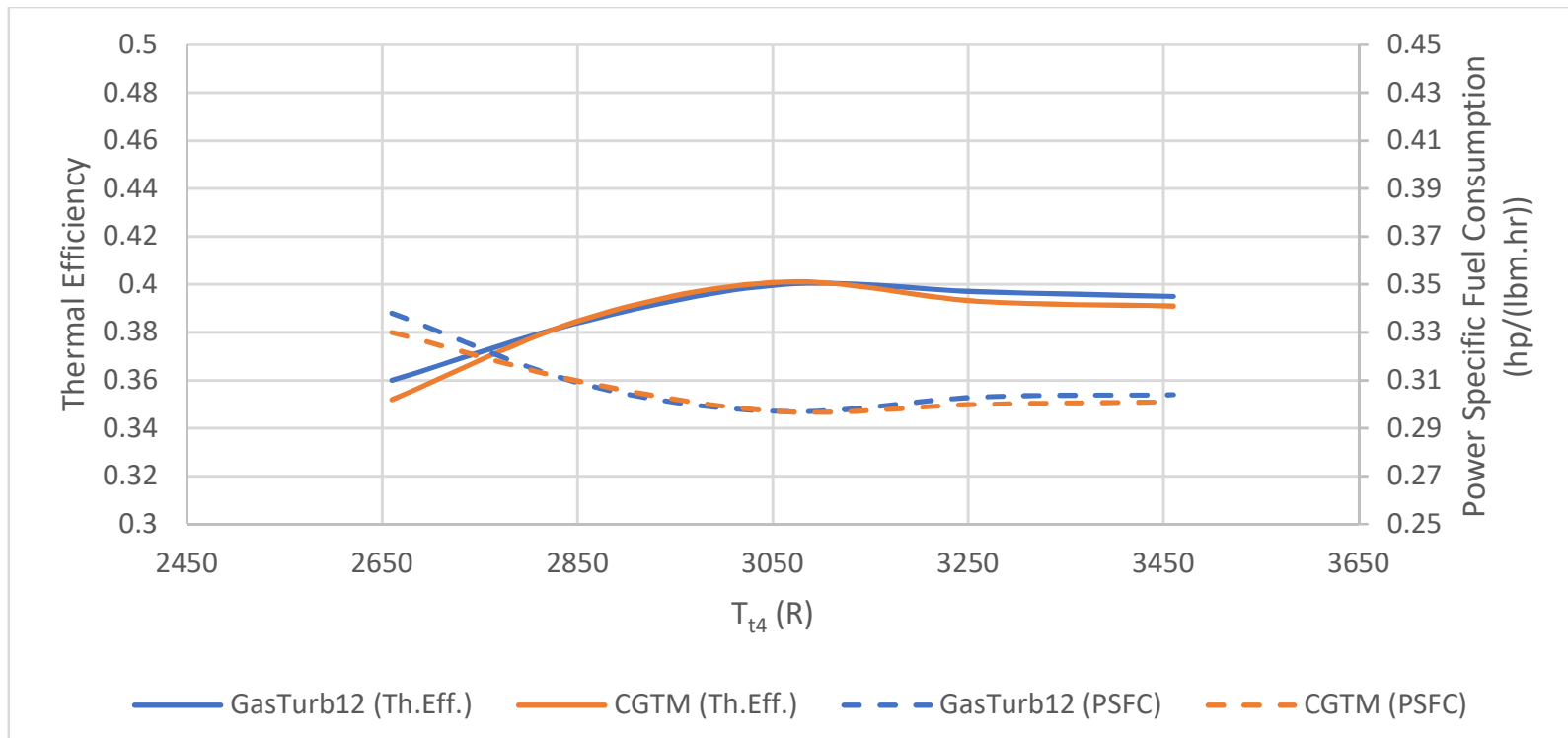

Figure 40: Change in Thermal Efficiency and Power Specific Fuel Consumption calculated for the cooled engine with the turbine inlet temperature

Similar to turbofan engine case, there exists an optimum point around a certain turbine inlet temperature $\left(\sim 3050^{\circ} \mathrm{R}\right)$, which was predicted the same by both the CGTM and GasTurb12. Above this optimum point, the advantages obtained from increasing the turbine inlet temperature are offset by the increased turbine losses and compressor work and result in a reduction in engine performance.

\subsection{Ambient Temperature}

For a fixed back pressure ratio, as the ambient temperature is increased it is expected to have a decrease in shaft power delivered. This can be explained with an analysis of temperature-entropy chart of the entire engine, which follows Brayton cycle principles as given in Figure 41.

The temperature-entropy diagram can also give information about the engine power produced. From Brayton cycle principles, increasing the engine inlet temperature will result in an increase in required compressor work, while the turbine is operating between the same pressure difference. In addition, increased coolant supply temperatures from the compressor will also reduce cooling performance in the turbine and results in a decrease in turbine performance as well. 


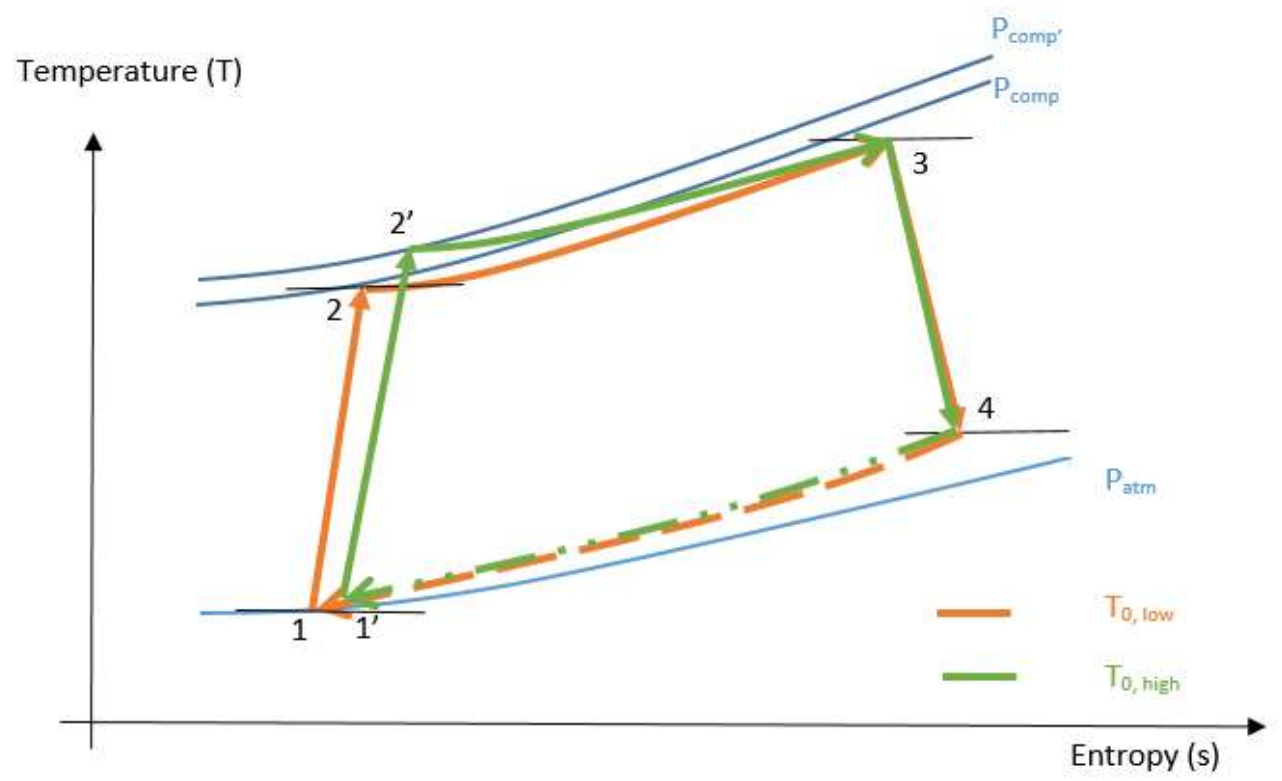

Figure 41: Temperature-entropy chart for a gas turbine; low ambient temperature case shown in orange, high ambient temperature case is shown in green

The comparison of the variation in the shaft power delivered and thermal efficiency as the ambient temperature is increased from $469{ }^{\circ} \mathrm{R}$ to $569{ }^{\circ} \mathrm{R}$ is shown in Figure 42.

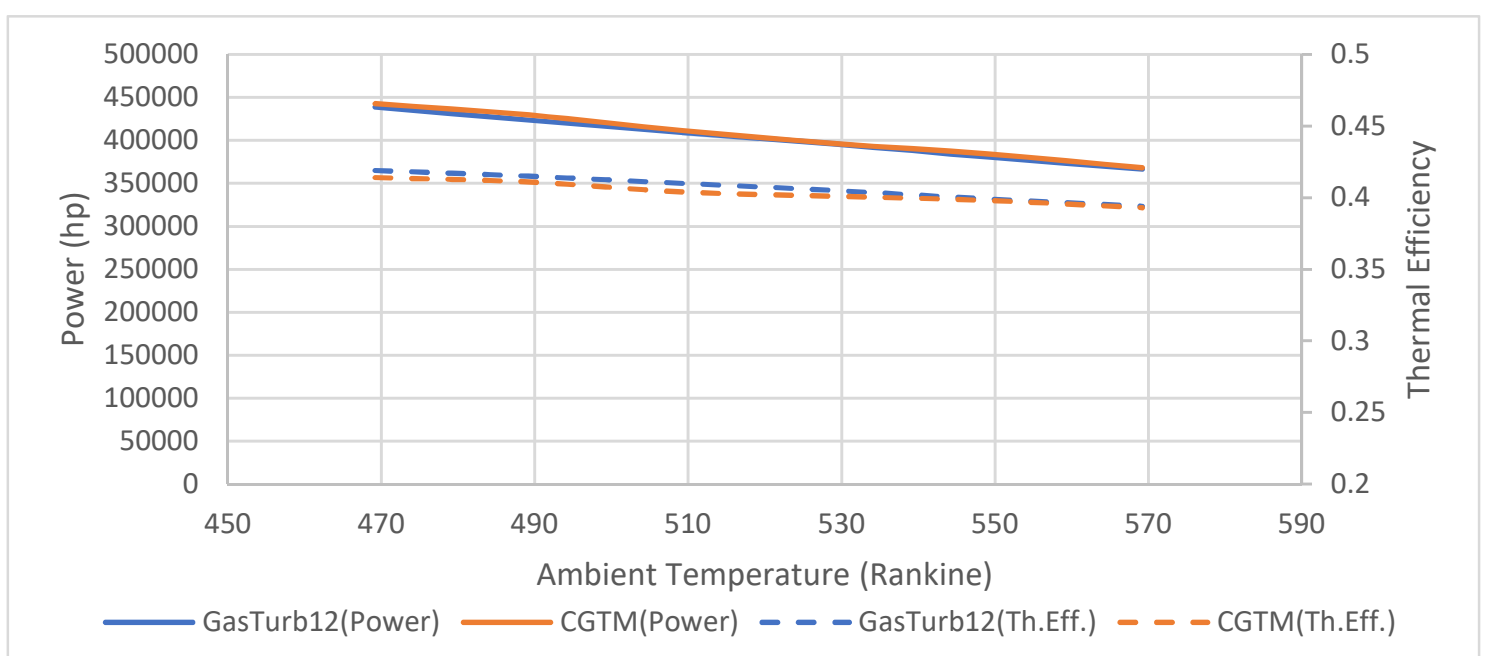

Figure 42: Change in Power and Thermal Efficiency calculated for the cooled engine with the ambient temperature

The comparison made for this test case in Figure 42 shows the expected trends, and the differences between the results obtained from CGTM and GasTurb12 are within $2 \%$.

\subsection{Back-Pressure Ratio}

For fixed turbine inlet temperature, as the engine back pressure ratio is increased, it is expected to have lower work production from the turbine, due to the increase in turbine exit pressure. Following up the thermodynamic analysis in Section 1.1, this would result in an increased turbine exit enthalpy and for a fixed maximum blade temperature, this would result in an increase in the coolant flows. Increased coolant flow fractions tend to 
reduce the increment in exhaust temperature but if the increase in the back pressure is higher, the effect of the increased cooling cannot compensate this effect, and thus a reduction in turbine work should occur.

In these tests, the back-pressure ratio is increased from 1.01 to 1.1 to observe the expected trend. The change in shaft power delivered and power specific fuel consumption are compared in Figure 43.

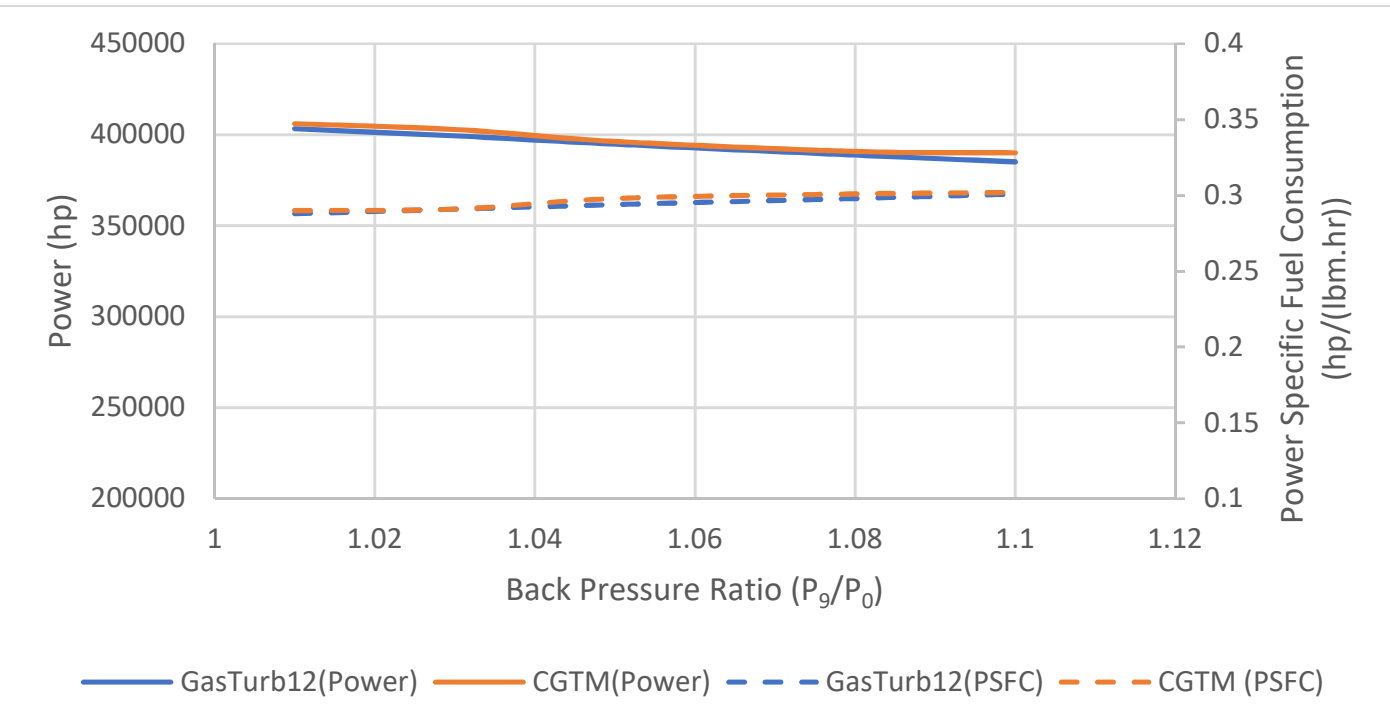

Figure 43: Change in Power and Thermal Efficiency calculated for the cooled engine with the back-pressure ratio

The comparison made for this test case in Figure 43 shows the expected trends, and the differences between the results obtained from CGTM and GasTurb12 are within $2 \%$. 


\section{CHAPTER 3: SENSITIVITY ANALYSIS ON GENERAL COOLING PARAMETERS}

In this section, some of the general cooling parameters used as inputs to the cooled engine models were changed within an interval to observe their effect on overall engine performance. Following cooling input parameters were used in this study:

- Blade Metal Biot Number $\left(\mathrm{Bi}_{\mathrm{m}}\right)$

- Thermal Barrier Coating Biot Number (Bi $\left.\mathrm{i}_{\mathrm{TBC}}\right)$

- Internal Cooling Efficiency $\left(\varepsilon_{c}\right)$

- Film Cooling Efficiency $\left(\varepsilon_{\mathrm{fc}}\right)$

- Film Cooling Effective Injection Angle $(\Phi)$

- Film Cooling Blowing Ratio (BR)§

All the input parameters considered above are changed within possible technological limits and the coolant configuration is fixed to be Internal + Film + TBC type for the first two stages, Internal + TBC for the third stage and Internal Only for the rest of the cooled stages.

\subsection{Analyzing the Effects of Individual Cooling Parameters}

To comment on the effects of different cooling parameters on the performance, CEDM and CGTM were used in this analysis for aero-engine and industrial gas turbine cases, respectively. The cooling inputs were then changed within the intervals shown in Table 24, and the change in key engine performance parameters are analyzed.

Table 24: Cooling parameters used in the Sensitivity Analysis are shown with their reference values and intervals

\begin{tabular}{|c|c|c|c|}
\hline Parameter & $\begin{array}{l}\text { Reference Value } \\
\text { (CEDM) }\end{array}$ & $\begin{array}{l}\text { Reference } \quad \text { Value } \\
\text { (CGTM) }\end{array}$ & $\begin{array}{l}\text { Range for } \\
\text { Analysis }\end{array}$ \\
\hline Metal Biot Number $\left(\mathrm{Bi}_{\mathrm{m}}\right)$ & 0.15 & 0.16 & {$[0.13,0.186]$} \\
\hline $\begin{array}{l}\text { Thermal Barrier Coating Biot } \\
\text { Number }\left(\mathrm{Bi}_{\mathrm{tbc}}\right)\end{array}$ & 0.35 & 0.37 & {$[0.17,0.57]$} \\
\hline $\begin{array}{l}\text { Maximum Blade Temperature } \\
\left(\mathrm{T}_{\mathrm{b}, \max }\left[{ }^{0} \mathrm{R}\right]\right.\end{array}$ & 2100 & 2000 & {$[1900,2020]$} \\
\hline $\begin{array}{l}\text { Adiabatic Film Cooling } \\
\text { Effectiveness }\left(\varepsilon_{f c}\right)\end{array}$ & 0.35 & 0.4 & {$[0.2,0.55]$} \\
\hline $\begin{array}{l}\text { Internal Cooling Effectiveness } \\
\qquad\left(\varepsilon_{c}\right)\end{array}$ & 0.6 & 0.7 & {$[0.45,0.85]$} \\
\hline $\begin{array}{l}\text { Effective Film Cooling Flow } \\
\text { Angle }(\Phi) \text { [deg] }\end{array}$ & 30 & 30 & {$[15,50]$} \\
\hline Film Cooling Blowing Ratio (BR) & 0.8 & 0.8 & {$[0.07,1.32]$} \\
\hline
\end{tabular}

§Film Cooling Blowing Ratio: The mass flux ratio between the coolant flow and the mainstream gas flow in film cooling 
The reference values of Table 24 were used from the values given in a study by Wilcock et al. [32] for "advanced cooling technology" levels for CGTM, and "recent cooling technology levels" for CEDM, and upper limits of the ranges were determined by considering the values given as "super-advanced technology". The results of reducing those parameters were also investigated, to observe the effect on engine performance when cooling techniques are negatively impacted during operations by conditions such as erosion, corrosion, sand/dust particles etc.

In all cases, the variation of required coolant flow rates with the change in certain cooling parameter was also traced. The following results were obtained by changing the indicated parameter independent of the other cooling parameters (i.e. the results are assuming an isolated effect on performance coming from a single parameter). All input parameters other than cooling related ones of CEDM and CGTM were used from the inputs for generic aero-engine case from Table 8, and generic $\mathrm{H}$-Class engine case from Table 22, respectively.

\subsubsection{Metal Biot Number $\left(\mathrm{Bi}_{\mathrm{m}}\right)$}

The definition of the Biot number of the blade material is defined as in Table-2 from Young and Wilcock [7] and given as follows (in ref. to Figure 4).

$$
B i_{m}=\frac{\alpha_{g} t_{m}}{k_{m}}=\frac{T_{m, e x t}-T_{m, i n t}}{T_{a w}-T_{w}}
$$

A decrease in metal Biot number can be achieved (assuming the wall thickness is fixed by the structural design) by using blade materials having higher resistance to conductive heat transfer (l.e. using higher $\mathrm{k}_{\mathrm{m}}$ ). Per the considered heat transfer scheme from Figure 4, if the blade material has a higher conductivity (thus a lower $\mathrm{Bi}_{m}$ ) it can be cooled easier from the inside; resulting in a lower coolant flow requirement by external cooling techniques. Using less external coolant flow would eventually result in a decrease in turbine losses (mixing associated losses will be reduced) and the engine performance would be affected positively

It should be noted that this expected trend is valid only when the cooling configuration has a TBC on the blade metal, which is the assumed cooling configuration for this analysis. If there is no TBC on the blades, reducing the blade metal conductivity would reduce the resistance of the blade metal to the heat flux from the hot gas side, and for such a case lower $\mathrm{Bim}_{\mathrm{m}}$ would reduce engine performance (i.e. opposite of the expected trend with TBC).

\subsubsection{High By-Pass Turbofan Engine}

In this analysis with a turbofan engine with 1 cooled HPT stage, metal Biot number is changed from 0.13 to 0.17 to analyze the effects of this parameter on turbofan performance. The effect on the engine thrust is shown in Figure 44. 


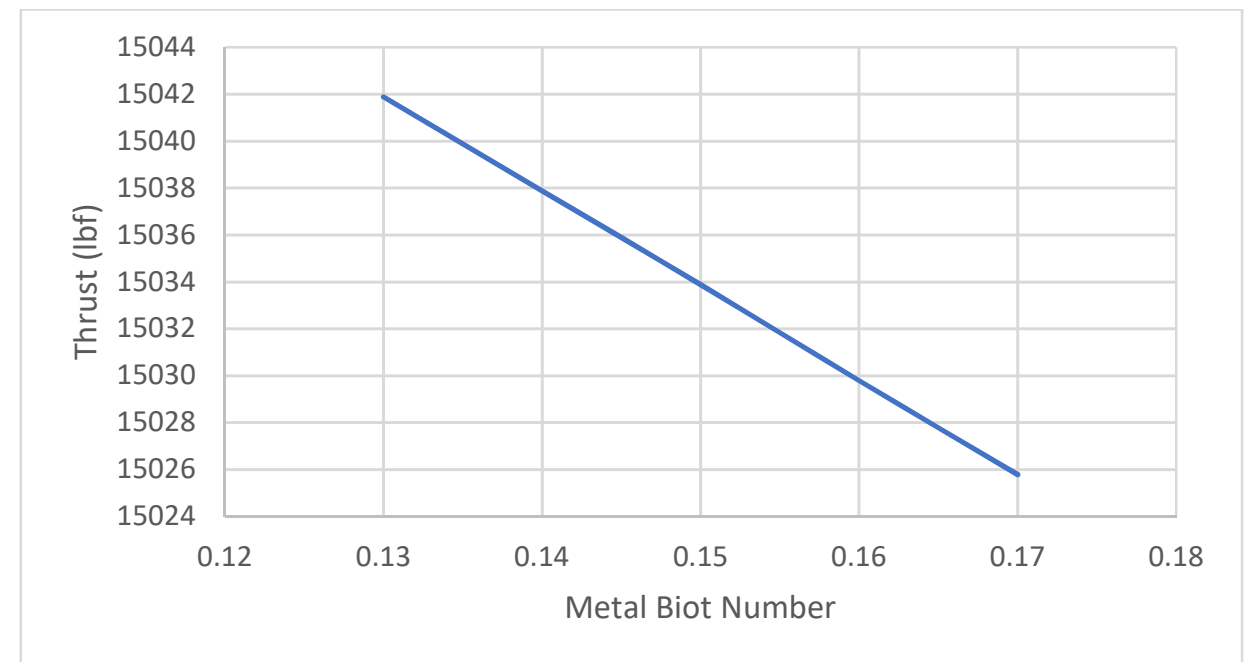

Figure 44: Change in the engine thrust when the metal Biot number is increased

As the metal Biot number is increased, thrust from the engine is reduced by $0.11 \%$. Corresponding change in the thrust specific fuel consumption is shown in Figure 45.

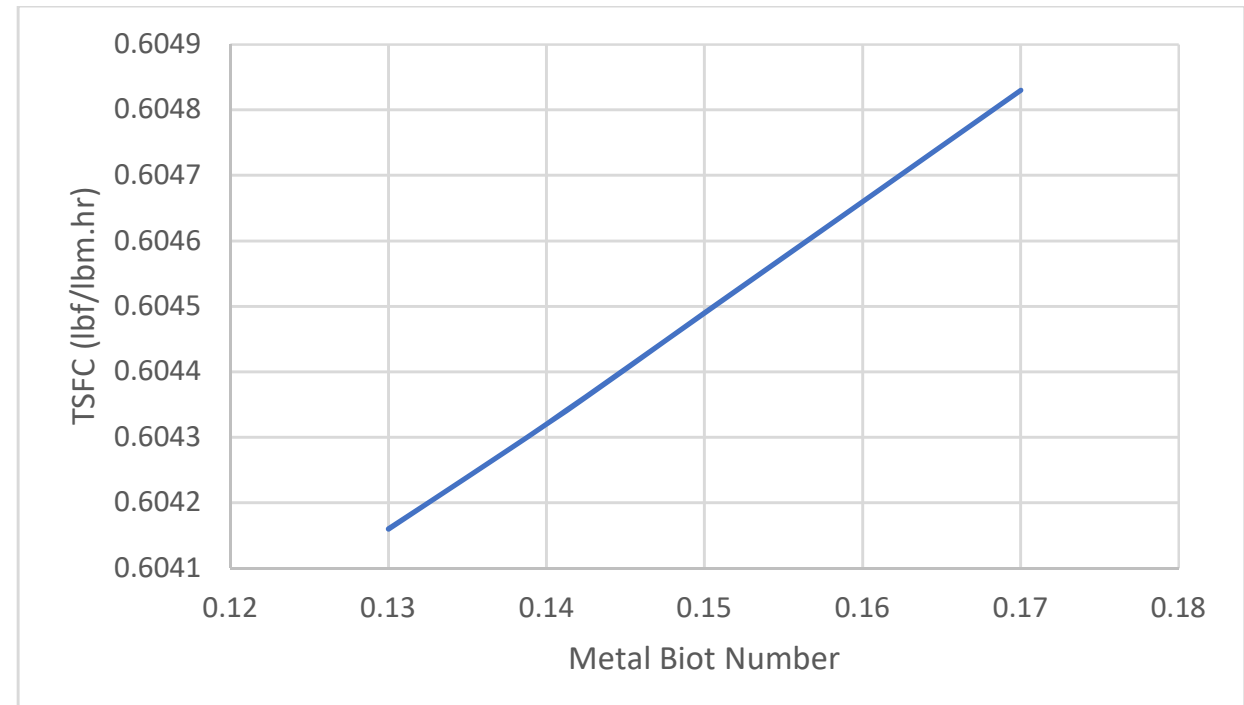

Figure 45: Change in the thrust specific fuel consumption when the metal Biot number is increased

The increase in the thrust specific fuel consumption occurred as $0.11 \%$, which indicates that the increase is totally caused by the reduction in thrust due to increased cooling losses in turbine (increase in fuel consumption is $0.01 \%$ ). The reduction in the thermal efficiency is therefore mostly caused by the reduction in the thrust and shown in Figure 46. 


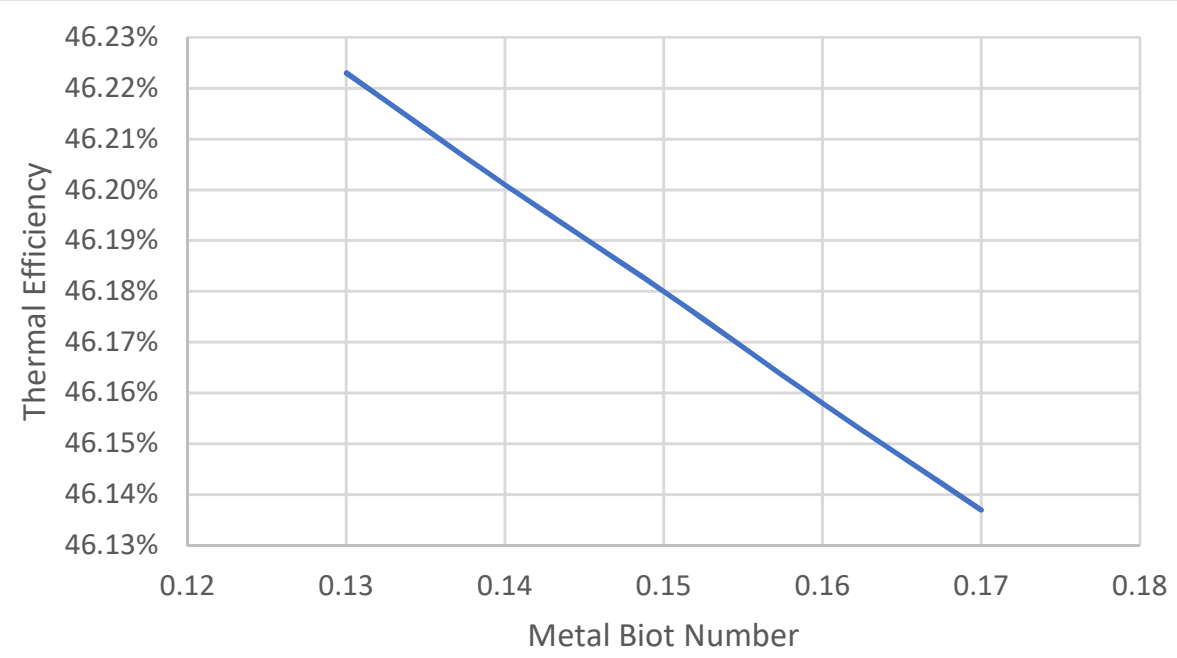

Figure 46: Change in the thermal efficiency when the metal Biot number is increased

The reduction in thermal efficiency occurred as $0.09 \%$. The reduction in engine performance is directly related with the increase in the total coolant flow rates. The corresponding change in stator and rotor coolant fractions are shown in Figure 47 for this analysis.

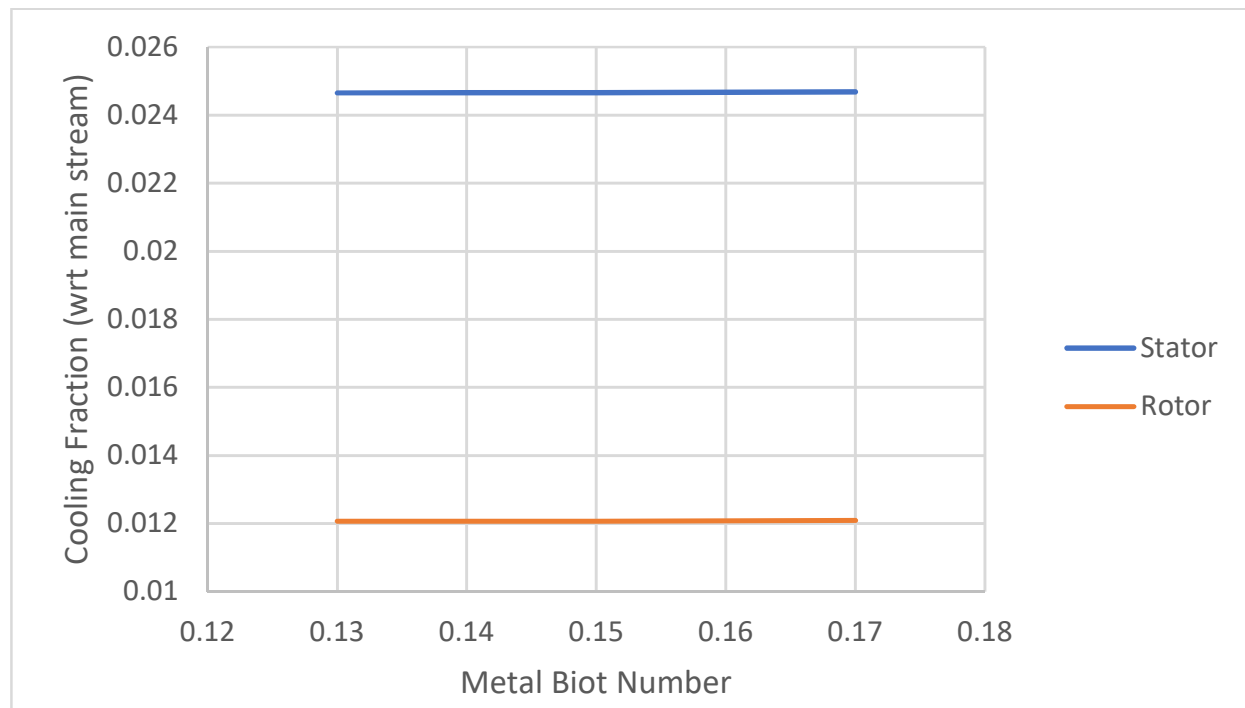

Figure 47: Change in the total coolant fractions when the metal Biot number is increased

\subsubsection{Industrial Gas Turbine}

In this analysis with an industrial gas turbine with 4 cooled turbine stages, metal Biot number is changed from 0.146 to 0.186 to analyze the effects of this parameter on the industrial gas turbine performance. The effect on the shaft power delivered is shown in Figure 48. 


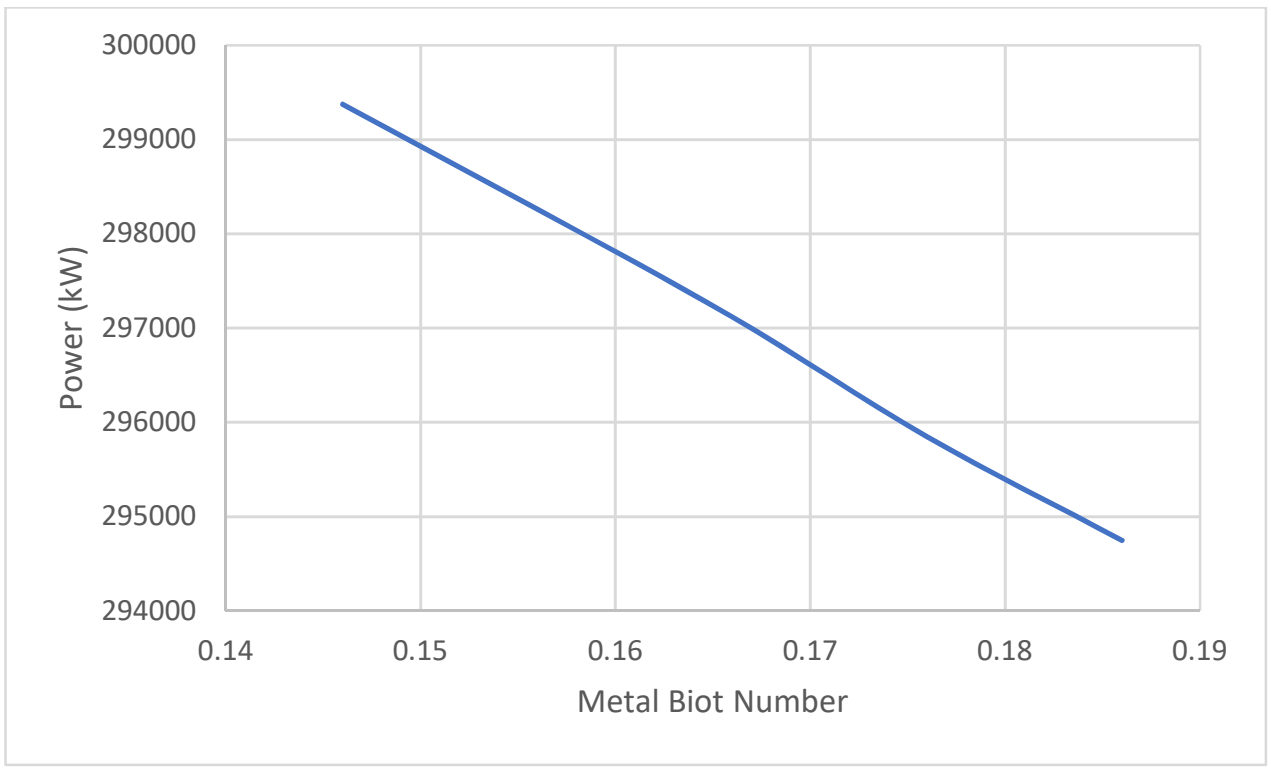

Figure 48: Change in the shaft power delivered when the metal Biot number is increased

The reduction in the shaft power delivered occurred as $1.55 \%$, greater than the reduction rate seen in aeroengine case due to having multi-staged cooling in this engine type. Corresponding change in the heat rate is shown in Figure 49.

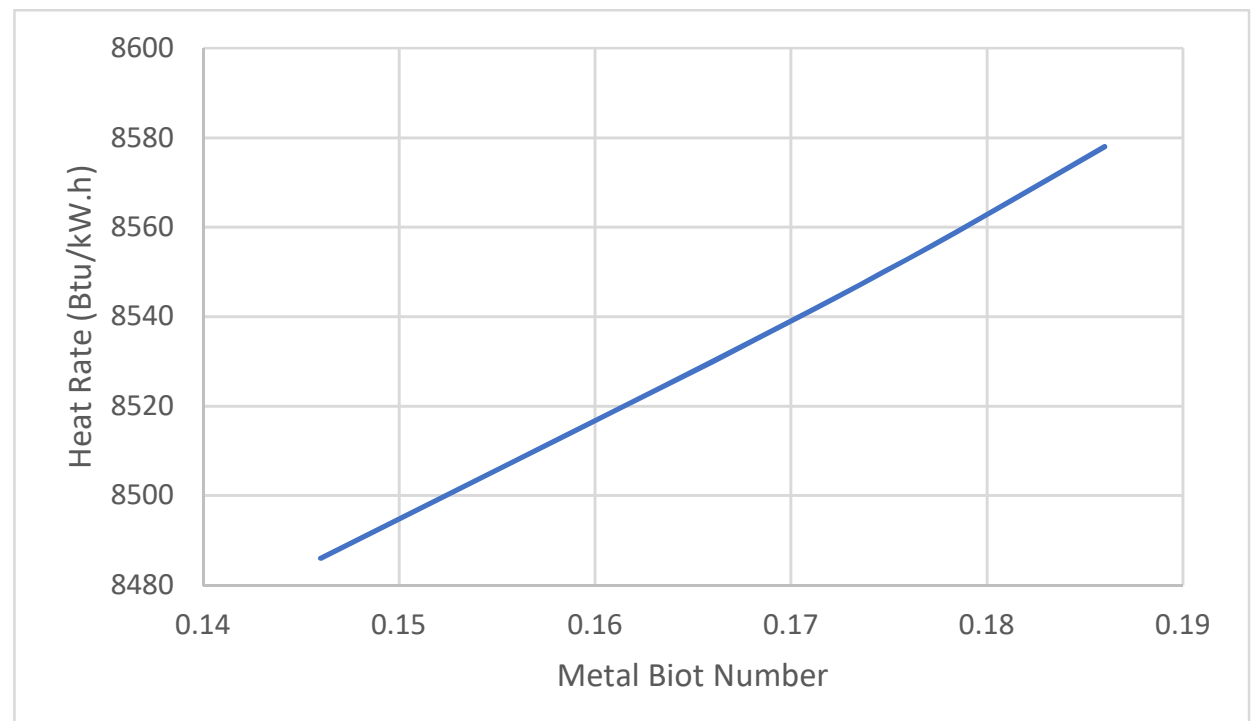

Figure 49: Change in the heat rate when the metal Biot number is increased

The increase in the heat rate occurred as $1.08 \%$, which is realized with an increase in fuel consumption by $1.67 \%$ (more than the reduction in power). Corresponding reduction in the thermal efficiency is shown in Figure 50. 


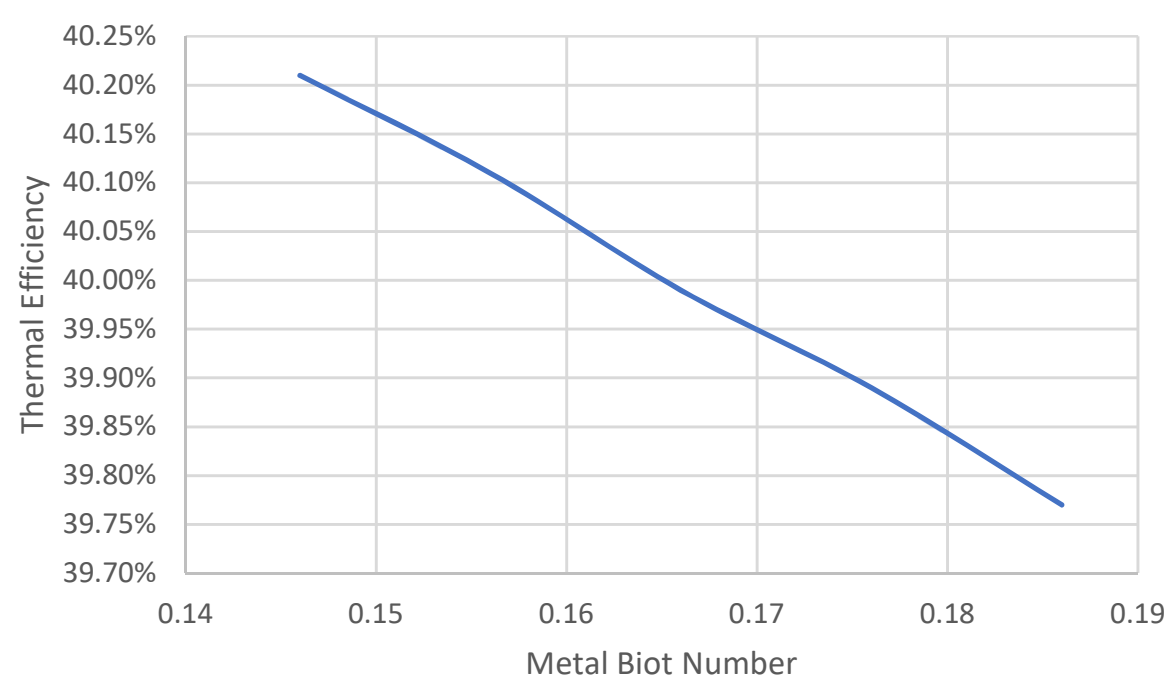

Figure 50: Change in the thermal efficiency when the metal Biot number is increased

Same trend in the reduction of thermal efficiency with the increase in metal Biot number was also noted by Wilcock et al. [32], in which the metal Biot number was changed from 0.1 to 0.2 . The reduction in the engine performance is due to an increase in total coolant flow rates, which are shown for the stators and rotors separately in Figure 51.

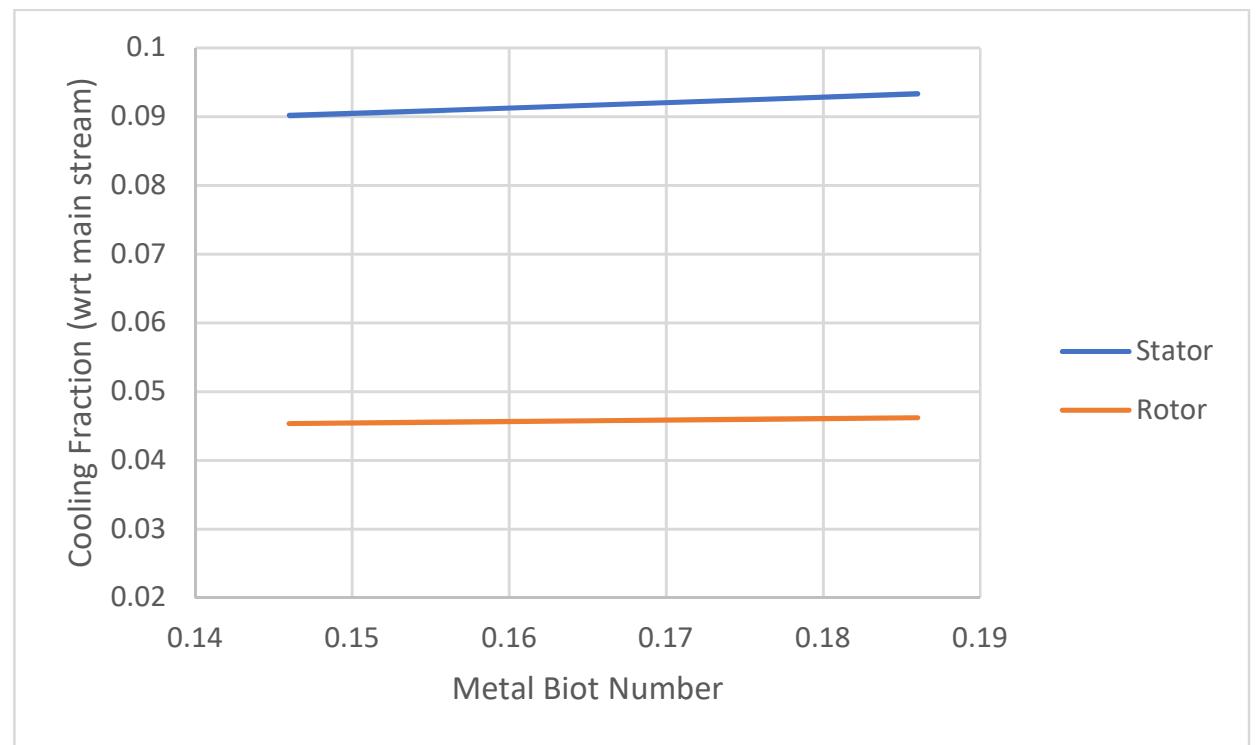

Figure 51: Change in the total coolant flow rates when the metal Biot number is increased

Total stator cooling flow rate is increased by $3.5 \%$, whereas the rotor coolant flow rate is increased by $1.87 \%$ (about the half of stators). This is due to having higher temperature gradients on stator blades than the rotor blades in all turbine stages.

Increasing the Biot number of the metal, while keeping the external metal temperature ( $T_{\mathrm{m} \text {,ext }}$ from Figure 4 ) constant at maximum allowable blade temperature $\left(T_{b}\right.$,max $)$ means increasing the temperature difference across the metal by reducing the coolant side wall temperature $\left(T_{m, \text { int }}\right.$ from Figure 4$)$ further. This promotes 
increased conductive heat transfer across the metal, which increases the "internal conduction losses" $\left(\dot{\sigma}_{m e t}\right)$, which is calculated from Equation (36).

$$
\dot{\Sigma}_{m e t}=\dot{Q}_{s}\left(\frac{1}{T_{m, i n t}}-\frac{1}{T_{m, e x t}}\right)
$$

This loss translates to loss of available turbine work due to the reduction of more heat to the blade by increasing the heat transfer across it, instead of using this heat to produce work from the turbine. Reducing the coolant side temperature of the metal with fixed internal cooling efficiency also requires more coolant flow. Because of this increase in internal conduction losses in addition to the increased amount of coolant flow rates, the power (or thrust) generated by the cooled gas turbine (or aero-engine) is reduced, causing a reduction in thermal efficiency. Heat rate (or thrust specific fuel consumption) is inversely proportional to the power (or thrust) produced; therefore, the resulting effect was an increase in this parameter.

\subsubsection{TBC Biot Number $\left(\mathrm{Bi}_{\mathrm{TBC}}\right)$}

The Biot number for the thermal barrier coating material is defined as in Table-2 from Young and Wilcock [7] and given as follows (in ref. to Figure 4).

$$
B i_{t b c}=\frac{\alpha_{g} t_{t b c}}{k_{t b c}}=\frac{T_{w}-T_{m, e x t}}{T_{a w}-T_{w}}
$$

In the cooling configurations that make use of thermal barrier coating, an increase in TBC Biot number can be achieved either by increasing the thickness of the TBC layer or using a lower conductivity TBC material. In the development of new cooled turbine components, using advanced TBC materials that offers low conductivity and longer durability is usually preferred among increasing the thickness, in order to avoid the risk of increasing structural loads on the blades.

\subsubsection{High By-Pass Turbofan Engine}

In this analysis with the turbofan engine with 1 cooled HPT stage, TBC Biot number is changed from 0.2 to 0.5. The effect on the engine thrust is shown in Figure 52.

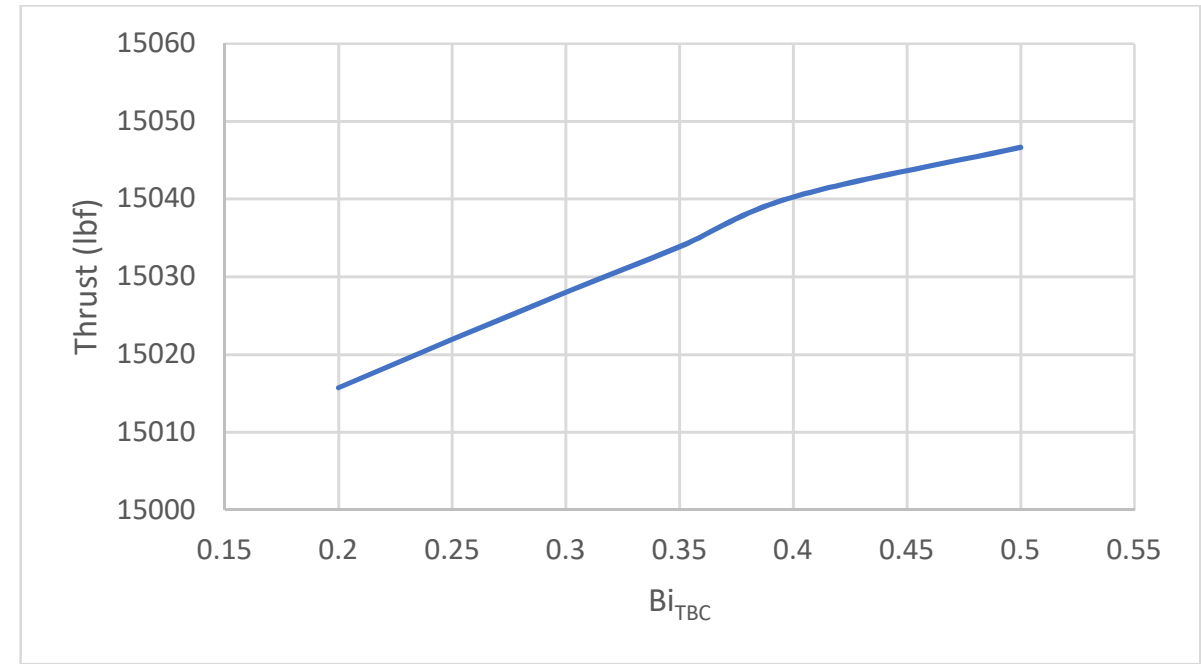

Figure 52: Change in the engine thrust when the TBC Biot number is increased 
The change in the engine thrust occurred as $0.21 \%$, when the TBC Biot number is increased. Corresponding change in the thrust specific fuel consumption is shown in Figure 53.

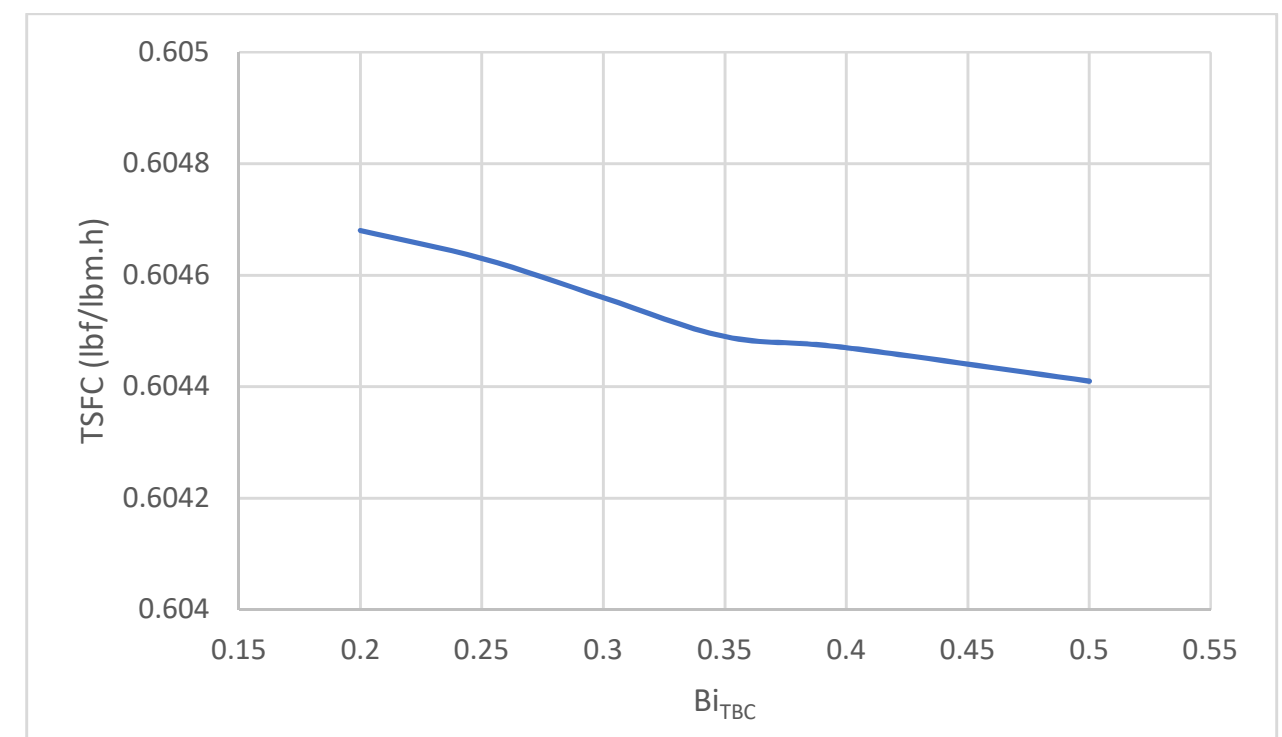

Figure 53: Change in the thrust specific fuel consumption when the TBC Biot number is increased

The reduction in the thrust specific fuel consumption occurred as $0.05 \%$, which is lower than the increase in the thrust indicating that the fuel consumption is nearly unaffected (reduced by $0.01 \%$ ) with this change. The effect on the thermal efficiency is shown in Figure 54.

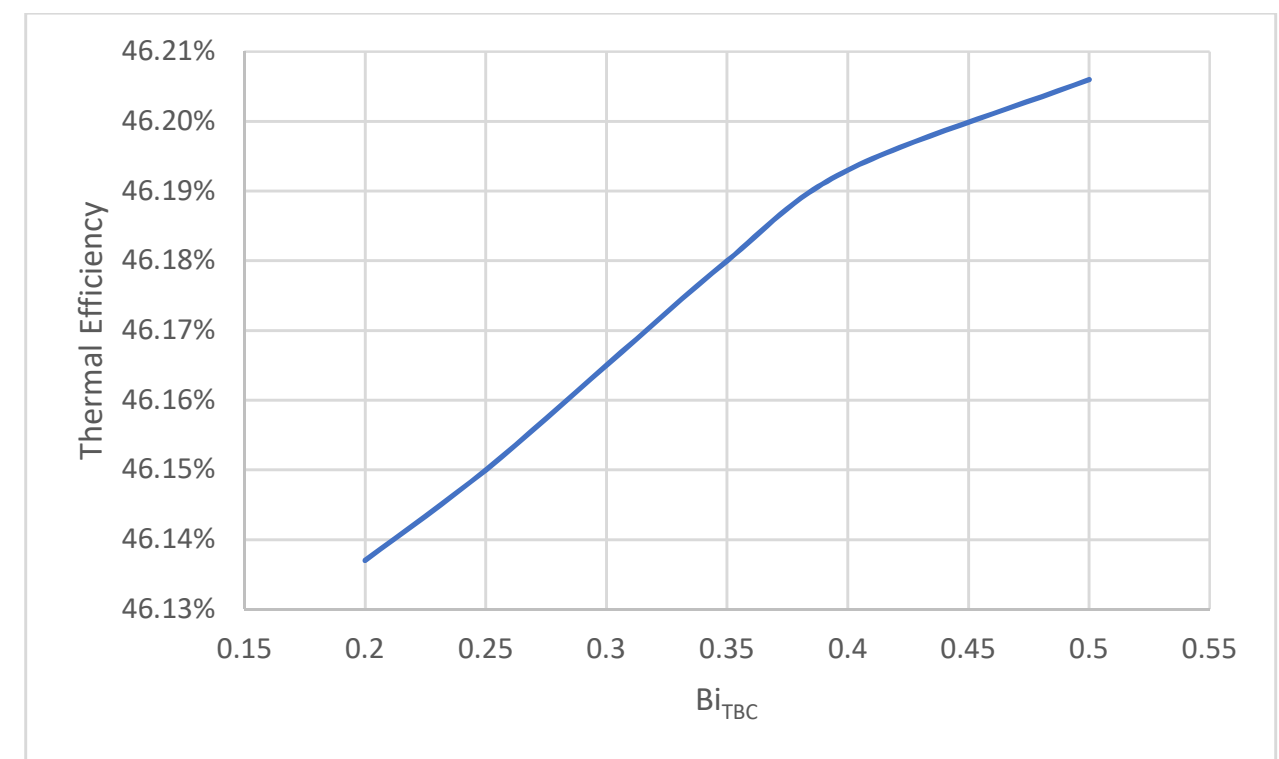

Figure 54: Change in the thermal efficiency when the TBC Biot number is increased

As the required coolant fractions are reduced, thrust specific fuel consumption is reduced while the thrust is increased. This resulted in an increase in the thermal efficiency by $0.07 \%$. The change in the total coolant fractions is shown in Figure 55. 


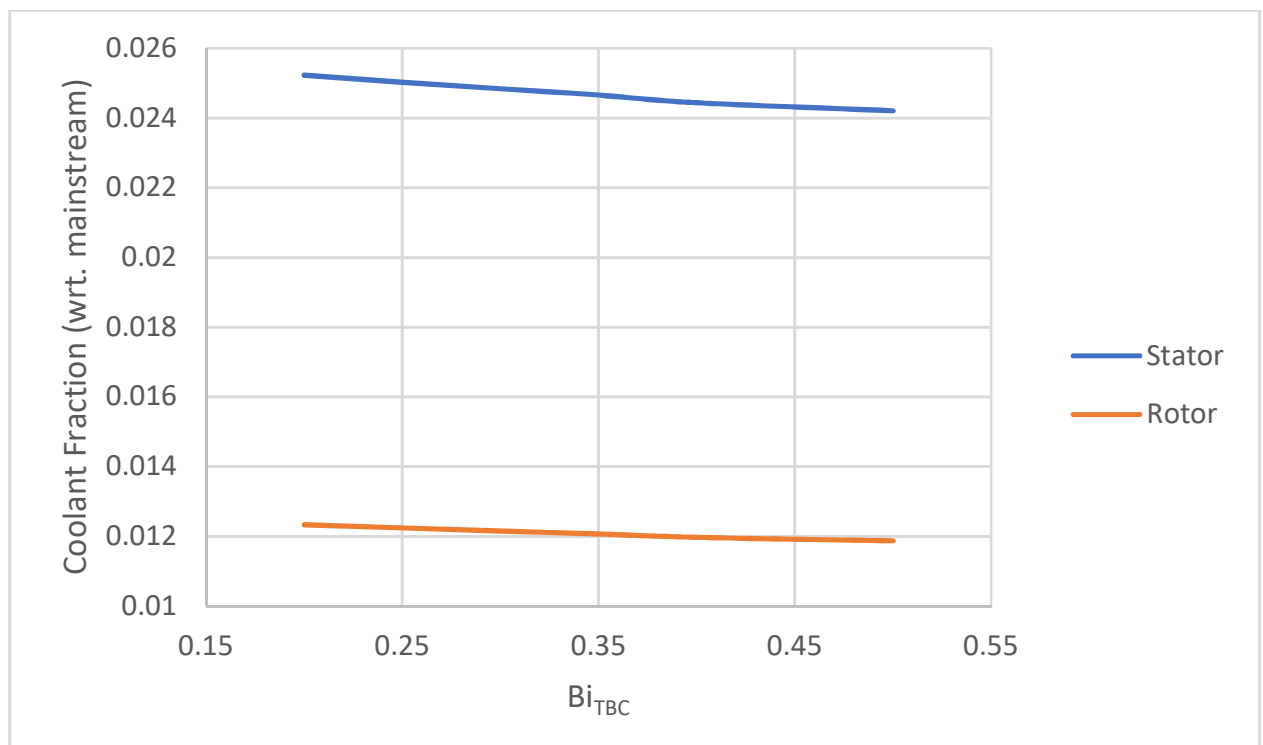

Figure 55: Change in the total coolant fractions when the TBC Biot number is increased

Stator coolant fraction is reduced by $4.04 \%$, and the rotor coolant fraction is reduced by $3.73 \%$, when the TBC Biot number is increased from 0.2 to 0.5 .

\subsubsection{Industrial Gas Turbine}

In the analysis with industrial gas turbine, which has 4 cooled turbine stages, TBC Biot number is changed from 0.17 to 0.57 to analyze the effects of this parameter on engine performance. The effect on the shaft power delivered is shown in Figure 56.

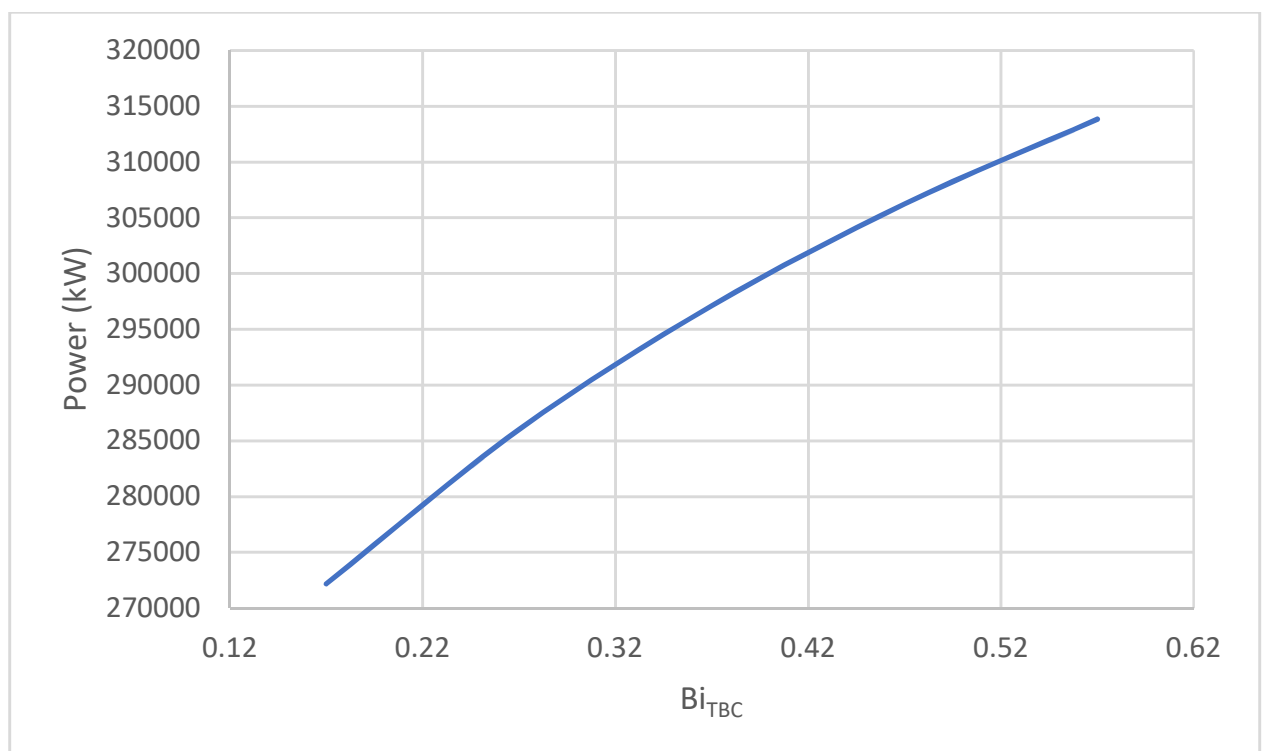

Figure 56: Change in the shaft power delivered when the TBC Biot number is increased

The increase in the shaft power delivered occurred as $15.3 \%$ with this change. Corresponding effect on the heat rate is shown in Figure 57. 


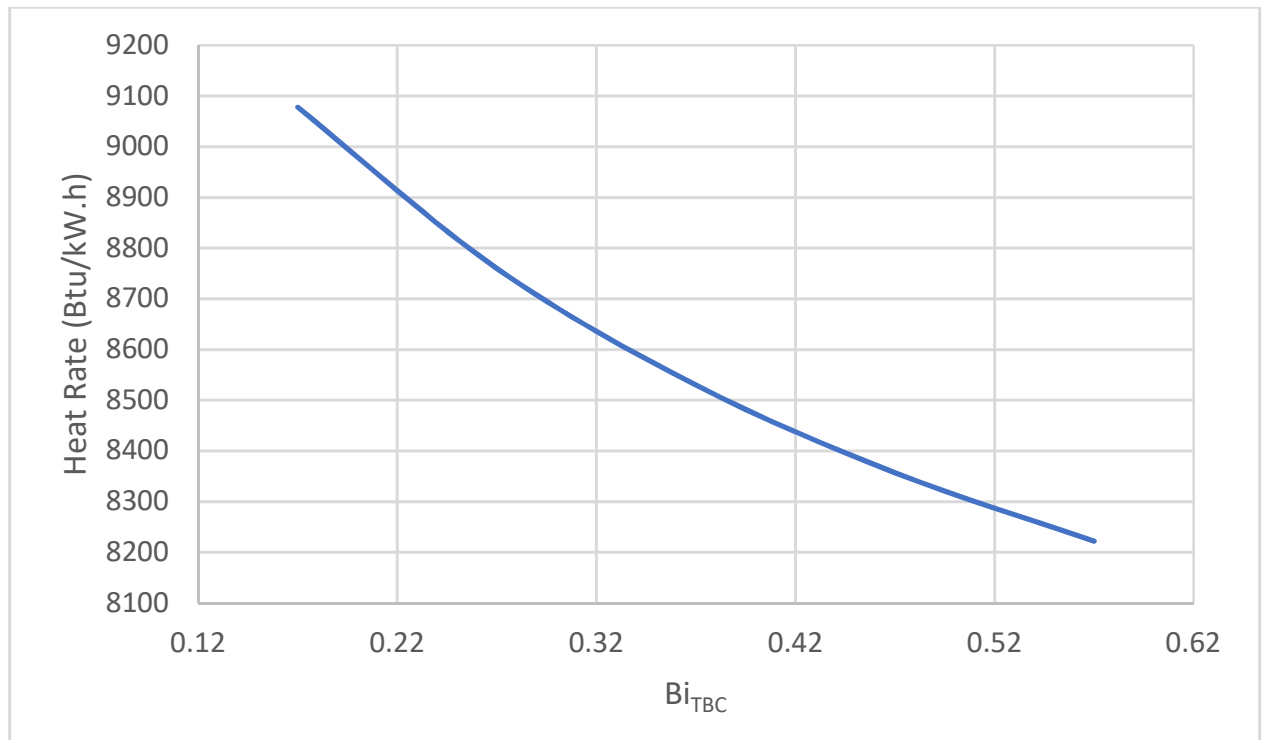

Figure 57: Change in the heat rate when the TBC Biot number is increased

The reduction in the heat rate is $9.43 \%$, which is dominated by the reduction in fuel consumption. The overall effect is therefore, an increase in the thermal efficiency as shown in Figure 58.



Figure 58: Change in the thermal efficiency when the TBC Biot number is increased

The increase in the thermal efficiency occurred as 3.91\%. Similar trend in thermal efficiency with the increase in TBC Biot number was also noted in a study by Wilcock et al. [32], in which it was changed from 0.2 to 0.4 . As with the turbofan engine, these changes in the engine performance are realized by the reduction of total coolant flows, which are shown in Figure 59. 


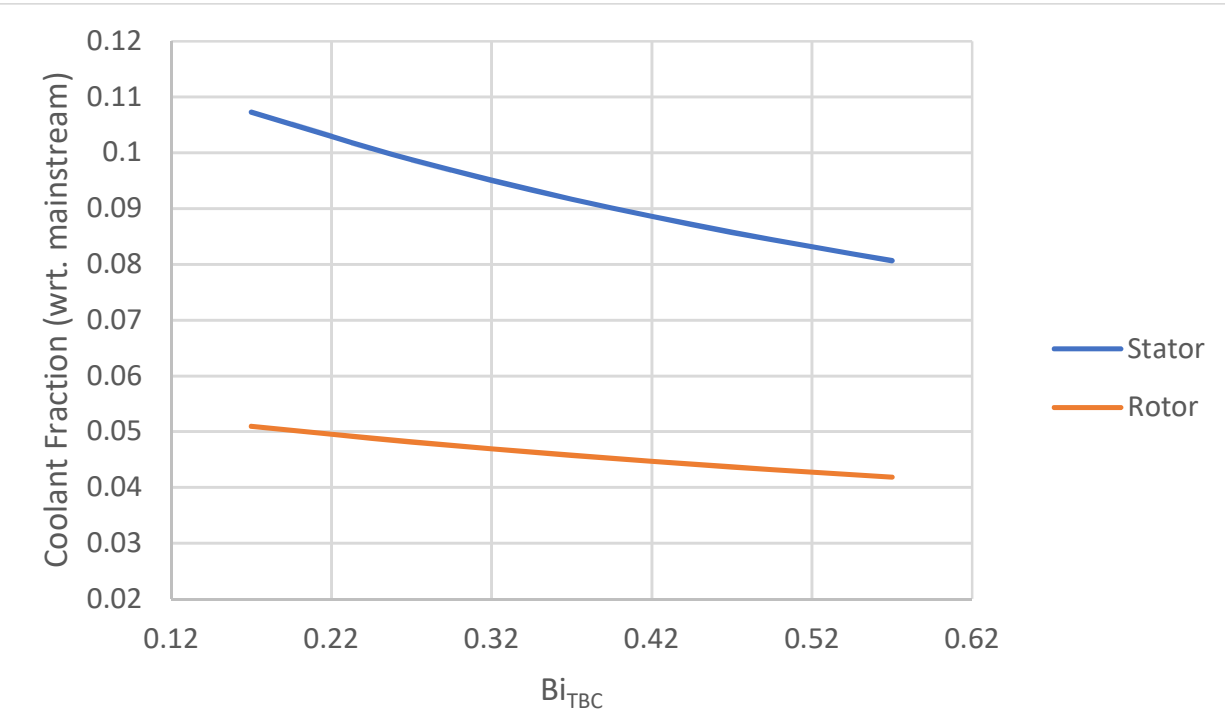

Figure 59: Change in the total coolant fractions when the TBC Biot number is increased

The reduction in the total coolant fraction for stator stages is $24.8 \%$, and in the rotor stages is $17.87 \%$.

As the TBC Biot number is increased, while keeping other cooling parameters constant, the wall temperature of the blade ( $T_{w}$ of Figure 4 ) is allowed to have a temperature greater than the external metal temperature ( $T_{\mathrm{m}}$, ext of Figure 4), which is kept constant at the maximum allowable blade metal temperature ( $T_{b}$, max). Having a temperature difference between two ends of TBC layer would increase TBC losses $\left(\dot{\sigma}_{t b c}\right)$.

However, in both engines, analyzed cooling configurations include film cooling. Fixing internal cooling flow efficiency and adiabatic cooling effectiveness of film cooling fixes the adiabatic wall temperature ( $T_{\text {aw }}$ of Figure 4). Using the definitions of internal cooling flow and film cooling effectiveness in the semi-empirical coolant mass flow rate relation from Equation (14), would give the proportionality relation given in Equation (72).

$$
\frac{\dot{m}_{c l}}{\dot{m}_{g}} \propto \frac{T_{a w}-T_{w}}{T_{t c l, x}-T_{t c l, i}}
$$

As the TBC Biot number is increased, wall temperature $T_{w}$ increases from a value closer to $T_{b, m a x}$ to a value close to gas temperature $\left(T_{\mathrm{tg}}\right)$. Since the gas temperature is always greater than the maximum allowable

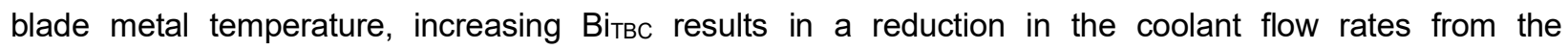
proportionality in Equation (72). The effect of the reduction in the coolant flow rates overcomes the increase in TBC conduction losses and the engine performance is increased.

\subsubsection{Maximum Allowable Blade Metal Temperature $\left(T_{b, \max }\right)$}

This temperature indicates the maximum allowable temperature on the blade metal, which might be lower than the blade wall temperature and/or the adiabatic wall temperature, if TBC and/or film cooling is used in the cooling configuration. If TBC and/or film cooling do not exist on the blade, then this temperature indicates a limit on the wall temperature.

From Equation (14), the maximum allowable blade temperature dictates the amount of the coolant flow required directly and $\mathrm{T}_{\mathrm{m} \text {,ext }}$ is set equal to this temperature, in order the coolant calculations be in accordance 
with this limit. Increasing this upper limit through the usage of advanced blade materials will reduce the required coolant flow rates that will also result in the reduction of all $2^{\text {nd }}$ Law losses defined in Section 1.1.3.

The expected trend with an increase in this temperature is, therefore, to have an increase in power production (or thrust), reduction in fuel consumption (reduces TSFC and heat-rate) and an increase in thermal efficiency.

\subsubsection{High By-Pass Turbofan Engine}

In the analysis made with a turbofan engine with 1 cooled HPT stage, maximum allowable blade metal temperature is changed between $2051^{\circ} \mathrm{R}$ to $2171^{\circ} \mathrm{R}\left(1139{ }^{\circ} \mathrm{K}\right.$ to $\left.1206^{\circ} \mathrm{K}\right)$. The effect on the engine thrust is shown in Figure 60.



Figure 60: Change in the engine thrust when the maximum allowable blade metal temperature is increased

The increase in the engine thrust is occurred as $2.55 \%$. Corresponding change on the thrust specific fuel consumption is shown in Figure 61.

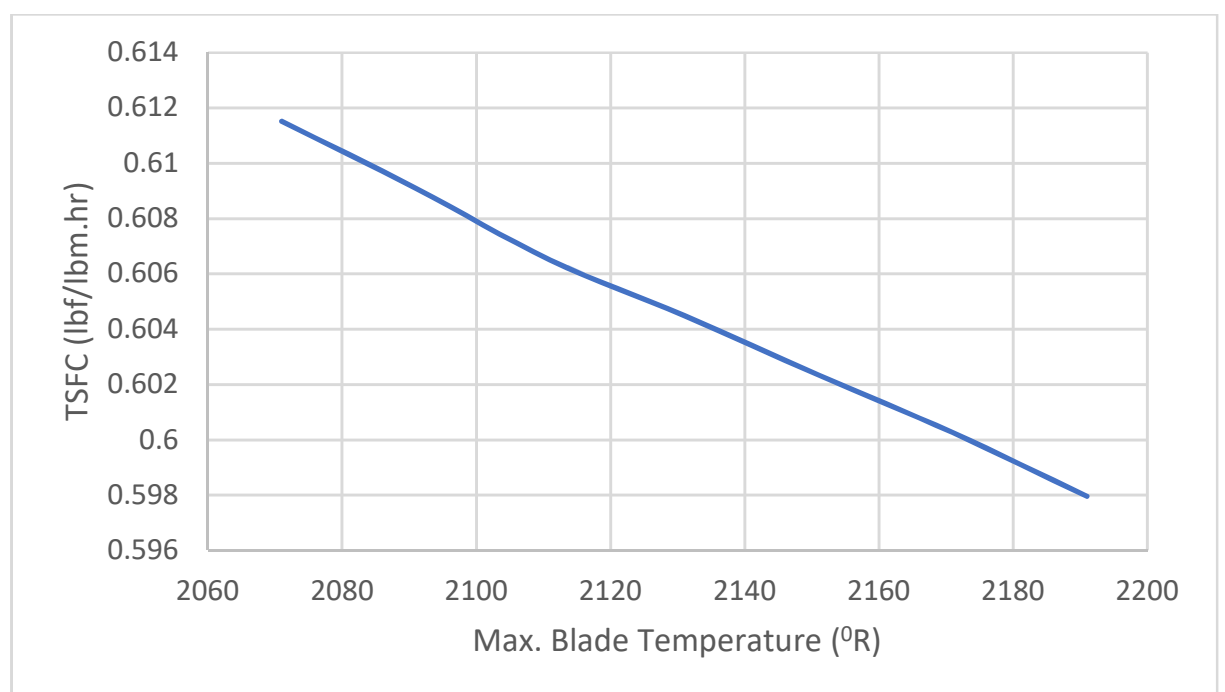

Figure 61: Change in the thrust specific fuel consumption when the maximum allowable blade metal temperature is increased 
The reduction in TSFC is occurred as $2.22 \%$, which indicates a reduction in the fuel consumption by $5.66 \%$. Resulting increase in the thermal efficiency is shown in Figure 62.

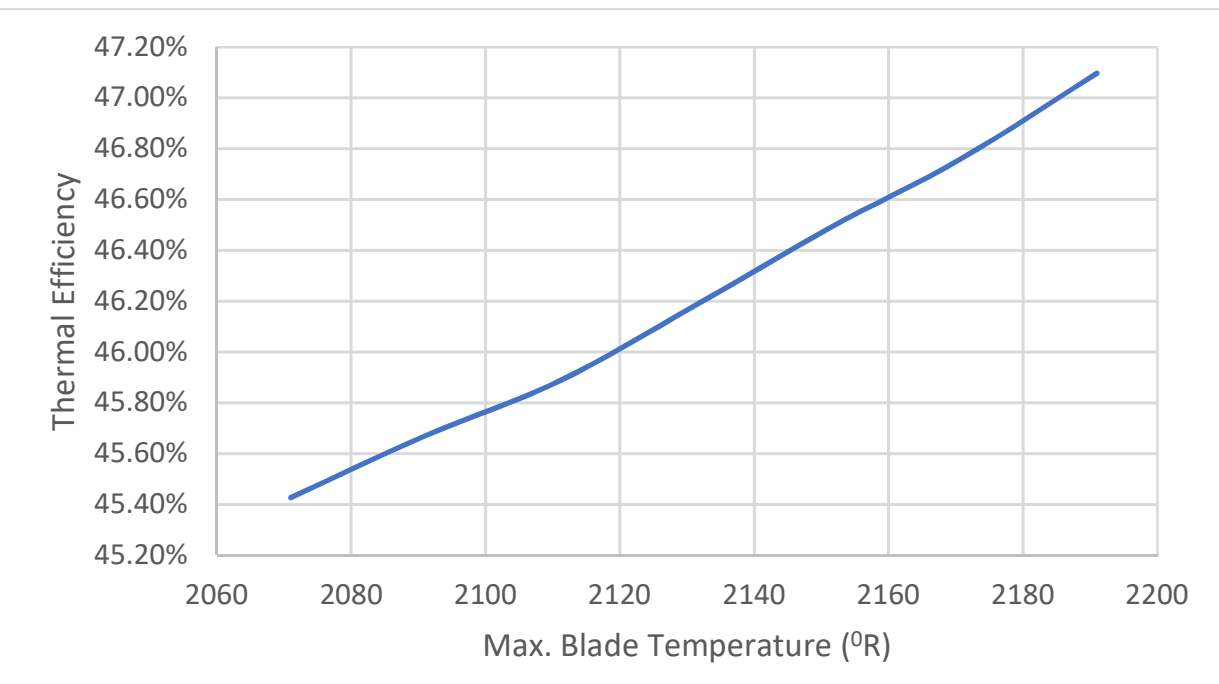

Figure 62: Change in the thermal efficiency when the maximum allowable blade metal temperature is increased

Thermal efficiency is increased by $2.05 \%$ when the maximum allowable blade temperature is increased. This increase in engine performance is realized by the reduction in total coolant fractions, which are shown in Figure 63.

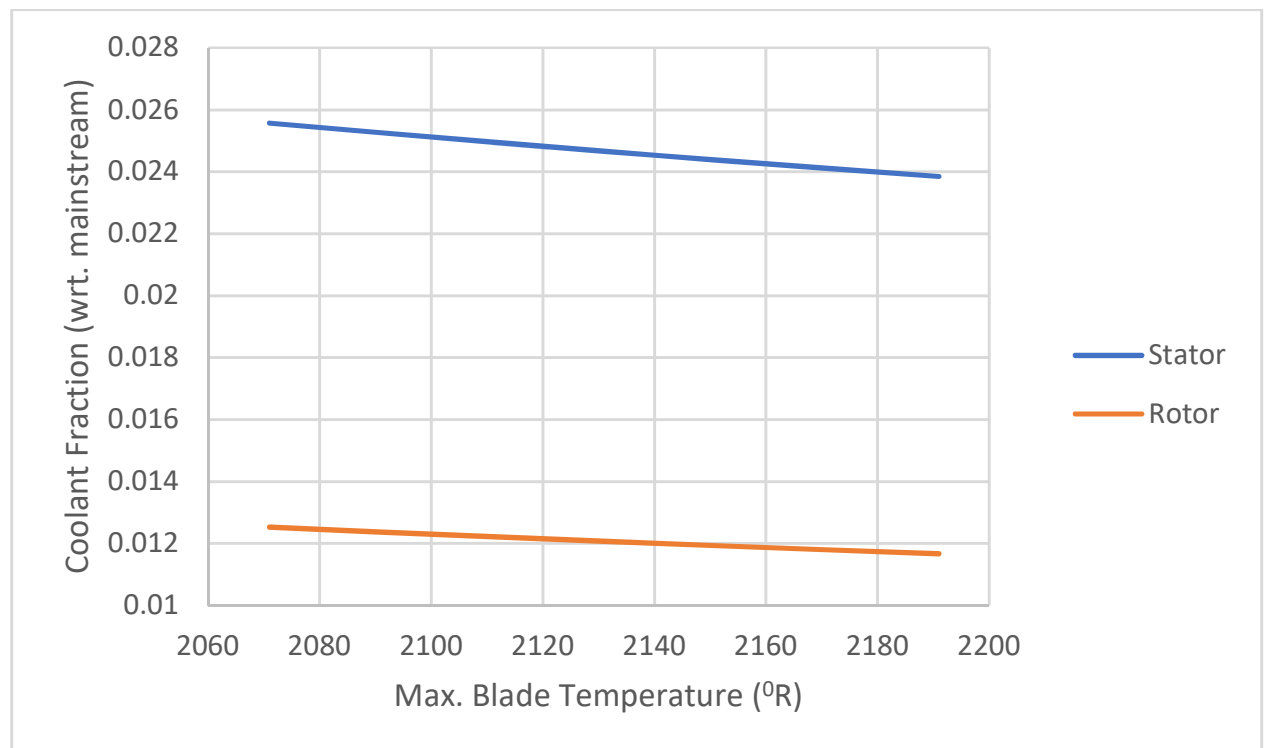

Figure 63: Change in the total coolant flow rates when the maximum allowable blade metal temperature is increased

Increasing the maximum allowable blade metal temperature resulted in a $6.73 \%$ decrease in the stator coolant flow, and $6.64 \%$ decrease in the rotor coolant flow. 


\subsubsection{Industrial Gas Turbine}

Maximum allowable blade temperature is changed between $1900{ }^{\circ} \mathrm{R}$ to $2020{ }^{\circ} \mathrm{R}$ (1056 ${ }^{\circ} \mathrm{K}$ to $1122{ }^{0} \mathrm{~K}$ ) to analyze the effects of this parameter on the performance of the industrial gas turbine with 4 cooled turbine stages. The effect on the shaft power delivered is shown in Figure 64.

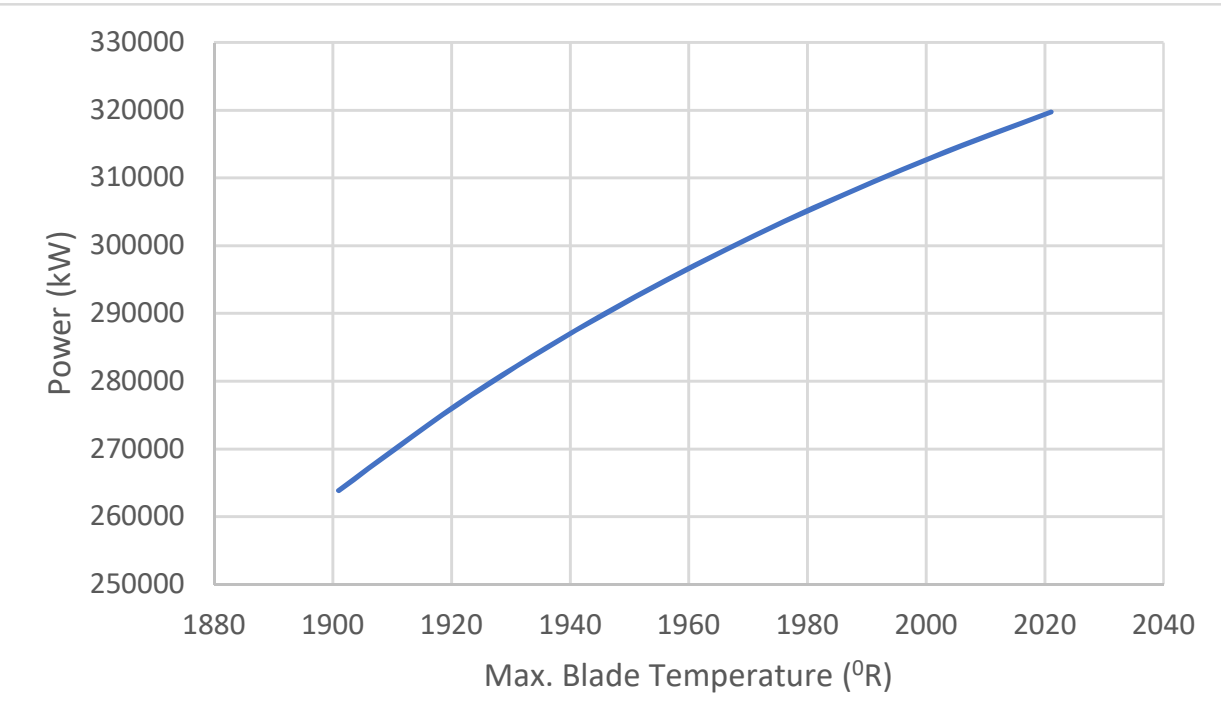

Figure 64: Change in the shaft power delivered when the maximum allowable blade metal temperature is increased

The increase in the shaft power delivered occurred as $21.16 \%$. Corresponding change in the heat rate is shown in Figure 65.

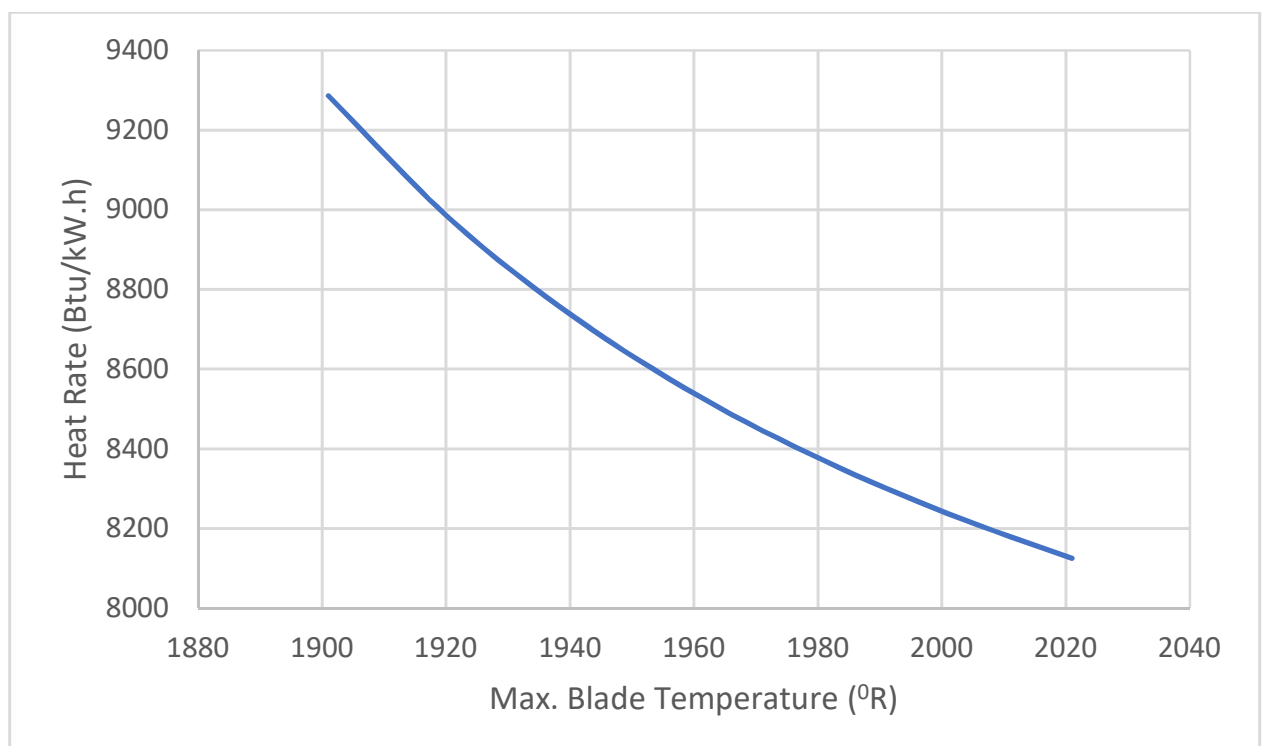

Figure 65: Change in the heat rate when the maximum allowable blade metal temperature is increased

The reduction in the heat rate occurred as $12.5 \%$, which also dominated by a reduction in fuel consumption, as in the turbofan case. Resulting increase in the thermal efficiency is shown in Figure 66. 


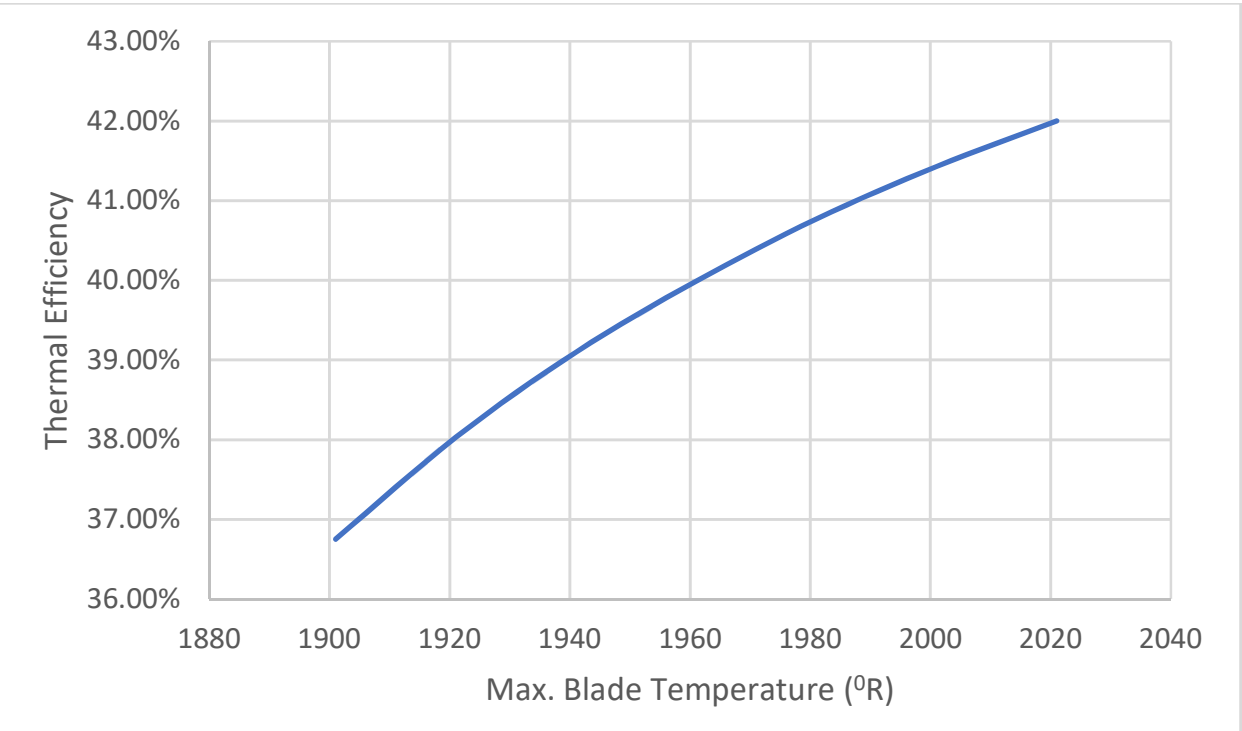

Figure 66: Change in the thermal efficiency when the maximum allowable blade metal temperature is increased

The positive trends in engine performance are realized with the reduction in the coolant flow fractions, which are shown for this engine in Figure 67.

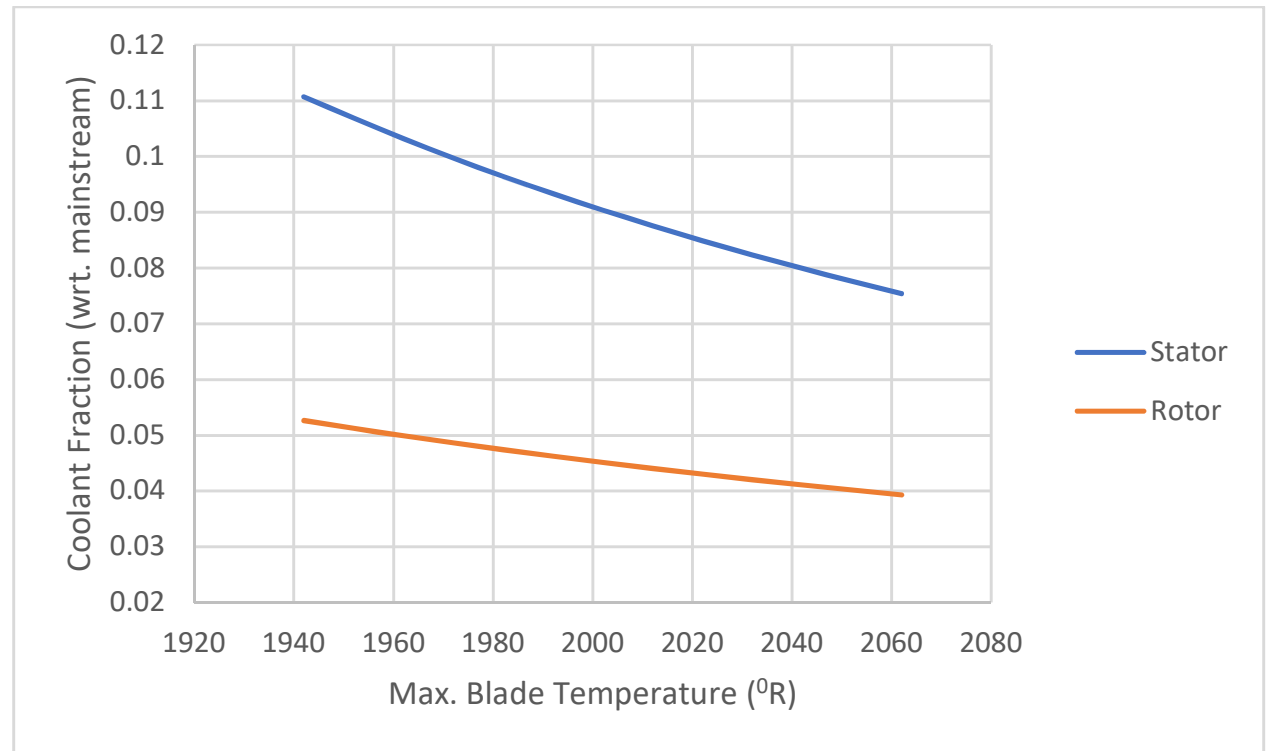

Figure 67: Change in the total coolant flow rates when the maximum allowable blade metal temperature is increased

The reduction in the total stator coolant fraction occurred as $31.9 \%$, and in the total rotor coolant fraction as $25.3 \%$.

\subsubsection{Internal Cooling Flow Effectiveness $\left(\varepsilon_{c}\right)$}

This effectiveness definition is from Young and Wilcock [7] and it differs from internal cooling effectiveness definition, by using the blade internal temperature $\left(T_{m}\right.$, int of Figure 4$)$ instead of the metal external temperature ( $T_{m}$, ext of Figure 4$)$ in the denominator. The definition is used from Table 2 and given as follows. 


$$
\varepsilon_{c}=\frac{T_{t c, x}-T_{t c, i}}{T_{m, i n t}-T_{t c, i}}
$$

Per the definition used for internal cooling flow efficiency, an increase in this efficiency is obtained if the internal cooling technique cools the blade to a lower wall temperature from the inside (lower $T_{m \text {, int). }}$.

An increase in this effectiveness can be achieved by internal cooling designs that allow higher convection heat transfer coefficient or enhanced heat transfer area through several internal cooling design improvements. From the semi-empirical coolant flow correlations of Equation (14), higher efficiency in internal cooling also means cooling the blade with less amount of coolant, since the metal Biot number is kept fixed in this analysis. Then the reduction in coolant flow rates, would reduce the entropy rates from the cooled turbine stage, which results in an increase in engine performance parameters.

\subsubsection{High By-Pass Turbofan Engine}

In this analysis with a turbofan engine having a single cooled HPT stage, internal cooling efficiency is increased from 0.45 to 0.85 . The corresponding change in the engine thrust is shown in Figure 68.

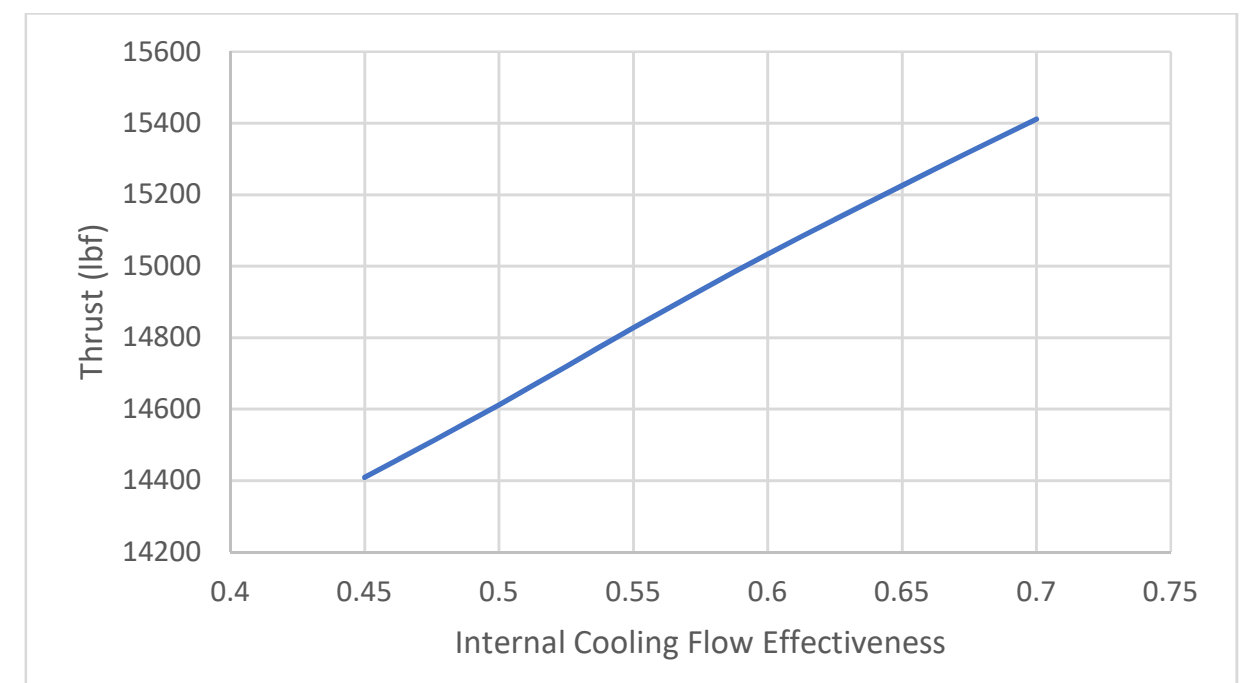

Figure 68: Change in the engine thrust when the internal cooling flow effectiveness is increased

The increase in engine thrust occurred as $6.95 \%$. Corresponding change in the thrust specific fuel consumption is given in Figure 69. 


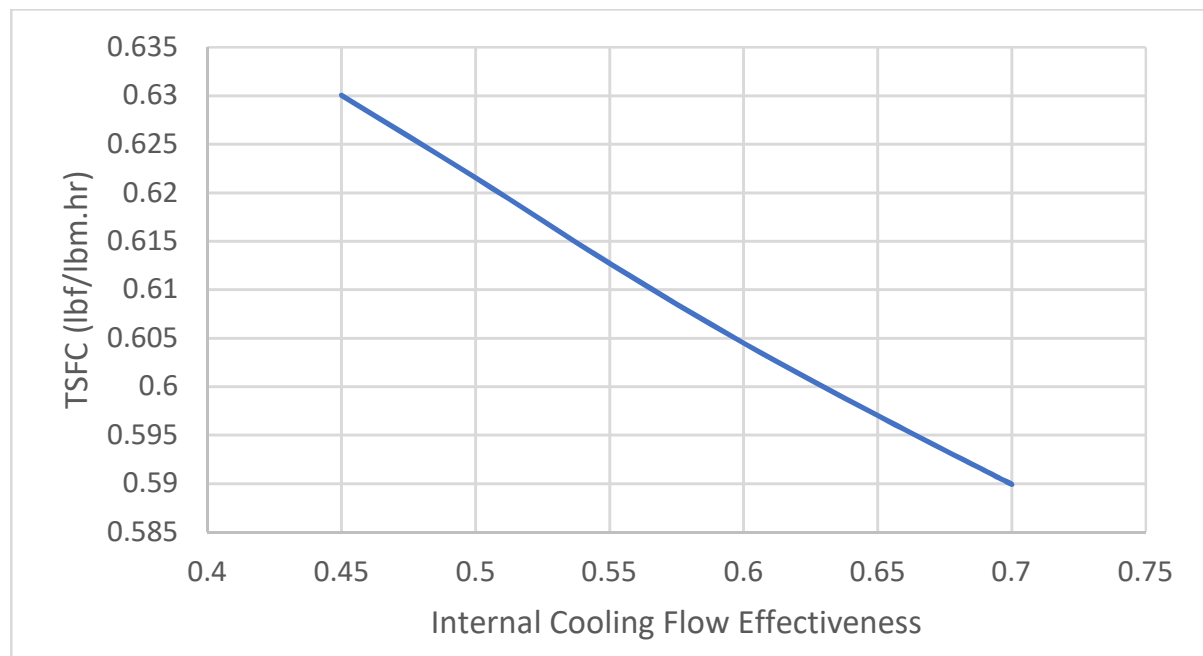

Figure 69: Change in the thrust specific fuel consumption when the internal cooling flow effectiveness is increased

Reduction in TSFC occurred as $6.37 \%$, which indicates a reduction in fuel consumption by $44.3 \%$. Therefore, the thermal efficiency is increased, as shown in Figure 70.



Figure 70: Change in the thermal efficiency when the internal cooling flow effectiveness is increased

Thermal efficiency is increased by $4.73 \%$, when the internal cooling flow effectiveness is increased to 0.7 from 0.45 . This is realized with the reduction in the coolant flow rates, which are shown in Figure 71. 


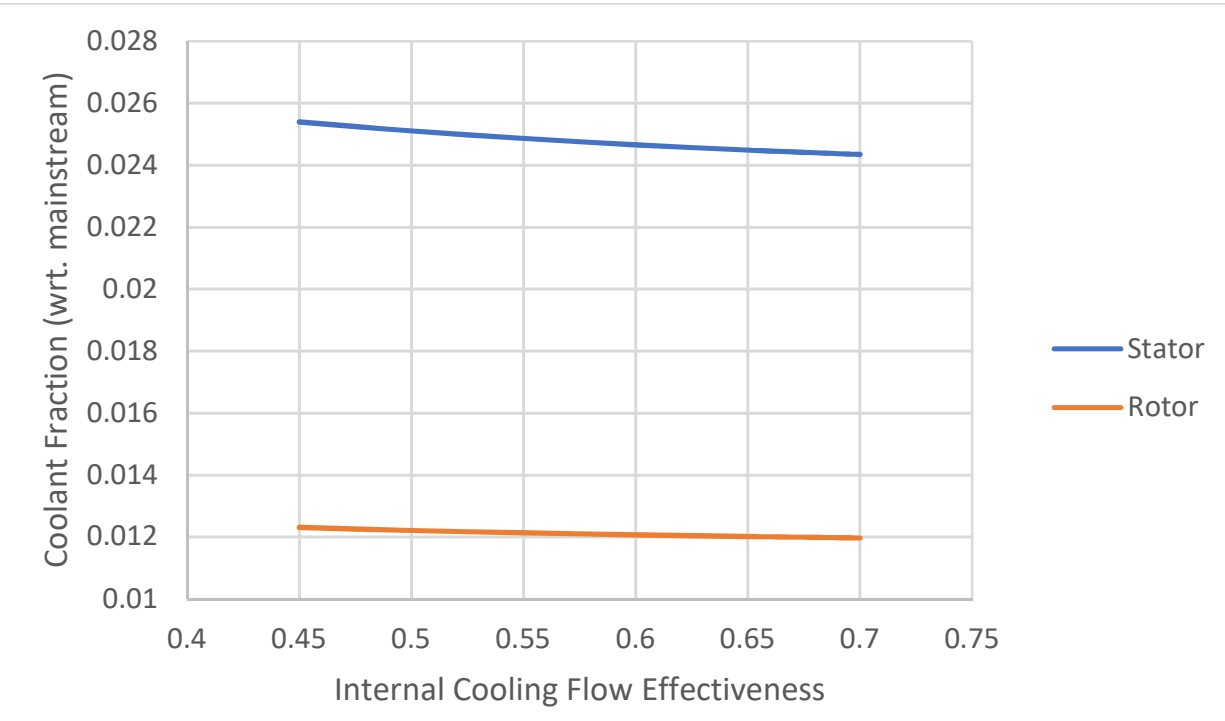

Figure 71: Change in the total coolant flow rates when the internal cooling flow effectiveness is increased

The reduction in stator cooling flow occurred as $4.13 \%$ and rotor cooling flow as $2.76 \%$.

\subsubsection{Industrial Gas Turbine}

In the analysis with industrial gas turbine having 4 cooled turbine stages, internal cooling flow effectiveness is changed from 0.45 to 0.85 . The effect on the shaft power delivered is shown in Figure 72.

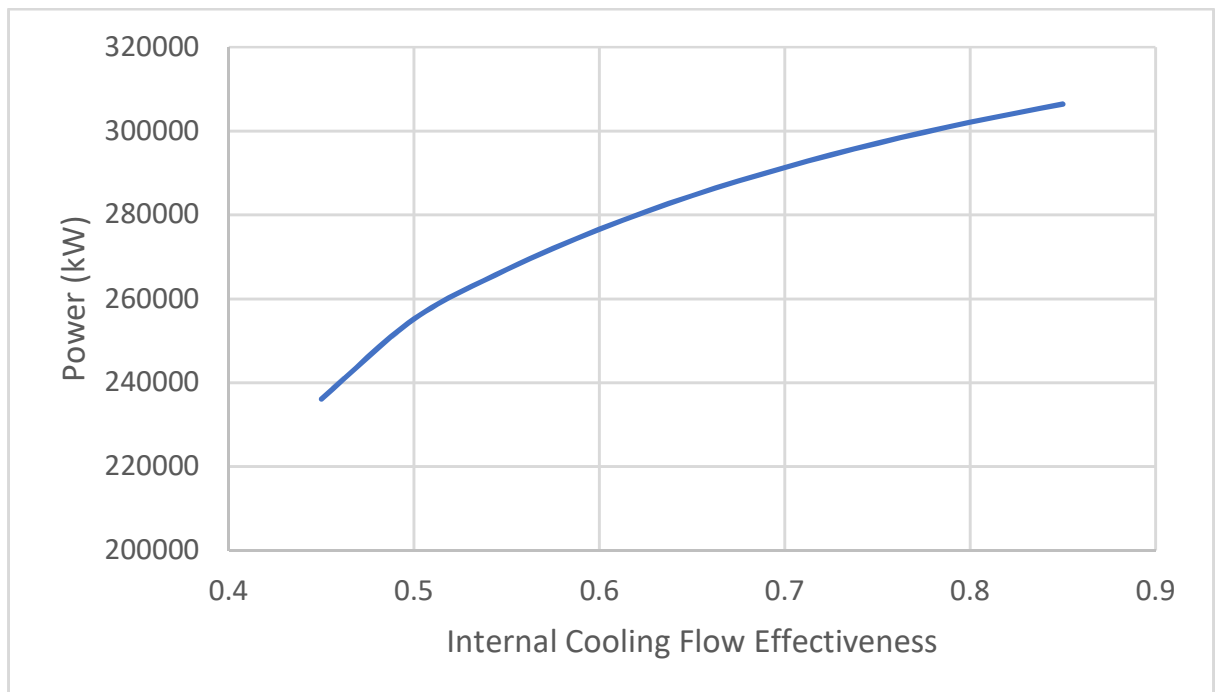

Figure 72: Change in the shaft power delivered when the internal cooling flow effectiveness is increased

The increase in the shaft power delivered occurred as $29.8 \%$. Reduction in the required coolant flow rates reduces internal losses and this causes an increase in the power output, due to having less pressure loss in the turbine. The effect on the heat rate is shown in Figure 73. 


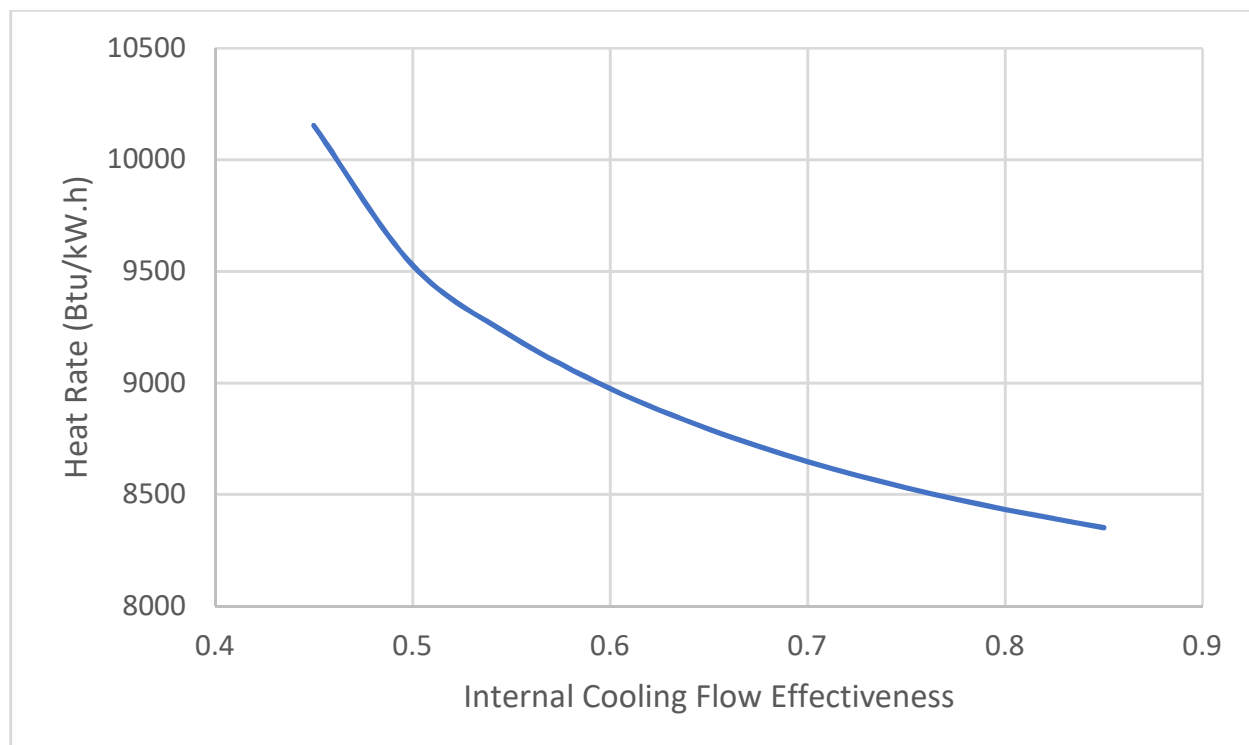

Figure 73: Change in the heat rate when the internal cooling flow effectiveness is increased

The reduction in the heat rate occurred as $17.8 \%$, which is dominated by the reduction in the fuel consumption. Corresponding increase in the thermal efficiency is shown in Figure 74.

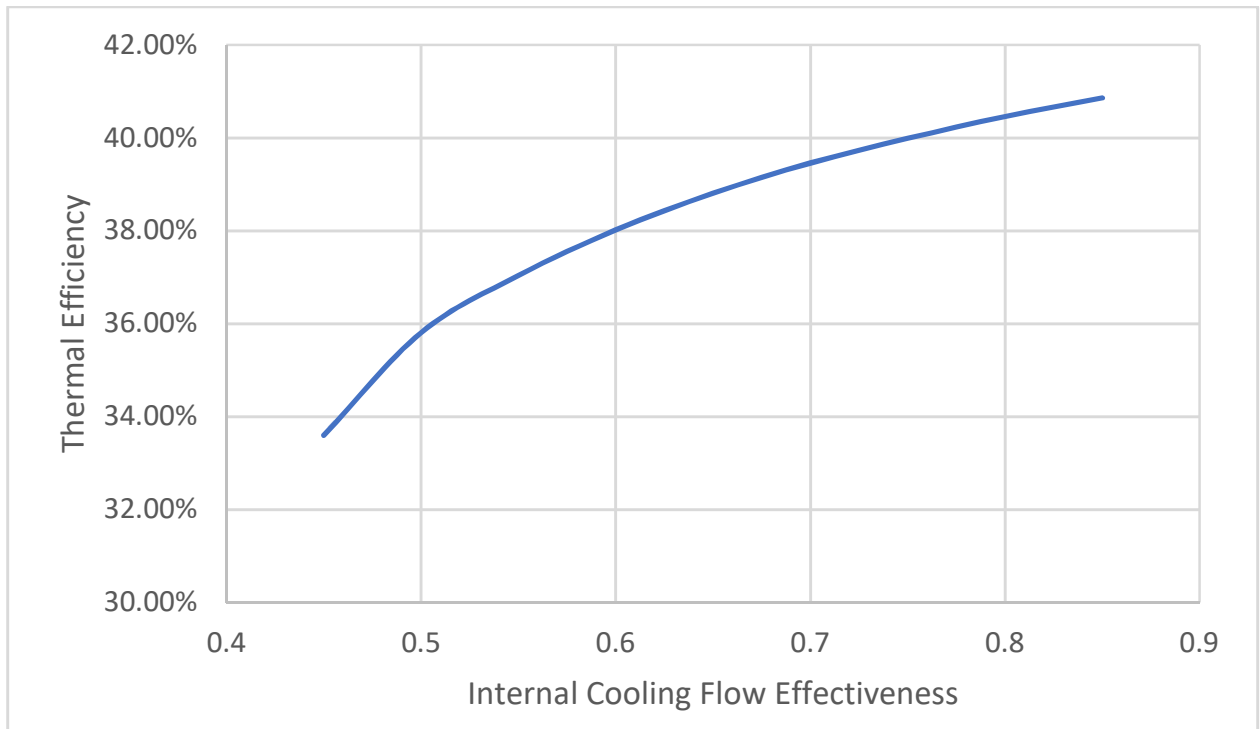

Figure 74: Change in the thermal efficiency when the internal cooling flow effectiveness is increased

The increase in the thermal efficiency by $7.26 \%$ occurred due to the reduction in the total coolant flow rates, which are shown in Figure 75. 


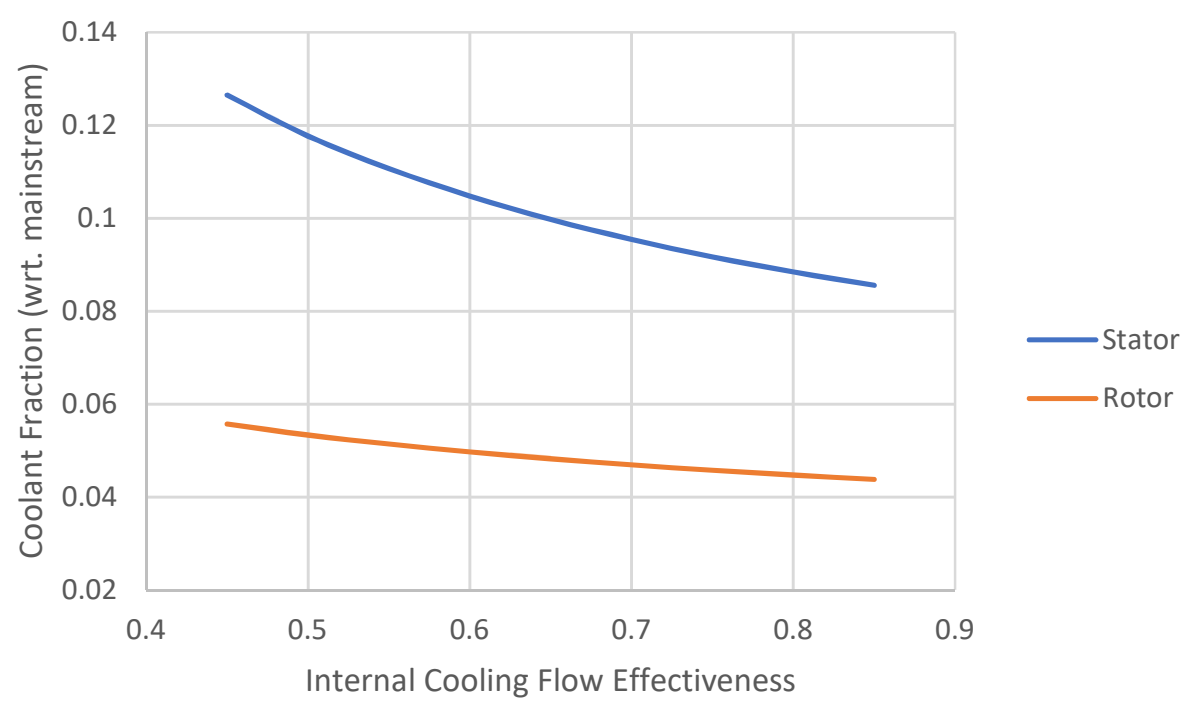

Figure 75: Change in the coolant flow rates when the internal cooling flow effectiveness is increased

Corresponding change in the total stator coolant flow rate is occurred as $32.36 \%$, and in the rotor coolant flow rate as $21.36 \%$.

\subsubsection{Film Cooling Parameters}

In this section, the effect on the engine performance due to a change in film cooling related parameters of the cooling analysis inputs are investigated.

For the turbofan engine cases, in which the only cooled stage is already equipped with film cooling, the effects of these parameters are more drastic than the industrial gas turbine cases, in which only first two stages are equipped with film cooling. Because of this fact, the effects of the blowing ratio and the effective film cooling angle showed extremely small to no impact on engine performance parameters and these parameters were investigated only for turbofan engine case.

\subsubsection{Blade Adiabatic Film Cooling Effectiveness $\left(\varepsilon_{\mathrm{fc}}\right)$}

Film cooling effectiveness definition used follows the definition from Young and Wilcock [7] from Table 2 and is the same as the definitions used in heat transfer literature as given below.

$$
\varepsilon_{f c}=\frac{T_{t g}-T_{a w}}{T_{t g}-T_{t c, x}}
$$

Temperatures used in the definition follows from Figure 4 and it is a measure of the effectiveness of the applied film cooling technique. In CTM, this value rather corresponds to the adiabatic film cooling efficiency for the entire blade, than representing the effectiveness for a single film cooling hole. Therefore, the results of this analysis correspond to the overall film cooling effectiveness obtained for the entire blade, which has multiple rows of film cooling holes.

Film cooling reduces the blade outer wall temperature by forming a boundary layer over it and reducing the gas side convective heat transfer coefficient; resulting in reduced heat flux to the blade from the hot gas side. 
In general, a highly effective film cooling method provides the required cooling by reducing adiabatic wall temperature less than or equal to the maximum blade temperature with the least amount of coolant possible.

As being an external cooling method, per the $2^{\text {nd }}$ Law analysis on losses (see Section 1.1.3.2.4), film cooling methods that require higher momentum flux ratios (and blowing ratios), and higher effective injection angles, could result in increased pressure losses that will affect the power generated from the turbine. Because of these effects, increasing the film cooling effectiveness is expected to improve the engine performance due to reduced coolant flow rates (from Equation (14)) and reduced mixing losses, which are the dominant loss terms as can be seen from Figures Figure 16 and Figure 17.

\subsection{High By-Pass Turbofan Engine}

In the analysis with the turbofan engine having a single cooled turbine stage with film cooling, the adiabatic film cooling efficiency is increased from 0.2 to 0.55 . The effect of increasing the adiabatic film cooling effectiveness resulted in an increase in the engine thrust, as shown in Figure 76.

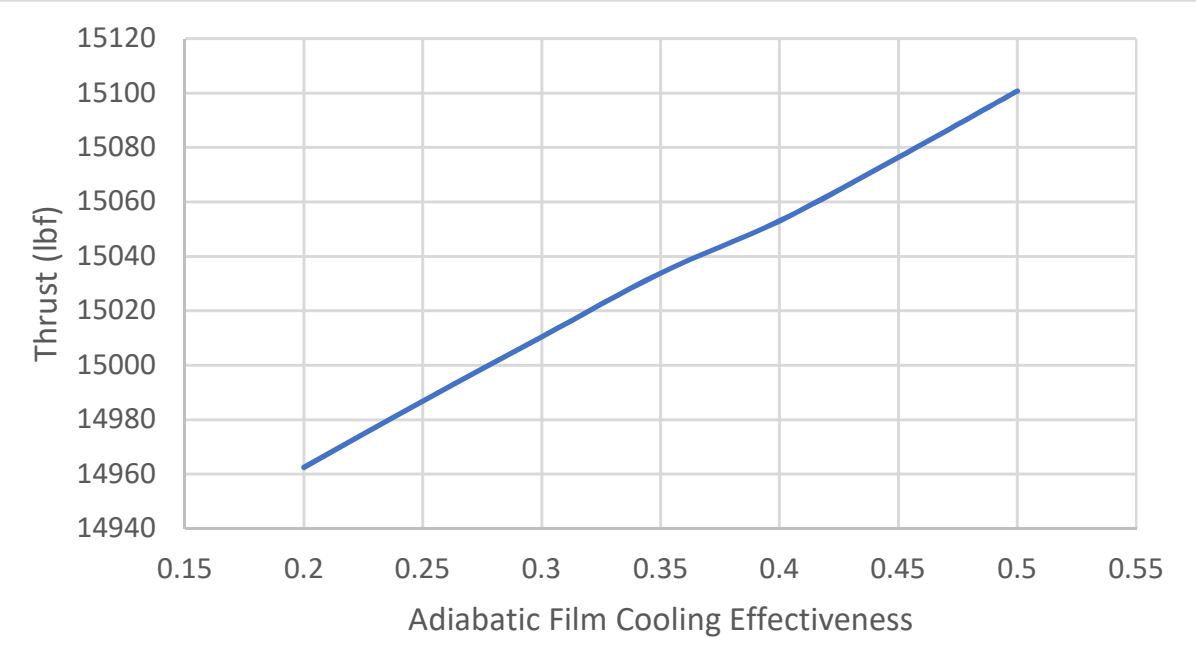

Figure 76: Change in the engine thrust when the blade adiabatic film cooling effectiveness is increased

The increase in the engine thrust occurred as $0.93 \%$. Reduction in required coolant flow rates and the increase in thrust resulted in a decreasing trend in thrust specific fuel consumption as shown in Figure 77. 


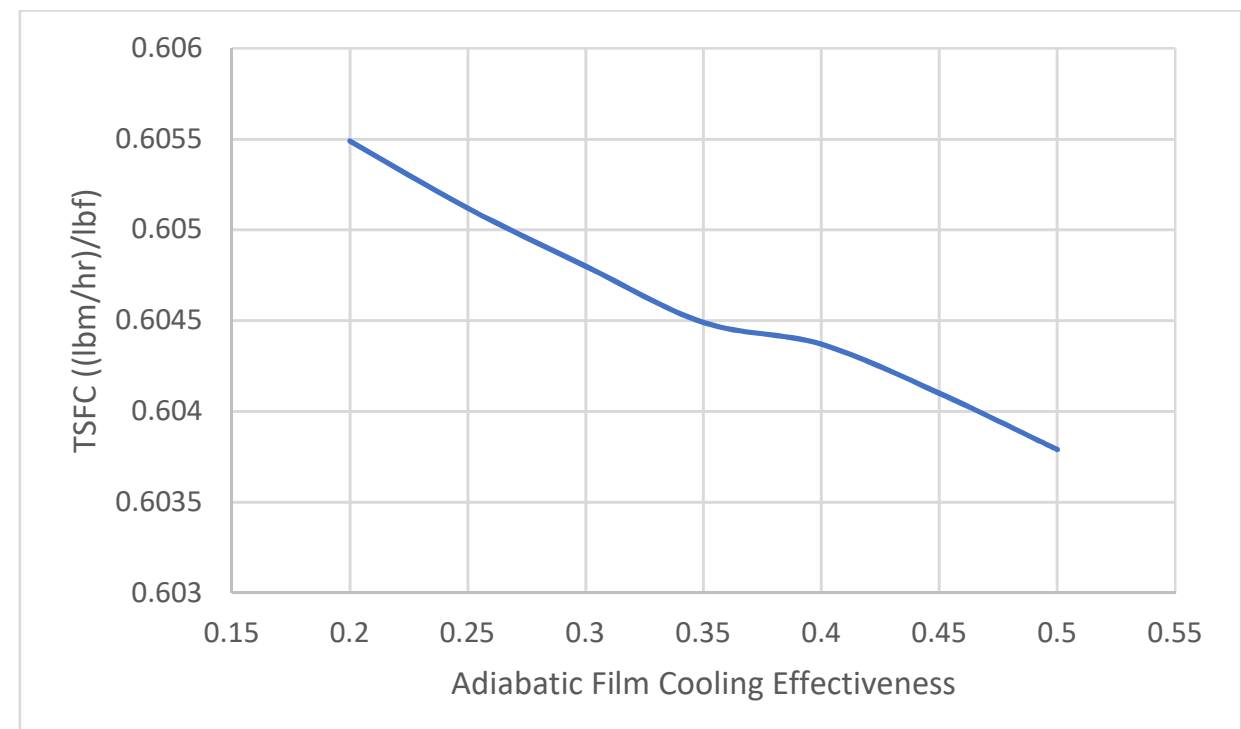

Figure 77: Change in the thrust specific fuel consumption when the blade adiabatic film cooling effectiveness is increased

The reduction in the thrust specific fuel consumption occurred as $0.28 \%$, indicating a reduction in fuel consumption by $0.26 \%$. The thermal efficiency is increased with this change, as shown in Figure 78.

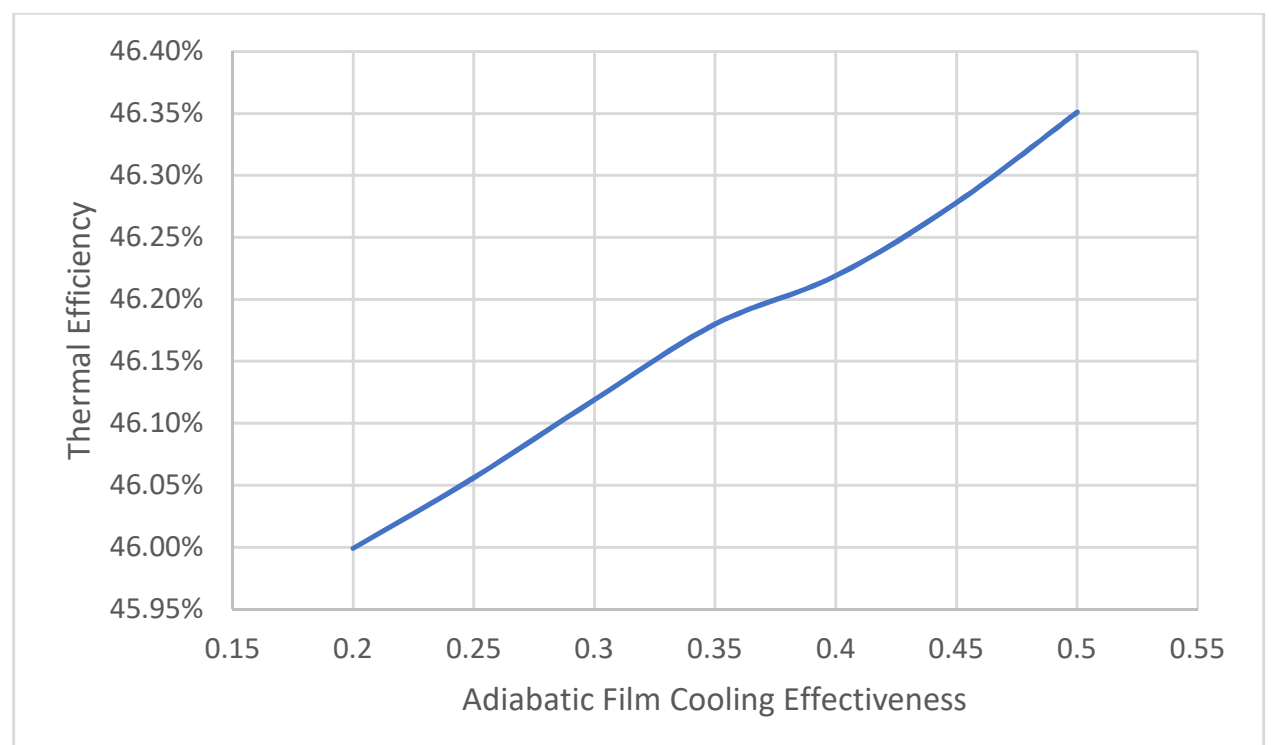

Figure 78: Change in the thermal efficiency when the blade adiabatic film cooling effectiveness is increased

The increase in the thermal efficiency occurred as $0.35 \%$., which is a result of the reduction in the required coolant flow rates due to increased film cooling effectiveness. Corresponding change in the coolant flow rates are shown in Figure 79. 


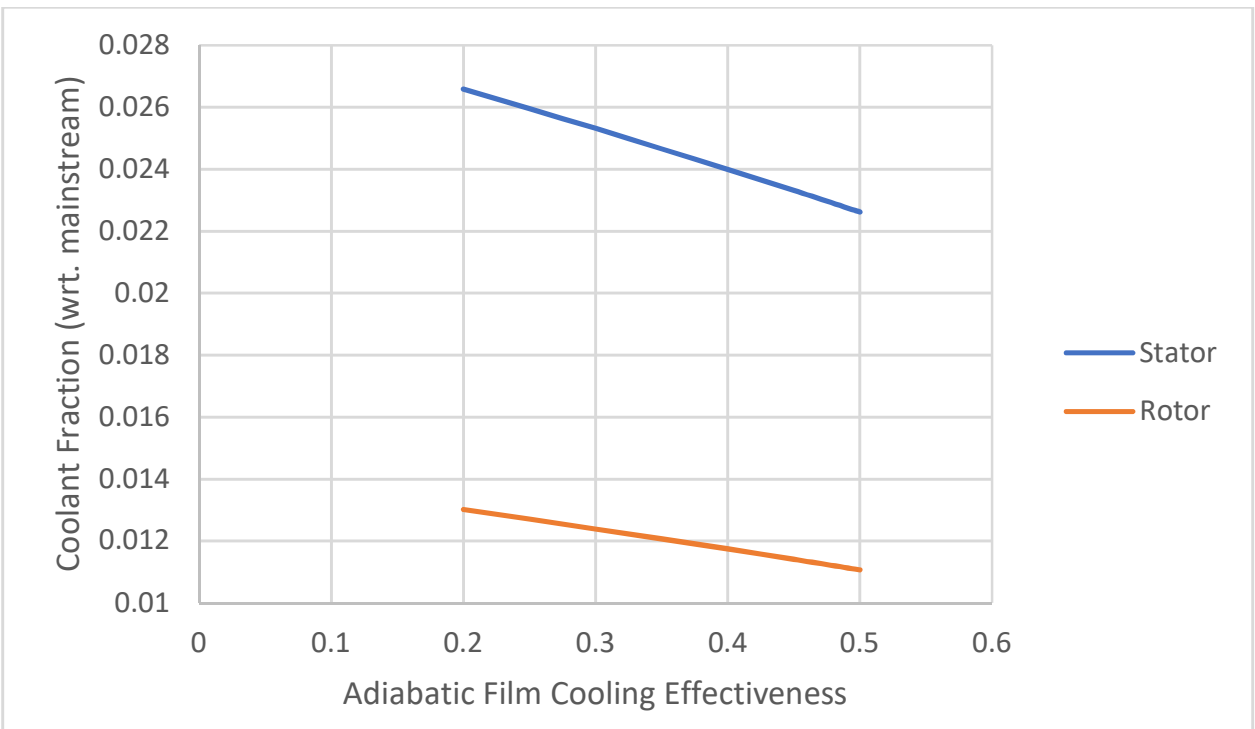

Figure 79: Change in the coolant flow rates when the blade adiabatic film cooling effectiveness is increased

With the increase in the blade adiabatic film cooling effectiveness from 0.2 to 0.5 , both the stator and rotor coolant flow rates are reduced by $14.9 \%$. Although this reduction is higher than the reduction observed when the internal cooling flow effectiveness is increased, the effect on the engine performance is lower.

\subsection{Industrial Gas Turbine}

In this section, the effect of increasing the adiabatic film cooling effectiveness on the industrial gas turbine having first two cooled turbine stages (out of 4 cooled stages) equipped with film cooling, is analyzed by increasing the effectiveness from 0.2 to 0.55 . The corresponding change on the shaft power delivered is shown in Figure 80.

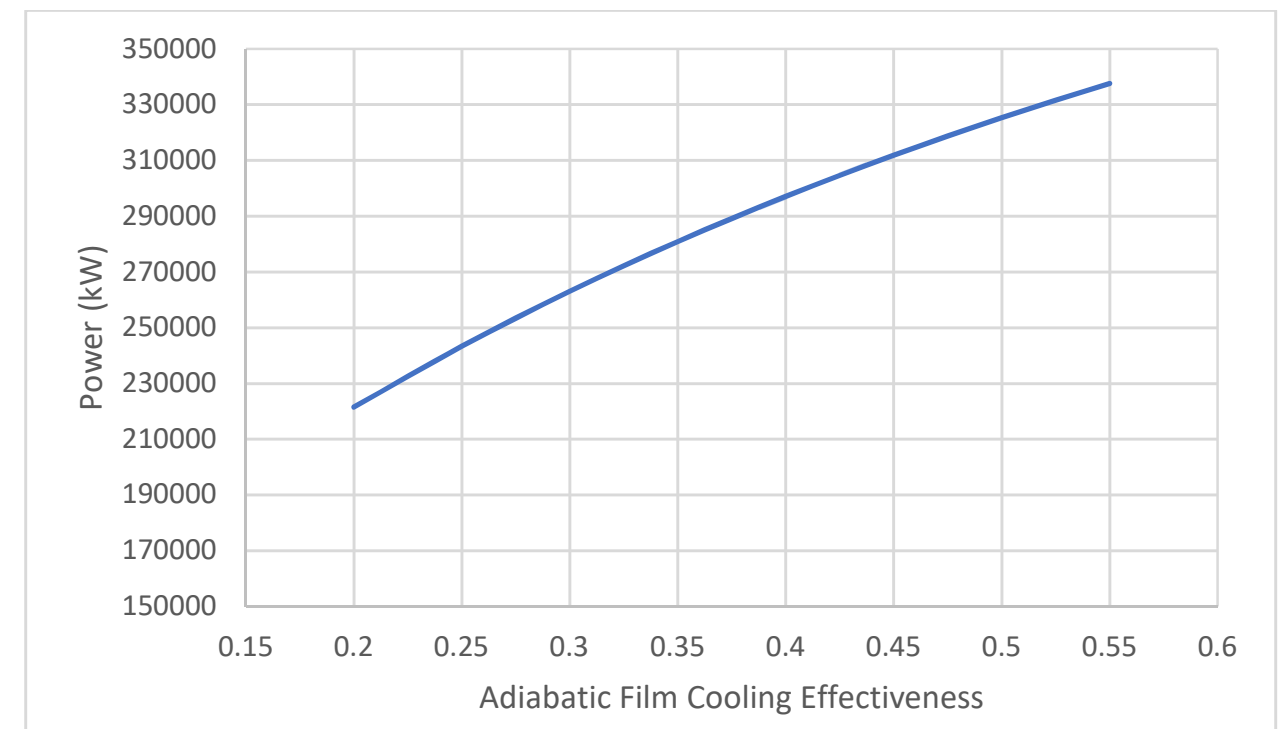

Figure 80: Change in the shaft power delivered when the blade adiabatic film cooling effectiveness is increased 
The parabolic increase in power generated is occurred as $52.4 \%$ and related to the reduction of losses as the required coolant flow rates are reduced when the film cooling effectiveness is increased. Corresponding change in the heat rate is shown in Figure 81.

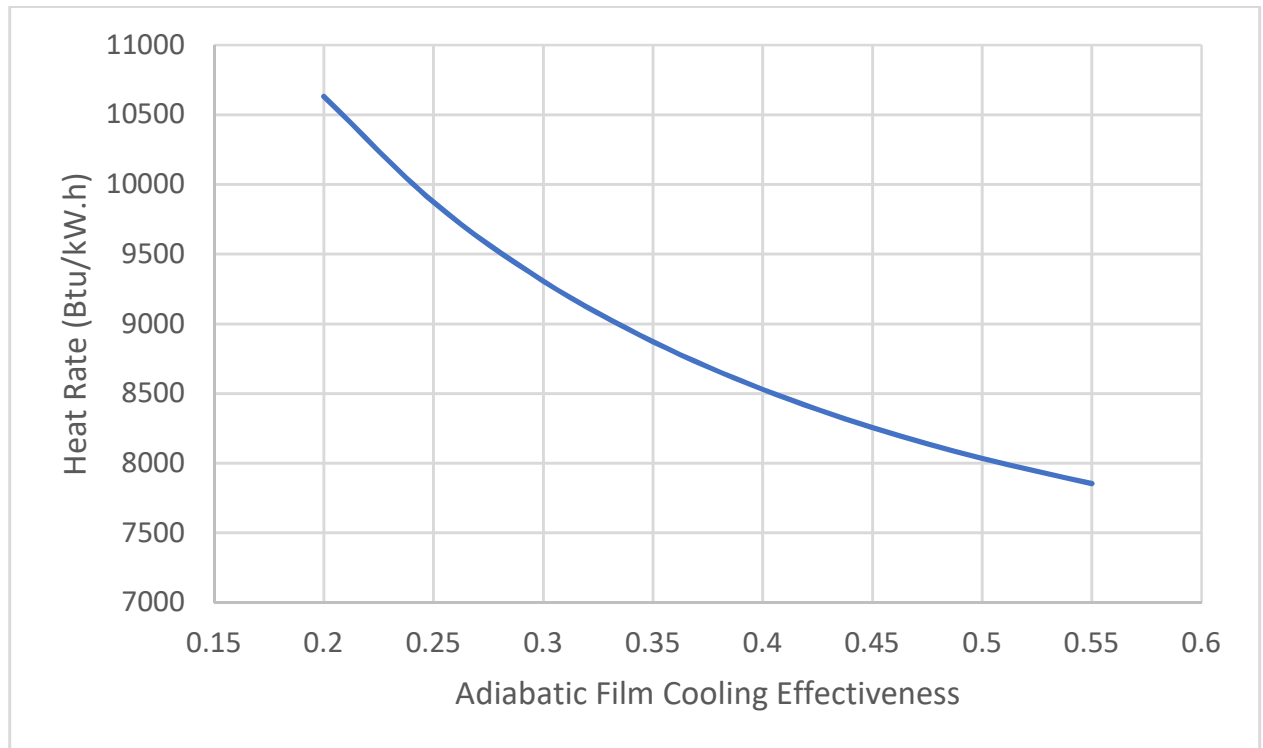

Figure 81: Change in the heat rate when the blade adiabatic film cooling effectiveness is increased

The reduction in the heat rate occurred as $26.1 \%$, which is dominated by the reduction in fuel consumption as the coolant flows are reduced. These changes result in an increase in thermal efficiency as shown in Figure 82.

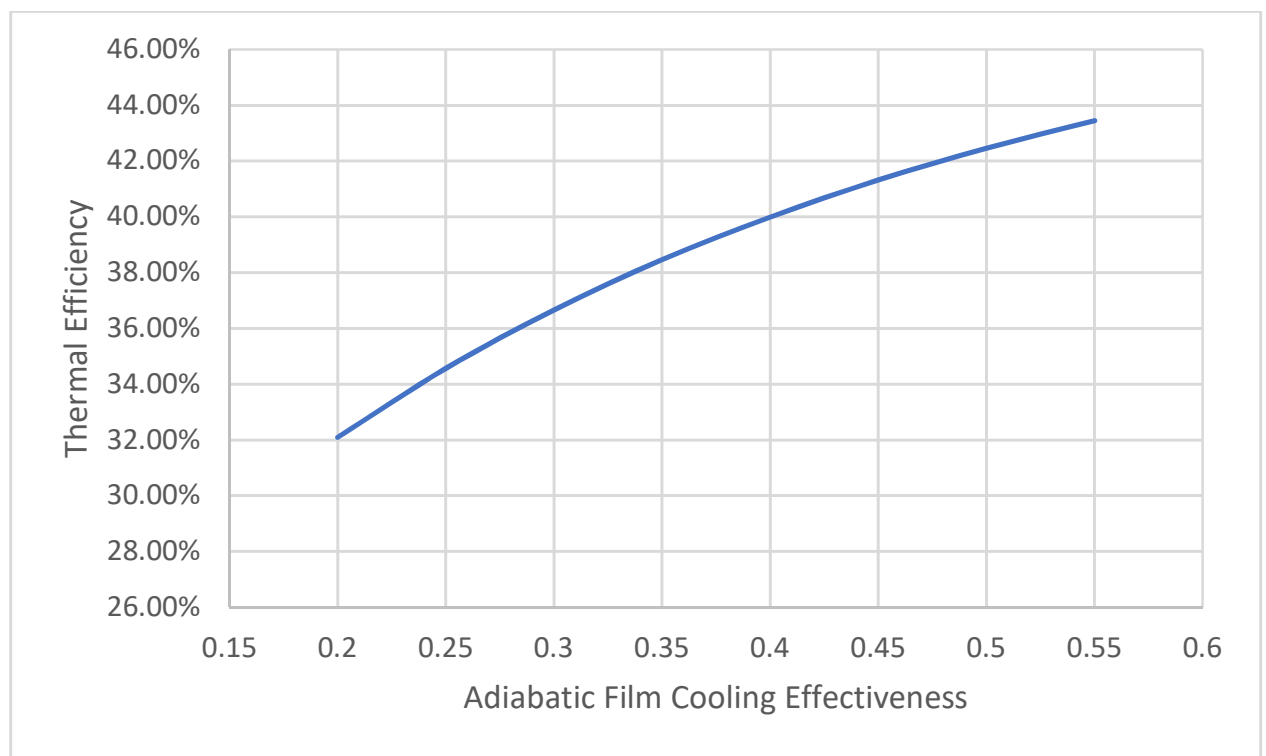

Figure 82: Change in the thermal efficiency when the blade adiabatic film cooling effectiveness is increased

The increase in the thermal efficiency occurred as $11.4 \%$, which results from the reduction in the coolant flow rates with the adiabatic film cooling efficiency as shown in Figure 83. 


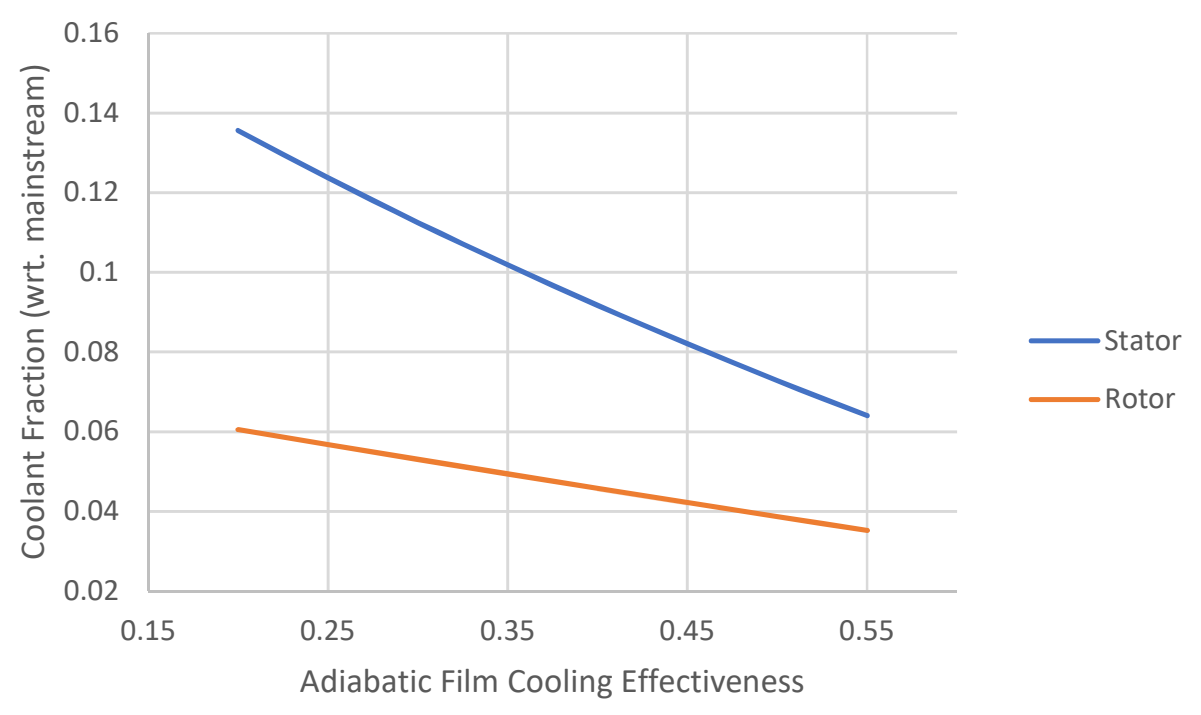

Figure 83: Change in the coolant flow rates when the blade adiabatic film cooling effectiveness is increased

When the blade adiabatic film cooling effectiveness Is increased from 0.2 to 0.55 , the reduction in the stator and the rotor coolant flow rates occurred as $52.8 \%$, and $41.8 \%$ respectively.

Increase in the adiabatic film cooling efficiency, while other cooling parameters kept fixed, means that the film cooling technique will need less amount of coolant to cool the blade surface to the same temperature. Reduction in required coolant flows would eventually result in an increase in engine performance due to reduction in entropy generation across the cooled stage and reduced compressor power demand.

For fixed maximum allowable blade temperature and fixed TBC Biot number, an increase in the film cooling effectiveness is only possible if the adiabatic wall temperature gets closer to the coolant exit temperature $T_{t c, x}$ (of Figure 4). It should be noted here that, the coolant exit temperature is much lower than the hot gas temperature and reducing the wall temperature more, will enhance the convective heat flux through the thermal boundary layer, which will increase the external heat transfer $\left(\dot{\sigma}_{\text {ext }, Q}\right)$ and mixing heat transfer losses $\left(\dot{\sigma}_{\text {mix }, Q}\right)$. From the results of Section 1.3.2, the latter loss parameter is the highest loss contributor and therefore outweighs the advantage coming from the reduction in coolant flow rates after a certain point. This could cause the non-linear trends seen in the performance curves when the film cooling effectiveness is increased.

\subsubsection{Effective Film Cooling Flow Angle (Ф)}

This angle is defined as the effective mixing angle of the coolant flow with the mainstream, which might be different than the actual hole inclination angle, due to vortex reduction techniques in advanced holes, the turbulent mixing and/or the differences in the coolant flow speed, and density ratio. The definition of this angle is shown in Figure 84.

Film cooling techniques that reduces the effective flow angle has a well-known positive impact on film cooling performance [33]. Any reduction in this angle will yield in an increased coolant coverage on the blade, reduce the coolant flow separation and reduce the $2^{\text {nd }}$ law related losses per the associated loss term $\left(\dot{\sigma}_{m i x, K E}\right)$ given as follows from Equation (46). 


$$
\dot{\Sigma}_{m i x, K E}=\dot{m}_{c l}\left[\frac{V_{g *}{ }^{2}+V_{c l}{ }^{2}-2 V_{g *} V_{c l} \cos \phi}{2 T_{g *}}\right]
$$

Per the definition from Equation (46), it is expected to have an increase in the mixing losses when the film cooling flow angle is increased. This physically corresponds to reducing main stream kinetic energy due to the reduction in mixture flow speed. Turbine performance would be affected negatively due to the decrease in flow speed over the blades. Therefore, the expected trend on the engine performance parameters should be negative.

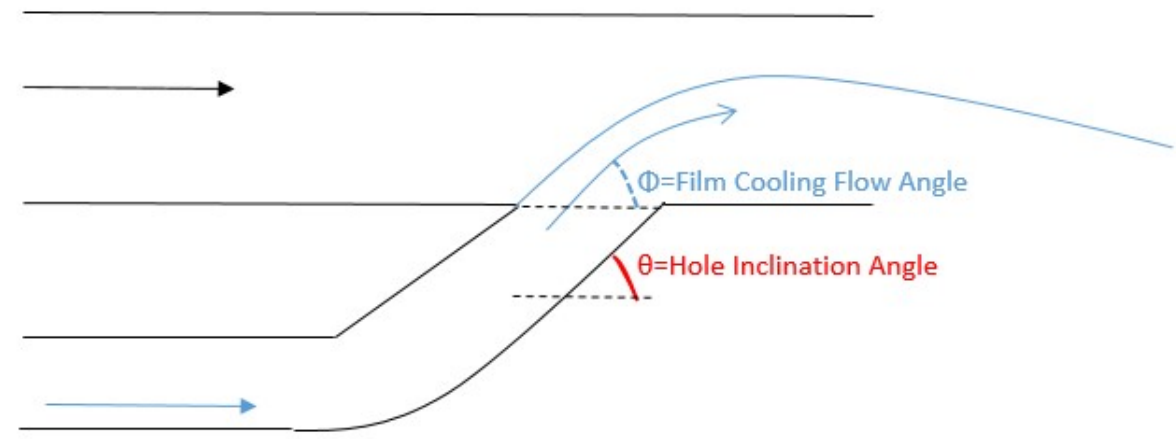

Figure 84: Definition of the effective film cooling flow angle is shown schematically

In order to analyze this effect, the film cooling flow angle is changed from $15^{\circ}$ to $50^{\circ}$. Due to having higher purge fractions and having more than one cooled stage, the effect on the industrial gas turbine performance was extremely small. However, the effect on the turbofan engine performance was more notable, possibly due to having lower amount of purge fractions and lower number of cooled stages.

The effect on the engine thrust was negative as shown in Figure 85, due to increased losses in the cooled turbine resulting in reduced work output.

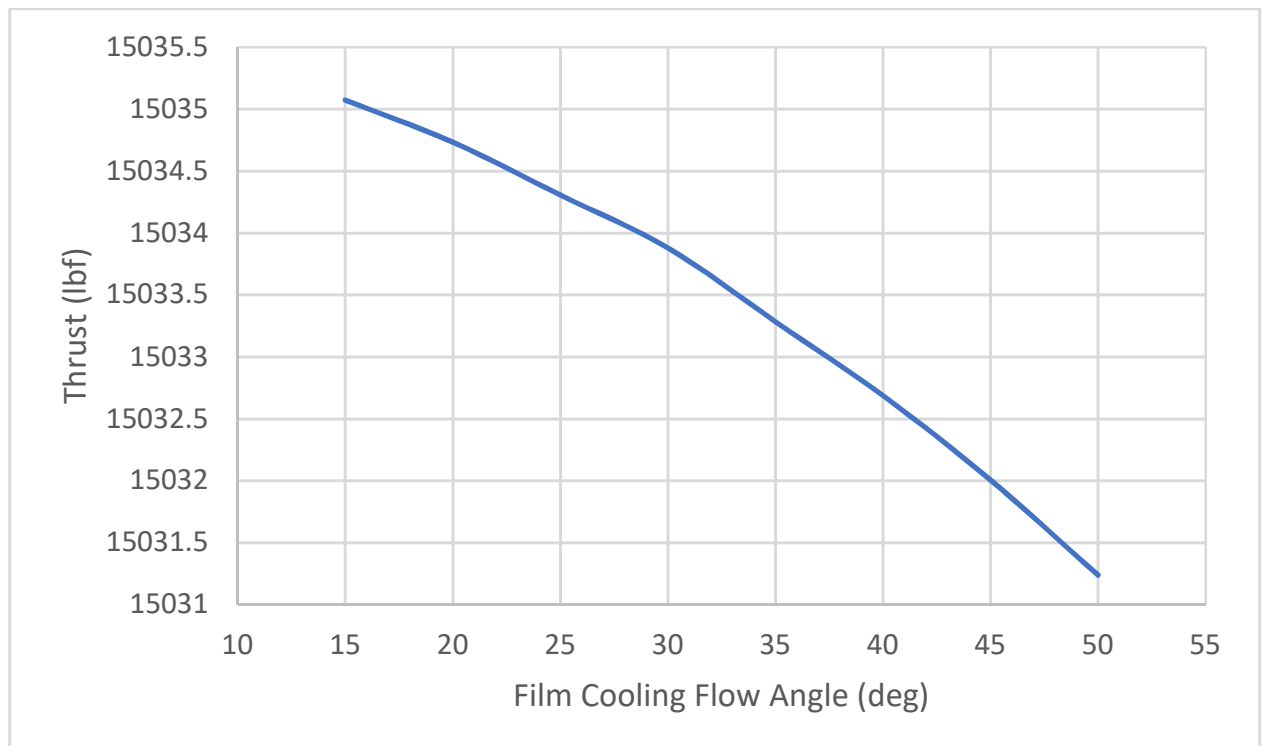

Figure 85: Change in the engine thrust when the effective film cooling flow angle is increased 
It should be noted that the reduction in thrust is very small; occurred as $0.03 \%$ for the change interval considered. Calculated coolant flow rates were the same for the entire analysis. A reduction in the turbine work increases the fuel consumption, which will result in an increasing trend for the thrust specific fuel consumption as shown in Figure 86.

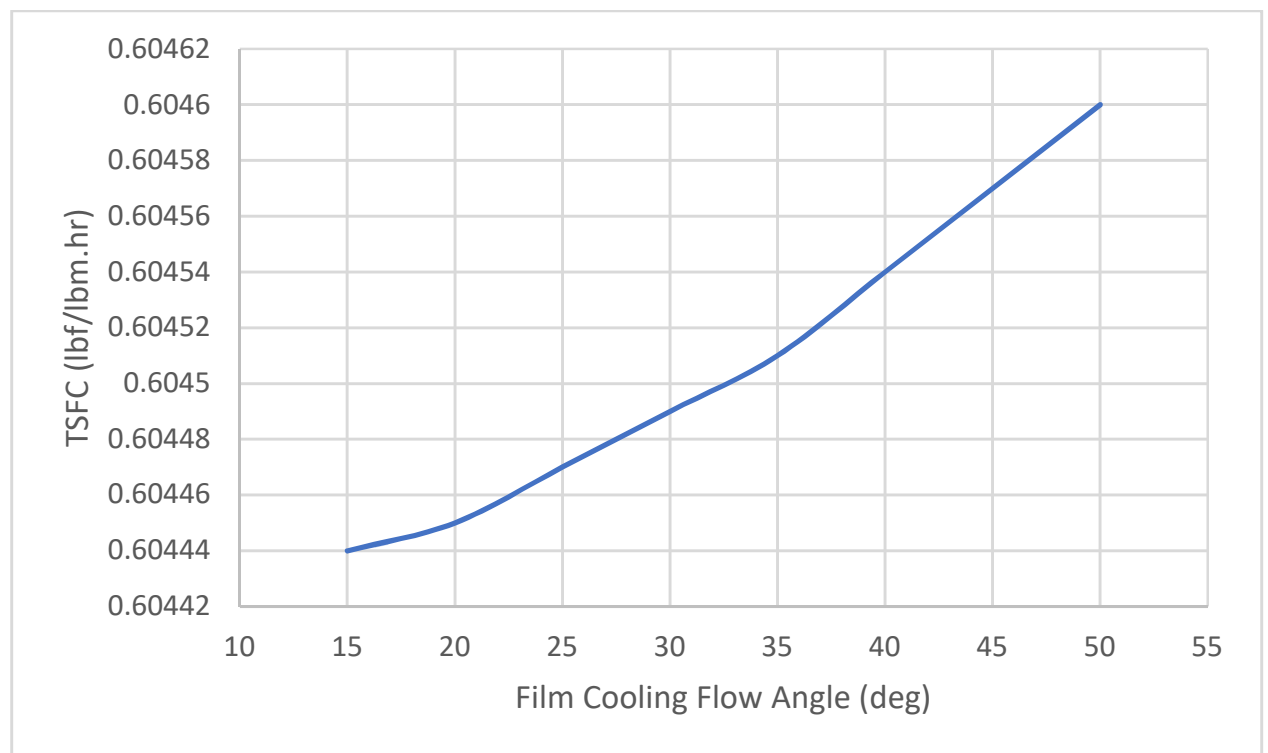

Figure 86: Change in the thrust specific fuel consumption when the effective film cooling flow angle is increased

The increase in the thrust specific fuel consumption occurred as $0.03 \%$, from an increase in the fuel consumption by $9 \mathrm{E}-04 \%$ which is negligible. Therefore, the increase in TSFC is entirely due to the reduction in engine thrust. Reduction in the engine thrust also caused reduction in the thermal efficiency as shown in Figure 87.

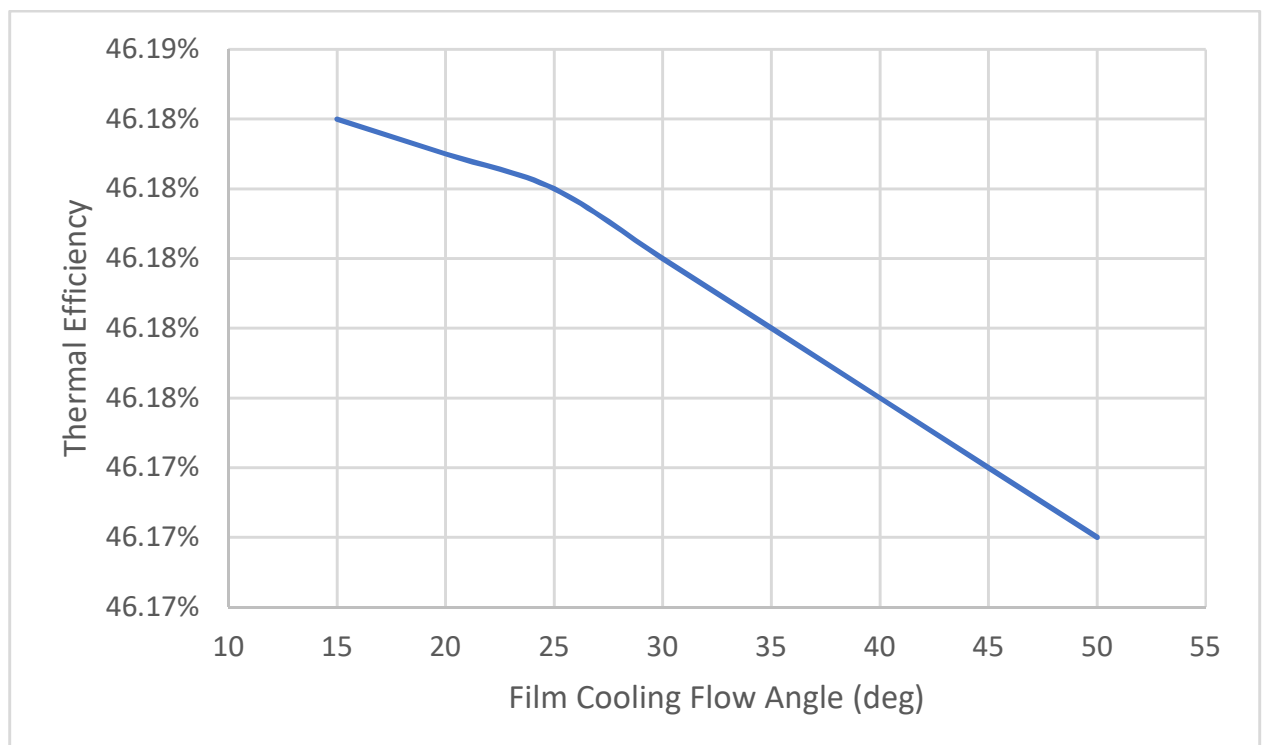

Figure 87: Change in the thermal efficiency when the effective film cooling flow angle is increased

Reduction in the thermal efficiency occurred as $0.01 \%$. Although the impact by the film cooling flow angle found almost negligible in this analysis, it should be noted that the combined effect of this parameter with 
another cooling parameter (i.e. increased metal Biot number) might increase the negative impact more on the engine performance. Conversely, having high TBC Biot number and high internal flow cooling efficiency might totally eliminate the negative effect of increased coolant flow angle, which is another possible explanation to not observing the impact of this parameter in the industrial gas turbine case.

\subsubsection{Blowing Ratio (BR)}

The blowing ratio of film cooling flow is defined as in Equation (73).

$$
B R=\frac{\rho_{c l, x} V_{c l, x}}{\rho_{g, x} V_{g, x}}
$$

The momentum flux ratio of the film cooling flow is defined as in Equation (74) from Young and Wilcock [7], which is the same definition used in the film cooling heat transfer literature.

$$
M F R=\frac{\rho_{c l, x} V_{c l, x}^{2}}{\rho_{g, x} V_{g, x}^{2}}=B R \frac{V_{c l, x}}{V_{g, x}}=\frac{\gamma_{c l, x} M_{c l, x}^{2}}{\gamma_{g, x} M_{g, x}^{2}}
$$

In Equation (74), $V_{c, x}$ is the coolant exit speed and $V_{g, x}$ is the hot gas flow speed on the blade, which are known from the turbomachinery design section results. The momentum flux ratio is related to coolant to mainstream total pressure ratio $\left(\frac{P_{t c l, x}}{P_{t c, i}}\right)$, which is included in the internal fiction losses parameter $\left(\dot{\sigma}_{i n t, F}\right)$, given as follows from Equation (40).

$$
\dot{\sigma}_{\text {intF }}=\dot{m}_{c l} c_{p, c l}\left[\ln \left(\frac{T_{t c l, x}}{T_{t c l, k}}\right)-\left(1+\frac{\gamma_{c l}-1}{2} M_{c l}^{2}\right) \ln \left(\frac{T_{t c l, x}}{T_{t c l, i}}\right)\right]-\dot{m}_{c} R_{c} \ln \left(\frac{P_{t c l, x}}{P_{t c l, i}}\right)
$$

Increasing the momentum flux ratio by increasing the coolant flow speed will result in an increase in the coolant to mainstream total pressure ratio [7], which will reduce the internal friction losses logarithmically. Increasing the coolant flow speed will also reduce the mixing kinetic energy losses (Equation (46)), which will yield an increase in the turbine performance. Therefore, it is expected that increasing momentum flux ratio would increase engine performance.

In order to analyze this effect, the momentum flux ratio is changed from 0.05 to 0.8 by increasing the blowing ratio from 0.07 to 1.32 . This can be accomplished by reducing the coolant hole diameter while keeping other film cooling parameters constant. Similar to film cooling flow angle, the effects on the industrial gas turbine performance parameters were negligible. However, the results for turbofan engine case showed more notable changes in the performance.

The effect on the engine thrust was positive as shown in Figure 88, due to reduced losses in the cooled turbine internal friction losses. 


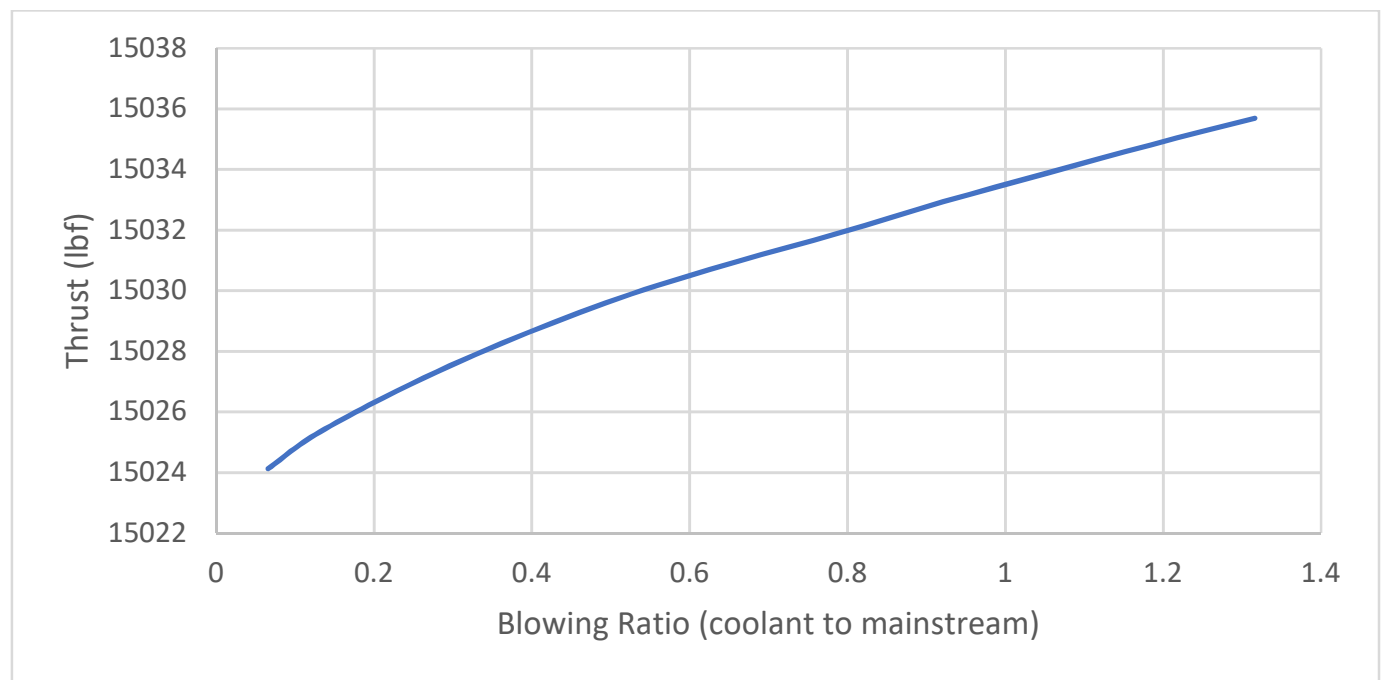

Figure 88: Change in the engine thrust when the blowing ratio is increased

Calculated coolant flow rates were the same for the entire analysis. Increase in turbine work, occurred as $0.08 \%$, reduces the fuel consumption, which will result in a decrease in thrust specific fuel consumption as shown in Figure 89.

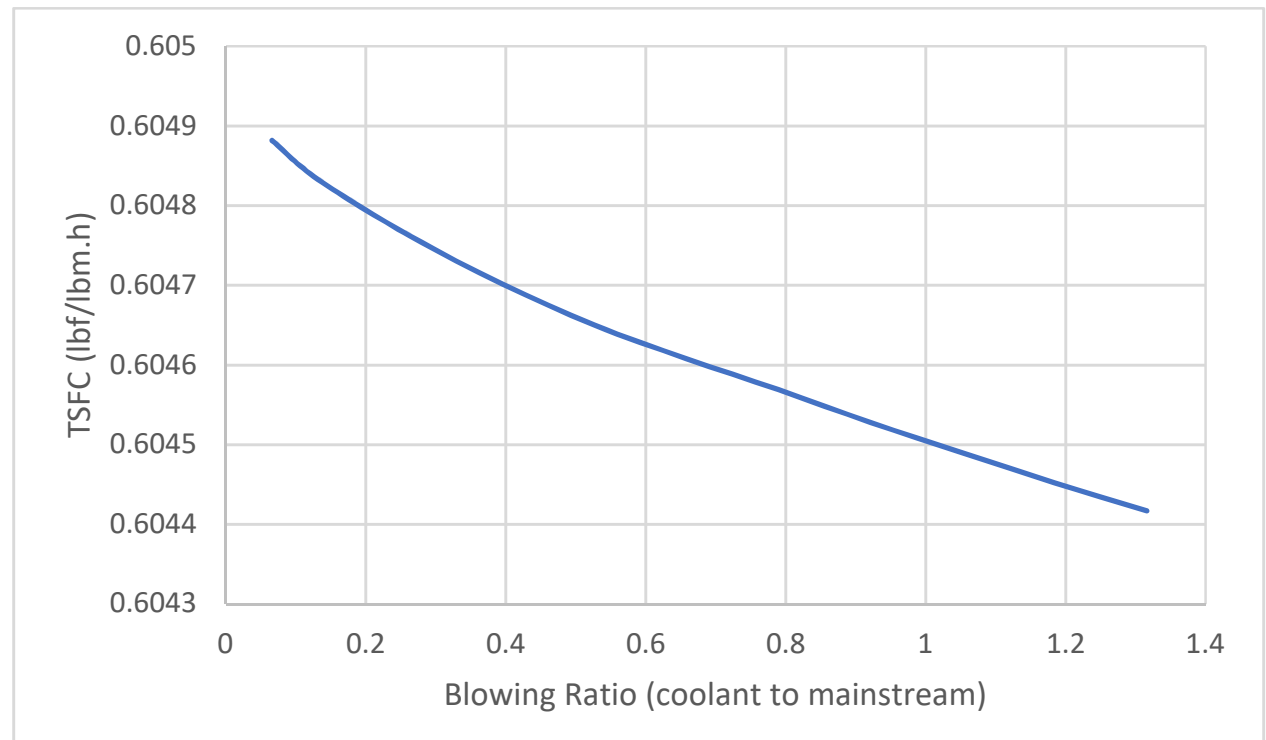

Figure 89: Change in the thrust specific fuel consumption when the blowing ratio is increased

The reduction in the thrust specific fuel consumption occurred as $0.08 \%$, from a reduction in fuel consumption by $0.006 \%$ which is negligible. Therefore, the reduction in TSFC resulted entirely from the increase in the thrust. Corresponding change on the thermal efficiency is shown in Figure 90. 


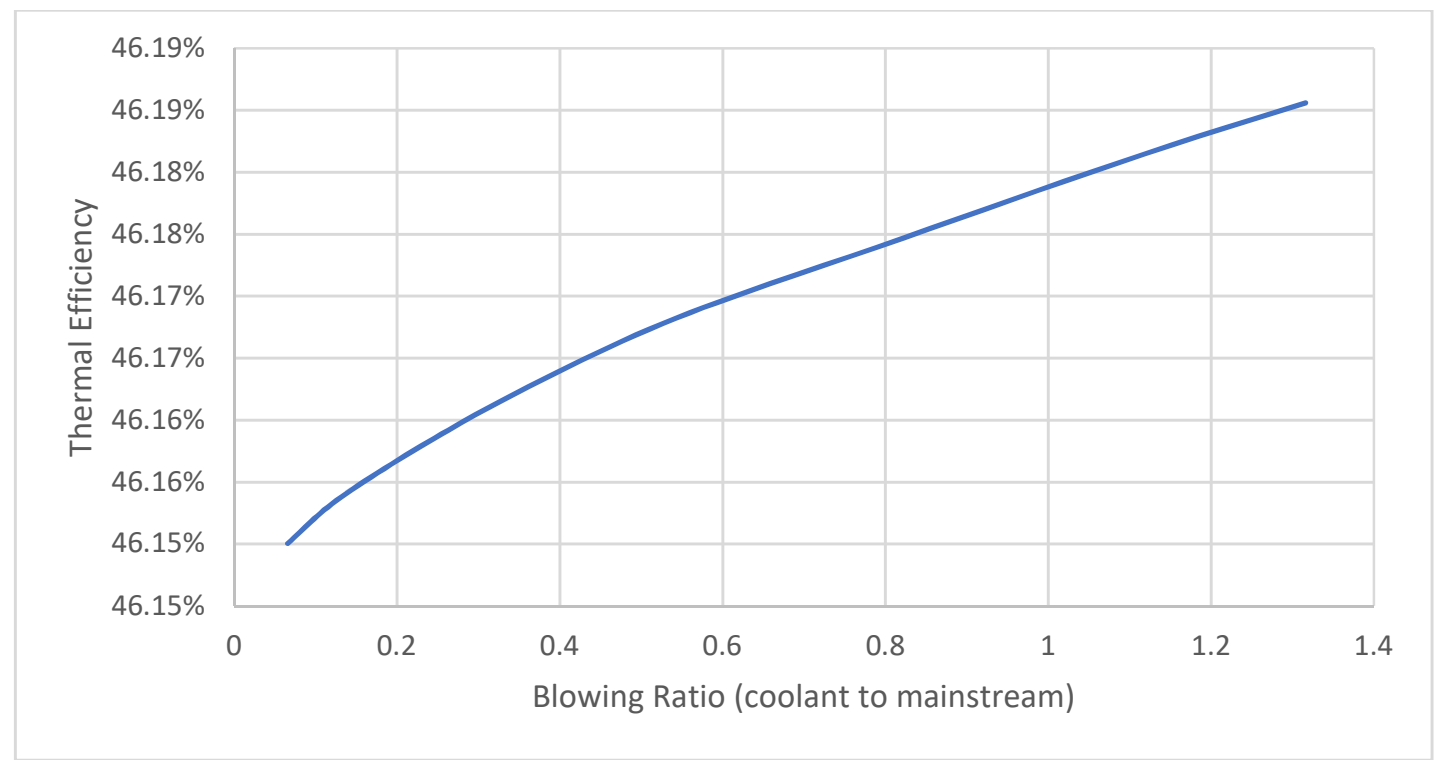

Figure 90: Change in the thrust specific fuel consumption when the blowing ratio is increased

The increase in the thermal efficiency occurred as $0.03 \%$. As for the film cooling flow angle, the impact of BR on the performance parameters was small but the combined effect of this parameter together with another positive impacting parameter, could result in notable increase in engine performance. (i.e. having higher BR with high adiabatic film cooling efficiency)

\subsection{Sensitivity Analysis}

In the previous section, the effects on the engine performance parameters from different cooling parameters were analyzed separately. In order to compare the strength of each cooling parameter on changing the engine performance parameters, a sensitivity analysis is conducted in this section with the data obtained in Section 3.1 for aero-engine and industrial gas turbine cases.

A preliminary parameter dependency analysis is made for each cooling parameter by using the respective interval of analysis from Table 24 and using the mathematical approach given by Equation (75) as follows.

$$
\frac{\partial Y}{\partial \theta}=\frac{\left(Y_{2}-Y_{1}\right) / Y_{1}}{\left(\theta_{2}-\theta_{1}\right) / \theta_{1}}
$$

In Equation (75), $Y$ represents the selected performance parameter and $\theta$ represents the cooling parameter considered. The magnitude of $\frac{\partial Y}{\partial \theta}$ indicates the strength of dependency of $Y$ on $\theta$, and a positive sign indicates increasing $\theta$ will increase $Y$, whereas a negative sign indicates a negative impact on $Y$ when $\theta$ is increased. The increase in $\delta \theta$ is selected in the following analyses to satisfy the $\delta \theta \ll \theta$ condition. For the high by-pass turbofan aero-engine case of Section 3.1, parameter dependency analysis results are given in Table 25. 
Table 25: Results of the parameter dependency study for the aero-engine case

\begin{tabular}{|c|c|c|c|}
\hline Parameter & Impact on Thrust & Impact on TSFC & Impact on Thermal Efficiency \\
\hline Metal Biot Number $\left(\mathrm{Bi}_{\mathrm{m}}\right)$ & -0.0039 & +0.0040 & -0.0067 \\
\hline TBC Biot Number (Bí & +0.0021 & -0.0004 & +0.0016 \\
\hline $\begin{array}{lr}\text { Max. } & \text { Allowable Blade } \\
\text { Metal } & \text { Temperature } \\
\left(\mathrm{T}_{\mathrm{b}, \text { max }}\right) & \end{array}$ & +0.4464 & -0.3952 & +0.6411 \\
\hline $\begin{array}{l}\text { Internal Flow Cooling } \\
\text { Effectiveness }\left(\varepsilon_{c}\right)\end{array}$ & +0.1479 & -0.1428 & +0.2301 \\
\hline $\begin{array}{l}\text { Adiabatic Film Cooling } \\
\text { Effectiveness }\left(\varepsilon_{\mathrm{fc}}\right)\end{array}$ & +0.0099 & -0.0030 & +0.0083 \\
\hline $\begin{array}{l}\text { Film Cooling Flow Angle } \\
(\Phi)\end{array}$ & -0.0002 & +0.0002 & -0.0002 \\
\hline $\begin{array}{l}\text { Film Cooling Blowing } \\
\text { Ratio (BR) }\end{array}$ & +0.0003 & -0.0003 & +0.0003 \\
\hline
\end{tabular}

From the results of the dependency analysis of Table 25, increasing the maximum allowable blade metal temperature improves all 3 performance parameters most (i.e. increases thrust, reduces TSFC and increases thermal eff.), followed by increasing the internal flow cooling effectiveness, film cooling effectiveness, TBC Biot number and blowing ratio. Most negative impact on performance (i.e. decreases thrust, increases TSFC and reduces thermal eff.) occurs when the metal Biot number is increased, followed by increasing the film cooling flow angle.

The results of the parameter dependency study made for the generic $\mathrm{H}$-Class industrial gas turbine case are given in Table 26.

The results from Table 26 indicates that the performance of an industrial gas turbine is affected positively (i.e. increased power, reduced HR, and increased thermal eff.) when the maximum allowable blade temperature is increased, this is followed by the internal cooling flow effectiveness, then film cooling efficiency and TBC Biot number. The impact is negative when the metal Biot number is increased.

Table 26: Results of the parameter dependency study for the industrial gas turbine case

\begin{tabular}{|c|c|c|c|}
\hline Parameter & Impact on Power & Impact on Heat Rate & Impact on Thermal Efficiency \\
\hline Metal Biot Number $\left(\mathrm{Bi}_{\mathrm{m}}\right)$ & -0.063 & +0.044 & -0.044 \\
\hline TBC Biot Number (Bi $\left.\mathbf{T}_{\mathrm{TBC}}\right)$ & +0.105 & -0.070 & +0.072 \\
\hline $\begin{array}{lr}\text { Max. } & \text { Allowable Blade } \\
\text { Metal } & \text { Temperature } \\
\left(T_{b, \max }\right) & \end{array}$ & +3.160 & -2.134 & +2.186 \\
\hline $\begin{array}{l}\text { Internal Flow Cooling } \\
\text { Effectiveness }\left(\varepsilon_{c}\right)\end{array}$ & +0.375 & -0.268 & +0.277 \\
\hline $\begin{array}{l}\text { Adiabatic Film Cooling } \\
\text { Effectiveness }\left(\varepsilon_{\mathrm{fc}}\right)\end{array}$ & +0.396 & -0.264 & +0.276 \\
\hline
\end{tabular}

It should be noted here that, the dependency analysis made in Tables Table 25 and Table 26 are the average values of $\frac{\partial Y}{\partial \theta}$ over the entire cooling parameter interval and it does not provide information about how the influence of these parameters are changing on the interval of interest.

The sensitivity of performance parameters on selected cooling parameters was sought by comparing their percent change from the reference engine performance when the selected cooling parameter is increased or 
decreased from the reference values given at Table 24. In this type of analysis, the slope shows the comparative impact strength of various cooling parameters on the engine performance variables.

\subsubsection{High By-Pass Turbofan Engine}

In this analysis, sensitivity charts were obtained for engine thrust, thrust specific fuel consumption, coolant flow rates, and thermal efficiency by plotting the percent change in these parameters against the percent change in each selected cooling parameter of Table 24.

Sensitivity of thrust on the selected cooling parameters is shown in Figure 91 . Thrust sensitivity follows the same trend obtained from parameter dependency analysis, having the highest impact from maximum allowable blade temperature, followed by the internal flow cooling effectiveness, film cooling effectiveness, TBC Biot number and the blowing ratio. Film cooling injection angle and metal Biot number impacts thrust negatively but their relative impact strength is very small and therefore not shown in the following sensitivity charts.

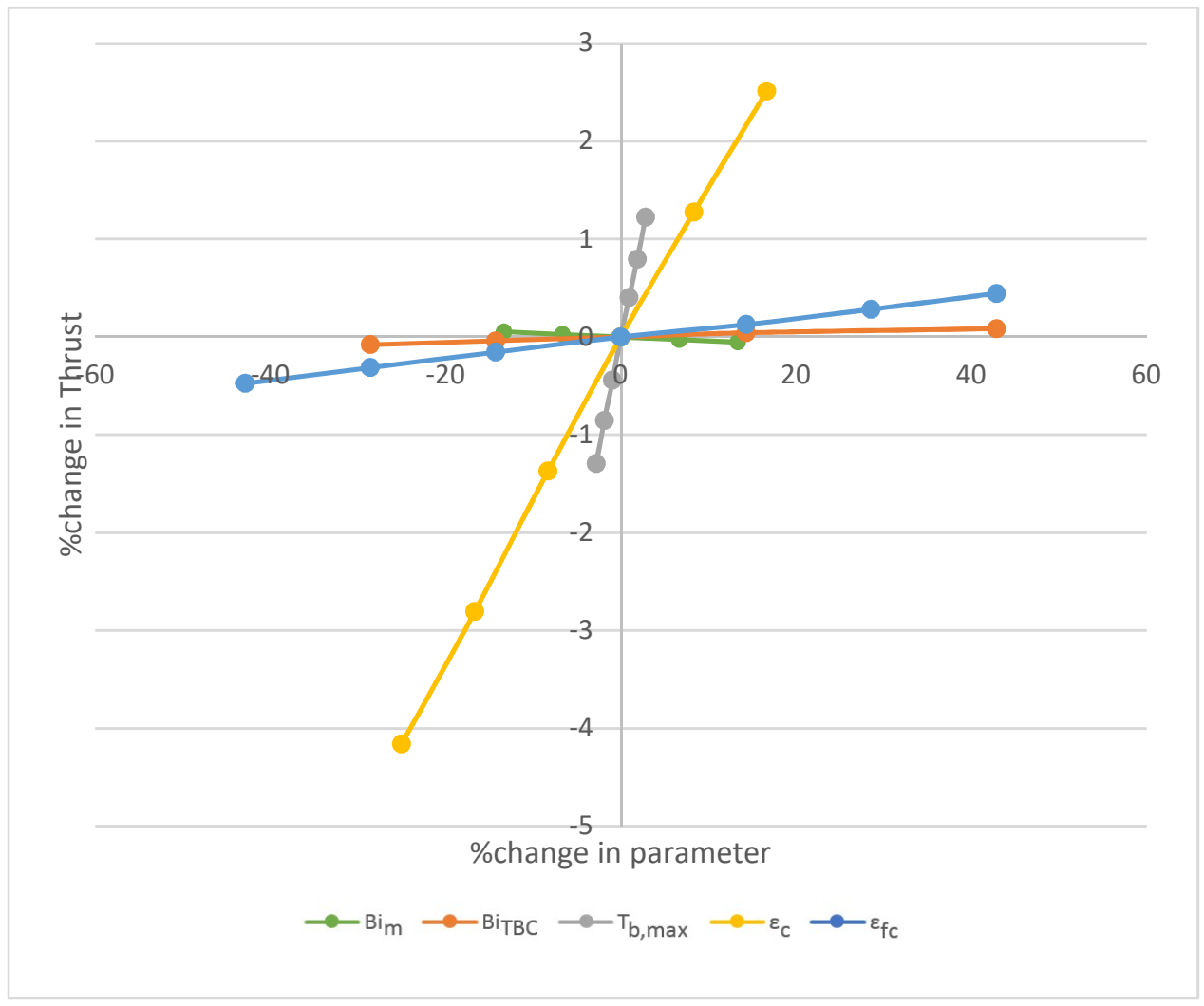

Figure 91: Sensitivity of engine thrust on the general cooling parameters

Sensitivity for Thrust Specific Fuel Consumption is obtained as in Figure 92. For TSFC comparison, the technique that reduces the TSFC more means it could increase the thrust more while causing more reduction in the fuel consumption; therefore, in this sensitivity graph negative slopes indicates the improvement in the performance. 


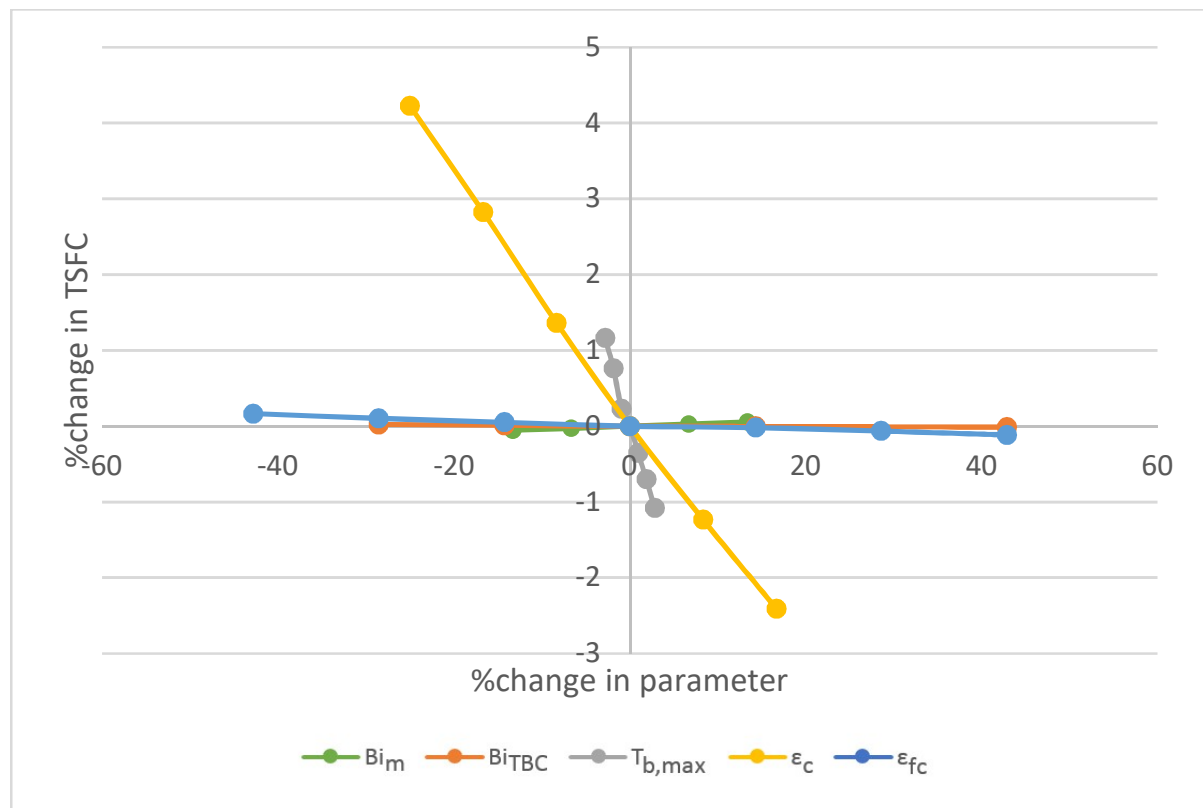

Figure 92: Sensitivity of thrust specific fuel consumption on the general cooling parameters

Thrust specific fuel consumption is reduced mostly when the maximum allowable blade metal temperature is increased, followed by the increase in internal cooling flow effectiveness, film cooling effectiveness, TBC Biot number and blowing ratio and impacted negatively when the film cooling flow angle and metal Biot number is increased.

Changes in the cooling parameters affects the amount of required coolant flows. In the sensitivity analysis, the parameter that reduces the coolants most, is generally expected to have the highest impact on the engine performance, but the existence of secondary losses might change this conclusion. The sensitivity of the total coolant flow rates on cooling parameters is plotted in Figure 93.

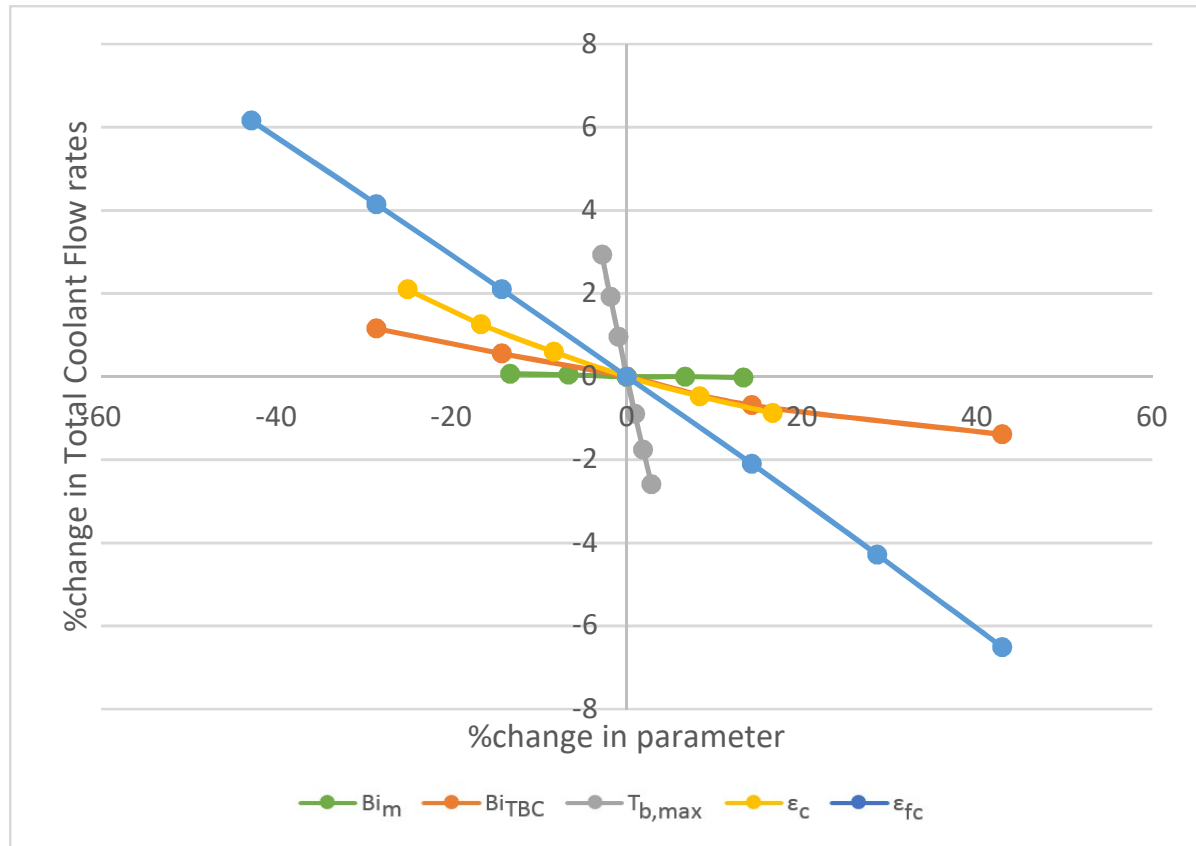

Figure 93: Sensitivity of the total coolant flow fraction on the general cooling parameters 
The sensitivity chart in Figure 93 shows that film cooling techniques has the highest reduction rate for the coolant flows, which is obtained similarly in the study by Young et al [7].

The impact on the engine thermal efficiency follows the same trends with the sensitivities obtained for thrust and also the parameter dependency analysis, as shown in Figure 94.

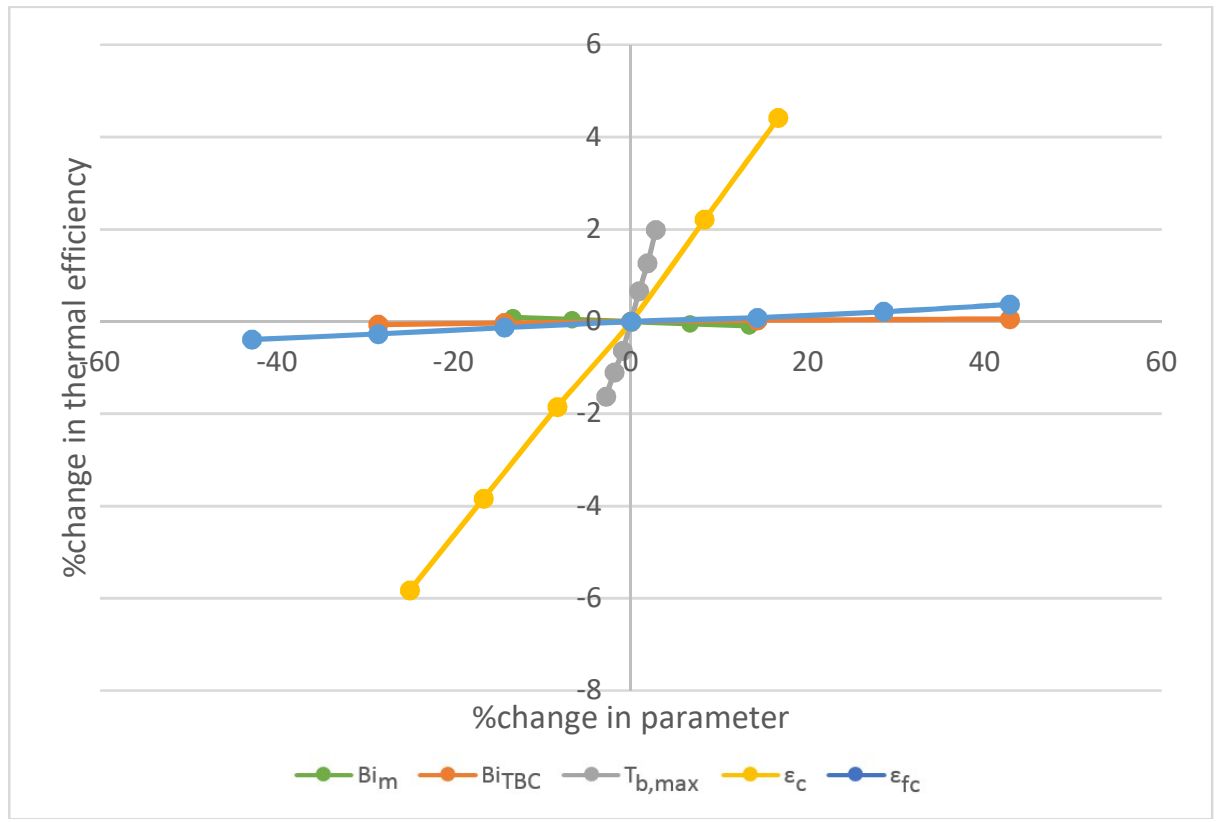

Figure 94: Sensitivity of thermal efficiency on the general cooling parameters

The slopes of sensitivity plots of Figure 94 indicate that advancements in blade materials (through an increase in maximum allowable blade metal temperature, $T_{b}, \max$ ) have the highest impact on the thermal efficiency, which is expected since it is the primary parameter that affects the overall blade cooling effectiveness. Advancements in internal cooling techniques (through improvements in internal cooling flow effectiveness) have the second highest impact followed by advanced film cooling techniques, using of advanced TBC materials, and film cooling techniques having low flow inclination angles in high blowing ratios. According to the heat transfer model used from Young and Wilcock [7], if the blade material has a higher conductivity (thus a lower $\mathrm{Bi}_{\mathrm{m}}$ ) it can be cooled easier from the inside; resulting in a lower coolant flow requirement by external cooling techniques. Using less external coolant flow would eventually result in a decrease in turbine losses (mixing associated losses will be reduced) and the engine performance would be affected positively.

The sensitivities obtained in this analysis also provide information about negative changes in cooling parameters as well. For instance, erosion of TBC material during operation might reduce the TBC thickness and reduce Вітвс. Sand or dust particles in the incoming airflow might fill the internal and external cooling channels, resulting in lower effectiveness values for internal cooling flow and film cooling effectiveness, respectively. Erosion of TBC materials might also change the external cooling hole geometry, resulting in a decrease in the design blowing ratio and increase in cooling flow angle. Corrosion of the blade materials might reduce the maximum allowable blade metal temperature. In such cases, the effects on the engine performance would be strongly influenced by changes in blade metal properties, then followed by negative effects on internal and external cooling channels and TBC erosion. 


\subsubsection{Industrial Gas Turbine}

In this analysis, sensitivity charts were obtained for shaft power generated, heat rate, coolant flow rates, and thermal efficiency by plotting the percent change in these parameters against the percent change in each selected cooling parameter of Table 24 .

The sensitivity curves obtained for the industrial gas turbine cases are non-linear due to having more cooled turbine stages than the aero-engine and more increase in compressor power demand due to inter-stage coolant extractions. Because of this reason, sensitivity curves show different impact order than the parameter dependency study for the heat rate and thermal efficiency.

Positive part of the sensitivity of shaft power delivered follows the same order with parameter dependency analysis and is shown in Figure 95.

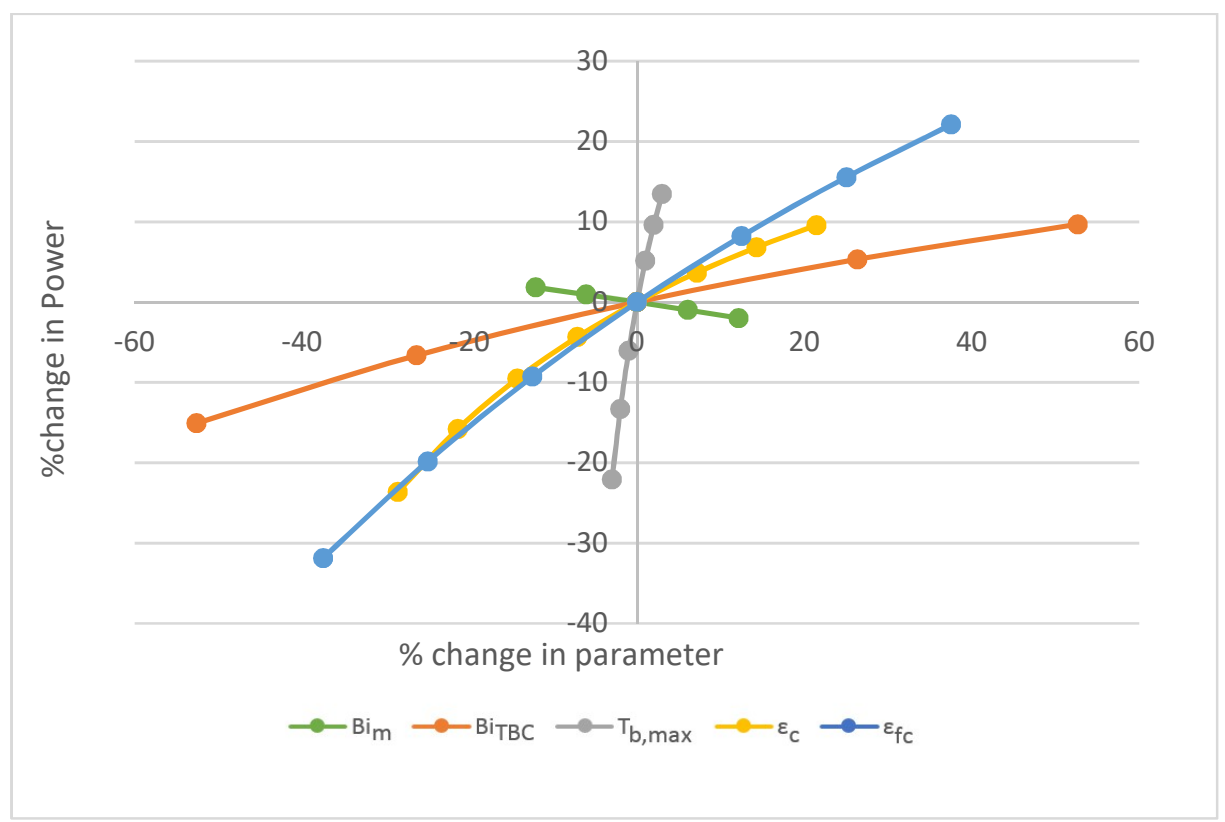

Figure 95: Sensitivity of shaft power delivered on the general cooling parameters

From the trends in Figure 95, engine power is highly dependent on the changes in maximum blade temperature, followed by changes in film cooling efficiency, internal cooling efficiency and TBC Biot number, respectively. The impact is negative when the metal Biot number is increased. It should be noted that reduction in internal cooling flow efficiency affects the performance slightly larger than the reduction in film cooling efficiency.

The sensitivity of heat rate on the general cooling parameters is shown in Figure 96. 


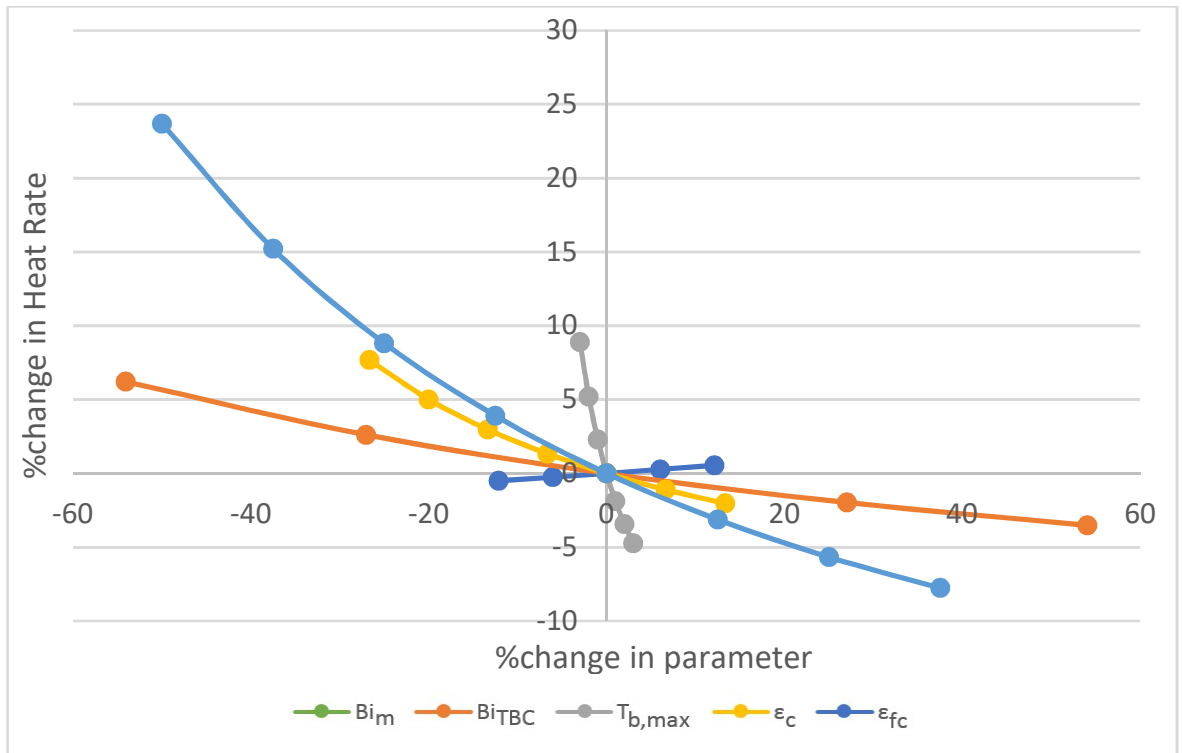

Figure 96: Sensitivity of heat rate on the general cooling parameters

In a gas turbine design, it is desired to reduce the heat rate as much as possible, because this parameter is a measure of fuel consumption per shaft power generated. From the sensitivity results, heat rate is highly sensitive on maximum allowable blade temperature, then film cooling efficiency, internal cooling efficiency and least sensitive to TBC Biot number increase. It is negatively affected by an increase in the metal Biot number.

For an industrial gas turbine, the sensitivities are directly dependent on how much a certain cooling technology is able to reduce the required coolant flow rates to cool the blade to the same maximum allowable temperature. Thus, the sensitivities of the coolant flow rates on considered cooling parameters are shown in Figure 97.

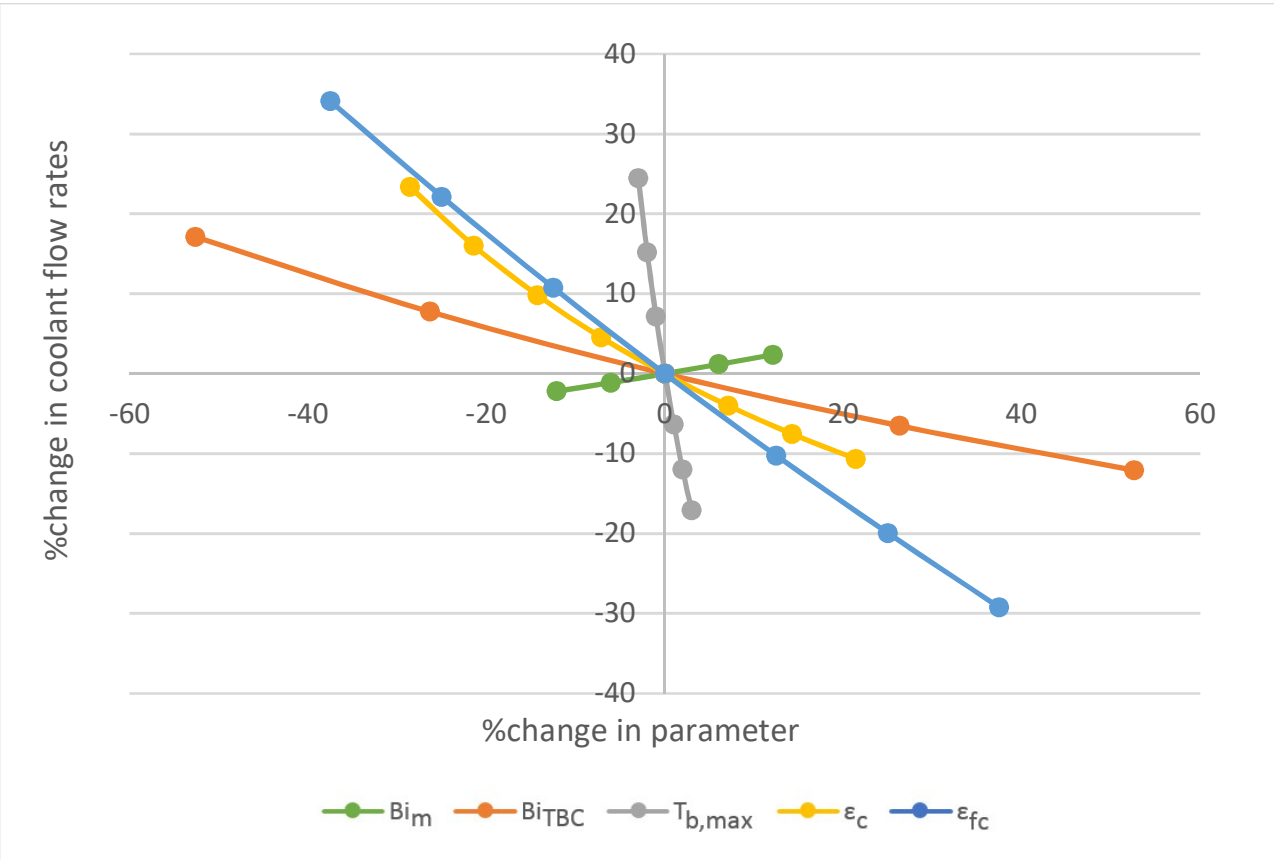

Figure 97: Sensitivity of total coolant flow rates on the general cooling parameters 
Aside from the maximum allowable blade temperature, film cooling performance is highly effective on the required coolant flow rates. It is followed by internal cooling performance and TBC Biot number. The trend is opposite, if the metal Biot number is increased.

The impact on the engine thermal efficiency follows the same trends with the sensitivities obtained for the reduction in required coolant flows, as shown in Figure 98.

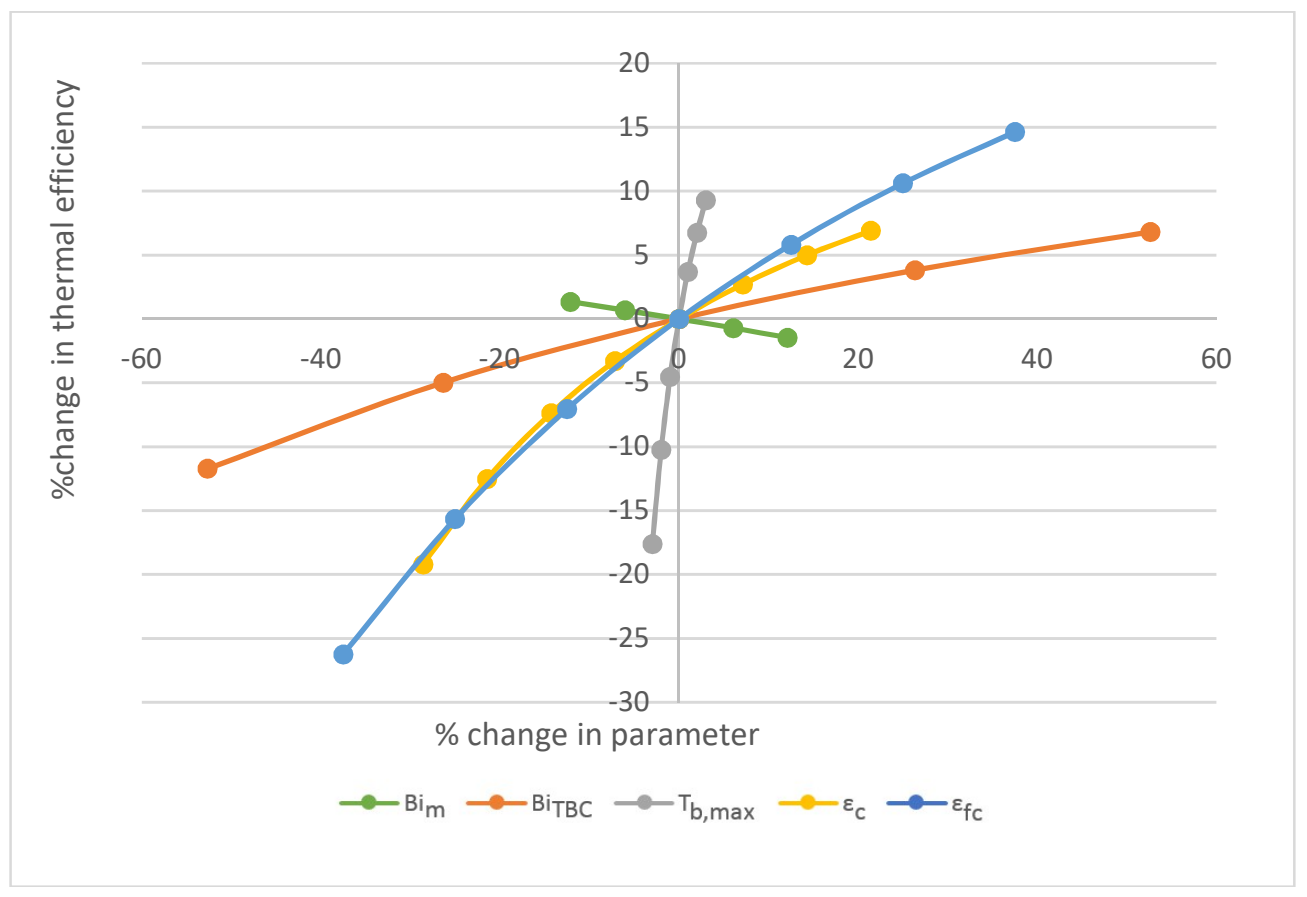

Figure 98: Sensitivity of thermal efficiency on the general cooling parameters

The positive part of the sensitivity charts gives information about the improvements that can be made to improve the engine performance, whereas the negative part gives information about how much reduction in the engine performance will occur if one of the analysis parameters is reduced. According to the results in Figure 98, for an industrial gas turbine, advancements in blade materials (through an increase in maximum allowable blade metal temperature, $T_{b}$, max) have the highest impact on the thermal efficiency, which is similar with the aero-engine case. Advancements in film cooling techniques have the second highest impact followed by the advancements in internal cooling techniques and advancements in TBC materials. Increasing the metal Biot number has a negative impact.

The effect of reduction in engine performance parameters follows a different order, indicating that reduction in the maximum allowable blade metal temperature (for instance, through corrosion or thermal fatigue) would reduce the engine performance most, followed by the reduction in internal cooling effectiveness (for instance through the filling of internal cooling channels with sand or dust particles), reduction in film cooling performance through filling of the external cooling channels and erosion of TBC materials.

\subsubsection{Differences in Sensitivity Trends for Different Engine Types}

Comparing the sensitivity analysis results of the aero-engine case and the industrial gas turbine case shows that internal cooling effectiveness has more impact than film cooling effectiveness for the aero-engine, which is different from the industrial gas turbine case. 
In order to investigate this difference, some of the engine parameters compared for aero-engine case when the internal and film cooling effectiveness is increased by the same amounts as shown in Table 27.

Table 27: Results of the parameter dependency study for the industrial gas turbine case

\begin{tabular}{|c|c|c|c|c|c|c|c|c|c|}
\hline Parameters & $\pi_{\mathrm{tH}}$ & $\mathbf{T}_{\mathrm{tH}}$ & $\pi_{\mathrm{tL}}$ & $\mathbf{T}_{\mathrm{tL}}$ & $\begin{array}{l}T_{\text {bw }} \\
\left({ }^{0} R\right)\end{array}$ & $\begin{array}{l}T_{t 4.5} \\
\left({ }^{0} R\right)\end{array}$ & $\begin{array}{l}\mathrm{T}_{9} \\
\left({ }^{0} \mathrm{R}\right)\end{array}$ & $V_{9}(\mathrm{ft} . / \mathrm{s})$ & Sp. Thrust \\
\hline \multicolumn{10}{|c|}{ Increasing Film Cooling efficiency by $14 \%$} \\
\hline 0.35 & 0.2792 & 0.7243 & 0.3796 & 0.7861 & 2210 & 2080 & 1467 & 1682 & 17.6431 \\
\hline \multirow[t]{2}{*}{0.4} & 0.2799 & 0.7255 & 0.3803 & 0.7866 & 2197 & 2083 & 1470 & 1684 & 17.6660 \\
\hline & $+0.25 \%$ & $+0.17 \%$ & $+0.18 \%$ & $+0.06 \%$ & $\overline{0} .59 \%$ & $\begin{array}{l}+0.14 \\
\%\end{array}$ & $+0.2 \%$ & $+0.12 \%$ & $+0.13 \%$ \\
\hline \multicolumn{10}{|c|}{ Increasing Internal Cooling Efficiency by $14 \%$} \\
\hline 0.6 & 0.2792 & 0.7243 & 0.3796 & 0.7861 & 2210 & 2080 & 1467 & 1682 & 17.6431 \\
\hline \multirow[t]{2}{*}{0.68} & 0.2812 & 0.7514 & 0.393 & 0.7937 & 2217 & 2149 & 1532 & 1719 & 17.9995 \\
\hline & $+0.72 \%$ & $+3.74 \%$ & $+3.53 \%$ & $+0.97 \%$ & $\begin{array}{l}+0.32 \\
\%\end{array}$ & $\begin{array}{l}+3.32 \\
\%\end{array}$ & $\begin{array}{l}+4.43 \\
\%\end{array}$ & $+2.20 \%$ & $+2.02 \%$ \\
\hline
\end{tabular}

Increasing the film cooling effectiveness will reduce the ambient wall temperature, which enhances the convection heat transfer from hot gas to film layer. Removing more heat from the hot gas side to cool the blade will result in reduced HPT exhaust temperature ( $T_{t 4.5}$ in Table 27$)$

In the dual spool turbofan engine, existence of the low pressure turbine behind the high pressure turbine causes a difference in the effects on engine performance. Because of reduced inlet temperature, with constant polytropic efficiency low pressure turbine will produce less power (observed with changes in $\pi_{t L}$ in Table 27)

Cooling the blade to the same temperature by increasing the internal cooling effectiveness with fixed film cooling efficiency means cooling the blade more from inside, without causing a significant change in adiabatic wall temperature. Due to removing less heat from the hot gas side but being able to cool the blade from inside to the same maximum allowable metal temperature will result in higher HPT exit gas temperature ( $T_{t 4.5}$ in Table 27). Having higher HPT exit temperature will result in higher power production from LPT; resulting in an increase in the engine power produced (Specific Thrust in Table 27).

Thrust equation is affected from exhaust to ambient total pressure and temperature ratios (changes in $T_{9}$ of Table 27), as well as the primary exhaust gas flow velocity ( $V_{9}$ in Table 27). Having higher pressure ratio from the turbines will result in higher exhaust pressure which gives higher exhaust flow velocity and temperature from isentropic flow equations. Resulting effect is a higher impact on thrust when the internal cooling effectiveness is increased by the same amount with film cooling effectiveness.

From the thermal efficiency definition used for aero-engines, having higher thrust results in higher thermal efficiency. Although the coolant mass flow rates were reduced by film cooling more, the increase in thrust with internal cooling efficiency was more than with film cooling efficiency and resulted in a lower thrust specific fuel consumption.

It should be noted that similar trends might be seen in combined cycle performance of industrial gas turbines, in which a steam turbine is connected to the exit of the turbine and its performance is directly affected by the changes in the gas turbine exhaust temperature $\left(T_{t 9}\right)$. Aero-derivative industrial gas turbines with dual spool could also show the same trends with the trends obtained with turbofan engine case. 


\section{CHAPTER 4: USING DETACHED EDDY SIMULATION TECHNIQUE TO GENERATE AN ADVANCED FILM COOLING HOLE GEOMETRY DATA}

The Sensitivity Analysis made in the previous section showed that highly efficient film cooling techniques can provide improvements to gas turbine performance. For the aero-engine case, although improving internal cooling effectiveness provided more positive impact on engine performance, it should be noted that such an improvement can be attained by changing the internal cooling configuration of the turbine blade, which could be a longer and more expensive process than using an advanced film cooling technique together with an existing internal cooling design.

In film cooling of turbine blades, a cooler fluid (supplied from the compressor) is injected under the boundary layer formed by the flow over the blade surface. Conventionally, this cooling is applied through a set of single cylindrical holes aligned perpendicular to the flow direction on the blade. One of the drawbacks of this technique is the generation of Counter Rotating Vortex (CRV) pair, which reduces the effectiveness. As a remedy, an advanced film cooling hole named as "Anti-Vortex Hole" developed by Heidmann and Ekkad [34] to eliminate this effect by introducing two side holes which generate vortices that neutralizes the CRV effect.

The literature on analyzing film cooling flow for conventional holes under different flow conditions is very broad both in experimental $[33,38,42,43]$ and numerical aspects [35, 40, 41], which allows researchers to use these resources to validate their numerical tools that they developed for advanced cooling hole configurations. Anti-Vortex Hole configuration also has a developing literature in both experimental $[39,44]$ and numerical analysis $[36,37,45]$.

Numerical simulations for film cooling methods uses different solution techniques involving steady Reynolds Averaged Numerical Solver (RANS) [37, 46], unsteady RANS [37], Detached Eddy Simulations (DES) [35, 41] and Large Eddy Simulations (LES) [40]. Among these numerical solution techniques, DES and LES simulations are proven to be more successful over the other techniques for conventional holes [41], but do not have broad applications for AVH holes yet due to their increased numerical cost. In this section, DES type of numerical simulation is used for AVH film cooling analysis, to generate adiabatic film cooling effectiveness performance data under various turbulence intensity conditions at large length scales $\left(\Lambda_{x} / d_{m}=6\right)$ and high blowing ratio $(\mathrm{BR}=2.0)$, which are close to engine-like conditions for the turbine sections of aero-engines and industrial gas turbines.

\subsection{Numerical Setup}

Star-CCM+ $₫$ Software by CD-ADAPCO was used in this study. One of the main reasons for the consideration of this software was the Detached Eddy Simulation (DES) module combined with several built-in meshing tools. Numerical setup is determined by using conventional hole case, because of the high availability of comparable experimental and numerical studies in the literature. The determined setup in this step is then adapted to AVH cases, with individual grid and time step independence studies performed prior to each AVH simulation presented in the following sections. This part consists of selecting a turbulence model, grid refinement and a time step refinement study. The aim is to find the best analysis conditions that will be used in the subsequent steps of the study. 


\subsubsection{Geometry}

The geometries used for conventional and Anti-Vortex Hole cases were compatible with the literature and experimental studies used in the comparisons. For DES simulations, the cooling hole geometry should be modelled as full domain because of the unsymmetrical nature of the expected flow field. Therefore, full geometry was used and the boundary conditions were set in accordance with the literature and previous work. The vertical extension of the domain was determined such that a free stream boundary layer condition can be applied to the top surface. Solution geometry is shown in Figure 99 with all dimensions normalized to main film cooling hole diameter $\left(\mathrm{d}_{\mathrm{m}}\right)$.

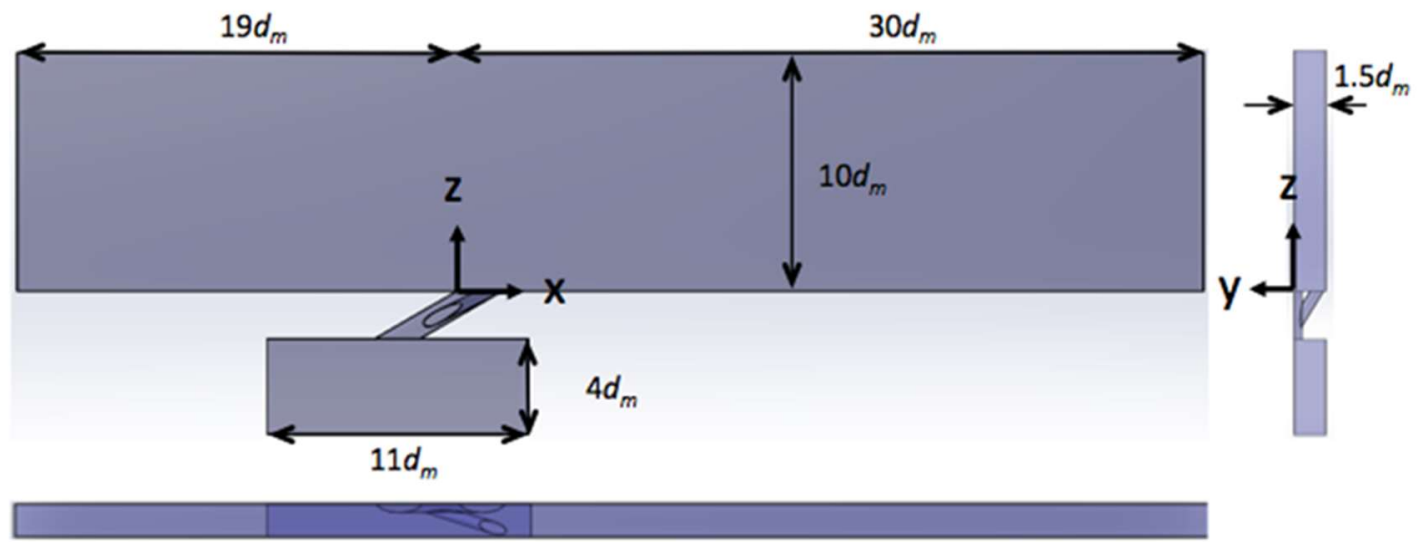

Figure 99: Half of the used solution geometry is shown for the AVH case, conventional hole case has the same geometry except for the side holes

\subsubsection{Boundary Conditions}

Boundary conditions were set in accordance with similar other CFD studies using conventional and AVH film cooling configurations ([33], [35]- [37]). The boundary types used in the simulations are given in Table 28.

Table 28: Boundary types that are applied to several sections of the solution domain

\begin{tabular}{|l|l|}
\hline Boundary & Type \\
\hline Top Surface & Freestream \\
\hline Inlet & Velocity Inlet \\
\hline Outlet & Pressure Outlet \\
\hline Plenum & Stagnation Inlet \\
\hline Wall Surfaces & Wall (no-slip) \\
\hline Sides & Symmetry Plane \\
\hline
\end{tabular}

\subsubsection{Flow Conditions}

The flow physics models and flow conditions were selected in accordance with several numerical ([35] and [37]) and experimental [38] studies. Unsteady, 3D, turbulent flow with ideal gas properties was used in all simulations. The flow conditions at the defined boundaries to match the physical conditions in those resources were set in accordance with the values given in Table 29. These flow conditions generate temperature ratio as 0.5 , and blowing ratio as 1.0 . 
At the inlet, the velocity boundary condition was set as a boundary layer velocity profile which is a curve fit function (exponential) of measured velocity boundary layer profile at WVU wind tunnel in the experimental study with AVH holes by Hayes [39] for the same flow conditions considered in Table 29.

Table 29: Flow Conditions at the solution domain boundaries

\begin{tabular}{|l|l|}
\hline Flow Variable & Value Set at the Boundary \\
\hline Inlet & $300 \mathrm{~K}$ \\
\hline Static Temperature & 0.05 \\
\hline Turbulence Intensity & 10.0 \\
\hline Turbulent Viscosity Ratio & $\mathrm{BL}$ Input ${ }^{\star \star}$ \\
\hline Velocity Magnitude & \\
\hline Outlet & $101320.0 \mathrm{~Pa}$ \\
\hline Pressure (reference) & $300 \mathrm{~K}$ \\
\hline Static Temperature & 0.01 \\
\hline Turbulence Intensity & 10.0 \\
\hline Turbulent Viscosity Ratio & \\
\hline Plenum & $101595.0 \mathrm{~Pa}$ \\
\hline Total Pressure (reference) & $150 \mathrm{~K}$ \\
\hline Total Temperature & 0.01 \\
\hline Turbulence Intensity & 10.0 \\
\hline Turbulent Viscosity Ratio & \\
\hline
\end{tabular}

\subsubsection{Numerical Solvers}

Implicit unsteady, and segregated flow and energy solvers were selected in the study. Temporal discretization was increased to second order after considerable amount of decay was seen in residuals, which is a suggested practice for the usage of these solvers. Maximum inner iterations per time step was chosen to be 30 and maximum physical time was set to $0.09 \mathrm{~s}$, in accordance with other similar numerical studies [35].

\subsubsection{Turbulence Model and Initial Meshing}

Built-in meshing tools of Star-CCM+ was used in meshing the geometry with tetrahedral type of meshing. The number of cells were arranged so as to be in accordance with the number of cells suggested by Kim and Hassan [35], in which it is shown that DES simulations gave more accurate predictions to experimental data when more than 1.3E06 number of cells is used. The Surface Growth Rate, and Prism Layer Stretching values were selected to be 1.14, which were in accordance with [35] and [40]. As a result, a mesh with $y+\approx 1.0$ was obtained.

For DES simulations, only k- $\omega$ SST and Spalart-Allmaras types of turbulence models were available from the analysis software. From literature ([35] and [41]), it is shown that k- $\omega$ SST models usually predict the film cooling flows better, but due to using a different numerical analysis software than these resources, a selection process involving a comparative study on centerline effectiveness predictions from both models is made and the results are compared with the experimental data from Sinha [38].

The centerline effectiveness predictions by using these turbulence models is compared in Figure 100 for the same number of cells. Although Spalart-Allmaras based DES gave closer approximation to the experimental data in the near hole region, the difference from the experimental data is increased along the downstream. The situation is the opposite for the $k-\omega$ based DES, which has a better agreement in the downstream region. 

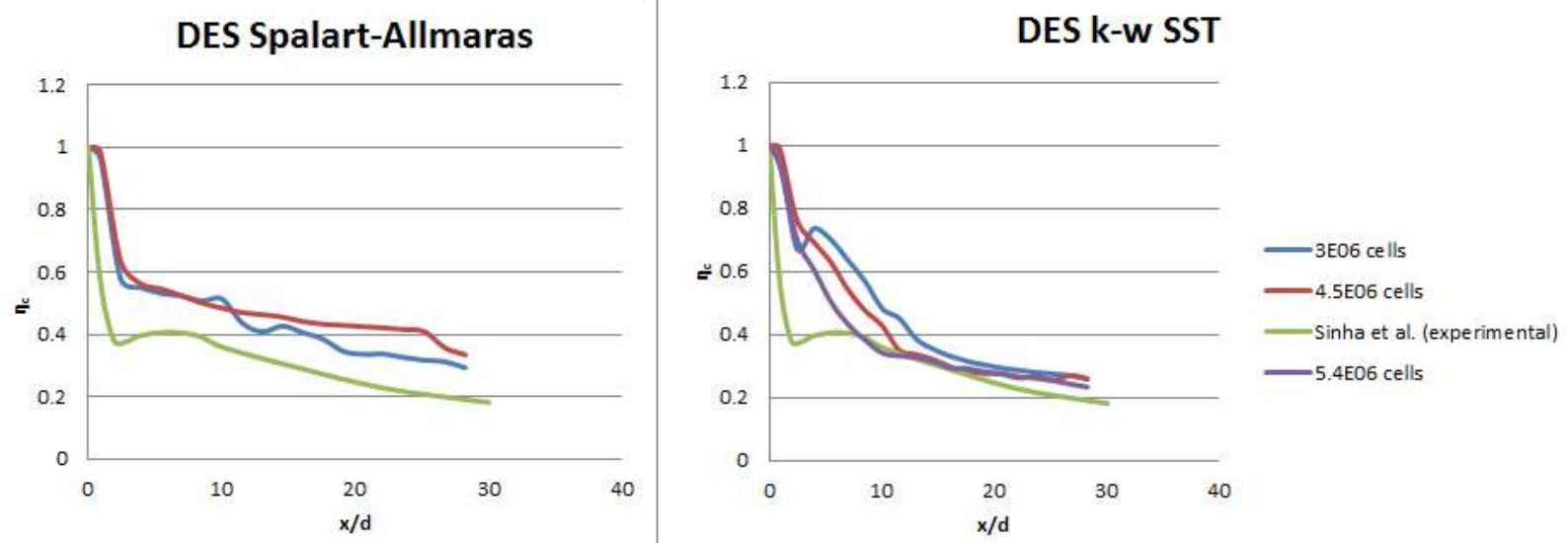

Figure 100: Grid density is increased in both turbulence models to decide over the performance of the models. The response of increasing mesh quality was better in $k-\omega$ SST model

In order to be able to give a decision on a turbulence model, grid density was increased from 3E06 cells to 5.4E06 cells in order to see whether the disagreement regions (downstream for S-A and near hole region for $k-\omega)$ are reduced with this improvement. Increasing the grid density is generally expected to lower the errors, therefore if Spalart-Allmaras model performs better with this improvement it could be selected over $k-\omega$. However, improvement in the grid did not resulted in a better prediction in the downstream region for SpalartAllmaras case, while the predictions were improved in k- $\omega$ case, as shown in Figure 100. As a result, $k-\omega$ SST model is selected to perform the following validation steps. The disagreement in the near hole region was expected to be reduced with the grid and time step refinement studies.

\subsubsection{Time-Step}

An important numerical error source in the CFD simulations is the time step, which affects the accuracy of numerical predictions significantly. In order to determine a time step that will provide numerical solutions not vulnerable to such errors, a time-step sensitivity analysis is made starting with 5.4E06 cell grid with k- $\omega$ SST turbulence model from the previous section.

The time step is changed from $1.0 \mathrm{E}-03 \mathrm{sec}$ to $5.0 \mathrm{E}-04$ and $1.0 \mathrm{E}-04 \mathrm{sec}$. The change in the centerline effectiveness approximations is compared in Figure 101.

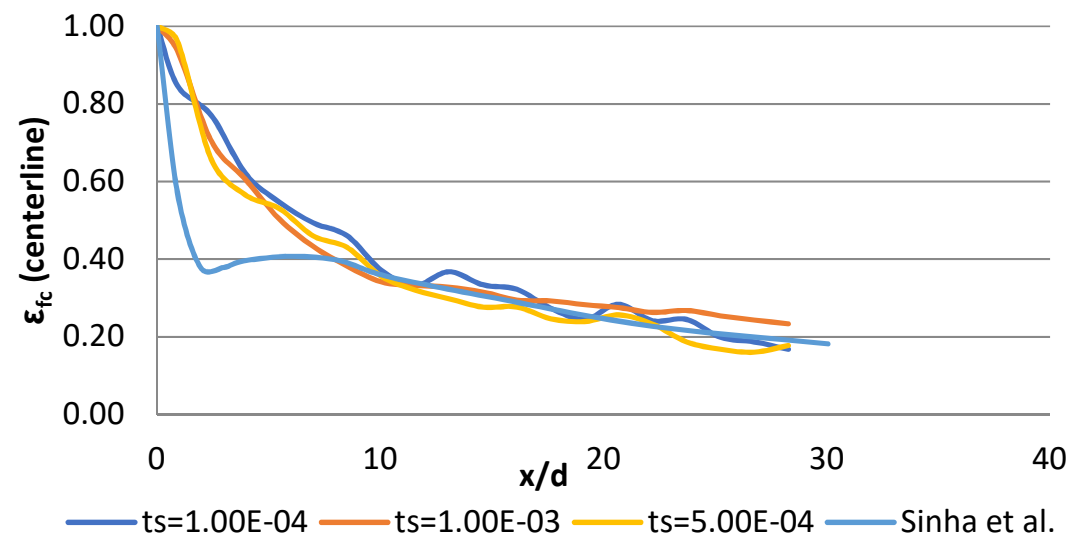

Figure 101: Centerline cooling effectiveness predictions with different time steps are compared with experimental data from [38] 
By reducing the time step size, the downstream region approximations became closer to the experimental data. As can be seen from the graph, the difference in predictions between $5.0 \mathrm{E}-04 \mathrm{sec}$ and $1.0 \mathrm{E}-04 \mathrm{sec}$ time steps are negligible; therefore, time step size of $5.0 \mathrm{E}-04 \mathrm{sec}$ was chosen by considering the overall solution time.

\subsubsection{Mesh Refinement Study}

The mesh refinement study is pursued with the time step size selected in the previous part. In this study, the number of cells would be increased from 5.0E06 cells to above, which would have the potential of increasing the overall solution time further. In order to accomplish this work more efficiently, adaptive meshing technique is applied, by using the temperature gradients from the results obtained with 5.0E06 cell case from initial meshing made in Section 4.1.5. Adaptive meshing provided an upgraded grid with cells that are concentrated on the region of interest for film cooling, which allowed to obtain 10.4E06 and 13.5E06 cell grids more efficiently.

The necessary condition for the adaptive meshing is to determine a "meshing function", which is usually selected either by using the pressure, velocity or temperature gradients depending on the application. In this study, a meshing function that captures the temperature gradients that are greater than a specified threshold was used. The resulting advanced mesh has denser region in the film cooling region, as shown in Figure 102.



Figure 102: Adaptive meshing with temperature gradients increased mesh concentration in the film cooling flow region

The runs with adaptive meshes with increased number of cells were made with 5.0E-04 sec time step size and the resultant centerline effectiveness values are compared in Figure 103.

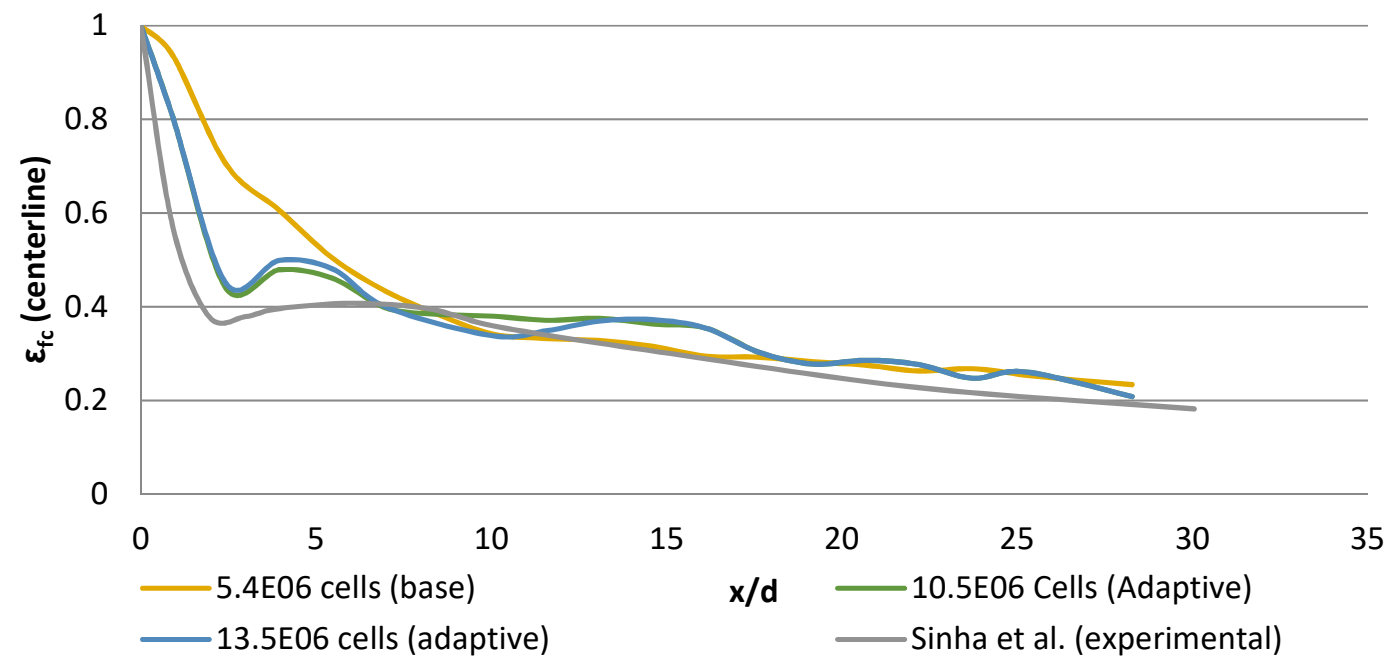

Figure 103: Grid Independence tests made with adaptive meshes are compared with experimental data from [38] 
As can be seen from Figure 103, the centerline effectiveness results of the adaptive grids are close enough to reach a conclusion for mesh independence. By considering the overall solution wall clock time, adaptive mesh with 10.4E06 cells is chosen to be used in the subsequent sections.

\subsection{Validation}

\subsubsection{Conventional Hole Cases}

For the validation of the DES simulations to generate conventional hole data, the span averaged effectiveness results obtained with the selected turbulence model, selected grid and the time step were compared with the experimental conventional hole data obtained by Hayes [39] at WVU Wind Tunnel. The flow conditions in the CFD simulations for this part are determined to match the experimental conditions and are given in Table 30.

Table 30: Flow Conditions used in DES validations made with experimental conventional hole data from [39]

\begin{tabular}{|l|l|}
\hline Parameter & Value \\
\hline $\mathbf{R e}_{\mathbf{D}}$ & 10000 \\
\hline $\mathbf{V}_{\mathbf{g}}$ & $15 \mathrm{~m} / \mathrm{s}$ \\
\hline $\mathbf{T}_{\text {tg }} / \mathbf{T}_{\mathbf{C}}$ & 1.06 \\
\hline Density Ratio & 1.05 \\
\hline Turbulence Intensity & $1 \%$ \\
\hline $\mathbf{T}_{\text {tg }}$ & $300 \mathrm{~K}$ \\
\hline
\end{tabular}

The experiment with blowing ratio of 1.0 and turbulence intensity of $1 \%$ was chosen for the validations, which is the same blowing ratio and turbulence intensity value used in determining the numerical setup. The iterations were stopped once the blowing ratio and hole exit velocity values are stabilized for about 1000 iterations, which corresponded to $0.09 \mathrm{sec}$ of physical time. The corresponding effectiveness distribution obtained on the adiabatic wall surface is shown in Figure 104.
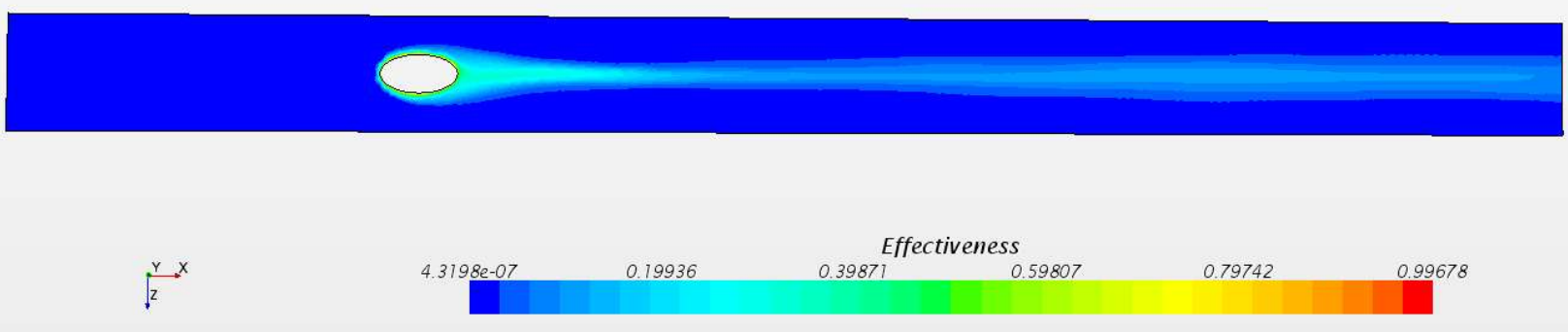

Figure 104: Effectiveness distribution obtained with the experimental conditions given in Table 30 for the conventional hole shows the expected cooling effectiveness distribution

The temperature distribution obtained on the center plane is given in Figure 105, which provides information about the observed decay in the effectiveness distribution after $\sim 3-3.5 \mathrm{dm}$ distance from the hole, which is due to the flow separation. There is a slight reattachment observed around $10 \mathrm{~d}_{\mathrm{m}}$ distance from the hole, which caused the slight increase in the cooling effectiveness distribution after this location. 


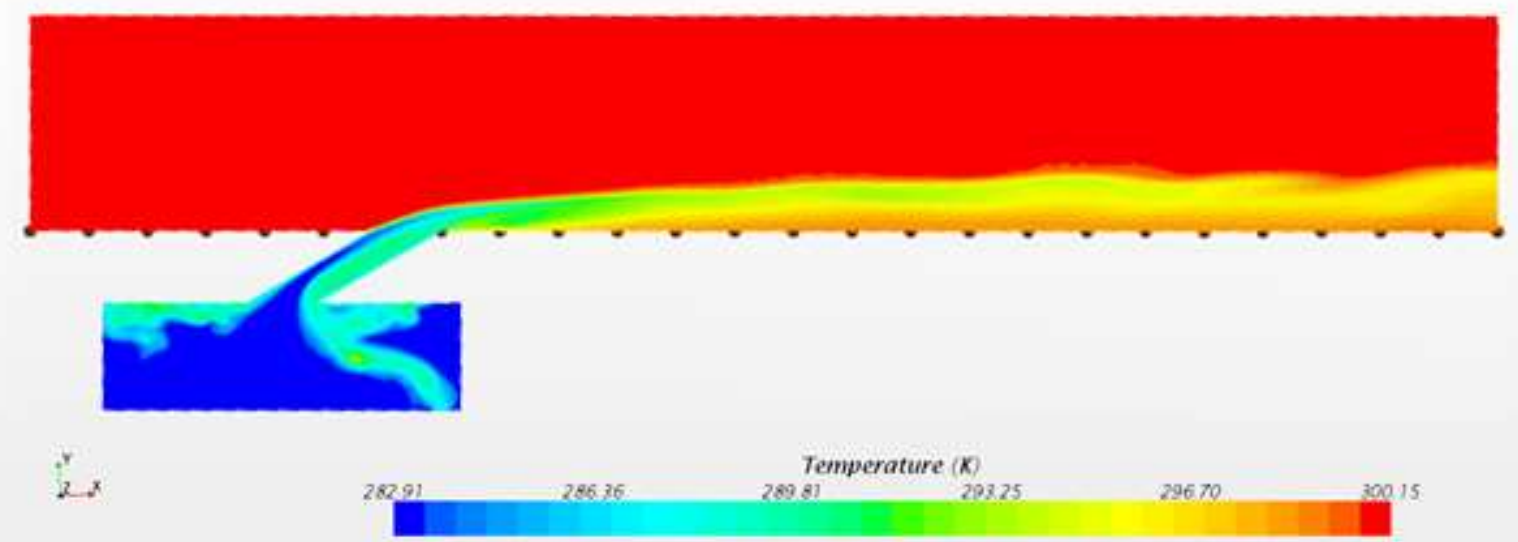

Figure 105: Temperature distribution plot for the center plane allows to identify the flow separation and reattachment regions to explain the fluctuations in the effectiveness predictions

The centerline effectiveness values from DES simulations is then compared with experimental data from Hayes [39], Dhungel et al. [44] and LeBlanc [47] in Figure 106.

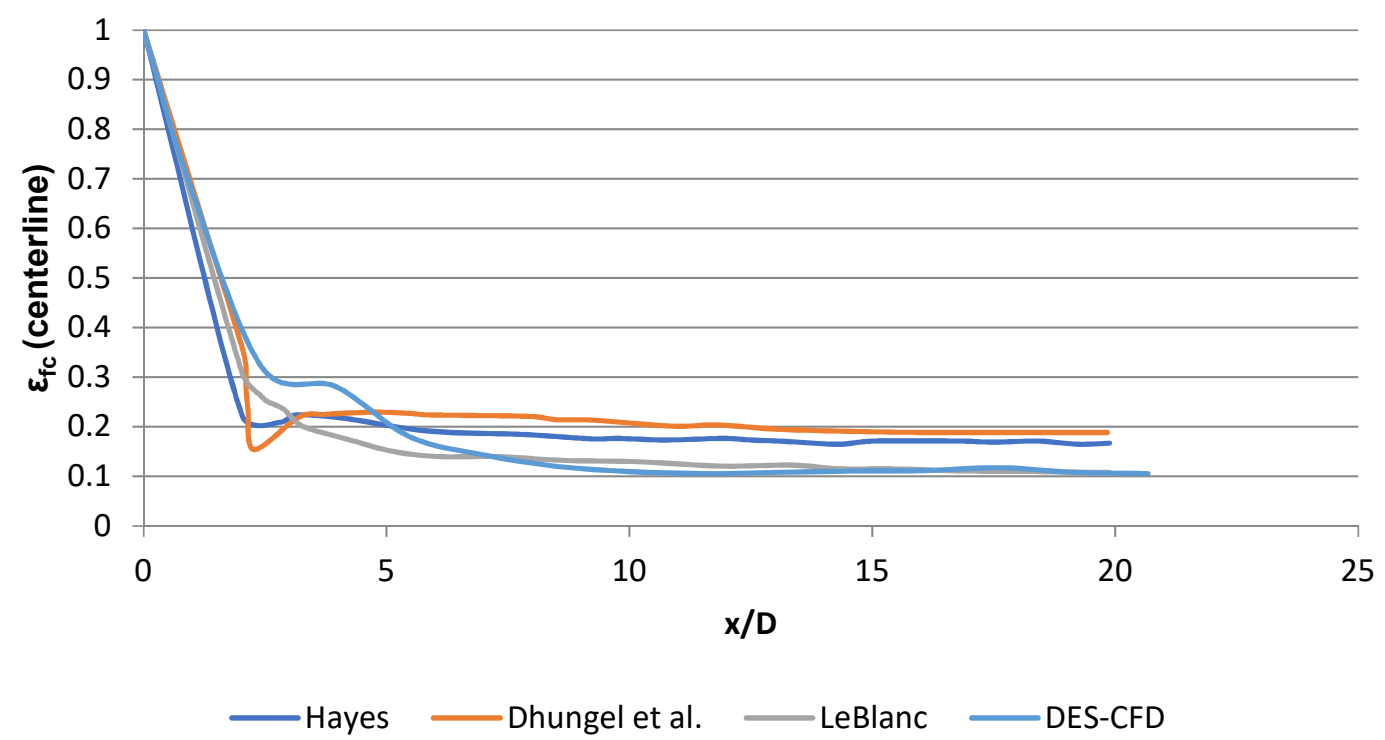

Figure 106: Centerline effectiveness data obtained for conventional hole DES simulation is compared with experimental data

The difference in centerline cooling effectiveness data by CFD with experimental data in the comparison of Figure 106 can be explained as follows:

- According to Bons et al. [48], in film cooling experiments the turbulence intensity changes non-linearly as the flow progresses along the downstream, whereas in CFD simulations the intensity and length scale of the main flow is changed from inlet to exit linearly, which is a possible explanation to the differences observed in the far downstream region. 
- According to the study by Kalghatigi and Acharya [40], CRV strength is strongly affected from the boundary layer profile inside the coolant delivery tube. Therefore, the difference between the estimated boundary layer profile by the CFD simulation and the actual profile occurred in compared experiments for the coolant delivery tube causes a difference in the CRV strength calculated by CFD. Differences in CRV strength causes the flow separation and re-attachment locations to be different, which is the possible explanation of the difference observed in the near-hole region.

By considering the aforementioned effects, and obtaining a cooling effectiveness trend that is coherent with the experimental data, the numerical setup is validated to be used in generating conventional hole effectiveness data valid for engine-like conditions.

\subsubsection{Anti-Vortex Hole Cases}

AVH simulations were generated by using full domain as in the conventional hole case, in order to be able to capture the unsteady flow field accurately. The mesh was generated by using the same mesh type used in the single hole case. The generated mesh was aimed to have number of cells close to 5E06, since this was the number of cells that produced closest results to adaptive mesh results in single hole section of the study. The resultant mesh had 4.5E06 cells, which formed the basis of the adaptive mesh generated by using the same technique considered in conventional hole case.

Same type of boundary conditions described in Section 4.1.2 for the single hole case were used in the AVH geometry case. The boundary condition types were kept as the same in this study to be in accordance with the convergence conditions obtained in single hole case. Same flow conditions given in Table 30 were used in the validations.

The values at the boundaries were set as given in Table 31, some of the boundary values were needed to be changed from the values used in Section 4.1.3 for the conventional hole case in order to match the same Blowing Ratio of 1.0, and a temperature ratio of 1.06.

Table 31: Flow Conditions at the solution domain boundaries for AVH case

\begin{tabular}{|l|l|}
\hline Flow Variable & Value set at the boundary \\
\hline Inlet & $300 \mathrm{~K}$ \\
\hline Static Temperature & 0.05 \\
\hline Turbulence Intensity & 1 \\
\hline Turbulent Length Scale (wrt. $\mathbf{d}_{\mathbf{m}}$ ) & BL Input (same with the previous section) \\
\hline Velocity Magnitude & \\
\hline Outlet & $101320.0 \mathrm{~Pa}$ \\
\hline Total Pressure & $300 \mathrm{~K}$ \\
\hline Static Temperature & 0.01 \\
\hline Turbulence Intensity & 1 \\
\hline Turbulent Length Scale $\left(\mathbf{w r t} . \mathbf{d}_{\mathbf{m}}\right)$ & \\
\hline Plenum & $102120.0 \mathrm{~Pa}$ \\
\hline Total Pressure & $150 \mathrm{~K}$ \\
\hline Total Temperature & 0.01 \\
\hline Turbulence Intensity & 10.0 \\
\hline Turbulent Viscosity Ratio & \\
\hline
\end{tabular}


Same type of solvers described in Section 4.1.4 for single hole case were used for AVH analysis. Stopping criteria were needed to be modified due to the highly unsteady nature of the flow field at early time steps. The maximum inner iterations were determined to begin with 60 , and reduced to 40 after 10 time steps by considering the rate of decrease of all residuals by 4 orders of magnitude. Comparison with the single hole case showed that AVH geometry needs more time steps (hence needs more physical time) to reach steady flow, which resulted in increasing of the maximum physical time to $0.219 \mathrm{~s}$ from $0.09 \mathrm{~s}$ used in conventional hole case.

After obtaining initial results with 4.5E06 cell mesh for AVH geometry, adaptive meshing method described in Section 4.1.7 is applied to refine the initial mesh and obtain 7E06 and 12E06 cell adaptive meshes. Mesh independence study made with the centerline effectiveness predictions using the adaptive meshes is shown in Figure 107.

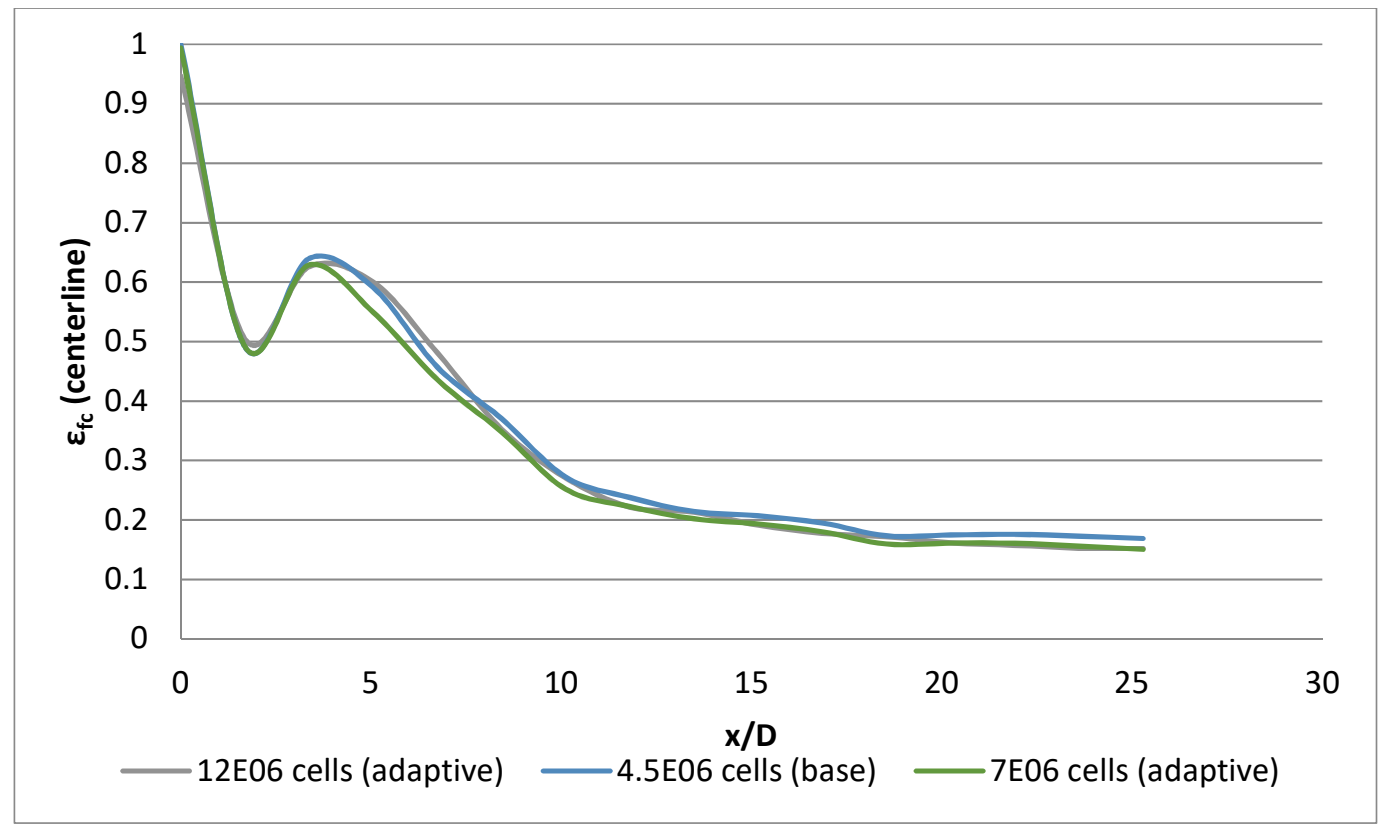

Figure 107: Grid Independence tests made with adaptive meshes for AVH cases

From Figure 107, the mesh independence of the simulation can be concluded. By the consideration of solution wall clock time, adaptive mesh with 7E06 cells is chosen.

The experiment by Hayes [39] for AVH cases with blowing ratio of 1.0 and turbulence intensity of $1 \%$ was chosen to be used in DES result validations. Span averaged cooling effectiveness is computed from AVH DES results by taking average of the effectiveness values from the probes equally spaced across the main hole in the lateral direction. The resulting average effectiveness values are compared with corresponding experimental data from [39] in Figure 108. 


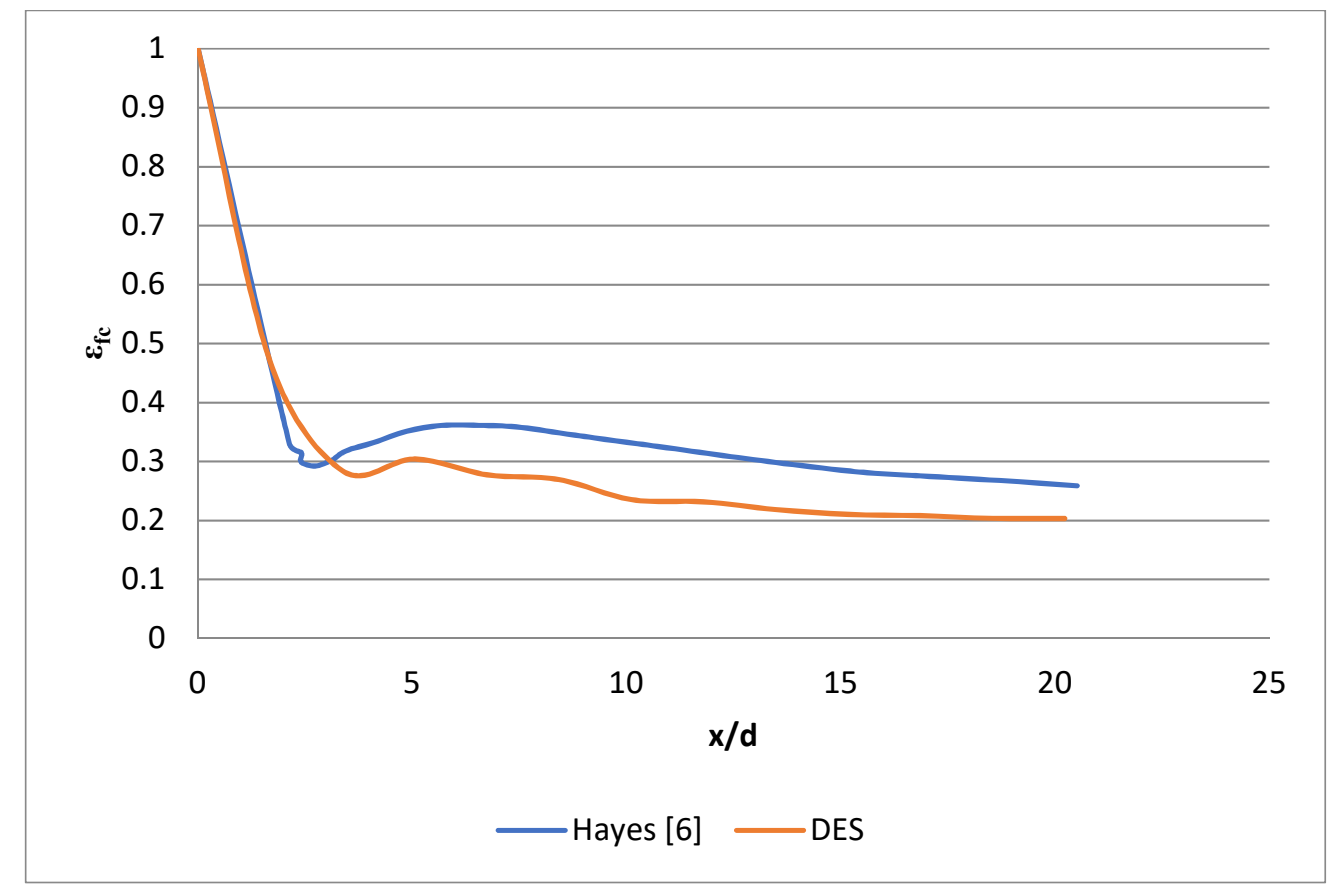

Figure 108: Span averaged effectiveness from DES simulations was compared with the experimental data for the same flow conditions

High centerline effectiveness at the near hole region indicates less lateral spread than the experimental data due to conservation of energy, therefore the magnitude of the span averaged cooling effectiveness values from DES are lower than the experimental data. The minimum of the span averaged effectiveness curves corresponds to the flow separation location and the local maximum reached after this location indicates the flow re-attachment, in accordance with the results obtained for centerline effectiveness. By considering the aforementioned differences between the DES and compared experimental data, and successfully capturing the overall effectiveness trend with DES, the numerical setup is validated to be used in generating AVH effectiveness data valid for engine-like conditions.

\subsection{Data Generation}

If the CFD simulations give accurate estimations to film cooling performance of a film cooling hole under the same flow conditions from an experiment, they can be used to analyze the change in the film cooling performance when the flow conditions are changed to a level that is not easily be attained in an experimental facility. An example of such flow conditions are high turbulence intensity and large turbulence length scale flow, which is often encountered in the turbine sections of aero-engines and industrial gas turbines.

Estimation of film cooling effectiveness with DES methods is validated with the experimental data for conventional and Anti-Vortex Hole for small turbulent length scale $\left(\Lambda_{x} / \mathrm{d}_{m}=1\right)$ and low turbulence intensity $(1 \%)$ in Section 4.2. Comparison of the centerline effectiveness and the span averaged effectiveness values obtained from DES with the experimental data showed good agreement and the validated numerical setup is used to generate large length scale $\left(\Lambda_{x} / d_{m}=6\right)$ and high turbulence intensity $(5 \%, 10 \%$ and $20 \%)$ data for the conventional and Anti-Vortex hole geometries. The details of these procedures are described separately in the following sub-sections for each hole type. 


\subsubsection{Conventional Hole Cases}

For the conventional hole geometry, numerical setup from Section 4.1 that is validated with experimental data in Section 4.2, was used to generate span averaged effectiveness data for three different mainstream turbulence intensities, which are 5, 10 and 20\%, respectively. Aside from generating data for span averaged effectiveness, temperature distribution on the adiabatic wall and the velocity contours at half plane are also analyzed to comment on the changes observed in film cooling performance, when the turbulence intensity is increased. The flow conditions at the boundaries, that gives the blowing ratio as 2.0 and the density ratio as 1.0 in all considered flow cases, were set to the values given in Table 32.

Table 32: Flow variables set at the boundaries for data generation of conventional hole under engine-like flow conditions

\begin{tabular}{|l|l|}
\hline Flow Variable & Value set at the boundary \\
\hline Inlet & $300 \mathrm{~K}$ \\
\hline Static Temperature & $0.05 / 0.1 / 0.2$ \\
\hline Turbulence Intensity & 6 \\
\hline Turbulent Length Scale $\left(\mathbf{w r t} . \mathbf{d}_{\mathbf{m}}\right.$ ) & BL Input (same profile used in Section 4.2) \\
\hline Velocity Magnitude & \\
\hline Outlet & $101320.0 \mathrm{~Pa}$ \\
\hline Total Pressure & $300 \mathrm{~K}$ \\
\hline Static Temperature & 0.01 \\
\hline Turbulence Intensity & 6 \\
\hline Turbulent Length Scale $\left(\mathbf{w r t}_{\mathbf{m}} \mathbf{d}_{\mathbf{m}}\right)$ & \\
\hline Plenum & $101595.0 \mathrm{~Pa}$ \\
\hline Total Pressure & $150 \mathrm{~K}$ \\
\hline Total Temperature & 0.01 \\
\hline Turbulence Intensity & 10.0 \\
\hline Turbulent Viscosity Ratio & \\
\hline
\end{tabular}

When the turbulence intensity of the main flow is changed, the time step of each simulation was reduced accordingly, to keep the average CFL number less than 1.0 at all locations in the solution domain.

From Kadotani and Goldstein [42], in the case of high blowing ratio (defined as $B R \geq 1.5$ ) and large length scales, which corresponds to the considered case in data generation, both centerline and span averaged effectiveness were increased at all downstream locations as the mainstream turbulence intensity is increased. This trend is the opposite when the turbulence length scale of the main stream flow is small [43]. In the case of large turbulence length scales with high blowing ratio, the boundary layer thickness and the penetration height of the injected flow are the main parameters that are governing the cooling effectiveness distribution. In the case of high blowing ratio, the coolant jet is penetrated further into the mainstream than the boundary layer thickness which makes the injection flow become less sensitive to the turbulence level of the main flow. The increase in the penetration height when the turbulence intensity is increased, can be seen in the velocity contour plots given for each turbulence intensity case in Figure 109. 

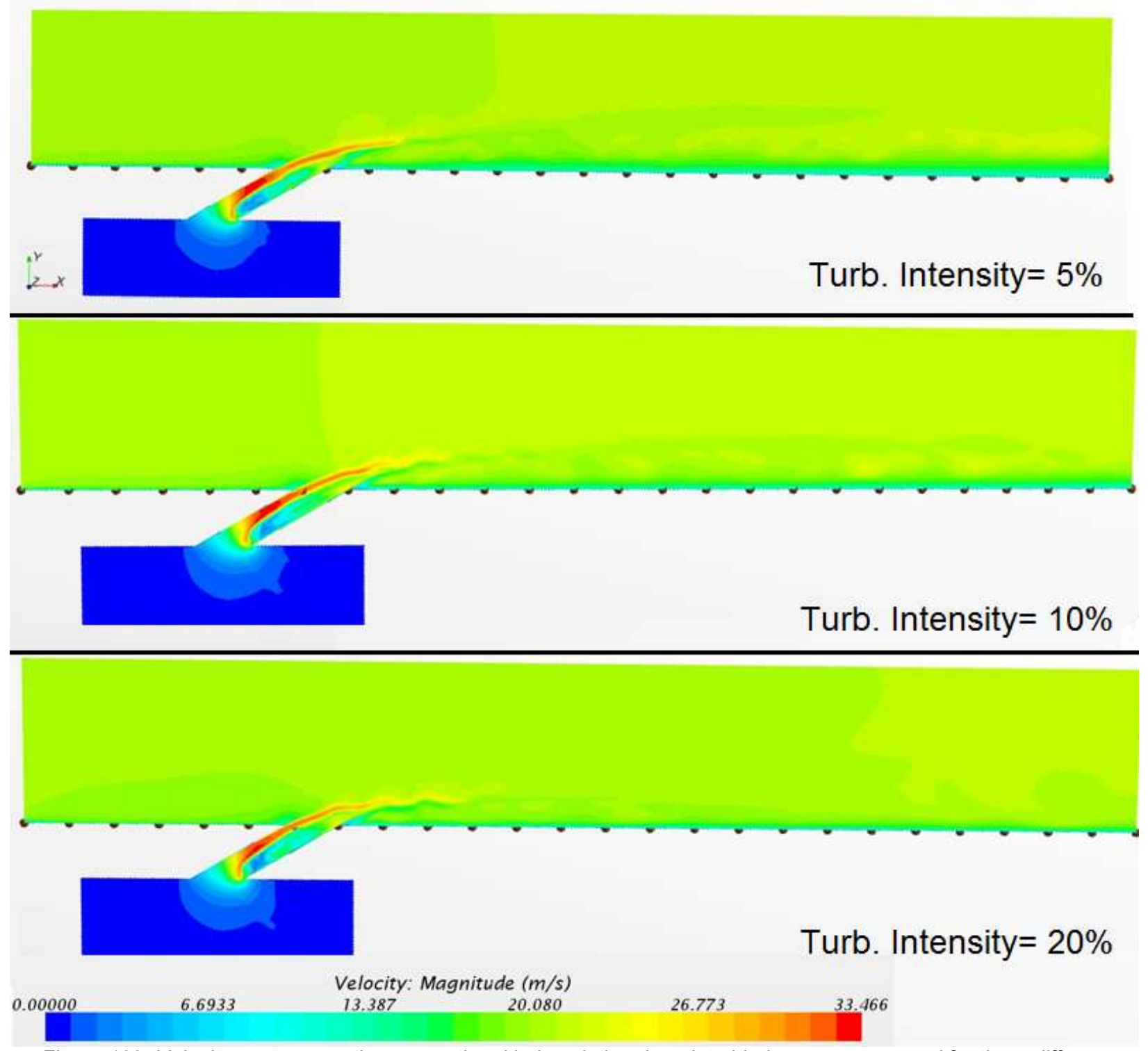

Figure 109: Velocity contours on the conventional hole solution domain mid-plane are compared for three different turbulence intensities

As the turbulence intensity of the main flow is increased, the increased kinetic energy content of the main stream flow reduces the vertical dissipation of the coolant flow further and the flow tends to spread laterally [48], which is a possible explanation for the differences observed in temperature distributions on the adiabatic wall surface as shown in Figure 110 for three different turbulence intensity cases. 


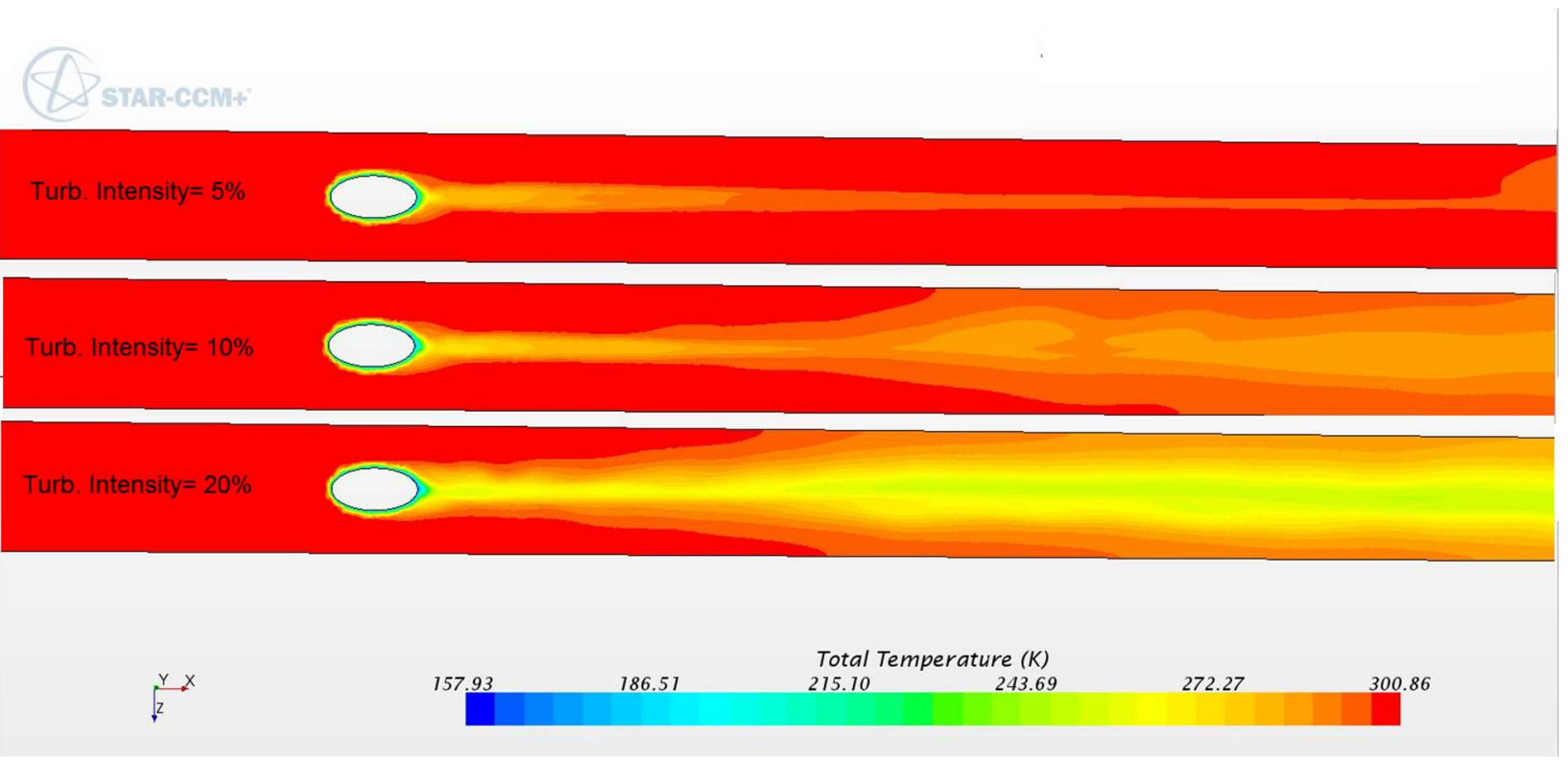

Figure 110: Temperature distribution on the adiabatic wall surface is compared for three different turbulence intensities for the conventional hole case

At larger length scales boundary layer displacement thickness is smaller, which causes the injected flow to lose its momentum and stay close to the wall that results in the increase in the centerline effectiveness. For large blowing ratios, the effect of boundary layer thickness on the jet penetration increases (i.e. the flow with the smallest boundary layer thickness has the highest centerline effectiveness). As the turbulence intensity is increased, mainstream boundary layer thickness is reduced and the injected jets penetrate less due to increased mixing, which causes the centerline effectiveness to increase, resulting in higher span-averaged effectiveness distribution when the turbulence intensity is increased as shown in Figure 111.

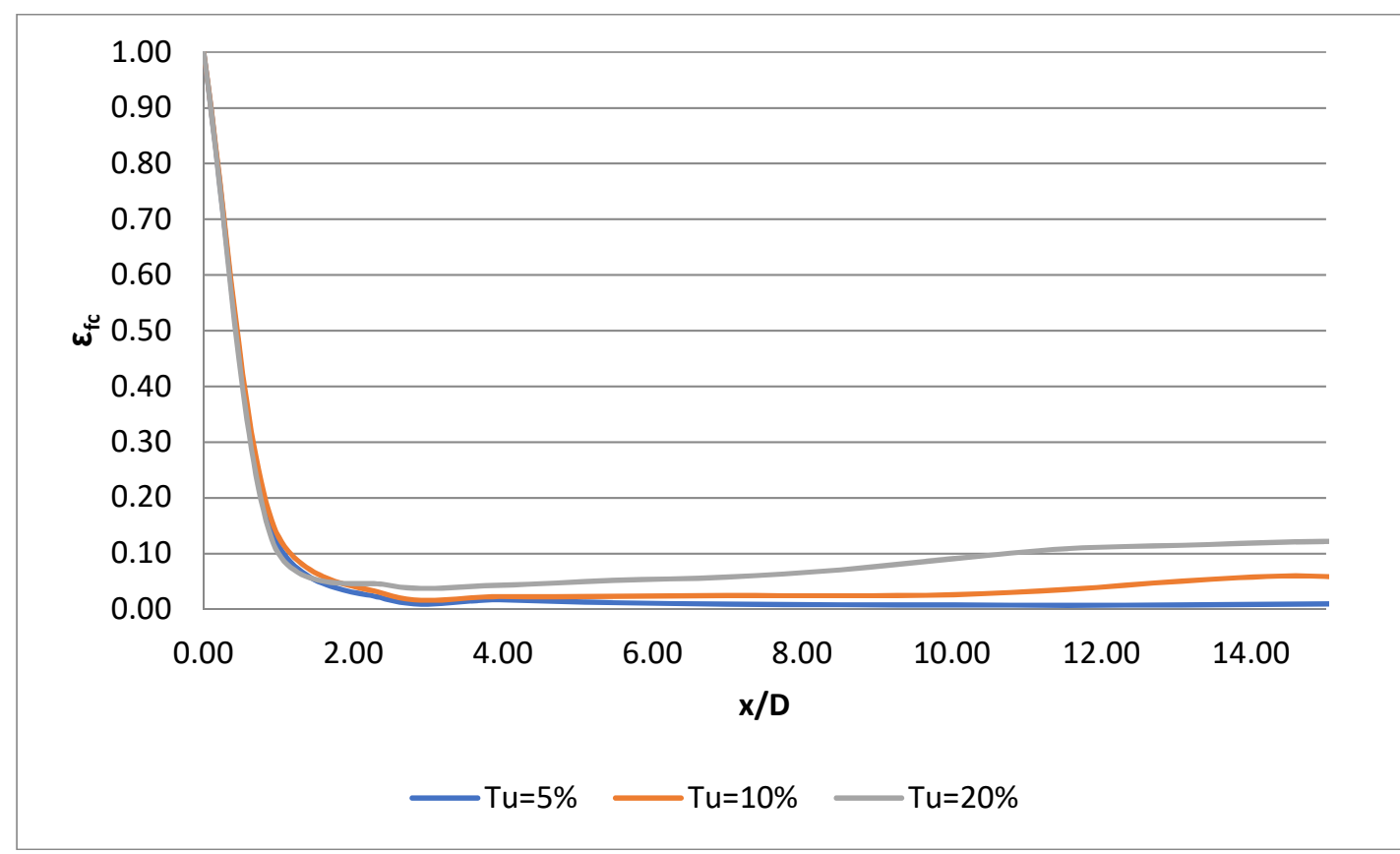

Figure 111: Span averaged cooling effectiveness data from DES simulations for high blowing ratio, high turbulence intensity and large length scale conditions for conventional hole geometry 


\subsubsection{Anti-Vortex Hole Cases}

For the anti-vortex hole geometry cases, numerical setup from Section 4.1, that is validated in Section 4.2, was used to generate span averaged film cooling data for 5,10 and $20 \%$ mainstream flow turbulence intensities. Temperature distribution on the adiabatic wall and the velocity contours at half plane are used to analyze and comment on the changes in film cooling performance when the turbulence intensity is changed. The flow conditions at the boundaries, that gives the blowing ratio as 2.0 and density ratio as 1.0 in all considered flow cases, were set to the values given in Table 33 .

Table 33: Flow variables set at the boundaries for data generation of AVH under engine-like flow conditions

\begin{tabular}{|l|l|}
\hline Flow Variable & Value set at the boundary \\
\hline Inlet & $300 \mathrm{~K}$ \\
\hline Static Temperature & $0.05 / 0.1 / 0.2$ \\
\hline Turbulence Intensity & 6 \\
\hline Turbulent Length Scale (wrt. $\mathbf{d}_{\mathbf{m}}$ ) & BL Input (same profile used in Section 4.2) \\
\hline Velocity Magnitude & \\
\hline Outlet & $101320.0 \mathrm{~Pa}$ \\
\hline Total Pressure & $300 \mathrm{~K}$ \\
\hline Static Temperature & 0.01 \\
\hline Turbulence Intensity & 6 \\
\hline Turbulent Length Scale (wrt. $\mathbf{d}_{\mathbf{m}}$ ) & \\
\hline Plenum & $102120.0 \mathrm{~Pa}$ \\
\hline Total Pressure & $150 \mathrm{~K}$ \\
\hline Total Temperature & 0.01 \\
\hline Turbulence Intensity & 10.0 \\
\hline Turbulent Viscosity Ratio & \\
\hline
\end{tabular}

As for the conventional hole cases, when the turbulence intensity is changed the time step of each simulation was reduced accordingly, to keep the average CFL number less than 1.0 at all locations in the solution domain.

The velocity contours on the main hole centerline of three different turbulence intensity cases are shown in Figure 112, which shows the reduction in the jet penetration vertical height with respect to the mainstream boundary layer thickness as the turbulence intensity is increased. 

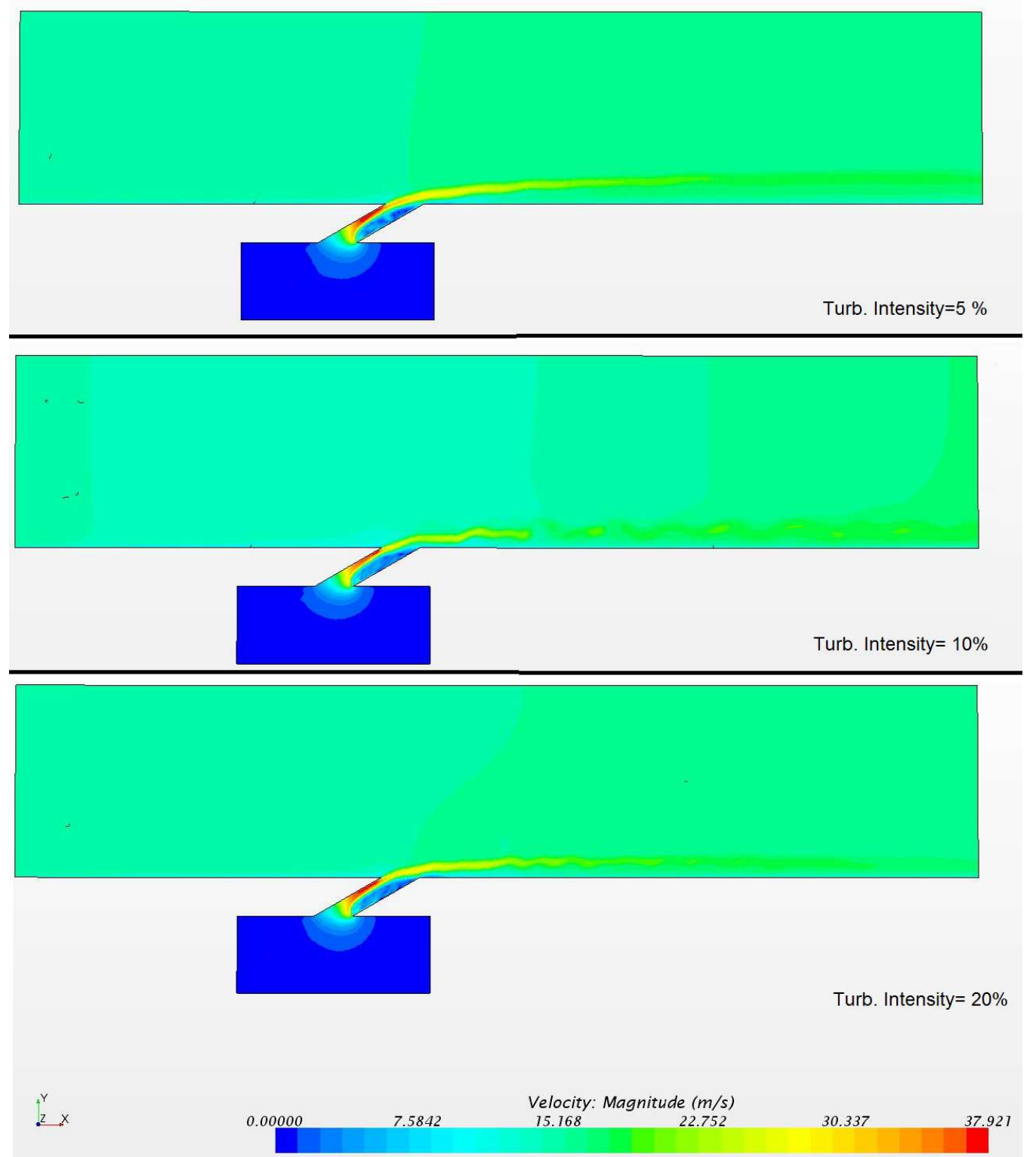

Figure 112: Velocity contours on the AVH solution domain mid-plane, are compared for three different turbulence intensities

According to Bons et al. [48], increasing the turbulence intensity of the main flow increases the effectiveness distribution in the midline region between adjacent single holes. For the AVH hole geometry, Repko et al. [37] showed that the orientation of the vortices generated from the main hole and the side holes of the AVH configuration will be the same with the case of adjacent single holes.

In terms of the flow considered on the cooled surface, the AVH concept can be seen as three single cylindrical holes that are placed apart at very close span wise locations. Counter-rotating vortex strength from the main hole of $\mathrm{AVH}$ is therefore reduced by the vortices generated from the side holes, which cause the vertical penetration of the coolant jet to be reduced and cause the energy of the exiting coolant jet to be directed laterally. As a result, jet is penetrated further towards the adiabatic wall, which resulted in the increased coolant coverage when the turbulence intensity is increased, as shown in the comparative temperature contours in Figure 113. 

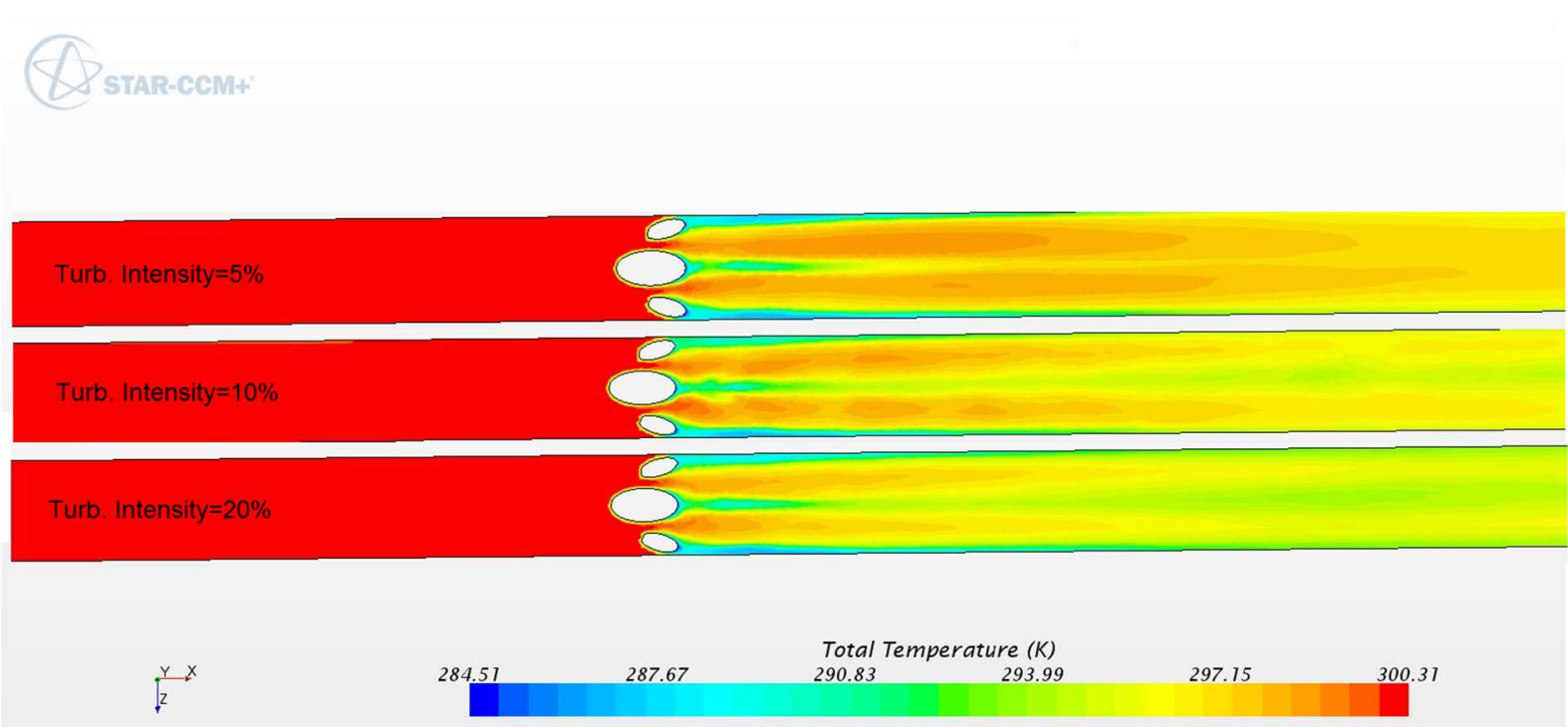

Figure 113: Temperature distribution on the adiabatic wall surface is compared for three different turbulence intensities for the AVH case

For the three turbulence intensity levels studied in AVH cases, corresponding span-averaged cooling effectiveness data is obtained as shown in Figure 114, which are higher in magnitude for all downstream locations than the corresponding values obtained with the conventional holes. The improvement in the spanaveraged cooling effectiveness with AVH geometry is due to more reduction in the coolant jet lift-off, and hence the reduced vortex strength, by the existence of the vortices from the side-holes. As the mainstream turbulence intensity is increased, increased flow kinetic energy pushes the weakened coolant jet easier to the adiabatic wall, resulting in an increased lateral coolant coverage.

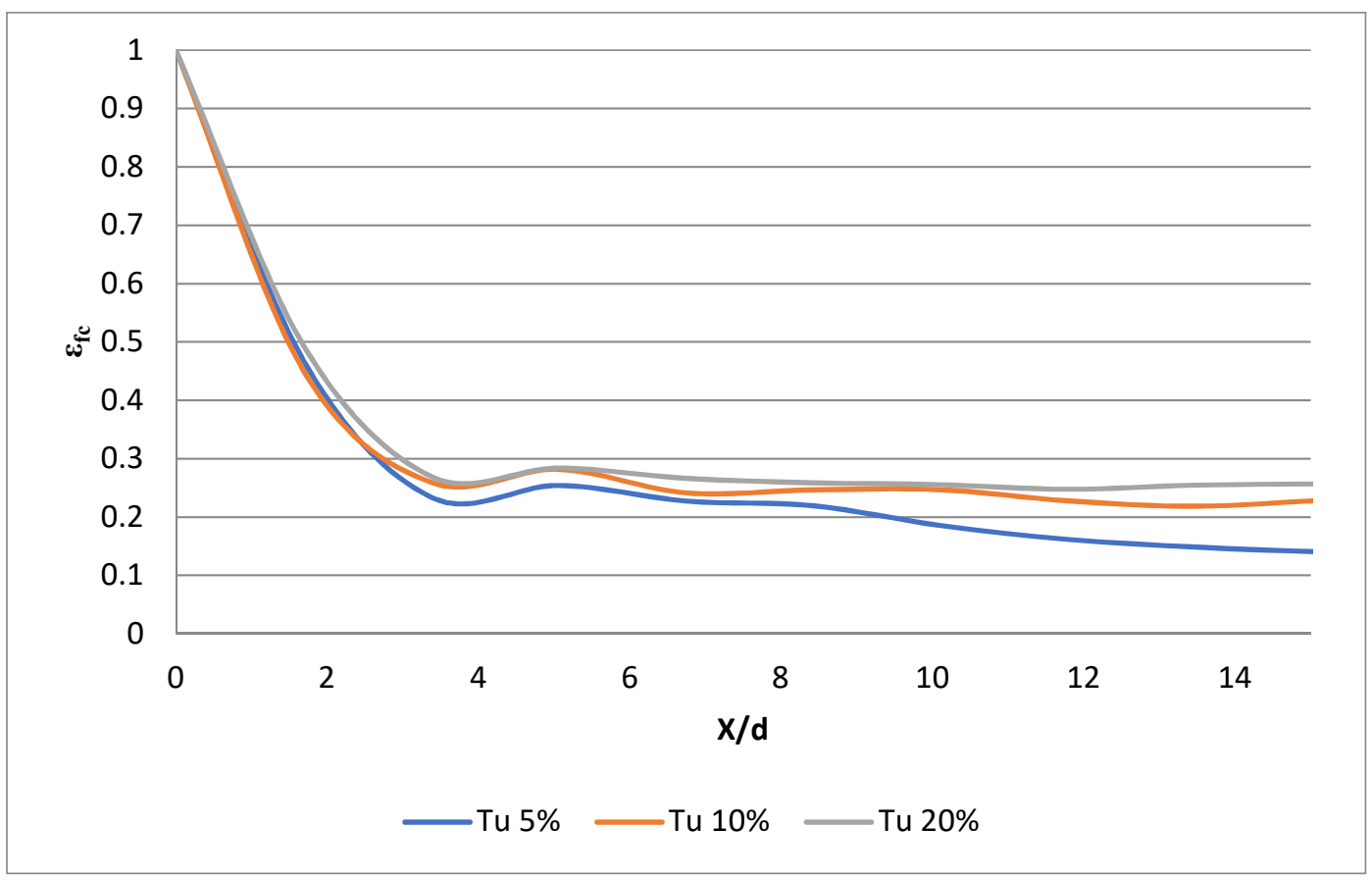

Figure 114: Span averaged cooling effectiveness data from DES simulations for high blowing ratio, high turbulence intensity and large length scale conditions for AVH geometry 


\section{CHAPTER 5: DETERMINING THE PERFORMANCE OF COOLED GAS TURBINES USING DIFFERENT COOLING CONFIGURATIONS}

The methodology employed in the "Coolant Calculator Block" developed in Section 1.2.1, which finds the coolant fractions by using Equation (14) is based on Holland and Thake Theory $[7,49]$ and it is a semiempirical approach to model the cooled turbine blade through a heat resistance network. This approach is easy to model, can give realistic estimations in coolant fraction calculations, and has low number of user inputs.

However, when the effects of individual internal and external cooling techniques on the engine performance wanted to be analyzed, correlations and/or experimental data for these techniques need to be used. Existing coolant calculation methodology of Equation (14) (Section 1.1.1) does not allow to use this case specific information because of lumping some of the cooling configuration specific parameters into simulation constants (i.e. Kcool etc.).

Per the aerothermal modelling of the cooled turbine blade used, when the cooling configuration of a turbine blade is changed, this change can only be reflected to the performance calculations through coolant mass flow rates and coolant exit enthalpies. Using the coolant flow rate and coolant exit enthalpy information calculated with a method that can differentiate the cooling configurations, could be used in the $1^{\text {st }}$ Law Analysis and $2^{\text {nd }}$ Law Analysis sub-systems (see Sections 1.2 .2 and 1.2.3, respectively) to calculate further turbine performance parameters without requiring a change in the algorithms and equations used in these subsystems.

In this section, a new coolant calculator that replaces the existing coolant calculator sub-system of Section 1.2.1 without changing the types of outputs provided by the previous algorithm $\left(\varepsilon_{1}, \varepsilon_{2}\right.$ and $\left.h_{t c x}\right)$ is developed and used with different turbine blade cooling configurations (internal and external) to estimate their impact on aero-engine and industrial gas turbine key performance parameters.

\subsection{Literature Review on Advanced Coolant Calculation Methods}

In the study by Torbidoni and Horlock [49], which compares and introduces some of the applicable advanced coolant fraction calculation methods to analytical models similar to CEDM and CGTM, the semi-empirical calculation method used in CEDM/CGTM of the coolant calculator block is compared with advanced coolant calculation methods developed by Ainley [50], Consonni [1] and Torbidoni and Horlock [49], which could allow specification of a certain cooling configuration through the internal Stanton number and Number of Thermal Units (NTU) values.

Ainley [50] introduced an advanced coolant calculation method that treats the cooled blade as a heat exchanger. The required coolant fractions are obtained by solving 2D convection heat transfer equations valid for a heat exchanger and integrated from blade hub to tip. The differential element considered in this derivation is shown in Figure 115. 


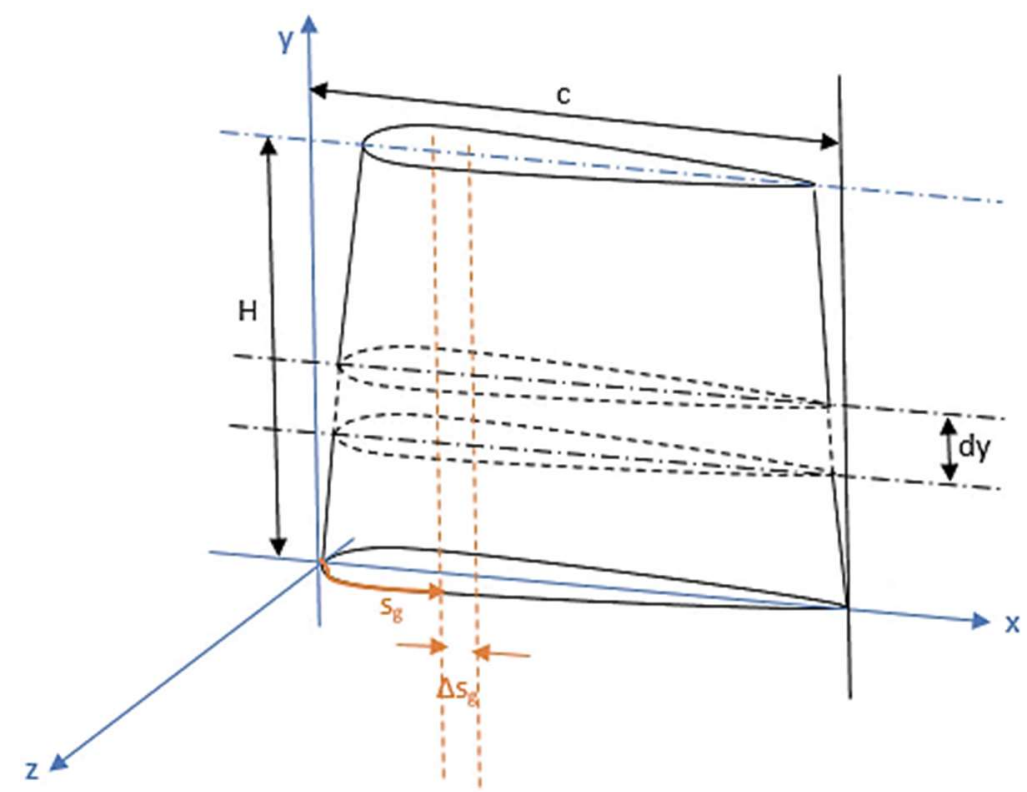

Figure 115: The differential area considered in the derivation of advanced coolant calculation methods is shown with the blade coordinates

In Figure 115, dimension $S_{g}$ is named as "the external heat transfer perimeter", which is defined as the $x-$ dimension of the differential element wetted onto the airfoil surface. The relation between the wetted dimension and the actual $\mathrm{x}$-coordinate is given with Equation (76).

$$
S_{g}=\varphi_{g} x
$$

In Equation-14, $\varphi_{\mathrm{g}}$ is the local blade curvature, which depends on the airfoil geometry at the considered vertical location. In the derivations that will be made in the following sections, it is assumed that the same airfoil profile is used from hub to tip. Therefore, dimension $S_{g}$ becomes independent of the $y$-coordinate and the projection of the differential area onto $x-y$ plane becomes a rectangle, as shown in Figure 116.

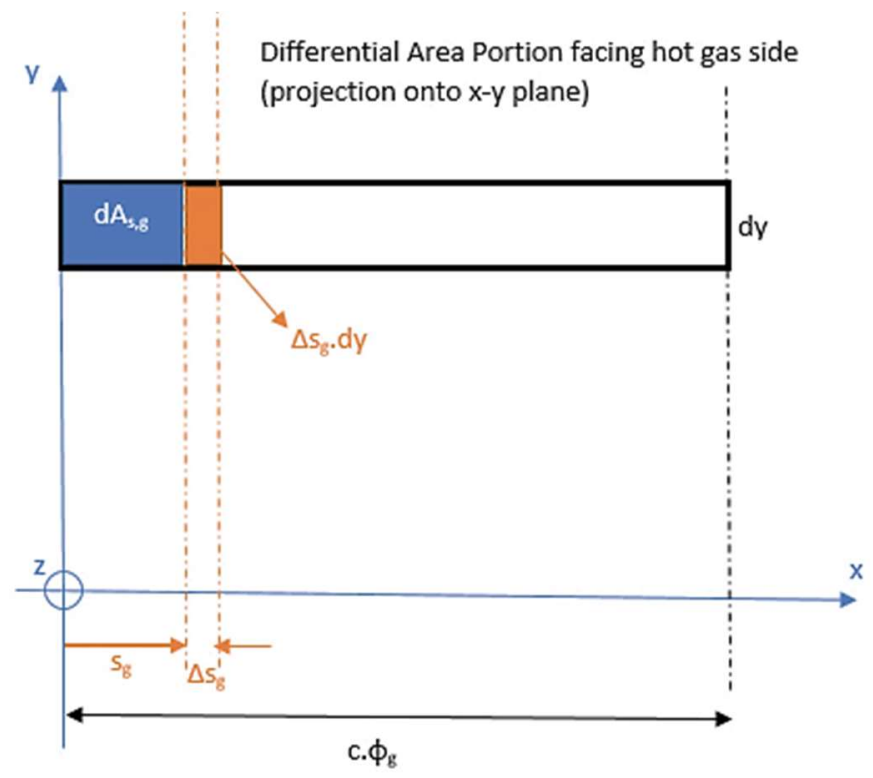

Figure 116: The projection onto the $x-y$ plane of the differential area used in the derivations 
The projection of the considered differential element onto $x-z$ plane is shown in Figure 117. Another wetted dimension, denoted as $S_{c l}$, is also defined as the dimension measured from the leading edge of the inner blade wall to the same $x$-location with the corresponding $S_{g}$ length. This dimension is named as "the internal heat transfer perimeter". The inner walls may or may not have the same curvature with the outer wall; depending on the internal cooling channel design.

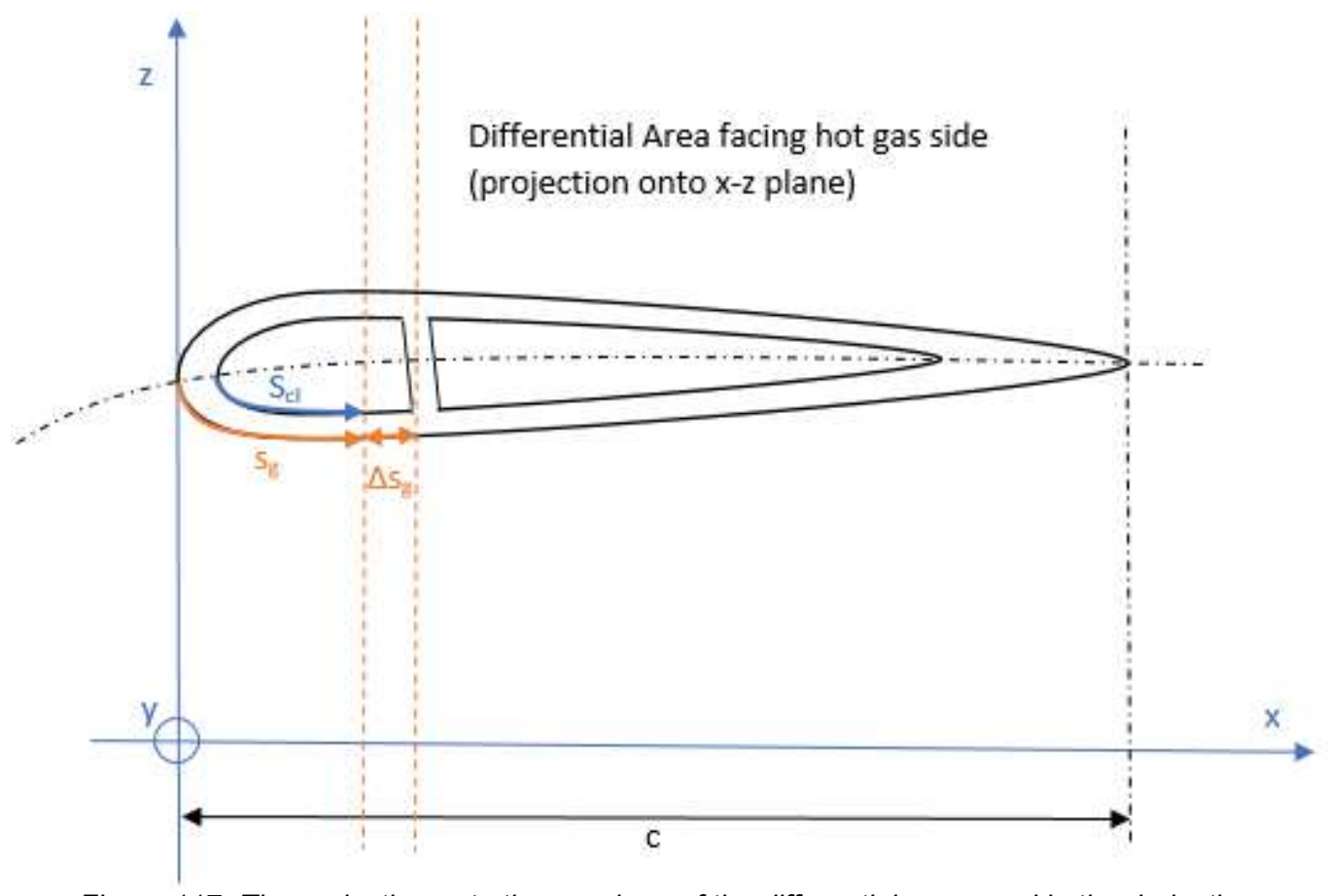

Figure 117: The projection onto the $x-z$ plane of the differential area used in the derivations

The 2D convection heat transfer equations valid for the considered differential area with steady flow assumption (both internal and external) are given in Equation(s) (77).

$$
\begin{aligned}
& \text { Continuit }: \quad \dot{m}_{c l} c_{p, c l} \frac{d T_{c l}}{d y}+\alpha_{c l} S_{c l}\left(T_{c l}-T_{m, e x t}\right)=0 \\
& \text { Energy: } \quad k_{m} A_{\text {surf }} \frac{d^{2} T_{m, e x t}}{d y^{2}}+\alpha_{g} S_{g}\left(T_{g}-T_{m, \text { ext }}\right)+\alpha_{c l} S_{c l}\left(T_{c l}-T_{m, \text { ext }}\right)=0
\end{aligned}
$$

Assuming that the heat transfer occurs only along the cord (i.e. no conduction heat transfer is occurring along the $y$-direction) then the heat transfer equations of Equation(s) (77) is simplified as shown in Equation(s) (78).

$$
\begin{array}{lc}
\text { Continuity: } & \dot{m}_{c l} c_{p, c l} \frac{d T_{c l}}{d y}+\alpha_{c l} S_{c l}\left(T_{c l}-T_{m, \text { ext }}\right)=0 \\
\text { Energy: } & \alpha_{g} S_{g}\left(T_{g}-T_{m, \text { ext }}\right)+\alpha_{c l} S_{c l}\left(T_{c l}-T_{m, \text { ext }}\right)=0
\end{array}
$$

Define the parameter $\Theta$ as the ratio of convection heat transfer coefficients of the coolant to gas side ( $\alpha_{c l}$ and $\alpha_{g}$, respectively) multiplied with the ratio of the inner to outer heat transfer perimeters ( $\mathrm{S}_{\mathrm{cl}}$ and $\mathrm{S}_{g}$, respectively) as given in Equation (79). 


$$
\Theta=\frac{\alpha_{c l} S_{c l}}{\alpha_{g} S_{g}}
$$

For convenience in notations, define a parameter $\mathrm{w}^{+}$as shown in Equation (80).

$$
w^{+}=\frac{\dot{m}_{c l} c_{p, c l}}{\alpha_{g} S_{g} H}
$$

In the derivation procedure by Ainley [50] (which is partially reproduced here), it is assumed that the blade metal temperature is constant along the y-direction (i.e. $\frac{d T_{m, e x t}(y)}{d y}=0$ ), and the convection heat transfer coefficients can be used from their span-wise averaged values, which allows replacing the $\Theta$ definition of Equation (79) with $\bar{\Theta}$ in the following sections of the derivation.

In Equation (80), $\mathrm{H}$ defines the blade height, and $\mathrm{c}_{\mathrm{p}, \mathrm{cl}}$ is the heat capacity of the coolant and other parameter definitions are used as same from the previous sections. Then the Equation (s) (78) becomes a linear differential system, of which the two solutions are the temperature distributions $T_{m, e x t}(y)$ and $T_{d}(y)$, which are the blade metal temperature and coolant temperature variations in the y-direction, respectively. Then using the definitions of $\Theta$ and $w^{+}$in Equation (78a) will give a multi-variable first order differential equation as given in Equation (81).

$$
H w^{+} \frac{d T_{c l}}{d y}+T_{m, e x t}(y)-T_{g}(y)=0
$$

Using $\bar{\Theta}$ definition in Equation (78b) will reduce Equation (81) into a single variable linear differential equation with respect to $T_{c l}(y)$, as given in Equation (82).

$$
H \frac{d T_{c l}}{d y}=\left(T_{t g *}-T_{c l}(y)\right) \xi
$$

In Equation (82) $\xi$ is defined as shown in Equation (83), and it is a constant parameter.

$$
\xi=\frac{\bar{\Theta}}{(1+\bar{\Theta}) w^{+}}
$$

Integrating Equation (82) and applying the boundary conditions given for the coolant temperatures at the blade hub $(y=0)$ as $T_{t c l}$, in and the blade tip $(y=H)$ as $T_{t c l}$, out will result in the expression given in Equation (84), which also gives the definition of "internal cooling efficiency", $\varepsilon_{c}$, ext.

$$
\varepsilon_{c, \text { ext }}=\frac{T_{t c l, o u t}-T_{t c l, \text { in }}}{T_{m, \text { ext }}-T_{t c l, \text { in }}}=1-e^{-\bar{\Theta} / w^{+}}
$$

The derivation made by Ainley [50] was made for only internally cooled blade configurations. Consonni [1], later extended this method to be also used in film cooled and TBC applied blade configurations. This is done by regarding the entire blade (now includes the blade wall and the film cooling flow) as the heat exchanger that is subject to a constant external gas temperature (and constant adiabatic wall temperature ( $\left.T_{a w}\right)$, if film cooling exists) and following the same derivation procedure by Ainley but using $\mathrm{w}^{+}$to include the film cooling flow, results in a different form of internal cooling efficiency definition as shown in Equation (85). 


$$
\frac{T_{t c l, o u t}-T_{t c l, \text { in }}}{T_{t g *}-T_{t c l, i n}}=1-e^{-\frac{1}{W^{+}}}
$$

Equation (85) is the definition of "internal cooling flow efficiency" (denoted as $\varepsilon_{c}$ ) in the previous sections. The definition of $\mathrm{W}^{+}$in this case now includes the overall heat transfer coefficient through the metal of finite thickness, which is denoted as $\bar{U}_{h}$, and given in Equation (86),

$$
W^{+}=\frac{\dot{m}_{c l} c_{p, c l}}{\bar{U}_{h} A_{c l, s}}
$$

The span-averaged overall heat transfer coefficient $\bar{U}_{h}$ used in the efficiency definition is obtained from the span-averaged values of the heat transfer parameters shown in Equation (87).

$$
\bar{U}_{h}=\left[\frac{A_{c l, s}}{A_{g, s}}\left(\frac{1}{\alpha_{g}}+\frac{t_{m}}{k_{m}}\right)+\frac{1}{\alpha_{c l}}\right]^{-1}
$$

In the heat exchanger literature, Number of Thermal Units (NTU) represents a measure of the effectiveness of the heat exchanger and for the cooled turbine blade, it is equal to the inverse of $\mathrm{W}^{+}$defined as in Equation (88).

$$
N T U=\frac{1}{W^{+}}=\frac{\bar{U}_{h} A_{c l, s}}{\dot{m}_{c l} c_{p, c l}}
$$

Then for the entire cooled blade, using the NTU definition from Equation (85) in Equation (88) gives an important relation between two types of internal cooling efficiencies that will be later used in the derivation of the new coolant calculator, and is given in Equation (89).

$$
\varepsilon_{c, \text { ext }}\left(\frac{T_{m, e x t}-T_{t c l, i n}}{T_{t g *}-T_{t c l, i n}}\right)=\varepsilon_{c}=1-e^{-N T U}
$$

In Equation (89), the blade wall temperature $T_{b w}$ is a constant value attained on the blade metal wall and it is dependent on the metal Biot number, TBC Biot number, internal cooling flow and the film cooling efficiency, depending on the cooling configuration used on the blade. Consonni used a heat resistance network similar to the one in Figure 4 to derive the blade wall temperature equations that is compared with the maximum allowable blade metal temperature.

In the algorithm by Consonni [1], the coolant mass flow rate $\left(\dot{m}_{c l}\right)$ is treated as a known parameter; the value of which is calculated in an iterative loop that checks $T_{m, \text { ext }} \leq T_{b}$, max condition and increases the coolant fraction until it finds an NTU value that satisfies the blade wall temperature criteria. In the calculation of the NTU, the relation in Equation (90) is used (shown here in expanded form).

$$
N T U=\frac{4 \overline{S t_{l n}} \vartheta_{i} n_{p}\left(\frac{H}{d}\right)}{1+\left(\Lambda_{c} \overline{S t_{i n}} \frac{c_{p, c l}}{c_{p, g}} C_{g} n_{p} \frac{\dot{m}_{c l}}{\alpha_{h}}\right)\left(1+B i_{m}\right)}
$$

In Equation (90), $\vartheta_{i}$ represents a "cooling channel efficiency" and taken as a constant, $\mathrm{n}_{\mathrm{p}}$ is the number of passes per cooling channel, $\mathrm{H}$ is the blade height, $\mathrm{d}$ is the cooling channel hydraulic diameter and $\Lambda_{c}$ is the 
ratio of inner to outer heat transfer perimeters $\left(S_{g} / S_{c l}\right.$ of Equation (79)). In Consonni's algorithm, the average internal Stanton Number $\left(\overline{S t_{l n}}\right)$ is replaced with Colburn Equation [1] and a technology parameter defined to be able to distinguish the performance of different rib cooling configurations.

In the calculation of the adiabatic wall temperature for film cooled configurations, the span averaged film cooling effectiveness value is replaced with a built-in film cooling correlation for conventional film cooling holes [1].

\subsection{Development of the Advanced Coolant Calculator}

In the development of the new coolant calculator algorithm, a hybrid approach between the semi-empirical method and the NTU method from Consonni [1] is used, in order to have a coolant calculation method with minimum increase in the number of input parameters but includes the effects of different internal and external cooling configurations with higher accuracy.

The major drawback in using the Consonni's algorithm directly as the new coolant calculator of CEDM/CGTM is the existence of built-in internal and external cooling correlations that would allow only to use a rib-type of cooling for internal cooling with convectional film cooling holes.

The newly developed algorithm allows using different correlations for the average internal Stanton Number calculation $\left(\overline{S t_{l n}}\right)$ through various built-in correlations requiring less input parameters from the user and the span-averaged film cooling effectiveness value $\left(\varepsilon_{f c}\right)$ is calculated from using film cooling effectiveness data for the specified film cooling hole geometry. The algorithm also differs in the execution of the coolant fraction calculations, treating NTU as a known parameter, and the internal cooling flow efficiency $\left(\varepsilon_{c}\right)$ as the iteration variable, which always takes values between 0.6 to 0.8 , regardless of the specific internal cooling configuration [7]. Changing of the iteration variable to $\varepsilon_{c}$ instead of using the coolant fraction instead, removes possible dependency of the calculated coolant fraction value on the initial value of the coolant fraction used, which might differ from one internal cooling configuration to another.

\subsubsection{Development of the Algorithm}

In the development of the new coolant calculation algorithm, following assumptions were made:

i. The basic analysis element is a blade strip that is shown schematically in Figure 118.

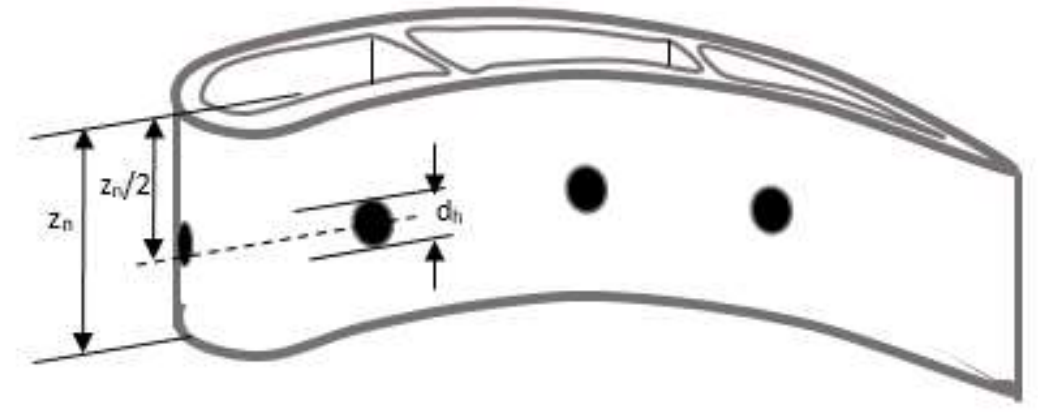

Figure 118: The basic analysis element is a strip with length $\mathrm{z}_{n}$ which is equal to the lateral hole spacing. Depending on the user-input cooling configuration, number of holes and the shown internal cooling type could be different. 
If film cooling exists in the cooling configuration, each analysis strip contains only one row of film cooling holes. If impingement cooling is applied, strip can contain more than one impingement hole, depending on the impingement diameter.

ii. Within each blade, the cooling flow is equally divided between the pressure and suction sides

iii. Blade curvature and compressibility effects are negligible (this allows flat plate impingement correlations to be used for the blade body section)

iv. No flow and temperature variations along the radial direction (i.e. adjacent blade strips)

v. Cross-flow effects between adjacent strips are negligible

vi. Changes in the coolant supply temperature is covered in the internal cooling flow effectiveness parameter $\left(\varepsilon_{c}\right)$ used as same with the definition from Consonni [1] and the semi-empirical model [7]. It is assumed that all internal cooling segments will have the same $\varepsilon_{c}$ so that the coolant heating within the blade is same for all internal segments.

vii. Coolant fraction required by the film cooling is supplied entirely by the internal cooling. The analysis strip is assumed to be configured accordingly.

viii. Average adiabatic film cooling effectiveness for the blade strip can be obtained by the Seller's Method [70]

ix. Average internal cooling Stanton number can be found by a weighted average based on the chord length fractions of each applied internal cooling method.

$x$. Pressure losses in the internal cooling are included in the performance calculations through the "internal cooling friction loss" term from Equation (40).

In the new algorithm, the value of NTU became a "known" parameter by using Equation (89) with the iteration variable $\varepsilon_{c}$, which takes values starting from 0.55 up to 0.85 (5\% tolerance is added to both sides of the interval from Young and Wilcock [7]). Then the definition of NTU in Equation (90) is used with calculated NTU to find the required coolant fraction.

A transformation of the NTU definition is required to obtain an equation that can be used to calculate the coolant fractions once the NTU, gas side Stanton number, average internal Stanton number and the ratio of coolant to gas side specific heats are known. The resulting equation after the transformation is given in Equation (91).

$$
\frac{\dot{m}_{c l}}{\dot{m}_{g}}=\left(\frac{4 \overline{S t}_{i n} \vartheta_{i} n_{p}\left(\frac{H}{d}\right)}{N T U}-1\right) \frac{1}{\left(1+B i_{m}\right)} \frac{\alpha_{h}}{C_{g}} \frac{S t_{g}}{\overline{S t}_{i n}} \frac{c_{p, g}}{c_{p, c l}}
$$

In Equation (91), NTU value is calculated by using Equation (92), which is obtained by solving Equation (89) for NTU.

$$
N T U=-\ln \left(1-\varepsilon_{c, \text { ext }} \frac{T_{m, \text { ext }}-T_{t c l, i n}}{T_{t g *}-T_{t c l, i n}}\right)
$$

In Equation (92), the value of $T_{m, e x t}$ is unknown and its value is dependent on the metal and TBC Biot numbers, film cooling, internal blade wall temperature and outer wall temperature ( $\left.T_{w}\right)$. The formula to calculate $T_{m, e x t}$ changes depending on the TBC application, and it is given in Equation (93a) and (93b). 


$$
\begin{cases}T_{m, \text { ext }}=B i_{m}\left(T_{t g *}-T_{w}\right)+T_{m, \text { int }} & \text { no } T B C \\ T_{m, \text { ext }}=\frac{B i_{m} T_{w}+B i_{T B C} T_{m, i n t}}{\left(B i_{m}+B i_{T B C}\right)} & \text { with } T B C\end{cases}
$$

The outer wall temperature $T_{w}$ in Equation (93) is dependent on the application of film cooling and calculated from Equation (94a) and (94b).

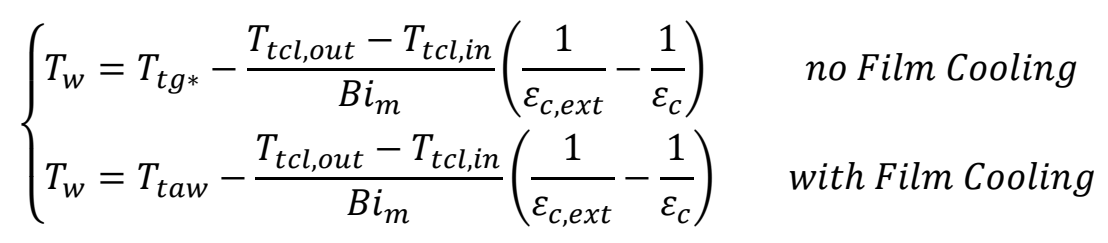

The adiabatic wall temperature is calculated by using the blade span averaged film cooling effectiveness value as shown in Equation (95).

$$
T_{t, a w}=T_{t g *}-\varepsilon_{f c}\left(T_{t g *}-T_{t c l, x}\right)
$$

The value of the internal cooling efficiency, $\varepsilon_{c, \text { ext }}$ depends on the internal cooling flow efficiency $\varepsilon_{c}$ through the coolant exit temperature value. This dependency requires an iterative solution approach to calculate $\varepsilon_{c, e x t}$ with the procedure given in Appendix-A.

An iterative solution technique using $\varepsilon_{c}$ as the iteration variable and calculates $\varepsilon_{c, \text { ext }}$ from the algorithm in Appendix-A can be used to solve the described heat transfer problem. With known values for $\varepsilon_{c, e x t}$, the outer wall temperatures $\left(T_{w}\right)$ can be found, which allows the calculation of the metal external wall temperature from Equation (94). It should be noted that the metal external wall temperature should always satisfy $T_{m, e x t} \leq T_{b, \max }$ condition, therefore an outer loop controlling this criterion is required to decide on the increment that will be made to $\varepsilon_{c}$.

\subsubsection{Development of the Model}

The new algorithm is implemented as a new coolant calculator for cooled turbine models, that has the same number of outputs with the previous method, which were the coolant mass flow rates, the coolant exit enthalpies and the blade temperatures. Number of inputs were increased, due to the additional information needed by the new method from the turbomachinery section. A flowchart of the advanced coolant calculator is given in Figure 119. 


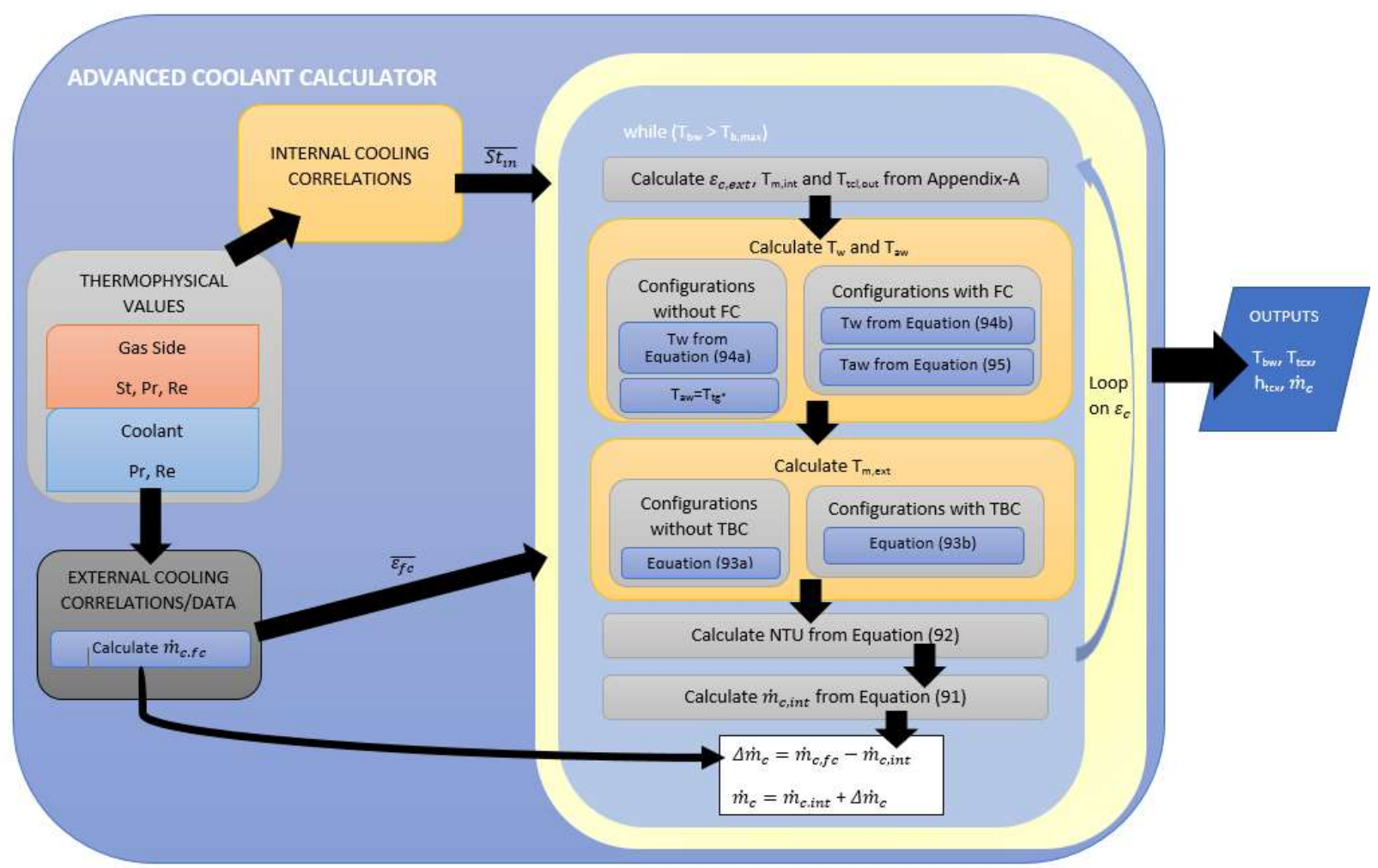

Figure 119: Flowchart of the advanced coolant calculator

In the flowchart of Figure 119, the "while loop" block has the advanced coolant calculation methodology as described in the previous section. Internal and External Cooling blocks includes correlations and/or data obtained for a specific cooling methods to calculate the average Stanton number and blade adiabatic film cooling effectiveness. Thermophysical values such as Stanton Number, Prandtl Number and Reynolds Number that are needed by the correlations are calculated in a separate sub-system.

Figure 120 shows the considered internal and external cooling types that are modelled through correlations in the advanced coolant calculator. A user input indicates what types of internal and external cooling is applied to each cooled blade, and the algorithm picks the corresponding correlation and/or data. 


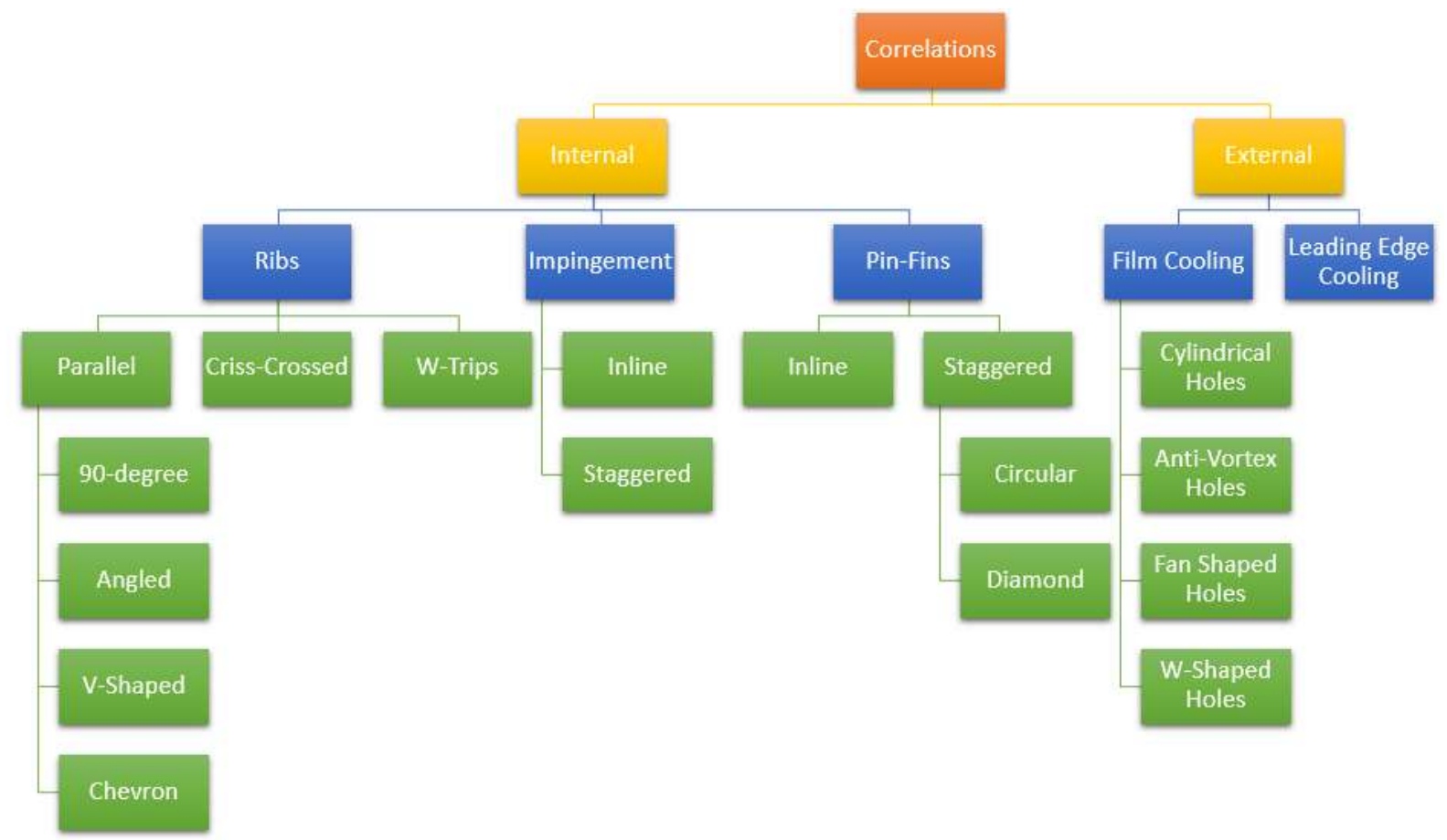

Figure 120: Internal and External Cooling Types that are included in the advanced coolant calculator

The details of the calculation methodology that makes use of these correlations are discussed in the following sections.

\subsubsection{Internal Cooling Sub-System}

In this sub-system, a blade is assumed to be consisted from three main sections, which are the leading edge, the body and the trailing edge as shown in Figure 121. Calculations in this module assumes that the coolant Stanton Number (Stin) do not have variations between blade sections, therefore a single chordwise average Stin value is used for the entire blade. Depending on the internal cooling schedule (a user input) applied to each blade, algorithm picks the regarding correlation and calculates a local internal Stanton Number for each blade section.

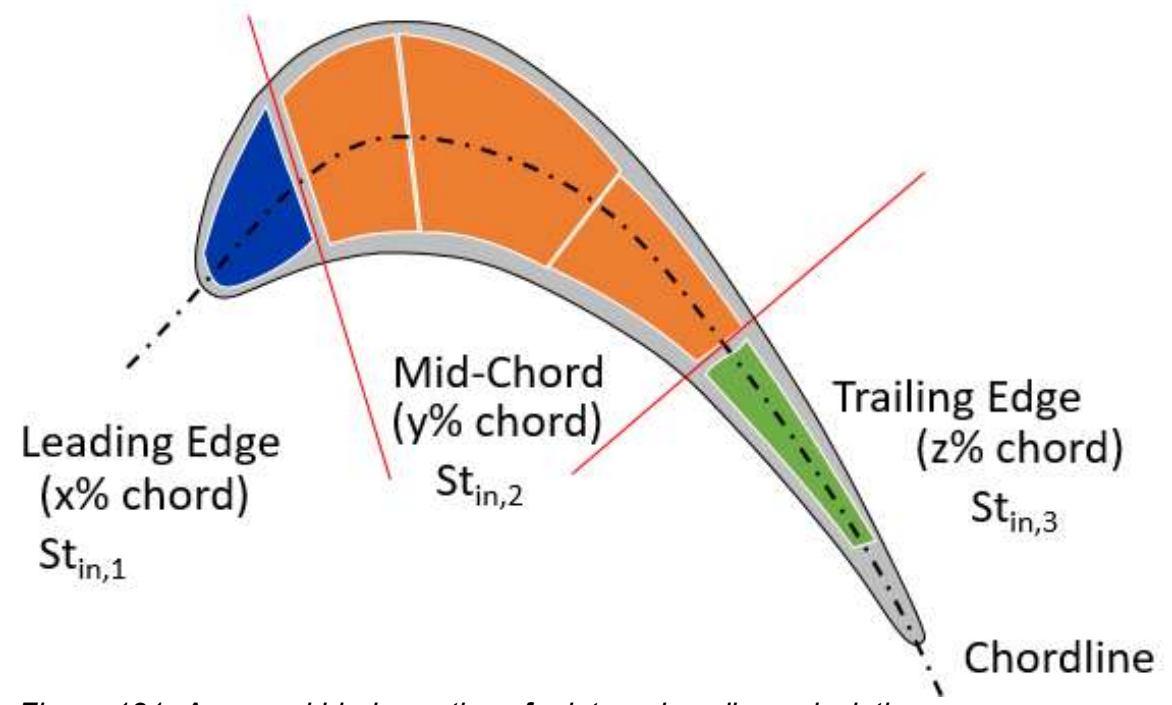

Figure 121: Assumed blade sections for internal cooling calculations 
A user input indicating the chord fractions for each blade section is used in the averaging that will be used to calculate the $\overline{S t_{l n}}$ value for each blade. By default, these fractions are assumed to be $20 \%$ chord for leading edge, $60 \%$ chord for the mid-chord region and $20 \%$ for the trailing edge. Averaging is done by using Equation (96) (variables are referenced to Figure 121).

$$
\overline{S t_{\text {ln }}}=\frac{x S t_{i n, 1}+y S t_{i n, 2}+z S t_{i n, 3}}{100}
$$

Possible cooling types that can be used for each blade section is shown with the flowchart of the "internal cooling" subsystem that includes these correlations and carries out the calculations for $\overline{S t_{l n}}$ is shown in Figure 122.

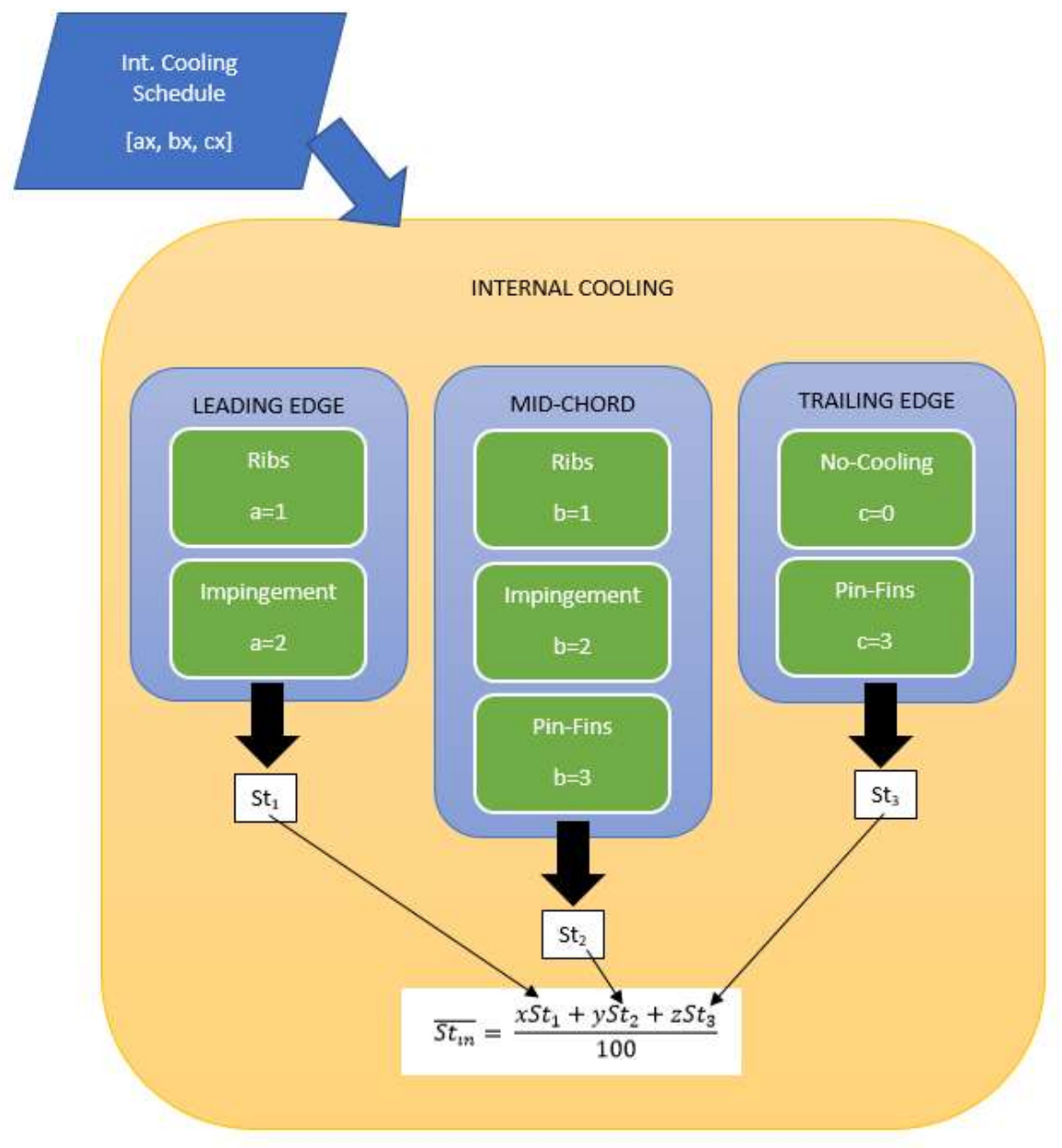

Figure 122: Flowchart of the Internal Cooling sub-system is shown with possible internal cooling types that can be specified for each blade section

The indices $a, b$, and $c$ in the internal cooling matrix indicates which type of internal cooling is applied, and index $x$ indicates the specific type of the cooling method.

It is important to use correlations that are applicable to the typical Reynolds number ranges that are valid for the internal cooling of gas turbine blades. NETL Gas Turbine Handbook [51] provides correlations for some of the rib configurations that are valid for rib types widely used in the gas turbine applications. Oates [52] and 
Khan [53] provide comprehensive information on pin-fin cooling technique and regarding correlations that are valid for typical gas turbine internal flow conditions. Sundberg [54] provides a comprehensive list for possible correlations that can be used for various types of rib and impingement cooling, as well as includes a comparison of the performances of these correlations within gas turbine flow Reynolds number ranges. Correlations that are used in this sub-system are selected from these resources and are given with their references in Table 34. See Appendix-E for the used correlation equations, validity ranges and parameter descriptions.

Table 34: Used correlations, their references and corresponding internal cooling schedule indices

\begin{tabular}{|c|c|c|c|}
\hline & Correlation Reference & Index & Type \\
\hline \multicolumn{4}{|l|}{ Ribs } \\
\hline Parallel $90^{\circ}$ ribs & NETL GT Handbook [51] & 11 & St\# correlation \\
\hline Parallel Angled Ribs & NETL GT Handbook [51] & 12 & St\# correlation \\
\hline Parallel V-Shaped & NETL GT Handbook [51] & 13 & St\# correlation \\
\hline Criss-Crossed & NETL GT Handbook [51] & 14 & St\# correlation \\
\hline $\begin{array}{l}\text { Discrete } \\
\text { (Chevron) }\end{array}$ & Wright et al. [55] & 15 & Polynomial fit to experimental data on Nu\# \\
\hline W-Shaped & Wright et al. [55] & 16 & Polynomial fit to experimental data on Nu\# \\
\hline \multicolumn{4}{|l|}{ Impingement } \\
\hline Inline & $\begin{array}{l}\text { Florschütz et al. [56] or } \\
\text { Chupp et al. [57] }\end{array}$ & 21 & $\begin{array}{l}\text { Nu\# corr. from [56] for impingement on body } \\
{[57] \text { for LE impingement }}\end{array}$ \\
\hline Staggered & $\begin{array}{l}\text { Florschütz et al. [56] or } \\
\text { Chupp et al. [57] }\end{array}$ & 22 & $\begin{array}{l}\text { Nu\# corr. from [56] for impingement on body } \\
{[57] \text { for LE impingement }}\end{array}$ \\
\hline \multicolumn{4}{|l|}{ Pin-Fins } \\
\hline No-Cooling & $* * *$ & 30 & \\
\hline Inline & Hausen et al. [58] & 31 & Nu\# correlation \\
\hline Staggered (circular) & Hausen et al. [58] & 32 & Nu\# correlation \\
\hline Staggered (diamond) & Jaswal and Ames [59] & 33 & Nu\# correlation from experimental data \\
\hline
\end{tabular}

Nusselt number correlations were converted to Stanton numbers by using Equation (97).

$$
S t=\frac{N u}{\operatorname{RePr}}
$$

\subsubsection{External Cooling Sub-System}

In the external cooling sub-system, the film cooling efficiencies that are obtained either through experiments or CFD is used to obtain blade average adiabatic film cooling efficiency $\left(\overline{\varepsilon_{f c}}\right)$. This sub-system requires the following information to be able to calculate a film cooling effectiveness distribution over the blade:

- Number of cooling hole rows

- Blade chord length

- Hole diameter

Blade chord length (c) is calculated by using incidence angles and velocity triangles in the turbomachinery section for each turbine stage. Number of cooling rows are needed to distribute the holes evenly along the chord, and the hole diameter is needed to understand up to which (c/d) value the film cooling data will be used.

In this sub-system, it is assumed to have 1 less number of cooling rows on the suction side than the pressure side, due to the common application in such blades to prevent flow separation. For instance, if the number of 
cooling hole rows is entered as 3, algorithm assumes there are 3 cooling rows on the pressure side, and 2 rows on the suction side. Algorithm assumes a showerhead cooling represented by a single conventional hole located on the stagnation point of the blade. This scheme is schematized in Figure 116.

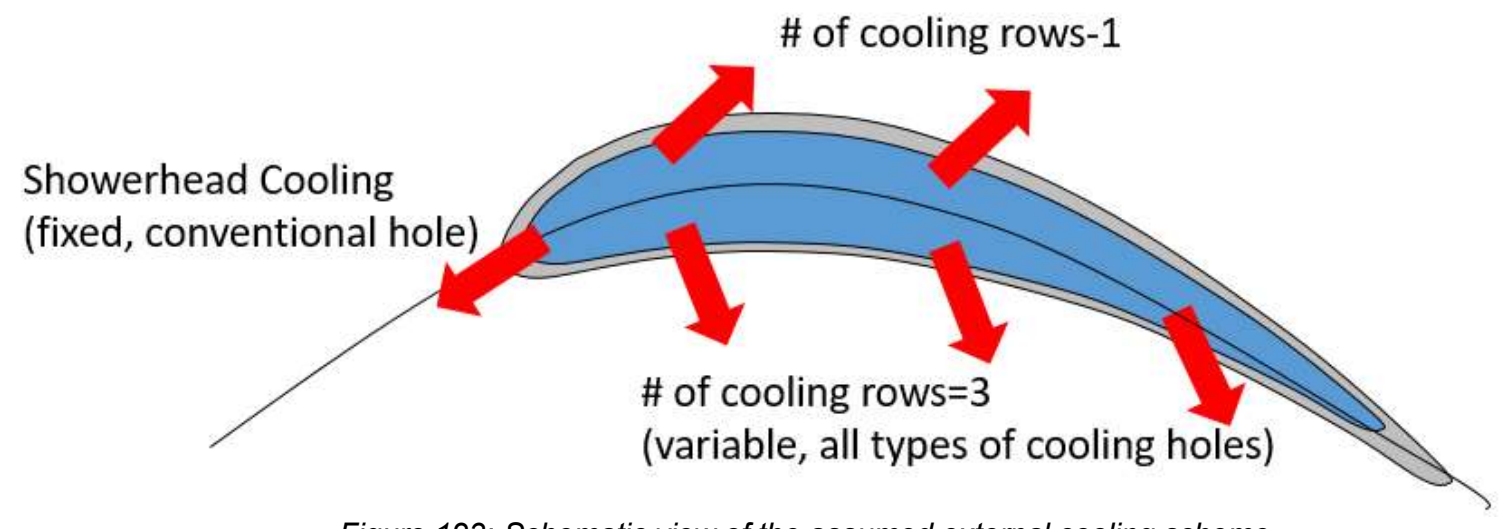

Figure 123: Schematic view of the assumed external cooling scheme

With chord length, hole diameter and the number of cooling rows are known, an average film cooling effectiveness value can be found for pressure and suction sides of the blade. By using thin airfoil assumption, the chord length of the blade can be divided into sub-sections accordingly. The averaging of the spanaveraged film cooling effectiveness data is then done with a 10-point linear averaging of the data up to the $\mathrm{x} / \mathrm{d}_{\mathrm{h}}$ value that corresponds to the location of the next cooling hole.

Algorithm picks the corresponding film cooling data based on user input specifications. Regardless of the user specification, algorithm assumes a conventional hole on the showerhead. Averaging of the film cooling data procedure is shown in schematized in Figure 124 for 3 cooling rows case. This procedure is repeated for both pressure and suction sides independently.

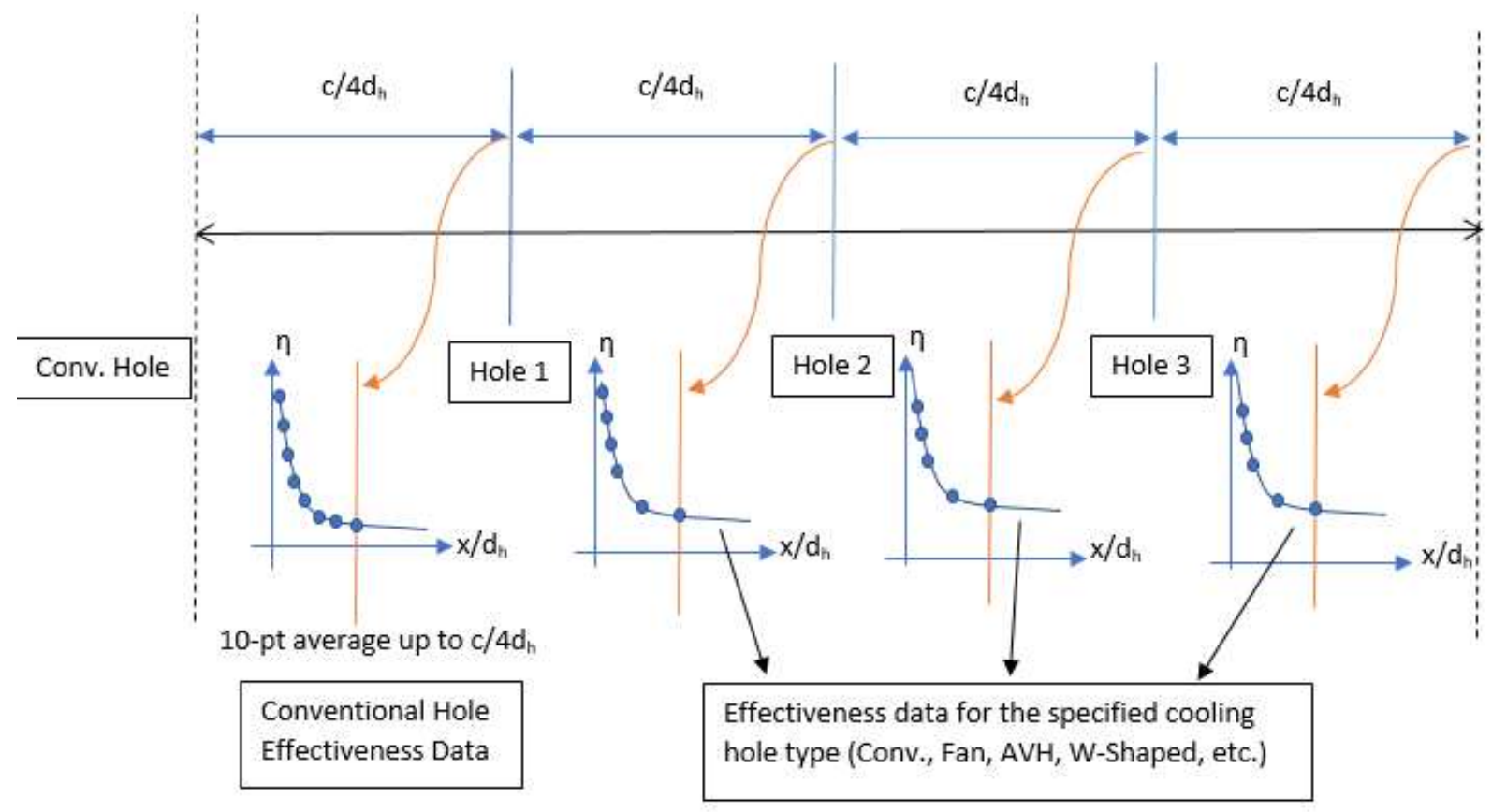

Figure 124: Schematic view of the assumed external cooling scheme 
At the end of this averaging procedure, a local span-averaged adiabatic film-cooling effectiveness can be found for each portion shown in Figure 124. Equation (98) from Seller's Method [70] can then be used to find average effectiveness values for pressure and suction sides separately.

$$
\bar{\varepsilon}_{f c, \text { pressure/suction }}=\sum_{i=1}^{\text {\#of cooling rows }} \varepsilon_{f c, i}\left[\prod_{j=0}^{i-1}\left(1-\varepsilon_{f c, j}\right)\right]
$$

The values obtained for the pressure and suction sides can then later be averaged to obtain a blade averagefilm cooling effectiveness value, which is denoted by $\overline{\varepsilon_{f c}}$. The flowchart of the algorithm is shown in Figure 125.



Figure 125: Flowchart of the external cooling sub-system that calculates average adiabatic effectiveness for the blade 
Correlations and data used for considered hole types that are used in this sub-system are given with their references in Table 35. For the conventional holes and Anti-Vortex Holes, data generated with DES methods in Chapter 4 are used. For the fan shaped holes, the experimental correlation method by Colban et al. [60] is used to generate film cooling hole data for a range of blowing ratio values. Another advanced film cooling hole design is investigated by Shih et al. [61] and the film cooling effectiveness data was extracted through plot digitizing from this resource. The hole type can be specified through user inputs and the algorithm picks the corresponding data set to perform the calculation procedure indicated in Figure 125. See Appendix-E for the details of used film cooling data for the Fan Shaped and W-Shaped holes.

Table 35: Used correlation/film cooling data, their references and corresponding external cooling type index

\begin{tabular}{|l|l|l|l|l|l|}
\hline Hole Type & Index & BR Range & Data Source Type & Notes & Reference \\
\hline $\begin{array}{l}\text { Conventional } \\
\text { (Cylindrical) }\end{array}$ & Hole & 1 & {$[0.5-2.0]$} & CFD Tu\% Data & Chapter 4 \\
\hline $\begin{array}{l}\text { Anti-Vortex Hole } \\
\text { Fan Shaped Hole }\end{array}$ & 2 & $\begin{array}{l}{[1.0-2.0]} \\
{[0.5-2.0]}\end{array}$ & $\begin{array}{l}\text { CFD } \\
\begin{array}{l}\text { Experimental } \\
\text { (Correlation) }\end{array}\end{array}$ & $\begin{array}{l}\text { Low Tu\% Data } \\
\text { Corr. used for } \\
\text { Fan Shaped } \\
\text { Holes }\end{array}$ & $\begin{array}{l}\text { Colban et } \\
\text { [60] }\end{array}$ \\
\hline W-Shaped Hole & 4 & {$[0.31-1.4]$} & CFD & Plot-Digitized & Shih et al. [61] \\
\hline
\end{tabular}

\subsubsection{Calculation of the Film Cooling Coolant Flow rates}

Calculation of the coolant mass flow rates, coolant exit enthalpies and blade temperatures are carried out in the while loop of Figure 119. This part applies the iterative approach described in Section 5.2.1 to calculate the coolant mass fractions required by the internal cooling configuration. The calculated value is the total coolant flow rate, if the cooling configuration does not involve film cooling. If the cooling configuration involves film cooling (Int.+Film and Int.+ Film + TBC), an additional calculation step is needed to obtain the final coolant flow rates.

The need to calculate the external coolant fraction separately, stems from the fact that the new algorithm is not using a fixed correlation for $\overline{\varepsilon_{f c}}$ that relates the effectiveness to the overall coolant fraction as in the original method by Consonni [1], in which $\overline{\varepsilon_{f c}}$ in blade metal wall temperature calculations is replaced with a coolant massflow correlation specific to cylindrical cooling hole geometries under certain blowing ratios. In the new algorithm, $\overline{\varepsilon_{f c}}$ is used as a numerical value obtained for each different cooling hole geometry, and the necessary link between the film cooling hole effectiveness and the coolant flow rate is obtained by using the definition of blowing ratio.

The blowing ratio is defined as in Equation (73) in the heat transfer literature.

$$
B R=\frac{\rho_{c l} V_{c l}}{\rho_{g} V_{g}}
$$

Multiplying both sides of Equation (73) with the film cooling area $\left(A_{c, f c}\right)$ to gas flow area per blade $\left(A_{g, p b}\right)$ gives the required film cooling mass flow rate per blade as in Equation (99).

$$
\frac{A_{c, f c}}{A_{g, p b}} B R=\frac{\rho_{c l} V_{c l}}{\rho_{g} V_{g}} \frac{A_{c, f c}}{A_{g, p b}}=\frac{\dot{m}_{c, f c}}{\dot{m}_{g}}
$$


In Equation (99), the film cooling area can be found by considering the analysis strip shown in Figure 118 with Equation (100).

$$
\left(A_{c, f c}\right)_{\text {strip }}=(\# \text { of FC rows }) \frac{\pi d_{h}{ }^{2}}{4}
$$

For a single film cooling row, the number of cooling holes $\left(n_{h}\right)$ is calculated by using Equation (101) and the blade height $(\mathrm{H})$ for that stage from turbomachinery design section, assuming that the film cooling holes are spaced equally from each other with vertical spacing $z$, which is taken from user inputs in terms of film cooling hole diameter $\mathrm{d}_{\mathrm{h}}$.

$$
n_{h}=\left(\frac{H}{d_{h}}-z\right) \frac{1}{z+1}
$$

Then for the entire blade, the film cooling coolant mass fraction can be found by using Equation (102).

$$
\frac{\dot{m}_{c, f c}}{\dot{m}_{g}}=B R \frac{n_{h}(\# \text { of FC rows })}{\pi\left({R_{T}}^{2}-{R_{H}}^{2}\right) / N B} \frac{\pi d_{h}{ }^{2}}{4}=N B . B R \cdot \frac{n_{h}(\# \text { of FC rows })}{{\left(R_{T}{ }^{2}-R_{H}{ }^{2}\right)}_{\frac{d_{h}}{}{ }^{2}}}
$$

In Equation (103), number of blades (NB), blade tip radius from shaft axis ( $\left.R_{T}\right)$, blade hub radius from shaft axis $\left(R_{H}\right)$ are calculated by the turbomachinery design section. Blowing Ratio $(B R)$, hole diameter $\left(d_{h}\right)$, vertical hole spacing $(z)$ and number of film cooling rows are user inputs.

If the cooling configuration includes film cooling, the algorithm computes the difference $\left(\Delta m_{c}\right)$ between the film cooling coolant requirement $\left(\dot{m}_{c, f c}\right)$ and the supplied coolant for the internal cooling $\left(\dot{m}_{c, \text { int }}\right)$. Per the assumptions in the algorithm development, all coolant required for the film cooling is supplied by the internal cooling system. Therefore:

- If $\dot{m}_{c, f c}>\dot{m}_{c, i n t}$ then film cooling requires more coolant than supplied; add the difference to $\dot{m}_{c, i n t}$ to obtain stage cooling flow fraction

- If $\dot{m}_{c, f c} \leq \dot{m}_{c, i n t}$ then film cooling performance can be satisfied with the supplied coolant for the internal cooling, the stage cooling flow fraction is equal to $\dot{m}_{c, \text { int }}$

\subsubsection{Thermophysical Property Calculations}

This sub-system calculates the gas side Reynolds, Stanton, and Prandtl numbers that are required in the correlations and the coolant flow rate calculations. The gas side Reynolds number is calculated with Equation (103).

$$
R e_{g}=\frac{\rho_{g} V_{g} H}{\mu_{g}}
$$

In Equation (103), properties are used from the engine station prior to the stage component to calculate the density $(\rho)$, speed of the flow $\left(V_{g}\right)$, and the viscosity $\left(\mu_{g}\right)$. Blade height $(H)$ is obtained from turbomachinery design results and used from the blade height information for the stage.

Prandtl number $(\mathrm{Pr})$ and the viscosity data for various temperatures are generated for air and air-fuel mixtures by using REFPROP Software [20]. The Stanton number for the hot gas side is calculated by Equation (104), which is a correlation for internal turbulent flows and used from Consonni [1]. 


$$
S t_{g}=0.45 R e_{g}^{-0.37} \operatorname{Pr}_{g}^{-2 / 3}
$$

For the coolant side, the internal cooling correlation sub-system calculates the coolant Reynolds number from Equation (105).

$$
R e_{c l}=\frac{\rho_{c l} V_{c l} d}{\mu_{c l}}
$$

In Equation (106), the hydraulic diameter $d$ is calculated with Equation (106).

$$
\left\{\begin{array}{lr}
d=\frac{w_{e} h_{e}}{2\left(w_{e}+h_{e}\right)} & \text { for ribs } \\
d=d_{i m p} & \text { for impingement } \\
d=\frac{w_{e} h_{e}}{2\left(w_{e}+h_{e}\right)} & \text { for pin }- \text { fins }
\end{array}\right.
$$

In Equation (106), $\mathrm{w}$ is the width and $\mathrm{h}$ is the height of the indicated internal cooling element. For impingement cooling, the impingement diameter is used for the hydraulic diameter. The correlations in the internal cooling sub-system use the coolant Reynolds number and/or the hydraulic diameter to calculate the internal Stanton number for each internal cooling segment.

For the coolant speed information $\left(\mathrm{V}_{\mathrm{cl}}\right)$, the compressor turbomachinery information about the coolant extraction stage rotor exit vertical flow speed component is used. This flow speed information is also used in the calculation of compressor pressure losses (see Appendix-C).

\subsubsection{Validation of the Algorithm}

The validation of the developed algorithm is carried out by comparing it's results with the semi-empirical formula given in Equation (14). In the calculations with the semi-empirical formula' the blade film cooling effectiveness $\left(\varepsilon_{\mathrm{fc}}\right)$, and internal cooling flow effectiveness $\left(\varepsilon_{\mathrm{c}}\right)$ are used from the results of the new coolant calculation algorithm. Biot numbers for the metal and TBC layer are used as same in both calculations.

From the description by Young and Wilcock [7], the parameter $\mathrm{K}_{\text {cool }}$ in Equation (14) is a composite parameter that includes the coolant to gas side heat capacity ratio, gas side Stanton number and the ratio of the total cooled area to the hot gas side area. The expression used to compute this parameter is given in Equation (107).

$$
K_{\text {cool }}=S t_{g} \frac{c_{p, g}}{c_{p, c l}} \frac{A_{t o t, \text { cooled }}}{A_{g}}
$$

In the advanced coolant calculator and in the other parts of the cooling analysis, the computations are done per blade, and the coolant fraction value obtained is used in the stage coolant fraction calculations. The gas side area is the flow area is per blade and therefore $\mathrm{K}_{\text {cool }}$ is proportional to the number of blades in the stage. For the rotor section, $\mathrm{K}_{\mathrm{cool}}$ value is used as 0.045 , which is the value used in the validations in Section 1.3 and from the validation inputs used by Young and Wilcock [7]. For the stator section, this value is proportioned with the number of blades. Input parameters used in the validation with semi-empirical formula (Equation (14)) is given in Table 36. 
Table 36: Inputs used in the validation of the new coolant calculation algorithm with semi-empirical formula

\begin{tabular}{|c|c|c|}
\hline Parameter & Stator & Rotor \\
\hline $\mathrm{Bi}_{\mathrm{m}}$ & 0.15 & 0.15 \\
\hline $\mathbf{B i}_{\mathrm{TBC}}$ & 0.35 & 0.35 \\
\hline$\varepsilon_{\mathrm{fc}}{ }^{*}$ & 0.336 & 0.336 \\
\hline$\varepsilon_{c}^{*}$ & 0.594 & 0.599 \\
\hline $\mathrm{T}_{\mathrm{tg}^{*}}\left({ }^{0} \mathrm{R}\right)^{*}$ & 2890 & 2707 \\
\hline$T_{\text {tcl,in }}\left({ }^{0} R\right)^{*}$ & 1227 & 1227 \\
\hline$T_{b, \max }\left({ }^{0} R\right)$ & 2115 & 2115 \\
\hline Number of Blades* & 66 & 85 \\
\hline $\mathbf{K}_{\text {cool }}$ & 0.035 & 0.045 \\
\hline
\end{tabular}

In Table 38, the values indicated with an asterisk $\left(^{*}\right)$ indicates that those values are calculated from the new coolant calculation algorithm. Comparison of the coolant fraction results computed by two approaches is given in Table 39.

Table 37: Comparison of the coolant fraction calculations by the new algorithm with the semi-empirical formula

\begin{tabular}{|c|c|c|c|c|c|c|}
\hline & \multicolumn{2}{|c|}{ Semi-Empirical Formula } & \multicolumn{2}{|c|}{$\begin{array}{l}\text { Coolant } \\
\text { Algorithm }\end{array}$} & \multicolumn{2}{|c|}{$\begin{array}{l}\text { Difference wrt. semi- } \\
\text { emp. Formula (\%) }\end{array}$} \\
\hline & Stator & Rotor & Stator & Rotor & Stator & Rotor \\
\hline$\dot{m}_{c} / \dot{m}_{g}$ & 0.01893 & 0.017114 & 0.0189 & 0.01709 & $0.16 \%$ & $0.14 \%$ \\
\hline
\end{tabular}

The percent differences from the calculated coolant fractions with semi-empirical formula using the inputs from new coolant algorithm are within acceptable limits.

\subsection{The Effect of Different Cooling Configurations on Gas Turbine Performance}

The integration of the new coolant algorithm to CEDM and CGTM eliminated some of the cooling analysis user inputs from Table 8 and Table 22, respectively because they are already calculated within the new calculator but some others are added to identify the cooling configuration and some geometric parameters, if required by the correlation used. The user input list for the cooling analysis inputs for both CEDM and CGTM is given in Table 38 . 
Table 38: User Inputs for Cooling Analysis with Advanced Coolant Calculator

\begin{tabular}{|c|c|}
\hline Blade Metal Biot Number (Bim) & $\star \star * *$ \\
\hline TBC Biot Number (Bitbc) & $\star * \star *$ \\
\hline Maximum Allowable Blade Metal Temperature $\left(T_{b, \max }\right)\left[{ }^{0} R\right]$ & {$\left[0, T_{t 4.1}\right]$} \\
\hline Purge Fractions [\%] & *** \\
\hline Cooling Configuration & $\begin{array}{l}\text { Internal + Film + TBC, Internal + Film, } \\
\text { Internal + TBC, or Internal Only }\end{array}$ \\
\hline Film Cooling Effective Injection Angle (deg) & {$[0,90]$} \\
\hline Film Cooling Blowing Ratio (BR) & *** \\
\hline Film Cooling Hole Type & Cylindrical, AVH, Fan, or W-Shaped \\
\hline Film Cooling Hole Diameter $\left(d_{h}\right)[i n]$ & $* * *$ \\
\hline Film Cooling Rows (\# of FC rows) & $\star * *$ \\
\hline Film Cooling Hole Vertical Spacing $\left(z_{n}\right)$ [per $d_{h}$ ] & $* * *$ \\
\hline Internal Cooling Configuration & $\begin{array}{c}\text { [Rib or Impg.Types, Rib or } \\
\text { Impingement Types, Pin-Fin Types] }\end{array}$ \\
\hline Blade Chord Percentile for each Internal Cooling Type (\%) & [\%LE, \%mid-chord, \%TE] \\
\hline Rib Geometry Input [in/in, in/in, in/in, deg] & $\begin{array}{l}\text { [channel hyd. Diameter }(\mathrm{d}) \text {, rib } \\
\text { spacing per } \mathrm{d} \text {, rib height per } \mathrm{d} \text {, rib } \\
\text { angle] }\end{array}$ \\
\hline Impingement Geometry Input & $\begin{array}{l}\text { [LE Curvature Radius/d, hyd. diameter } \\
\text { (d), xn/d, yn/d, zn/d] }\end{array}$ \\
\hline Pin-Fin Geometry Input & [channel hyd. diameter] \\
\hline
\end{tabular}

With the inputs given in Table 38, it is possible to specify blade cooling configurations for a cooled turbine blade shown schematically in Figure 126.

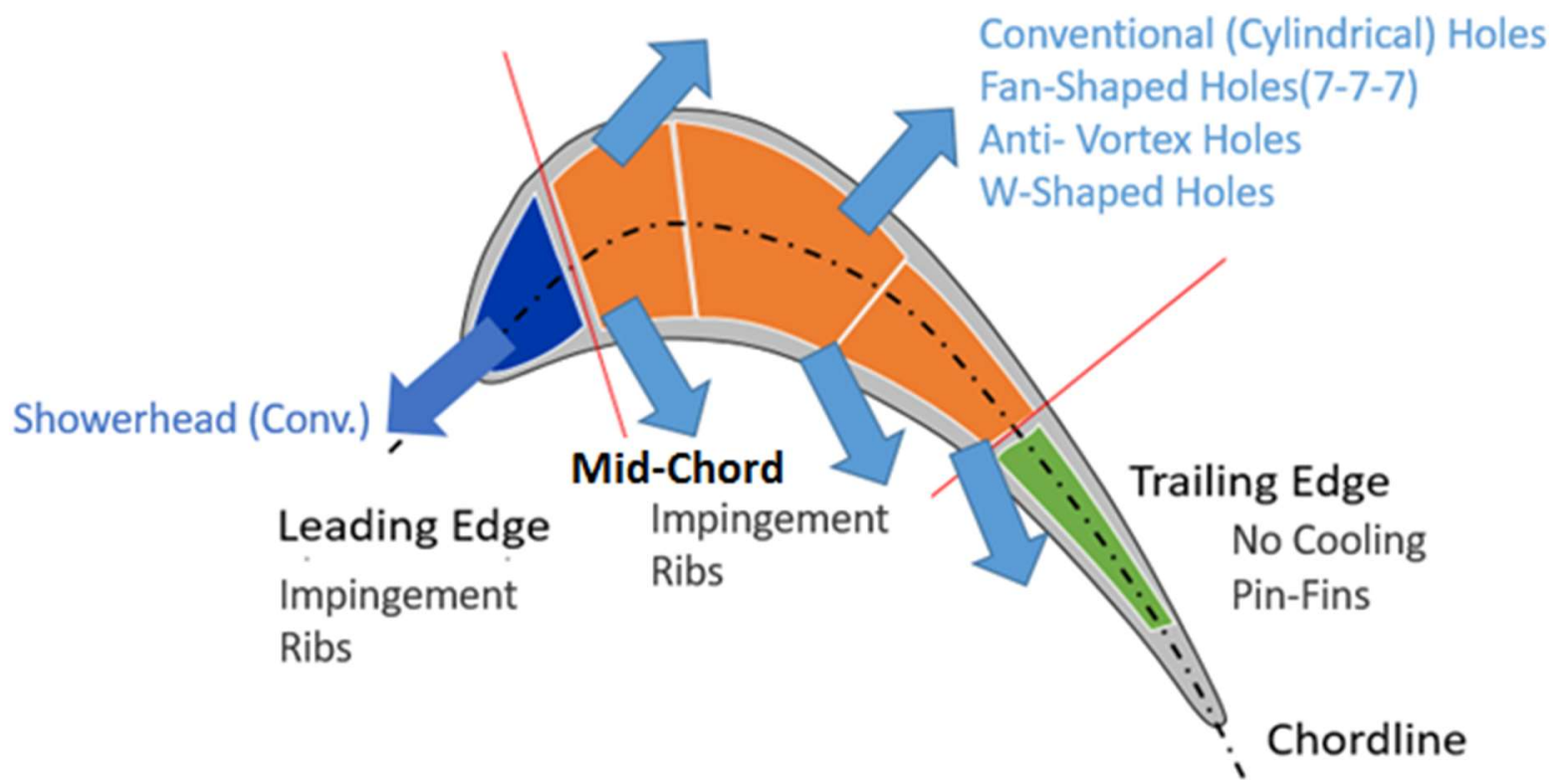

Figure 126: Schematic view of the cooled turbine blade modelled in advanced cooling calculator

With the internal and external correlations and CFD data built-in to the advanced coolant calculator, it is possible to analyze 1024 logical blade cooling configurations with the input list given in Table 38. However, to reduce the number of analysis cases, the blade cooling configurations were determined from the configurations mostly used in recent advanced gas turbines [62]; which has impingement cooling on the 
leading edge, rib cooling on the mid-chord and pin-fins on the trailing edge. The configurations for the analysis in the following sections are shown in Table 41.

Table 39: Blade Cooling Configurations used for Analysis

\begin{tabular}{|c|c|c|c|c|}
\hline Configuration Name & LE (Impingement) & Mid-Chord (Ribs) & $\begin{array}{l}\text { TE (Pin- } \\
\text { Fins) }\end{array}$ & External Cooling \\
\hline FC Test AVH & Curved Plate Imp. & V-Shaped (Chevron) & Inline & $\mathrm{AVH}$ \\
\hline FC Test Conventional & Curved Plate Imp. & V-Shaped (Chevron) & Inline & Conventional \\
\hline FC Test Fan Shaped & Curved Plate Imp. & V-Shaped (Chevron) & Inline & Fan Shaped \\
\hline FC Test W-Shaped & Curved Plate Imp. & V-Shaped (Chevron) & Inline & W-Shaped \\
\hline MC Test W-Trips AVH & Curved Plate Imp. & W-Shaped Ribs & Inline & AVH \\
\hline MC Test W-Trips Conv. & Curved Plate Imp & W-Shaped Ribs & Inline & Cylindrical \\
\hline $\begin{array}{l}\text { MC Test Angled Trips } \\
\text { AVH }\end{array}$ & Curved Plate Imp & Angled Ribs $\left(45^{\circ}\right)$ & Inline & AVH \\
\hline $\begin{array}{l}\text { MC Test Angled Trips } \\
\text { Conv. }\end{array}$ & Curved Plate Imp & Angled Ribs $\left(45^{\circ}\right)$ & Inline & Cylindrical \\
\hline $\begin{array}{l}\text { TE Test Staggered } \\
\text { AVH }\end{array}$ & Curved Plate Imp. & V-Shaped (Chevron) & Staggered & $\mathrm{AVH}$ \\
\hline $\begin{array}{l}\text { TE Test Staggered } \\
\text { Conv. }\end{array}$ & Curved Plate Imp. & V-Shaped (Chevron) & Staggered & Cylindrical \\
\hline TE Test Diamond AVH & Curved Plate Imp. & V-Shaped (Chevron) & $\begin{array}{l}\text { Diamond } \\
\text { Pedestal }\end{array}$ & AVH \\
\hline $\begin{array}{l}\text { TE Test Diamond } \\
\text { Conv. }\end{array}$ & Curved Plate Imp. & V-Shaped (Chevron) & $\begin{array}{l}\text { Diamond } \\
\text { Pedestal }\end{array}$ & Cylindrical \\
\hline
\end{tabular}

The configurations are determined here with a similar methodology in a study that determines advanced cooling configurations for aero-engines and gas turbines from Town et al. [62]; in which a baseline configuration is determined and other configurations were generated by changing one cooling technology at a time to see the relative impact of a certain cooling technology in a test rig that can measure the impacts of different cooling techniques on the turbine performance [63].

The analyses are made separately with the cycle and turbomachinery design inputs from Table 8 and Table 22 for the generic aero-engine and generic $\mathrm{H}$-Class engine cases, respectively.

For both aero-engine and industrial gas turbine cases, the effects of film cooling hole type, mid-chord internal cooling type, trailing edge internal cooling type, and the number of film cooling rows are studied by changing the input parameters and comparing their impact on key performance parameters such as thrust (or power), thrust specific fuel consumption (or heat rate), thermal efficiency and total coolant fractions.

For the aero-engine cases, cooling analysis inputs used in CEDM are given in Table 40. 
Table 40: Cooling Analysis inputs used in High By-Pass Turbofan Engine cases

Cooling Analysis Inputs for CEDM with advanced coolant calculator

\begin{tabular}{|c|c|}
\hline Blade Metal Biot Number ( $\left.\mathrm{Bi}_{\mathrm{m}}\right)$ & 0.15 \\
\hline TBC Biot Number (Bitbc) & 0.35 \\
\hline Maximum Allowable Blade Metal Temperature $\left(T_{b}, \max \right)\left[{ }^{0} R\right]$ & 2100 \\
\hline Purge Fractions [\%] & 0.2 and 0.1 \\
\hline Cooling Configuration & Internal + Film + TBC \\
\hline Film Cooling Effective Injection Angle (deg) & [varied] \\
\hline Film Cooling Blowing Ratio (BR) & 1.0 \\
\hline Film Cooling Hole Type & [varied] \\
\hline Film Cooling Hole Diameter $\left(d_{h}\right)$ [in] & 0.03 \\
\hline Film Cooling Rows (\# of FC rows) & 2 \\
\hline Film Cooling Hole Vertical Spacing $\left(z_{n}\right)$ [per $d_{h}$ ] & 3 \\
\hline Internal Cooling Configuration & [Impg., Ribs, Pin-Fin] \\
\hline Blade Chord Percentile for each Internal Cooling Type (\%) & {$[\% 20, \% 40, \% 20]$} \\
\hline Rib Geometry Input [ in/in, in/in, in/in, deg] & {$[0.0795,0.05,0.6$, varied $]$} \\
\hline Impingement Geometry Input & {$[30,0.08,5,5,1]$} \\
\hline Pin-Fin Geometry Input & [0.094] \\
\hline Blade Chord Length (stator, rotor) [in, in] ${ }^{*}$ & $0.7,0.9$ \\
\hline Blade Height (stator, rotor) [in, in] * & $2.08,2.601$ \\
\hline Number of Blades in the Stage (stator, rotor) * & 75,85 \\
\hline
\end{tabular}

The baseline internal cooling configuration is determined to have a hydraulic diameter of 0.0795 for the midchord (V-Shaped (Chevron) Ribs) and 0.094 for the trailing edge (Inline Circular Pin-Fins). In Table 40, the values indicated with asterisk $\left(^{*}\right)$ indicates that those values are computed by turbomachinery design section of CEDM.

For the industrial gas turbine cases, cooling analysis inputs used in CGTM are given in Table 41.

Table 41: Cooling Analysis inputs used in the industrial gas turbine case

\begin{tabular}{|c|c|}
\hline ooling Analysis Inputs for CGTM with advanced coolan & ulator \\
\hline Blade Metal Biot Number ( $\left.\mathbf{B i}_{\mathrm{m}}\right)$ & 0.16 \\
\hline TBC Biot Number (Bitbc) & 0.37 \\
\hline Maximum Allowable Blade Metal Temperature $\left(T_{b, \max }\right)\left[{ }^{0} R\right]$ & 2000 \\
\hline Purge Fractions [\%] & 0.5 (for both stations) \\
\hline Cooling Configuration & $\begin{array}{l}\text { Internal + Film + TBC (on first two stages) } \\
\text { Internal+ TBC (on third stage) } \\
\text { Internal Only (on fourth stage) }\end{array}$ \\
\hline Film Cooling Effective Injection Angle (deg) & [varied] \\
\hline Film Cooling Blowing Ratio (BR) & 1.0 \\
\hline Film Cooling Hole Type & [varied] \\
\hline Film Cooling Hole Diameter $\left(d_{h}\right)[i n]$ & 0.06 \\
\hline Film Cooling Rows (\# of FC rows) & 3 \\
\hline Film Cooling Hole Vertical Spacing $\left(z_{n}\right)$ [per $\left.d_{h}\right]$ & 3 \\
\hline Internal Cooling Configuration & [Impg., Ribs, Pin-Fin] \\
\hline Blade Chord Percentile for each Internal Cooling Type (\%) & {$[\% 20, \% 40, \% 20]$} \\
\hline Rib Geometry Input [ in/in, in/in, in/in, deg] & {$[0.0795,0.05,0.6$, varied $]$} \\
\hline Impingement Geometry Input & {$[30,0.08,5,5,1]$} \\
\hline Pin-Fin Geometry Input & [0.094] \\
\hline
\end{tabular}


Table 41 continued: Cooling Analysis inputs used in the industrial gas turbine case

\section{Cooling Analysis Inputs for CGTM with advanced coolant calculator}

Blade Chord Length (stator, rotor) [in, in] *

Blade Height (stator, rotor) [in, in] *

Number of Blades in the Stage (stator, rotor) *
$(2.98,3.00),(5.97,6.00),(6.84,6.86),(8.34,8.36)$

(8.96, 8.98), (17.9, 18.1), (20.5, 20.6), (25.0, 25.1)

$(66,120),(48,80),(44,60),(40,56)$

Same baseline internal cooling configuration with the aero-engine, which has a hydraulic diameter of 0.0795 for the mid-chord (V-Shaped (Chevron) Ribs) and 0.094 for the trailing edge (Inline Circular Pin-Fins). In Table 41 the values indicated with asterisk $\left({ }^{*}\right)$ indicates that those values are computed by the turbomachinery design section of CGTM.

\subsubsection{The Effect of Film Cooling Geometry}

The effect of using different film cooling geometries on the engine performance parameters are analyzed by using the FC Test configurations in Table 39 by also considering the changes in the effective flow injection angle when the film cooling hole type is changed. Film Cooling data for the conventional and AVH holes are used from low turbulence intensity and 1.0 blowing ratio results (from Hayes [39] and Figure 108) to be able to compare with the results for the other hole geometries [60,61], which are obtained under such conditions.

In this analysis, the engine performance is affected mainly from the changes in the blade film cooling effectiveness and its impact on the internal cooling flow effectiveness. A comparison of the span-averaged film cooling data for the considered film cooling geometries is shown in Figure 127.

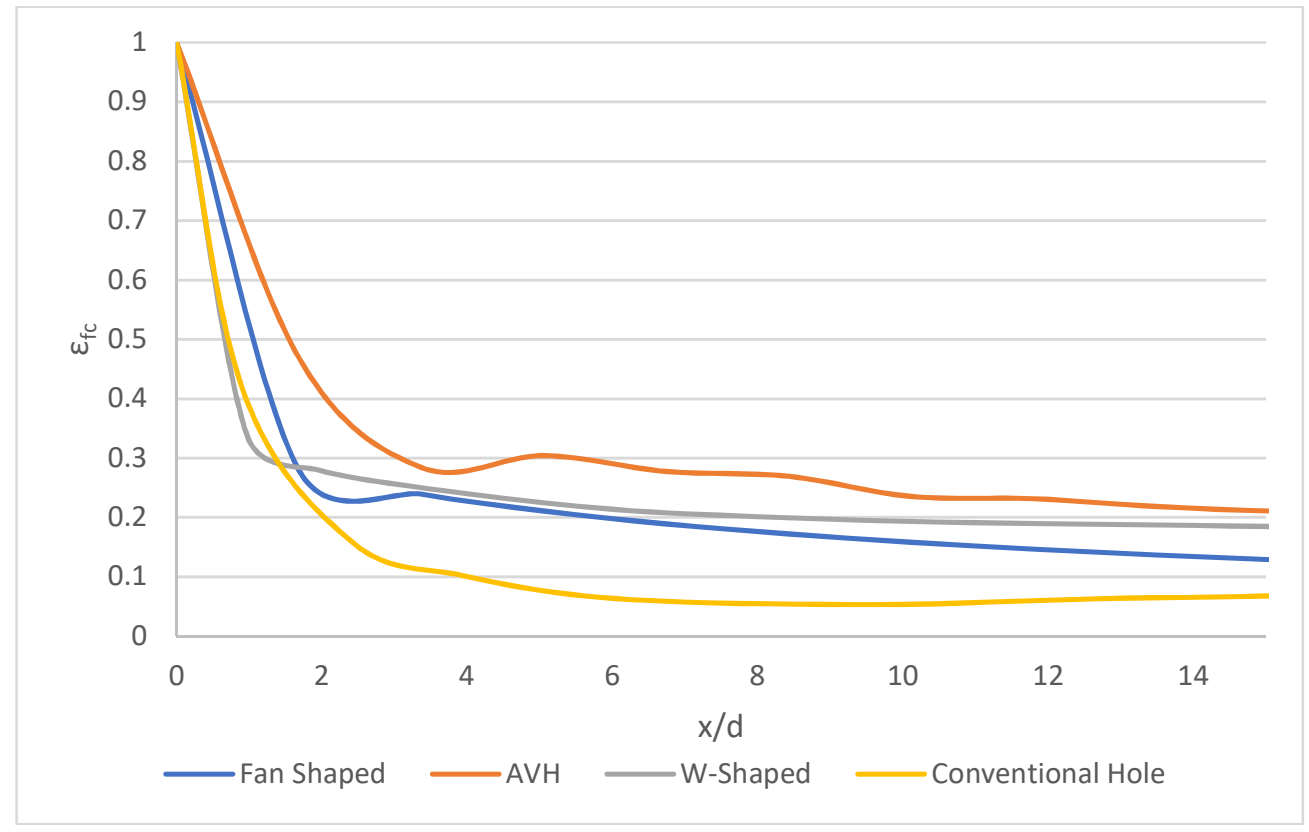

Figure 127: Comparing the span-averaged film cooling effectiveness values of different hole geometries used in the analysis 
For the same blowing ratio, the method that gives the highest film cooling effectiveness would reduce the adiabatic wall temperature most, which also improves the internal cooling flow effectiveness through reducing the coolant heating. Therefore, it is expected to obtain higher engine performance when the film cooling effectiveness is higher.

\subsubsection{High By-Pass Turbofan Engine}

For the generic case of high by-pass turbofan engine, the results are obtained by fixing the high pressure turbine exit temperature to $2030{ }^{\circ} \mathrm{R}$ from [22]. Cooling analysis inputs in this analysis are used from Table 40.

From Figure 127 and using Seller's Method [70] given in Equation (98) with the blade chord lengths in Table 40 , it is expected to obtain highest blade film cooling effectiveness with $\mathrm{AVH}$, followed by the fan shaped hole, $\mathrm{W}$-shaped hole and conventional hole cases of the aero-engine turbine blade with 3 holes on the pressure side and 2 holes on the suction side. Calculated values of film cooling effectiveness and the internal cooling flow effectiveness with different film cooling hole geometry cases are shown in Table 42.

Table 42: Variations in the blade film cooling and internal cooling flow effectiveness with different film cooling configurations

\begin{tabular}{|c|c|c|c|c|}
\hline Parameter & Conventional & Anti-Vortex & Fan Shaped & W-Shaped \\
\hline \multicolumn{5}{|c|}{ Stator } \\
\hline$\varepsilon_{\mathrm{fc}}$ & 0.218 & 0.441 & 0.364 & 0.336 \\
\hline$\varepsilon_{\mathrm{c}}$ & 0.603 & 0.61 & 0.608 & 0.604 \\
\hline \multicolumn{5}{|c|}{ Rotor } \\
\hline$\varepsilon_{\mathrm{fc}}$ & 0.218 & 0.441 & 0.364 & 0.336 \\
\hline$\varepsilon_{c}$ & 0.609 & 0.618 & 0.614 & 0.611 \\
\hline
\end{tabular}

From the results of the sensitivity analysis, it is expected to have the lowest coolant fraction with the highest internal cooling flow and film cooling effectiveness values. The results obtained for the coolant fractions follows this expected trend, and is shown in Figure 128.



Figure 128: Total coolant fractions are compared for different film cooling hole geometry cases of the aero-engine case 
As the coolant fractions are reduced, the turbine losses are also reduced resulting in an increase in thrust generated through an increase in cooled turbine polytropic efficiency. The result comparison for the specific thrust obtained shows this trend as shown in Figure 129.

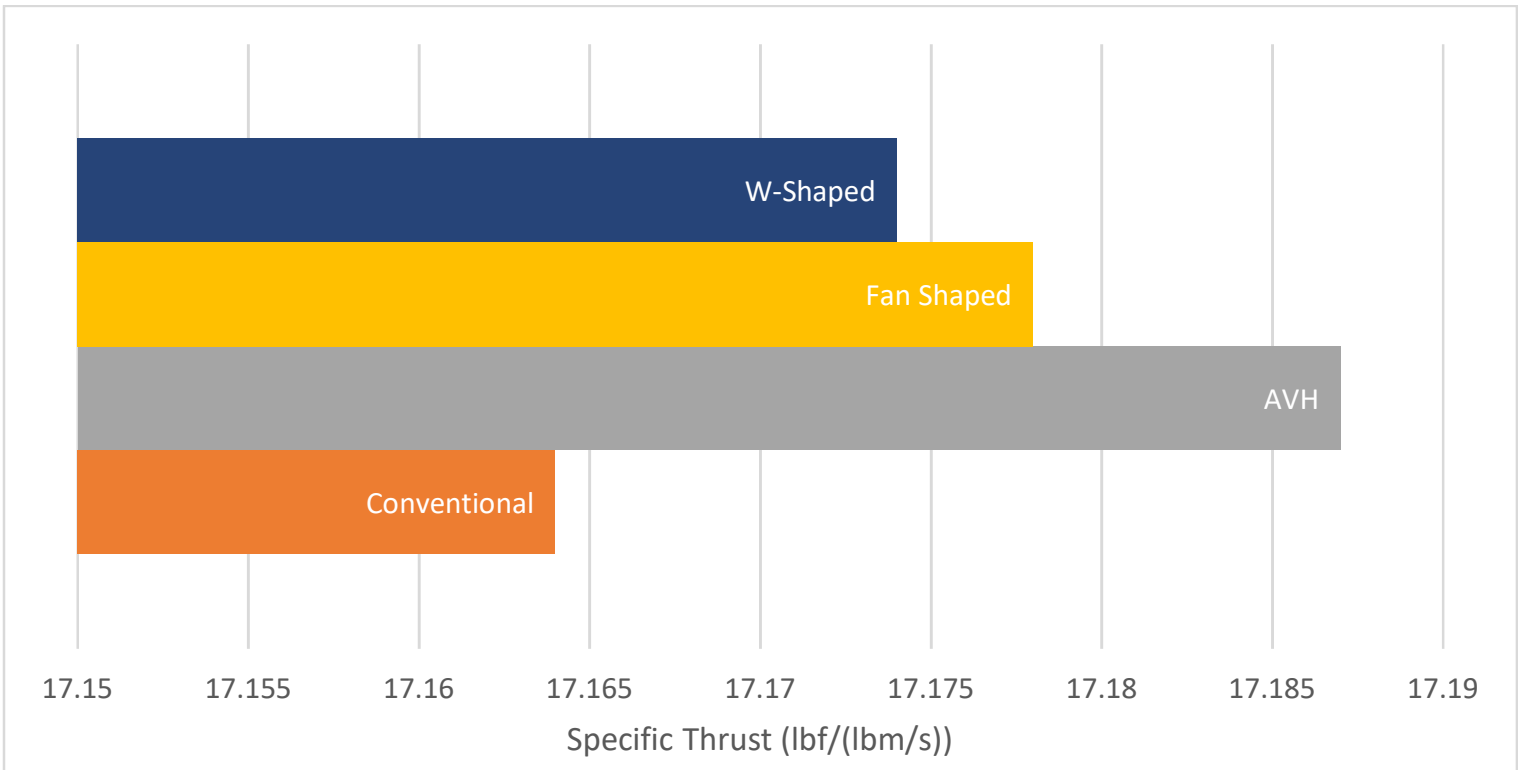

Figure 129: Specific Thrust values are compared for different film cooling hole geometry cases of the aero-engine case

The change in specific thrust is occurred within $0.14 \%$, which is in parallel to the results obtained in sensitivity analysis and considering the cross-effects of cooling parameters to each other. When the coolant fractions are reduced, due to increased air flow to the combustor, a slight increase in the fuel consumption occurs. However, if the rate of increase in the thrust due to the increase in turbine performance is higher than the rate of increase in the fuel consumption, then the thrust specific fuel consumption decreases. Comparison of the thrust specific fuel consumption with different film cooling configurations are shown in Figure 130.

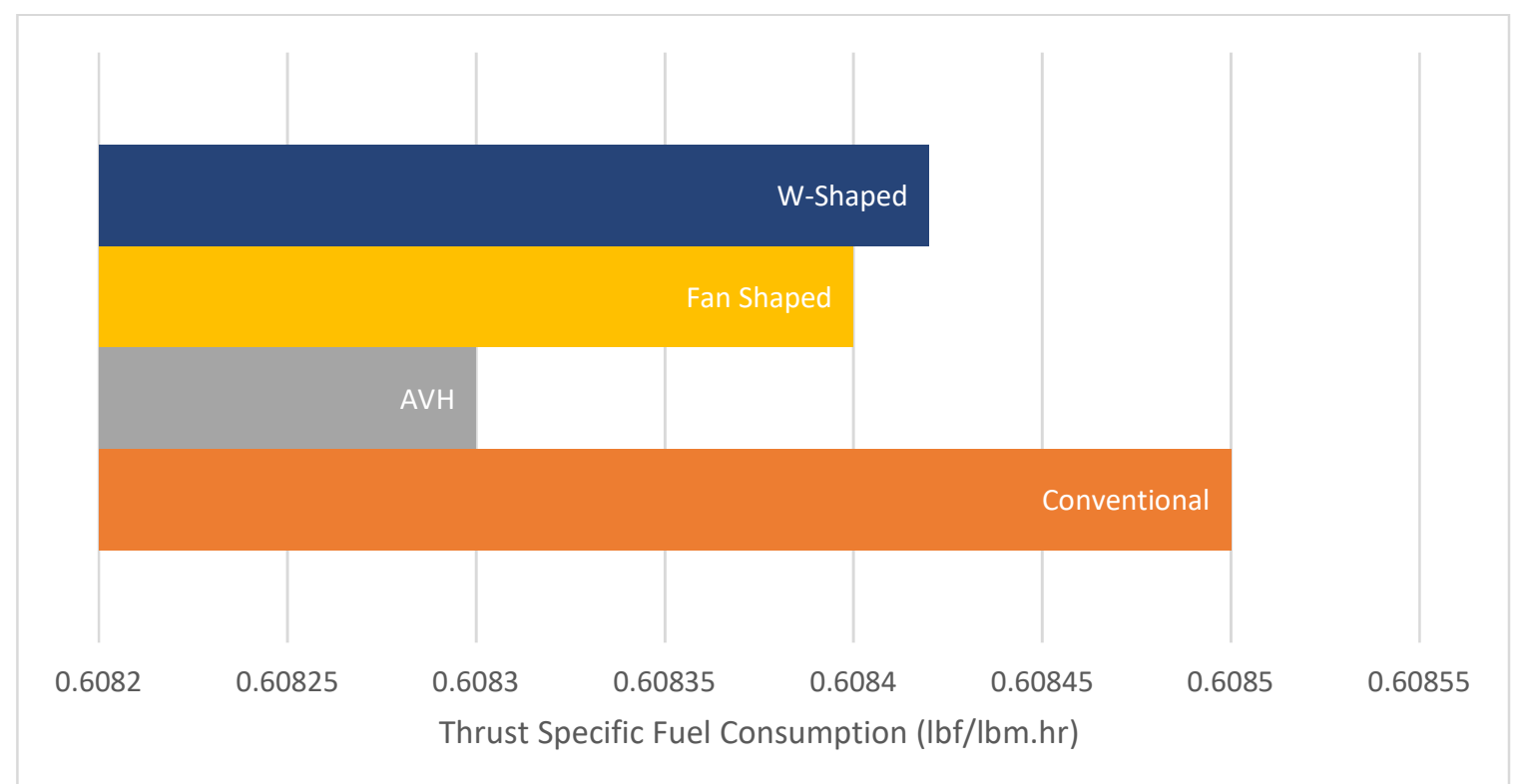

Figure 130: Thrust Specific Fuel Consumptions are compared for different film cooling hole geometry cases of the aeroengine case 
Thermal efficiency follows the same trend with the thrust; highest thrust producing engine with the lowest fuel consumption gives highest thermal efficiency. This trend is also observed in the comparison made for thermal efficiency obtained with different film cooling configurations and shown in Figure 131.

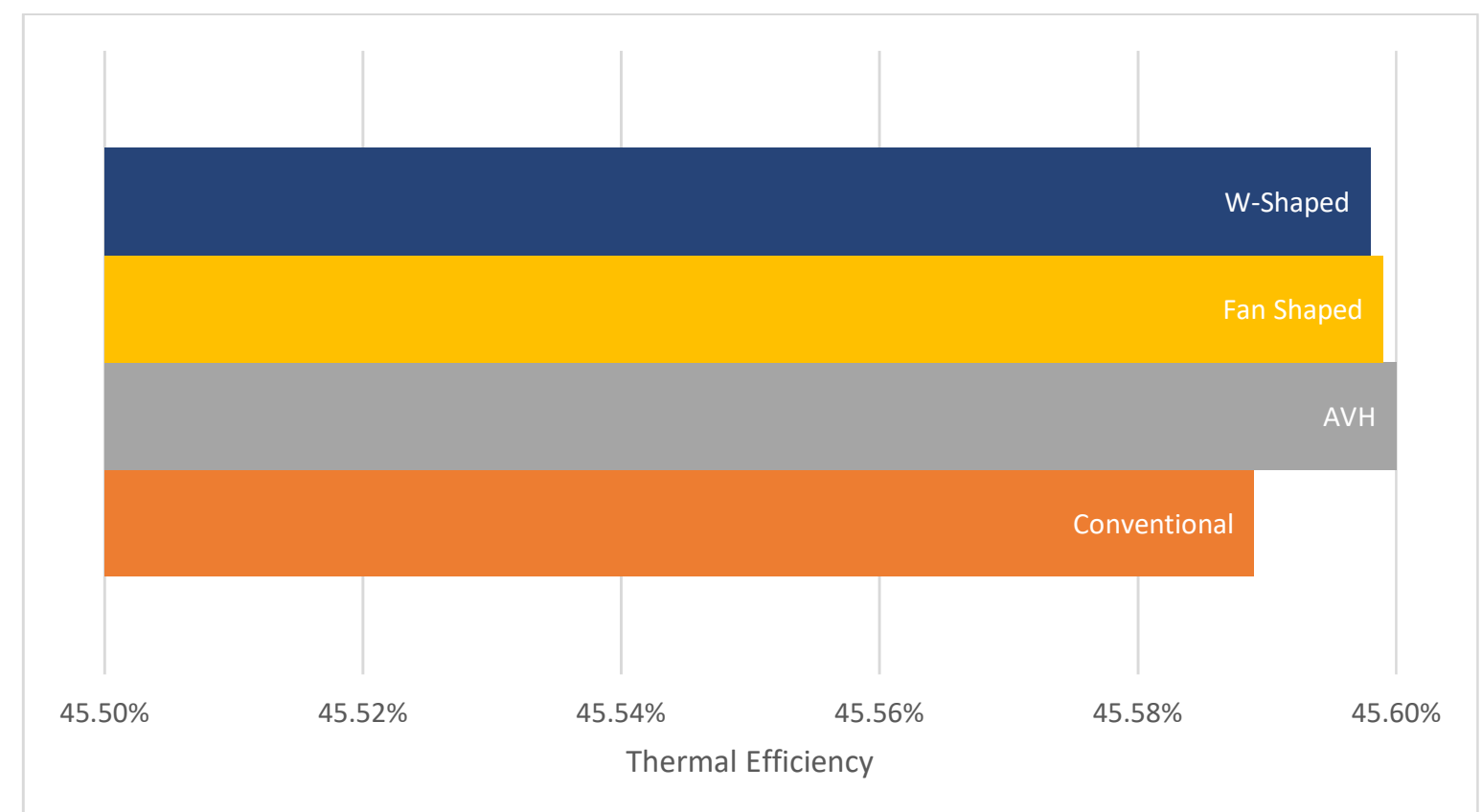

Figure 131: Thermal efficiencies compared for different film cooling hole geometry cases of the aero-engine case

The magnitude of the changes in the engine thermal efficiencies obtained with different film cooling geometries are within $0.15 \%$.

\subsubsection{Industrial Gas Turbine}

For the generic $\mathrm{H}$-Class industrial gas turbine case, the results are obtained by fixing turbine exit temperature to $1620{ }^{\circ} \mathrm{R}$. Cooling analysis inputs used in this analysis are given in Table 41.

Due to having longer chords than the aero-engine blades, the maximum span ( $\mathrm{x} / \mathrm{d})$ location used for each film cooling hole is higher in the gas turbine case than the aero-engine case, which results in differences in the calculated blade film cooling effectiveness values from the aero-engine case. Calculated values of film cooling effectiveness and the internal cooling flow effectiveness for the first two turbine stages with film cooling for different film cooling hole geometry cases are shown in Table 43.

Table 43: Variations in the blade film cooling and internal cooling flow effectiveness with different film cooling configurations

\begin{tabular}{|l|c|c|c|c|c|c|c|c|}
\hline Parameter & Conv. & $\begin{array}{l}\text { Anti- } \\
\text { Vortex }\end{array}$ & $\begin{array}{l}\text { Fan } \\
\text { Shaped }\end{array}$ & W-Shaped & Conv. & $\begin{array}{l}\text { Anti- } \\
\text { Vortex }\end{array}$ & $\begin{array}{l}\text { Fan } \\
\text { Shaped }\end{array}$ & W-Shaped \\
\hline \multicolumn{8}{c|}{ Stage 1 Stator } \\
\hline$\varepsilon_{\mathrm{fc}}$ & 0.275 & 0.569 & 0.426 & 0.487 & 0.2437 & 0.502 & 0.352 & 0.451 \\
\hline$\varepsilon_{\mathrm{c}}$ & 0.57 & 0.59 & 0.57 & 0.58 & 0.57 & 0.59 & 0.57 & 0.58 \\
\hline \multicolumn{3}{|c|}{ Stage 1 Rotor } \\
$\boldsymbol{\varepsilon}_{\mathrm{fc}}$ & 0.275 & 0.569 & 0.426 & 0.487 & 0.2437 & 0.502 & 0.352 & 0.451 \\
\hline$\varepsilon_{\mathrm{c}}$ & 0.57 & 0.59 & 0.57 & 0.58 & 0.57 & 0.59 & 0.57 & 0.58 \\
\hline
\end{tabular}


In Table 43, the ranking in terms of the blade film cooling and the internal cooling flow effectiveness are the same with the aero-engine case, except for the W-Shaped hole case. Analyzing Figure 127 shows that WShaped hole has higher span-averaged effectiveness than fan shaped hole after $\sim 6$ hole diameters, makes the computed blade film cooling effectiveness with Equation (98) higher than the fan shaped hole. This difference is due to the fact that, in the industrial gas turbine case the film cooling data read per hole is longer than 6 hole diameters due to having longer chords than the aero-engine blade.

Similar to the aero-engine case, having higher internal cooling flow and film cooling flow effectiveness results in lower coolant fractions from the sensitivity analysis in Chapter 3 . The results obtained for the coolant fractions follows this expected trend, and is shown in Figure 128

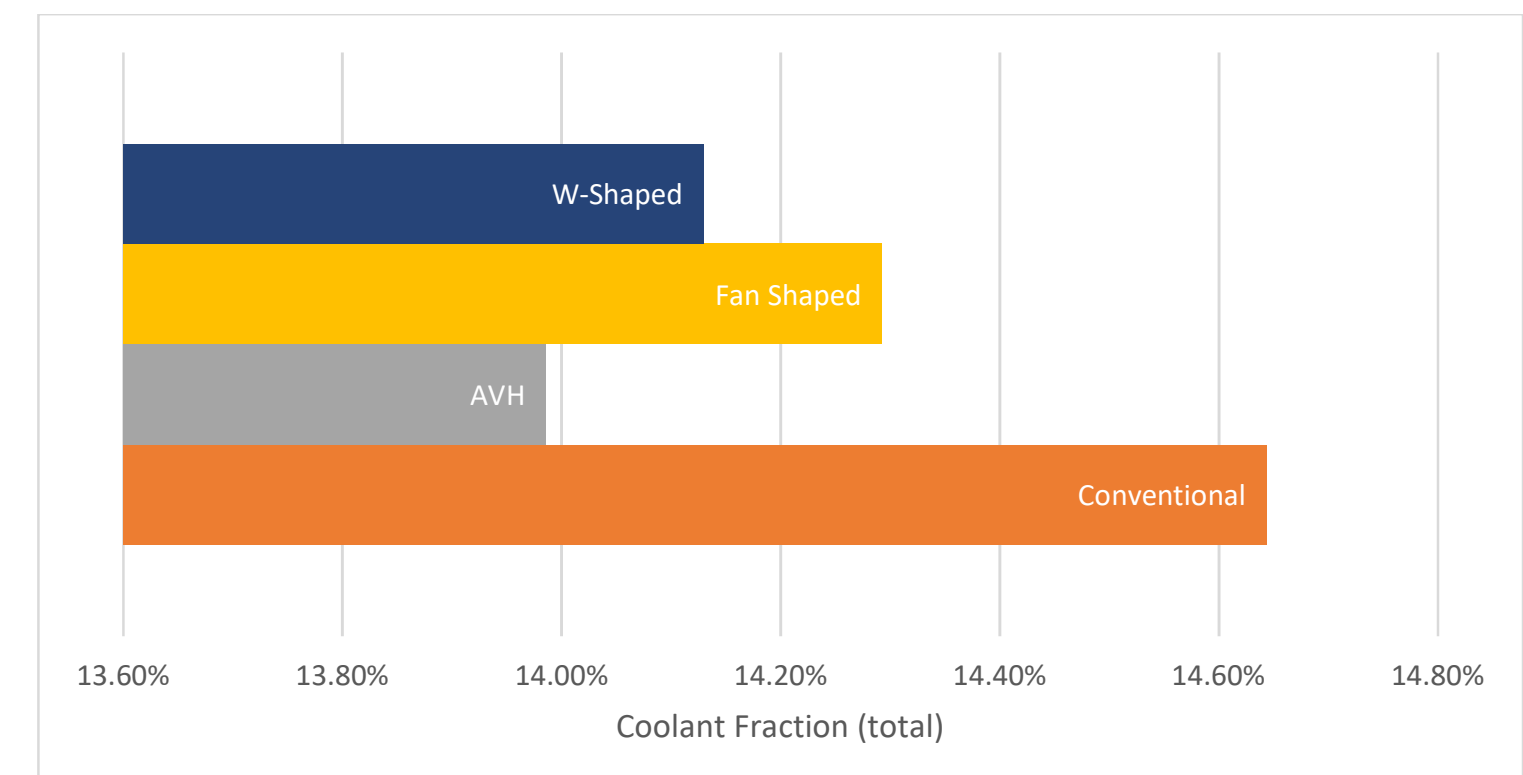

Figure 132: Total coolant fractions are compared for different film cooling hole geometry cases of the industrial gas turbine case

The change in the required coolant fractions occurred within $0.65 \%$. When the required coolant fractions are reduced, turbine cooling losses are reduced, and the cooled turbine polytropic efficiency is increased. In addition, due to the interstage coolant extraction in the compressor, reducing the coolant fractions also improves compressor performance, resulting in less power demand from the turbine. Overall impact is an increase in the shaft power produced, as shown in Figure 133. 


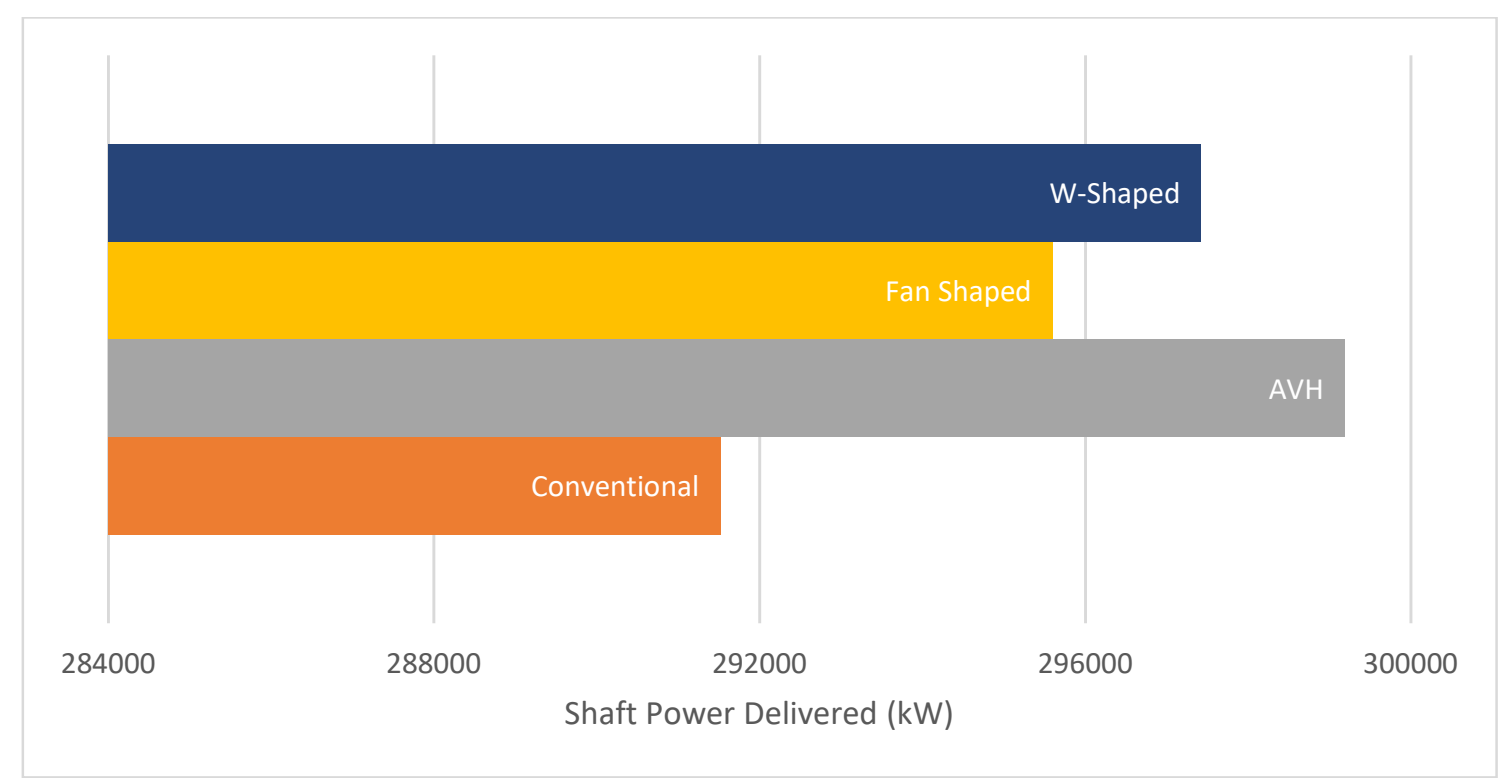

Figure 133: Shaft power values are compared for different film cooling hole geometry cases of the industrial gas turbine case

The change in the shaft power delivered occurred within $2.6 \%$ (relative to conventional hole case), which is within acceptable limits when the sensitivity analysis and the cross-effects between cooling parameters are considered. Similar to the aero-engine, reducing the coolant flows results in a slight increase in fuel consumption but if this increase is outweighed with the improvement in the turbine power production, the heat rates are lower for the higher film cooling performance as shown in Figure 134.

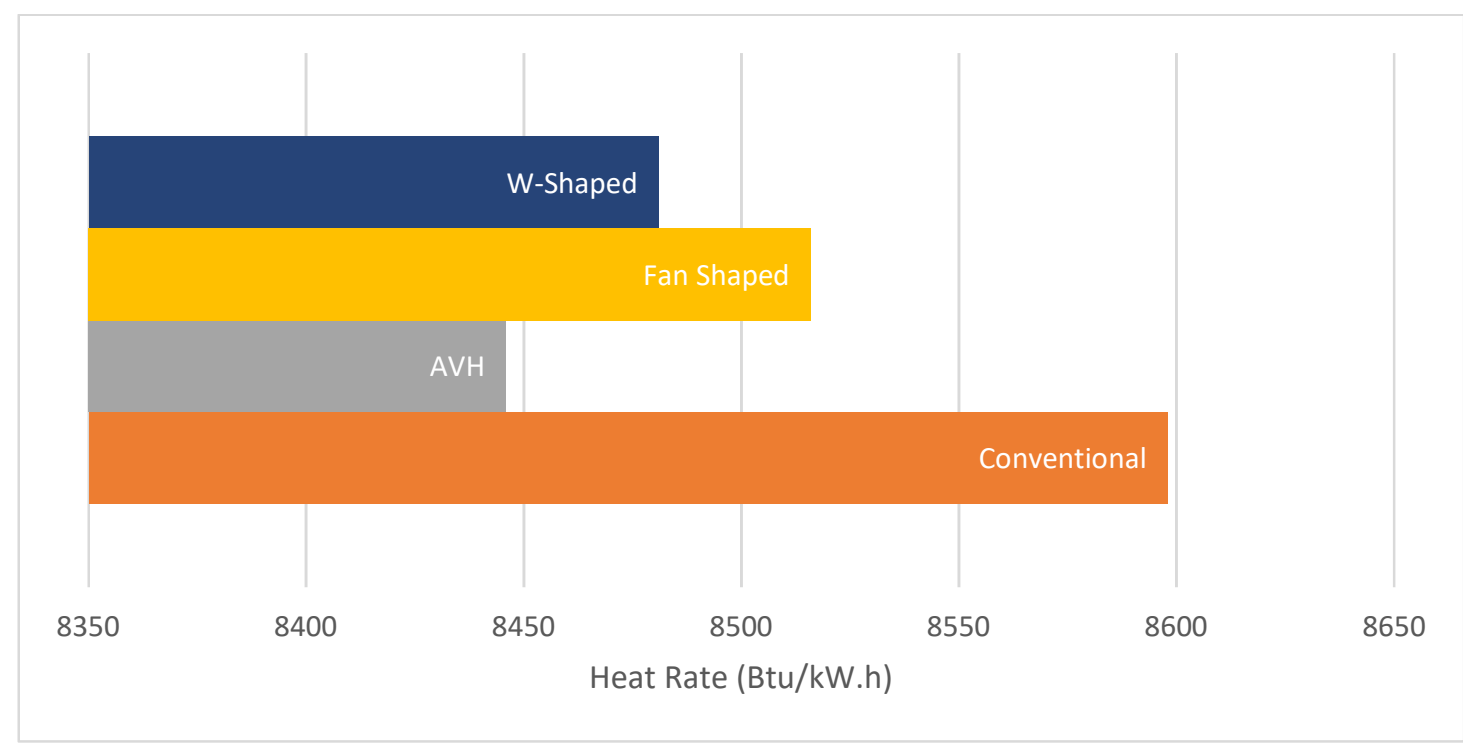

Figure 134: Heat rates are compared for different film cooling hole geometry cases of the industrial gas turbine case

Thermal efficiency of an industrial gas turbine is a measure of how effectively the fuel energy is used to generate power output., therefore it is expected to obtain highest thermal efficiency with the configuration that gives the highest film cooling performance. Hence, the ranking of the configurations with respect to thermal efficiencies is expected to follow the same order with the power, as shown in Figure 135. 


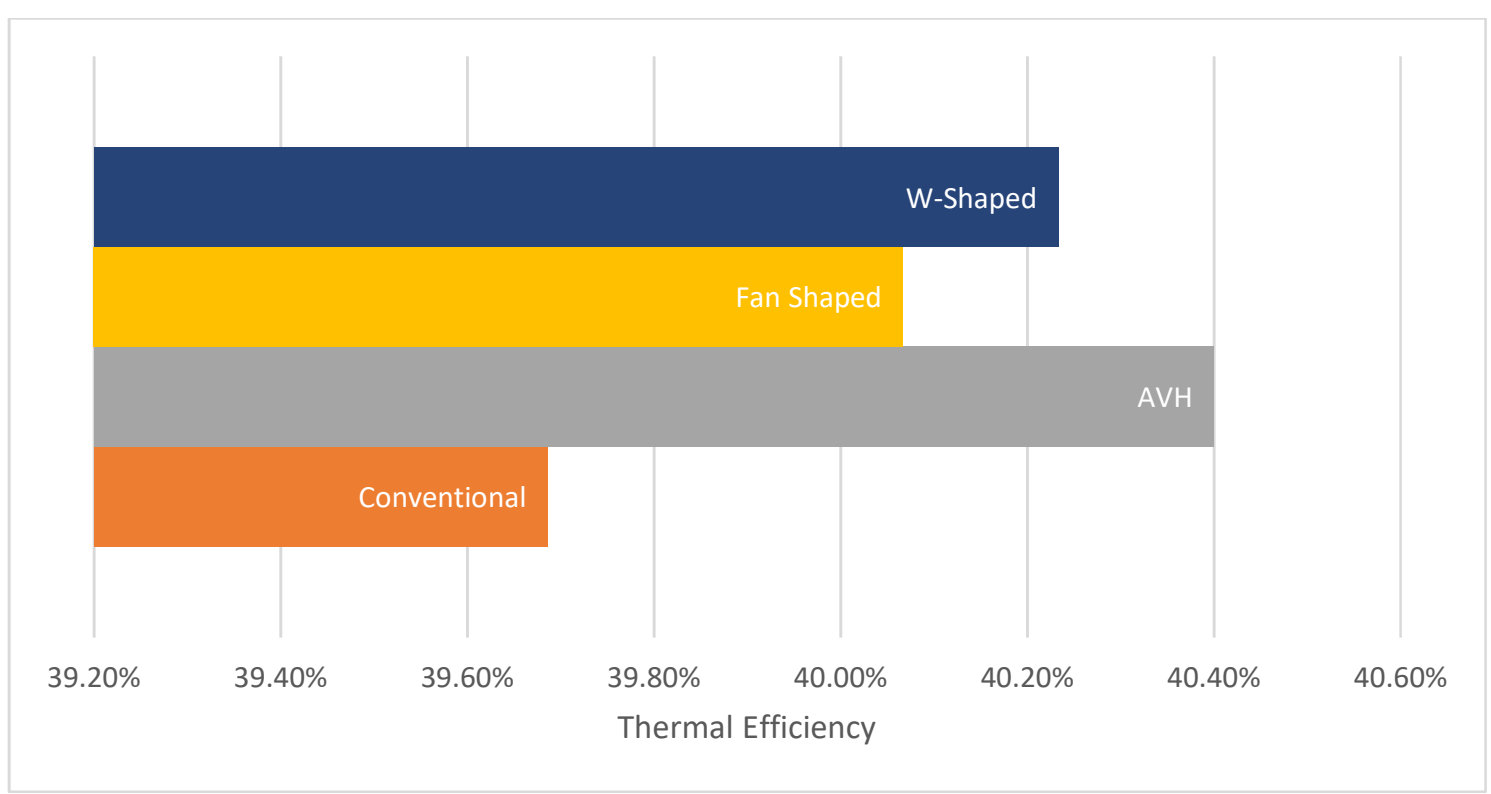

Figure 135: Thermal efficiencies are compared for different film cooling hole geometry cases of the industrial gas turbine case

The magnitude of the changes in the engine thermal efficiencies obtained with different film cooling geometries are within $0.7 \%$.

\subsubsection{The Effect of Internal Cooling Configuration}

The effect of internal cooling configuration on the engine performance is analyzed by changing the mid-chord and trailing edge cooling test configurations, named as MC Test and TE Test configurations in Table 39, respectively. The results of the analysis for aero-engines and industrial gas turbines are presented in the following sub-sections.

\subsubsection{Mid-Chord Cooling Configuration}

Three different mid-chord rib cooling methods, which are the $\mathrm{V}$-Shaped (Chevron), W-Shaped and parallel angled, are used in this analysis. Same hydraulic diameter value of 0.08 was used in all configurations. In this analysis, the changes in the engine performance is caused by the changes in the mid-chord internal Stanton number that directly affects the internal cooling flow effectiveness. The analyses in this section are made for two different film cooling cases (i.e. conventional hole and AVH) in order to observe the effect of using different adiabatic wall temperatures on mid-chord cooling performance.

The internal cooling correlations used for the mid-chord cooling cases for the same hydraulic diameter of 0.08 and within the same Reynolds number ranges are compared in Figure 136. 


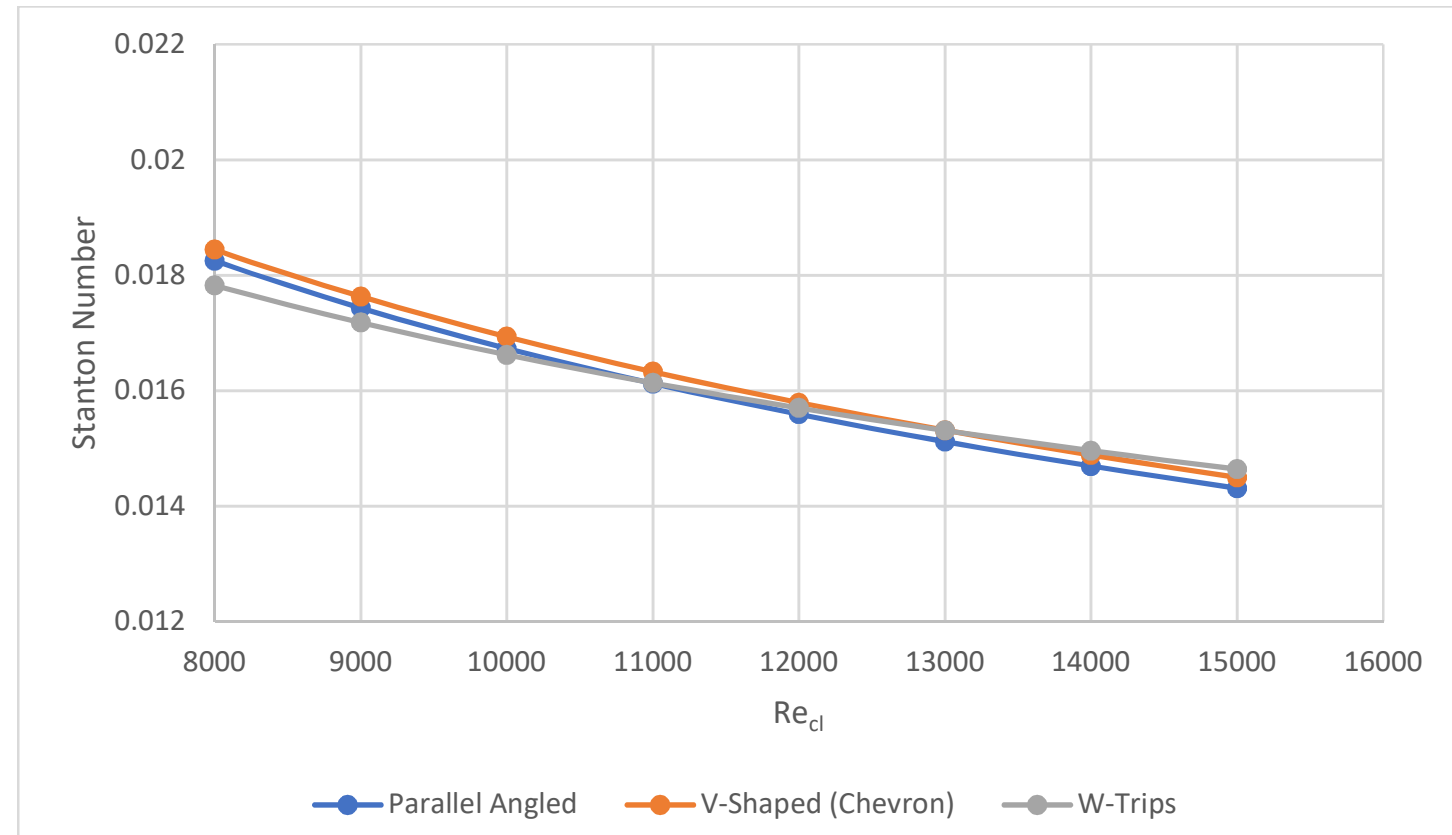

Figure 136: Mid-Chord Rib cooling performance of the analysis cases are compared by using their correlations

From the definition of the Stanton number, the internal cooling method that provides the lowest Stanton number at the same Reynolds number provides lowest coolant heating, due to reduced convection heat transfer rate. From Figure 136, up to a coolant Reynolds number of 12000, W-Trips provides better performance, whereas for the higher coolant Reynolds numbers, V-Shaped (Chevron) and Parallel Angled configurations provides better performance than W-Trips.

\subsection{High By-Pass Turbofan Engine}

For the generic case of high by-pass turbofan engine, the results are obtained by fixing the high pressure turbine exit temperature to $2030^{\circ} \mathrm{R}$ and using cooling analysis inputs from Table 40.

Calculated values for coolant Reynolds numbers with the coolant supply temperatures, Stanton numbers and the internal cooling efficiencies for different rib cooling configurations are given in Table 44.

Table 44: Variations in the mid-chord Stanton number and cooling flow effectiveness with different rib cooling configurations

\begin{tabular}{|l|l|l|l|l|l|l|}
\hline & \multicolumn{3}{|c|}{ Conventional } & \multicolumn{3}{c|}{ AVH } \\
\hline & $\begin{array}{l}\text { V-Shaped } \\
\text { (Chevron) }\end{array}$ & W-Trips & $\begin{array}{l}\text { Parallel } \\
\text { Angled }\left(45^{\circ}\right)\end{array}$ & $\begin{array}{l}\text { V-Shaped } \\
\text { (Chevron) }\end{array}$ & W-Trips & $\begin{array}{l}\text { Parallel } \\
\text { Angled } \\
\left(45^{0}\right)\end{array}$ \\
\hline $\mathbf{R e}_{\mathrm{cl}}$ & 11115 & 11115 & 11115 & 11115 & 11115 & 11115 \\
\hline $\mathbf{S t}_{\text {midchord }}$ & 0.01624 & 0.01608 & 0.01625 & 0.01624 & 0.01608 & 0.01625 \\
\hline $\begin{array}{l}\boldsymbol{\varepsilon}_{\mathrm{c}} \\
\text { (stator/rotor) }\end{array}$ & $0.603 / 0.609$ & $0.605 / 0.611$ & $0.604 / 0.61$ & $0.61 / 0.618$ & $0.613 / 0.621$ & $0.611 / 0.619$ \\
\hline
\end{tabular}

From the results of the sensitivity analysis, it is expected to have lowest coolant fraction with the method that has the highest internal cooling flow effectiveness. Therefore, it is expected to obtain the lowest cooling fractions with the W-Trips, followed by parallel angled and V-shaped, as shown in Figure 137. For the configurations with $\mathrm{AVH}$, due to higher blade film cooling effectiveness coolant fractions are lower. 


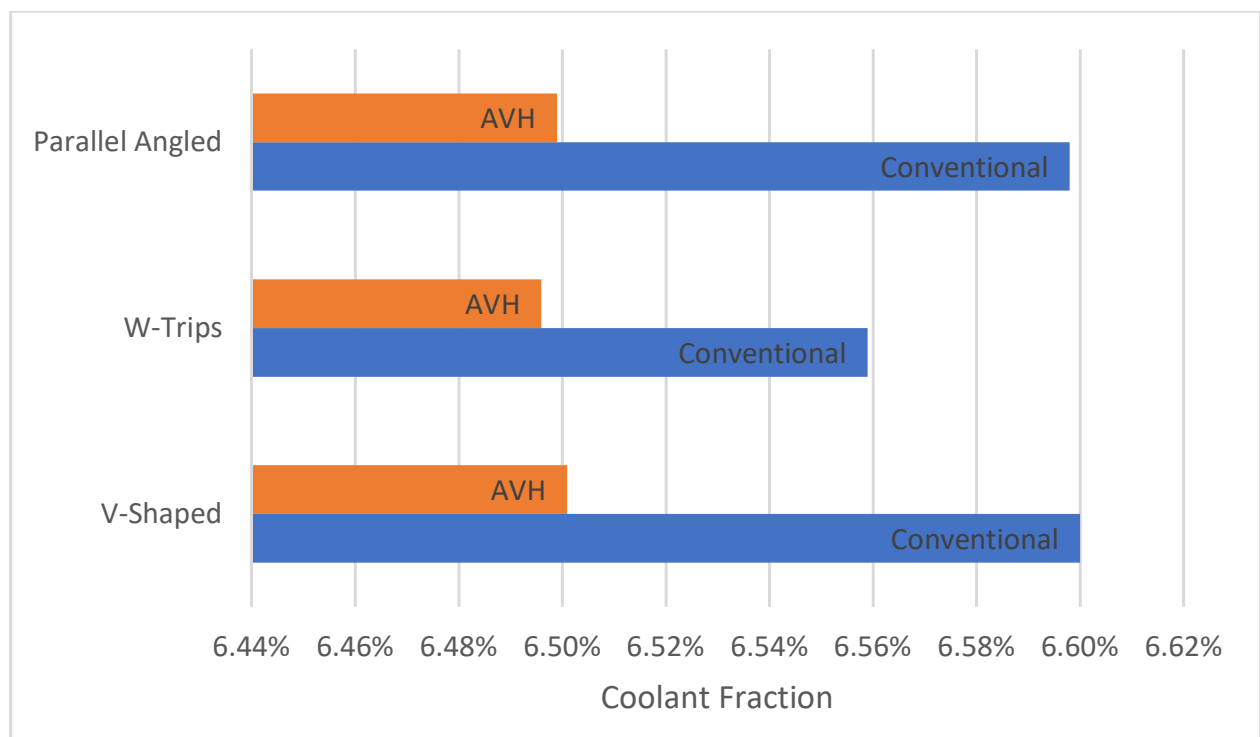

Figure 137: Total coolant fractions are compared for different rib cooling cases of the aero-engine case

The change in the total coolant fractions is within $0.04 \%$ and occurred same for both film cooling cases. As the coolant fractions are reduced, reduction in the turbine cooling losses results in higher engine thrust. This expected trend is obtained for both conventional hole and AVH equipped cases, as shown in Figure 138.

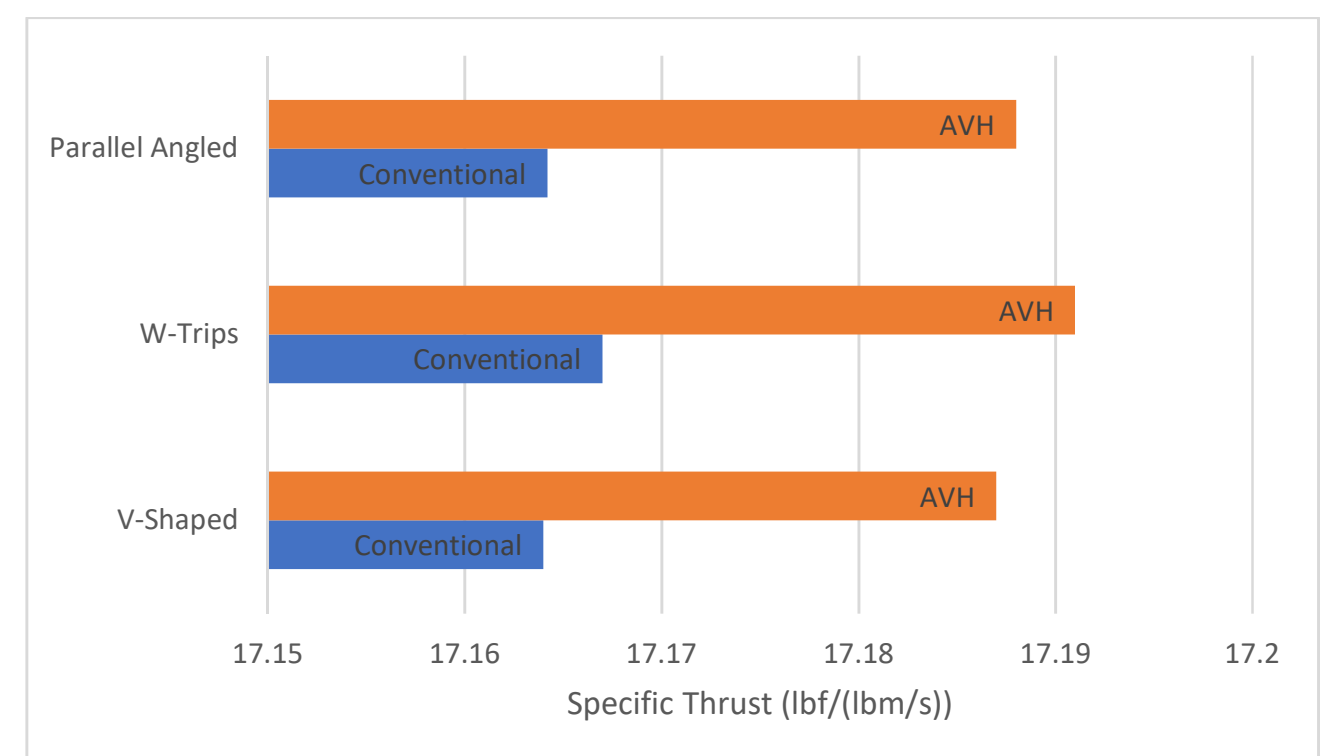

Figure 138: Specific thrust values are compared for different rib cooling cases of the aero-engine case

The changes in the specific thrust with the change in rib configuration is occurred within $0.02 \%$ and it is same for both film cooling cases. Reduction in the coolant fractions are very small, therefore the configuration with the highest thrust production is expected to have the lowest thrust specific fuel consumption, as shown in Figure 139. 


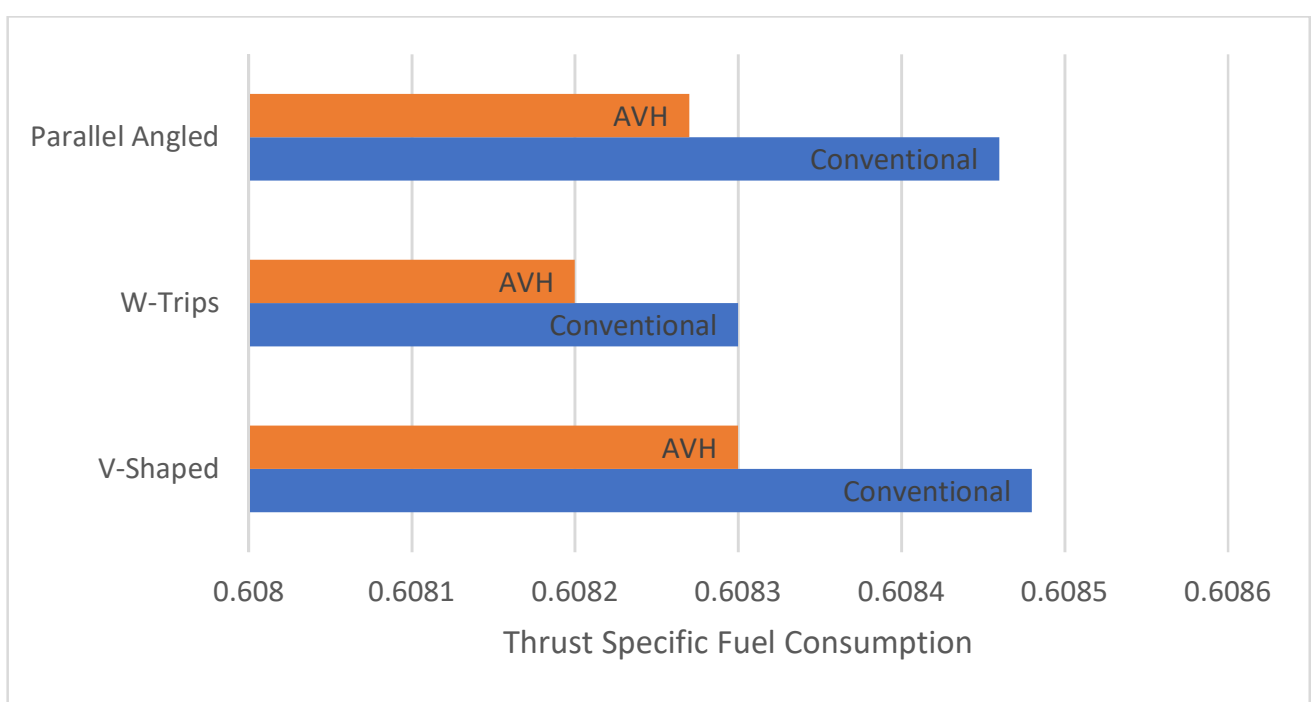

Figure 139: Thrust Specific Fuel Consumption values are compared for different rib cooling cases of the aero-engine case

Changes in the thrust specific fuel consumption occurred within $0.02 \%$ and same for both film cooling cases, indicating that the increase in fuel consumption is negligible. Corresponding changes in the engine thermal efficiencies are compared in Figure 140.

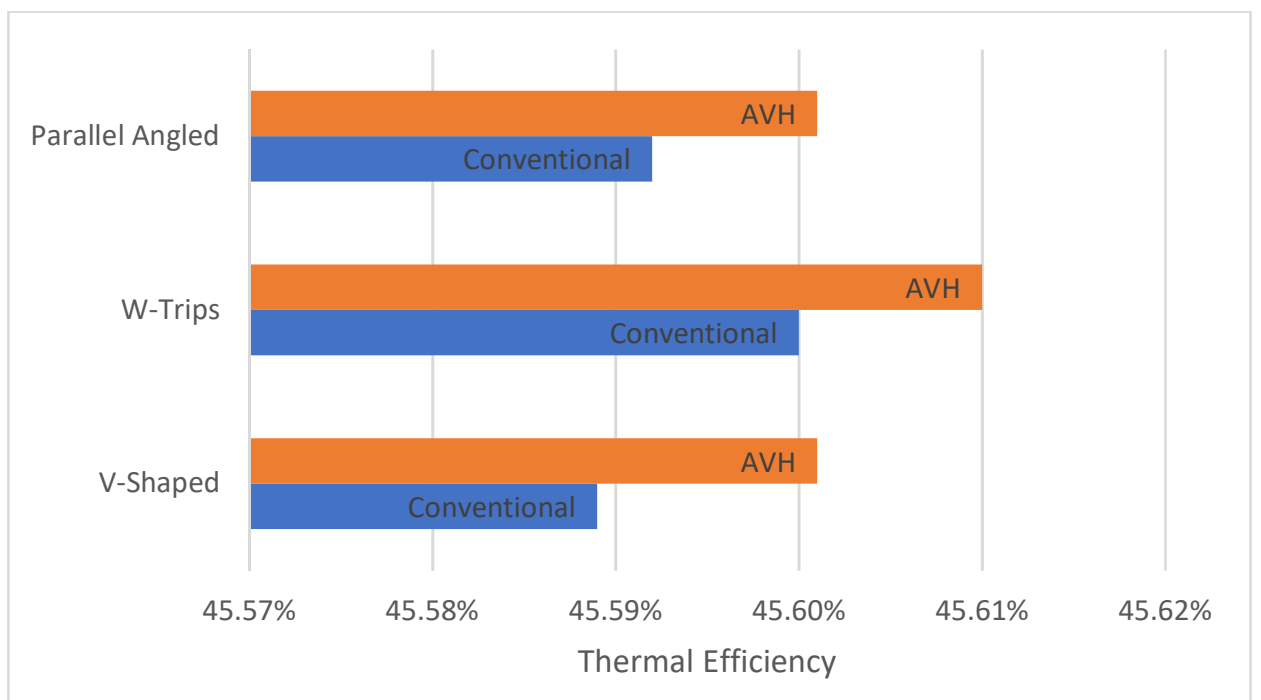

Figure 140: Thermal Efficiencies are compared for different rib cooling cases of the aero-engine case

The effect on the thermal efficiency from using different rib cooling techniques occurred within $0.01 \%$ for both film cooling configurations.

Although the relative changes to key engine performance parameters are very small, the results obtained in this section gives information about the rankings of the overall impact from different rib cooling techniques.

\subsection{Industrial Gas Turbine}

For the generic case of industrial gas turbine, the results are obtained by fixing the turbine exit temperature to $1620^{\circ} \mathrm{R}$ and using cooling analysis inputs from Table 41. 
In the case of industrial gas turbines, the number of cooled stages are higher than aero-engines and the coolant Reynolds numbers are higher in later stages due to reduction in the coolant supply temperatures (due to earlier stage extraction). For the particular case considered in this analysis, coolant Reynolds number for the second, third and fourth stages are obtained higher than 12000, which resulted in a change in the ranking of mid-chord Stanton numbers obtained for different rib cooling methods. For each stage, the coolant Reynolds numbers, Stanton numbers and cooling flow efficiencies are provided in Table 47.

Table 45: Variations in the mid-chord Stanton number and cooling flow effectiveness with different rib cooling configurations

\begin{tabular}{|c|c|c|c|c|c|c|c|}
\hline \multirow[b]{2}{*}{ Stage\# } & \multirow{2}{*}{ Parameter } & \multicolumn{3}{|c|}{ Conventional } & \multicolumn{3}{|c|}{ AVH } \\
\hline & & $\begin{array}{l}\text { V-Shaped } \\
\text { (Chevron) }\end{array}$ & W-Trips & $\begin{array}{l}\text { Parallel } \\
\text { Angled } \\
\left(45^{\circ}\right)\end{array}$ & $\begin{array}{l}\text { V-Shaped } \\
\text { (Chevron) }\end{array}$ & W-Trips & $\begin{array}{l}\text { Parallel } \\
\text { Angled } \\
\left(45^{\circ}\right)\end{array}$ \\
\hline \multirow{3}{*}{1} & $\operatorname{Re}_{c l}$ & 9719 & 9719 & 9719 & 9719 & 9719 & 9719 \\
\hline & St $t_{\text {midchord }}$ & 0.01712 & 0.01677 & 0.01692 & 0.01712 & 0.01677 & 0.01692 \\
\hline & $\begin{array}{l}\varepsilon_{\mathrm{c}} \\
\text { (stator/rotor) }\end{array}$ & $0.57 / 0.59$ & $0.571 / 0.591$ & $0.57 / 0.59$ & $0.57 / 0.59$ & $0.571 / 0.591$ & $0.57 / 0.59$ \\
\hline \multirow{3}{*}{2} & $\operatorname{Re}_{c l}$ & 12530 & 12530 & 12530 & 12530 & 12530 & 12530 \\
\hline & $S t_{\text {midchord }}$ & 0.01533 & 0.01549 & 0.01533 & 0.01533 & 0.01549 & 0.01533 \\
\hline & $\begin{array}{l}\varepsilon_{\mathrm{c}} \\
\text { (stator/rotor) }\end{array}$ & $0.573 / 0.593$ & $0.572 / 0.592$ & $0.573 / 0.593$ & $0.573 / 0.593$ & $0.572 / 0.592$ & $0.573 / 0.593$ \\
\hline \multirow{3}{*}{3} & $\operatorname{Re}_{c l}$ & 14270 & 14270 & 14270 & 14270 & 14270 & 14270 \\
\hline & St $t_{\text {midchord }}$ & 0.01478 & 0.01487 & 0.01459 & 0.01478 & 0.01487 & 0.01459 \\
\hline & $\begin{array}{l}\varepsilon_{\mathrm{c}} \\
\text { (stator/rotor) }\end{array}$ & $0.574 / 0.594$ & $0.572 / 0.592$ & $0.575 / 0.595$ & $0.574 / 0.594$ & $0.572 / 0.592$ & $0.575 / 0.595$ \\
\hline \multirow{3}{*}{4} & $\operatorname{Re}_{c l}$ & 14270 & 14270 & 14270 & 14270 & 14270 & 14270 \\
\hline & St $t_{\text {midchord }}$ & 0.01478 & 0.01487 & 0.01459 & 0.01478 & 0.01487 & 0.01459 \\
\hline & $\begin{array}{l}\varepsilon_{\mathrm{c}} \\
\text { (stator/rotor) }\end{array}$ & $0.574 / 0.594$ & $0.572 / 0.592$ & $0.575 / 0.595$ & $0.574 / 0.594$ & $0.572 / 0.592$ & $0.575 / 0.595$ \\
\hline
\end{tabular}

From Table 47, although the W-Trips provided higher internal cooling flow efficiencies in the first stage, increase in the coolant Reynolds number in the later stages caused other two rib configurations to perform better, resulting in an overall internal cooling performance ranking as parallel angled to be the highest, followed by the V-Shaped and the W-Trips being the last. From the results of the sensitivity analysis, the reduction in the total coolant requirement should follow the opposite order with internal cooling performance, which is shown in Figure 141. 


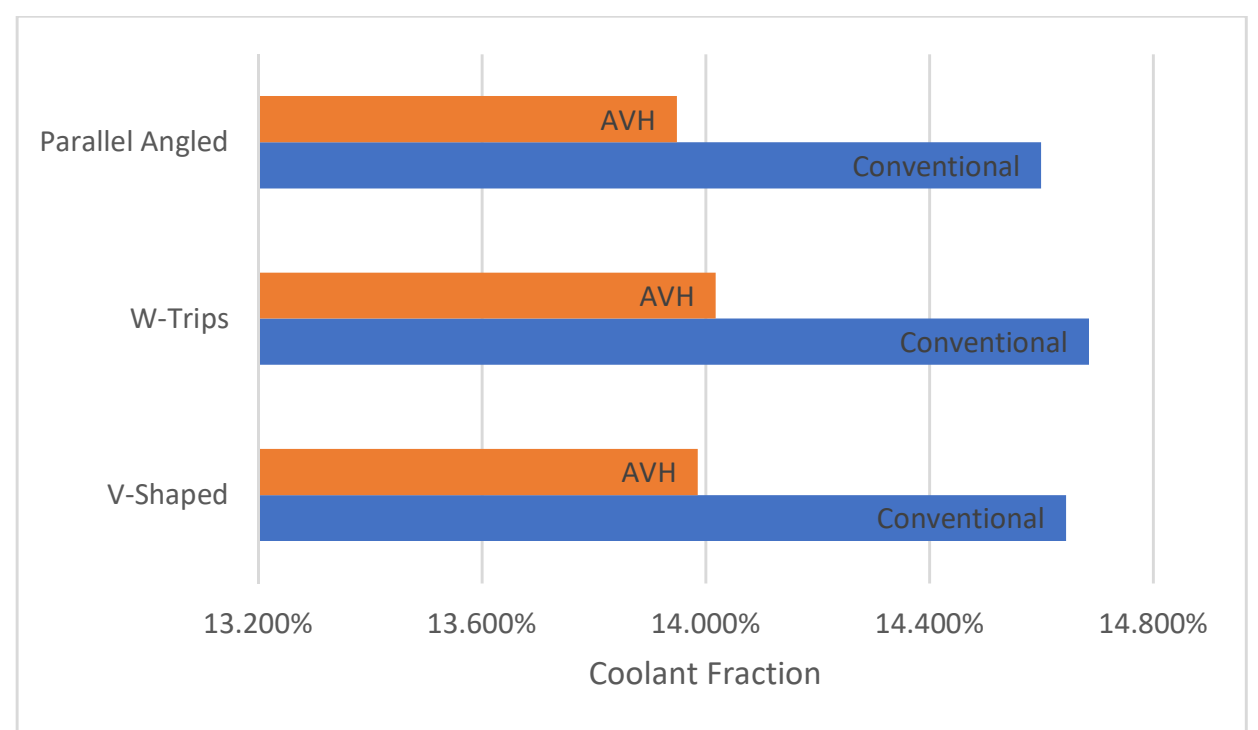

Figure 141: Total coolant fractions are compared for different rib cooling cases of the industrial gas turbine case

The reduction in the total coolant fraction occurred as $0.09 \%$ and $0.07 \%$ for the conventional and AVH cases, respectively. The impact of the reduction in the coolant flow rates indicates a positive change due to reduced turbine losses and increased compressor performance. Resulting changes on the shaft power delivered is shown in Figure 142.

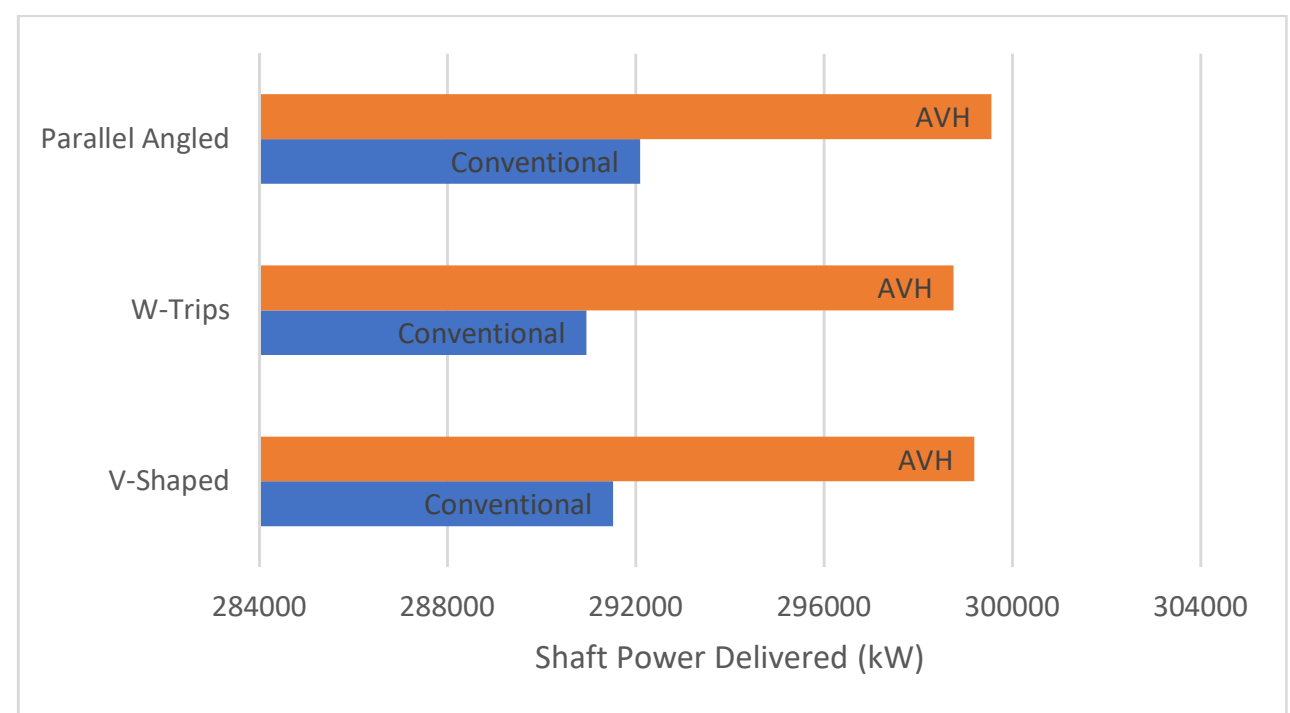

Figure 142: Shaft power values are compared for different rib cooling cases of the industrial gas turbine case

The changes in the power production occurred as $0.39 \%$ and $0.33 \%$ for the conventional and AVH cases, respectively. The impact of the rib cooling type on the heat rate is compared in Figure 143. 


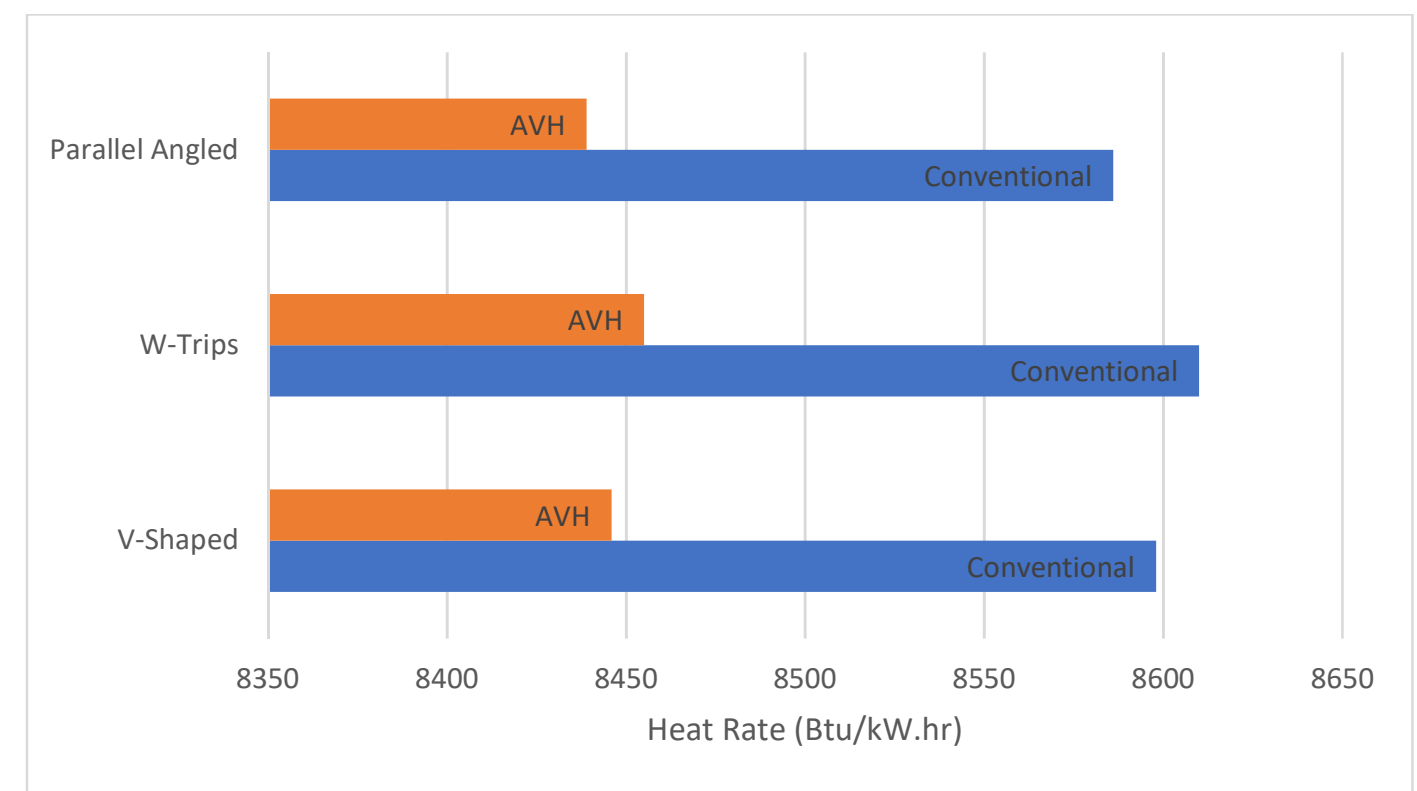

Figure 143: Heat Rate values are compared for different rib cooling cases of the industrial gas turbine case

The impact on the heat rates occurred within $0.3 \%$ and $0.2 \%$ for the conventional hole and AVH cases, respectively. Corresponding changes in the thermal efficiencies are shown in Figure 144.



Figure 144: Thermal Efficiencies are compared for different rib cooling cases of the industrial gas turbine case

The changes in thermal efficiencies with different rib cooling techniques are occurred within $0.11 \%$ and $0.07 \%$ for the conventional and $\mathrm{AVH}$ cases, respectively.

\subsubsection{Trailing Edge Cooling Configuration}

For the trailing edge cooling configurations, three different methods are selected for the analysis, which are inline circular, staggered circular and inline diamond pedestal configurations. Hydraulic diameter value is used as the same in all configurations and taken as 0.08 . Similar to the mid-chord analysis, the changes in the engine performance is caused by the changes in the trailing edge internal Stanton number that affects the 
internal cooling flow effectiveness. However, due to lower chord percentile used for the trailing edge cooling than the mid-chord cooling, the impact from this parameter on the engine performance is expected to be lower. The analyses in this section are made for two different film cooling cases (i.e. conventional hole and $\mathrm{AVH}$ ) in order to observe the effect of using different adiabatic wall temperatures on the trailing edge cooling performance.

The internal cooling correlations used for the trailing edge cooling cases for the same hydraulic diameter of 0.08 and within the same Reynolds number ranges are compared in Figure 145.



Figure 145: Trailing edge pin-fin cooling performance of the analysis cases are compared by using their correlations

Similar to the mid-chord cases, the method that provides the lowest Stanton number for the same coolant Reynolds number gives the highest internal cooling flow effectiveness. Unlike for the mid-chord, due to not having a pin-fin performance trend change after a certain coolant Reynolds number, same impact on the engine performance parameters is expected in both aero-engine and industrial gas turbine cases.

\subsection{High By-Pass Turbofan Engine}

For the generic case of high by-pass turbofan engine, the results are obtained by fixing the high pressure turbine exit temperature to $2030^{\circ} \mathrm{R}$ and other cooling analysis inputs are used from Table 40 . In the advanced coolant calculator, same coolant Reynolds number is used for the entire blade, therefore the coolant Reynolds number is 11115 as in the rib analysis cases. Calculated values for coolant Stanton number and internal cooling flow efficiencies for different pin-fin cooling cases are shown in Table 46.

Table 46: Variations in the trailing edge Stanton number and cooling flow effectiveness with different pin-fin cooling configurations

\begin{tabular}{|l|l|l|l|l|l|l|}
\hline & \multicolumn{3}{|c|}{ Conventional } & \multicolumn{3}{c|}{ AVH } \\
\hline & $\begin{array}{l}\text { Circular } \\
\text { (inline) }\end{array}$ & $\begin{array}{l}\text { Circular } \\
\text { (staggered) }\end{array}$ & $\begin{array}{l}\text { Diamond } \\
\text { Pedestal }\end{array}$ & $\begin{array}{l}\text { Circular } \\
\text { (inline) }\end{array}$ & $\begin{array}{l}\text { Circular } \\
\text { (staggered) }\end{array}$ & $\begin{array}{l}\text { Diamond } \\
\text { Pedestal }\end{array}$ \\
\hline $\mathbf{R e}_{\mathrm{cl}}$ & 11115 & 11115 & 11115 & 11115 & 11115 & 11115 \\
\hline $\mathbf{S t}_{\text {trailingedge }}$ & 0.01353 & 0.01279 & 0.01128 & 0.01353 & 0.01279 & 0.01128 \\
\hline $\begin{array}{l}\boldsymbol{\varepsilon}_{\mathrm{c}} \\
\text { (stator/rotor) }\end{array}$ & $0.603 / 0.609$ & $0.6032 / 0.6091$ & $0.6033 / 0.6092$ & $0.61 / 0.618$ & $0.6102 / 0.6182$ & $0.6103 / 0.6183$ \\
\hline
\end{tabular}


Reduction in the coolant Stanton number improves internal cooling flow effectiveness due to reduced coolant heating in the trailing edge region. Similar to the mid-chord analysis results, it is expected to obtain the lowest coolant requirement with the method that provides lowest Stanton number. This expected trend in the coolant fractions are obtained similar in this analysis, and the coolant fraction comparison is shown in Figure 146.



Figure 146: Total coolant fractions are compared for different pin-fin cooling cases of the aero-engine case

The change in the total coolant fractions with the changes in pin-fin cooling method is occurred within $0.02 \%$ for both hole geometry cases. The improvement in the turbine performance with these changes results in obtaining highest thrust with the diamond pedestal case, as shown in Figure 147.

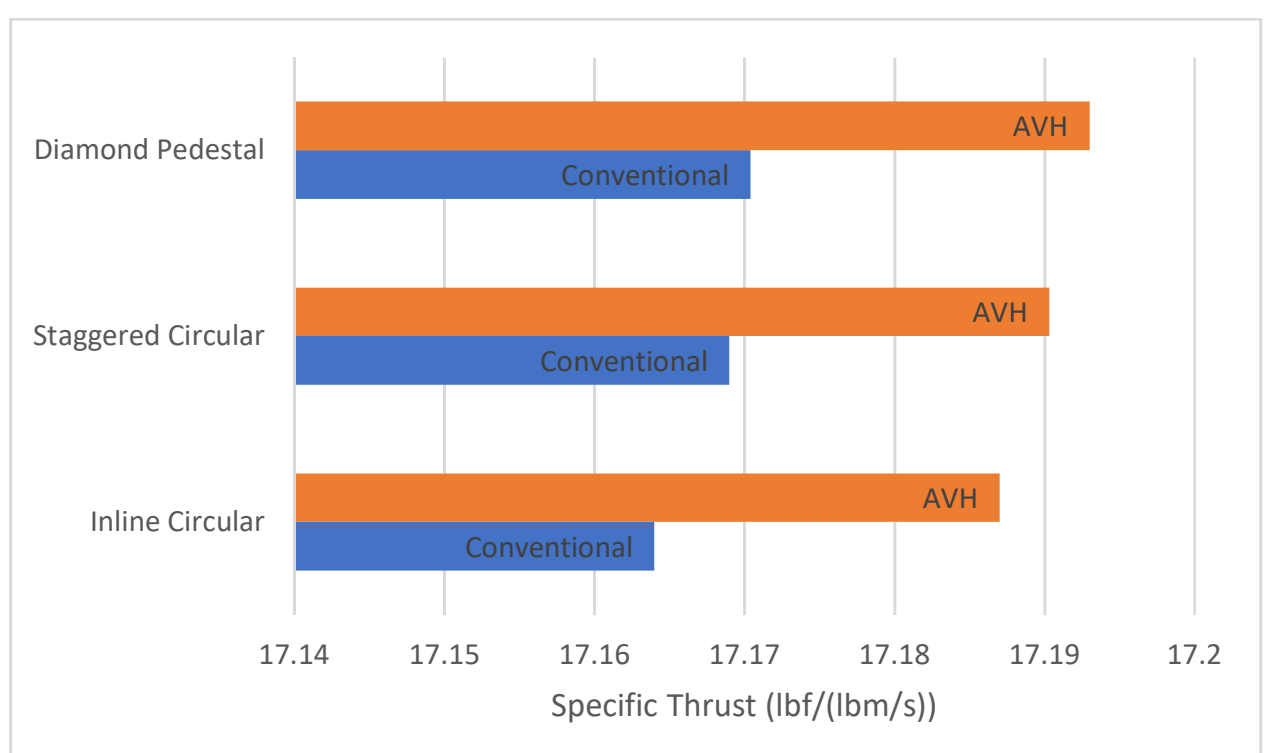

Figure 147: Specific thrust values are compared for different pin-fin cooling cases of the aero-engine case

The improvement in the specific thrust from inline circular configuration to diamond pedestal configuration occurred as $0.03 \%$ for both hole geometry cases. Similar for the mid-chord cases, it is expected to have lowest thrust specific fuel consumption with the highest thrust providing method, as shown in Figure 148. 


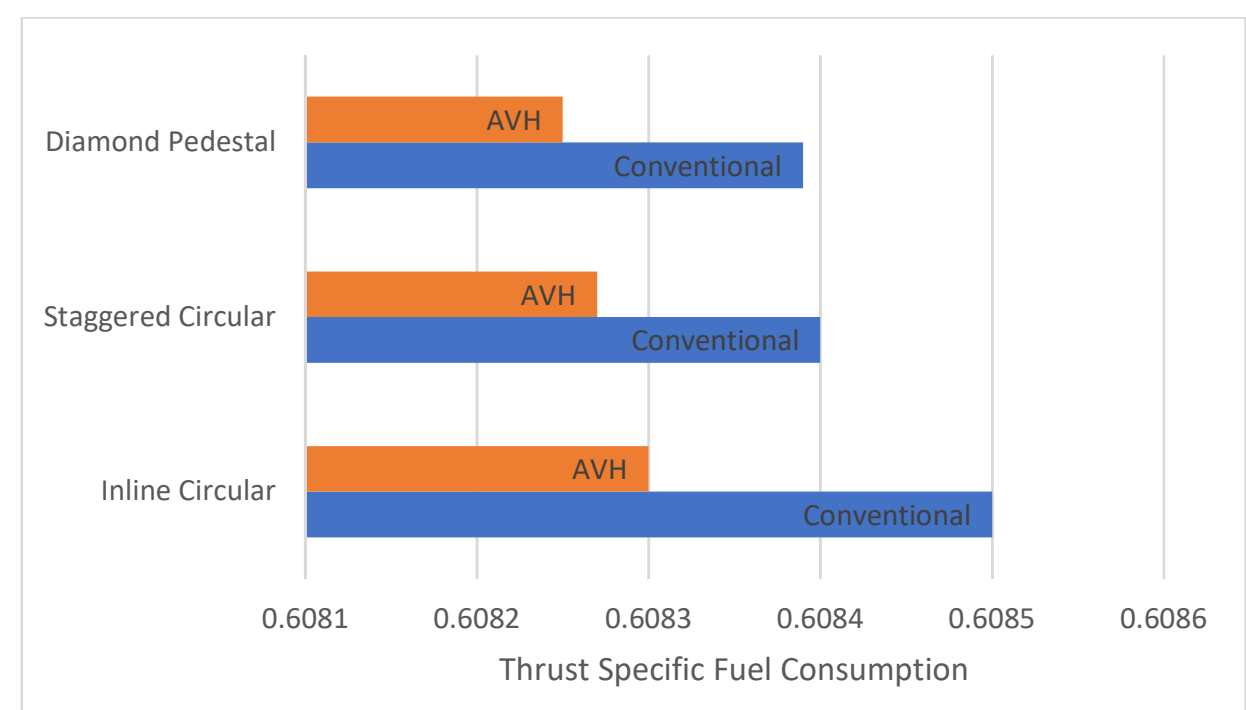

Figure 148: Thrust Specific Fuel Consumption values are compared for different pin-fin cooling cases of the aero-engine case

The reduction in the thrust specific fuel consumption from circular inline configuration to diamond pedestal is within $0.01 \%$ for both film cooling hole cases. Improvement in the engine thermal efficiency is shown in Figure 149.



Figure 149: Thermal efficiencies are compared for different pin-fin cooling cases of the aero-engine case

The changes in the thermal efficiency occurred as $0.01 \%$ for both hole geometry cases, indicating the improvement comes entirely from the reduction in the thrust specific fuel consumption.

Although the relative changes to key engine performance parameters are very small, the results obtained in this section gives information about the rankings of the overall impact from different pin-fin cooling techniques. 


\subsection{Industrial Gas Turbine}

For the generic case of industrial gas turbine, the results are obtained by fixing the turbine exit temperature to $1620^{\circ} \mathrm{R}$ and using cooling analysis inputs from Table 39. For each cooled stage, calculated values for coolant Stanton numbers and internal cooling flow efficiencies for different pin-fin cooling cases are shown in Table 47.

Table 47: Variations in the mid-chord Stanton number and cooling flow effectiveness with different pin-fin cooling configurations

\begin{tabular}{|c|c|c|c|c|c|c|c|}
\hline \multirow[b]{2}{*}{ Stage\# } & \multirow[t]{2}{*}{ Parameter } & \multicolumn{3}{|c|}{ Conventional } & \multicolumn{3}{|c|}{ AVH } \\
\hline & & $\begin{array}{l}\text { Circular } \\
\text { (inline) }\end{array}$ & $\begin{array}{l}\text { Circular } \\
\text { (staggered) }\end{array}$ & $\begin{array}{l}\text { Diamond } \\
\text { Pedestal }\end{array}$ & $\begin{array}{l}\text { Circular } \\
\text { (inline) }\end{array}$ & $\begin{array}{l}\text { Circular } \\
\text { (staggered) }\end{array}$ & $\begin{array}{l}\text { Diamond } \\
\text { Pedestal }\end{array}$ \\
\hline \multirow{3}{*}{1} & $\operatorname{Re}_{c 1}$ & 9719 & 9719 & 9719 & 9719 & 9719 & 9719 \\
\hline & $S t_{\text {midchord }}$ & 0.01425 & 0.01355 & 0.0119 & 0.01425 & 0.01355 & 0.0119 \\
\hline & $\begin{array}{l}\varepsilon_{\mathrm{c}} \\
\text { (stator/rotor) }\end{array}$ & $0.57 / 0.59$ & $0.5701 / 0.59$ & $0.5702 / 0.5902$ & $0.57 / 0.59$ & $0.5701 / 0.59$ & $0.5702 / 0.5902$ \\
\hline \multirow{3}{*}{2} & $\mathrm{Re}_{\mathrm{cl}}$ & 12530 & 12530 & 12530 & 12530 & 12530 & 12530 \\
\hline & Stmidchord & 0.01291 & 0.01215 & 0.01075 & 0.01533 & 0.01549 & 0.01533 \\
\hline & $\begin{array}{l}\varepsilon_{\mathrm{c}} \\
\text { (stator/rotor) }\end{array}$ & $0.5701 / 0.5901$ & $0.5701 / 0.5901$ & $0.5703 / 0.5903$ & $0.5701 / 0.5901$ & $0.5701 / 0.5901$ & $0.5703 / 0.5903$ \\
\hline \multirow{3}{*}{3} & $\operatorname{Re}_{\mathrm{cl}}$ & 14270 & 14270 & 14270 & 14270 & 14270 & 14270 \\
\hline & $S t_{\text {midchord }}$ & 0.01227 & 0.01149 & 0.01021 & 0.01478 & 0.01487 & 0.01459 \\
\hline & $\begin{array}{l}\varepsilon_{\mathrm{c}} \\
\text { (stator/rotor) }\end{array}$ & $0.5702 / 0.5902$ & $0.5703 / 0.5903$ & $0.5703 / 0.5904$ & $0.5702 / 0.5902$ & $0.5703 / 0.5903$ & $0.5703 / 0.5904$ \\
\hline \multirow{3}{*}{4} & $\operatorname{Re}_{c 1}$ & 14270 & 14270 & 14270 & 14270 & 14270 & 14270 \\
\hline & $S t_{\text {midchord }}$ & 0.01227 & 0.01149 & 0.01021 & 0.01478 & 0.01487 & 0.01459 \\
\hline & $\begin{array}{l}\varepsilon_{\mathrm{c}} \\
\text { (stator/rotor) }\end{array}$ & $0.5702 / 0.5902$ & $0.5703 / 0.5903$ & $0.5703 / 0.5904$ & $0.5702 / 0.5902$ & $0.5703 / 0.5903$ & $0.5703 / 0.5904$ \\
\hline
\end{tabular}

Due to not having a different trend after a certain coolant Reynolds number, the impact from using different pin-fin cooling techniques on the performance parameters will be the same with aero-engine cases. Diamond pedestal technique is therefore expected to require the less coolant fractions, as shown in Figure 150.

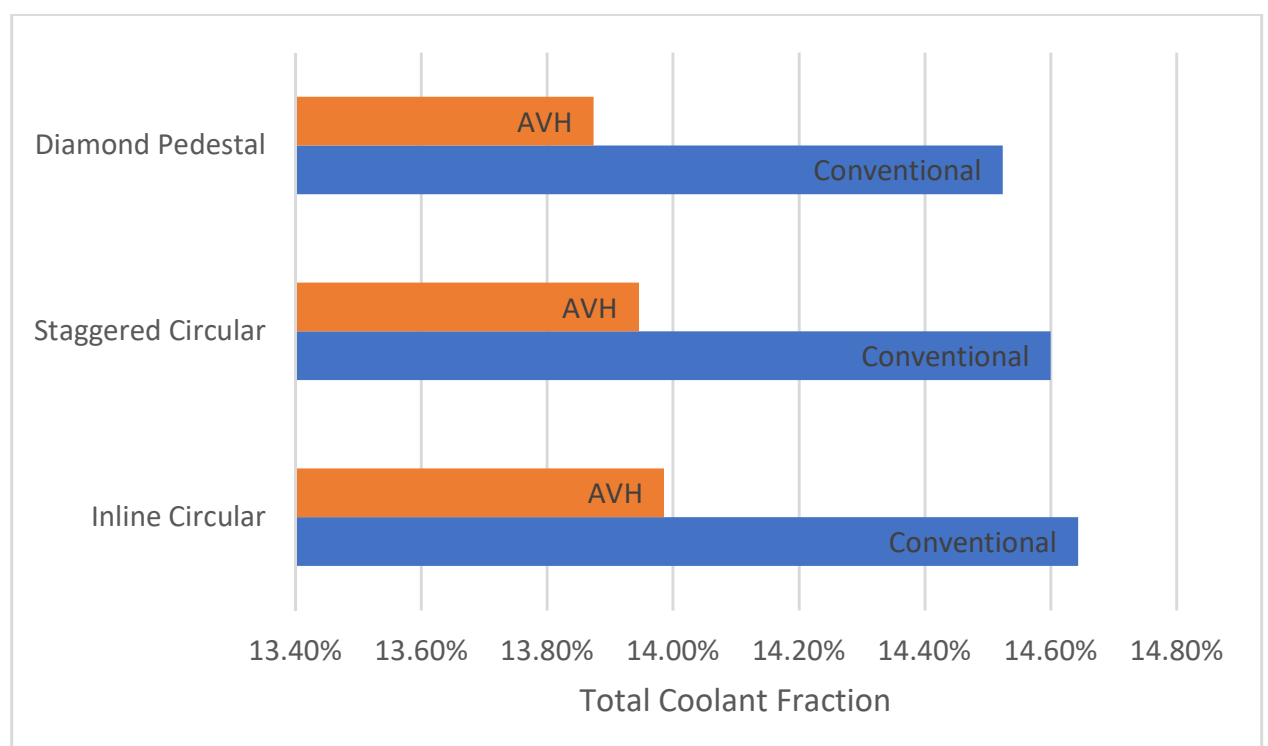

Figure 150: Total coolant fractions are compared for different pin-fin cooling cases of the industrial gas turbine case 
Using the diamond pedestal configuration instead of inline circular configuration reduces the total coolant fraction by $0.11 \%$ for both hole geometry cases. Reduction in the coolant flow rates results in an increase in the turbine polytropic efficiency and compressor performance, resulting in the trends shown for shaft power delivered in Figure 151.

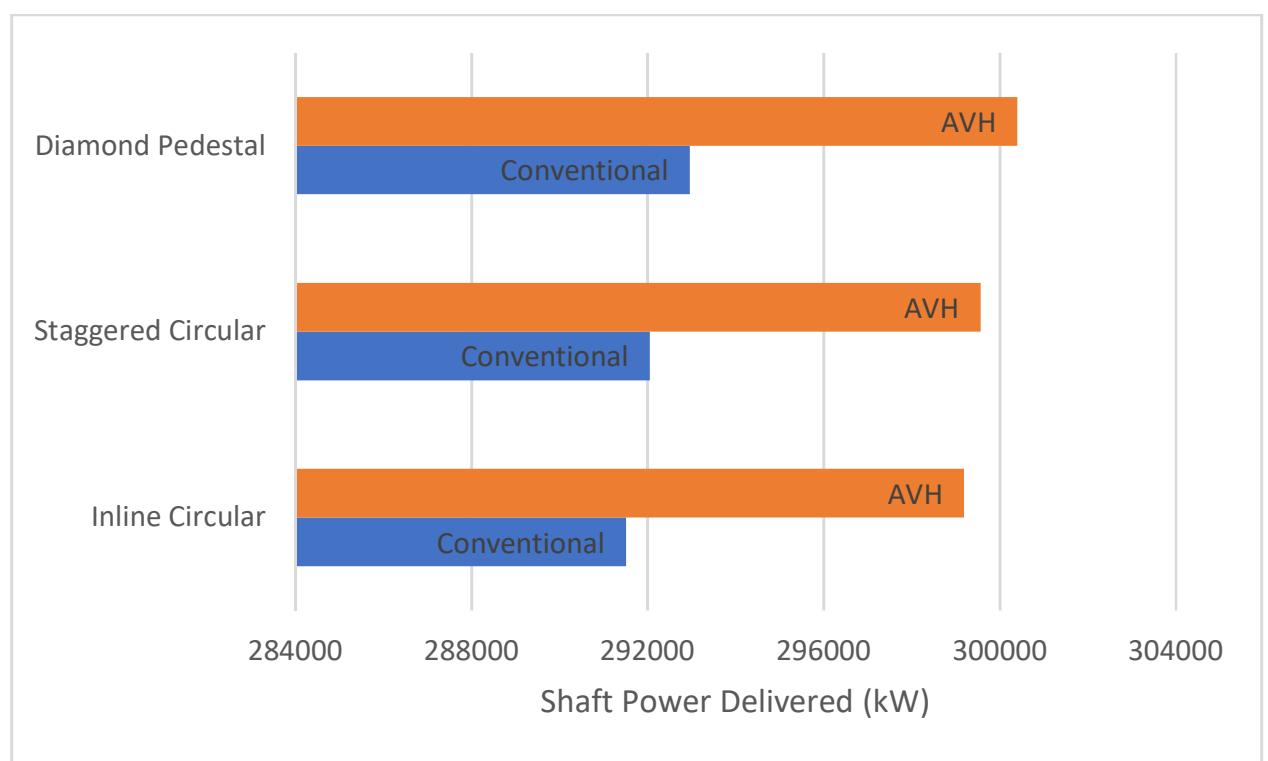

Figure 151: Shaft power values are compared for different pin-fin cooling cases of the industrial gas turbine case

The increase in the power production changing the pin-fin cooling method from inline circular to diamond pedestal type provided $0.4 \%$ increase in both film cooling types. Corresponding changes in the heat rate are compared in Figure 152.

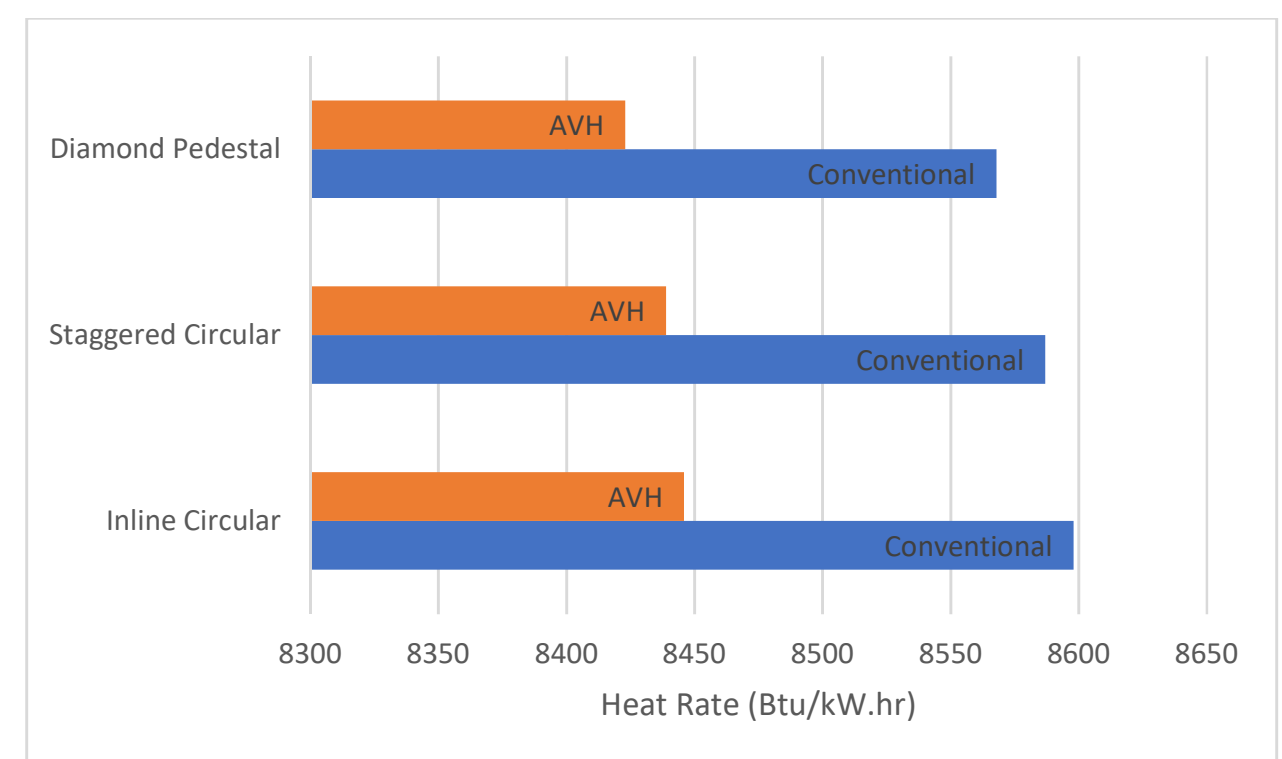

Figure 152: Heat rate values are compared for different pin-fin cooling cases of the industrial gas turbine case

The changes in the heat rate between different pin-fin configurations occurred within $0.3 \%$. Thermal efficiencies of the different pin-fin blade cooling configurations are compared in Figure 153. 


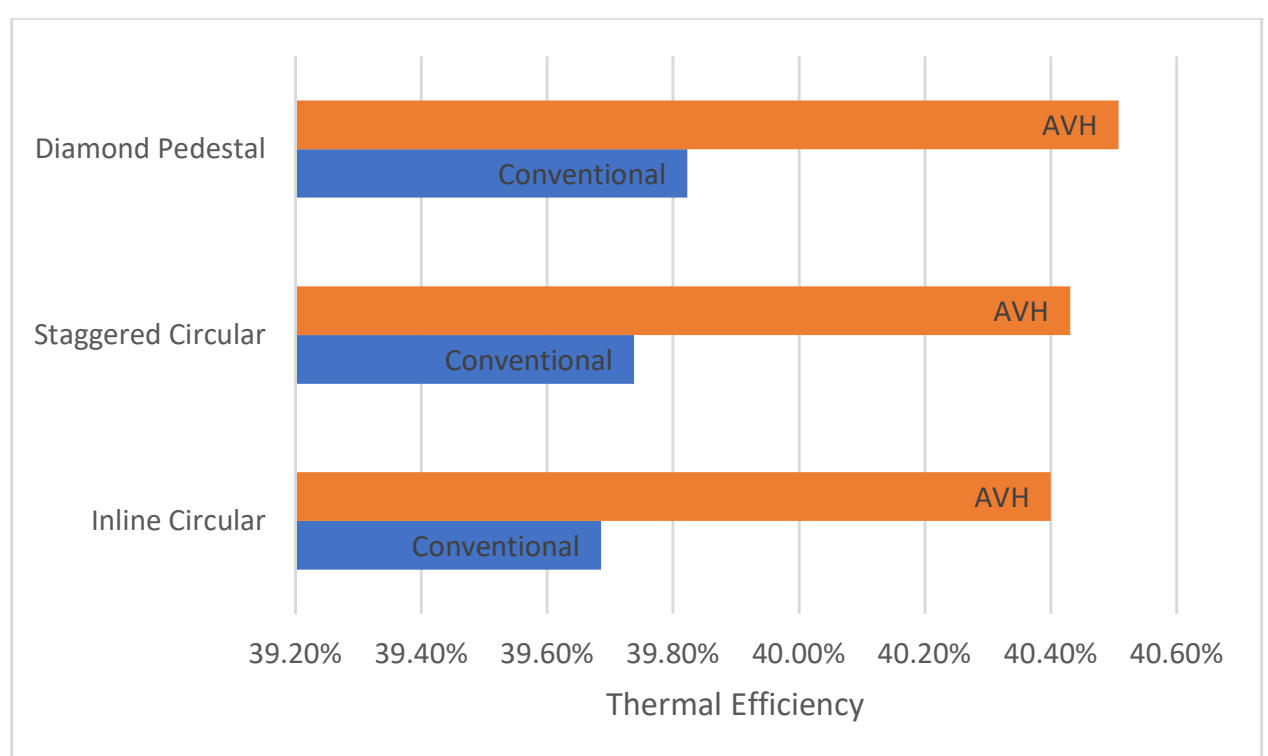

Figure 153: Thermal efficiencies are compared for different pin-fin cooling cases of the industrial gas turbine case

Improvement in the thermal efficiency by changing the pin-fin configuration from inline circular to diamond pedestal configuration is occurred as $0.13 \%$ and $0.11 \%$ for the conventional hole and AVH cases, respectively.

\subsection{Finding the Optimum Number of Film Cooling Rows}

Regardless of the engine type, increasing the number of film cooling hole rows up to a certain value would increase the engine performance due to increased blade film cooling effectiveness. This improvement is caused by locating a new coolant hole at an earlier blade span location, which is preferably the location where the coolant separation starts. However, using too many film cooling holes would also increase the required coolant flow rates, which in turn result in a reduction in the engine performance.

In this analysis, the optimum number of film cooling rows are found by increasing this value from 1 to 4 in the aero-engine blade for the pressure side ( 0 to 3 on the suction side), and 2 to 5 in the pressure side of the film cooled stages of the industrial gas turbine blade (1 to 4 on the suction side). In each case, showerhead cooling exists on the leading edge of the blade, therefore changing the number of rows affects only the number of film cooling rows used on the remainder of the blade. For the high by-pass turbofan engine, high turbulence intensity data for both conventional and AVH cases (from Figure 111 and Figure 114, respectively) are used, whereas for the industrial gas turbine case mid turbulence intensity data are used due to having slower flow speeds and larger flow area.

\subsubsection{High By-Pass Turbofan Engine}

For the generic case of high by-pass turbofan engine, the results are obtained by fixing the high pressure turbine exit temperature to $2030{ }^{\circ} \mathrm{R}$ and other cooling analysis inputs are used from Table 40 . The changes in the thermal efficiency and specific thrust are compared in Figure 154 for conventional hole and AVH film cooling on the blade body. 


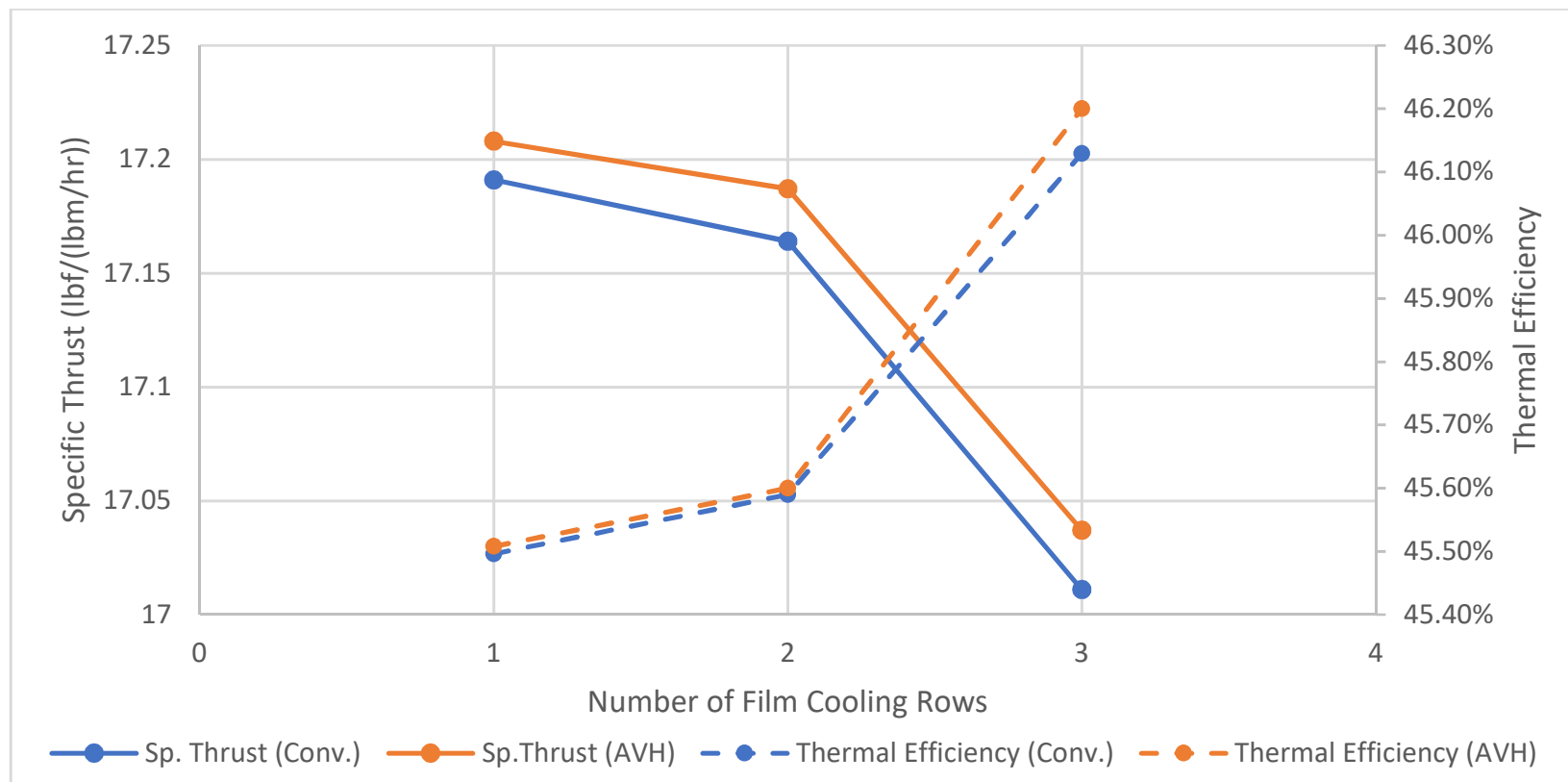

Figure 154: Specific thrust and thermal efficiencies obtained by increasing the number of film cooling rows are compared for aero-engine blade

In Figure 154, an optimum point occurred at 2 film cooling hole rows, determined by the point where the reduction in the specific thrust is lowest and the thermal efficiency is increasing. The increase in the thermal efficiency occurred due to the increase in the blade film cooling effectiveness, but the reduction in the specific thrust after 2 rows is resulting from the increased compressor power demand due to increased coolant fractions. The change in the coolant fractions and the thrust specific fuel consumption also indicates an optimum value at 2 film cooling rows as shown in Figure 155.

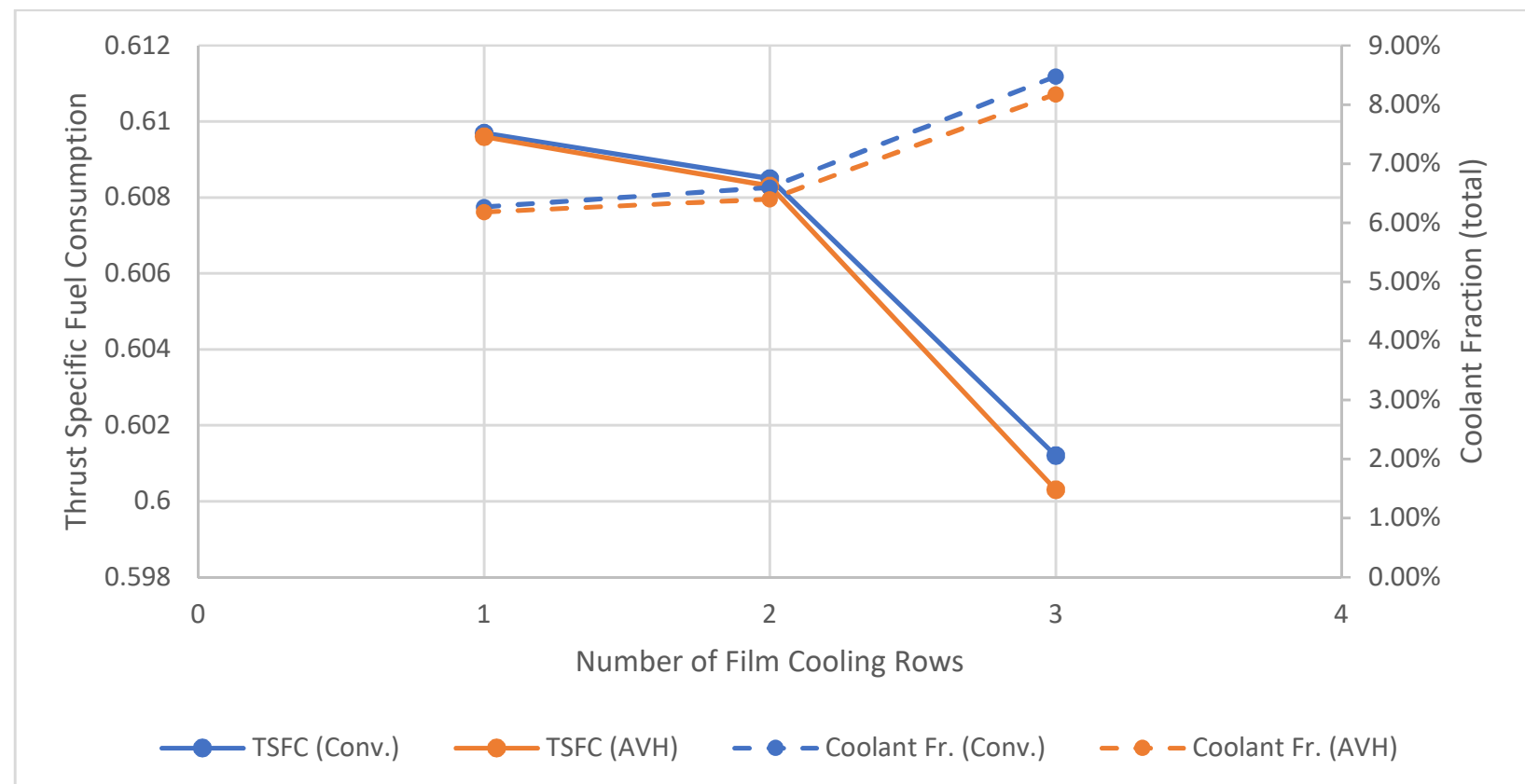

Figure 155: Changes in thrust specific fuel consumption and total coolant fractions when the number of film cooling rows are increased are compared for aero-engine blade 
The optimum number of film cooling rows is found as 2 , which is the same for both conventional and antivortex hole geometry cases. The impact on the engine performance is higher with AVH holes, due to providing higher film cooling effectiveness than the conventional hole.

\subsubsection{Industrial Gas Turbine}

For the generic case of industrial gas turbine, the results are obtained by fixing the turbine exit temperature to $1620^{\circ} \mathrm{R}$ and using cooling analysis inputs from Table 41. The changes in the shaft power delivered and the heat rate are compared in Figure 156 for conventional hole and $\mathrm{AVH}$ film cooling configurations.

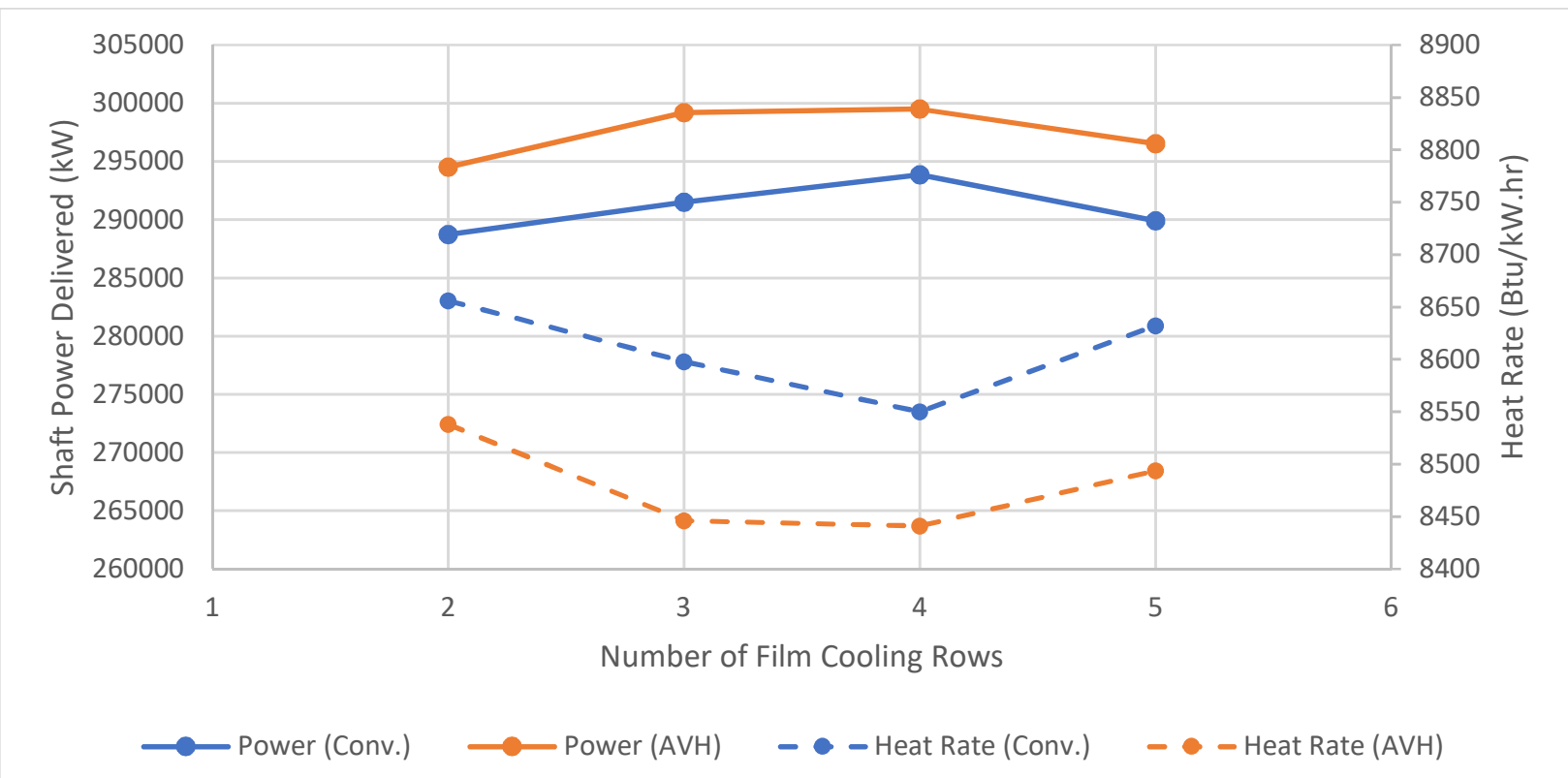

Figure 156: Shaft power delivered and heat rates obtained by increasing the number of film cooling rows are compared for industrial gas turbine blades

For the conventional hole case, a distinct optimum at 4 cooling rows occurs at the point where the power production is maximum and the heat rate is the lowest. Whereas for the AVH blades, the change in the performance between 3 and 4 holes is very small, but by considering the manufacturing costs and flow separation on the blades, using $3 \mathrm{AVH}$ rows would give the best performance. The changes in the engine power and the heat rate is affected from the changes in the required coolant fractions, which is compared with the changes in the thermal efficiency as shown in Figure 157.

Analyzing the changes in Figure 157 indicates an optimum at 4 cooling rows for conventional hole configuration, whereas the flat change between 3 and 4 rows for the $\mathrm{AVH}$ case indicates reducing the number of holes from 4 to 3 will not degrade the engine performance. 


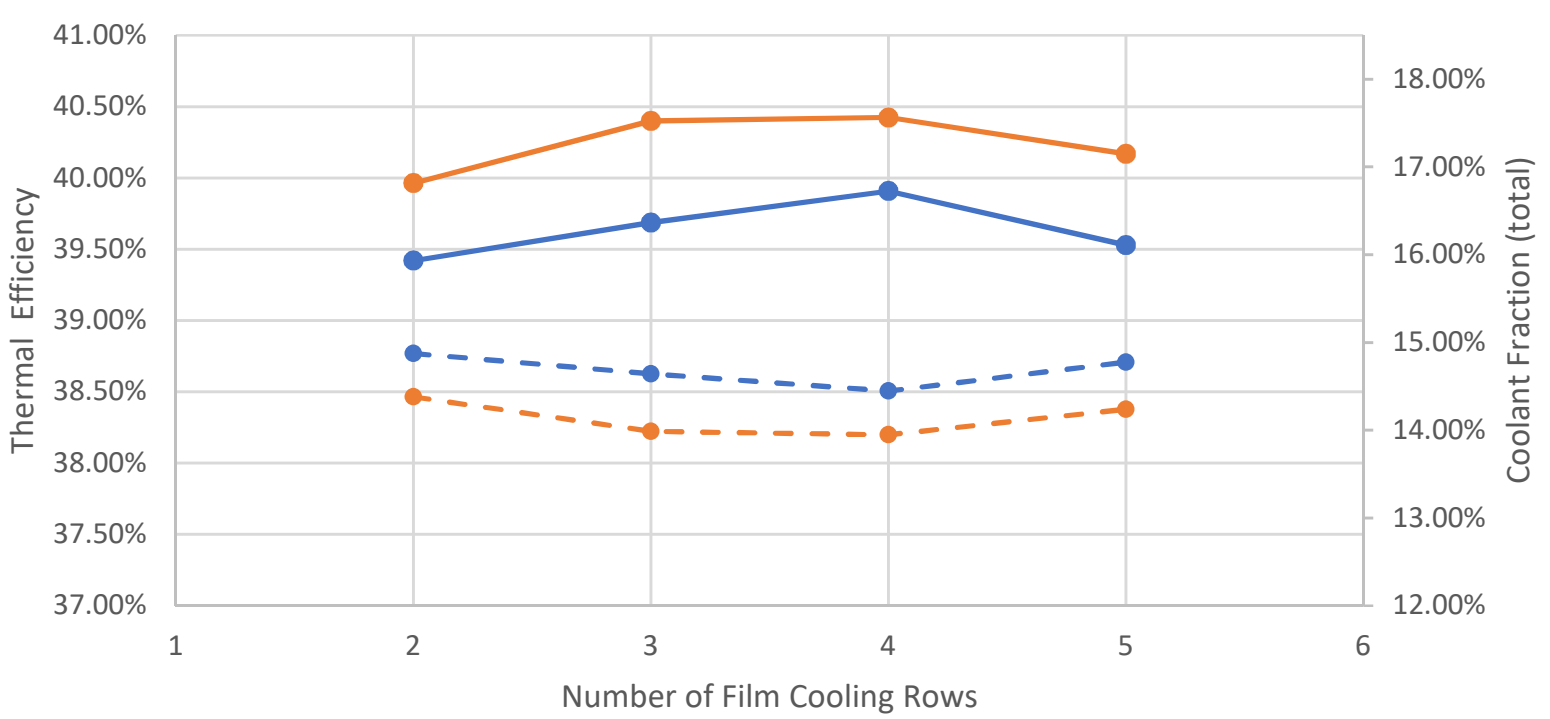

$\longrightarrow$ Thermal Eff. (Conv) - Thermal Eff. (AVH) - - Coolant Fr. (Conv.) - - Coolant Fr. (AVH)

Figure 157: Thermal efficiency and coolant fractions when the number of film cooling rows are increased are compared for industrial gas turbine blade 


\section{CONCLUSION}

In this research, a multi-stage cooled turbine model was developed from the theory for a single cooled turbine stage of Young and Wilcock [7]. The model was then integrated into engine performance calculation models for high by-pass turbofan engines (the Cooled Engine Design Model, CEDM) and industrial gas turbines (the Cooled Gas Turbine Model, CGTM).

The CEDM was developed from an earlier model [13] developed for the same engine type by changing the usage of the coolant flows in the cycle calculations and defining the cooled engine case as an off-design point with modifications to the related section accordingly. A compressor model with coolant extraction losses was also included in CEDM, to capture the effects of cooling extraction on the compressor performance. The CEDM, including the cooled turbine model, was then validated with GasTurb12 Software [17] with casespecific tests and continuity tests, in which the effects of cooling the turbine on several aero-engine performance parameters were also analyzed. The effects of increasing turbine inlet temperature, by-pass ratio and flight Mach number on the performance parameters show the expected trends for such engines.

The CGTM, as being the industrial gas turbine version of the model, was developed from CEDM by including a methane combustion model, removal of aero-engine specific components, and modification to cycle calculations as well as the mean-line turbomachinery design section. The validation of the CGTM was made with several published available $\mathrm{H}$-Class gas turbine data and with case specific and continuity tests made with GasTurb12 [17]. In the continuity tests, validations made with turbine inlet temperature, ambient temperature and the back-pressure ratio show good agreement with referenced software and provided expected trends.

A parameter sensitivity analysis made by using the developed cooled engine models to analyze the strength of the general cooling parameters on the key engine performance parameters. The results of this analysis showed that using a blade material that allows a higher maximum allowable blade temperature has the highest impact on increasing the gas turbine performance for both aero-engines and industrial gas turbines, which is not unexpected. The second highest impact differed for the different engine types considered; for the industrial gas turbines, this was the advancements in film cooling techniques (through increased adiabatic efficiency), and advancements in internal cooling technologies for the aero-engines. For the aero-engine case, this difference in the impact trend from the industrial gas turbine was explained by the negative impact of reducing the hot gas temperature through reduced adiabatic wall temperatures by film cooling on the low pressure spool performance. Advancements in TBC materials ranked as the fourth highest impact parameter on the engine performance for both engines.

The sensitivity analysis that assumes the isolated impacts of the cooling parameters on the engine performance, showed that highly efficient film cooling techniques can provide improvements to gas turbine performance. Although the impact from this improvement ranked behind the advancements in internal cooling effectiveness for the aero-engines, such an improvement might require longer and more expensive upgrade process than using an advanced film cooling technique with an existing internal cooling configuration. An advanced film cooling hole design, named as Anti-Vortex Hole, is analyzed by using Detached Eddy Simulation Techniques and the simulations were validated with the previous experimental studies made at West Virginia University (WVU) [39]. The validated model was then used to generate span averaged film cooling effectiveness data for mid to high turbulence intensity flow conditions with large length scales, which is the condition that exists in the actual engines. 
To analyze the impacts of using a specific film cooling technique (AVH) on the engine performance parameters, the existing cooling calculation methodology using a semi-empirical equation from Young and Wilcock [7] was needed to be modified. Giving the cooled engine models the ability to analyze the impacts of different blade cooling configurations on engine performance, was made by developing an advanced coolant calculation algorithm, which is a modified version of the advanced methods from Consonni [1] and Torbidoni [49]. The developed algorithm includes a database from correlations and experimental data for several different internal and external cooling methods. This advanced coolant calculation algorithm is then used in an analysis that used different blade configurations determined from open literature for advanced turbine blades [62], and the performance of the blades equipped with AVH film cooling geometry is compared with other external cooling methods. Results of this analysis showed that the blade configuration with AVH geometry gave the highest performance for both aero-engine and industrial gas turbine cases. Comparison of the blade configurations using film cooling effectiveness data under engine-like flow conditions were then used in another analysis comparing the changes in the engine performance with different internal cooling configurations. Results of this analysis showed that for different engine types, the best performing internal cooling configuration might be different due to the differences in coolant supply conditions and the blade geometries. Cooled engine models with the advanced coolant calculation method were then used in a small optimization problem determining the number of cooling hole rows, which gave different optimal values for different engine types but the $\mathrm{AVH}$ equipped blades required the lowest number of holes, in both cases.

The primary outcome from this research was to develop a cooled turbine model that can be used to identify the impacts of different cooling techniques on overall engine performance parameters within the limits of assumptions made through the development of cooled engine analysis models for turbofan and industrial gas turbine engines. Due to the losses introduced by the cooled turbine, it is often not viable to assume that a cooling method, having a proven efficiency in a micro scale, will have the same impact on the engine parameters such as power or thrust, fuel consumption and engine efficiencies, which are quantities on a macro scale. The impact may not be negative, but it might provide no significant increase in engine performance either. In such a case, engine designers and investors might want to consider another technique, which provides a significant impact on performance parameters. At that point, the developed cooled engine models could serve as a connection between experimental and/or computational data and a macro-scale system model.

Using the models within a combined cycle plant or aircraft simulation can provide information regarding the impacts from the cooling system design on plant or aircraft performance. For instance, reducing the exhaust gas temperature of an industrial gas turbine through a certain technique would result in a higher engine thermal efficiency, but this might negatively affect the performance of Rankine Cycle in combined cycle operation, yielding the possibility of a decrease in combined cycle efficiency. Such impacts on higher-level systems may not be easy to assess without a cooled engine simulation.

The algorithms and the models developed in this research can also be used with optimization algorithms to decide what range a specific cooling parameter should be selected to provide a desired engine performance level. Designers might use these outputs to determine that level of cooling technique performance, which could save a considerable amount of time and research effort should the desired engine performance be already satisfied with a less enhanced cooling design.

The results of this research also provide information about the relative impacts of several cooling techniques on engine performance parameters, which will be useful in the decision for technology developers to decide on which direction the future of cooling research should steer toward. 


\section{APPENDIX-A}

\section{An Algorithm to Find the Coolant Exit Temperature (and Enthalpy)}

The coolant exit temperature value refers to $T_{t c l}, x$ temperature of Figure 4 and includes the effect of coolant heating within the blade. This value is dependent on the known parameters listed in Table 2, and can be calculated in an iterative way as described in this section.

Assuming that the internal cooling system is designed to keep the blade metal external temperature ( $\left.T_{m, \text { ext }}\right)$ fixed to maximum allowable blade temperature ( $T_{b, \max }$ ) at all times(i.e. $T_{m, \text { ext }}=T_{b}$, max $)$, the coolant exit temperature can be found by using Equation (A.1) from the definition of internal cooling efficiency ( $\varepsilon_{c, \text { ext }}$ ) by Young and Wilcock [7]. It should be noted that the value of $\varepsilon_{c, \text { ext }}$ is unknown at the beginning of the calculations.

$$
T_{t c l, x}=T_{t c l, \text { in }}+\varepsilon_{c, e x t}\left(T_{b, \max }-T_{t c l, i n}\right)
$$

For the calculation of the coolant exit temperature, the coolant supply temperature $\left(T_{t c l, i n}\right)$ is also a known parameter, which is the air temperature at the coolant extraction stage in the compressor plus the temperature increase due to frictional losses in the coolant supply pipes from compressor to turbine.

The internal flow cooling efficiency $\left(\varepsilon_{c}\right)$ is a known parameter, which usually takes values between 0.6 to 0.8 , depending on the level of internal cooling technology [7]. It is defined as in Equation (A.2).

$$
\varepsilon_{c}=\frac{T_{t c l, x}-T_{t c l, i n}}{T_{m, i n t}-T_{t c l, i n}}
$$

Aside from the coolant exit temperature ( $\left.T_{t c l, x}\right)$, the other unknown parameter in Equation (A.2) is the internal metal temperature ( $\left.T_{m, i n t}\right)$ and its value can be found by Equation (A.3), that is obtained from the definition of metal Biot number and the heat flux from the hot gas side to the blade.

$$
T_{m, \text { int }}=T_{b, \max }-B i_{m}\left(T_{a w}-T_{b w}\right)
$$

If this is a film cooled blade, the adiabatic wall temperature ( $\left.T_{a w}\right)$ can be calculated from the definition of adiabatic film cooling efficiency $\left(\varepsilon_{f c}\right)$, which is a known parameter, with Equation (A.4); otherwise this temperature is equal to the gas temperature $\left(T_{\mathrm{tg}^{*}}\right)$.

$$
T_{a w}=T_{t g *}+\varepsilon_{f c}\left(T_{t g *}-T_{t c l, x}\right)
$$

The value of blade wall temperature ( $\left.T_{b w}\right)$ in Equation (A.3) depends on whether the cooling configuration includes a TBC layer or not. If there is no TBC layer, than this temperature is equal to the maximum allowable blade temperature ( $\left.T_{b, \max }\right)$; otherwise its value can be found from Equation (A.5), which is derived from the definition of TBC Biot number and the heat flux from the hot gas side to the blade.

$$
T_{b w}=\frac{B i_{T B C} T_{a w}+T_{b, \max }}{\left(1+B i_{T B C}\right)}
$$


The equation system from A.1 to A.5 has two unknowns, $T_{t c l, x}$ and $\varepsilon_{c, \text { ext }}$, which are linked through the internal flow cooling efficiency. Assuming an initial value for $\varepsilon_{c, \text { ext,INIT }}=\varepsilon_{c}$ and reducing the value of $\varepsilon_{c, \text { ext }}$ until the calculated $T_{t c l, x}$ value satisfies the known $\varepsilon_{c}$ value will give the coolant exit temperature value that will be used within a thermodynamic property table to obtain the regarding enthalpy value $\left(\mathrm{h}_{\mathrm{tcl}, \mathrm{x}}\right)$. 


\section{APPENDIX-B}

\section{Derivation of the Rotor Exit Enthalpy Equation}

For the rotor stage of the turbine, $1^{\text {st }}$ Law of Thermodynamics with the assumptions listed in Section 1.1.2 gives the rotor energy equation as in Equation (B.1).

$$
\dot{m}_{4.3 i}\left(h_{t 4.3 i}-h_{t 4.4 i}\right)+\dot{m}_{r c}\left(h_{t c l, i}-h_{t 4.4 i}\right)=\dot{W}_{t i}
$$

The Euler Turbine Equation that relates stage work $\dot{W}_{t i}$ of the cooled turbine stage to rotor wheel speed $(\omega R)$ and the swirl velocity $(v)$ is given in Equation (B.2).

$$
\dot{W}_{t i}=\dot{m}_{4.3 i}\left(\omega R v_{4.3 i}-\omega R v_{4.4 i}\right)+\dot{m}_{r c}\left(\omega R v_{c l, i}-\omega R v_{4.4 i}\right)
$$

Equation (B.2) is written for a stream tube control volume at a radial location $\mathrm{R}$ from the shaft center that is rotating with a radial speed of $\omega$, and $v_{x i}$ are the swirl velocities (vertical component of the station $\mathrm{x}$ of $\mathrm{i}^{\text {th }}$ turbine stage velocity vector). Station 4.3 corresponds to stator exit and 4.4 corresponds to rotor exit stations. Replacing the work term $\dot{W}_{t i}$ of Equation (B.1) with the Euler Turbine Equation given in Equation (B.2) will result in the energy equation expressed in specific rothalpy $\left(i=h_{t}-\omega R v\right)$ and result in the rothalpy balance equation given in Equation (B.3).

$$
\dot{m}_{4.3 i}\left(i_{4.3 i}-i_{4.4 i}\right)+\dot{m}_{r c}\left(i_{c l, i}-i_{4.4 i}\right)=0
$$

Assuming adiabatic axial flow across the stage, results in the rothalpy to be conserved along the stream tube control volume that encompasses through the mean-line of the stator and rotor which allows having $i_{4.3 i}=$ $i_{4.4 i}$. From Equation (B.3), this implies having the rothalpy balance given in Equation (B.4).

$$
i_{c l, i}=i_{4.4 i}
$$

It should be noted here that, both the coolant exit station (cl,i) and the rotor exit station (4.4i) are on the relative coordinate frame, which is a coordinate frame that is attached to rotor blade and rotating with the same rotational speed. Therefore, using rothalpy definition for relative frame $\left(i=h_{t}^{r e l}-\frac{\omega R^{2}}{2}\right)$ in Equation (B.4) will result in the expression given in Equation (B.5).

$$
\begin{aligned}
h_{t c l, i}^{r e l}-\frac{(\omega R)^{2}}{2} & =h_{4.4 i}^{r e l}-\frac{(\omega R)^{2}}{2} \\
h_{t c l, i}^{r e l} & =h_{4.4 i}^{r e l}
\end{aligned}
$$

The stage loading is defined in the stationary frame of reference and given by Mattingly [12] in terms of the station numbering used in this thesis as in Equation (B.6).

$$
\psi=\frac{h_{t 4.3 i}-h_{t 4.4 i}}{(\omega R)^{2}}=\frac{\dot{W}_{t i}}{\dot{m}_{4.3 i}(\omega R)^{2}}
$$


The coolant relative to static total enthalpy is given by Young and Wilcock [7] for a 50\% reaction stage. When this equation is written for the general case of a rotor with degree of reaction ${ }^{0} R_{t}$, this relation becomes as in Equation (B.7).

$$
h_{t c l, i}^{r e l}=h_{t c l, i}-\omega R\left(v_{c l, i}-\omega R^{o} R_{t}\right)
$$

The coolant exit swirl velocity $v_{c l, i}$ is different than the rotor exit swirl velocity $v_{4.4 i}$ and it is approximated as $v_{c l, i}=K_{s w i r l} \omega R$ by Young and Wilcock [7] and the constant $\mathrm{K}_{\text {swirl }}$ actually represents the effect of the stationary swirl vanes that are usually mounted to the upstream of the coolant inlet to rotor and serves for increasing the coolant absolute swirl velocity. The constant value $\mathrm{K}_{\text {swir }}$ can take values within $(0,2.5)$ interval [7].

Equation (B.6) can be used to extract the wheel speed $(\omega R)$ if the stage loading and the stage work are treated to be known variables (i.e. $(\omega R)^{2}=\frac{\dot{W}_{t i}}{\dot{m}_{4.3 i} \psi}$ ). Using the aforementioned coolant exit swirl velocity approximation in Equation (B.7) will result in the useful expression given in Equation (B.8).

$$
h_{t c l, i}-h_{t c l, i}^{r e l}=(\omega R)^{2}\left(K_{\text {swirl }}-{ }^{o} R_{t}\right)=\frac{\dot{W}_{t i}}{\dot{m}_{4.3 i} \psi}\left(K_{\text {swirl }}-{ }^{o} R_{t}\right)
$$

From Equation (B.5) replace $h_{t c l, i}^{r e l}$ with $h_{4.4 i}^{r e l}$ and re-arrange equation (B.8) to obtain an expression for relative rotor exit enthalpy as in Equation (B.9).

$$
h_{t 4.4 i}^{r e l}=h_{t c l, i}-\frac{\dot{W}_{t i}}{\dot{m}_{4.3 i} \psi}\left(K_{\text {swirl }}-{ }^{o} R_{t}\right)
$$

To obtain an enthalpy value that can be used within the cycle calculations (which are carried out in stationary frame of reference), the relative rotor exit enthalpy term $h_{t 4.4 i}^{\text {rel }}$ should be transformed to stationary coordinates. An expression that can be used for such transformation valid for the considered streamtube control volume is given by Moustapha et al. [10] and is expressed as in Equation (B.10).

$$
h_{t}^{r e l}=h_{t}+\frac{v^{2}}{2}
$$

Using this transformation in Equation (B.9) will result in the static enthalpy expression for rotor exit station as given in Equation (B.11).

$$
h_{t 4.4 i}=h_{t c l, i}-\frac{\dot{W}_{t i}}{\dot{m}_{4.3 i} \psi}\left(K_{s w i r l}-{ }^{o} R_{t}\right)-\frac{v_{4.4 i}^{2}}{2}
$$

Replacing the stage work term $\dot{W}_{t i}$ in Equation (B.11) with its definition in Equation (B.1) will give the exact expression for rotor exit station enthalpy as in Equation (B.12).

$$
h_{t 4.4 i}=h_{t c l, i}-\frac{\dot{m}_{4.3 i}\left(h_{t 4.3 i}-h_{t 4.4 i}\right)+\dot{m}_{r c}\left(h_{t c l, i}-h_{t 4.4 i}\right)}{\dot{m}_{4.3 i} \psi}\left(K_{s w i r l}-{ }^{o} R_{t}\right)-\frac{v_{4.4 i}{ }^{2}}{2}
$$

Performing the division by $\dot{m}_{4.3 i} \psi$ in the second term and noting that $\dot{m}_{r c} / \dot{m}_{4.3 i}$ is the coolant fraction that is already calculated by semi-empirical methodology (i.e. a known parameter) described in Section 1.1.1, and assuming the rotor exit swirl velocity is negligible (i.e. $v_{4.4 i} \cong 0$ ), an approximate relation for rotor exit enthalpy can be found as given in Equation (B.13). 


$$
h_{t 4.4 i} \cong \frac{h_{t c l, i}-\frac{\left(K_{\text {swirl }}-{ }^{o} R_{t}\right)}{\psi}\left(h_{t 4.3 i}+\frac{\dot{m}_{r c}}{\dot{m}_{4.3 i}} h_{t c l, i}\right)}{1-\frac{\left(K_{\text {swirl }}-{ }^{o} R_{t}\right)}{\psi}\left(1+\frac{\dot{m}_{r c}}{\dot{m}_{4.3 i}}\right)}
$$

In some resources, a further assumption is made by considering the fact $\frac{\dot{m}_{r c}}{\dot{m}_{4.3 i}} \ll 1$, which results in another approximate form of rotor exit enthalpy given as in Equation (B.14).

$$
h_{t 4.4 i} \cong \frac{h_{t c l, i}-\frac{\left(K_{\text {swirl }}-{ }^{o} R_{t}\right)}{\psi}\left(h_{t 4.3 i}+\frac{\dot{m}_{r c}}{\dot{m}_{4.3 i}} h_{t c l, i}\right)}{1-\frac{\left(K_{\text {swirl }}-{ }^{o} R_{t}\right)}{\psi}}
$$




\section{APPENDIX-C}

\section{Development of a Compressor Model with the Effect of Coolant Air Extraction on the Compressor Performance}

Assume a steady-flow well-insulated compressor with $\mathrm{N}_{\text {comp }}$ number of stages with a polytropic efficiency $\mathrm{e}_{c}$ can be divided into $\mathrm{N}$ compressors, each having the same polytropic efficiency $\mathrm{e}_{c}$ where $\mathrm{N}$ indicates the number of different compressor stages that the coolant flows are extracted. The described modelling approach is shown schematically in Figure $\mathrm{C} 1$.

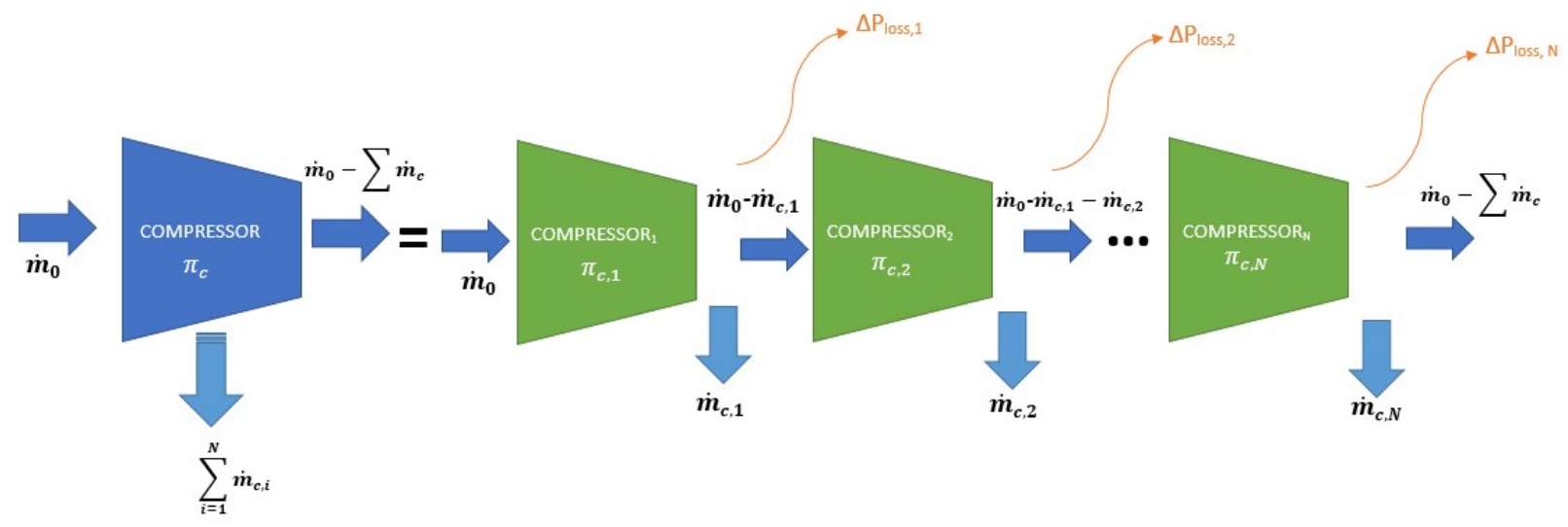

Figure C 1: Compressor modelling approach employed in the compressor model with losses

For instance, if the coolant flows are extracted from the $7^{\text {th }}, 9^{\text {th }}$ and $14^{\text {th }}$ stages of a 14-staged compressor, $\mathrm{N}$ would be equal to 3 and the compressor can be assumed to be formed by 3 consecutive compressors, each having polytropic efficiency $e_{c}$ but running with different air flow rates and has different number of stages that are 7,2 , and 5 , respectively.

Due to having different number of stages, each compressor will have different pressure ratios. The pressure ratio of the $\mathrm{j}^{\text {th }}$ imaginary compressor can be calculated by using Equation (C.1).

$$
\pi_{c, j}=\pi_{c}^{(i(j)-i(j-1))} / e_{c} N_{c o m p} \Delta P_{\text {loss }, i}
$$

In Equation (C.1), i is a vector that has the coolant extraction stage numbers (i.e. $i=[7,9,14]$ for the example case), $\pi_{c}$ is the compressor pressure ratio, and $\Delta \mathrm{P}_{\text {loss }}$ is a pressure loss due to coolant extraction, of which the details on calculation will be given in this section.

With the assumption described above the pressure loss $\Delta \mathrm{P}_{\text {loss }}$ occurs only in the intermediate region where the coolant is extracted from, which includes no stator or rotor components. Then the control volume to derive the pressure loss term, shown in Figure $\mathrm{C} 2$ ) is a basic flow separation region with no work or heat transfer passing through the control volume boundaries. 




Figure C 2: Control Volume boundaries encompasses the coolant extraction region that is after the stator of the extraction stage and before the rotor of the next compressor stage

The coolant extraction is usually made with an angle almost perpendicular to the compressor outer walls; then the momentum and energy equations for the control volume in Figure C2, will be given as in Equation(s) (C.2) and (C.3), respectively.

$$
\begin{aligned}
& \text { Momentum: } \quad \dot{m}_{g}\left(\frac{\delta P_{g}}{\rho_{g}}+V_{g} \delta V_{g}\right)-\delta \dot{m}_{c} V_{g}{ }^{2}=0 \\
& \text { Energy: } \quad \dot{m}_{g}\left(V_{g} \delta V_{g}\right)+\delta \dot{m}_{c l} \frac{\left(V_{g}^{2}-V_{c}^{2}\right)}{2}=0
\end{aligned}
$$

Inserting $\dot{m}_{g}\left(V_{g} \delta V_{g}\right)$ term from energy equation (Equation (C.3)) into the momentum equation (Equation (C.2)) and re-arranging will result in a relation that gives the pressure change in the compressor flow in terms of coolant velocities and mass flow rates as shown in Equation (C.4).

$$
\frac{\delta P_{g}}{\rho_{g}}=-\frac{\delta \dot{m}_{c l}}{\dot{m}_{g}}\left(\frac{V_{g}^{2}}{2}-\frac{V_{c}^{2}}{2}\right)
$$

Assuming ideal gas for the compressor air flow allows replacing the gas density $\rho_{g}$ in Equation (C.4) with $P_{g} / R T_{g}$, which gives the relation in Equation (C.5) after re-arranging.

$$
\frac{\delta P_{g}}{P_{g}}=-\frac{\delta \dot{m}_{c l}}{\dot{m}_{g}} \frac{1}{R T_{g}}\left(\frac{V_{g}^{2}}{2}-\frac{V_{c}^{2}}{2}\right)
$$

Multiplying and dividing the right-hand side of Equation (C.5) with gas side heat capacity ratio $\mathrm{Yg}_{\mathrm{g}}$ and using the definition of the speed of sound for the gas side from $a=\sqrt{\gamma_{g} R T_{g}}=\sqrt{\gamma_{c} R T_{c}}$ (since $\mathrm{T}_{\mathrm{g}}=\mathrm{T}_{\mathrm{c}}$ ) will give Equation (C.6).

$$
\frac{\delta P_{g}}{P_{g}}=-\frac{\delta \dot{m}_{c l}}{\dot{m}_{g}} \frac{\gamma_{g}}{a^{2}}\left(\frac{V_{g}^{2}}{2}-\frac{V_{c}^{2}}{2}\right)
$$

Using the definition for the Mach number from $M=V / a$, where $\mathrm{V}$ is the flow speed and $\mathrm{a}$ is the speed of sound, in Equation (C.6) will give a more useful form of the pressure difference relation as given in Equation (C.7).

$$
\frac{\delta P_{g}}{P_{g}}=-\gamma_{g} \frac{\delta \dot{m}_{c l}}{\dot{m}_{g}}\left(\frac{M_{g}{ }^{2}}{2}-\frac{M_{c}^{2}}{2}\right)
$$


Noting that $\epsilon \cong \frac{\Delta \dot{m}_{c l}}{\dot{m}_{g}}$, where $\varepsilon$ is the extracted coolant fraction for turbine cooling, integration of Equation (C.7) results in an approximate relation for the pressure loss due to coolant extraction as shown in Equation (C.8).

$$
\Delta P_{\text {loss }}=\Delta P_{g} \cong e^{-\gamma_{g} \epsilon\left(\frac{M_{g}^{2}}{2}-\frac{M_{c}{ }^{2}}{2}\right)}
$$

Then for each compressor, the compressor exit pressure is reduced by $\Delta P_{\text {loss }}$ and the exit pressure with losses can be used in a thermodynamic property calculator to compute other thermodynamic states, such as total enthalpy that is used in calculating the compressor work term. The Mach numbers of the compressor airflow and the coolant extraction flow in Equation (C.8) are calculated from the turbomachinery section results with free-vortex distribution assumption for the compressor blades.

For repeating compressor stages the axial flow speed is assumed the same for the entire compressor stage (i.e. $\mathrm{u}_{1}=\mathrm{u}_{2}=\mathrm{u}_{3}$ ) and this flow speed is used to calculate $\mathrm{M}_{\mathrm{g}}$ of Equation (C.8). Whereas the coolant speed and the Mach number $M_{c}$ can be calculated from the vertical component of the flow velocity at the exit of stator station, since the coolant is extracted with an angle almost perpendicular to central axis of the compressor. For the free-vortex distribution case, Mattingly [12] provides the relation given in Equation (C.9) to calculate this velocity component in terms of the radial distance from the compressor central axis ( $r$ ).

$$
v_{3, i}(r)=a \frac{r_{m}}{r}+b \frac{r_{m}}{r}
$$

In Equation (C.9), $\mathrm{r}_{\mathrm{m}}$ is the mean radius of the blade and calculated for each compressor extraction stage ' $i$ ' separately. Coefficients $a$ and b of Equation (C.9) are given in Equation (C.10).

$$
\begin{gathered}
a=\omega r_{m}\left(1-{ }^{0} R_{c}\right) \\
b=\frac{g_{c} c_{p} \Delta T_{t, i}}{2 \omega r_{m}}
\end{gathered}
$$

In Equation (C.10), $\omega$ is the rotational speed, ${ }^{0} R_{c}$ is the degree of reaction for the stator of the $\mathrm{i}^{\text {th }}$ stator stage, $\mathrm{g}_{\mathrm{c}}$ is 32.2 if $\mathrm{BE}$ units and 1 if SE units are used and $\Delta T_{t, i}$ is the temperature difference across the stator stage before the extraction location. 


\section{APPENDIX-D}

\section{Development of a Transition Cooling Model}

Transition cooling is an additional cooling section that is placed between the combustor and the turbine inlet region in the advanced industrial gas turbines. Existence of such an additional cooling region allows engine developers to use higher combustor exit temperatures, with minimum increase to turbine inlet temperature.

In the development of the transition cooling model for the CGTM, a coolant mixer control volume is used with the following assumptions:

- Adiabatic walls

- Steady-state flow

- The reduction in hot gas temperature by the transition cooling section is fixed to $200^{\circ} \mathrm{R}$.

- Pressure losses encountered in this region are negligible

- Changes in the flow speed from the entry to the exit of the mixer is negligible

The control volume, and flow fractions used in this analysis is shown in Figure D1.

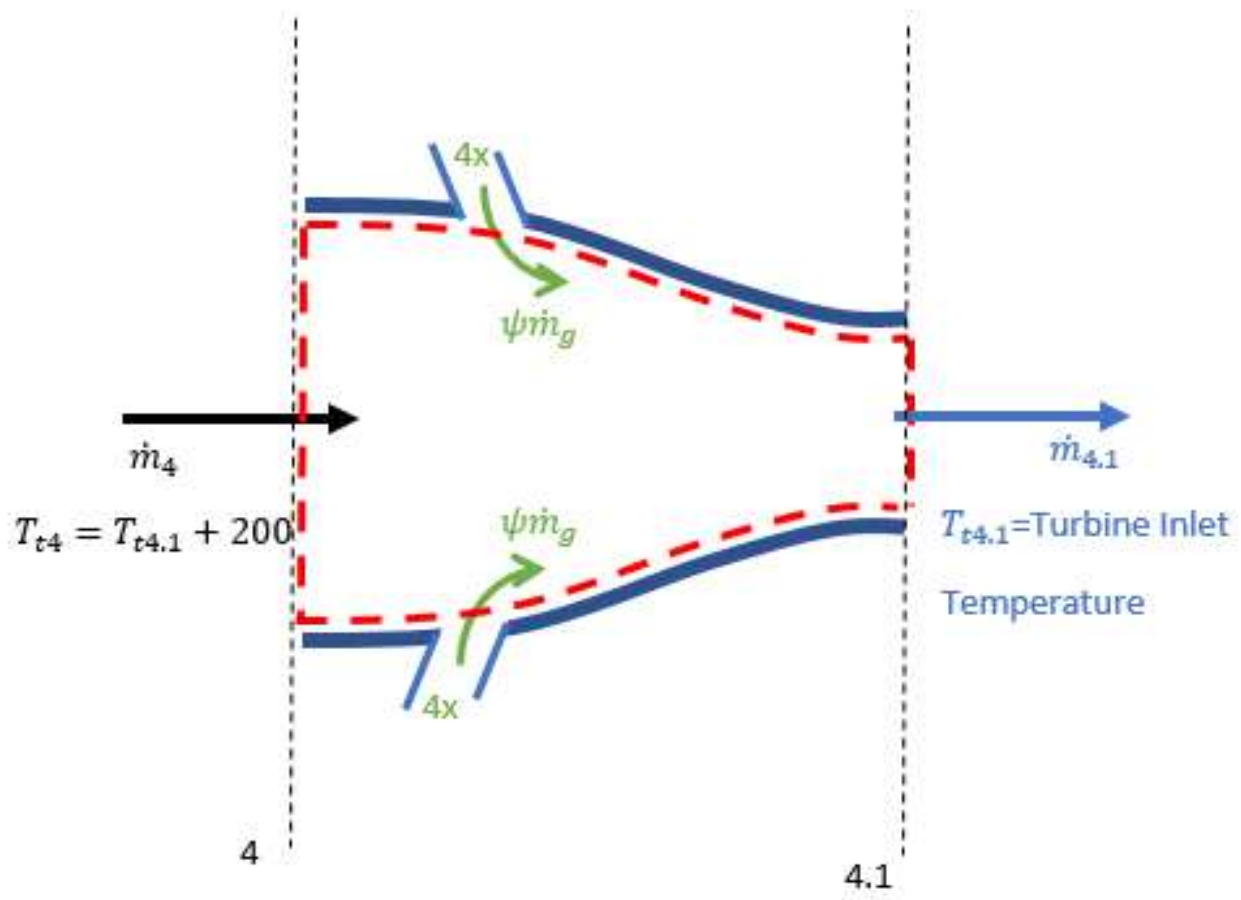

Figure D 1: Control Volume used in the transition cooling model development is shown with the engine station numbers used in CGTM

In Figure D1, station 4x represents the transition coolant exit location, while station 4 and station 4.1 are used from the station numbering given in Figure 29. The coolant used for transition cooling is extracted after the last stage of the compressor together with the bleed air $(\beta)$ and a portion of the coolant air used for turbine cooling. It is assumed here that the transition cooling air is heated due to similar reasons considered for the 
turbine cooling air heating and the gain in this temperature can be represented with the coolant air heating constant $\mathrm{K}_{\text {gain, }}$ which is calculated iteratively with the details given in the turbine cooling analysis (Section 2.1.1). Therefore, the enthalpy of the transition cooling air is given by Equation (D.1).

$$
h_{t 4 x}=h_{t 3} K_{t e m p, g a i n}
$$

And the mass flow rate in this station is given by Equation (D.2).

$$
\dot{m}_{4 x}=\dot{m}_{0} \psi
$$

The hot gas mass flow rate at Station 4 is equal to the sum of the fuel flow $\left(\dot{m}_{0} f\right)$ and turbine and transition cooling flow fractions $\left(\psi+\epsilon_{1}+\epsilon_{2}\right)$ subtracted from the intake air mass flow rate $\left(\dot{m}_{0}\right)$ as given by Equation (D.3).

$$
\dot{m}_{4}=\dot{m}_{f}+\dot{m}_{0}\left(1-\psi-\epsilon_{1}-\epsilon_{2}\right)
$$

Whereas for the exit station 4.1, the mass flow rate is given by Equation (D.4).

$$
\dot{m}_{4.1}=\dot{m}_{f}+\dot{m}_{0}\left(1-\epsilon_{1}-\epsilon_{2}\right)
$$

The $1^{\text {st }}$ Law of Thermodynamics written for the control volume of Figure D1 with the stated assumptions Is given in Equation (D.5).

$\dot{Q}_{\text {station }}-\dot{W}_{\text {station }}=\sum_{\text {st.ext }} \dot{m}_{\text {st.exit }}\left(h_{t, \text { st.ext }}+\frac{V_{\text {st.ext }}^{2}}{2}+g z_{\text {st.ext }}\right)-\sum_{\text {st.in }} \dot{m}_{s t . i n}\left(h_{t, \text { st. in }}+\frac{V_{\text {st.in }}^{2}}{2}+g z_{\text {st.in }}\right)$

In the transition section, no boundary or shaft work is produced and further assuming that the relative height differences between the inlet and exit stations are zero, reduces Equation (D.5) into the form given in Equation (D.6), which will be used in transition cooling fraction calculations.

$$
0=\sum_{\text {st.ext }} \dot{m}_{\text {st.exit }}\left(h_{t, \text { st.ext }}\right)-\sum_{\text {st.in }} \dot{m}_{\text {st.in }}\left(h_{t, \text { st.in }}\right)
$$

Inserting the inlet and exit stations to Equation (D.6) from Figure D1 will give:

$$
0=\dot{m}_{4.1} h_{t 4.1}-\left(\dot{m}_{4} h_{t 4}+\dot{m}_{4 x} h_{t 4 x}\right)
$$

Using the mass flow rates in equation (D.7) from Equations (D.2), (D.3), and (D.4) and enthalpy of the coolant from Equation (D.1) will result in an equation for the transition cooling fraction, as given in Equation (D.8).

$$
\psi=\frac{(1+f)\left(1-\epsilon_{1}-\epsilon_{2}-\beta\right)\left[h_{t 4.1}-h_{t 4}\right]}{\left(K_{\text {temp,gain }} h_{t 3}-(1+f) h_{t 4}+h_{t 4.1}\right)}
$$

In Equation (D.8), the compressor exit enthalpy $\left(h_{t 3}\right)$ is calculated by using the compressor exit temperature $\left(T_{t 3}\right)$ which is previously calculated in compressor section, the transition cooling exit enthalpy $\left(h_{t 4.1}\right)$ is calculated by using the user-input turbine inlet temperature $\left(T_{t 4.1}\right)$ and the transition cooling entry temperature is calculated by adding $200^{\circ} \mathrm{R}$ to user-input turbine inlet temperature $\left(\mathrm{T}_{t 4.1}\right)$ with the thermodynamic property calculators (F-AIRng from Section 2.1.3). 


\section{APPENDIX-E}

\section{Internal and External Cooling Correlations used in Advanced Coolant Calculator}

The correlations used in the advanced coolant calculator are selected from various resources on common blade cooling applications and from the experimental resources on selected advanced cooling concepts introduced by Town et al. [62]. The correlation formulae, their limitations and the source information are listed in the following sub-sections.

\section{E.1 Internal Cooling Correlations}

The correlation formulae for the cooling techniques listed in Table 34 are given in the following sub-sections.

\section{E.1.1 Rib Cooling Correlations}

\section{E.1.1.1 Parallel $90^{\circ}$ Ribs}

The correlation formula for these ribs are given by Han [64], with the correlation parameters shown in the schematic view given in Figure E.1.

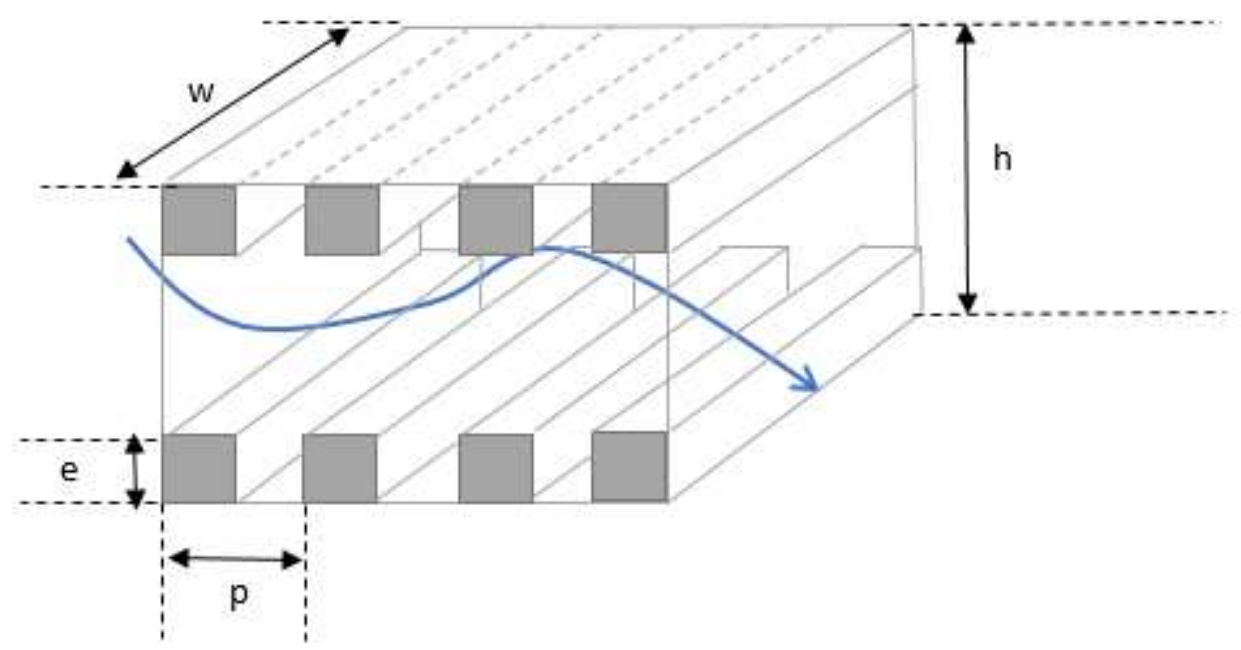

Figure E 1: Schematic view of the parallel $90^{\circ}$ ribs with correlation input parameters

A roughness function that models the rib channel pressure losses are given for this type of channels with Equation (E.1).

$$
R=3.2\left(\frac{p / e}{10}\right)^{0.35}
$$

The friction factor $\left(f_{f}\right)$ for $90^{\circ}$ parallel ribs are calculated by using Equation (E.2).

$$
f_{f}=\frac{w}{w+h}\left(\frac{h}{w} f_{s}+\frac{2}{\left(R-2.5 \ln \left(\frac{2 e}{d}\right)-2.5\right)^{2}}\right)
$$


Parameter d in Equation (E.2) is the hydraulic diameter and can be found by using Equation (E.3).

$$
d=\frac{w \cdot h}{2(w+h)}
$$

The parameter $f_{s}$ in Equation (E.2) is the smooth channel friction factor and can be found by using Equation (E.4).

$$
f_{s}=0.046 R e_{i n}^{-0.2}
$$

The thermal roughness function $(G)$ is given by Equation $(E .5)$ for these ribs.

$$
G=3.7\left(\frac{e}{d} R e_{i n} \sqrt{f_{f} / 2}\right)^{0.28}
$$

And the Stanton number correlation for this rib geometry is given by Equation (E.6).

$$
S t_{i n}=\frac{f_{f} / 2}{1+(G-R) \sqrt{f_{f} / 2}}
$$

\section{E.1.1.2 Parallel $\alpha$-Angled Ribs}

These ribs are similar to the parallel $90^{\circ}$ ribs, except for their angled layout with the oncoming channel flow with angle $\alpha$, as schematized in Figure E.2 with the correlation parameters.

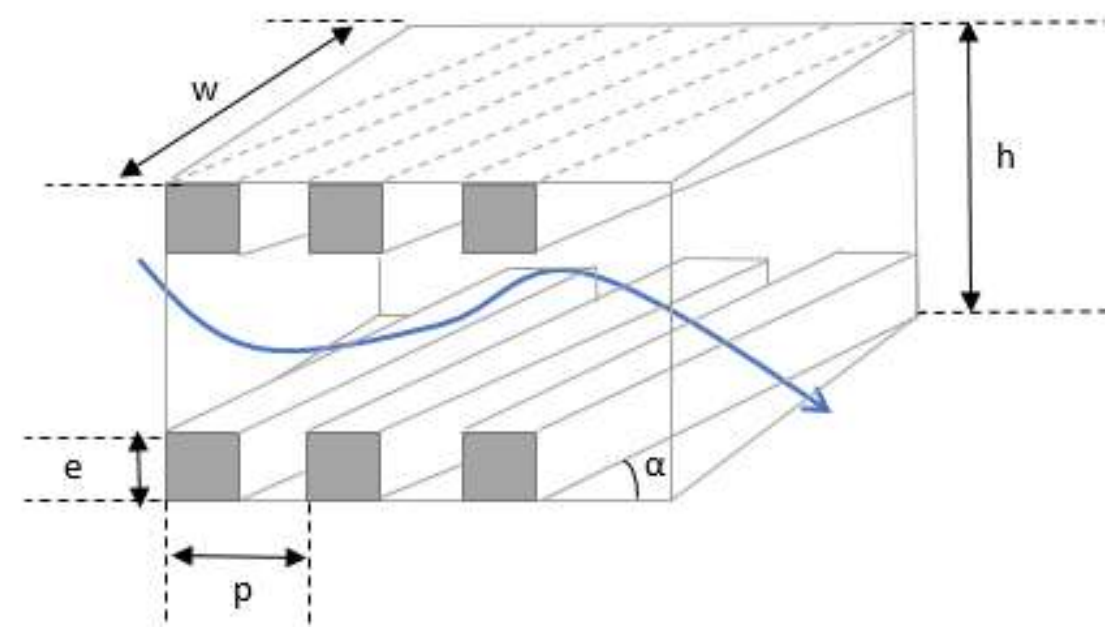

Figure E 2: Schematic view of the parallel angled ribs with correlation input parameters

The correlation for the Stanton number for these types of ribs are given by Han et al. [65], The roughness function for this rib types are given by Equation (E.7).

$$
R=\left(\frac{p / e}{10}\right)^{0.35}\left(0.03 \frac{e}{d} R e_{i n} \sqrt{\frac{f_{f}}{2}}\right)^{n}\left[15.6-31.6\left(\frac{\alpha}{90^{0}}+21.1\left(\frac{\alpha}{90^{0}}\right)^{2}\right)\right]
$$

In Equation (E.7), $\mathrm{n}=0$ for rib angles of $45^{\circ}$ or higher, and $\mathrm{n}=0.17$ for the ribs having $\alpha<45^{\circ}$. The friction factor $f_{f}$ is given by Equation (E.8). 


$$
f_{f}=2\left\{R-\left(2.5 \ln \left(\frac{2 e}{d}\right)+2.5\right)\right\}^{-2}
$$

In Equation (E.8), $d$ is the hydraulic diameter and can be found by Equation (E.3). The thermal roughness function $(G)$ is given by Equation (E.9).

$$
G=\left(\frac{p / e}{10}\right)^{0.14} 2.83\left(\frac{\alpha}{90^{0}}\right)^{3}\left(\frac{e}{d} R e_{i n} \sqrt{\frac{f_{f}}{2}}\right)^{0.28}
$$

Then the Stanton number can be found by using Equation (E.6) with the thermal roughness function from Equation (E.9), roughness function from Equation (E.7) and friction factor from Equation (E.8).

\section{E.1.1.2 V-Shaped $\alpha$-Angled Parallel Ribs}

This specific rib case has parallel ribs on the opposite faces of the rib channel and the angle a represents here the angle of the $V$ shape, as schematized in Figure E.3.

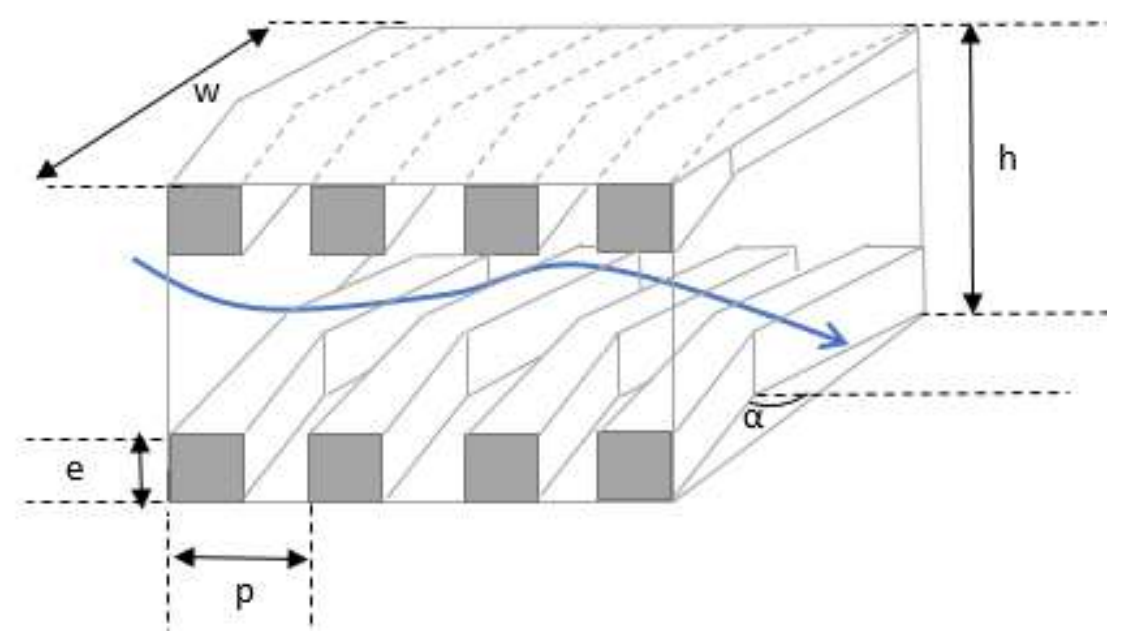

Figure E 3: Schematic view of the parallel V-Shaped ribs with correlation input parameters

The correlation for the Stanton number of these ribs are given by Han et al. [66], with the roughness function calculated from Equation (E.10).

$$
R=a\left(\frac{e}{d} R e_{i n} \sqrt{\frac{2 f_{f}-f_{s}}{2}}\right)^{b}
$$

In Equation (E.10), the values for the parameters $a$ and $b$ depends on the angle $\alpha$ and the ratio $p / e$. The values are given for common $\mathrm{V}$-Shaped rib p/e cases in Equation (E.11).

$$
\begin{gathered}
\text { for } \frac{p}{e}=10 \quad\left\{\begin{array}{l}
\text { if } \alpha=45^{0} ; a=1.605, b=0.0015 \\
\text { if } \alpha=60^{0} ; a=1.232, b=0.0454
\end{array}\right. \\
\text { for } \frac{p}{e}=20 \quad\left\{\text { if } \alpha=60^{\circ} ; a=3.687, b=-0.0571\right.
\end{gathered}
$$


In Equation (E.10), the value for the smooth channel friction factor $\left(f_{s}\right)$ can be calculated from Equation (E.4). Thermal roughness function $(G)$ is in a similar form with the roughness function, and is given by Equation (E.12).

$$
G=c\left(\frac{e}{d} R e_{i n} \sqrt{\frac{2 f_{f}-f_{s}}{2}}\right)^{g}
$$

Similar to the roughness function, the parameters $c$ and $g$ depend on the ratio for p/e and the rib angle $\alpha$. The values for these constants are given in Equation (E.13) for the cases shown in Equation (E.11).

$$
\begin{array}{cl}
\text { for } \frac{p}{e}=10 & \left\{\begin{array}{l}
\text { if } \alpha=45^{0} ; c=1.819, g=0.355 \\
\text { if } \alpha=60^{\circ} ; c=1.299, g=0.399
\end{array}\right. \\
\text { for } \frac{p}{e}=20 & \left\{\text { if } \alpha=60^{0} ; c=1.685, g=0.376\right.
\end{array}
$$

For this rib type, the friction factor $\left(f_{f}\right)$ is given by Equation (E.14), where the smooth channel friction factor $\left(f_{s}\right)$ can be found from Equation (E.4) and the hydraulic diameter from Equation (E.3).

$$
f_{f}=\frac{f_{s}}{2}+\left\{R-\left(2.5 \ln \left(\frac{2 e}{d}\right)+2.5\right)\right\}^{-2}
$$

The Stanton number correlation for this rib type is given by Equation (E.15).

$$
S t_{\text {in }}=\frac{\sqrt{0.5\left(2 f_{f}-f_{s}\right)}}{G-2.5 \ln \left(\frac{2 e}{d}\right)+2.5}
$$

\section{E.1.1.4 Criss-Crossed Parallel Ribs}

These ribs are parallel angled ribs that have opposite angular orientation between the opposite faces of the coolant channel. A schematic view of this rib type is given in Figure E.4, with correlation input parameters.

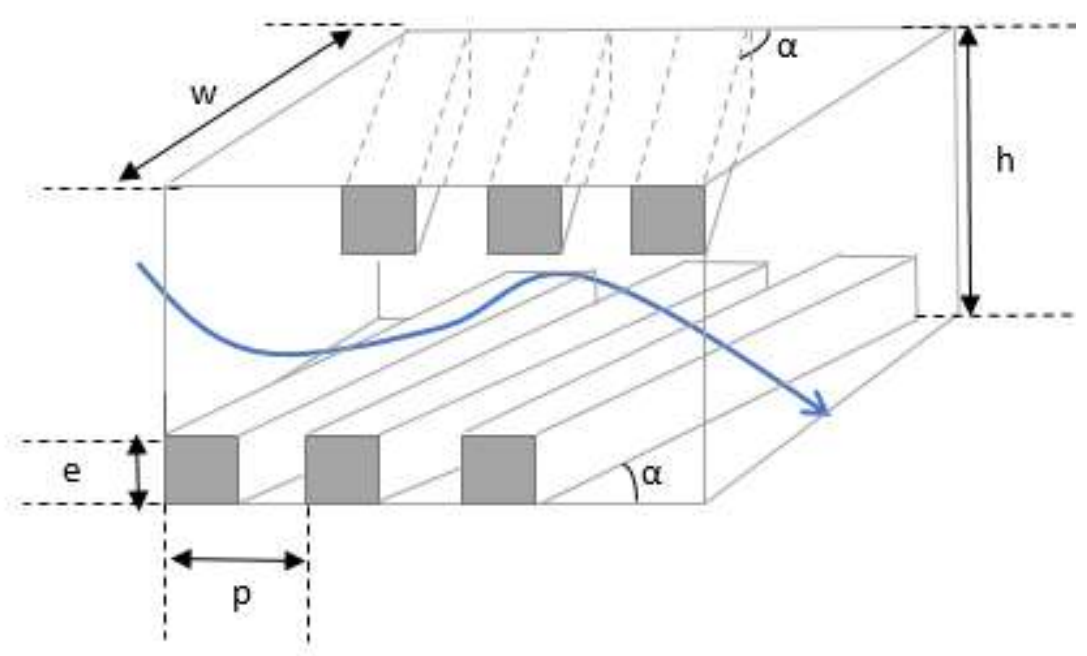

Figure E 4: Schematic view of the criss-crossed ribs with correlation input parameters 
Correlation for the Stanton number for these rib types are given by Lau et al. [67] with the friction factor given by Equation (E.14) by using a constant roughness function value as 3.18 (i.e. $R=3.18$ ). The thermal roughness function for this rib case is given in Equation (E.16).

$$
G=1.82\left(\frac{e}{d} R e_{i n} \sqrt{\frac{2 f_{f}-f_{s}}{2}}\right)^{0.41}
$$

The smooth channel friction factor $\left(f_{s}\right)$ can be found from Equation (E.4), and the Stanton number can be found from Equation (E.15), by using the thermal roughness function from Equation (E.16).

\section{E.1.1.5 Discrete Parallel V-Shaped Ribs (Chevron)}

The correlation for these ribs are obtained from a curve-fit to the results of an experimental study made with these rib types by Wright et al. [55] for the coolant Reynolds number range of 10000 to 40000 . These ribs differ from the parallel $V$-Shaped ribs with an offset in the two legs of the $V$-shape, shown schematically in Figure E.5.

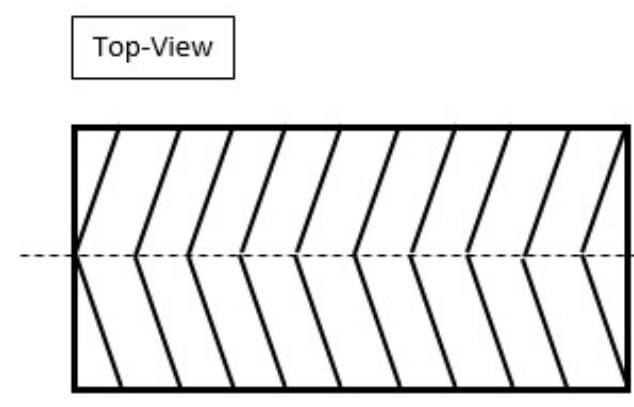

V-Shaped Parallel Angled

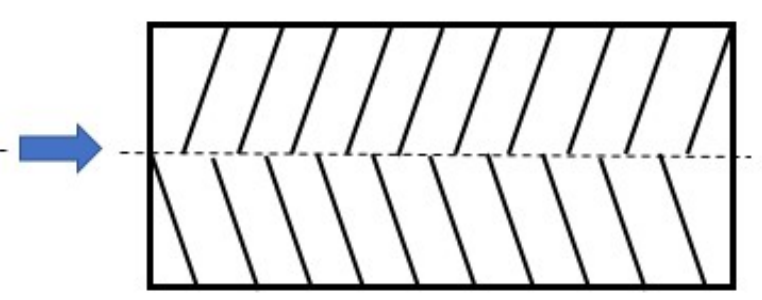

V-Shaped Discrete Parallel Angled

Figure E 5: Discrete V-Shaped ribs differ from the parallel V-Shaped ribs with an offset between two legs of the rib [55]

Plot digitization for the experimental results for this type of rib geometry from Wright et al [55] for the nonrotating case is used to obtain a curve-fit function, as shown in Figure E.6.

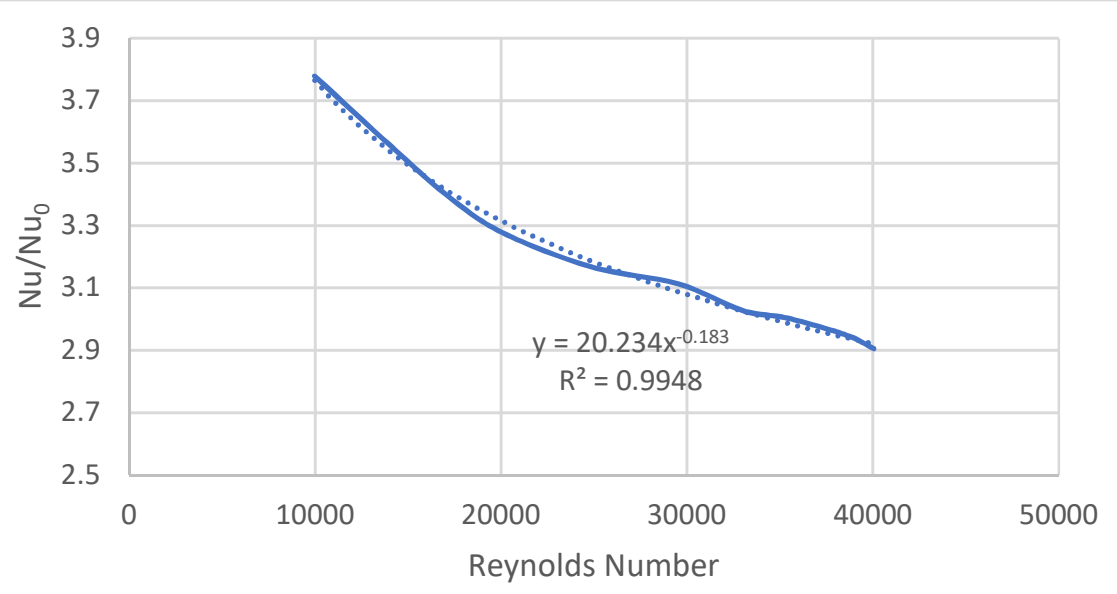

Figure E 6: Plot-digitization of the experimental data by Wright et al. [55] given for non-rotating case is used in obtaining a curve-fit function 
The curve-fit function is then used in the Internal Cooling correlations section of the new coolant calculation algorithm. The formula used for calculating the Nusselt number is given in Equation (E.17).

$$
N u=N u_{0} \cdot 20.234 \operatorname{Re}_{i n}{ }^{-0.183}
$$

In Equation (E.17), the Nusselt number for the flow in fully developed friction factor in non-rotating smooth tube $\left(\mathrm{Nu}_{0}\right)$ is found by using Equation (E.18).

$$
N u_{0}=0.023 \operatorname{Re}_{\text {in }}^{0.8} \operatorname{Pr}^{0.4}
$$

\section{E.1.1.6 W-Trips}

This type of ribs is proposed in the study by Town et al. [62] within one of the advanced blade configurations that will be analyzed in the START facilities [63]. The ribs are shaped in this concept forms a W shape, as shown schematically in Figure E.7.

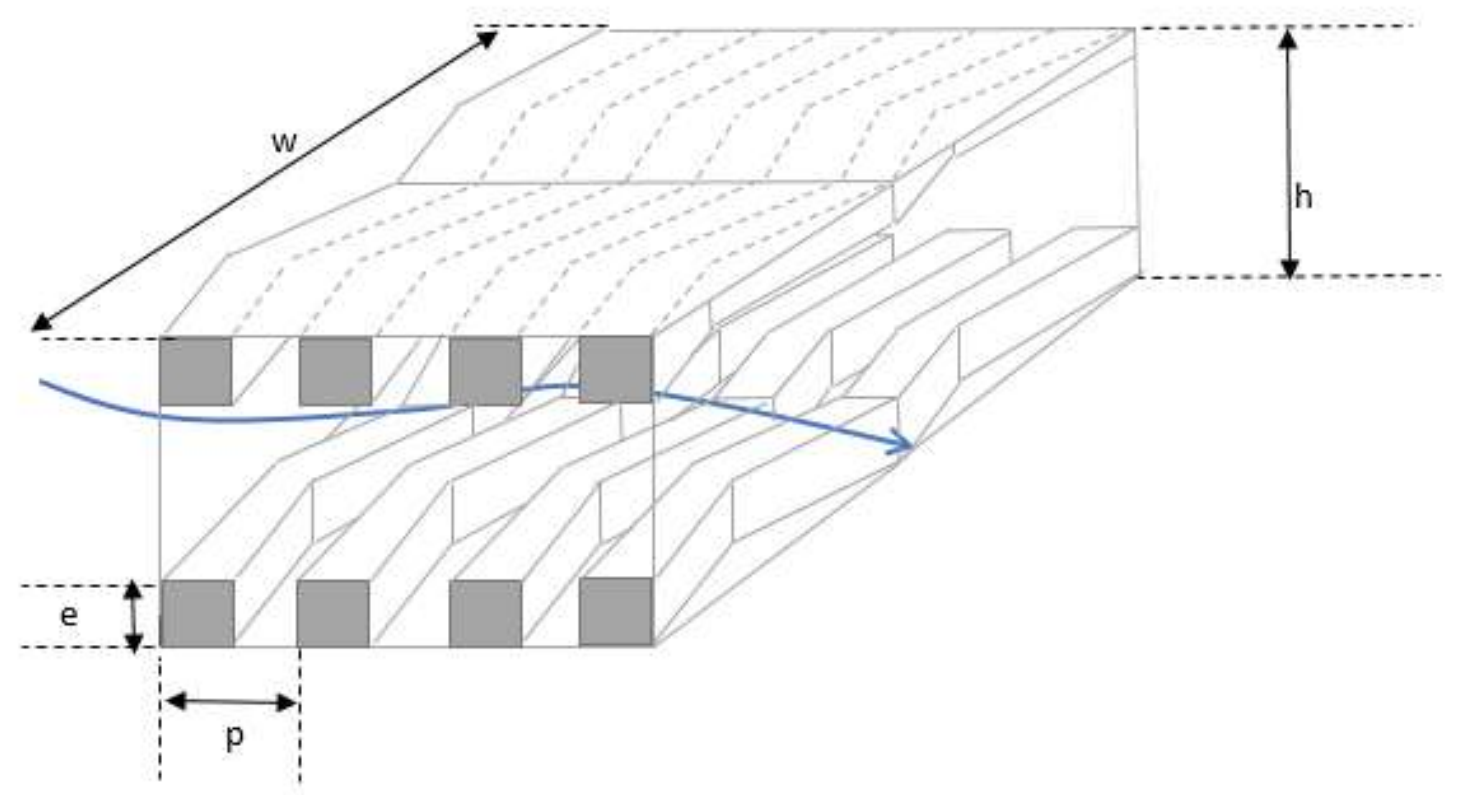

Figure E 7: Schematic view of W-Shaped ribs

Wright et al. [55] provides Nusselt number values obtained for this type of ribs from an experiment with coolant Reynolds number range of $[10000,40000]$. The results of these experiments for the non-rotating cases are plot-digitized as shown in Figure E.8 to obtain a curve-fit function that will be later used in the new coolant calculation algorithm correlations. 


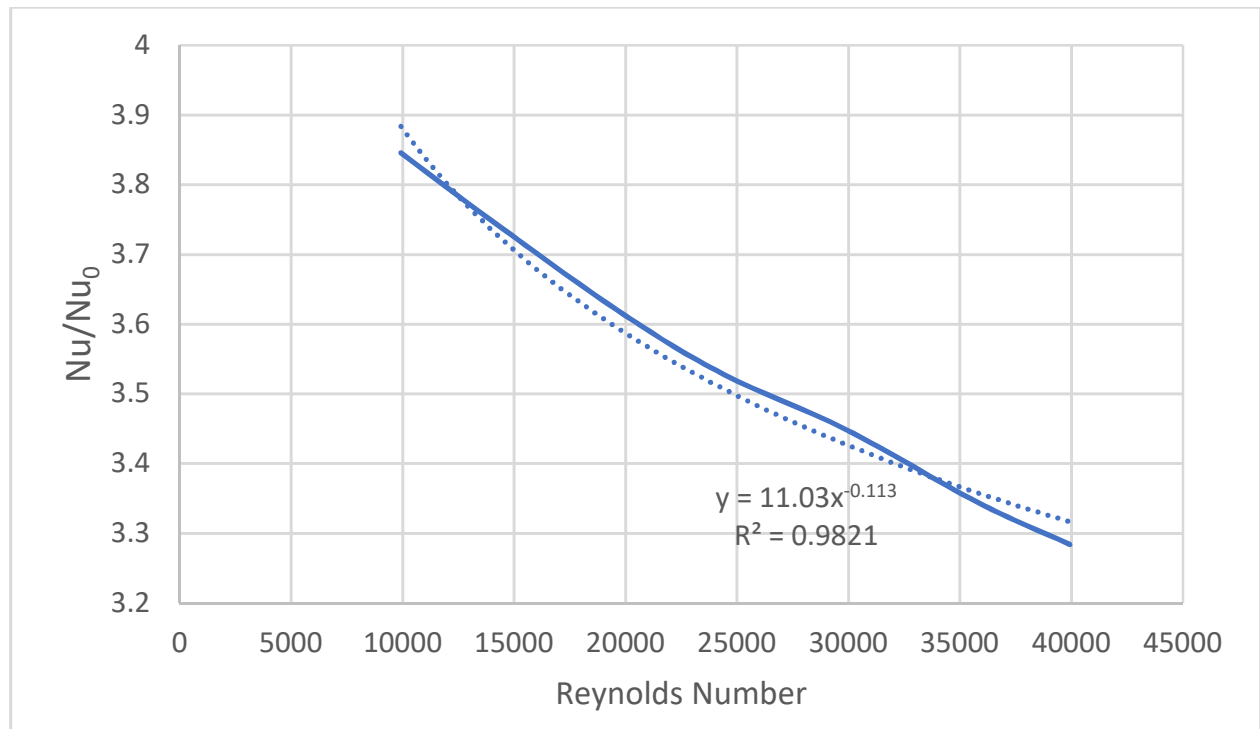

Figure E 8: Plot-digitization of the experimental data by Wright et al. [55] given for non-rotating case is used in obtaining a curve-fit function

The curve-fit function obtained for Nusselt number calculations is given by Equation (E.19).

$$
N u=N u_{0} .11 .03 R e_{\text {in }}{ }^{-0.113}
$$

Where the value for Nuo can be found from Equation (E.18).

\section{E.1.2 Impingement Cooling Correlations}

Impingement cooling is usually applied to the leading-edge region of the cooled turbine blades, which are generally represented by curved plate impingement correlations. However, some experimental studies also use impingement on the mid-chord region, in these cases such correlations are used from flat-plate correlations by neglecting the blade internal curvature. A list of various impingement correlation cases is given by Zuckermann and Lior [68] but the ones that are valid for the turbine blade cooling applications are selected from Sundberg [54] and are listed in the following sub-sections for the impingement types considered in the advanced coolant calculator.

\section{E.1.2.1 Flat Plate Correlations (for mid-Chord application)}

The correlation for the inline and staggered arrangements of the impingement cooling is given by Florshuetz et al. [56] for the schematic view with correlation inputs shown in Figure E.9.

The Nusselt number correlation is given by Equation (E.20).

$$
N u=N u_{0}\left(1-C\left(\frac{x_{n}}{d}\right)^{n x}\left(\frac{y_{n}}{d}\right)^{n y}\left(\frac{z_{n}}{d}\right)^{n z}\left(\frac{G_{c}}{G_{j}}\right)^{n}\right)
$$

In Equation (E.20), $x_{n}$ is the span wise spacing, $y_{n}$ is the lateral spacing and $z_{n}$ is the distance between the impingement hole and the target plate. In this correlation, $d$ is the hydraulic diameter, but it is taken equal to the impingement hole diameter, since it is a circular channel. 


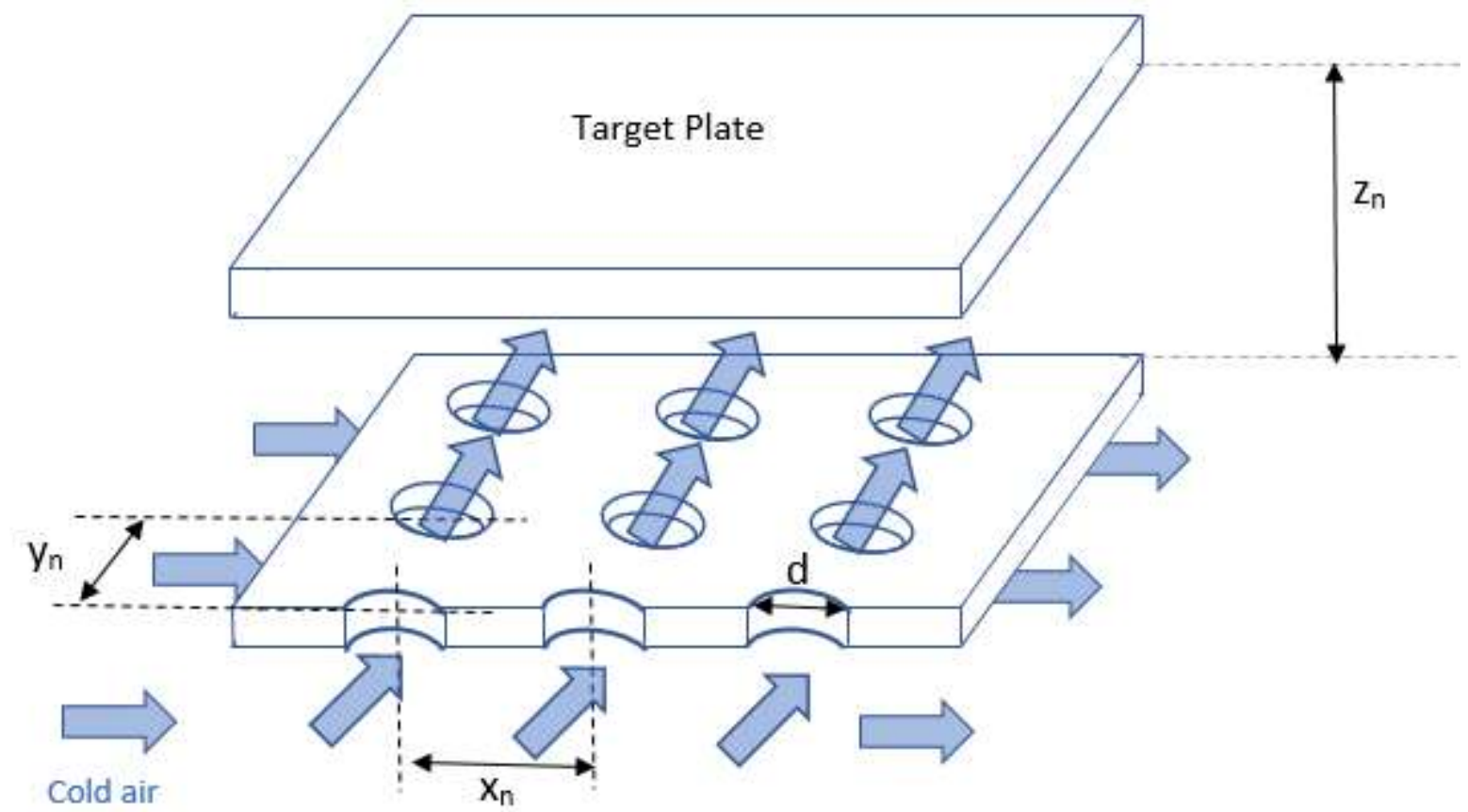

Figure E 9: Schematic view of the inline impingement geometry is shown with used input parameters in the correlation

The discharge coefficient $\left(\frac{G_{c}}{G_{j}}\right)$ in Equation (E.20) is calculated in the advanced coolant calculator with an algorithm from Consonni [1] that computes the number of impingement cooling holes by using the spanwise spacing and lateral spacing of impingement holes with the blade chord information.

The coefficient $C$ and the exponents $n x$, ny and $n z$ varies whether the arrangement is inline or staggered. The values of these coefficients are given in Table E.1.

Table E 1: Coefficients used in Florschütz correlation given by Equation (E.20)

\begin{tabular}{|l|l|l|l|l|l|}
\hline & $\mathbf{n x}$ & $\mathbf{n y}$ & $\mathbf{n z}$ & $\mathbf{n}$ & $\mathbf{C}$ \\
\hline Inline & 0.596 & -0.103 & -0.38 & 0.561 & 0.598 \\
\hline Staggered & -0.198 & -0.406 & 0.788 & 0.660 & 1.07 \\
\hline
\end{tabular}

The Nusselt number Nuo can be found from Equation (E.21) for both arrangement cases.

$$
N u_{0}=0.363\left(\frac{x_{n}}{d}\right)^{-0.554}\left(\frac{y_{n}}{d}\right)^{-0.422}\left(\frac{z_{n}}{d}\right)^{0.068} \operatorname{Re}_{i n}{ }^{0.727} \operatorname{Pr}^{1 / 3}
$$

\section{E.1.2.2 Curved Plate Correlation (for Leading Edge application)}

For the leading edge impingement, correlations given by Chupp et al. [57] is used. The correlation is given by Equation (E.22).

$$
N u=0.63 R e_{c l} 0.7 \sqrt{\left(\frac{y_{n}}{d}\right)^{-1}}\left(\frac{D_{p}}{d}\right)^{-0.6} e^{m}
$$


In Equation (E.22), the definitions given for $y_{n}$ and $z_{n}$ for the flat plate applies as the same for the curved plates. Parameter $D_{p}$ is the radius of curvature of the curved target plate and the exponent $m$ is given by Equation (E.23).

$$
m=-1.27\left(\frac{z_{n}}{d}\right) \sqrt{\left(\frac{y_{n}}{d}\right)^{-1}}\left(\frac{D_{p}}{d}\right)^{-1.2}
$$

\section{E.1.3 Pin-Fin Cooling Correlations}

For the pin-fin correlations, Oates [52] and Khan [53] provides thorough information on the correlations that can be used for cooled turbine blades using circular pin-fins. The results from the experimental study by Jaswal and Ames [59] is used for the advanced pin-fin configurations that is one of the suggested pin-fin configurations for advanced cooled turbine blade design [62].

\section{E.1.3.1 Circular Pin-Fins}

For the inline and staggered arrangements of circular pin-fins, a Nusselt number correlation from Hausen [58] is used. The form of the equation is the same for both arrangements, and is given by Equation (E.24).

$$
N u=C F_{a} \operatorname{Re}_{c l}{ }^{m} \operatorname{Pr}^{n}
$$

In Equation (E.24), $F_{a}$ is a shape factor, which should be between 0.7 and 1.2 for optimum performance [58], and the parameters $\mathrm{C}, \mathrm{m}$ and $\mathrm{n}$ are constants that are dependent on the type of the pin-fin arrangement. The values of these coefficients are given in Table E.2.

Table E 2: Coefficients used in circular pin-fin correlation given by Equation (E.24)

\begin{tabular}{|l|l|l|l|}
\hline & $\mathbf{C}$ & $\mathbf{m}$ & $\mathbf{n}$ \\
\hline Inline & 0.34 & 0.61 & 0.31 \\
\hline Staggered & 0.35 & 0.57 & 0.31 \\
\hline
\end{tabular}

\section{E.1.3.2 Diamond Pedestal Array Pin-Fins}

These pin-fin types are a special form of staggered pin-fins, which have distorted circular pin-fins that are in the shape of a diamond [59]. Jaswal and Ames [59] provides a curve-fit for their results of the experimental study conducted in constant and converging channels by using these pin-fin types. The correlation equation obtained for the constant channel height is given by Equation (E.25).

$$
N u=C R e_{c l}{ }^{m}
$$

In Equation (E.25), $\mathrm{C}$ is 0.329 and $\mathrm{m}$ is 0.6 . In the advanced coolant calculation algorithm, this correlation is also multiplied with the shape factor $F_{a}$, similar to the staggered circular arrangement.

\section{E.2 External Cooling Correlations}

The hole types considered in this analysis is compared in the illustration given in Figure E.10. 


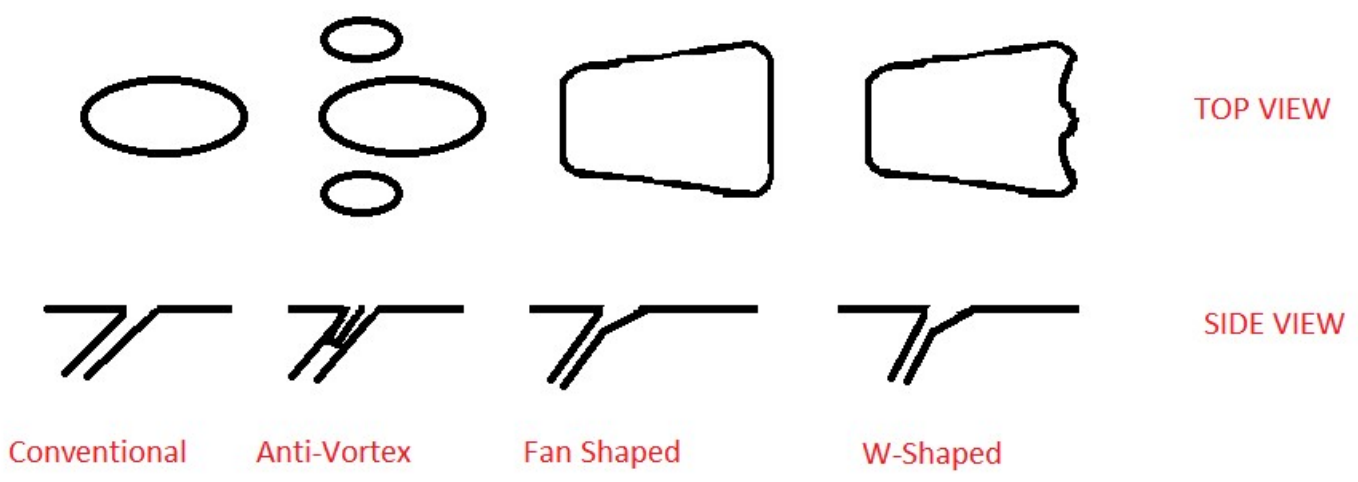

Figure E 10: Film cooling hole types used in the comparisons are shown schematically with their top and side views

For the span averaged film cooling effectiveness data of fan shaped and W-Shaped holes, a correlation by Colban et al. [60] and the data obtained from a CFD study by Shih and $\mathrm{Na}$ [61] are used with the details provided in the following sub-sections.

\section{E.2.1 Fan Shaped Hole}

A special form of the fan shaped holes, known as 7-7-7 holes, was developed by Schroeder and Thole [69] named after it's $7^{\circ}$ expansion in both lateral directions and in the laidback direction. Some of the geometric parameters of the 7-7-7 shaped hole are given in Table E.3.

Table E 3: 7-7-7 type fan shaped hole geometric parameters

\begin{tabular}{|l|l|}
\hline Parameter & Value \\
\hline Injection Angle, $\boldsymbol{\alpha}$ & $30^{\circ}$ \\
\hline $\boldsymbol{L}_{\boldsymbol{m}} / \boldsymbol{D}$ & 2.5 \\
\hline $\boldsymbol{L}_{\text {lat }} / \boldsymbol{D}, \boldsymbol{L}_{\boldsymbol{f} \boldsymbol{w d}} / \boldsymbol{D}$ & 3.5 \\
\hline $\boldsymbol{L} / \boldsymbol{D}$ & 6 \\
\hline Laidback Angle, $\boldsymbol{\beta}_{\boldsymbol{f} \boldsymbol{w d}}$ & $7^{\circ}$ \\
\hline Lateral Angle, $\boldsymbol{\beta}_{\text {lat }}$ & $7^{\circ}$ \\
\hline $\boldsymbol{P} / \boldsymbol{D}$ & 6 \\
\hline Coverage Ratio, $\boldsymbol{t} / \boldsymbol{P}$ & 0.35 \\
\hline Area Ratio, AR & 2.5 \\
\hline
\end{tabular}

An experimental study with fan shaped holes was carried out by Colban et al. [60] for different blowing ratios, area ratios, coverage ratios, and P/D values to obtain a correlation that can be used to estimate the span averaged film cooling performance of these cooling holes. The correlation equation obtained in this study that was used to generate span average effectiveness data for the advanced coolant calculator is given in Equation (E.26).

$$
\varepsilon_{f c}\left(\frac{x}{d_{h}}\right)=\frac{1}{\left(\frac{P}{t}\right)+0.1721(B R)^{-0.2664} \beth^{0.8749}}
$$


In Equation (E.26), the coverage ratio is used from Table E.3 and BR is the blowing ratio. The parameter $ב$ is given by Equation (E.27).

$$
\beth=\frac{4}{\pi} \frac{\frac{x}{d_{h}}\left(\frac{P}{D}\right)}{B R \cdot A R}
$$

The correlation equation is used for the blowing ratios from 0.5 to 2 from 0 to 20 hole diameters to generate a database for the advanced coolant calculator. The data generated for various blowing ratios are plotted in Figure E.11.

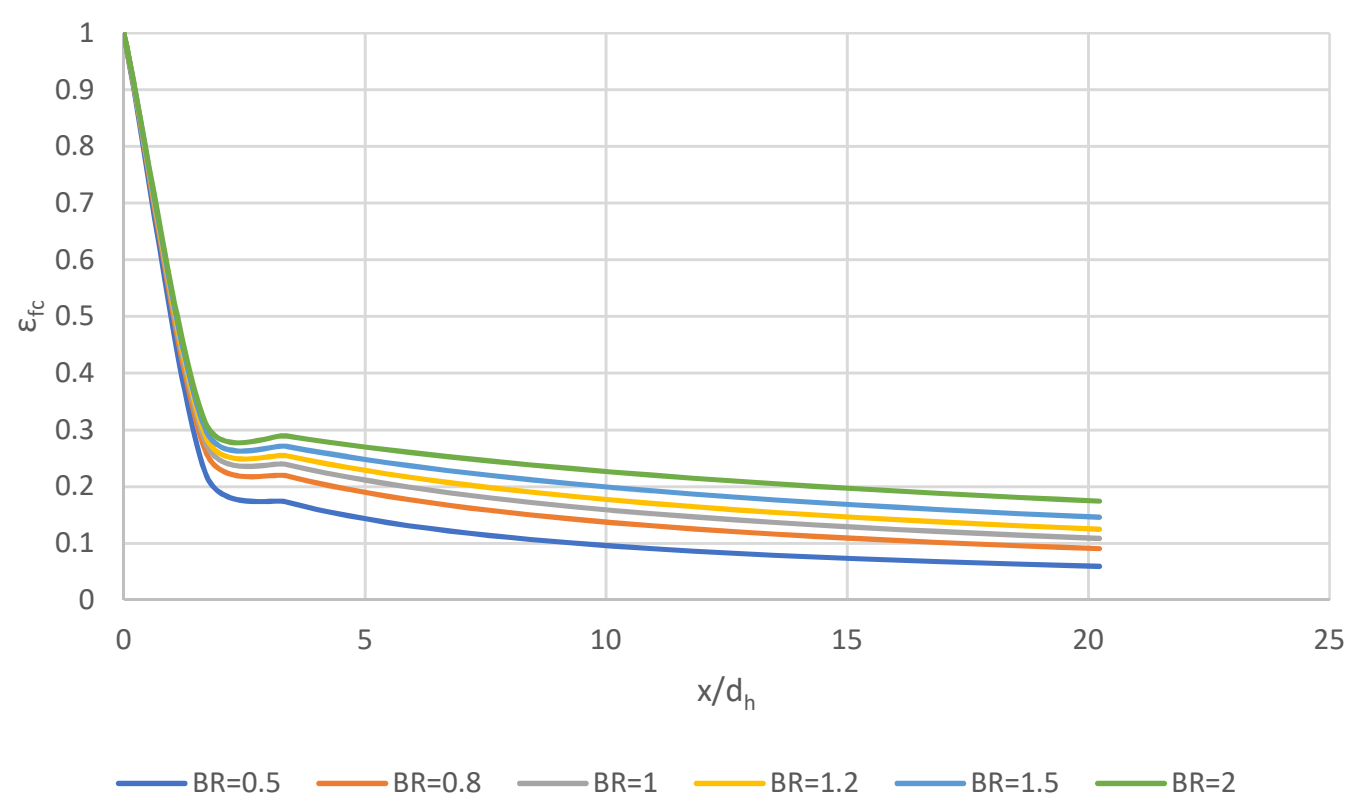

Figure E 11: Span averaged film cooling effectiveness database generated by using the correlation by Colban et al. [60]

\section{E.2.2 W-Shaped Hole}

W-shaped holes are another form of fan shaped holes that has a W-shaped cross-sectional area and the notch of the cross section protrudes upwards and widens laterally, to preserve the momentum of the coolant and direct it laterally to increase the cooled surface area.

This hole geometry is studied by Shih and $\mathrm{Na}$ [61] by using RANS with two equation realizable k- $\varepsilon$ turbulence model. The results presented for the lateral averaged film cooling effectiveness are plot-digitized and used to generate a database for W-Shaped holes in the advanced coolant calculator. The data used from Shih and $\mathrm{Na}$ [61] for various blowing ratios are plotted in Figure E.12. 


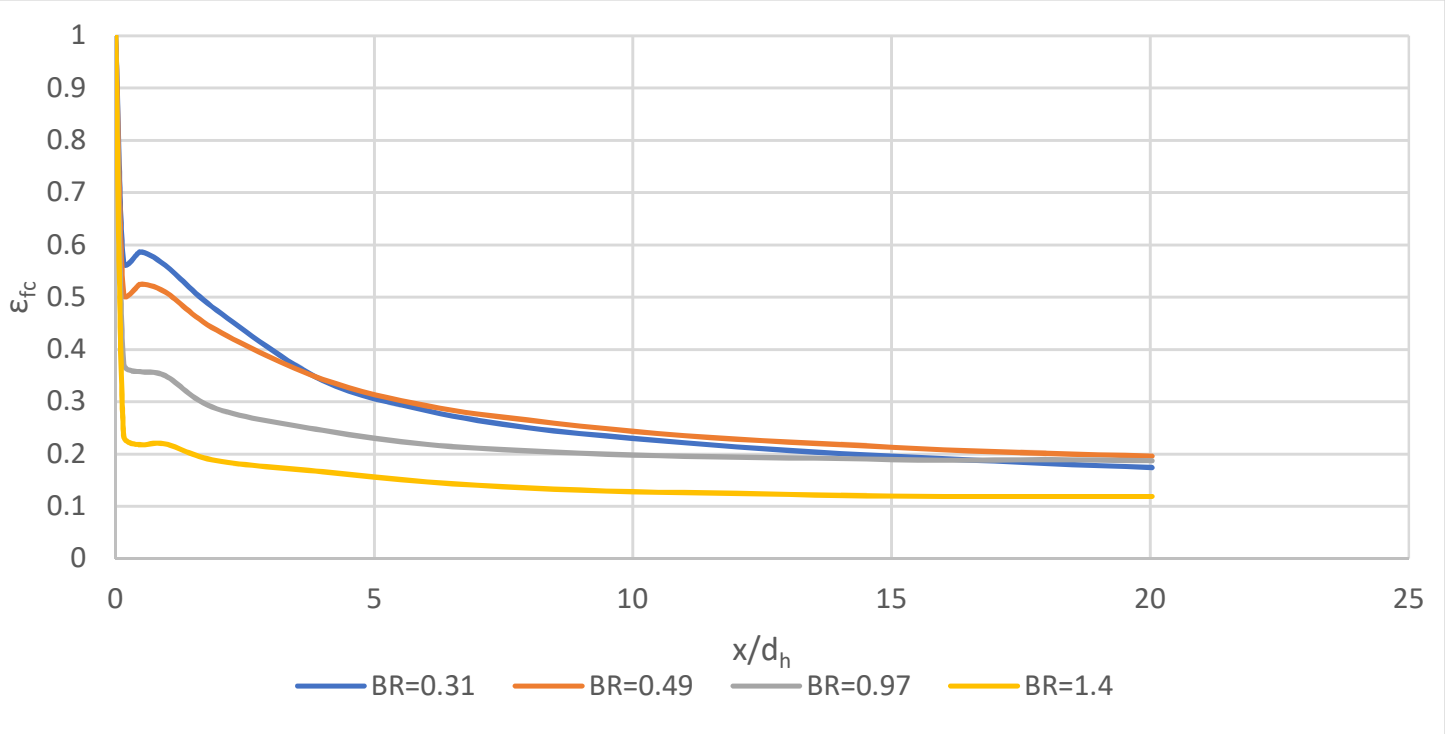

Figure E 12: Span averaged film cooling effectiveness database used from Shih and $\mathrm{Na}$ [61] 


\section{BIBLIOGRAPHY}

1) Consonni S.,(1992) "Performance Prediction of Gas/Steam Cycles for Power Generation", $\mathrm{PhD}$ Dissertation to Princeton University, Center for Energy and Environmental Studies

2) El-Marsi, M.B., (1988), "GASCAN- An Interactive Code for Thermal Analysis of Gas Turbine Systems", Journal of Engineering for Gas Turbines and Power, Vol.110, pp.201209

3) Gauntner, J.W., (1980), "Algorithm for Turbine Cooling Flow and the Resulting Decrease in Turbine Efficiency", NASA Technical Memorandum No.81453

4) Lallini, V., Janikovic, J., Pilidis, P., Singh, R., and Laskaridis, P., (2012), "A Calculation Tool of a Turbine Cooling Air Schedule for General Gas Turbine Simulation Algorithms", Journal of Turbomachinery, Vol.134, pp.041003/1-041003/8

5) Li, Z, Zhao, L., Wang, B., Chi J., Zhang, S. and Xiao,Y., (2014), "A Thermodynamic Evaluation of GTCC/IGCC Based on a Quasi-One Dimensional Turbine Cooling Model", ASME GT2014-26534

6) Sanjay, O. S., and Prasad, B.N., (2008), "Comparative Performance Analysis of Cogeneration Gas Turbine Cycle for Different Blade Cooling Means", International Journal of Thermal Science, Vol.48, pp.1432-1440

7) Young J.B., and Wilcock R.C. (2002), "Modeling the Air-Cooled Gas Turbine: Part 2Coolant Flows and Losses", ASME 2001-GT-392

8) Horlock, J.H., and Torbidoni, L., (2008), "Calculations of Cooled Turbine Efficiency", ASME GT2006-90424

9) Torbidoni, L. and Horlock, J.H., (2005), "A New Method to Calculate the Coolant Requirements of a High Temperature Gas Turbine Blade", Journal of Turbomachinery, Vol. 127, pp.191-199

10) Moustapha, H.Zelesky, M.F., Baines, N.C., and Japikse, D., (2000), "Axial and Radial Turbines", Concepts NREC, VT, USA

11) Moran Michael J., and Shapiro Howard N. (2006), "Fundamentals of Engineering Thermodynamics 5th Edition", John Wiley and Sons Inc., East Lothian, United Kingdom

12) Mattingly Jack D. (2006), "Elements of Propulsion: Gas Turbines and Rockets / Jack D. Mattingly foreword by Hans von Ohain", American Institute of Aeronautics and Astronautics Inc., Reston, Virginia, USA

13) Uysal, S.C., (2014), "High By-Pass Turbofan Engines Aerothermodynamic Design and Optimization", MSc. Thesis to Middle East Technical University Department of Aerospace Engineering, Ankara, Turkey

14) Horlock, J.H., and Torbidoni, L., (2008), "Calculations of Cooled Turbine Efficiency”, ASME GT2006-90424

15) Esgar J.B., and Ziemer R.R., (1955), "Effects of Turbine Cooling with Compressor Air Bleed on Gas Turbine Engine Performance", NACA Research Memorandum-E54L20, Lewis Flight Propulsion Laboratory, Cleveland, $\mathrm{OH}$

16) Saravanamutto, H.I.H., Rogers, G.F.C, and Cohen, H., "Gas Turbine Theory", 5th Edition, pp.58-61 
17) GasTurb12 Software and User Manual by GasTurb GmbH c/o Institute of Jet Propulsion and Turbomachinery Templergraben 55, 52062, Aachen, Germany, http://www.gasturb.de

18) Chappel, M.S., and Cockshutt,E.P., (1974), "Gas Turbine Cycle Calculations: Thermodynamic Data Tables for Air and Combustion Products for Three Systems of Units", National Research Council of Canada Division of Mechanical Engineering, Ottawa, ON

19) GASEQ Software-Chemical Equilibria in Perfect Gases, Chris Morley, retrieved from http://www.gaseq.co.uk

20) National Institute of Technology (NIST), REFPROP Software using NIST Standard Reference 23, Version 8.0

21) Guha, A., (2001), "An Efficient Generic Method for Calculating the Properties of Combustion Products", Proc. Instn. of Mech. Engrs., Vol.215, Part A, pp.375-387

22) Lufthansa Technical Training GmbH, (1999), "Training Manual A319/A320/A321 ATA 7180 Engine CFM56-5A", Lufthansa Base Frankfurt, Germany (retrieved from http://atconsortium-indonesia.com/wp-content/uploads/2012/06/Airbus_A320_CFM56Wartungs-Manual_71-80-30-20-CFM56-5A-L3-e.pdf )

23) Walsh, P.P., and Fletcher, P., (2004), "Gas Turbine Performance-2nd Edition", Blackwell Science Publishing, pp. 292-382

24) Giampaolo, T., (2003), "The Gas Turbine Handbook: Principles and Practices-2nd Ed.", The Fairmont Press Inc., Lilburn, GA

25) Wilson, D.G., and Korakianitis T., (2014), "The Design of High Efficiency Turbomachinery and Gas Turbines-2nd Ed.", The MIT Press, Cambridge, MA

26) Siemens Global Website-Pressebilder, retrieved from http://www.siemens.com/press/de/pressebilder/?press=/de/pressebilder/2016/powergas/im2016010336pgde.htm\&content []=PG

27) Gas Turbine World, "2015 Performance Specs", 31st Edition, January-February 2015, Volume 45, No.1, Pequot Publishing Inc.

28) Muktinutalapati, N.R. (2011), "Materials for Gas Turbines - An Overview, Advances in Gas Turbine Technology", Dr. Ernesto Benini (Ed.), ISBN: 978-953-307-611-9, InTech Europe

29) GE Power, "GE7HA.01/02 Gas Turbines (60Hz) Fact Sheet 2016", retrieved from https://powergen.gepower.com/products/heavy-duty-gas-turbines/7ha-gas-turbine.html on $08 / 16 / 2016$

30) Hada, S., Takata, K., Iwasaki, Y., Yuri, M., and Masada, J., (2015), "High Efficiency Gas Turbine Development applying 1600 C class 'J' Technology”, Mitsubishi Heavy Industries Technical Review, Vol.52, No.2

31) Yuri, M., Masada J., Tsukagoshi, K. Ito, E. and Hada, S., (2013), "Development of 1600 CClass High Efficiency Gas Turbine for Power Generation Applying J-Type Technology", Mitsubishi Heavy Industries Technical Review, Vol.50, No.3

32) Wilcock, R.C., Young, J.B., and Horlock J.H., (2005), "The Effect of Turbine Blade Cooling on the Cycle Efficiency of Gas Turbine Power Cycles", Journal of Engineering for Gas Turbines and Power, Vol.127, pp.109-120

33) Walters D.K., and Leylek J.H., (2000), "A Detailed Analysis of Film Cooling Physics: Part IStreamwise Injection with Cylindrical Holes", Journal of Turbomachinery, Vol.122, pp.102112, ASME 97-GT-269

34) Heidmann J.D., and Ekkad, S.V., (2007), "A Novel Anti-Vortex Turbine Film Cooling Hole Concept", ASME Paper No. GT2007-27528, pp. 487-496

35) Kim, S.I., and Hassan I., (2010), "Unsteady Simulations of a Film Cooling Flow from an Inclined Cylindrical Jet", Journal of Thermophysics and Heat Transfer, Vol.24, No.1, DOI: 10.2514/1.33167, AIAA

36) Yao Y., Zhang J., and Yang Y., (2013), "Numerical Study on Film Cooling Mechanism and Characteristics of Cylindrical Holes with Branched Jet Injections", Propulsion and Power Research, Vol.2(1), pp.30-37, Elsevier Inc. 
37) Repko, T., Nix, A.C., Uysal, S.C., and Sisler A.T., (2015), "Flow Visualization of Multi-Hole Film Cooling Flow under Varying Freestream Turbulence Levels", Journal of Flow Control, Measurement and Visualization, Vol.4, pp.13-29, Scientific Research Publishing

38) Sinha, A.K., Bogard D.G., and Crawford, M.E., (1991), "Film-Cooling Effectiveness Downstream of a Single Row of Holes with Variable Density Ratio", Journal of Turbomachinery, Vol.113, No.3, pp.442-449

39) Hayes, S.A., (2014), "An Experimental Investigation on the Effects of Freestream Turbulence Intensity on Film Cooling Effectiveness and Heat Transfer Coefficient for an Anti-Vortex Hole", MSc. Thesis, West Virginia University

40) Kalghatgi P., and Acharya, S., (2014), "Modal Analysis of Inclined Film Cooling Jet Flow", Journal of Turbomachinery, Vol.136, pp.081007/1-11, ASME

41) Martini, P., Schulz A., Bauer, H.-J., and Whitney C.F., (2006), "Detached Eddy Simulation of Film Cooling Performance on the Trailing Edge Cutback of Gas Turbine Airfoils", Journal of Turbomachinery, Vol.128, pp.292-299, ASME

42) Kadotani K., and Goldstein, R.J., (1979), "On the Nature of Jets Entering a Turbulent Flow Part B-Film Cooling Performance", Journal of Engineering for Power, Vol.101, pp.466-470, ASME

43) Schmidt, D.L., and Bogard, D.G., (1996), "Effects of Free-Stream Turbulence and Surface Roughness on Film Cooling", ASME 96-GT-462

44) Dhungel, A., Lu, Y., Phillips, W., Ekkad, S.V. and Heidmann, J.D., (2007), "Film Cooling from a Row of Holes Supplemented with Anti-Vortex Holes". ASME Paper GT2007-27419.

45) Nemdili, F., Nemdili, S. and Azzi, S., (2013), "Numerical Investigation on Film Cooling Effectiveness Using the Anti-Vortex Concept" Conference Paper for '21ème CongrèsFrançais de Mécanique'

46) Heidmann, J.D., (2008), "A Numerical Study of Anti-Vortex Film Cooling Designs at High Blowing Ratio", ASME Paper GT2008-50845

47) LeBlanc, C.N., (2012), "Design, Analysis and Development of a Tripod Film Cooling Hole Design for Reduced Coolant Usage", PhD Dissertation submitted to Virginia Polytechnic Institute and State University

48) Bons, J.P., MacArthur, C.D., and Rivir, R.B., (1996), "The Effect of High Free-Stream Turbulence on Film Cooling Effectiveness", Journal of Turbomachinery, Vol.118, pp.814824, ASME

49) Torbidoni L., and Horlock, J.H., (2005), "A New Method to Calculate the Coolant Requirements of a High-Temperature Gas Turbine Blade", Journal of Turbomachinery, Vol.127, pp.191-199

50) Ainley, D.G., (1957), "Internal Air Cooling for Turbine Blades: A General Design Survey", Aeronautical Research Council Reports and Memo. 3013

51) Cunha, F.J., (2002), "The Gas Turbine Handbook", Chapter 4.4, National Energy Technology Laboratories, pp. 389-410

52) Oates, G.C., (1985), "Aerothermodynamics of Aircraft Engine Components", $2^{\text {nd }}$ Edition, AIAA Inc., New York, NY, pp. 275-324

53) Khan, W.A., (2004), "Modeling of Fluid Flow and Heat Transfer for Optimization of Pin-Fin Heat Sinks", PhD Dissertation submitted to University of Waterloo, ON, Canada

54) Sundberg, J., "Heat Transfer Correlations for Gas Turbine Cooling", LITH-IKP-EX-05/2313-SE, Finspång, Sweden

55) Wright, L.M., Fu, W. and Han, J., (2004), "Thermal Performance of Angled, V-Shaped, and W-Shaped Rib Turbulators in Rotating Rectangular Cooling Channels (A.R. $=4: 1$ )", Journal of Turbomachinery, Vol.126, pp.604-614

56) Florschuetz, L.W., Truman C. R. and Metzger D.E., (1981), "Streamwise Flow and Heat Transfer Distributions for Jet Array Impingement with Crossflow", Journal of Heat Transfer, Vol. 103, pp. 337-342 
57) Chupp, R.E., Helms, H.E., McFadden, R.W., and Brown, T.R., (1969), "Evaluation of Heat Transfer Coefficients for Impingement Cooled Turbine Airfoils", Journal of Aircraft, Vol. 6, pp.203-208

58) Hausen, H., (1983), "Heat Transfer in Counterflow, Parallel Flow and Cross Flow," McGrawHill, Inc, New York, NY.

59) Jaswal, I., and Ames, F.E., (2009), "Heat Transfer and Pressure Drop Measurements in Constant and Converging Section Pin and Diamond Pedestal Arrays", Journal of Thermal Science and Engineering Applications, Vol.1, pp. (011006)01-07

60) Colban, W.F., Thole, K.A., and Bogard, D., (2011), "A Film Cooling Correlation for Shaped Holes on a Flat-Plate Surface", Journal of Turbomachinery, Vol.133, pp. (011002)1-11

61) Shih, T. and Na, S., (2007), "Momentum Preserving Shaped Holes for Film Cooling", ASMEGT2007-27600, Montreal, Canada

62) Town, J., Straub, D., Black, J., Thole, K., and Shih, T., (2017), "State-of-the-Art Cooling Technology for a Rotor Blade", ASME GT2017-64728, Charlotte, NC

63) Barringer, M., Coward, A., Clark, K., Thole, K., Schmitz, J., Wagner, J., Alvin, M.A., Burke, P., and Dennis, R., (2014), "The Design of a Steady Aero Thermal Research Turbine (START) for Studying Secondary Flow Leakages and Airfoil Heat Transfer", ASME GT201425570, Düsseldorf, Germany

64) Han, J.C., (1984), "Heat Transfer and Friction in Channels with Two Opposite RibRoughened Walls", Transactions of ASME, Journal of Heat Transfer, Vol.106, pp. 774-781

65) Han, J.C., Park, J.S., and Lei, C.K., (1985), "Heat Transfer Enhancements in Channels with Turbulence Promoters", Journal of Eng. Gas Turbine and Power, Vol.107, pp.628-635

66) Han, J.C., Zhang, Y.M., and Lee, V.P., (1991), "The Influence of Surface Heat Flux Ratio on Heat Transfer Augmentation in Square Channels with Parallel, Crossed and V-Shaped angled Ribs", ASME GT-91-003

67) Lau, S.C., Kukreja, R.T., and McMillin, R.D., (1991), "Effects of V-Shaped Rib Arrays on Turbulent Heat Transfer and Friction of Fully Developed Flow in a Square Channel", International Journal of Heat and Mass Transfer, 34, 7, pp.1605-1616

68) Zuckerman, N.and Lior, N., (2006),"Jet Impingement Heat Transfer: Physics, Correlations, and Numerical Modelling" ,Advances in Heat Transfer, Vol.39, ISSN 0065-2717, pp.610619

69) Schroeder, R. P., and Thole, K. A., (2014), "Adiabatic Effectiveness Measurements for a Baseline Shaped Film Cooling Hole," ASME GT2014-25992

70) Sellers, J. P., Jr., (1963), "Gaseous Film Cooling With Multiple Injection Stations," AIAA J., 1(9), pp. 2154-2156.

71) Soares C., (2014) "Gas Turbines: A Handbook of Air, Land and Sea Applications (2nd Edition)". Jordan Hill, GBR: Elsevier Science \& Technology 


\section{INDEX}

\section{1}

$1^{\text {st }}$ Law, v, x $1,4,8,9,18,19,21,24,35,115,162,169$

\section{2}

$2^{\text {nd }}$ Law, v, 1, 2, 4, 10, 17, 18, 19, 20, 21, 24, 35, 58, 72, 81, 115

A

adiabatic film cooling efficiency, 80, 81, 84, 85, 90, 126, 160

adiabatic wall temperature, 71,81

Anti-Vortex Hole, 1, 99, 100, 106, 108, 112, 129, 158, 184

AVH, ii, vii, x, 99, 100, 101, 106, 107, 108, 112, 113, 114,

$133,134,136,137,142,143,144,146,147,148,149$, $152,154,156,159$

\section{B}

back-pressure ratio, vi, 44, 60, 158

Basic Loss, 13

Biot Number, 7, 37, 45, 61, 62, 67, 91, 133, 135

blade material, 58, 62, 94, 158

blade metal external temperature, 160

bleed air, 54, 57, 168

blowing ratio, vii, $80,88,89,90,91,92,93,94,99,100,104$, $107,109,111,112,114,129,136,137,180$

by-pass ratio, v, 40, 158

\section{C}

case-specific tests, $x, 47,48,56,158$

channel friction, 171, 173, 174

Combustor, 32, 33, 37, 42, 43, 45, 56

compressor, vi, ix, 2, 9, 23, 24, 35, 41, 44, 46, 48, 50, 52, 53, $55,57,58,85,95,99,131,140,147,153,155,158,160$, $165,166,167,168,169$

continuity tests, 36, 46, 55, 158

Coolant Calculator, v, x, 18, 21, 115, 120, 133, 170

coolant exit temperature, 9, 85, 122, 160, 161

coolant fraction, $x, 1,18,21,69,71,75,115,119,120,121$, $129,131,132,137,143,147,150,153,163,167$

coolant heating, $121,137,143,150,160$

Cooled Turbine, 1, v, xii, 3, 10, 18, 21, 23, 33, 182, 185

cooled turbine stage, $v, 5,6,11,22,23,36,76,81,158$, 162

Cooling Effectiveness, xii, 7, 37, 45, 61, 80, 91, 184

Cooling Loss, 13
Detached Eddy Simulation, ii, iv, 99, 158, 184

\section{E}

entropy, v, vi, 3, 4, 5, 11, 12, 13, 14, 15, 16, 17, 19, 21, 22, $24,28,29,35,58,59,76,85$

External Cooling, viii, 16, 123, 124, 126, 134, 170, 178

\section{$\mathbf{F}$}

Fan Shaped Hole, 129, 179

\section{H}

Heat Rate, viii, 44, 54, 55, 57, 91, 148

Heat Transfer Loss, 14, 16

I

Impingement, 126, 133, 134, 135, 176, 184, 185

Internal Cooling, viii, 15, 45, 61, 75, 98, 124, 125, 133, 135, 142, 170, 175

Isentropic Efficiency, xii

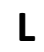

Losses, 1, 15, 16, 17, 182

\section{M}

Mach number, 39, 43, 158, 166

maximum allowable blade temperature, ii, $66,71,73,85$, $91,92,96,97,158,160$

\section{$\mathbf{N}$}

Nusselt number, 126, 175, 176, 177, 178

\section{$\mathbf{P}$}

Pin-Fin, 133, 135, 178, 184

polytropic efficiency, 3, 5, 10, 13, 26, 35, 55, 98, 138, 140, 153,165

Power Specific Fuel Consumption, vi, 44, 47, 48, 58

Propulsive Efficiency, v, 38, 39, 41

purge, $v, 5,6,8,9,10,11,12,54,86$ 
$\mathbf{R}$

rib, viii, xi, 120, 125, 133, 134, 142, 143, 144, 145, 146, 147, $148,149,170,171,172,173,174$

rothalpy, 9,162

rotor, v, ix, 1, 5, 8, 9, 10, 11, 12, 13, 18, 22, 23, 38, 50, 53, 64, $66,69,71,73,75,78,80,83,85,131,135,136,143,146$, $149,152,162,163,164,165,166$

\section{$\mathbf{S}$}

semi-empirical, $v, x, 7,8,18,71,76,115,120,121,131$, $132,159,163$

Sensitivity Analysis, iv, x, 61, 90, 99

Stanton number, xi, 115, 121, 123, 130, 131, 142, 143, 146, $148,149,150,152,171,172,173,174$

stator, 5, 8, 135, 136
$\mathbf{T}$

TBC, v, vi, 7, 8, 14, 36, 37, 45, 54, 56, 61, 62, 67, 68, 69, 70, $71,85,88,91,92,93,94,95,96,97,118,119,121,129$, $131,133,135,158,160$

Thermal Barrier Coating, 61

Thermal Efficiency, v, vi, 44, 47, 48, 49, 54, 55, 57, 58, 59, 60,91

transition cooling, ix 44, 168, 169

turbine inlet temperature, vi, 41, 44, 48, 54, 55, 57, 58, 59, $158,168,169$

\section{W}

wall temperature, $17,66,71,76,80,85,98,118,119,120$, $121,122,129,137,160$ 


\title{
CURRICULUM VITAE
}

\author{
Selçuk Can Uysal \\ Aerospace Engineer \\ canuysal@msn.com
}

\section{Education}

January 2015- May 2017

West Virginia University, Morgantown, West Virginia, USA

Graduate School of Engineering

Department of Mechanical and Aerospace Engineering

$\mathrm{PhD}$ Program in Aerospace Engineering

Advisor: Dr. Andrew C. Nix

Dissertation Title: "Analytical Modelling of the Effects of Different Gas Turbine Cooling Techniques on Engine Performance"

Cumulative GPA: 4.00/4.00

\section{September 2014- January 2015}

Syracuse University, Syracuse, New York, USA

(TRANSFER-OUT)

Graduate School of Engineering and Computer Sciences

Department of Mechanical and Aerospace Engineering

PhD Program in Mechanical Engineering

Cumulative GPA: $3.33 / 4.00$

\section{September 2011-February 2014}

Middle East Technical University, Ankara, Turkey

Graduate School of Natural and Applied Sciences,

Department of Aerospace Engineering

Master of Science Program in Aerospace Engineering

Thesis Title: "High By-Pass Ratio Turbofan Engines Aerothermodynamic Design and Optimization"

Advisor: Dr. I. Sinan Akmandor

Cumulative GPA: $3.58 / 4.00$

September 2005- June 2009

Middle East Technical University, Ankara, Turkey

Faculty of Engineering, Department of Aerospace Engineering

Bachelor of Science program in Aerospace Engineering

Cumulative GPA: 3.23/4.00 (Honor Degree)

\section{Work Experience}

\section{January 2016-Present}

Postgraduate Research Associate (ORISE Contract), National Energy Technology Laboratories, U.S. Department of Energy, Morgantown, WV 
Internal and External Turbine Cooling Techniques, Developing Aircraft and Industrial Gas Turbine Engine Models with cooling system, Impact of different cooling techniques on engine performance/ combined cycle performance

\section{January 2015-December 2015}

Graduate Research Assistant, Department of Mechanical and Aerospace Engineering, West Virginia University, Morgantown, WV

CFD simulations of advanced multi-hole turbine film cooling geometries under varying freestream turbulence levels cooling blowing ratios and boundary layer thickness

Experimental film cooling measurements in flat plate low-speed film cooling wind tunnel

EcoCAR3 Modelling \& Simulation Team Leader

\section{August 2014-December 2014}

Graduate Assistant, Department of Mechanical and Aerospace Engineering, Syracuse University, Syracuse, NY

Computational Fluid Dynamics, Propulsion and Turbomachinery Design

June 2009-June 2014

Engineer, ROKETSAN Missiles Industries Inc., Ankara, Turkey, Systems Design and Testing Division

Flight mechanics and simulations of rockets under the influence of aerodynamic forces, 3DoF-6DoF trajectory simulations, firing calculation software in various programming languages, embedded software

\section{February 2009-June 2009}

Part-time Engineer, ROKETSAN Missiles Industries Inc., Ankara, Turkey, Systems Design and Testing Division

\section{Projects}

\section{MATLAB}

- Aeroacoustics noise calculation codes [Aeroacoustics noise calculations for a compact source, Turbomachinery Noise Calculation]

- GPS Receiver algorithm code [By using the given satellite positions, obtaining the location of an object]

- Vehicle Dynamics Calculation codes [Bicycle Model Car Model, Articulated Vehicle Model, 3DoF Car Model, Suspension Model]

- Basic Finite Element Calculation code (B.Sc. Project) [Force calculation on a simple truss by using FEM method]

- Viscid and Inviscid Euler Equation Solver (application to a 2D nozzle and axisymmetric flow nozzle)

\section{Simulink}

- $\quad 3$ DoF and 6DoF Models of unguided regular and spin stabilized land- to land rockets

- 3DoF and 6DoF Models of an unguided Anti-Submarine Warfare Rocket (two-phased flight simulation including the flight, water entry, and the motion under the water)

- 3DoF Car Vehicle Dynamics Modelling

- Design and Optimization of a Turbofan Engine (M.Sc. Thesis)

- Design and Analysis Model for cooled Turbofan Engine (PhD Thesis)

- Design and Analysis Model for cooled Industrial Gas Turbine (PhD Thesis) 


\section{$\mathrm{C} / \mathrm{C}++$ and $\mathrm{C \#}$ Programming Languages}

- Firing Table Calculation Software for various land-to land rockets in MS Windows operating systems (programming, embedding and testing) (C, $\mathrm{C}++$ and $\mathrm{C \#}$ )

- Embedded 6DoF flight simulation software for various land-to land rockets that is used by Fire Control Programs running in MS Windows operating systems (programming, embedding and testing) (C, C++ and $\mathrm{C \#}$ )

- Embedded 6DoF flight simulation software for Anti-Submarine Warfare rockets in WindRiver VXWorks real-time operating system (modeling, programming, embedding and testing)

- DLL interactions, from managed and/or unmanaged $\mathrm{C}++$ codes

- User Interface Software for Firing Calculations of several land-to-land rockets (.NET C++)

\section{FORTRAN Programming Language}

- Embedded 6DoF flight simulation programs for various land-to land rockets in MS Windows operating systems (programming, embedding and testing)

\section{Star-CCM+}

- Turbine Cascade Flow simulation for the heat transfer analysis of internally cooled blades

- Conventional turbine film cooling flow and heat transfer simulations

- Advanced multi-hole turbine film cooling flow and heat transfer simulations

\section{Publications}

- Uysal S.C., and Akmandor I.S., (2013), "Engine Design Model for Separate Flow Turbofan Engine", AlAC-2013-025, $7^{\text {th }}$ Ankara International Aerospace Conference, Ankara, Turkey

- Repko T.W., Nix A.C., Uysal S.C., and Sisler A.T., (2015), "Flow Visualization of Multi-Hole Film Cooling Flow under Varying Freestream Turbulence Levels", Journal of Flow Control, Measurement \& Visualization, Scientific Research, USA

- Repko T.W., Nix A.C., Uysal S.C., and Heidmann J., (2016), "Numerical Study on the Effects of Freestream Turbulence on Anti-Vortex Film Cooling Design at High Blowing Ratio", Journal of Thermal Science and Engineering Applications, Vol.9, Issue 1, ASME TSEA-15-1359

- Uysal S.C., Liese E.A., Black J.B., and Nix A.C., (2017), "A Thermodynamic Model to Quantify the Impact of Cooling Improvements on Gas Turbine Efficiency", Proceedings in ASME TurboExpo 2017: Turbomachinery Technical Conference and Exposition, ASME GT2017-63480

\section{Languages}

- Turkish: Native Language

- German: Intermediate Level (testDaF: 15/20 (09.06.2009))

- English: Advanced Level (TOEFL iBT: 105/120 (25.04.2014))

\section{Honors/Awards}

- January 2006-January 2009: METU Honor List

- June 2009: METU High Honor List

- January 2016-December 2016: US Dept. of Energy ORISE Fellowship

- January 2017-July 2017: US Dept. of Energy ORISE Fellowship 